\title{
Development of a Fully Three-Dimensional Groundwater Flow Model for the A/M Area Using Data Fusion
}

by

D. Jackson

Westinghouse Savannah River Company

Savannah River Site

Aiken, South Carolina 29808

RECEIVEO

FFR 26 1958

OSTI

\section{Distribution of THIS DOCAMENT is UNLMUTED}

DOE Contract No. DE-AC09-89SR18035

This paper was prepared in connection with work done under the above contract number with the U.S.

Department of Energy. By acceptance of this paper, the publisher and/or recipient acknowledges the U.S. Government's right to retain a nonexclusive, royalty-free license in and to any copyright covering this paper, along with the right to reproduce and to authorize others to reproduce all or part of the copyrighted paper. 


\title{
DEVELOPMENT OF A FULEY THREE-DIMENSIONÁL GROUNDWATER FLOW MODEL FOR THE A/M AREA USING DATA FUSION
}

\author{
Final Report
}

Prime Contract No. DE-AC09-89SR18035

Subcontract No. AA82250N

Prepared for:

Westinghouse Savannah River Company

Aiken, SC 29808

Prepared by:

HydroGeoLogic, Ine.

1155 Herndón Parkway, Suite 900

Herndon, VA 22070

Tel: (703) $478-5186$

November 18,1996 
RECEIVED

FFR 261998

OSTI

\section{DEVElopMENT OF A Fully Three-Dimensional GROUNDWATER FLOW MODEL FOR THE A/M AREa Using Data Fusion}

\section{Final Report}

Prime Contract No. DE-AC09-89SR18035

Subcontract No. AA82250N

Prepared for:

Westinghouse Savannah River Company

Aiken, SC 29808

Prepared by:

HydroGeoLogic, Inc.

1155 Herndon Parkway, Suite 900

Herndon, VA 22070

Tel: (703) 478-5186

November 18, 1996 


\section{DISCLAIMER}

Portions of this document may be illegible electronic image products. Images are produced from the best available original document. 


\section{TABLE OF CONTENTS}

Page

EXECUTIVE SUMMARY

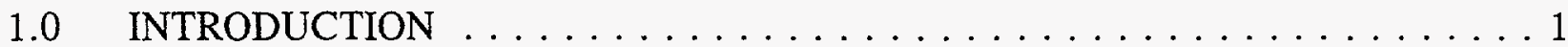

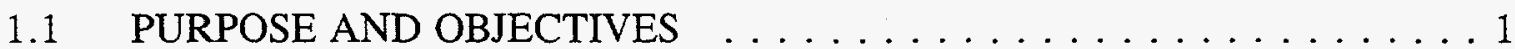

1.2 DESCRIPTION OF MODELED SITE $\ldots \ldots \ldots \ldots \ldots \ldots \ldots \ldots \ldots$

1.3 PREVIOUS GROUNDWATER MODELING INVESTIGATIONS . . . . . . 3

2.0 HYDROGEOLOGICAL SETTING $\ldots \ldots \ldots \ldots \ldots \ldots \ldots \ldots \ldots \ldots$

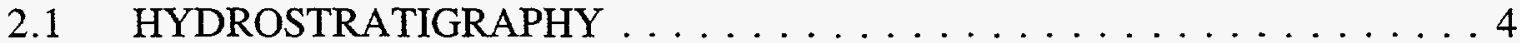

2.2 GROUNDWATER FLOW $\ldots \ldots \ldots \ldots \ldots \ldots \ldots \ldots \ldots$

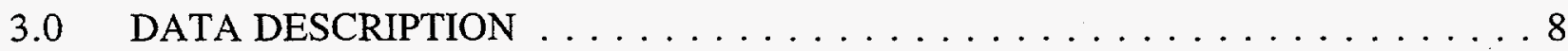

3.1 MONITORING WELL AND BOREHOLE $\ldots \ldots \ldots \ldots \ldots \ldots$

3.2 PUMP TEST $\ldots \ldots \ldots \ldots \ldots \ldots \ldots \ldots \ldots \ldots \ldots \ldots \ldots \ldots \ldots$

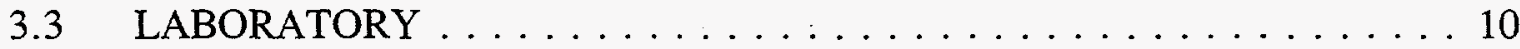

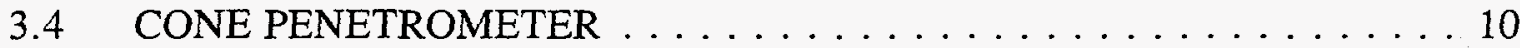

4.0 NUMERICAL, GEOSTATISTICAL, AND MEASUREMENT MODELS . . . . 14

4.1 GROUNDWATER FLOW $\ldots \ldots \ldots \ldots \ldots \ldots \ldots \ldots \ldots \ldots$

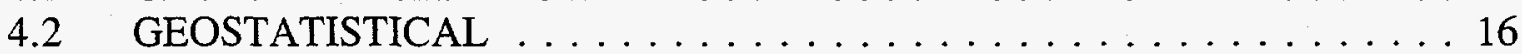

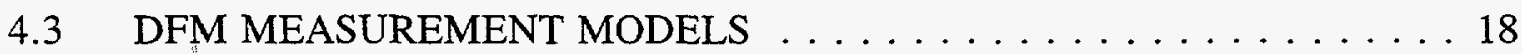

4.4 MUD FRACTION TO LOG CONDUCTIVITY MAPPING $\ldots . . . . .19$

5.0 DELINEATION OF HYDROSTRATIGRAPHIC UNITS USING DFM $\ldots \ldots$

6.0 GROUNDWATER MODEL CALIBRATION USING DFM . . . . . . . . . . 52

6.1 DATA FUSION MODELING METHODOLOGY $\ldots \ldots \ldots \ldots \ldots$

6.2 APPLICATION OF DFM TO SRS A/M AREA . . . . . . . . . . . 54

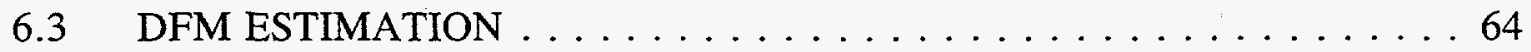

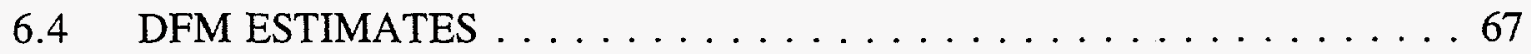

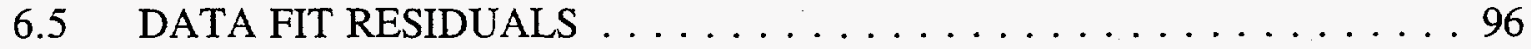

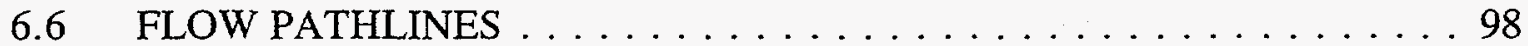

$7.0 \quad$ SPATIAL VARIABILITY IN FLOW PATTERNS $\ldots \ldots \ldots \ldots$

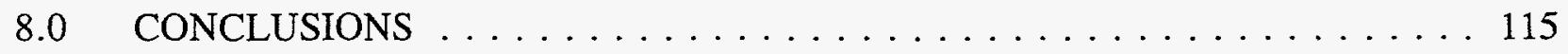

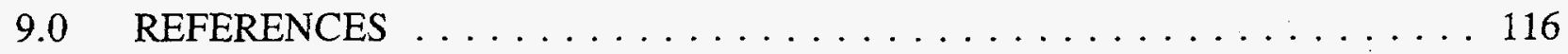

APPENDIX A: $\quad$ Hydraulic Head Measurements

APPENDIX B: $\quad$ Cores and Hydrostratigraphic Data

APPENDIX C: $\quad$ Core Mud Fraction Plots

APPENDIX D: $\quad$ Geological Residuals

APPENDIX E: Hydrualic Head Residuals 


\section{LIST OF FIGURES}

Page

Figure $1.1 \quad \mathrm{~A} / \mathrm{M}$ Area recovery wells and sector boundaries. . . . . . . . . . .

Figure $2.1 \quad$ A/M Area topography and drainage divide. $\ldots \ldots \ldots \ldots \ldots$

Figure 2.2 Schematic north-south hydrostratigraphic section of the A/M Area (Aadland et al. 1995). . . . . . . . . . . . . . . . . . . 6

Figure 3.1 Monitoring well and borehole data. . . . . . . . . . . . . 9

Figure 3.2 Location of the cone penetrometer tests. . . . . . . . . . . . . 12

Figure 4.1 Continuous autocorrelation functions in one, two, and three dimensions. . . 17

Figure $4.2 \quad$ Mud fraction to $\log$ conductivity mapping. . . . . . . . . . . . . 21

Figure 4.3 Residual plot for mud fraction to $\log$ conductivity fit. . . . . . . . . . 21

Figure 4.4 Process of translating mud fraction data to horizontal and vertical conductivity measurements. . . . . . . . . . . . . . 22

Figure 5.1 DFM estimated top elevation $(\mathrm{ft})$ of the "green clay." . . . . . . . . . 26

Figure 5.2 DFM estimated bottom elevation $(\mathrm{ft}$ ) of the "green clay." . . . . . . . . 27

Figure 5.3 DFM estimated top elevation $(\mathrm{ft})$ of the "upper clay.” . . . . . . . . . 28

Figure 5.4 DFM estimated bottom elevation (ft) of the "upper clay." . . . . . . . . . . 29

Figure 5.5 DFM estimated top elevation $(\mathrm{ft})$ of the lower clay. . . . . . . . . . . . 30

Figure 5.6 DFM estimated bottom elevation $(\mathrm{ft})$ of the "lower clay." . . . . . . . 31

Figure 5.7 DFM estimated thickness ( $\mathrm{ft}$ ) of "Crouch Branch confining unit." . . . . . 32

Figure $5.8 \quad$ Geological DFM estimated layers at $\mathrm{y}=99,500(\mathrm{ft}) \ldots \ldots \ldots$. . . . . . 44

Figure $5.9 \quad$ Geological DFM estimated layers at $\mathrm{y}=102,000(\mathrm{ft}) . \ldots \ldots 45$

Figure 5.10 Geological DFM estimated layers at $\mathrm{y}=105,500(\mathrm{ft})$. . . . . . . . . . 46

Figure 5.11 Geological DFM estimated layers at $\mathrm{y}=108,500$ (ft.) . . . . . . . . 47

Figure 6.1 Fine grid at $\mathrm{SRS}$ Easting $50,000(\mathrm{ft}) \ldots \ldots \ldots \ldots$

Figure 6.2 Prior estimate of surface recharge. . . . . . . . . . . . . 61

Figure 6.3 Head contours and data for Crouch Branch Aquifer. . . . . . . . . . . 62

Figure 6.4 Estimated heads (ft) and measurement locations for "M-area" aquifer. . . . 71

Figure 6.5 Estimated heads (ft) and measurement locations for "Upper Lost Lake" aquifer. .............................. 72

Figure 6.6 Estimated heads (ft) and measurement locations for "Lower Lost Lake"

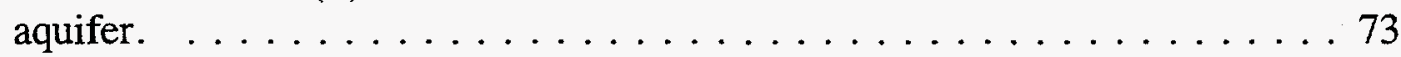

Figure 6.7 Estimated heads (ft) and measurement locations for "middle sand" aquifer . 74

Figure 6.8 Estimated pore velocities for "Upper Lost Lake" aquifer. . . . . . . . . . . 82

Figure 6.9 Estimated pore velocities for "Lower Lost Lake" aquifer. . . . . . . . . . 83

Figure 6.10 Head estimates, head measurements, and pore velocities at $\mathrm{x}=49,750(\mathrm{ft}) .84$

Figure $6.11 \mathrm{~K}_{\mathrm{v}}(\mathrm{ft} / \mathrm{d})$ estimates for "green clay." . . . . . . . . . . . . . 86

Figure $6.12 \mathrm{~K}_{\mathrm{v}}(\mathrm{ft} / \mathrm{d})$ estimates for "upper clay." $\ldots \ldots \ldots \ldots \ldots \ldots \ldots$ 


\section{LIST OF FIGURES}

Page

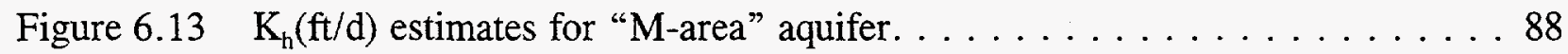

Figure $6.14 \mathrm{~K}_{\mathrm{h}}(\mathrm{ft} / \mathrm{d})$ estimates for "Upper Lost Lake" aquifer. . . . . . . . . . . . . . . . 89

Figure $6.15 \mathrm{~K}_{\mathrm{h}}(\mathrm{ft} / \mathrm{d})$ estimates for "Lower Lost Lake" aquifer. . . . . . . . . . . . . . 90

Figure 6.16 $\mathrm{K}_{\mathrm{h}}(\mathrm{ft} / \mathrm{d})$ estimates and measurement locations at $\mathrm{x}=53,000(\mathrm{ft}) . \ldots \ldots 91$

Figure 6.17 "M-area" aquifer head residuals. . . . . . . . . . . . . . . . . 99

Figure 6.18 "Lost Lake" aquifer head residuals. . . . . . . . . . . . . . . . . . . . 100

Figure 6.19 "Crouch Branch" Confining Unit head residuals. . . . . . . . . . . . . . 101

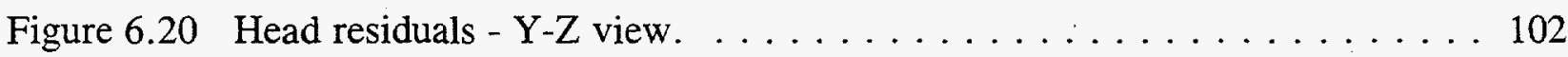

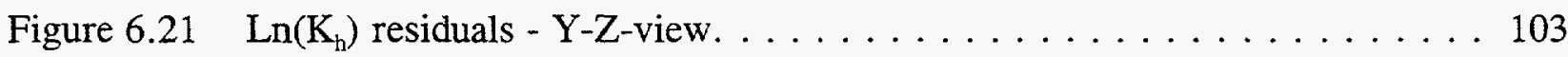

Figure $6.22 \ln \left(\mathrm{K}_{\mathrm{v}}\right)$ residuals - $\mathrm{Y}-\mathrm{Z}$ view. . . . . . . . . . . . . . . . . . . 104

Figure 6.23 3D pathlines - plan view (markers at 10-year intervals). . . . . . . . . . 105

Figure 6.24 3D pathlines - Y-Z view (markers at 10-year intervals). . . . . . . . . 106

Figure 6.25 Capture zone for recovery wells RWM-1 through RWM-12 . . . . . . 107

Figure 7.1 Estimated head and pore velocity vectors for the "Upper Lost Lake" aquifer. . . . . . . . . . . . . . . . . . . . . 109

Figure 7.2 Estimated head and pore velocity vectors at $\mathrm{x}=50,000(\mathrm{ft}) \ldots \ldots \ldots 110$

Figure 7.3 Estimated $\ln (\mathrm{Kh})$ at $\mathrm{x}=50,000(\mathrm{ft}) \ldots \ldots \ldots \ldots \ldots \ldots \ldots \ldots$

Figure $7.4 \quad 3 \mathrm{D}$ pathlines with 10 -year markers (plan view) . . . . . . . . . . . 112

Figure 7.5 3D pathlines with 10 -year markder $(\mathrm{Y}-\mathrm{Z}$ view). $\ldots \ldots \ldots \ldots \ldots \ldots$

Figure 7.6 Variation in 3D pathlines. . . . . . . . . . . . . . . 114 


\section{LIST OF TABLES}

Page

Table 3.1. Selected Soil Properties from the Integrated Demonstration Site (IDS) Data Set (modified from Riha $(1993)$ ). . . . . . . . . . . . . . . . . . 11

Table $3.2 \quad$ Cone Penetrometer Data . . . . . . . . . . . . . . . . . . 13

Table 5.1 Prior Estimate and Uncertainty of Zone Trend Parameters . . . . . . . . . . 24

Table $5.2 \quad$ Zone Parameters Random Model . . . . . . . . . . . . . . . . . . . . . . 24

Table $5.3 \quad$ Total Residual RMS by Layer . . . . . . . . . . . . . . . . . 25

Table 5.4 Geological Data for Layer 1 (Top of "Green Clay") . . . . . . . . . . . . 33

Table 5.5 Geological Data for Layer 2 (Bottom of "Green Clay") . . . . . . . . . . . 36

Table 5.6 Geological Data for Layer 3 (Top of "upper clay") . . . . . . . . . . . . . . 39

Table 5.7 Geological Data for Layer 4 (Bottom of "upper clay") . . . . . . . . . . 41

Table 5.8 Geological Data for Layer 5 (Top of "lower clay") . . . . . . . . . . . . . 42

Table 5.9 Geological Data for Layer 6 (Bottom of "lower clay") . . . . . . . . . . 44

Table $5.10 \quad$ Geological Data at $\mathrm{y}=99500(\mathrm{ft}) \ldots \ldots \ldots \ldots$. . . . . . . . 48

Table $5.11 \quad$ Geological Data at $\mathrm{y}=102000(\mathrm{ft}) \ldots \ldots \ldots$. . . . . . . . . . . 49

Table $5.12 \quad$ Geological Data at $\mathrm{y}=105500(\mathrm{ft}) \ldots \ldots \ldots \ldots \ldots$

Table 5.13 Geological Data at $\mathrm{y}=108500(\mathrm{ft}) \ldots \ldots \ldots \ldots \ldots$

Table $6.1 \quad$ Model Layers per Hydrostratigraphic Unit . . . . . . . . . . . . . . 5 54

Table $6.2 \quad$ Crouch Branch Aquifer Head Data . . . . . . . . . . . . . . . 57

Table 6.3 Prior Estimate and Uncertainty of Trend Log Conductivity States . . . . . 58

Table 6.4 Prior Estimate and Uncertainty of Measurement Bias and Recharge States . 58

Table 6.5 Prior Estimate and Uncertainty of North Boundary Source Head States . . 59

Table 6.6 Prior Estimate and Uncertainty of South Boundary Source Head States ... 59

Table 6.7 Prior Estimate and Uncertainty of East Boundary Source Head States . . . 59

Table 6.8 Prior Estimate and Uncertainty of West Boundary Source Head States . . . 60

Table 6.9 Location and Pumping Rates (cu-ft/day) of Recovery Wells . . . . . . . 63

Table 6.10 Statistics of Random Field Model for $\ln (\mathrm{Kh}) \ldots \ldots$. . . . . . . . . . 64

Table 6.11 Posterior Estimate and Uncertainty of Mean Log Conductivity States . . . 68

Table 6.12 Posterior Estimate and Uncertainty of Measurement Bias and Recharge

States .......................... 68

Table 6.13 Posterior Estimate and Uncertainty of North Boundary Source Head States . 68

Table 6.14 Posterior Estimate and Uncertainty of South Boundary Source Head States . 69

Table 6.15 Posterior Estimate and Uncertainty of East Boundary Source Head States . . 69

Table 6.16 Posterior Estimate and Uncertainty of West Boundary Source Head States . 70

Table 6.17 M-Area Aquifer Hydraulic Head Data . . . . . . . . . . . . . . . 75

Table $6.18 \quad$ Upper Lost Lake Aquifer Hydraulic Head Data . . . . . . . . . . . . . . 77 


\section{LIST OF TABLES}

Page

Table 6.19 Lower Lost Lake Aquifer Hydraulic Head Data . . . . . . . . . . . . . . . . 79

Table $6.20 \quad$ Middle Sand Aquifer Hydraulic Head Data ...................... 81

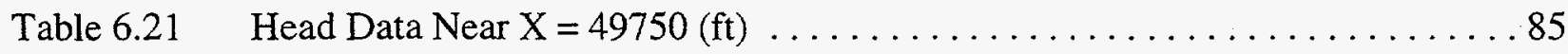

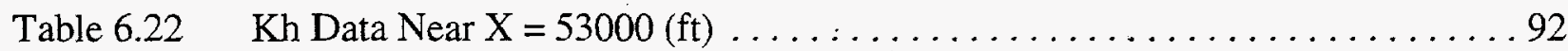

Table 6.23 Residual Sum-of-Square Contributions ........................ 97

Table 6.24 Measurement Residual Statistics ........................ 97 


\section{EXECUTIVE SUMMARY}

SRS was established in the 1950 's to produce plutonium, tritium, and other nuclear materials. The A/M Area contained fabrication facilities where assemblies were degreased using industrial solvents, primarily trichloroethylene.(TCE) and tetrachloroethylene (PCE). From 1952 to 1982, about 3.5 million pounds of solvents were released to the environment as process waste. The DFM calibrated groundwater flow model will contribute to the cleanup and containment of the dissolved plume. The DFM delineation of clay layers has the potential to contribute to the cleanup of DNAPL's.

Data Fusion Modeling (DFM) was used in the A/M Area to provide the distribution of parameters that effect groundwater flow and to provide a calibrated model to be used for development of corrective action programs. DFM was developed by EM-50's Characterization Monitoring Sensor Technology Program and was demonstrated at the Savannah River Site (SRS) Old Burial Ground(Gibbs and Jones (1995)). DFM provided a better fit to the data in less time than conventional methods and also quantified parameter uncertainty.

In the $\mathrm{A} / \mathrm{M}$ Area, lithological data on subsurface heterogeneity was incorporated into model calibration using DFM. Since DFM provides rapid model updates, alternative conceptual models were able to be considered in order to select the best model. Groundwater flow patterns were determined using particle tracking methods.

Prior to model calibration, DFM was used to delineate the hydrostratigraphy. A combination of picks from well data and cone penetrometer data were used by DFM to map the interfaces between clay layers and aquifers. The hydrostratigraphy was then used as input to model calibration.

SRS used previous models to aid in the siting of recovery wells. The DFM calibration effort builds on the results of previous model calibrations. The most recent model prior to the DFM calibration is the first fully three-dimensional model for the A/M Area using the FACT code. The model incorporates local discontinuities and regional variations of the aquifers and confining units. Hydraulic head data were used to calibrate the model. Hydraulic properties and boundary conditions were manually adjusted to reduce the residual difference between the measured head and the model predicted head. Modeling activities before the FACT model used a quasi-three-dimensional approach that restricted the horizontal flow to the aquifer zones and vertical flow to the confining zones.

DFM model calibration builds on the FACT model by incorporating lithological data on subsurface heterogeneities. Mud fraction data, aquifer pump tests, and laboratory permeability tests were used to determine local hydraulic conductivities treated as measured data by DFM. Head data were also used by DFM where the VAM3DF code (similar to the FACT code) was used to provide the groundwater numerical model. In addition, prior information was used on recharge/boundary conditions that were to be estimated by DFM. Conductivity data provide information on a local scale while head data provide information on a larger scale. DFM geostatistical random field models were used in each hydrostratigraphic unit in order to combine data on different scales. 
The result is a model that uses conductivity data to provide a better distribution of hydraulic conductivity model while still fitting the hydraulic head data. In fact, the root-mean-squared (RMS) head residual for DFM is $1.0 \mathrm{ft}$. The heterogeneity in hydraulic conductivity is particularly evident in the "Lost Lake" Aquifer Zone as shown in the $2 \mathrm{D}$ visualization of hydraulic conductivity shown in Figure 6.12. Groundwater flow patterns for the "Lost Lake" Aquifer Zone are shown by the particle pathlines of Figure 6.23.

Because DFM processing is efficient, a number of conceptual models were considered in order to select the best. For example, boundary conditions were treated at different times as prescribed head, no flow, no accumulation, and head-dependent flux (HDF) boundary. It was ultimately decided that the best model was to use HDF boundary and to let DFM estimate the source head parameter in the HDF as part of calibration.

Data files were delivered for the calibrated model so that the model can be used by SRS investigators. The model was produced for the purpose of being employed by SRS investigators for development of corrective action programs for the Southern Sector, Western Sector, and the Northern Sector groundwater remediation activities. DFM software modifications made in the course of the work were delivered. The software modifications provide the capability to perform rapid model updates with additional data that becomes available, thus supporting remediation decisions and monitoring purposes.

Different combinations of parameters sometimes give nearly the same fit to the data, producing uncertainties in parameter estimates. DFM quantifies the uncertainties and computes the impact of the uncertainties on the variability in model predictions. For example, Figure 7.4 shows the variability in a particle pathline in the "Lost Lake" Aquifer Zone. The variability is displayed as Monte Carlo pathlines all starting from the same point, but each experiencing different random realizations of parameter estimate errors.

DFM delineation of the top of the "Green Clay" Confining Zone has been observed by SRS investigators to correlate better with the known distribution of DNAPL's than previous maps of clay surfaces. DFM estimates the clay surfaces as part of determining the hydrostratigraphic layers by interpolating picks using geostatistical models for the spatial variability of layer width. Due to the ability of DFM to incorporate physical models, it is possible to constrain the estimation to be consistent with the known distribution of DNAPL's. Potential future work would be to customize DFM software to estimate DNAPL target zones for remediation. Building on the success of groundwater model calibration, other potential areas of future work are to customize DFM software to incorporate contaminant data for the calibration of flow and transport parameters and to calibrate multi-phase models in the vadose zone. 


\subsection{INTRODUCTION}

\subsection{PURPOSE AND OBJECTIVES}

The purpose of the work was to obtain the distribution of the parameters that effect groundwater flow and to calibrate a model for subsequent use in the development of corrective action programs in the $\mathrm{A} / \mathrm{M}$ Area. This lead to the following objectives:

- Delineate hydrostratigraphy with DFM using picks from borehole data and cone penetrometer data and define numerical grid conformal with hydrostratigraphic layers.

- Incorporate mud fraction data, aquifer pump tests, and laboratory permeability tests into DFM with 1995 head data, VAM3DF, geostatistical models in each hydrostratigraphic layer for spatial variability of hydraulic conductivity, and recharge/boundary condition prior information.

- Calibrate model considering alternative conceptual models.

- Determine parameter uncertainties and impact of uncertainties on spatial variability of flow patterns.

\subsection{DESCRIPTION OF MODELED SITE}

The Savannah River Site (SRS) operated by Westinghouse Savannah River Company (WSRC) is located in South Carolina along the banks of the Savannah River. The A/M Area is located in the northern section of the SRS and consists of those facilities that fabricated reactor fuel and target assemblies for the SRS reactors (M-Area), Savannah River Technology Center (SRTC), and administrative and support facilities (A-Area). The vadose zone and aquifers beneath the A/M Area have been contaminated with tetrachloroethylene (PCE) and trichloroethylene (TCE). In order to remove the subsurface contamination, SRS has installed a groundwater recovery well network in the A/M Area. In 1985, pump-and-treat remediation began in the central A/M Area with eleven operating recovery wells, RWM-1 through RWM-11 (M1 system). Later, recovery well RWM-12 in the northern sector of the A/M Area began pumping. In 1996, remediation began at recovery wells, RWM-13B, RWM-13C, RWM-14B, RWM-14C, and RWM-15.

The modeled area is a 6.7 square mile rectangle which is aligned with the SRS plant coordinates. The rectangle covers an area from 43,500 (ft) to 56,000 (ft) SRS easting and from 95,000 (ft) to $110,000(\mathrm{ft}) \mathrm{SRS}$ northing. Computational limitations preclude an areal extent similar to previous models of the A/M Area. SRS north is $36^{\circ}$ to the west of true north. Figure 1.1 shows the modeled area with roads, buildings, sector boundaries, and recovery well in the A/M Area. 


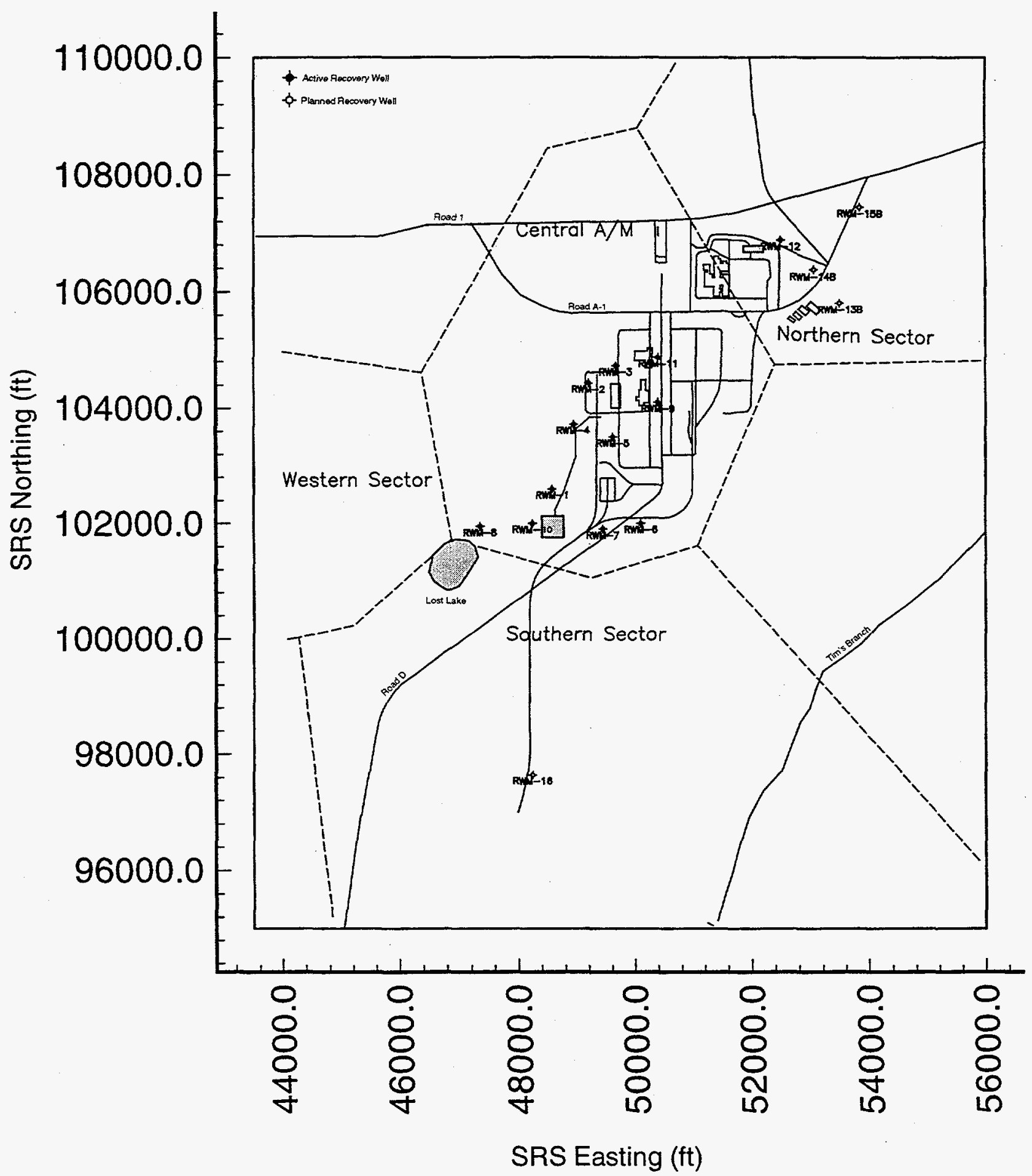

Figure 1.1 A/M Area recovery wells and sector boundaries. 


\subsection{PREVIOUS GROUNDWATER MODELING INVESTIGATIONS}

SRS has continually supported the development of numerical models that simulate groundwater flow in the A/M Area. The numerical models have been used to determine the effectiveness of the existing and planned recovery well systems. Papadopulos and Associates (1987) studied the effectiveness of the M1 system by applying the United States Geological Survey's groundwater flow model, MODFLOW, of McDonald and Harbaugh (1983). Beaudoin et al. (1991) updated the A/M Area MODFLOW model with new hydrologic information and calibrated to 1989-1990 groundwater levels. Intera (1993) extended the A/M Area MODFLOW model to include a transient flow model. The Intera model was calibrated both to steady-state heads before the M1 system was fully operational, and to transient heads from the fourth quarter of 1985 through the fourth quarter of 1991. Jackson (1994) developed the first fully three-dimensional groundwater flow model for the A/M Area using the WSRC flow and contaminant transport code, FACT (WSRC (1995a)). 


\subsection{HYDROGEOLOGICAL SETTING}

The SRS is located on the Aiken Plateau in the Upper Atlantic Coastal Plain of South Carolina. The unconsolidated and semi-consolidated sediments underlying the SRS are part of a Tertiary to Late Cretaceous wedge that dips and thickens in a southeasterly direction. The sediments mainly consist of unconsolidated interbedded sands, silts, and clays. The Aiken Plateau is deeply incised by surface water drainage features. Even though the plateau is well drained, several depressions or poorly-drained areas exist (Carolina Bays). The topography in the model area ranges from 380 feet mean sea level (MSL) in the A/M Area to 200 feet (MSL) along Tim's Branch (Figure 2.1).

\subsection{HYDROSTRATIGRAPHY}

The hydrostratigraphic representation utilized in this report is presented in Aadland et al. (1995). Aadland and Bledsoe (1990) developed a hydrostratigraphic classification system for the SRS. The classification system is divided into 4 ranks: (1) hydrogeologic province, (2) system, (3) unit, and (4) zone. Figure 3 shows the hydrostratigraphic classification for the A/M Area as presented by Aadland et al. (1995). The SRS is within the southeastern Central Plain hydrogeologic province. Layers in this province tend to thin in the direction of the ancestral coastline to the northwest. The hydrostratigraphic units beneath the A/M Area are part of the Floridan-Midville Aquifer System and include the Steed Pond Aquifer Unit, the Crouch Branch Confining Unit, the Crouch Branch Aquifer, the McQueen Branch Confining Unit, and the McQueen Branch Aquifer. The Steed Pond Aquifer Unit is divided into upper and lower aquifer units, the "M-Area" Aquifer Zone and the "Lost Lake" Aquifer Zone by the "Green Clay" Confining Zone. In the underlying Crouch Branch Confining Unit, the transmissive "middle sand" zone splits the unit into an "upper clay" zone and a "lower clay" zone. The "green clay", "upper clay" and "lower clay" confining zones thin and eventual pinchout north of the A/M Area as indicated in Figure 2.2. One significant feature is not shown in Figure 2.2. The "Lost Lake" Aquifer Zone is divided into "upper" and "lower" subzones by a thin discontinuous clay in some parts of the A/M Area. The groundwater flow model presented in this report includes the Steed Pond Aquifer and the Crouch Branch Confining Unit.

\subsection{GROUNDWATER FLOW}

The major drainage divide in the model area is along the ridge on which the A/M Area is located (Figure 2.1). Surface water on the west side of the ridge drains into the wetlands to the west of the A/M Area, while surface water on the east side of the ridge drains into Tim's Branch which flows south into Upper Three Runs Creek. Since the water table elevation mimics the surface topography, water entering the "M-Area" Aquifer Zone from the vadose zone flows west towards the wetlands, or east towards Tim's Branch. Most of the recharge on the A/M Area ridge flows through the "M-Area" Aquifer Zone and "Green Clay" Confining Zone into the "Lost Lake" Aquifer Zone. Once water enters the "Lost Lake" Aquifer Zone, it ignores the drainage divide and flows southwest towards the Savannah River or south towards Upper Three Runs Creek. Water that flows through the Crouch Branch Confining Unit into the Crouch Branch Aquifer then flows southwest toward the Savannah River. The A/M Area ridge is a recharge area where hydraulic head decreases with increasing depth. Discharge areas along Tim's Branch and along the low, swampy area west of the A/M Area have hydraulic heads increasing with increasing depth. 


\section{A/M Area Topographic Contours in Feet (MSL)}

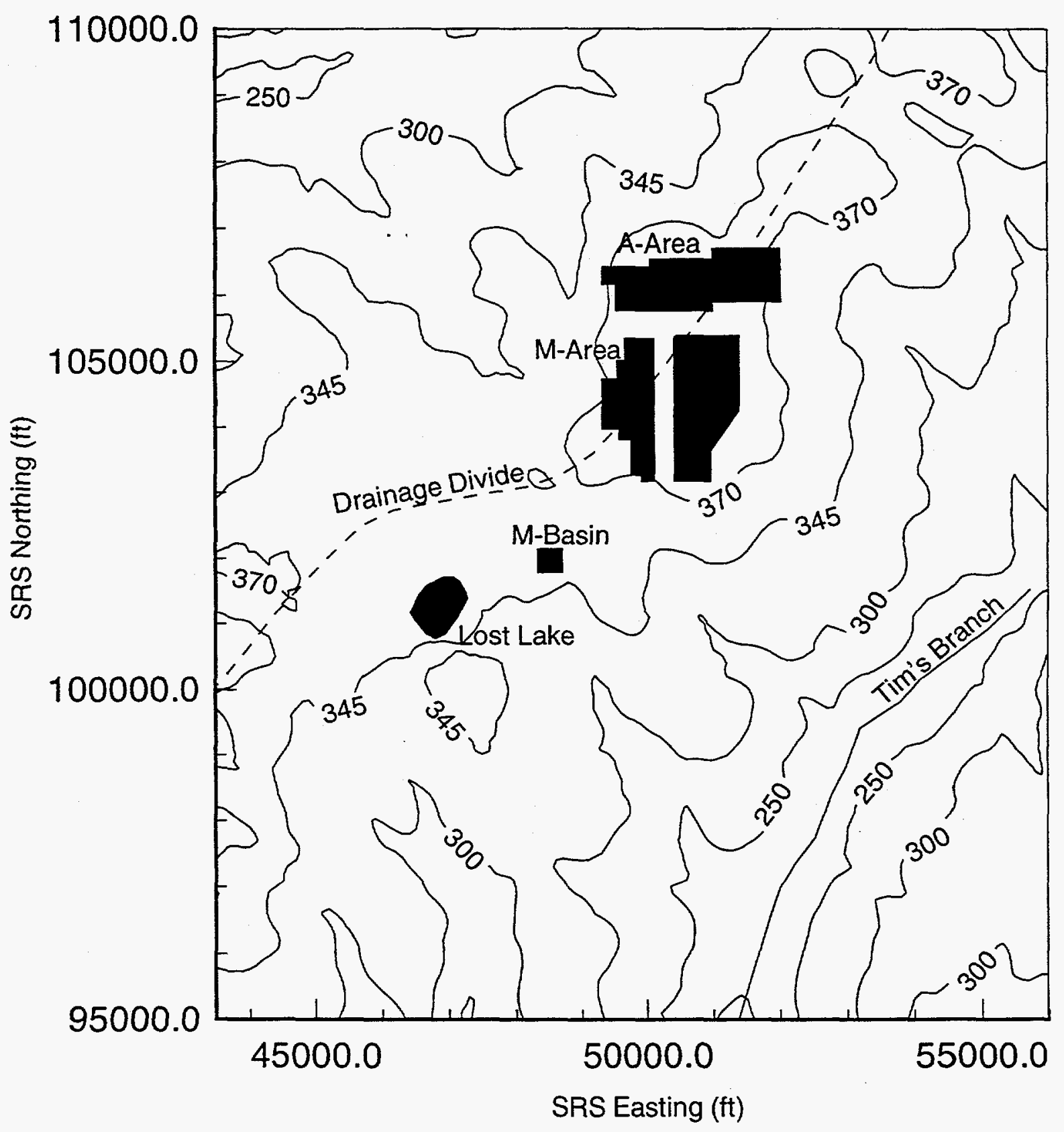

Figure 2.1 A/M Area topography and drainage divide. 
- (\$66I

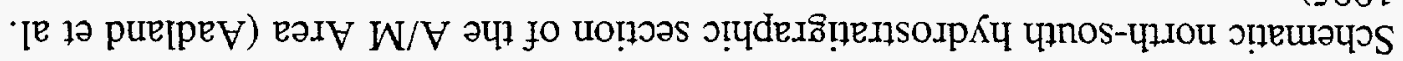
$\tau \cdot \tau$ ว.กริ!

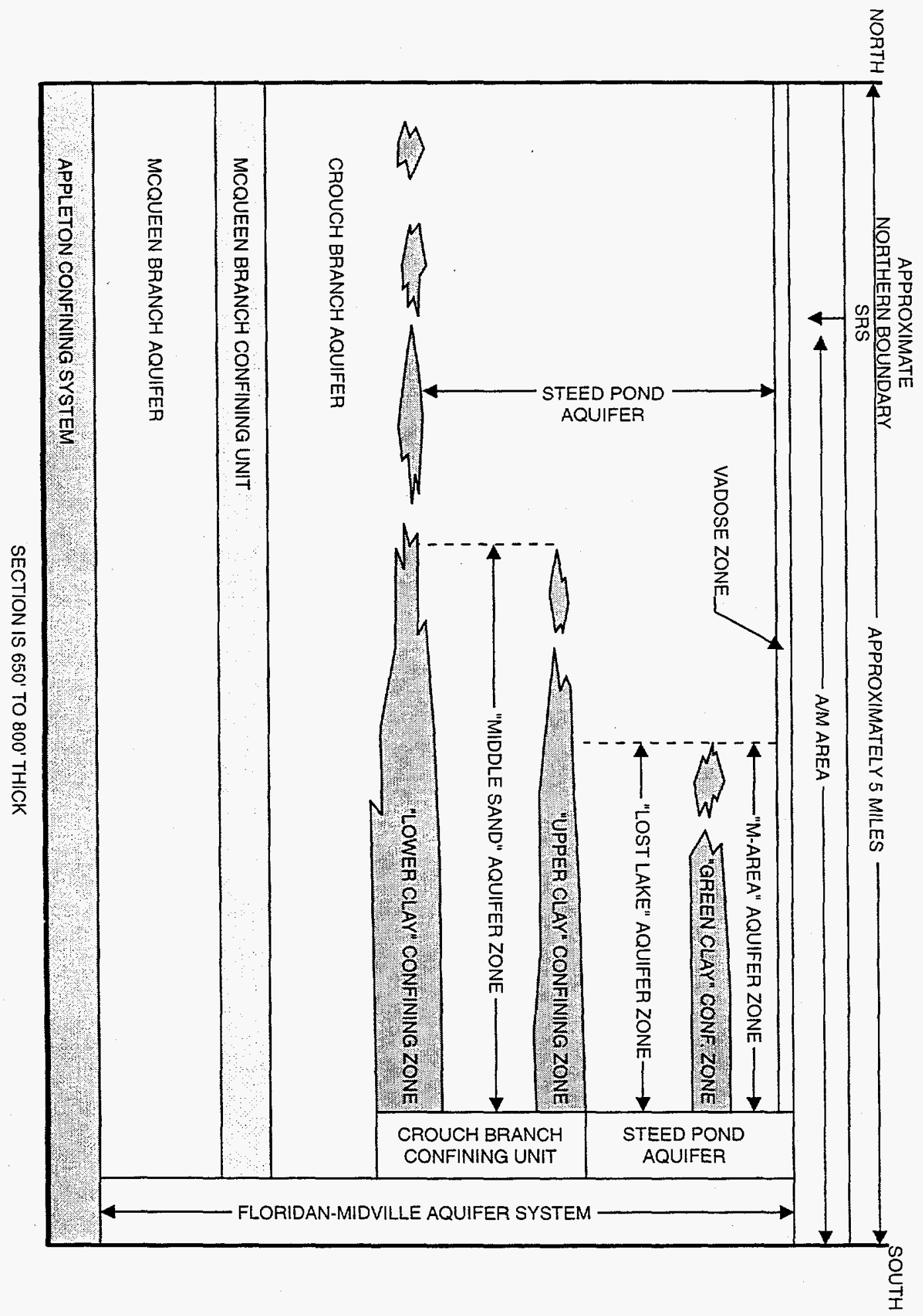


Most of the recharge to the groundwater flow system is due to infiltration of precipitation. A large proportion of the precipitation runs off to drainageways and then flows out of the region or evaporates back into the atmosphere. Some of the precipitation is absorbed by plants and transpired back into the atmosphere. At the SRS, it was estimated by Looney et al. (1987) that the water table receives an average annual recharge rate of $15 \mathrm{in} / \mathrm{yr}$. The actual recharge may vary significantly from this estimate since soil type, ground slope, vegetative cover and man-made structures will affect how water enters the subsurface.

Additional recharge to the groundwater flow system is due to the process water discharged at the A-1 and A-14 Outfalls. The drainageways lose water to the subsurface from the outfall point to the point where the drainageways reach a discharge area.

Natural discharge from the groundwater flow system occurs in low lying areas near streams and wetlands. Additional discharge is due to pumping in the $\mathrm{A} / \mathrm{M}$ Area. Of the seven production wells which pump water from the Crouch Branch Aquifer and McQueen Branch Aquifer, only 905-53A and 905-20A were used in 1995. Twelve recovery wells pumped water from the Steed Pond Aquifer in 1995. 


\subsection{DATA DESCRIPTION}

\subsection{MONITORING WELL AND BOREHOLE}

Since 1979 , monitoring well water levels have been monitored on a quarterly basis in the $\mathrm{A} / \mathrm{M}$ Area. The 1995 quarterly monitoring well data (WSRC (1995b), (1995c), (1995d), (1995e)) was used to compute an average 1995 water level of each monitoring well. The average 1995 water levels were calibration targets for the groundwater flow model. In an attempt to eliminate water levels that are resulting from perched water, monitoring wells that were pumped less than 50 gallons were discarded. Monitoring well AMB 10DD was discarded since it is located near a Carolina Bay and has a depth to water of six feet. Appendix A contains a list of the 348 accepted 1995 average water levels. The hydraulic head measurements were specified at the middle of the screens.

Hydrologic data was obtained from continuous drill cores at 103 boreholes in the $\mathrm{A} / \mathrm{M}$ Area. The foot-by-foot description of mud fraction (silt and clay sized material) at 98 cores inside the model domain were used to produce conductivity measurements. Appendix B contains a list of the cores and hydrostratigraphic data modified from Aadland et al. (1995). Figure 3.1 shows the location of the monitoring well and borehole data along with a basemap of the modeled area. Notice lack of data in the northwest and southeast corners of the modeled area.

Appendix $\mathrm{C}$ contains plots of mud fraction (smoothed) versus elevation ( $\mathrm{ft}$ ) corresponding to the 103 cores. The dashed lines represent the hydrostratigraphic picks modified from Aadland et al. (1995). The raw mud fraction data was smoothed using the following filter which rejects frequencies in the upper half of the spectrum (Hamming):

$$
h_{k}=\frac{f_{k-3}+2 f_{k-2}+3 f_{k-1}+3 f_{k}+2 f_{k+1}+f_{k+2}}{12} \text {, }
$$

where $f_{k}$ is the raw mud fraction data and $h_{k}$ is the smoothed mud fraction. The no recovery zones found in the raw mud fraction data are assumed to be sandy soils with an average mud fraction of $3 \%$. The no recovery zones were replaced with a $3 \%$ mud fraction.

\subsection{PUMP TEST}

Geraghty and Miller (1987) analyzed recovery wells RWM-4 through RWM-11 in the central A/M Area. Step-drawdown and constant-rate pump tests were performed to estimate well efficiency, specific capacity, maximum potential pumping rates, and aquifer transmissivity. Since the pumping wells are screened across the "M-Area" and "Lost Lake" Aquifer Zones, transmissivity estimates for specific hydrostratigraphic units were not obtained. The total transmissivity of the "M-Area" and "Lost Lake" Aquifer Zones varied between $174 \mathrm{ft}^{2} / \mathrm{d}$ near RWM-7 to over $14703 \mathrm{ft}^{2} / \mathrm{d}$ near RWM-9. 


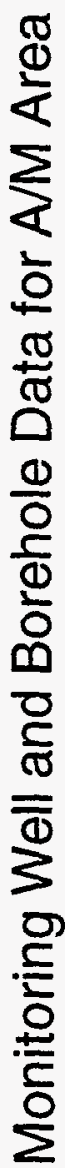

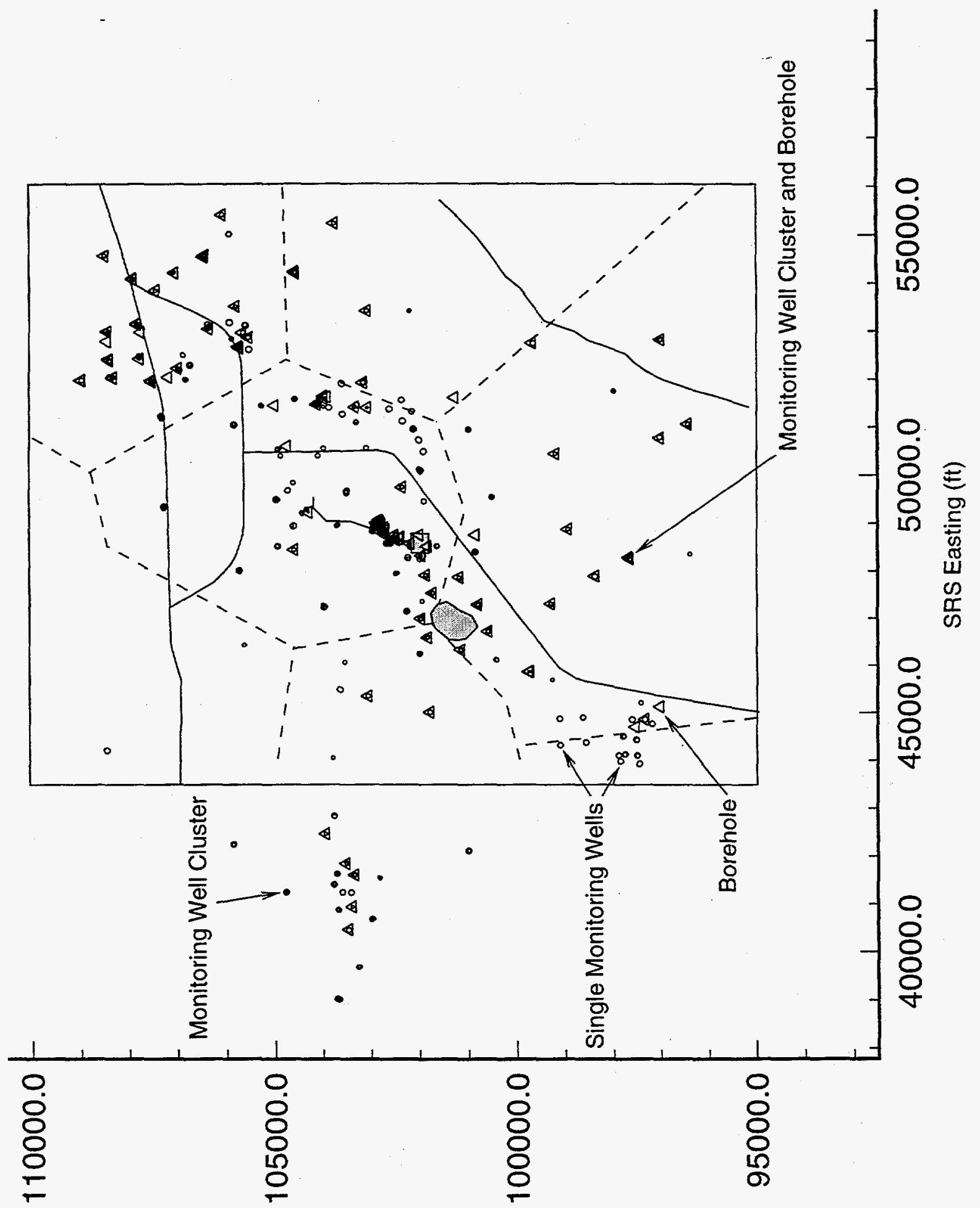

(H) 6u!4นON SUS

Figure 3.1 Monitoring well and borehole data. 
An aquifer pumping test was performed on recovery well RWM-16 which is screened in the "Lost Lake" Aquifer Zone (Hiergesell (1993)). The horizontal hydraulic conductivity estimates ranged from $28 \mathrm{ft} / \mathrm{d}$ to $35.5 \mathrm{ft} / \mathrm{d}$ with an aquifer thickness of $40 \mathrm{ft}$. Estimates of the vertical hydraulic conductivity of the "Green Clay" Confining Zone ranged from $1.6 \times 10^{-3} \mathrm{ft} /$ day to $9.1 \times 10^{-3}$ $\mathrm{ft} /$ day with an aquitard thickness of six feet.

An aquifer pumping test was also performed on recovery well RWM-12 which is screened in the "Upper Lost Lake" and "Lower Lost Lake" aquifer zones (Hiergesell (1994)). Since there was no response to pumping the "Upper Lost Lake" aquifer zone, all water flow was assumed to originate in the "Lower Lost Lake" aquifer zone. The horizontal hydraulic conductivity estimates for the "Lower Lost Lake" aquifer zone ranged from $43 \mathrm{ft} / \mathrm{d}$ to $82 \mathrm{ft} / \mathrm{d}$ with an aquifer thickness of $20.5 \mathrm{ft}$. The vertical hydraulic conductivity estimates for the aquitard dividing the "Lost Lake" Aquifer Zone ranged from $2.9 \times 10^{-3} \mathrm{ft} / \mathrm{d}$ to $6.4 \times 10^{-3} \mathrm{ft} / \mathrm{d}$.

Hiergesell et al. (1994) performed aquifer pumping tests on recovery wells RWM-13B, RWM13C, RWM-14B, RWM-14C, and RWM-15B which are screened in the "Lost Lake" Aquifer Zone. Estimates of horizontal hydraulic conductivity ranged from $17 \mathrm{ft} / \mathrm{d}$ near RWM-14C to 434 $\mathrm{ft} / \mathrm{d}$ near RWM-15B.

Hiergesell and Pemberton (1995) performed aquifer pumping tests on five 2-inch monitoring wells in the Southern Sector of the A/M Area. The horizontal hydraulic conductivity estimates for the "Lost Lake" Aquifer Zone ranged from $21 \mathrm{ft} / \mathrm{d}$ to $119 \mathrm{ft} / \mathrm{d}$. The horizontal hydraulic conductivity of the "M-Area" Aquifer Zone was estimated to be $53 \mathrm{ft} / \mathrm{d}$.

\subsection{LABORATORY}

Riha (1993) presented laboratory permeability data from soil samples of the Integrated Demonstration Site (IDS), which is located immediately north of the M-Basin. The laboratory testing was performed by Law Environmental, Inc. under contract to O'Brien and Gere Engineers, Inc. Table 3.1 lists selected soil properties for 17 soil samples.

\subsection{CONE PENETROMETER}

Cone penetrometer tests were conducted adjacent to the M-Basin in order to delineate the geologic layering and determine the location of dense non-aqueous liquid-phase (DNAPL) contaminated soils (Shinn and Bratton (1992)). As the penetrometer probe is forced into the ground, the conical tip and friction sleeve independently measure vertical resistance beneath the tip as well as frictional resistance on the side of the probe as functions of depth. The penetrometer probe also has a pressure transducer in the cone that measures the pore water pressure and a resistivity module that measures variance in soil conductance. The tip resistance and friction ratio values from the cone penetrometer test were used to determine soil classification versus depth.

The cone penetrometer test (CPT) results presented by Shinn and Bratton (1992) were analyzed to determine the elevation of the top and bottom of the "Green Clay" Confining Zone. Figure 3.2 shows the location of the cone penetrometer tests and Table 3.2 lists the analysis results for 23 cone penetrometer tests. Only 12 of the cone penetrometer tests showed evidence of the "Green Clay" Confining Zone. 
Table 3.1. Selected Soil Properties from the Integrated Demonstration Site (IDS) Data Set (modified from Riha (1993)).

\begin{tabular}{|l|c|c|c|c|c|c|c|}
\hline Sample & $\begin{array}{c}\% \\
\text { Sand }\end{array}$ & $\begin{array}{c}\% \\
\text { Silt }\end{array}$ & $\begin{array}{c}\% \\
\text { Clay }\end{array}$ & $\begin{array}{c}\text { Mud } \\
\text { Fraction }\end{array}$ & Porosity & $\begin{array}{c}\text { Vertical K } \\
\text { (ft/d) }\end{array}$ & $\begin{array}{c}\text { Horizontal K } \\
\text { (ft/d) }\end{array}$ \\
\hline IDS 2 & 36.3 & 26.7 & 37.0 & 0.636 & 0.43 & $9.9 \times 10^{-5}$ & $1.19 \times 10^{-4}$ \\
IDS 3 & 78.3 & 16.7 & 5.0 & 0.217 & 0.42 & .94 & 0.22 \\
IDS 4 & 91.4 & 3.6 & 5.0 & 0.086 & 0.37 & 4.3 & NT \\
IDS 5 & 90.4 & 4.6 & 5.0 & 0.096 & 0.42 & 1.6 & NT \\
IDS 6 & 30.1 & 31.9 & 38.0 & 0.699 & 0.43 & 1.1 & NT \\
IDS 7 & 93.8 & 3.2 & 3.0 & 0.062 & 0.4 & 3.1 & NT \\
IDS 8 & 44.0 & 28.0 & 28.0 & 0.56 & 0.39 & $1.1 \times 10^{-3}$ & $1.04 \times 10^{-3}$ \\
IDS 9 & 80.3 & 8.1 & 7.0 & 0.151 & 0.39 & 1.6 & NT \\
IDS 10 & 72.6 & 15.4 & 12.0 & 0.274 & 0.42 & $1.1 \times 10^{-1}$ & $5.38 \times 10^{-2}$ \\
IDS 11 & 92.2 & 4.4 & 3.0 & 0.074 & 0.39 & 4.0 & NT \\
IDS 12 & 48.1 & 24.9 & 27.0 & 0.519 & 0.7 & $1.6 \times 10^{-5}$ & $3.4 \times 10^{-5}$ \\
IDS 13 & 80.8 & 14.2 & 5.0 & 0.192 & 0.36 & $9.6 \times 10^{-4}$ & $9.6 \times 10^{-4}$ \\
IDS 14 & 68.2 & 21.8 & 10.0 & 0.318 & 0.35 & $1.1 \times 10^{-3}$ & $1.55 \times 10^{-3}$ \\
IDS 15 & 93.3 & 3.7 & 3.0 & 0.067 & 0.39 & 0.12 & 0.127 \\
IDS 16 & 50.8 & 32.9 & 16.0 & 0.489 & 0.37 & $5.4 \times 10^{-4}$ & $1.58 \times 10^{-2}$ \\
IDS 17 & 93.1 & 3.9 & 3.0 & 0.069 & 0.39 & 0.71 & 0.24 \\
IDS 18 & 95.6 & 4.4 & 0.0 & 0.044 & 0.37 & 1.1 & 0.34 \\
\hline
\end{tabular}

$\mathrm{NT}=$ not tested 


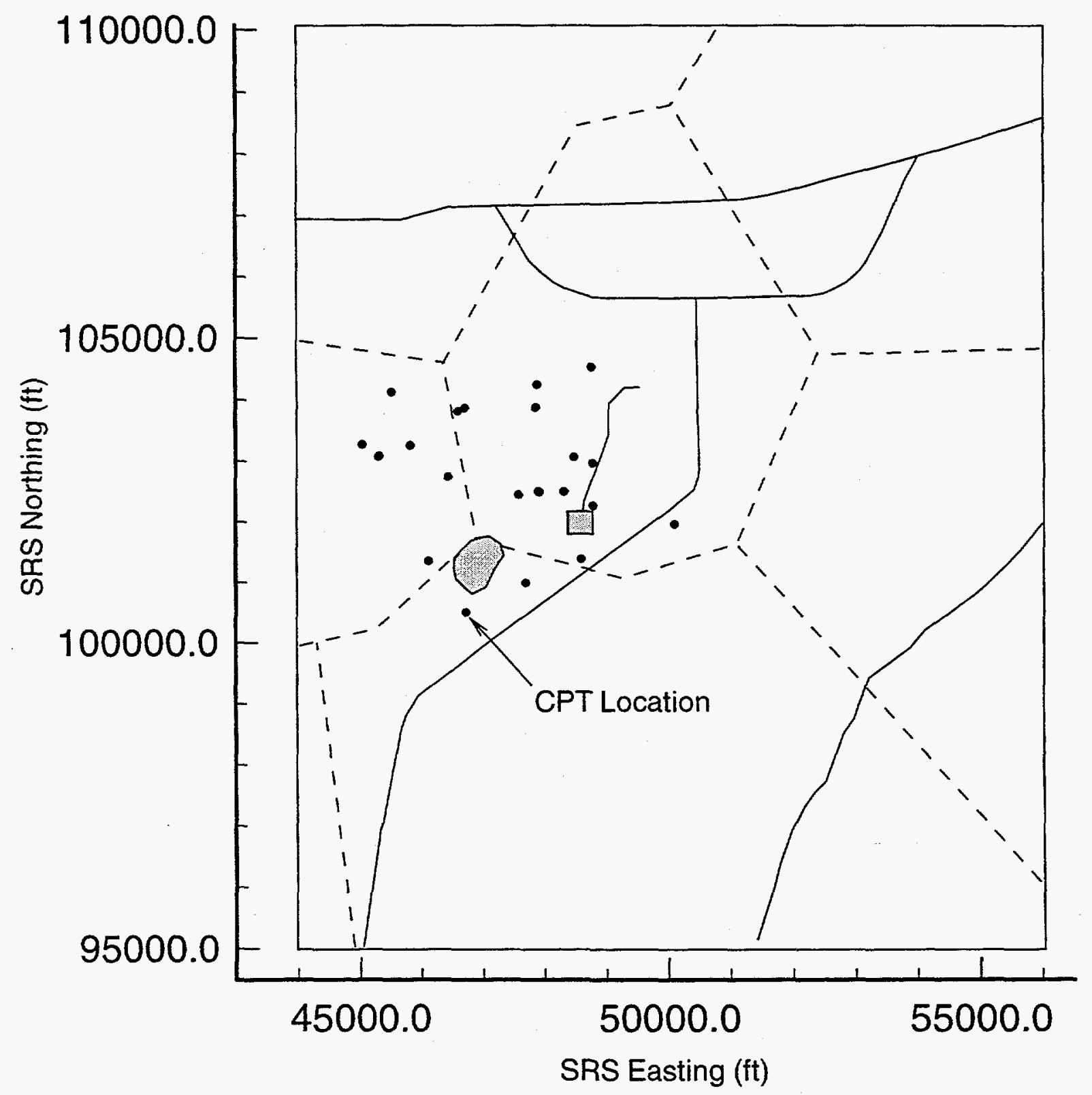

Figure 3.2 Location of the cone penetrometer tests. 
Table 3.2 Cone Penetrometer Data

\begin{tabular}{|c|c|c|c|c|c|}
\hline Name & $\begin{array}{l}\text { SRS Easting } \\
\text { (ft) }\end{array}$ & $\begin{array}{l}\text { SRS Northing } \\
\text { (ft) }\end{array}$ & $\begin{array}{c}\text { Bottom of } \\
\text { push } \\
\text { (ft) }\end{array}$ & $\begin{array}{l}\text { Top of } \\
\text { "green } \\
\text { clay" } \\
\text { (ft) }\end{array}$ & $\begin{array}{c}\text { Bottom of } \\
\text { "green } \\
\text { clay" } \\
\text { (ft) }\end{array}$ \\
\hline CPT-1 & 48761.45 & 104527.29 & 215.00 & & \\
\hline СРT-2 & 47884.75 & 04243.57 & 180.00 & 192. & 187. \\
\hline СРТ-3 & 45819.40 & 103251.70 & 180.00 & 187. & 185 . \\
\hline CPT-4 & 45512.11 & 104120.50 & 192.00 & 205. & 199. \\
\hline CPT-5 & 47863.29 & 103874.72 & 174.00 & 195. & 190. \\
\hline СРТ-6 & 48469.71 & 103064.78 & 207.00 & & \\
\hline СРТ-7 & 47586.21 & 102444.36 & 165.00 & 203. & 200 . \\
\hline СРТ-9 & 47696.68 & 100993.00 & 180.00 & 207. & 200. \\
\hline CPT-10 & 46714.64 & 100505.84 & 194.00 & 204. & 202. \\
\hline СРТ-11 & 46114.51 & 101349.24 & 184.00 & 198. & 195. \\
\hline CPT-12 & 45036.43 & 103267.58 & 190.00 & & \\
\hline CPT-13A & 45297.14 & 103066.76 & 210.00 & & \\
\hline CPT-13B & 45312.23 & 103083.19 & 180.00 & & \\
\hline CPT-14 & 46433.01 & 102736.28 & 190.00 & 198. & 195. \\
\hline CPT-15A & 48778.89 & 102963.77 & 208.00 & & \\
\hline CPT-17 & 50104.06 & 101955.15 & 190.00 & 206. & 200. \\
\hline CPT-19A & 48785.38 & 102265.49 & 204.00 & & \\
\hline CPT-20A & 47921.33 & 102488.19 & 232.00 & & \\
\hline CPT-20B & 47906.81 & 102500.20 & 240.00 & & \\
\hline CPT-21 & 48590.50 & 101383.04 & 192.00 & 209. & 202. \\
\hline CPT-22 & 48316.81 & 102495.85 & 238.00 & & \\
\hline CPT-23A & 46704.50 & 103862.03 & 230.00 & & \\
\hline CPT-23B & 46596.17 & 103812.47 & 184.00 & 205 . & 201. \\
\hline
\end{tabular}




\subsection{NUMERICAL, GEOSTATISTICAL, AND MEASUREMENT MODEL S}

\subsection{GROUNDWATER FLOW}

DFM incorporates a modified version of the HydroGeoLogic, Inc., variably saturated flow and transport code VAM3DCG (Huyakorn and Panday (1992)). The modified code, called VAM3DF (Huyakorn and Panday(1995)), was specifically developed for DFM. Enhanced features of VAM3DF include:

- $\quad$ Nodal hydraulic soil properties.

- More efficient assembly of the variably saturated flow equation.

- Head-dependent flux boundary conditions.

When used for steady-state flow modeling within DFM, VAM3DF does not invert the flow equation to compute the steady-state heads (since this is done by DFM). Thus, the Orthomin and PCG solvers are not used. Rather, VAM3DF only computes the steady-state flow error at each node. Partial derivatives of the flow error with respect to estimated parameters are computed numerically by repeated calls to VAM3DF.

Adopting the assumptions of Huyakorn et al. (1984) and using hydraulic head as the dependent variable, the governing equation for water flow in a variably saturated porous medium can be written as

$$
\begin{aligned}
\frac{\partial}{\partial x_{i}}\left[K_{i j} k_{m} \frac{\partial h}{\partial x_{j}}\right] & =S_{w} S_{s} \frac{\partial h}{\partial t}+\phi \frac{\partial S_{w}}{\partial t}-q \quad i, j=1,2,3 \\
& =\eta \frac{\partial h}{\partial t}-q
\end{aligned}
$$

where $\eta=S_{w} S_{s}+\phi \frac{\partial S_{\psi}}{\partial h}$ and $\mathrm{h}$ is the hydraulic head, $\mathrm{K}_{\mathrm{ij}}$ is the saturated hydraulic conductivity tensor for the porous thedium, $\mathrm{k}_{\mathrm{rw}}$ is the relative permeability of water, $\mathrm{x}_{\mathrm{i}}$ represents an orthogonal coordinate system, $q$ is the volumetric flow rate (due to sources or sinks) per unit volume of the porous medium, $S_{w}$ is the water saturation, $S_{s}$ is the specific storage, and $\phi$ is the effective porosity.

The hydraulic head is related to the water saturation in the variably saturated zone by the moisture retention function

$$
S_{w}=S_{w}(\psi)
$$

where the pressure head $\psi$ is given as

$$
\psi=h-z
$$

and $z$ is the elevation above a given datum. The relative permeability of the unsaturated zone is a function of the water saturation [i.e., $k_{n v}=k_{n w}\left(S_{w}\right)$ ]. Appropriate initial and boundary conditions required to solve the governing equations, including evaporation, plant root uptake, and seepage faces, are discussed by Huyakorn et al. (1986). 
When the soil retention and relative permeability functions of a soil are unknown and the saturated zone moisture behavior is unimportant to the simulated scenario, the pseudo-soil relations may be used to predict the water table levels. VAM3DF utilizes simple linear relations to account for changes in element saturated thickness and relative permeability due to the transient movement of the water table in the aquifer.

These linear pseudo-soil moisture relations are given by

$$
S_{w}=1-\left(\left|\psi-\psi_{s}\right|\right) / \cdot b \geq 0.01
$$

and

$$
k_{r w}=S_{w}
$$

where $S_{w}$ is the saturation of the element defined such that $S_{w}=1$ when the water table is above the top of the element, and $S_{w} \approx 0$ when the water table is below the bottom of the element. $\Psi$ is the pressure head at the element centroid, which governs the pseudo-relation; $b$ is the average elemental thickness; and $\psi_{\mathrm{s}}=\mathrm{b} / 2$ is the pressure head scaling parameter. The pseudo-relative permeability function ensures that the vertically integrated transmissivity of the element is adjusted to its saturated thickness as in done in the solution to the vertically integrated areal 2-D aquifer flow equations.

The flow equation (4.1) is approximated in three-dimensional space using the Galerkin finiteelement method, resulting in a system of differential equations of the form

$$
A_{I J} h_{J}+B_{I J} \frac{d h_{j}}{d t}-F_{I}=0
$$

The Galerkin approximation process is detailed in Huyakorn et al. (1984). The global coefficient matrices $A_{\mathrm{IJ}}, \mathrm{B}_{\mathrm{IJ}}$, and $\mathrm{F}_{\mathrm{IJ}}$ are assembled as a sum of the element matrices for a general 8 noded orthogonal curvilinear element. Influence coefficient techniques presented by Huyakorn et al. (1984) and Huyakorn et al. (1986) can be effectively used for assembling 8 noded prism elements in order to avoid integration of each of the element level matrices. They are presented here as

$$
\begin{aligned}
A_{I J}^{e} & =<K_{x x}^{\prime} k_{n w}>\frac{m H}{2 \ell}\left[A_{I J}^{x x}\right]^{e}+<K_{y y}^{\prime} k_{r w}>\frac{\ell H}{2 m}\left[A_{I J}^{y y}\right]^{e} \\
& +<K_{z z}^{\prime} k_{n w}>\frac{\ell m}{2 H}\left[A_{I J}^{z z}\right]^{e}+<K_{x y}^{\prime} k_{n w}>\frac{H}{2}\left[A_{I J}^{x y}\right]^{e} \\
& =<K_{y z}^{\prime} k_{n w}>\frac{\ell}{2}\left[A_{I J}^{y z}\right]^{e}+<K_{z x}^{\prime} k_{r w}>\frac{m}{2}\left[A_{I J}^{z x}\right]^{e}
\end{aligned}
$$




$$
B_{I J}^{e}=\frac{\ell m H}{8}<\eta>\left[M_{I J}\right]^{e}
$$

where $A_{I J}^{e}$ is the seepage influence matrix; $B_{I J}^{e}$ is the storage matrix; $\mathrm{F}^{e}$ represents all elemental sources, sinks and other boundary conditions; and $\eta$ represents the storage term of equation (4.1), represented in the original mixed form of the equation. The superscript $e$ in equations (4.2) and (4.3) represents an element level matrix; $\mathrm{m}, \ell$, and $\mathrm{H}$ are the mean lengths of the element in its local $x_{i}{ }^{\prime}(i=1,2,3)$ coordinate system, and the absolute conductivities are represented in the local coordinate system. The quantities in angular brackets refer to centroidal values of the respective hydraulic properties and the seepage influence coefficient matrices $\left[A_{I J}^{x x}\right]^{e},\left[A_{I J}^{y v}\right]^{e},\left[A_{I J}^{z z}\right]^{e},\left[A_{I J}^{x y}\right]^{e}$, $\left[A_{I J}^{y z}\right]^{e}$, and $\left[A_{I J}^{z x}\right]^{e}$ may have a 7-point, an 11-point, or a 27-point connectivity in three dimensions.

\subsection{GEOSTATISTICAL}

DFM uses geostatistical models for spatial variability in order to interpolate and to combine information on different scales of variability. For example, DFM uses geostatistical models in the $\mathrm{A} / \mathrm{M}$ Area to delineate hydrostratigraphy and to perform model calibration. Well and cone penetrometer picks are interpreted as hydrostratigraphic layers using geostatistical models for interpolation. Hydraulic conductivity data on a local scale and hydraulic head data on a larger scale are combined using geostatistical models for the three-dimensional spatial variation of hydraulic conductivity within each hydrostratigraphic layer.

DFM uses nested structures for spatial variability at different scales as described by Gibbs and Hughes (1995). At the observational scale, measurement noise and microstructure appear as white noise. At intermediate scales larger than observational but smaller than the aquifer, spatial variability is modeled as a stationary process using the Markov Random Field (MRF). At the aquifer scale, nonstationary effects are taken into account with a deterministic trend having unknown coefficients that are estimated in DFM. The distinctions between scales are problem and objective dependent. If there are too few terms in the trend, then the Markov process will be forced to account for nonstationary behavior for which it is not well suited. On the other hand, too many terms in the trend leads to familiar problems with overparameterization. Our experience has been that decisions about problems of scale are a natural and reasonably straightforward part of modeling conceptual iteration.

Correlated spatial variability is modeled using state variables that are the sum of a spatial polynomial trend and an MRF. DFM estimates the trend polynomial coefficients as well as the random variation. For example, for a two-dimensional model using a first order autoregression and second order polynomial:

$$
\begin{gathered}
s_{i j}=p_{i j}+v_{i j} \\
p_{i j}=c_{0}+c_{1} x_{i}+c_{2} y_{j}+c_{3} x_{i}^{2}+c_{4} y_{j}^{2}+c_{5} x_{i} y_{j}
\end{gathered}
$$




$$
v_{i j}=a\left(v_{i+1, j}+v_{i-1, j}\right)+b \cdot\left(v_{i, j+1}+v_{i, j-1}\right)+w_{i j}
$$

where $s_{i j}$ is the state at position $x_{i}, y_{j} ; p_{i j}$ is the trend evaluated at position $x_{i}, y_{j} ; v_{i j}$ is the MRF at $\mathrm{x}_{\mathrm{i}}, \mathrm{y}_{\mathrm{j}} ; \mathrm{c}_{\mathrm{i}}$ are the polynomial coefficients; and $\mathrm{a}, \mathrm{b}$ are the autoregressive coefficients. If $\mathrm{a}=\mathrm{b}$, then this is isotropic; otherwise it is anisotropic. $w_{i j}$ is the white noise input with zero mean and variance computed from specified variance on $v_{\mathrm{i}}$.

The autoregressive coefficients ( $\mathrm{a}$ and $\mathrm{b}$ ) indicate the extent of the surrounding states influence on the value at a given state. As the grid points are taken closer together, the coefficients will be larger. In DFM, the autoregressive coefficients are computed internally from other inputs (Yancey (1994)).

There are two inputs relating to the spatial variability model that the user supplies. The spatial variability model has a spatial autocorrelation function which is exponential in three dimensions but deviates from exponential in one or two dimensions (see Figure 4.1). In two dimensions, except near zero, we have

$$
E\left[\left(v\left(x_{1}, y_{1}\right)-\bar{v}\left(x_{1}, y_{1}\right)\right)\left(v\left(x_{2}, y_{2}\right)-\bar{v}\left(x_{2}, y_{2}\right)\right)\right] \approx \sigma^{2} \exp \left\{-\frac{\sqrt{\left(x_{1}-x_{2}\right)^{2}+\left(y_{1}-y_{2}\right)^{2}}}{\tau}\right\}
$$

where the average value $\bar{v}$ is obtained from the polynomial (4.5). The user must input $\tau$ which is the distance at which the correlation coefficient falls to $1 / \mathrm{e}=0.37$ (possibly different for different directions), and input $\sigma$ which is the standard deviation of the output of (4.6).

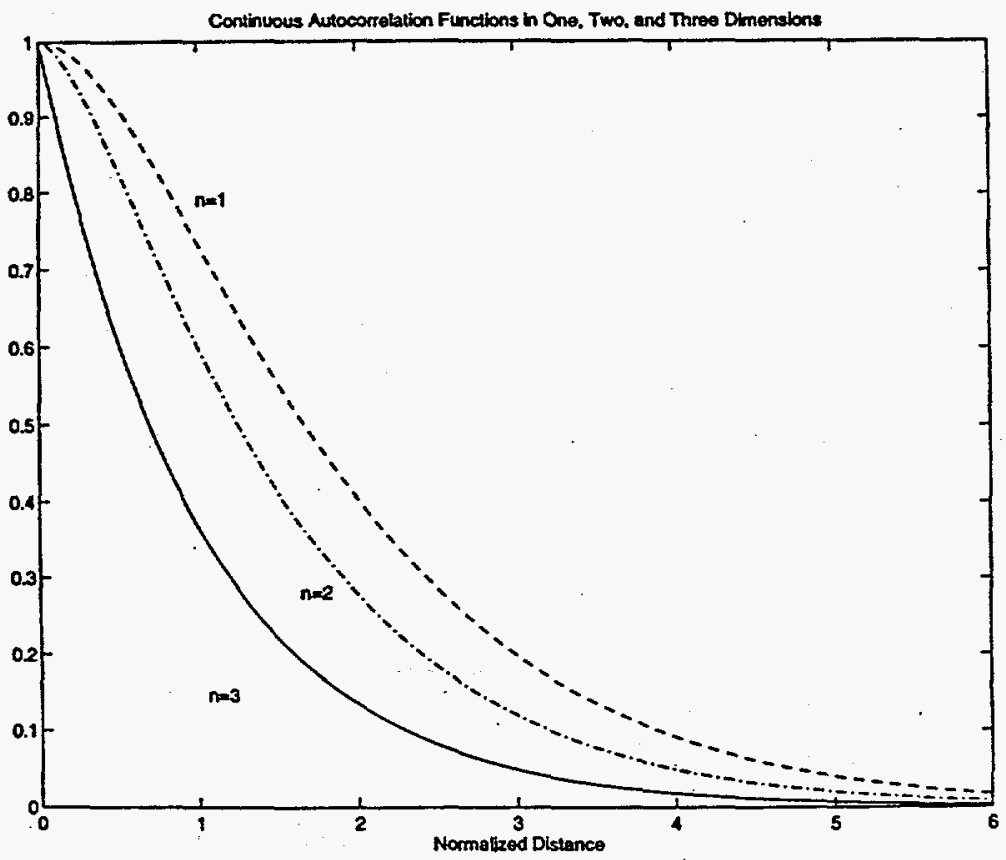

Figure 4.1 Continuous autocorrelation functions in one, two, and three dimensions. 
Ideally, the correlation distance $\tau$ and variance $\sigma^{2}$ should be chosen from statistical analysis of data. However, the data may not be initially available or may not be in a form suitable for easy interpretation. Thus the scientists' and engineers' prior insight and experience must often be used to select initial $\tau$ and $\sigma^{2}$. As additional data are acquired, $\tau$ and $\sigma^{2}$ should be updated based on residuals. The statistical likelihood function is computed by fusion. Statistically optimal maximum likelihood parameter estimates are found by selecting $\tau$ and $\sigma^{2}$ to maximize the likelihood.

Grid spacing should be selected to accommodate the correlation distance. It is expected that the ratio of correlation distance to grid spacing should be greater than 1.0. Note that the fusion software internally computes the relationship between the output variance, the correlation distances, the autoregressive coefficients, and the white noise input variance.

The user should also note that very small $\sigma$ 's can be used to constrain parameters in some situations. It might be easier to use a small $\sigma$ in a test fusion run, rather than to change all the input required to delete the parameter from the estimated states.

\subsection{DFM MEASUREMENT MODELS}

The geological DFM measurement model used for 2D layered-earth models (e.g. section 5.0) is simply linear interpolation of the state values at the four nodes surrounding the measurement location and contained in the same model layer. This interpolation involves linearly interpolating in the $\mathrm{y}$-direction at the two $\mathrm{x}$-values, and then linearly interpolating in $\mathrm{x}$. The appropriate layer is usually determined by the layer number specified on the measurement file, although other options are available for some measurement types. If the measurement type is "elevation" or "depth from the reference elevation" and the state at the specified layer is "layer thickness", the interpolations must be added across layers to obtain "elevation" or "depth".

The measurement model for 3D hydraulic conductivity is based on the same method. That is, it is basically a $2 \mathrm{D}$ interpolation. For $\log$ horizontal conductivity measurements $\ln (\mathrm{Kh})$, the model layer closest to the average well screen elevation (specified on the measurement file) is first calculated. Then the $2 \mathrm{D}$ interpolation described above is used to calculate the prediction of the measurement based on current model states. Since $\ln (\mathrm{Kh})$ is a nodal state in standard DFM, the interpolation is direct. Multi-grid DFM also stores $\ln (\mathrm{Kh})$ at each node, but this is interpolated from the coarse grid.

The computation is somewhat more complex for log vertical conductivity measurements $\ln (\mathrm{Kv})$, since this is not a nodal state in the model. Each of the four nodes surrounding the measurement are checked to determine if they lie within a zone in which $\ln (\mathrm{Kv})$ is directly specified. For nodes that are in $\ln (\mathrm{Kv})$ zones, those values are used. For other nodes, $\ln (\mathrm{Kv})$ is computed as $\ln (\mathrm{Kh})-\ln (\mathrm{Kh} / \mathrm{Kv})$ where $\ln (\mathrm{Kh})$ at the node is used and $\ln (\mathrm{Kh} / \mathrm{Kv})$ is evaluated from spatial polynomial coefficients. In all cases, the partial derivatives of the measurements with respect to the states are computed using a combination of analytic calculations plus the product rule (for the interpolation).

Calculation of the predicted head measurements is similar to that for the $\ln (\mathrm{Kh})$ measurements, since head is a nodal state, but involves interpolation in three dimensions. The average screen 
elevation listed on the measurement file should be the average of the minimum(well screen top, head elevation) and the screen bottom. The model layers above and below this elevation are used to interpolate the nodal head values. More accurately, the model head should be computed by integrating from the well screen bottom to top (or water level) using 3D interpolation of values from nodes surrounding the well. It is believed that the error resulting from the use of linear interpolation is small compared to the random measurement error.

The DFM software also has the ability to estimate biases on the various measurement types. This bias is added to the computed value to generate the predicted measurement, i.e. it represents the measured value minus the true value.

\subsection{MUD FRACTION TO LOG CONDUCTIVITY MAPPING}

In order to produce conductivity data at the core locations, the foot-by-foot mud fraction data must be transformed into horizontal and vertical conductivity data at nodes of the numerical grid. A mud fraction to log conductivity mapping was constructed which takes in account the available pump tests and laboratory permeability tests.

The functional form of the mud fraction to log conductivity mapping is a continuous piecewise linear function given by:

$$
f(x)= \begin{cases}P_{1} & \text { for } 0 \leq x \leq x_{1} \\ P_{1}-P_{2}^{2}\left(x-x_{1}\right) / \Delta x & \text { for } x_{1}<x \leq x_{2} \\ P_{1}-P_{2}^{2}-P_{3}^{2}\left(x-x_{2}\right) / \Delta x & \text { for } x_{2}<x \leq x_{3} \\ P_{1}-\sum_{i=2}^{m} P_{i}^{2} & \text { for } x_{m}<x \leq 1\end{cases}
$$

where $f$ is $\log$ conductivity, $x$ is mud fraction, $P_{1}$ through $P_{m}$ are parameters to be estimated, and $\Delta \mathrm{x}=\mathrm{x}_{\mathrm{i}}-\mathrm{x}_{\mathrm{i}-1}, \mathrm{i}=2, \ldots, \mathrm{m}$. The function $f$ is a decreasing function with $\mathrm{f}^{\prime}(0.0)=\mathrm{f}^{\prime}(1.0)=0.0$. The parameters $P_{1}$ to $P_{m}$ are found by solving a nonlinear least squares problem which minimizes

$$
\chi^{2}=\sum_{i=1}^{n}\left(\log \left(K_{i}\right)-\log \left(\bar{K}_{i}\right)\right)^{2}
$$

where $K_{i}$ is the conductivity derived from the $\mathrm{i}$ th pump test or laboratory permeability test, $\bar{K}_{i}$ is the average (arithmetic or harmonic) of $10^{f\left(x_{i}\right)}, \ldots, 10^{f\left(x_{j}\right)}$, where $x_{1} \ldots x_{j}$ is the mud fraction data corresponding to the $i$ th test. For a laboratory test, $j=1$, since laboratory tests are point data. Pump tests are integral data that estimates the transmissivity of an aquifer zone and the leakance of the overlying aquitard. For a pump test, mud fraction data $x_{1}, \ldots, x_{j}$ corresponds to the aquifer zone where the test was performed or the overlying aquitard. An arithmetic average is used for transmissivity data and a harmonic average is used for leakance data. 
The Hiergesell aquifer pumping test reports contain 14 pump test data. The reports contain transmissivity data at the following wells: ASB32C, MSB89B, RWM12, RWM13B, RWM14B, RWM16PA, and RWM16PB. The reports contain leakance data at the following wells: MSB32B, MSB32C, MSB89B, RWM12, RWM16PA, and RWM16PB. Riha (1993) presents 11 estimates of horizontal conductivity from IDS.

The Levenberg-Marquardt method (LMM) was used to minimize $\chi^{2}$ with $\mathrm{m}=6, \mathrm{x}_{1}=0.08$, and $\Delta x=0.143$. The initial guess of the parameters was $\left(P_{1}, P_{2}, P_{3}, P_{4}, P_{5}, P_{6}\right)=(1.0,0.1,0.5,0.5$, $0.5,0.1)$. The $L M M$ needed 5 iterations to converge to $\left(\mathrm{P}_{1}, \mathrm{P}_{2}, \mathrm{P}_{3}, \mathrm{P}_{4}, \mathrm{P}_{5}, \mathrm{P}_{6}\right)=(0.99,1.6$, $0.89,0.79,0.48,0.54)$ with $\chi^{2}=28.2$. Recall that the no recovery zones of the mud fraction data were replaced with a $3 \%$ mud fraction. Since $x_{1}=0.08$, the fit is not sensitive to any mud fraction less than or equal to $8 \%$. Figure 4.2 shows the resulting fit and Figure 4.3 shows the residuals. Since the pump tests gave higher estimates of conductivity than laboratory tests, the fit is lower than most of the pump test conductivity data and higher than most of the laboratory conductivity data.

The process for translating mud fraction data into horizontal and vertical conductivity measurements is shown in Figure 4.4. The above mapping is used to convert foot-by-foot mud fraction data to foot-by-foot conductivity data, which is then scaled-up to the numerical grid.

A given core penetrates several numerical cells and the top and bottom of each cell divides the foot-by-foot conductivity data into sections along the core length. Each section of conductivity data is averaged arithmetic and harmonic to produce horizontal and vertical conductivity values, respectively. The horizontal and vertical conductivity values are used as conductivity measurements at the core location with an elevation equal to the average of the top and bottom of the numerical cell. 


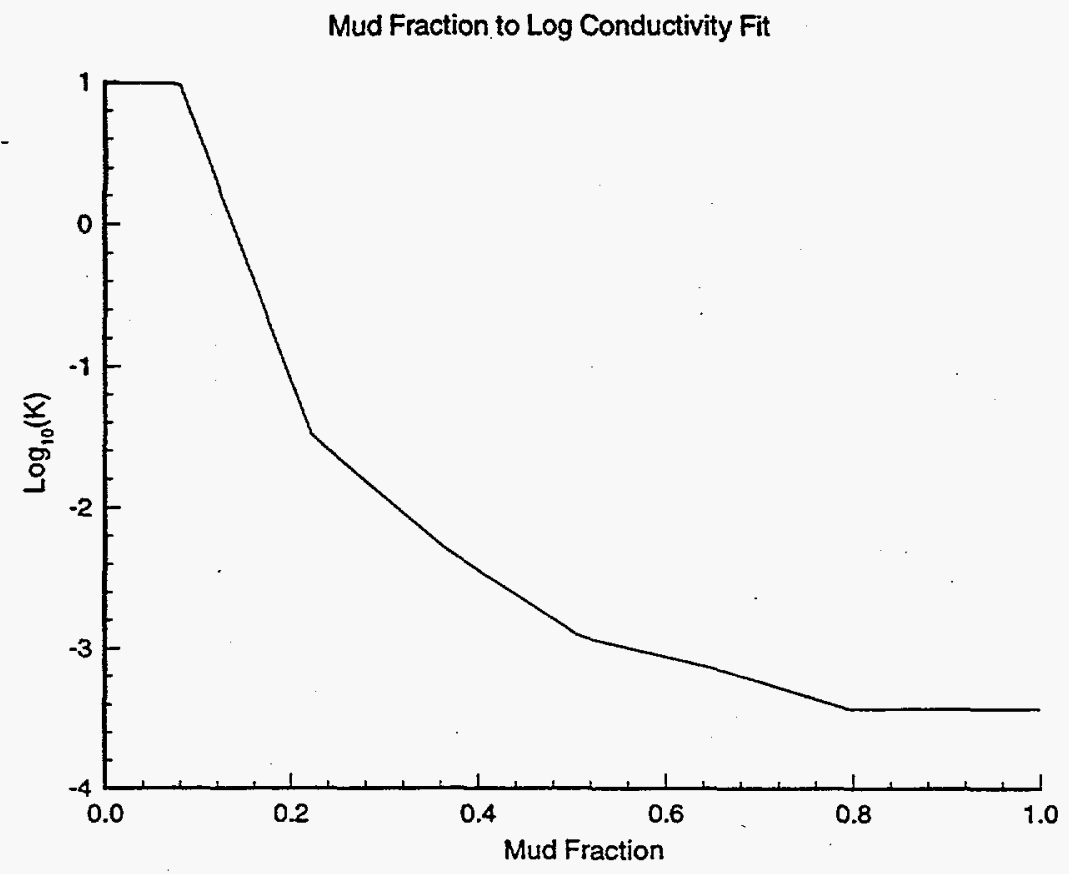

Figure 4.2 Mud fraction to log conductivity mapping.

Residual Plot

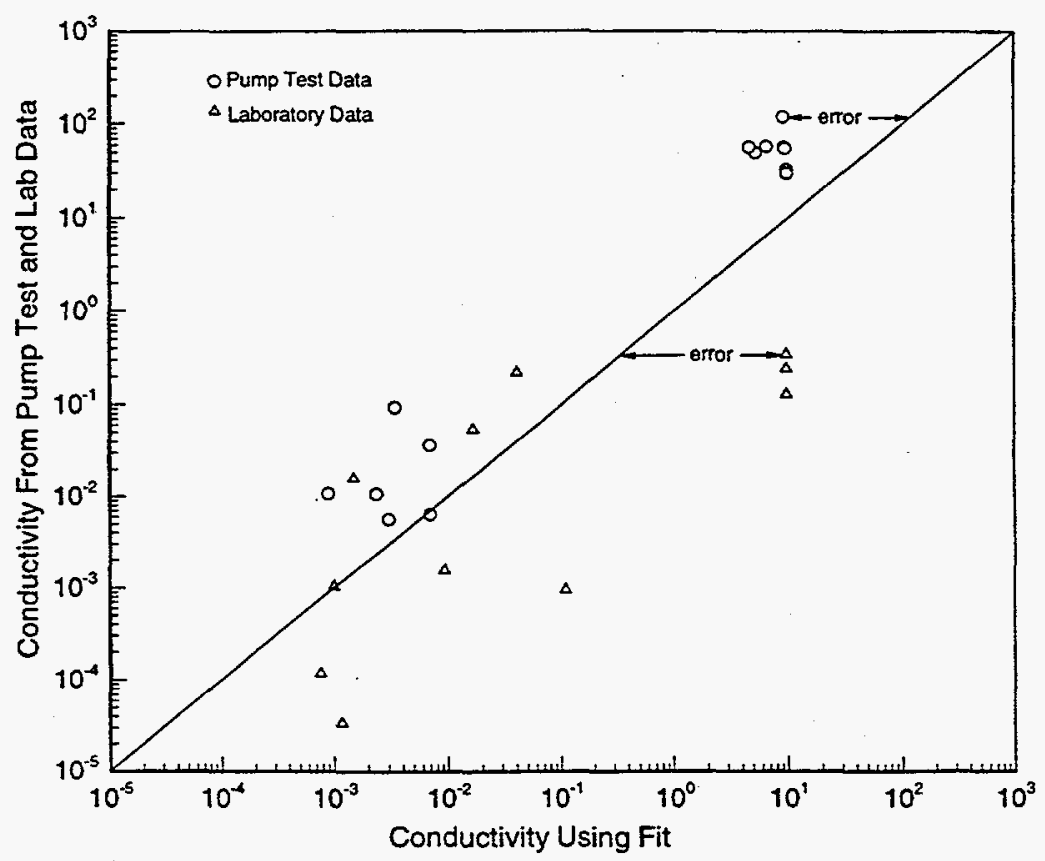

Figure 4.3 Residual plot for mud fraction to log conductivity fit. 


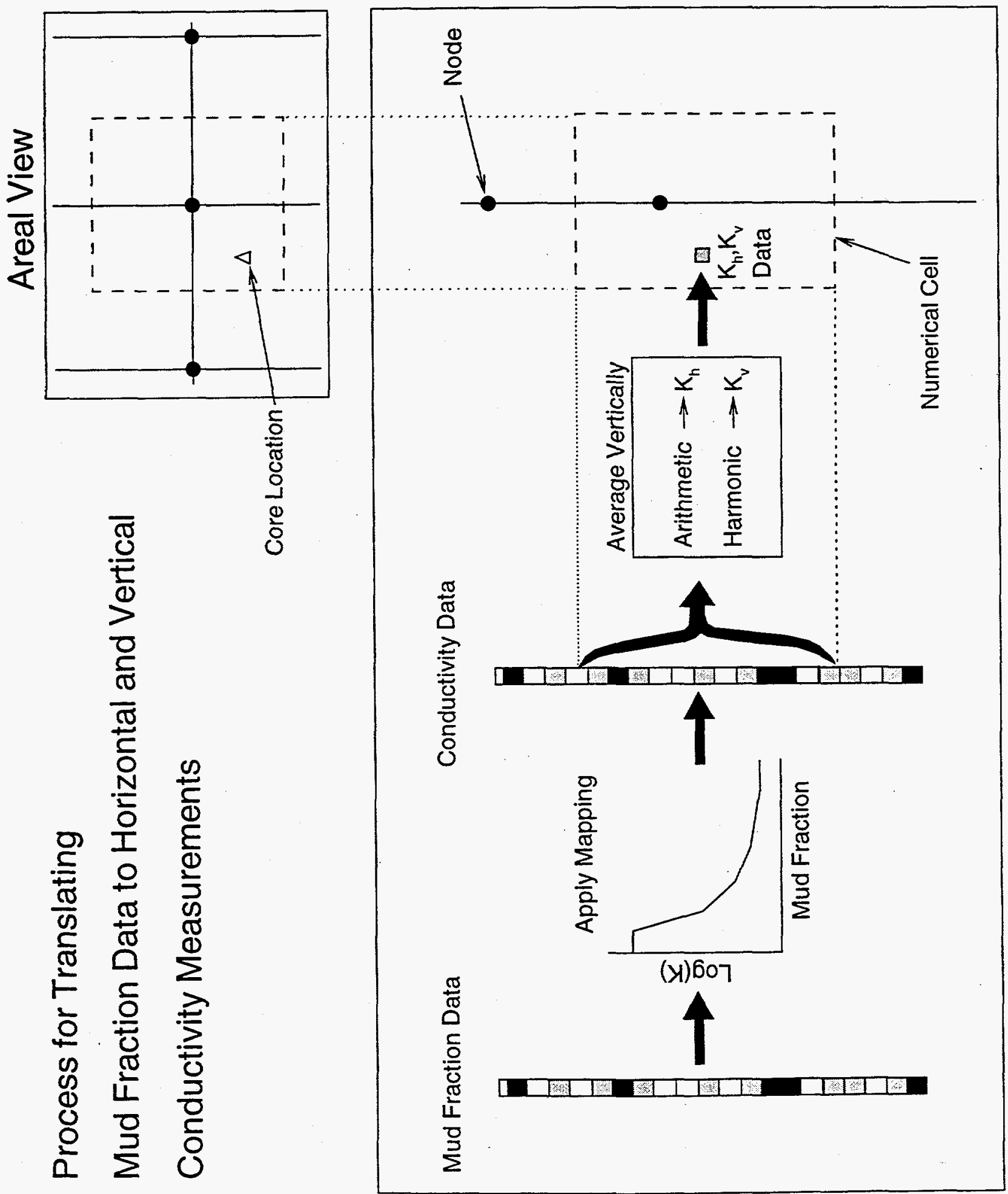

Figure 4.4 Process of translating mud fraction data to horizontal and vertical conductivity measurements. 


\subsection{DELINEATION OF HYDROSTRATIGRAPHIC UNITS USING DFM}

The goal of this effort was to develop a fully $3 \mathrm{D}$ hydrological model of the $\mathrm{A} / \mathrm{M}$ area where the hydraulic conductivity was treated as a heterogeneous field, computed from the core mud fraction data. This is in contrast to previous efforts which defined the model in hydrostratigraphic units. Although it would appear that there is no need to define any hydrostratigraphic units if conductivity is treated as a heterogeneous field, specification of these units can significantly improve the DFM estimates. If the entire domain is modeled as a single heterogeneous unit, the random field model must account for the significant discontinuities in conductivity which occur at the interfaces between the three "clays" ("green", "upper" and "lower") and the three "aquifers" ("M-Area", "Lost Lake" and "middle sand"). The first-order autoregressive model used in DFM will smooth these discontinuities. Thus the DFM process will be significantly aided if the conductivity is modeled as heterogeneous within each unit but independent between units. The trend models should also be modeled separately.

The primary disadvantage of this approach is the possible error introduced by incorrectly specifying the unit elevations, or in specifying unit locations where properties change (e.g. holes in clay layers, clay lenses within aquifers): However, this problem should not be great if the random field model is given enough flexibility to locally adjust the parameters where measurements indicate. In other words, it is better to give DFM an approximately correct structure (a framework for the model) rather than to give it no initial structure at all.

The data available for defining the unit top and bottom elevations consists of 341 layer picks as determined from borehole cores (modified from Aadland et al. (1995)), supplemented with 28 picks obtained from cone penetrometer picks for the "Green Clay" Confining Zone in the central region. This data is listed in Appendix B and Table 3.2. These layer elevations were input to the 2D geological DFM which models either layer depth, elevation or thickness as a random field plus trend. It was decided to estimate the top elevations of the "Green Clay" and "upper clay" zones, and the thicknesses of the "Green Clay", "upper clay", "middle sand" and "lower clay" zones. The choice of estimating layer elevations or thicknesses depends upon how the geologic unit was formed and on the thickness of the unit. Since the clay zones are relatively thin, it is better to estimate the unit thicknesses because DFM can constrain the thicknesses to be greater or equal to zero and because the zone top and bottom elevation will be highly correlated. Thus specification of the thickness as a trend plus spatially correlated random component is appropriate. The same argument also applies to the "middle sand" zone. However, the distances between the surface, the "Green Clay" top and the "upper clay" top are sufficiently large that these two zones are more probably independent than correlated. Thus the top elevations were estimated in DFM as a trend plus correlated random component. In all cases, the trend was specified as a bias plus linear $\mathrm{x}$ and $y$ terms, because of the significant slope to the area.

Table 5.1 lists the a prior trend coefficients and 1-sigma uncertainty used in DFM while Table 5.2 lists the 1-sigma and $\mathrm{x}$ and $\mathrm{y}$ correlation distances which define the random component. All values are in feet. 
Table 5.1 Prior Estimate and Uncertainty of Zone Trend Parameters

\begin{tabular}{|c|c|c|c|}
\hline $\begin{array}{c}\text { Parameter } \\
\text { elevation }\end{array}$ & Coefficient & Prior Value & Prior 1-sigma \\
\hline & bias & 220 & 20 \\
\hline & linear $\mathrm{x}$ & 0 & 10 \\
\hline green clay thickness & linear $\mathrm{y}$ & 0 & 10 \\
\hline & bias & 10 & 10 \\
\hline & linear $\mathrm{x}$ & 0 & 3 \\
\hline & linear $\mathrm{y}$ & 0 & 30 \\
\hline upper clay elevation & bias & 140 & 10 \\
\hline & linear $\mathrm{x}$ & 0 & 10 \\
\hline & linear $\mathrm{y}$ & 0 & 10 \\
\hline $\begin{array}{c}\text { upper clay } \\
\text { thickness }\end{array}$ & bias & 10 & 3 \\
\hline & linear $\mathrm{x}$ & 0 & 3 \\
\hline & linear $\mathrm{y}$ & 0 & 10 \\
\hline middle sand \\
thickness & bias & 20 & 3 \\
\hline & linear $\mathrm{x}$ & 0 & 3 \\
\hline & linear $\mathrm{y}$ & 0 & 10 \\
\hline lower clay \\
thickness
\end{tabular}

Table 5.2 Zone Parameters Random Model

\begin{tabular}{|c|c|c|c|}
\hline Parameter & 1-sigma & Correlation Distance - $\mathbf{x}$ & Correlation Distance - y \\
\hline $\begin{array}{c}\text { green clay top } \\
\text { elevation }\end{array}$ & 10 & 1500 & 1500 \\
\hline green clay thickness & 4 & 1500 & 1500 \\
\hline $\begin{array}{c}\text { upper clay top } \\
\text { elevation }\end{array}$ & 10 & 1500 & 1500 \\
\hline upper clay thickness & 4 & 1500 & 1500 \\
\hline middle sand thickness & 5 & 1500 & 1500 \\
\hline lower clay thickness & 4 & 1500 & 1500 \\
\hline
\end{tabular}


The model grid was 16000 feet in $\mathrm{x}$ and 15000 in $\mathrm{y}$ with a spacing of 250 feet ( 65 columns and 61 rows) where the origin was located at $x=40000$ and $y=95000$ in SRS site coordinates. The measurement standard deviation used to weight the measurements was 2 feet for well picks and 3 feet for CPT data. The model computes the predicted measurement by linearly interpolating the four nodes surrounding the measurement location, as explained in section 4.3.

Since the measurement and spatial continuity models are both linear, the DFM estimation should converge in one iteration. However, DFM required two iterations because one measurement was edited and the editing logic is nonlinear. In initial use of DFM, several measurements were edited, but it was found that these measurements were erroneous and were corrected.

The measurement residual RMS for the converged DFM estimate was 2.37 feet. The sum-of-squares for the three components of the cost function were 40 for the 18 prior trend coefficients, 443 for the 23790 spatial continuity nodes, and 476 for the 363 measurement residuals. Table 5.3 presents the residual RMS of each layer.

Figures 5.1 to 5.6 show the estimated unit surfaces and Tables 5.4-5.9 present measurements and residuals for each surface. Figure 5.7 shows the total thickness of the Crouch Branch Confining Unit. Figures 5.8 to 5.11 show several $x-z$ slices with the measurements and grid lines within $+/-300$ feet in y shown. Tables 5.10-5.13 present measurements and residuals for each $\mathrm{x}-\mathrm{z}$ slice. The surfaces generally follow the data except that the CPT data consistently shows the "Green Clay" Confining Zone to be thinner than the well picks. Since the well data is thought to be the more accurate, this was given more weight. Note that the "Green Clay" Confining Zone never thins out in the north as some references suggest - the data used here seems to indicate a relatively constant thickness, although a distinct facies change occurs. Also note that the "middle sand" zone almost disappears in the west and at several locations in the middle.

SRS personnel have indicated that the "Green Clay" Confining Zone elevation shown in Figure 5.1 is the only computed surface they have seen for which a gradient descent along the surface will generate pathlines starting at a known DNAPL (TCE) source and reach a location at which the DNAPL is believed to be present.

To summarize, the computed lithological layer elevations and thicknesses are believed to be reasonable bounds for defining the hydrostratigraphic units.

Table 5.3 Total Residual RMS by Layer

\begin{tabular}{|c|c|c|}
\hline Layer Number & Number of Data & RMS (ft) \\
\hline 1 & 99 & 3.0 \\
\hline 2 & 100 & 1.7 \\
\hline 3 & 73 & 1.6 \\
\hline 4 & 41 & 2.5 \\
\hline 5 & 33 & 3.2 \\
\hline 6 & 17 & 1.9 \\
\hline
\end{tabular}




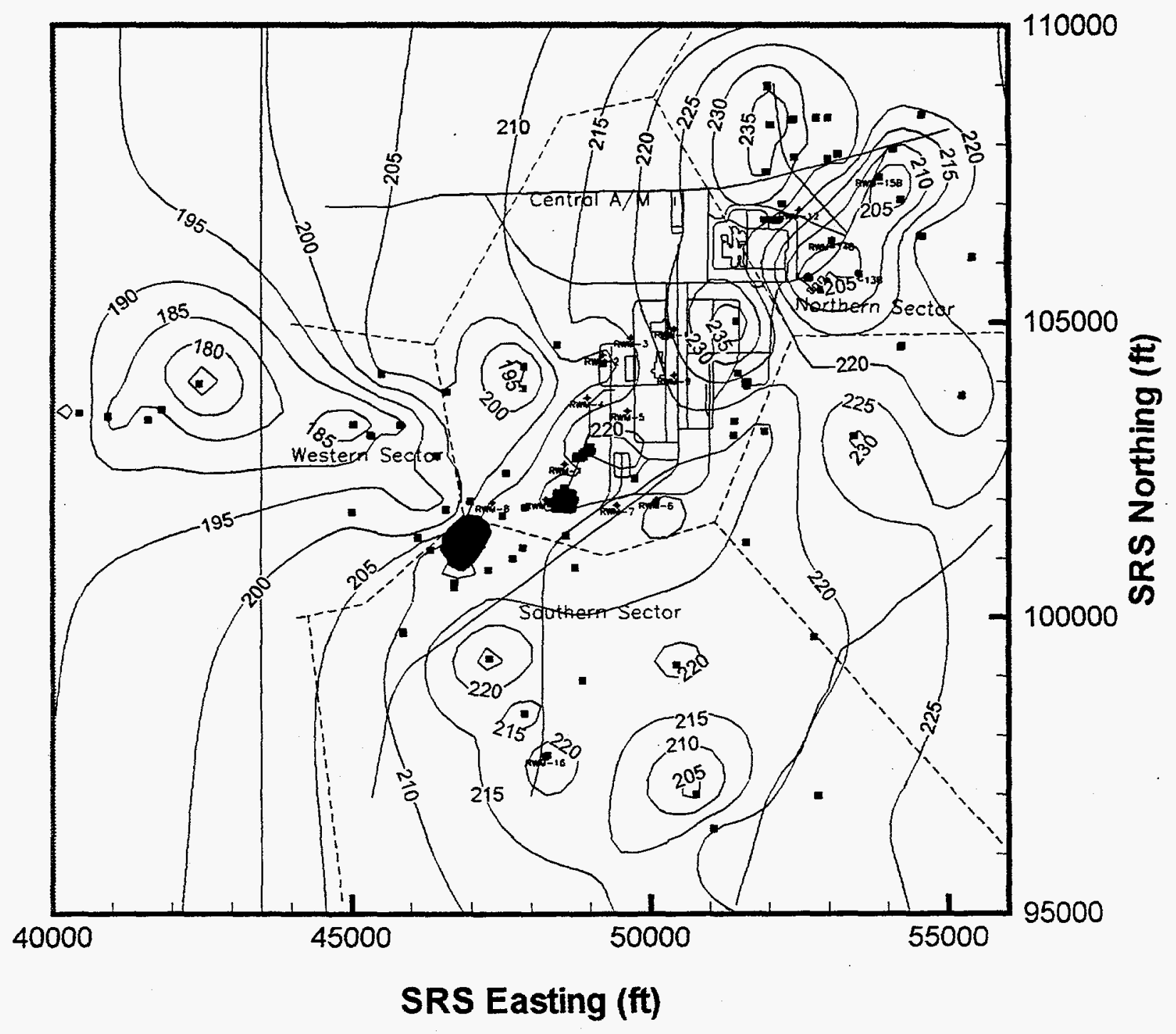

Figure 5.1- DFM Estimated Top Elevation (ft) of the "green clay" 


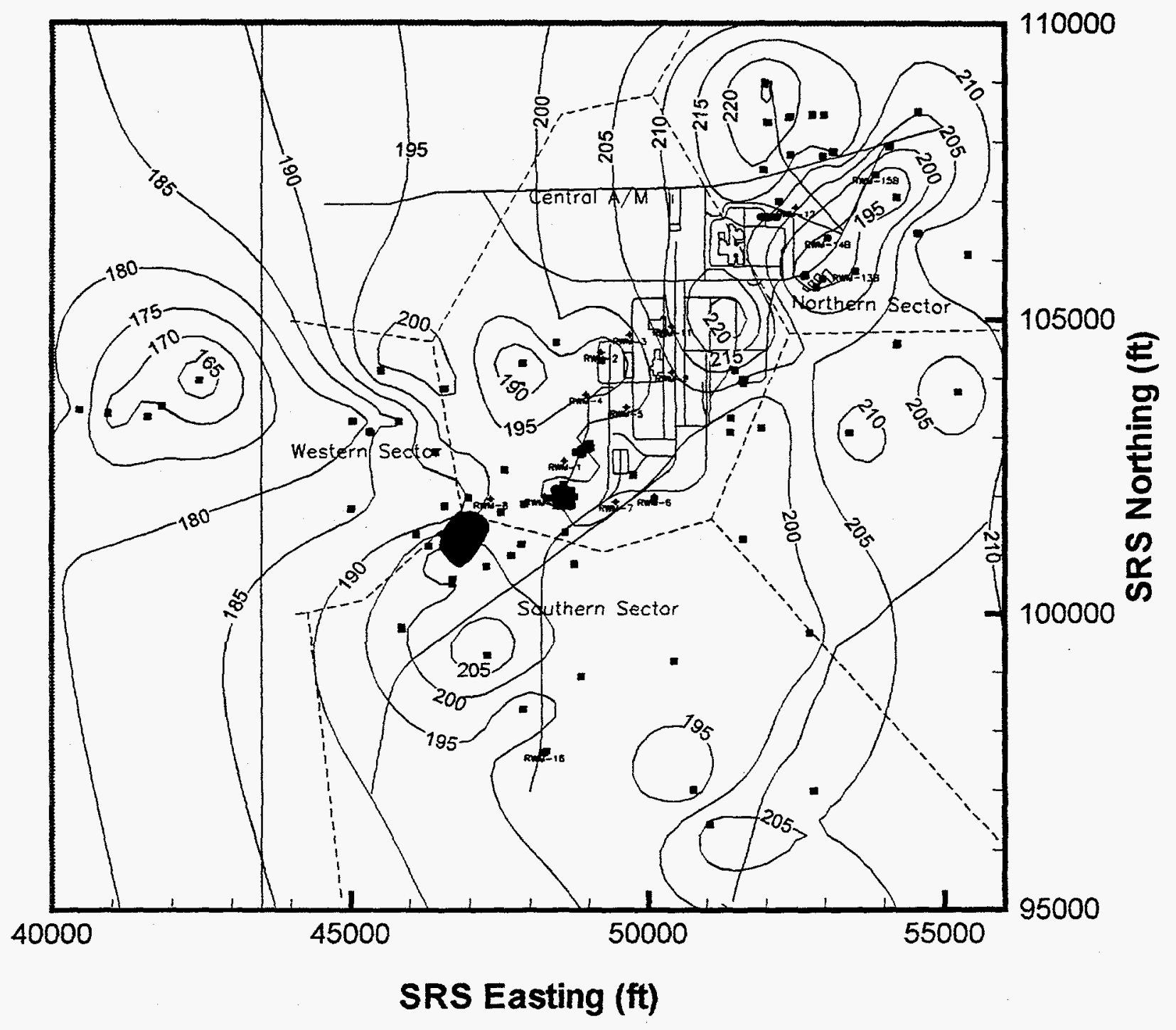

Figure 5.2- DFM Estimated Bottom Elevation (ft) of the "green clay" 


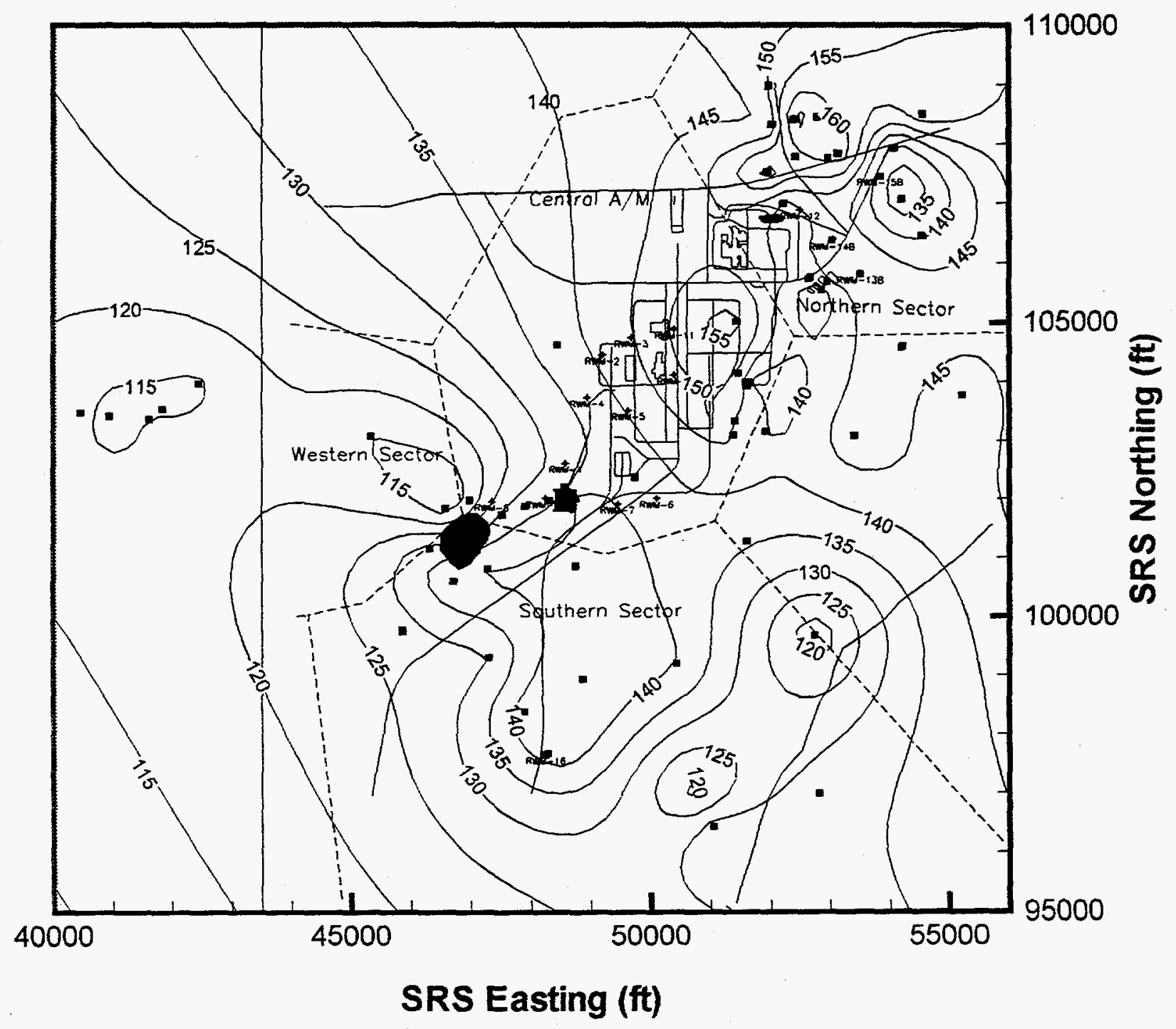

Figure 5.3- DFM Estimated Top Elevation ( $\mathrm{ft}$ ) of the "upper clay" 


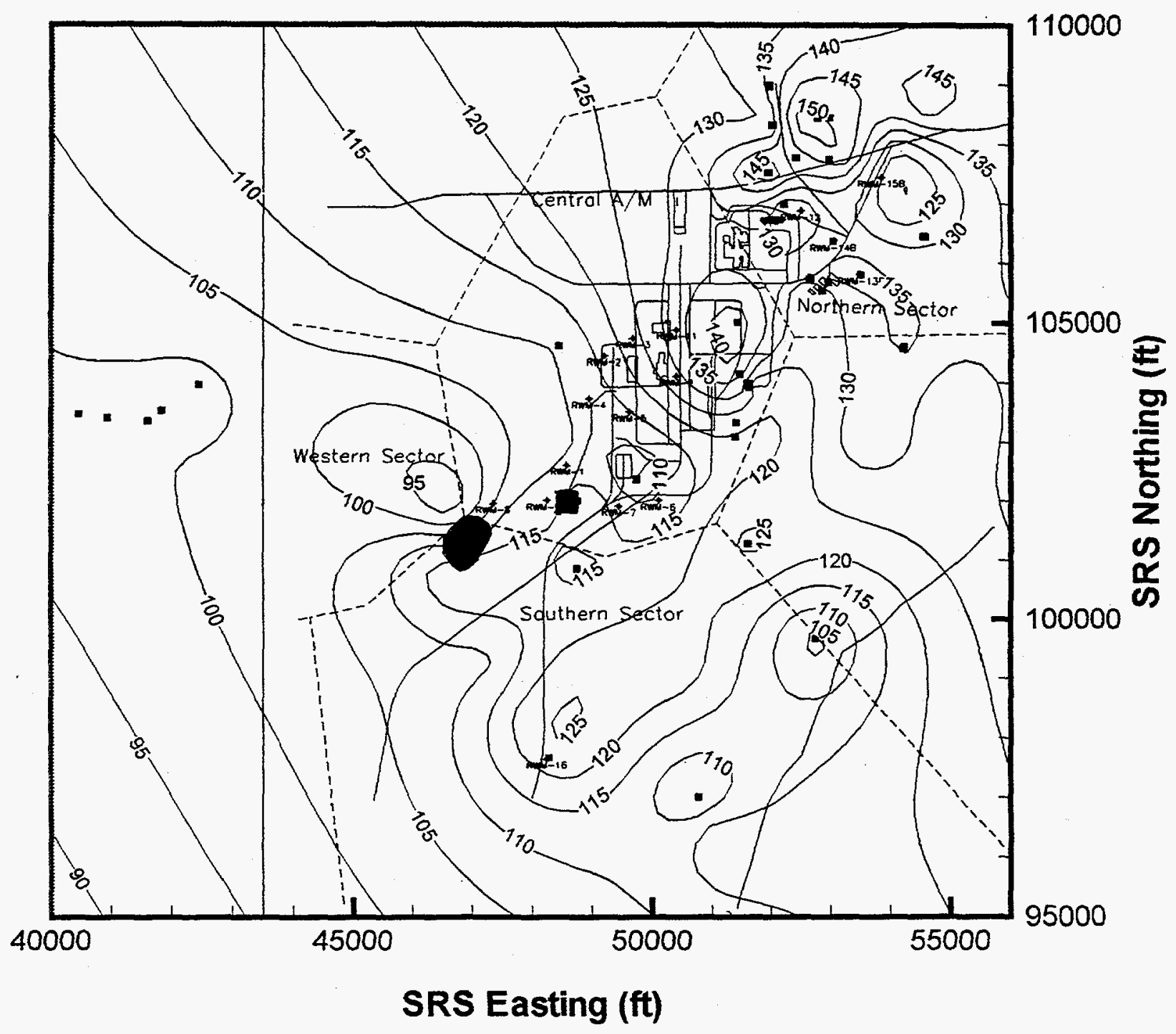

Figure 5.4- DFM Estimated Bottom Elevation (ft) of the "upper clay" 


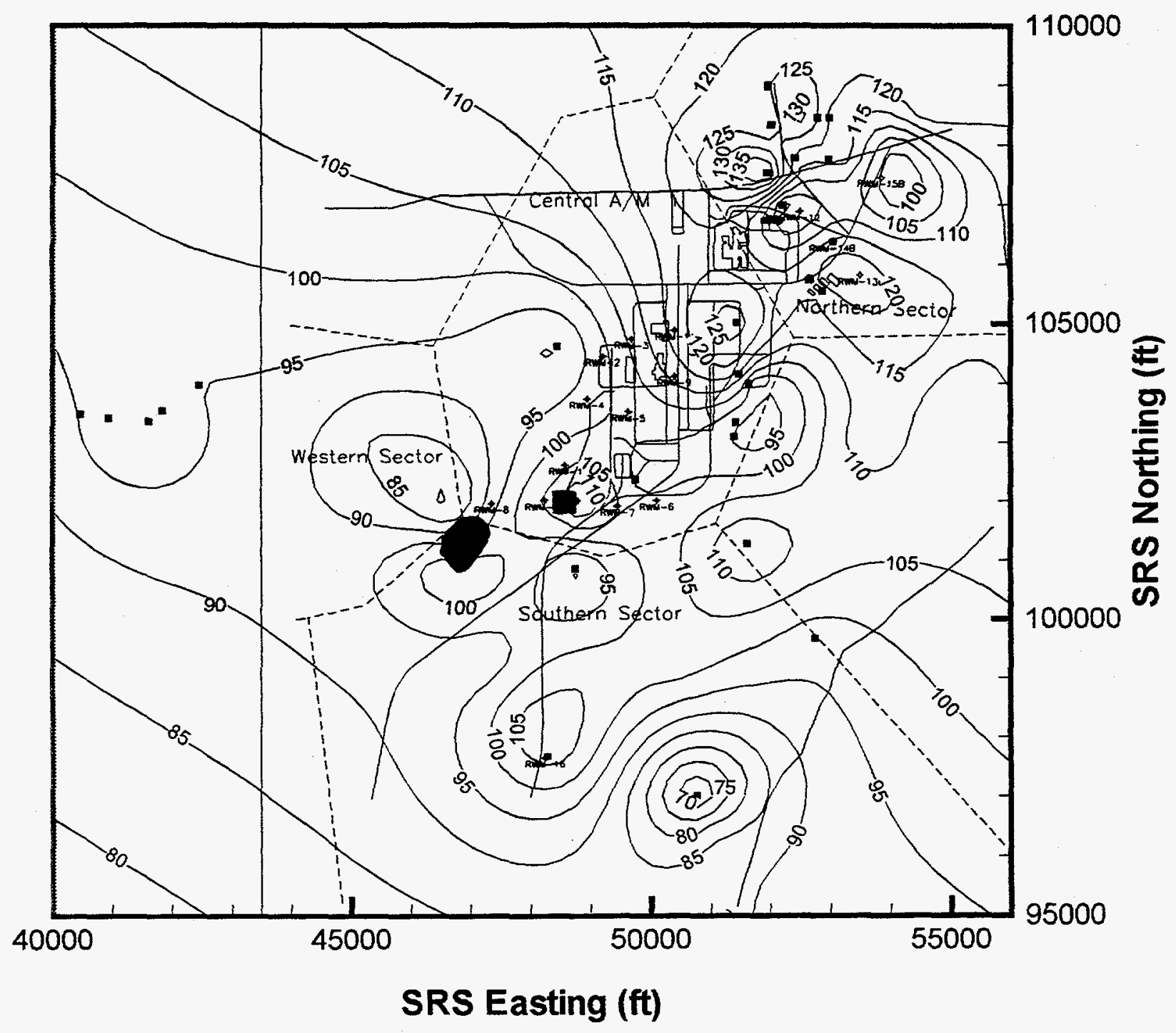

Figure 5.5- DFM Estimated Top Elevation ( $\mathrm{ft}$ ) of the "lower clay" 


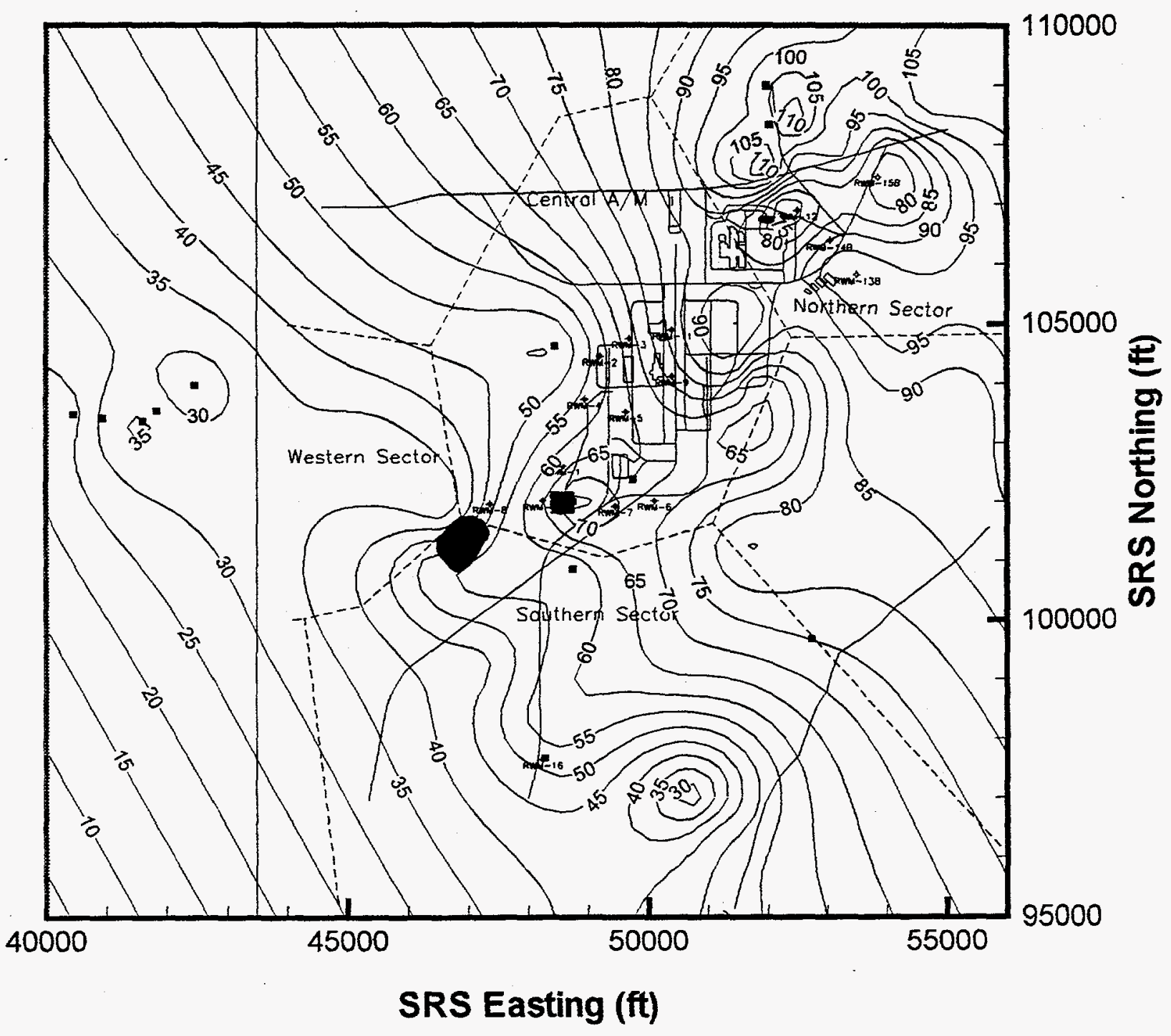

Figure 5.6 - DFM Estimated Bottom Elevation (ft) of the "lower clay" 


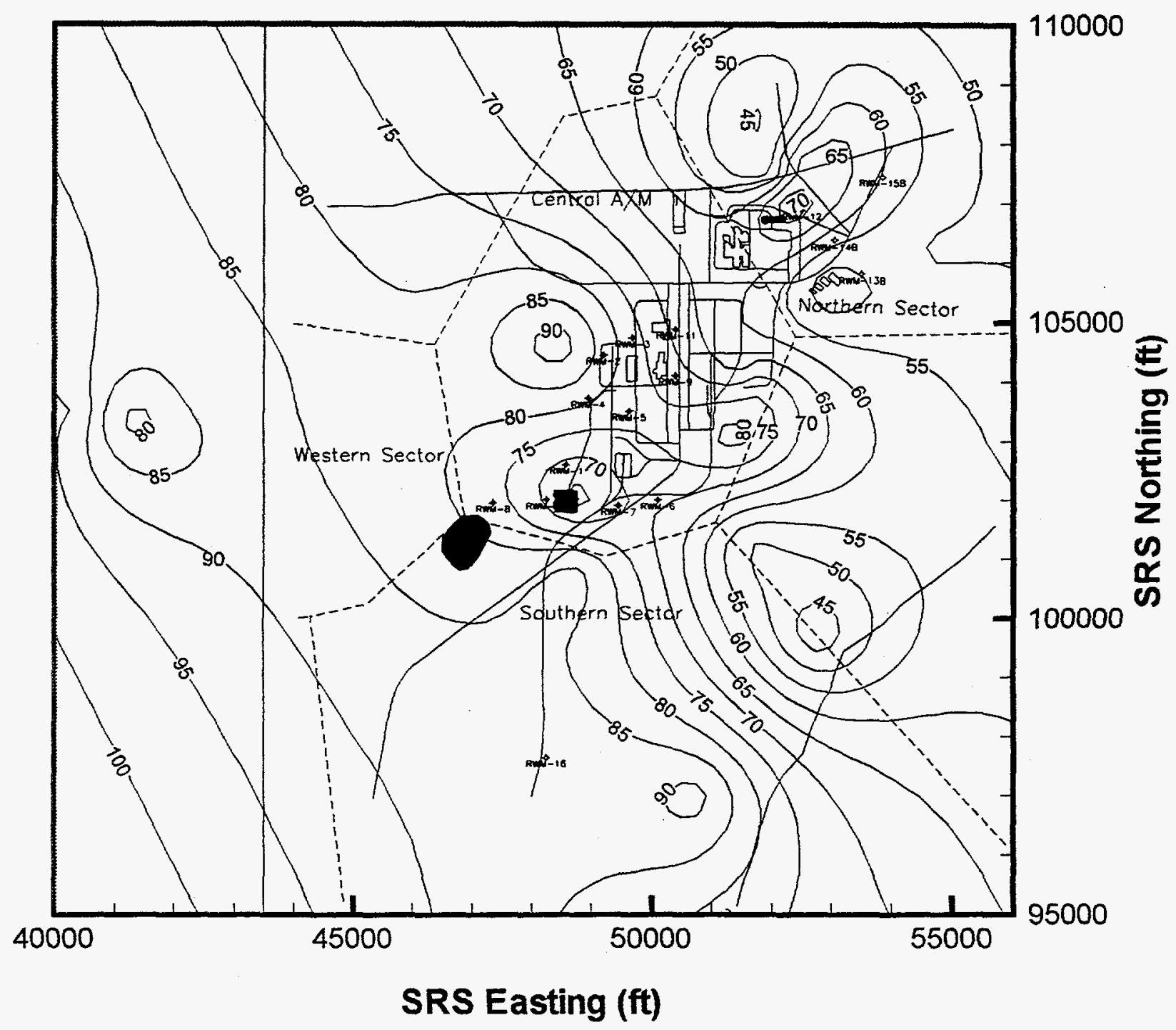

Figure 5.7- DFM Estimated Thickness (ft) of "Crouch Branch confining unit" 
Table 5.4 Geological Data for Layer 1 (Top of "Green Clay")

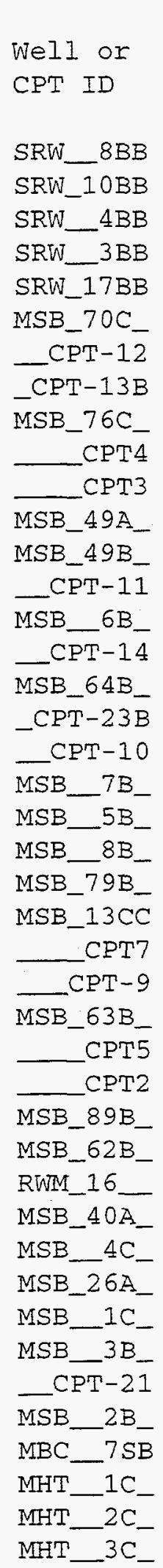

SRS

Easting

(ft)

40464.9

40940.5

41609.2

41845.6

42463.8

45012.0

45036.4

45312.2

45344.0

45512.1

45819.4

45864.6

45868.2

46114.5

46321.6

46433.0

46579.7

46596.2

46714.6

46718.1

46983.6

47281.9

47300.2

47525.7

47586.2

47696.7

47861.0

47863.3

47884.8

47889.9

47906.8

48244.8

48279.4

48313.6

48440.7

48512.7

48568.0

48590.5

48748.2

48750.0

48765.6

48780.3

48861.1

\section{SRS}

Northing

(Et)

103468.0

103399.1

103347.9

103526.6

103956.1

101785.2

103267.6

103083.2

103061.6

104120.5

103251.7

99759.0

99737.8

101349.2

101148.5

102736.3

101831.0

103812.5

100505.8

100597.6

101971.1

100805.8

99296.9

101728.8

102444.4

100993.0

101184.4

103874.7

104243.6

98374.1

101865.3

97647.2

97672.8

101963.2

104602.3

101832.5

102191.7

101383.0

101997.9

100850.0

102706.8

102747.1

102704.3 $\begin{array}{cc}\text { Elevation } & \text { Residual } \\ \text { (It) } & \text { (ft) }\end{array}$

197.00

1. 40

183.00

186.00

187.00

171.00

201.00

180.00

180.00

196.00

205.00

187.00

210.00

210.00

198.00

215.00

198.00

193.00

205.00

204.00

220.00

208.00

212.00

228.00

217.00

203.00

207.00

217.00

195.00

192.00

213.00

215.00

224.00

223.00

217.00

215.00

215.00

217.00

209.00

214.00

212.00

219.00

221.00

206.00
$-1.00$

$-0.10$

1.20

$-1.30$

1.20

$-1.20$

$-3.70$

3.90

0.40

$-0.90$

$-0.30$

$-0.40$

$-2.30$

2.70

$-0.30$

$-2.00$

0.10

$-3.70$

3.40

2.00

$-0.90$

1.00

1.00

$-2.00$

$-2.00$

2.40

$-0.80$

$-1.40$

$-0.60$

0.70

0.90

0.50

0.50

1.60

0.20

0.40

$-1.60$

$-1.00$

0.30

1.30

2.10

$-5.10$ 


\begin{tabular}{|c|c|c|c|c|}
\hline MHT_ $4 C_{-}$ & 48863.5 & 102778.9 & 217.00 & 0.00 \\
\hline MSB_75B_ & 48875.5 & 98937.4 & 218.00 & 0.00 \\
\hline $\mathrm{MHT}{ }_{-} 6 \mathrm{C}_{-}$ & 48900.0 & 102810.8 & 221.00 & 1.80 \\
\hline MHT_ $5 \mathrm{C}_{-}$ & 48905.9 & 102725.1 & 222.00 & 2.90 \\
\hline $\mathrm{MHT} \_8 \mathrm{C}_{-}$ & 48970.2 & 102880.7 & 222.00 & 1.60 \\
\hline $\mathrm{MHT} \_7 \mathrm{C}_{-}$ & 48977.5 & 102788.9 & 203.00 & -7.00 \\
\hline MHT_10C_ & 49011.6 & 102892.3 & 217.00 & -1.10 \\
\hline $\mathrm{MHT}$ _ $9 \mathrm{C}_{-}$ & 49015.6 & 102814.4 & 223.00 & 2.60 \\
\hline $\mathrm{MHT} \_9 \mathrm{~B}_{-}$ & 49031.8 & 102814.3 & 223.00 & 2.50 \\
\hline MSB_23TA & 49225.8 & 104298.8 & 208.00 & -0.10 \\
\hline MSB_38B_ & 49746.1 & 102360.8 & 220.00 & 1.00 \\
\hline CPT-17 & 50104.1 & 101955.1 & 206.00 & -1.80 \\
\hline MSB_74B_ & 50443.2 & 99197.4 & 223.00 & 1.10 \\
\hline MSB_88B_ & 50774.2 & 97013.0 & 200.00 & -2.20 \\
\hline $\mathrm{MSB}_{2} 50 \mathrm{~B}_{-}$ & 51053.5 & 96433.0 & 221.00 & 0.70 \\
\hline AMB_13AR & 51396.0 & 103082.0 & 216.00 & 0.30 \\
\hline AMB_10A_ & 51410.0 & 103326.4 & 217.00 & 0.10 \\
\hline $\mathrm{MBC} \_4 \mathrm{SB}$ & 51445.0 & 105008.0 & 239.00 & 0.70 \\
\hline$A M B$ & 51469.8 & 104131.6 & 226.00 & 0.60 \\
\hline $\mathrm{AMB}$ _ $7 \mathrm{~A}_{-}$ & 51591.0 & 103987.1 & 223.00 & 2.00 \\
\hline MSB_87B_ & 51607.0 & 101276.0 & 218.00 & 0.70 \\
\hline $\mathrm{AMB} \_7$ & 51624.9 & 103920.0 & 223.00 & 2.50 \\
\hline $\mathrm{MBC} \_5 \overline{\mathrm{SB}}$ & 51641.0 & 103983.0 & 206.00 & -5.80 \\
\hline$A M B_{-} 11 B_{-}$ & 51919.5 & 103154.2 & 214.00 & -0.10 \\
\hline MSB_82C_ & 51949.4 & 107521.9 & 236.00 & 0.40 \\
\hline $\mathrm{MSB} \_82 \mathrm{TA}$ & 51964.2 & 107525.7 & 236.00 & 0.40 \\
\hline MSB_84A_ & 51971.2 & 108982.1 & 234.00 & -0.20 \\
\hline MSB_84C_ & 51973.7 & 108967.9 & 234.00 & -0.30 \\
\hline MSB_55TA & 52014.7 & 108322.8 & 236.00 & -0.10 \\
\hline MSB_55C_ & 52029.7 & 108324.6 & 236.00 & 0.00 \\
\hline MSB_47TA & 52219.0 & 106987.7 & 228.00 & 0.60 \\
\hline MSB_83C_ & 52384.7 & 108405.3 & 236.00 & 1.10 \\
\hline MSB_83TA & 52410.9 & 108416.3 & 236.00 & 1.30 \\
\hline MSB_69TA & 52418.4 & 107772.5 & 229.00 & -0.80 \\
\hline$A S B \_6 A A$ & 52643.9 & 105727.0 & 201.00 & -2.30 \\
\hline$A S B \_6 T A$ & 52671.3 & 105749.5 & 201.00 & -1.70 \\
\hline MSB_32B_ & 52742.5 & 99676.0 & 218.00 & 0.00 \\
\hline $\mathrm{MBC} \_1 \mathrm{SB}$ & 52780.0 & 108450.0 & 222.00 & -2.10 \\
\hline MSB_51B_ & 52818.0 & 96992.7 & 224.00 & 0.30 \\
\hline$A S B \_2 C R$ & 52862.7 & 105540.2 & 225.00 & 5.20 \\
\hline ASB__ $10 C_{-}$ & 52965.5 & 105673.1 & 206.00 & -0.80 \\
\hline $\mathrm{MBC} \_2 \mathrm{SB}$ & 52975.0 & 107750.0 & 226.00 & -0.10 \\
\hline MSB_54TA & 52985.8 & 108446.3 & 227.00 & -0.40 \\
\hline RWM_14B_ & 53044.7 & 106362.1 & 206.00 & 0.00 \\
\hline MSB_85TA & 53137.2 & 107831.2 & 230.00 & 1.20 \\
\hline MSB_85C_ & 53151.4 & 107835.2 & 230.00 & 1.20 \\
\hline MSB_52B_ & 53418.4 & 103077.7 & 232.00 & 1.00 \\
\hline RWM_13B_ & 53516.3 & 105803.3 & 203.00 & -1.40 \\
\hline RWM_15B_ & 53849.0 & 107444.7 & 202.00 & -1.10 \\
\hline MSB_48C_ & 54077.0 & 107917.5 & 207.00 & -1.20 \\
\hline $\mathrm{MSB} \_48 \mathrm{TA}$ & 54089.2 & 107925.8 & 207.00 & -1.20 \\
\hline
\end{tabular}




$\begin{array}{llllr}\text { ASB_9C_- } & 54201.1 & 104568.1 & 218.00 & -0.30 \\ \text { MSB_77TA } & 54208.9 & 107053.8 & 205.00 & 0.40 \\ \text { ASB_9 } & 54226.2 & 104589.2 & 218.00 & -0.30 \\ \text { MSB_53C_- } & 54540.5 & 106456.2 & 222.00 & 0.50 \\ \text { MSB_86C_- } & 54560.5 & 108500.4 & 223.00 & 1.80 \\ \text { MSB_53B_- } & 54574.3 & 106443.6 & 222.00 & 0.40 \\ \text { MSB_81B_- } & 55230.4 & 103762.7 & 214.00 & -0.40 \\ \text { MSB_61C_ } & 55406.6 & 106091.1 & 222.00 & -0.60\end{array}$


Table 5.5 Geological Data for Layer 2 (Bottom of "Green Clay")

\begin{tabular}{|c|}
\hline PT ID \\
\hline I \\
\hline$\angle 10 \mathrm{BE}$ \\
\hline$W \_4 B B$ \\
\hline $\mathrm{KW} \_3 \mathrm{BB}$ \\
\hline $\mathrm{W} \_17 \mathrm{BB}$ \\
\hline$S B \_70 C_{-}$ \\
\hline _CPT-12 \\
\hline $\mathrm{CPT}-13 \mathrm{~B}$ \\
\hline$-76 \mathrm{C}$ \\
\hline CPT4 \\
\hline _CPT3 \\
\hline B_49 \\
\hline B $\_49 B$ \\
\hline _CPT-11 \\
\hline $\mathrm{SB}-6 \mathrm{~B}$ \\
\hline$-\overline{C P T}-1$ \\
\hline $1 S B \_64 B$ \\
\hline$C P T-23 B$ \\
\hline -CPT-10 \\
\hline $\mathrm{SB}-7 \mathrm{~B}$ \\
\hline $\mathrm{SB}_{-}-5 \mathrm{~B}$ \\
\hline $\mathrm{SB}-8 \mathrm{~B}$ \\
\hline$S B \_79 B$ \\
\hline$S B \_13 C$ \\
\hline - $\mathrm{CPT}^{\prime}$ \\
\hline - $\mathrm{CPT}-9$ \\
\hline \\
\hline _ $\mathrm{CPT}$ \\
\hline B_89B. \\
\hline$S B \_62 B$ \\
\hline WM_16 \\
\hline$B$ \\
\hline$S B \_4 C$ \\
\hline$S B \_26 A$ \\
\hline ISB $\quad 1 C$ \\
\hline$M S B-3 B$ \\
\hline$\ldots \mathrm{CPT}-21$ \\
\hline$S B \_2 B$ \\
\hline $\mathrm{BC} \_7 \mathrm{SE}$ \\
\hline $\mathrm{HT} \_2 \mathrm{C}$ \\
\hline $\mathrm{HT} \_3 \mathrm{C}$ \\
\hline ( \\
\hline
\end{tabular}

SRS

Easting

(ft)

40464.9

40940.5

41609.2

41845.6

42463.8

45012.0

45036.4

45312.2

45344.0

45512.1

45819.4

45864.6

45868.2

46114.5

46321.6

46433.0

46579.7

46596.2

46714.6

46718.1

46983.6

47281.9

47300.2

47525.7

47586.2

47696.7

47861.0

47863.3

47884.8

47889.9

47906.8

48244.8

48279.4

48313.6

48440.7

48512.7

48568.0

48590.5

48748.2

48750.0

48780.3

48861.1

48863.5
SRS

Northing

(Et)

103468.0

103399.1

103347.9

103526.6

103956.1

101785.2

103267.6

103083.2

103061.6

104120.5

103251.7

99759.0

99737.8

101349.2

101148.5

102736.3

101831.0

103812.5

100505.8

100597.6

101971.1

100805.8

99296.9

101728.8

102444.4

100993.0

101184.4

103874.7

104243.6

98374.1

101865.3

97647.2

97672.8

101963.2

104602.3

101832.5

102191.7

101383.0

101997.9

100850.0

102747.1

102704.3

102778.9
Elevation Residual

(ft)

(ft)

$177.00 \quad-0.80$

$170.00 \quad 0.20$

$172.00 \quad 0.40$

$168.00-1.00$

$164.00 \quad 0.70$

$182.00-1.10$

$179.00 \quad 0.70$

$179.00-1.90$

$188.00 \quad 2.20$

$199.00 \quad 0.10$

$185.00 \quad 0.00$

$200.00 \quad 0.30$

$200.00 \quad 0.30$

$195.00 \quad 1.60$

$194.00-1.80$

$195.00 \quad 0.60$

$189.00 \quad 1.20$

$201.00 \quad 0.30$

$202.00 \quad 1.00$

$200.00 \quad-0.80$

187.00

204.00

208.00

198.00

200.00

200.00

192.00

190.00

187.00

193.00

195.00

197.00

197.00

201.00

196.00

199.00

201.00

202.00

205.00

196.00

196.00

197.00

200.00
$-1.80$

1.00

$-0.40$

$-0.30$

1.70

1.50

$-2.20$

0.70

0.50

0.10

$-1.20$

$-0.50$

$-0.50$

0.10

$-0.70$

$-0.70$

$-0.10$

1. 60

1. 20

$-0.40$

$-1.40$

$-0.80$

0.40 


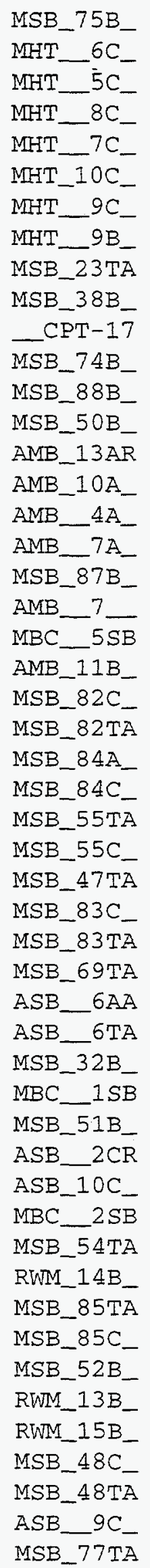

$\begin{array}{rr}48875.5 & 98937.4 \\ 48900.0 & 102810.8 \\ 48905.9 & 102725.1 \\ 48970.2 & 102880.7 \\ 48977.5 & 102788.9 \\ 49011.6 & 102892.3 \\ 49015.6 & 102814.4 \\ 49031.8 & 102814.3 \\ 49225.8 & 104298.8 \\ 49746.1 & 102360.8 \\ 50104.1 & 101955.1 \\ 50443.2 & 99197.4 \\ 50774.2 & 97013.0 \\ 51053.5 & 96433.0 \\ 51396.0 & 103082.0 \\ 51410.0 & 103326.4 \\ 51469.8 & 104131.6 \\ 51591.0 & 103987.1 \\ 51607.0 & 101276.0 \\ 51624.9 & 103920.0 \\ 51641.0 & 103983.0 \\ 51919.5 & 103154.2 \\ 51949.4 & 107521.9 \\ 51964.2 & 107525.7 \\ 51971.2 & 108982.1 \\ 51973.7 & 108967.9 \\ 52014.7 & 108322.8 \\ 52029.7 & 108324.6 \\ 52219.0 & 106987.7 \\ 52384.7 & 108405.3 \\ 52410.9 & 108416.3 \\ 52418.4 & 107772.5 \\ 52643.9 & 105727.0 \\ 52671.3 & 105749.5 \\ 52742.5 & 99676.0 \\ 52780.0 & 108450.0 \\ 52818.0 & 96992.7 \\ 52862.7 & 105540.2 \\ 52965.5 & 105673.1 \\ 52975.0 & 107750.0 \\ 52985.8 & 108446.3 \\ 53044.7 & 106362.1 \\ 53137.2 & 107831.2 \\ 53151.4 & 107835.2 \\ 53418.4 & 103077.7 \\ 53516.3 & 105803.3 \\ 53849.0 & 107444.7 \\ 54077.0 & 107917.5 \\ 54089.2 & 107925.8 \\ 54208.9 & 107053.8 \\ 540\end{array}$

$\begin{array}{rr}198.00 & 0.10 \\ 201.00 & 0.60 \\ 198.00 & -0.30 \\ 200.00 & -0.50 \\ 200.00 & 0.40 \\ 202.00 & 0.20 \\ 200.00 & 0.00 \\ 200.00 & -0.10 \\ 190.00 & -0.40 \\ 202.00 & -0.60 \\ 200.00 & 1.30 \\ 196.00 & -1.00 \\ 196.00 & 1.40 \\ 205.00 & -0.20 \\ 197.00 & -0.30 \\ 199.00 & -0.20 \\ 211.00 & 0.30 \\ 204.00 & -0.20 \\ 195.00 & -0.60 \\ 204.00 & 0.40 \\ 203.00 & 0.10 \\ 196.00 & -0.10 \\ 219.00 & -0.20 \\ 219.00 & -0.20 \\ 226.00 & 0.40 \\ 226.00 & 0.40 \\ 223.00 & 0.00 \\ 223.00 & 0.00 \\ 210.00 & -0.40 \\ 220.00 & -0.70 \\ 220.00 & -0.60 \\ 217.00 & 0.60 \\ 194.00 & 0.80 \\ 194.00 & 1.40 \\ 199.00 & 0.00 \\ 217.00 & 0.40 \\ 204.00 & -0.30 \\ 195.00 & -2.30 \\ 192.00 & -0.70 \\ 210.00 & -1.10 \\ 221.00 & 1.10 \\ 194.00 & 0.20 \\ 215.00 & -0.10 \\ 215.00 & -0.20 \\ 210.00 & -0.60 \\ 197.00 & 1.10 \\ 204.08 .00 & 0.50 \\ 191.00 & 1.10 \\ & -0.80\end{array}$




$\begin{array}{llllr}\text { ASB_9_- } & 54226.2 & 104589.2 & 208.00 & 0.30 \\ \text { MSB_53C_ } & 54540.5 & 106456.2 & 209.00 & 0.00 \\ \text { MSB_86C_ } & 54560.5 & 108500.4 & 202.00 & -1.70 \\ \text { MSB_53B_- } & 54574.3 & 106443.6 & 209.00 & -0.20 \\ \text { MSB_81B_- } & 55230.4 & 103762.7 & 201.00 & 0.00 \\ \text { MSB_61C_ } & 55406.6 & 106091.1 & 213.00 & 0.50\end{array}$


Table 5.6 Geological Data for Layer 3 (Top of "upper clay")

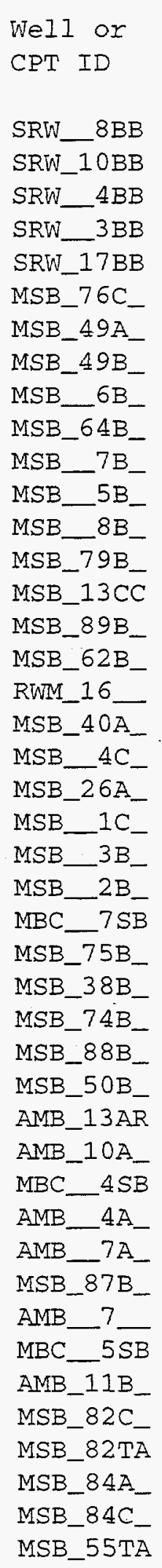

Well ox

SRS

Easting

(ft)

40464.9

40940.5

41609.2

41845.6

42463.8

45344.0

45864.6

45868.2

46321.6

46579.7

46718.1

46983.6

47281 .

47300.2

47525.7

47889.9

47906.8

48244.8

48279.4

48313.6

48440.7

48512.7

48568.0

48748.2

48750.0

48875.5

49746.1

50443.2

50774.2

51053.5

51396.0

51410.0

51445.0

51469.8

51591.0

51607.0

51624.9

51641.0

51919.5

51949.4

51964.2

51971.2

51973.7

52014.7
SRS

Northing

(ft)

103468.0

103399.1

103347.9

103526.6

103956.1

103061.6

99759.0

99737.8

101148.5

101831.0

100597.6

101971.1

100805.8

99296.9

101728.8

98374.1

101865.3

97647.2

97672.8

101963.2

104602.3

101832.5

102191.7

101997.9

100850.0

98937.4

102360.8

99197.4

97013.0

96433.0

103082.0

103326.4

105008.0

104131.6

103987.1

101276.0

103920.0

103983.0

103154.2

107521.9

107525.7

108982.1

108967.9

108322.8
Elevation Residual

(ft)

(ft)

118.00

111.00

115.00

0.70

$-1.40$

115.00

0.00

114.00

0.10

115.00

128.00

128.00

132.00

113.00

138.00

117.00

140.00

130.00

131.00

142.00

133.00

139.00

145.00

135.00

140.00

135.00

133.00

139.00

146.00

145.00

142.00

140.00

117.00

132.00

150.00

144.00

157.00

144.00

144.00

132.00

144.00

142.00

140.00

160.00

160.00

150.00

150.00

148.00
$-0.40$

$-0.20$

0.00

0.00

0.40

$-0.70$

0.10

$-0.20$

0.20

$-0.30$

0.20

0.20

0.10

$-1.30$

1. 50

0.30

1.20

$-0.60$

$-0.70$

$-0.80$

1.80

0.10

2.10

0.20

$-1.20$

0.50

2. 60

$-0.50$

0.80

$-2.30$

0.20

$-1.80$

0.50

$-0.10$

$-0.20$

$-0.40$

$-0.40$

$-0.10$

$-0.10$

$-0.30$ 


\begin{tabular}{|c|c|c|c|c|}
\hline MSB_55C_ & 52029.7 & 108324.6 & 148.00 & -0.60 \\
\hline MSB_47TA & 52219.0 & 106987.7 & 146.00 & 0.60 \\
\hline MSB_83C_ & 52384.7 & 108405.3 & 165.00 & 1.30 \\
\hline MSB_83TA & 52410.9 & 108416.3 & 165.00 & 0.90 \\
\hline MSB_69TA & 52418.4 & 107772.5 & 160.00 & 0.70 \\
\hline$A S B \_6 A A$ & 52643.9 & 105727.0 & 142.00 & 0.00 \\
\hline$A S B \_6 T A$ & 52671.3 & 105749.5 & 142.00 & -0.20 \\
\hline MSB_32B_ & 52742.5 & 99676.0 & 117.00 & -0.50 \\
\hline $\mathrm{MBC} \_1 \mathrm{SB}$ & 52780.0 & 108450.0 & 160.00 & -1.40 \\
\hline MSB_51B_ & 52818.0 & 96992.7 & 132.00 & 0.00 \\
\hline $\mathrm{ASB} \_2 \mathrm{CR}$ & 52862.7 & 105540.2 & 138.00 & -0.80 \\
\hline$A_{S B}{ }_{1} 10 C_{-}$ & 52965.5 & 105673.1 & 142.00 & -0.80 \\
\hline $\mathrm{MBC} \_2 \mathrm{SB}$ & 52975.0 & 107750.0 & 160.00 & -0.60 \\
\hline MSB_54TA & 52985.8 & 108446.3 & 160.00 & -0.50 \\
\hline RWM_14B_ & 53044.7 & 106362.1 & 148.00 & 0.60 \\
\hline MSB_85TA & 53137.2 & 107831.2 & 163.00 & 0.80 \\
\hline MSB_85C_ & 53151.4 & 107835.2 & 163.00 & 0.80 \\
\hline MSB_52B_ & 53418.4 & 103077.7 & 148.00 & 0.20 \\
\hline RWM_13B_ & 53516.3 & 105803.3 & 151.00 & 0.90 \\
\hline RWM_15B_ & 53849.0 & 107444.7 & 138.00 & -0.20 \\
\hline $\mathrm{MSB}_{4} 48 \mathrm{C}_{-}$ & 54077.0 & 107917.5 & 139.00 & -0.40 \\
\hline MSB_48TA & 54089.2 & 107925.8 & 139.00 & -0.40 \\
\hline ASB__ $9 C_{-}$ & 54201.1 & 104568.1 & 150.00 & 0.20 \\
\hline MSB_77TA & 54208.9 & 107053.8 & 132.00 & -0.40 \\
\hline $\mathrm{AsB}_{-}=$ & 54226.2 & 104589.2 & 150.00 & 0.20 \\
\hline MSB_53C_ & 54540.5 & 106456.2 & 139.00 & -0.20 \\
\hline MSB_86C_ & 54560.5 & 108500.4 & 159.00 & 0.60 \\
\hline MSB_53B_ & 54574.3 & 106443.6 & 139.00 & -0.40 \\
\hline MSB_81B_ & 55230.4 & 103762.7 & 140.00 & -0.30 \\
\hline
\end{tabular}


Table 5.7 Geological Data for Layer 4 (Bottom of "upper clay")

\begin{tabular}{|c|c|c|c|c|}
\hline $\begin{array}{l}\text { Well or } \\
\text { CPT ID }\end{array}$ & $\begin{array}{l}\text { SRS } \\
\text { Easting } \\
\text { (ft) }\end{array}$ & $\begin{array}{l}\text { SRS } \\
\text { Northing } \\
\text { (ft) }\end{array}$ & $\begin{array}{l}\text { Elevation } \\
\text { (ft) }\end{array}$ & $\begin{array}{l}\text { Residual } \\
\text { (ft) }\end{array}$ \\
\hline SRW__ $8 \mathrm{BB}$ & 40464.9 & 103468.0 & 99.00 & 0.00 \\
\hline SRW_10BB & 40940.5 & 103399.1 & 99.00 & 0.20 \\
\hline$S R W \_4 B B$ & 41609.2 & 103347.9 & 99.00 & -0.30 \\
\hline$S R W \_3 B B$ & 41845.6 & 103526.6 & 99.00 & 0.00 \\
\hline SRW_17BB & 42463.8 & 103956.1 & 99.00 & 0.10 \\
\hline $\mathrm{MSB}$ 40A_ & 48279.4 & 97672.8 & 122.00 & -0.50 \\
\hline MSB_26A_ & 48440.7 & 104602.3 & 110.00 & -0.40 \\
\hline $\mathrm{MSB} \_2 \mathrm{~B}_{-}$ & 48748.2 & 101997.9 & 121.00 & 0.30 \\
\hline $\mathrm{MBC} \_7 \mathrm{SB}$ & 48750.0 & 100850.0 & 114.00 & -0.20 \\
\hline $\mathrm{MSB}_{-} 38 \mathrm{~B}_{-}$ & 49746.1 & 102360.8 & 101.00 & -2.90 \\
\hline MSB_88B_ & 50774.2 & 97013.0 & 110.00 & 2.20 \\
\hline AMB_13AR & 51396.0 & 103082.0 & 116.00 & -1.80 \\
\hline$A M B_{2} 10 A_{-}$ & 51410.0 & 103326.4 & 123.00 & 0.50 \\
\hline $\mathrm{MBC} \_4 \mathrm{SB}$ & 51445.0 & 105008.0 & 140.00 & -0.70 \\
\hline $\mathrm{AMB} \_4 \mathrm{~A}_{-}$ & 51469.8 & 104131.6 & 138.00 & 1.30 \\
\hline $\mathrm{AMB}$ & 51591.0 & 103987.1 & 131.00 & 1.10 \\
\hline MSB_87B_ & 51607.0 & 101276.0 & 128.00 & 1.20 \\
\hline AMB 7 & 51624.9 & 103920.0 & 131.00 & 1.90 \\
\hline $\mathrm{MBC} \_5 \overline{\mathrm{SB}}$ & 51641.0 & 103983.0 & 123.00 & -2.00 \\
\hline MSB_82C_ & 51949.4 & 107521.9 & 145.00 & -1.20 \\
\hline MSB_82TA & 51964.2 & 107525.7 & 145.00 & -1.30 \\
\hline MSB_84A_ & 51971.2 & 108982.1 & 136.00 & -0.10 \\
\hline$M S B_{-} 84 C_{-}$ & 51973.7 & 108967.9 & 136.00 & -0.10 \\
\hline$M S B \_55 T A$ & 52014.7 & 108322.8 & 132.00 & -0.60 \\
\hline MSB_55C_ & 52029.7 & 108324.6 & 132.00 & -0.90 \\
\hline $\mathrm{MSB} \_47 \mathrm{TA}$ & 52219.0 & 106987.7 & 132.00 & 2.10 \\
\hline MSB_69TA & 52418.4 & 107772.5 & 146.00 & 1.40 \\
\hline $\mathrm{ASB} \_6 \mathrm{AA}$ & 52643.9 & 105727.0 & 132.00 & 0.50 \\
\hline $\mathrm{ASB} \_6 \mathrm{TA}$ & 52671.3 & 105749.5 & 132.00 & 0.30 \\
\hline MSB_32B_ & 52742.5 & 99676.0 & 102.00 & -1.20 \\
\hline $\mathrm{MBC} \_1 \mathrm{SB}$ & 52780.0 & 108450.0 & 149.00 & -0.80 \\
\hline $\mathrm{ASB} \_2 \mathrm{CR}$ & 52862.7 & 105540.2 & 125.00 & -2.00 \\
\hline$A S B \_10 C_{-}$ & 52965.5 & 105673.1 & 137.00 & 1.90 \\
\hline $\mathrm{MBC} \_2 \mathrm{SB}$ & 52975.0 & 107750.0 & 147.00 & 0.10 \\
\hline $\mathrm{MSB} \_54 \mathrm{TA}$ & 52985.8 & 108446.3 & 155.00 & 2.90 \\
\hline RWM_14B_ & 53044.7 & 106362.1 & 130.00 & -1.40 \\
\hline RWM_13B_ & 53516.3 & 105803.3 & 135.00 & -0.60 \\
\hline $\mathrm{ASB}$ & 54201.1 & 104568.1 & 135.00 & 0.00 \\
\hline $\mathrm{ASB} \_9$ & 54226.2 & 104589.2 & 135.00 & -0.10 \\
\hline MSB_53C_ & 54540.5 & 106456.2 & 129.00 & 0.40 \\
\hline MSB_53B_ & 54574.3 & 106443.6 & 129.00 & 0.20 \\
\hline
\end{tabular}


Table 5.8 Geological Data for Layer 5 (Top of "lower clay")

\begin{tabular}{|c|c|c|c|c|}
\hline & SRS & SRS & & \\
\hline Well or & Easting & Northing & Elevation & Residual \\
\hline CPT ID & $( \pm t)$ & $(f t)$ & $(f t)$ & $(f t)$ \\
\hline$S R W \_8 B B$ & 40464.9 & 103468.0 & 98.00 & 1.40 \\
\hline SRW_10BB & 40940.5 & 103399.1 & 98.00 & -0.10 \\
\hline $\mathrm{SRW}$ & 41609.2 & 103347.9 & 98.00 & -0.60 \\
\hline SRW__ $3 B B$ & 41845.6 & 103526.6 & 98.00 & 0.10 \\
\hline SRW_17BB & 42463.8 & 103956.1 & 98.00 & 1.40 \\
\hline MSB_40A_ & 48279.4 & 97672.8 & 110.00 & 1.70 \\
\hline MSB_26A_ & 48440.7 & 104602.3 & 90.00 & -0.20 \\
\hline $\mathrm{MSB} \_2 \mathrm{~B}_{-}$ & 48748.2 & 101997.9 & 120.00 & 2.00 \\
\hline $\mathrm{MBC} \_7 \mathrm{SB}$ & 48750.0 & 100850.0 & 85.00 & -2.90 \\
\hline MSB_38B_ & 49746.1 & 102360.8 & 100.00 & 0.20 \\
\hline $\mathrm{MSB}$ 88B_ & 50774.2 & 97013.0 & 63.00 & -1.70 \\
\hline$A M B \_13 A R$ & 51396.0 & 103082.0 & 94.00 & -0.50 \\
\hline $\mathrm{AMB}_{2} 10 \mathrm{~A}_{-}$ & 51410.0 & 103326.4 & 96.00 & -0.20 \\
\hline $\mathrm{MBC} \_4 \mathrm{SB}$ & 51445.0 & 105008.0 & 127.00 & 0.30 \\
\hline$A M B$ & 51469.8 & 104131.6 & 117.00 & 1.90 \\
\hline MSB_87B_ & 51607.0 & 101276.0 & 115.00 & 0.50 \\
\hline $\mathrm{MBC} \_5 \mathrm{SB}$ & 51641.0 & 103983.0 & 96.00 & -2.60 \\
\hline$M S B_{-} 82 C_{-}$ & 51949.4 & 107521.9 & 144.00 & 2.90 \\
\hline $\mathrm{MSB} \_82 \mathrm{TA}$ & 51964.2 & 107525.7 & 144.00 & 3.00 \\
\hline MSB_84A_ & 51971.2 & 108982.1 & 125.00 & 0.20 \\
\hline$M S B \_84 C_{-}$ & 51973.7 & 108967.9 & 125.00 & 0.20 \\
\hline $\mathrm{MSB} \_55 \mathrm{TA}$ & 52014.7 & 108322.8 & 120.00 & -0.60 \\
\hline MSB_55C_ & 52029.7 & 108324.6 & 120.00 & -0.80 \\
\hline MSB_47TA & 52219.0 & 106987.7 & 94.00 & -3.50 \\
\hline MSB_69TA & 52418.4 & 107772.5 & 114.00 & -2.50 \\
\hline $\mathrm{ASB} \mathrm{B}_{6 \mathrm{~A}}$ & 52643.9 & 105727.0 & 118.00 & 0.00 \\
\hline $\mathrm{ASB} \_6 \mathrm{TA}$ & 52671.3 & 105749.5 & 118.00 & -0.30 \\
\hline $\mathrm{MSB} \mathrm{B}_{3} 2 \mathrm{~B}_{-}$ & 52742.5 & 99676.0 & 98.00 & 0.20 \\
\hline $\mathrm{MBC} \_1 \mathrm{SB}$ & 52780.0 & 108450.0 & 128.00 & 1.70 \\
\hline$A S B \_2 C R$ & 52862.7 & 105540.2 & 120.00 & 1.10 \\
\hline $\mathrm{MBC}-2 \mathrm{SB}$ & 52975.0 & 107750.0 & 116.00 & -0.10 \\
\hline MSB_54TA & 52985.8 & 108446.3 & 114.00 & -2.60 \\
\hline RWM_14B_ & 53044.7 & 106362.1 & 118.00 & 0.90 \\
\hline
\end{tabular}


Table 5.9 Geological Data for Layer 6 (Bottom of "lower clay")

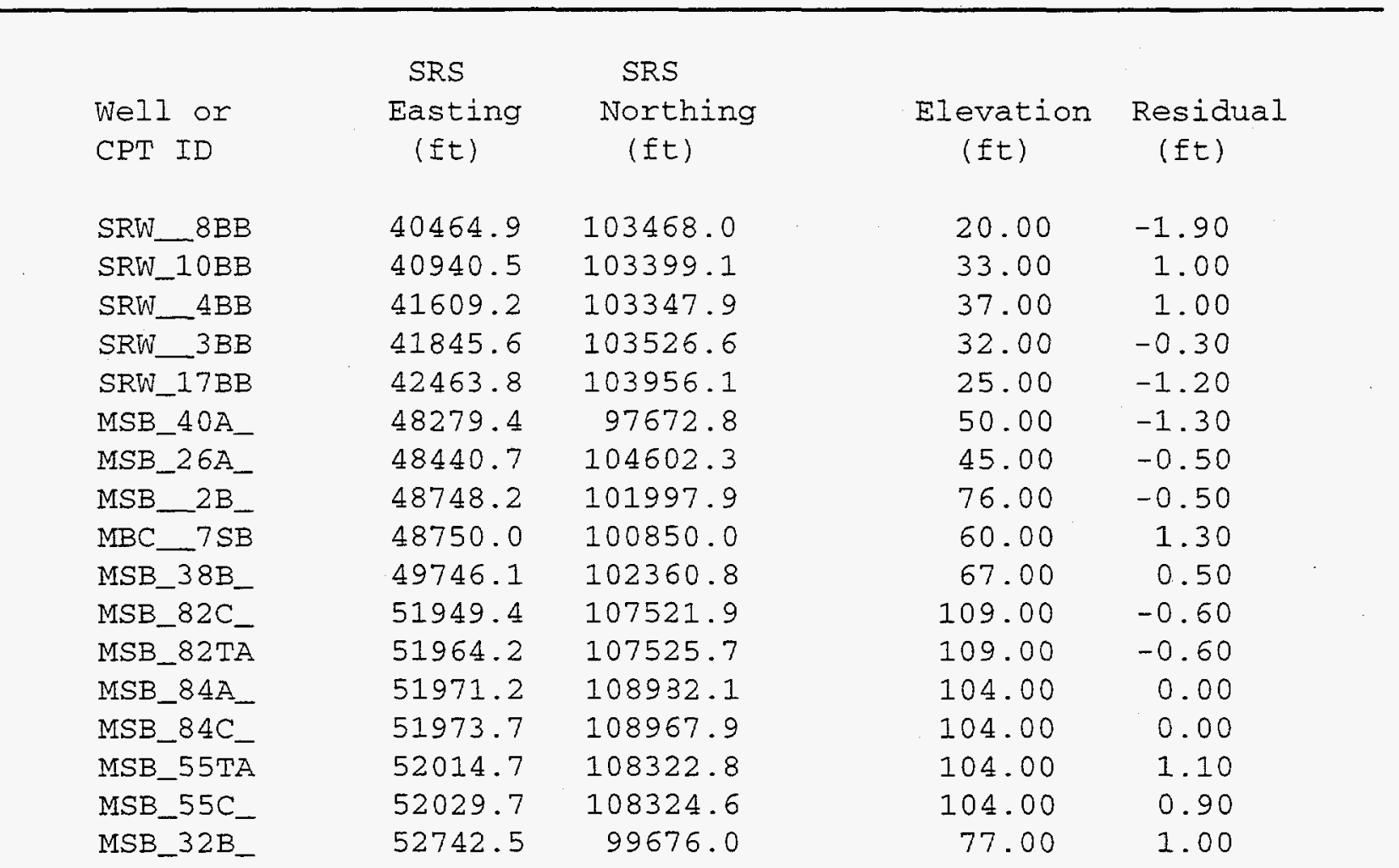




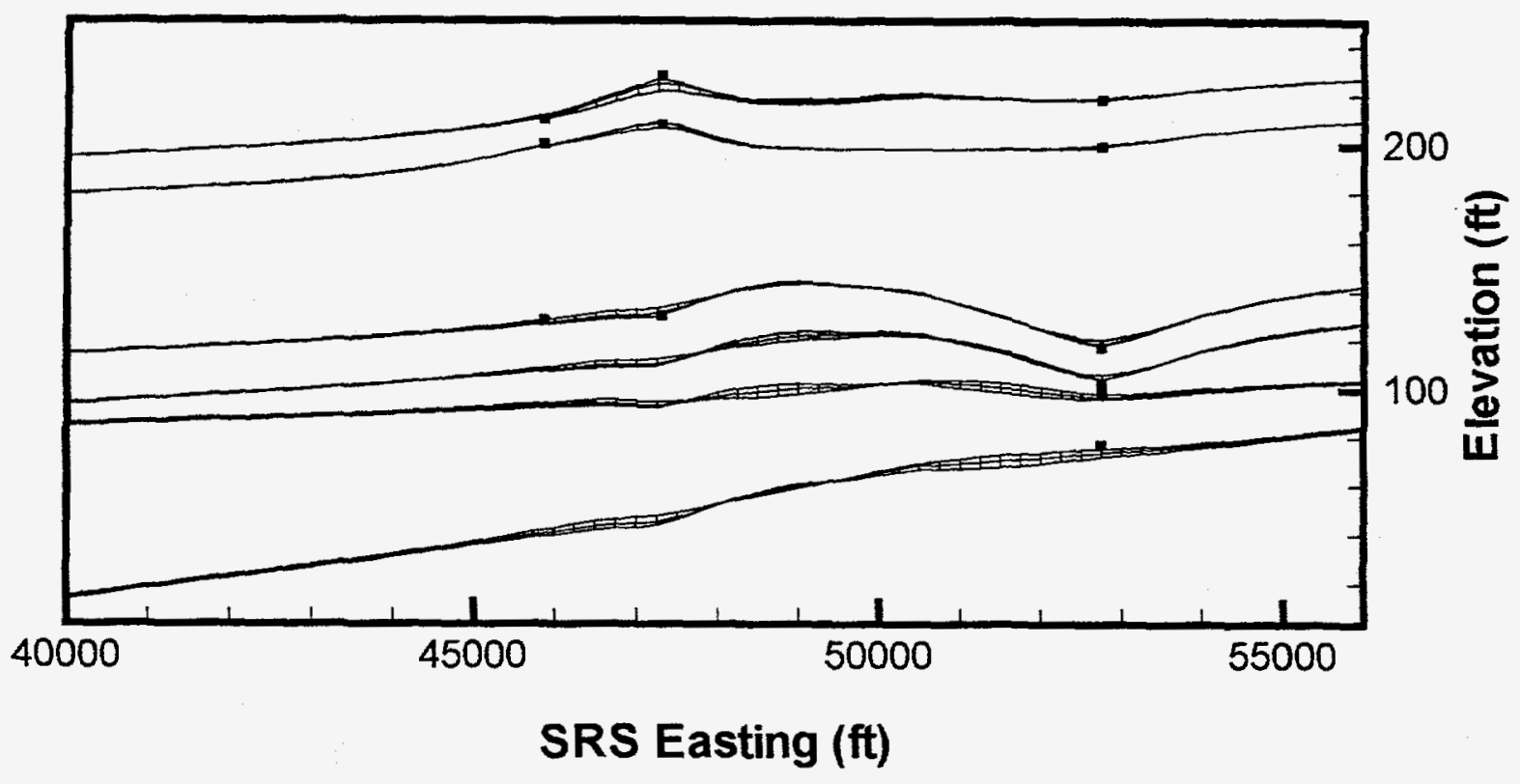

Figure 5.8 - DFM Estimated Layers at $y=99,500 \mathrm{ft}(+/-300)$ 


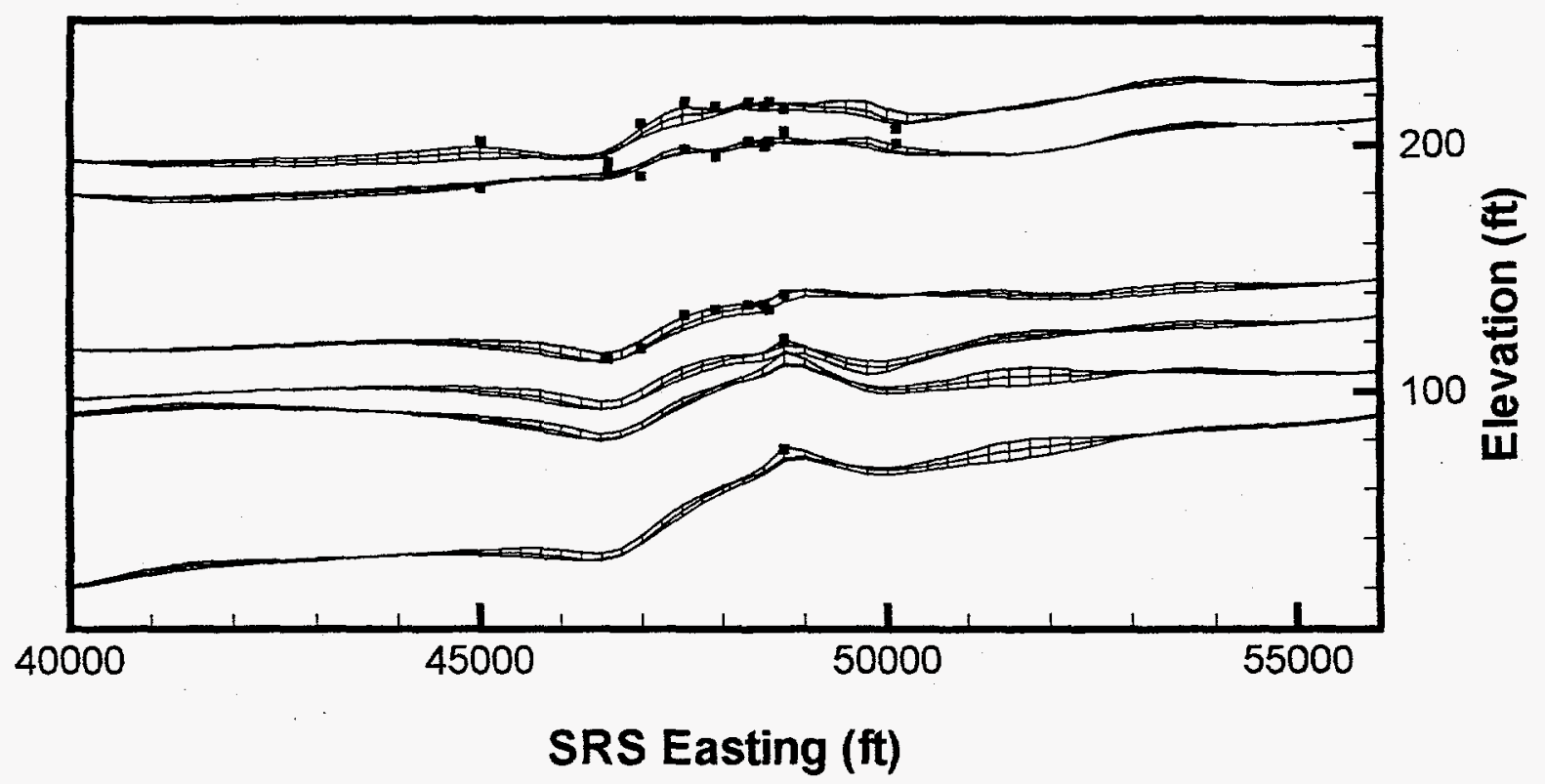

Figure 5.9 - DFM Estimated Layers at $y=102,000 \mathrm{ft}(+/-300)$ 


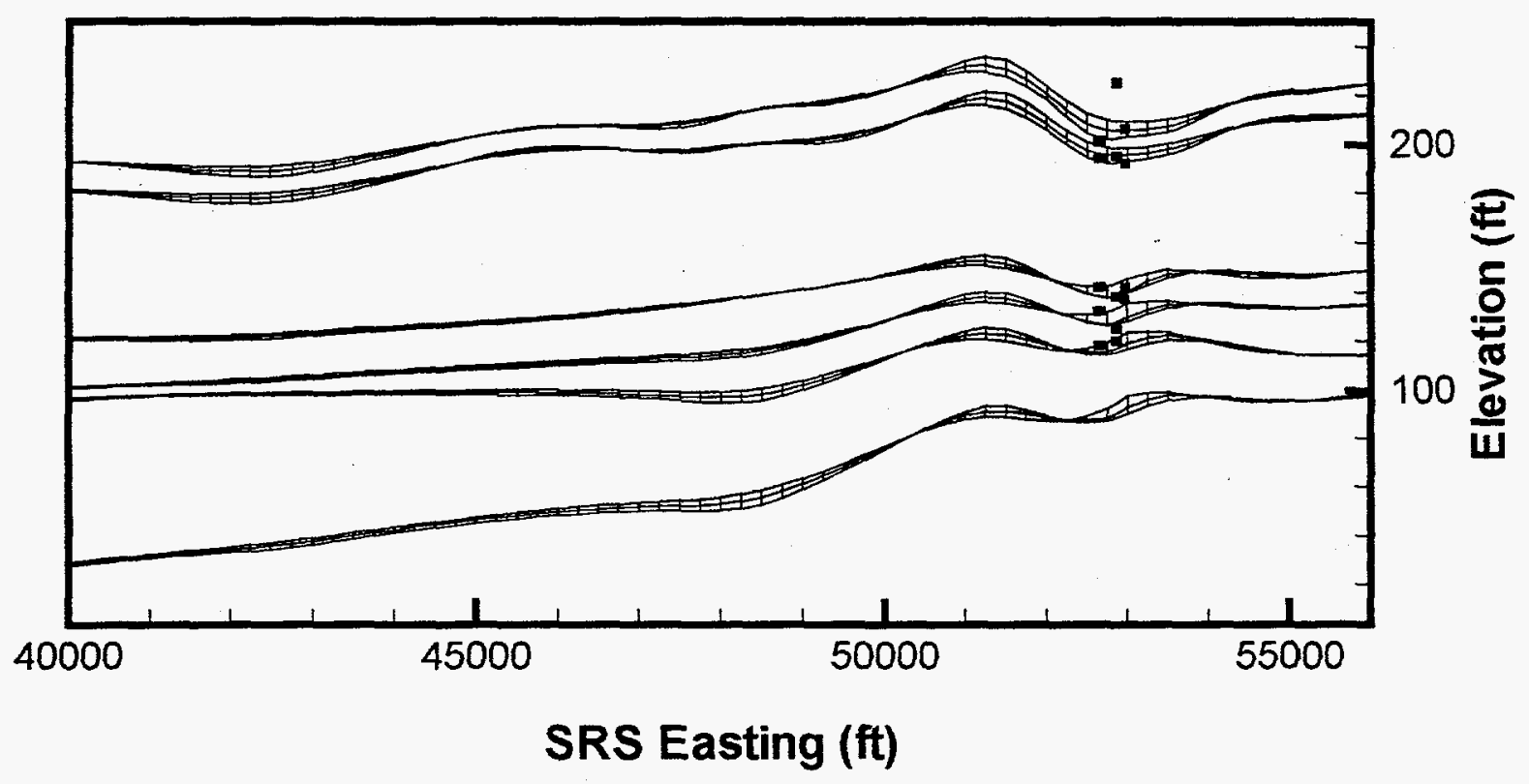

Figure 5.10 - DFM Estimated Layers at $y=105,500 \mathrm{ft}(+/-300)$ 


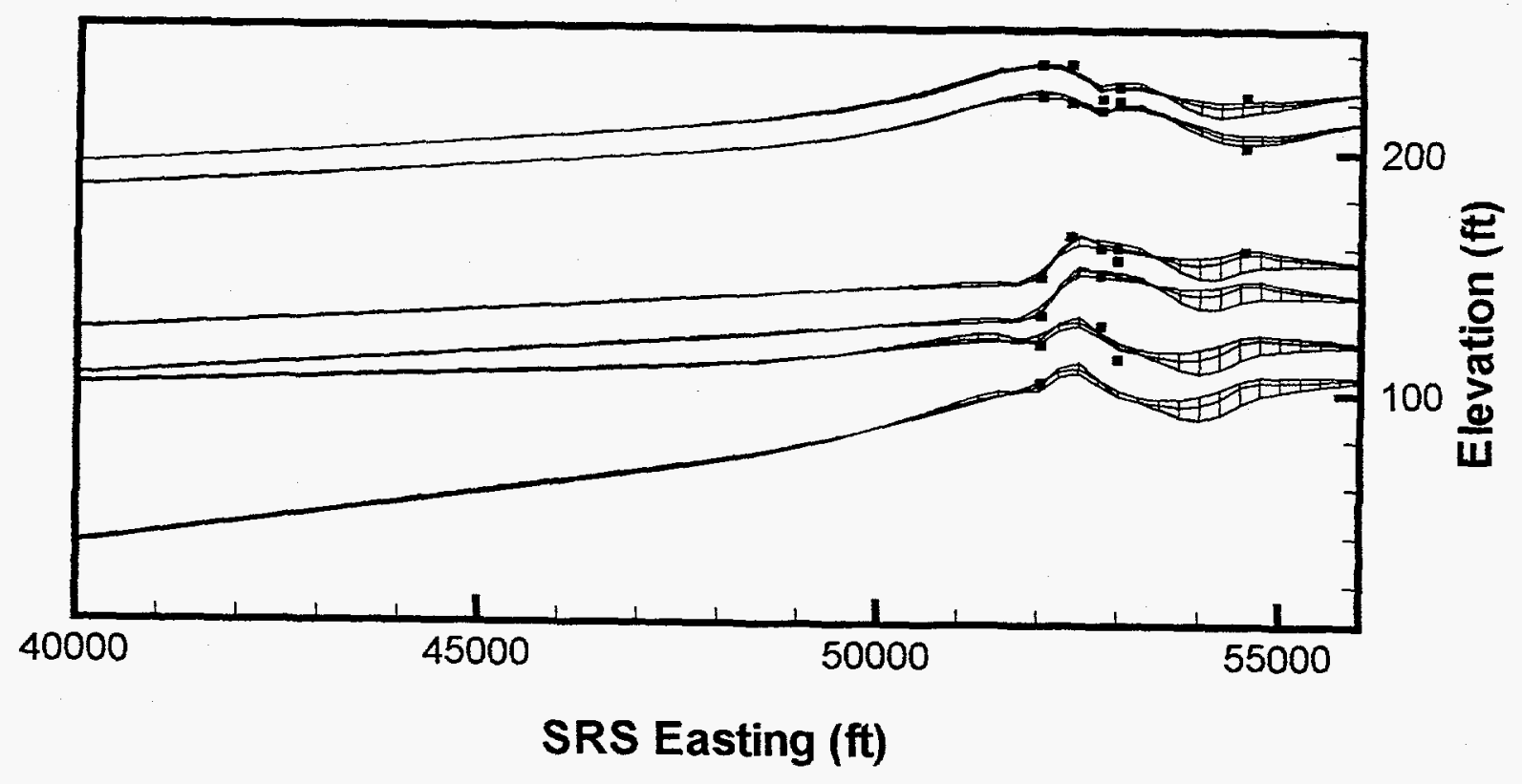

Figure 5.11 - DFM Estimated Layers at $y=108,500 \mathrm{ft}(+/-300)$ 
Table 5.10 Geological Data at y $=99500$ (ft)

\begin{tabular}{|c|c|c|c|c|c|}
\hline $\begin{array}{l}\text { Well or } \\
\text { CPT ID }\end{array}$ & $\begin{array}{l}\text { SRS } \\
\text { Easting } \\
(\mathrm{ft})\end{array}$ & $\begin{array}{l}\text { SRS } \\
\text { Northing } \\
\text { (ft) }\end{array}$ & Data Type & $\begin{array}{c}\text { Elevation } \\
\text { (ft) }\end{array}$ & $\begin{array}{c}\text { Residual } \\
\text { (ft) }\end{array}$ \\
\hline MSB_49A_ & 45864.6 & 99759.0 & GREEN_CLAY_TOP & 210.00 & -.62 \\
\hline MSB_49A_ & 45864.6 & 99759.0 & GREEN_CLAY_BOTTOM & 200.00 & .66 \\
\hline MSB_49A_ & 45864.6 & 99759.0 & UPPER_CLAY_TOP & 128.00 & .07 \\
\hline MSB_49B_ & 45868.2 & 99737.8 & GREEN_CLAY_TOP & 210.00 & -.71 \\
\hline $\mathrm{MSB}_{-} 49 \mathrm{~B}_{-}$ & 45868.2 & 99737.8 & GREEN_CLAY_BOTTOM & 200.00 & .61 \\
\hline $\mathrm{MSB}$ 49B_ & 45868.2 & 99737.8 & UPPER_CLAY_TOP & 128.00 & .00 \\
\hline MSB_79B_ & 47300.2 & 99296.9 & GREEN_CLAY_TOP & 228.00 & 2.05 \\
\hline MSB_79B_ & 47300.2 & 99296.9 & GREEN_CLAY_BOTTOM & 208.00 & -.79 \\
\hline MSB_79B_ & 47300.2 & 99296.9 & UPPER_CLAY_TOP & 130.00 & -.59 \\
\hline MSB_32B_. & 52742.5 & 99676.0 & LOWER_CLAY_BOTTOM & 77.00 & 1.91 \\
\hline MSB_32B_- & 52742.5 & 99676.0 & UPPER_CLAY_TOP & 117.00 & -.96 \\
\hline MSB_32B_ & 52742.5 & 99676.0 & UPPER_CLAY_BOTTOM & 102.00 & -2.33 \\
\hline MSB_32B_ & 52742.5 & 99676.0 & LOWER_CLAY_TOP & 98.00 & .49 \\
\hline MSB_32B_ & 52742.5 & 99676.0 & GREEN_CLAY_BOTTOM & 199.00 & -.06 \\
\hline MSB_32B_ & 52742.5 & 99676.0 & GREEN_CLAY_TOP & 218.00 & -.07 \\
\hline
\end{tabular}


Table 5.11 Geological Data at $y=102000(\mathrm{ft})$

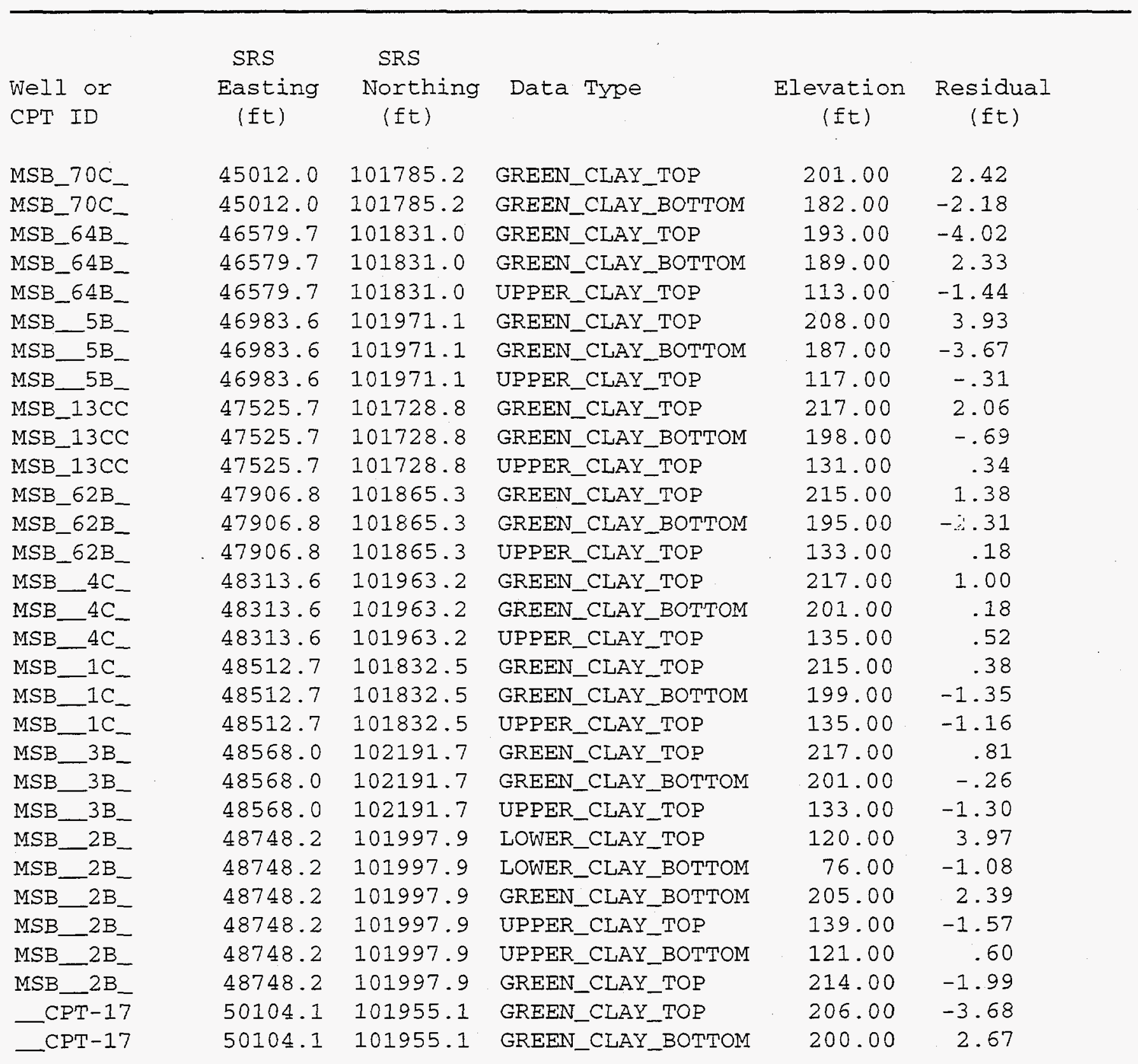


Table 5.12 Geological Data at $\mathrm{y}=105500(\mathrm{ft})$

\begin{tabular}{|c|c|c|c|c|c|}
\hline & SRS & SRS & & & \\
\hline $\begin{array}{l}\text { Well or } \\
\text { CPT ID }\end{array}$ & $\begin{array}{l}\text { Easting } \\
\quad(f t)\end{array}$ & $\begin{array}{l}\text { Northing } \\
\text { (Et) }\end{array}$ & Data Type & $\begin{array}{c}\text { Elevation } \\
\text { (ft) }\end{array}$ & $\begin{array}{c}\text { Residual } \\
\text { (ft) }\end{array}$ \\
\hline $\mathrm{ASB} \_6 \mathrm{AA}$ & 52643.9 & 105727.0 & GREEN_CLAY_TOP & 201.00 & -4.65 \\
\hline $\mathrm{ASB} \_6 \mathrm{AA}$ & 52643.9 & 105727.0 & GREEN_CLAY_BOTTOM & 194.00 & 1.59 \\
\hline $\mathrm{ASB} \_6 \mathrm{AA}$ & 52643.9 & 105727.0 & UPPER_CLAY_TOP & 142.00 & -.06 \\
\hline$A S B \_6 A A$ & 52643.9 & 105727.0 & UPPER_CLAY_BOTTOM & 132.00 & .98 \\
\hline $\mathrm{ASB} \_6 \mathrm{AA}$ & 52643.9 & 105727.0 & LOWER_CLAY_TOP & 118.00 & -.03 \\
\hline $\mathrm{ASB} \_6 \mathrm{TA}$ & 52671.3 & 105749.5 & UPPER_CLAY_TOP & 142.00 & -.43 \\
\hline $\mathrm{ASB}$ & 52671.3 & 105749.5 & GREEN_CLAY_TOP & 201.00 & -3.38 \\
\hline$A S B \_6 T A$ & 52671.3 & 105749.5 & GREEN_CLAY_BOTTOM & 194.00 & 2.75 \\
\hline $\mathrm{ASB} \_6 \mathrm{TA}$ & 52671.3 & 105749.5 & UPPER_CLAY_BOTTOM & 132.00 & .52 \\
\hline $\mathrm{ASB} \_6 \mathrm{TA}$ & 52671.3 & 105749.5 & IOWER_CLAY_TOP & 118.00 & -.54 \\
\hline $\mathrm{ASB} \_2 \mathrm{CR}$ & 52862.7 & 105540.2 & GREEN_CLAY_TOP & 225.00 & 10.48 \\
\hline $\mathrm{ASB} \_2 \mathrm{CR}$ & 52862.7 & 105540.2 & GREEN_CLAY_BOTTOM & 195.00 & -4.57 \\
\hline $\mathrm{ASB} \_2 \mathrm{CR}$ & 52862.7 & 105540.2 & UPPER_CLAY_TOP & 138.00 & -1.65 \\
\hline $\mathrm{ASB} \_2 \mathrm{CR}$ & 52862.7 & 105540.2 & UPPER_CLAY_BOTTOM & 125.00 & -3.91 \\
\hline $\mathrm{ASB} \_2 \mathrm{CR}$ & 52862.7 & 105540.2 & LOWER_CLAY_TOP & 120.00 & 2.27 \\
\hline$A S B \_10 C_{-}$ & 52965.5 & 105673.1 & UPPER_CLAY_TOP & 142.00 & -1.68 \\
\hline$A S B_{-} 10 C_{-}$ & 52965.5 & 105673.1 & GREEN_CLAY_TOP & 206.00 & -1.64 \\
\hline$A S B \_10 C_{-}$ & 52965.5 & 105673.1 & GREEN_CLAY_BOTTOM & 192.00 & -1.42 \\
\hline$A S B \_10 C_{-}$ & 52965.5 & 105673.1 & UPPER_CLAY_BOTTOM & 137.00 & 3.88 \\
\hline
\end{tabular}


Table 5.13 Geological Data at $y=108500(\mathrm{ft})$

\begin{tabular}{|c|c|c|c|c|c|}
\hline $\begin{array}{l}\text { Well or } \\
\text { CPT ID }\end{array}$ & $\begin{array}{l}\text { SRS } \\
\text { Easting } \\
\text { (ft) }\end{array}$ & $\begin{array}{l}\text { SRS } \\
\text { Northing } \\
(\mathrm{ft})\end{array}$ & Data Type & $\begin{array}{c}\text { Elevation } \\
\text { (ft) }\end{array}$ & $\begin{array}{c}\text { Residual } \\
\text { (ft) }\end{array}$ \\
\hline MSB_55TA & 52014.7 & 108322.8 & GREEN_CLAY_TOP & 236.00 & -.12 \\
\hline MSB_55TA & 52014.7 & 108322.8 & GREEN_CLAY_BOTTOM & 223.00 & -.04 \\
\hline MSB_55TA & 52014.7 & 108322.8 & UPPER_CIAY_TOP & 148.00 & -.51 \\
\hline MSB_55TA & 52014.7 & 108322.8 & UPPER_CLAY_BOTTOM & 132.00 & -1.10 \\
\hline $\mathrm{MSB} \_55 \mathrm{TA}$ & 52014.7 & 108322.8 & LOWER_CLAY_TOP & 120.00 & -1.28 \\
\hline $\mathrm{MSB} \_55 \mathrm{TA}$ & 52014.7 & 108322.8 & LOWER_CLAY_BOTTOM & 104.00 & 2.11 \\
\hline MSB_55C_ & 52029.7 & 108324.6 & UPPER_CLAY_TOP & 148.00 & -1.16 \\
\hline MSB_55C_ & 52029.7 & 108324.6 & GREEN_CLAY_TOP & 236.00 & -.10 \\
\hline MSB_55C_ & 52029.7 & 108324.6 & GREEN_CLAY_BOTTOM & 223.00 & -.02 \\
\hline MSB_55C_ & 52029.7 & 108324.6 & UPPER_CLAY_BOTTOM & 132.00 & -1.79 \\
\hline MSB_55C_ & 52029.7 & 108324.6 & LOWER_CLAY_TOP & 120.00 & -1.66 \\
\hline MSB_55C_ & 52029.7 & 108324.6 & LOWER_CLAY_BOTTOM & 104.00 & 1.72 \\
\hline MSB_83C_ & 52384.7 & 108405.3 & GREEN_CLAY_TOP & 236.00 & 2.14 \\
\hline MSB_83C_ & 52384.7 & 108405.3 & GREEN_CLAY_BOTTOM & 220.00 & -1.48 \\
\hline MSB_83C_ & 52384.7 & 108405.3 & UPPER_CLAY_TOP & 165.00 & 2.55 \\
\hline MSB_83TA & 52410.9 & 108416.3 & UPPER_CLAY_TOP & 165.00 & 1.83 \\
\hline MSB_83TA & 52410.9 & 108416.3 & GREEN_CLAY_TOP & 236.00 & 2.53 \\
\hline MSB_83TA & 52410.9 & 108416.3 & GREEN_CLAY_BOTTOM & 220.00 & -1.22 \\
\hline $\mathrm{MBC} \_1 \mathrm{SB}$ & 52780.0 & 108450.0 & LOWER_CLAY_TOP & 128.00 & 3.33 \\
\hline $\mathrm{MBC} \_1 \mathrm{SB}$ & 52780.0 & 108450.0 & UPPER_CIAY_BOTTOM & 149.00 & -1.64 \\
\hline $\mathrm{MBC} \_1 \mathrm{SB}$ & 52780.0 & 108450.0 & GREEN_CLAY_BOTTOM & 217.00 & .89 \\
\hline $\mathrm{MBC} \_1 \mathrm{SB}$ & 52780.0 & 108450.0 & UPPER_CLAY_TOP & 160.00 & -2.75 \\
\hline $\mathrm{MBC} \_1 \mathrm{SB}$ & 52780.0 & 108450.0 & GREEN_CLAY_TOP & 222.00 & -4.19 \\
\hline MSB_54TA & 52985.8 & 108446.3 & UPPER_CLAY_TOP & 160.00 & -1.00 \\
\hline MSB_54TA & 52985.8 & 108446.3 & UPPER_CLAY_BOTTOM & 155.00 & 5.77 \\
\hline MSB_54TA & 52985.8 & 108446.3 & LOWER_CLAY_TOP & 114.00 & -5.29 \\
\hline MSB_54TA & 52985.8 & 108446.3 & GREEN_CLAY_TOP & 227.00 & -.81 \\
\hline MSB_54TA & 52985.8 & 108446.3 & GREEN_CLAY_BOTTOM & 221.00 & 2.23 \\
\hline MSB_86C_ & 54560.5 & 108500.4 & UPPER_CLAY_TOP & 159.00 & 1.27 \\
\hline MSB_86C_ & 54560.5 & 108500.4 & GREEN_CLAY_TOP & 223.00 & 3.61 \\
\hline MSB_86C_ & 54560.5 & 108500.4 & GREEN_CI_AY_BOTTOM & 202.00 & -3.30 \\
\hline
\end{tabular}




\subsection{GROUNDWATER MODEL CALIBRATION USING DFM}

\subsection{DATA FUSION MODELING METHODOLOGY}

Details of the data fusion modeling (DFM) approach are given by Gibbs and Hughes (1995). Basically DFM is a Bayesean least squares estimation method which combines information from the measurements, prior information on model parameters, stochastic descriptions of the random variability (heterogeneity) of the hydraulic conductivity field, and the steady-state groundwater flow equations to compute a flow model which best fits all this information. Each source of information is weighted in the solution according to the expected accuracy of the data model. The groundwater flow model and stochastic model of conductivity heterogeneity are incorporated in the solution by treating these models as pseudo-measurements. That is, the output of these models at each node is treated somewhat like an ordinary measurement in that it has an expected value and random error for which the variance is known. Using this approach, all measurements and pseudo-measurements are processed as ordinary measurements in the data equation representation developed by Bierman (1977). Further details of the measurement processing are given by Gibbs and Hughes (1995).

DFM attempts to minimize the residuals (difference between the data and the model output) for all four sources of information described above by minimizing a nonlinear least squares cost function representing the weighted sum of residuals. i.e.

$$
J=\frac{1}{2} \sum_{i}\left[\frac{z_{i}-h_{i}(x)}{w_{z i}}\right]^{2}+\frac{1}{2} \sum_{i}\left[\frac{0-f_{i}(x)}{w_{f i}}\right]^{2}+\frac{1}{2} \sum_{i}\left[\frac{0-g_{i}(x)}{w_{g i}}\right]^{2}+\frac{1}{2} \sum_{i}\left[\frac{x_{p i}-x_{i}}{w_{x i}}\right]^{2}
$$

where $z_{i}$ is the measurement $i ; x$ is the state vector containing all model parameters to be estimated (nodal head and $\ln (\mathrm{Kh})$, anisotropy, recharge, source/drain heads of head-dependent flux boundary conditions, etc.); $h_{i}(x)$ is the nonlinear function which computes the expected value of measurement based on the state; $\mathrm{w}_{\mathrm{zi}}$ is the weighting of the measurement (standard deviation of measurement noise); $\mathrm{f}_{\mathrm{i}}(\mathrm{x})$ is the nonlinear function which computes the steady-state flow error based on $\mathrm{x} ; \mathrm{w}_{\mathrm{fi}}$ is the weighting of the flow measurement (standard deviation of model error set $=0.0002 \mathrm{ft} / \mathrm{day}) ; \mathrm{g}_{\mathrm{i}}(\mathrm{x})$ is the linear function which computes the expected value of the stochastic variability model based on state $\mathrm{x} ; \mathrm{w}_{\mathrm{gi}}$ is the weighting of the spatial variability model (standard deviation of model error); $\mathrm{x}_{\mathrm{p} i}$ is the prior expected value of component $\mathrm{i}$ of $\mathrm{x}$; $\mathrm{w}_{\mathrm{xi}}$ is the weighting of the prior estimate (standard deviation of prior error).

The models represented by $h_{i}(x), f_{i}(x)$, and $g_{i}(x)$ were described in Section 4 .

$\mathrm{J}$ can be minimized using an iterative Gauss-Newton method which re-linearizes about the current estimate at each iteration, using an approximate Hessian of the cost function. The linearized problem can be expressed in the information array form due to Bierman (1977).

Unfortunately, full Gauss-Newton steps may greatly exceed the linear region of the partial derivatives and thus the method may not converge. It is therefore necessary to restrict the 
Gauss-Newton steps. While many methods have been used for this purpose (e.g. Levenberg-Marquardt), we prefer either the trust-region methods (Vandergraft (1977)) or backtracking (Press (1992)). The trust region method may be the most robust, but it can also be computationally expensive. We have found that backtracking is quite robust and satisfactory for most applications.

The DFM software has several options for computing the step at each iteration: direct matrix solution (matrix triangularization), an iterative preconditioned conjugate gradient solution using iterative solutions of the flow and stochastic models, a conjugate gradient solution using the gradient only, or a quasi-Newton method based on the gradient only. The last option is restricted to the multi-grid DFM (described later) and the third option has not been overly successful. The direct solution is practical because all four sources of information can be formulated as finite-difference-like equations involving only nearest neighbor nodes. Thus the information array is extremely sparse and the direct method can be coded to take advantage of that sparseness. However, the direct method becomes increasing impractical in terms of computer memory, disk space and computations as the number of nodes increases beyond about 15000 to 20000 . Thus the second method, referred to as the "fast solver", is the preferred method for large problems. Unfortunately, while it computes the Gauss-Newton step, it does not compute the covariance: the direct method must be used to compute this for medium-sized problems or the multi-grid method (described later) for large problems.

One other important refinement of the basic Gauss-Newton iteration is the inclusion of automated data editing logic. While one hopes that the measurement data is accurate within about three times the specified standard deviation, real data sets always seem to include a few anomalous measurements which deviate significantly from the surrounding data. Often this is due to transcription errors in recording or transforming the data, or in specifying the location of the measurement. In other cases it is due to a local effect which cannot be modeled on the granularity of the numerical model (such as local drawdown near a pumping well). Thus the DFM software tests the residuals from each measurement set normalized by the specified standard deviation and by the overall residual RMS from the previous iteration. If that residual sum-of-squares exceeds at user specified threshold, the measurement set is edited. Since the residual RMS from the previous iteration is included in the test, large residuals on early iterations (caused by inaccurate initial estimates of the states) will not cause excessive editing. While this automated editing procedure cannot be based on rigorous statistical analysis, it has been used for many years in a variety of applications ranging from satellite orbit determination (Chin, et al. (1972)) to power plant modeling (Gibbs(1995)), and has proved satisfactory.

Most of the above DFM description refers to the standard DFM software. There is also an alternate version referred to as multi-grid. While standard hydrological DFM estimates hydraulic head and natural $\log \mathrm{y}$-direction conductivity $(\ln (\mathrm{Kh}))$ at each node in the numerical model, the multi-grid DFM uses a fine grid for the flow numerical model and a much coarser grid (e.g. less than 2000 nodes) for the random field model of $\ln (\mathrm{Kh})$. The basis for this approach is the belief that a fine numerical flow grid is necessary to accurately model flow (especially near pumping wells and other discontinuities), but even if the earth is truly heterogeneous on the scale of the numerical grid, a sufficient number of conductivity measurements is not available to estimate the conductivity at this scale. Thus the "standard DFM" user will specify a correlation distance for $\ln (\mathrm{Kh})$ much larger than the flow model grid spacing. In multi-grid DFM the conductivity is 
specified on a coarser grid where the spacing between nodes is comparable to the correlation distances which would be specified for standard DFM. Then interpolation (e.g. linear) can be used to compute the conductivity at the flow model nodes. Note that this approach is not simply a zonal specification of conductivity as is often used. Rather, $\ln (\mathrm{Kh})$ is modeled as a random field where $\ln (\mathrm{Kh})$ will differ at each node of the flow model.

While the multi-grid approach potentially suffers from the coarser specification of conductivity than standard DFM, it has several advantages. First, it is implemented using an exact solution to the steady-state flow equation so that some of the linearization problems encountered in standard DFM are minimized. Thus it tends to converge in fewer iterations than standard DFM and may be more robust. Secondly, it can be implemented using either a direct method or the quasi-Newton method: the computational requirements are approximately equal for the $\mathrm{A} / \mathrm{M}$ area model. Thus it is possible to compute the state error covariance matrix for much flow larger grids than for standard DFM. Furthermore, the method can be easily extended to include transient flow or contaminant transport measurements. Unfortunately, it suffers from one disadvantage which was not obvious. For small to medium sized grids, the computational burden is usually larger for multi-grid than standard DFM. However, the computations do not increase as rapidly with the number of grid nodes as for standard DFM, so that multi-grid will be more efficient for large grids. Most of the results in this section are based on standard DFM. The flow pattern variability analysis in section 7.0 used multi-grid DFM because of the requirement to compute the state error covariance matrix.

\subsection{APPLICATION OF DFM TO SRS A/M AREA}

As previously noted, the flow numerical model covers a horizontal area of 12,500 (ft) by 15,000 (ft). Numerical horizontal grid cell sizes of both 250 feet (fine grid) and 500 feet (coarse grid) were used in this study. The numerical model used 22 nodal layers distributed by hydrostratigraphic unit as follows:

Table 6.1 Model Layers per Hydrostratigraphic Unit

\begin{tabular}{|c|c|}
\hline Hydrostratigraphic Unit & Model Layers \\
\hline "M-Area" Aquifer Zone & 7 \\
\hline $\begin{array}{c}\text { "Green Clay" Confining plus "Lost } \\
\text { Lake" Aquifer Zones }\end{array}$ & 8 \\
\hline "Crouch Branch" Confining Zone & 7 \\
\hline
\end{tabular}

We initially used 6 hydrostratigraphic unit for the A/M Area: "M-Area" Aquifer Zone, "Green Clay" Confining Zone, "Lost Lake" Aquifer Zone, "upper clay" zone, "middle sand" zone, and "lower clay"zone. However, the poor definition and discontinuity of some clay layers made modeling difficult. It was found that DFM could better fit the measurements and produced more reasonable flow estimates using the three hydrostratigraphic units listed above. We also tried modeling the area as a single hydrostratigraphic unit, but the lack of a model structure caused convergence problems for DFM. 
The elevation of each layer node was determined by using a fixed ratio of the hydrostratigraphic unit upper and lower elevation. For example, the top layer was set to the surface elevation and layer 7 was set to the "Green Clay" top elevation plus $4 \%$ of the "M-Area" Aquifer Zone thickness. Layers 2 to 6 were computed as fixed ratios between layers 1 and 7 . Figure 6.1 shows the nodal layers for the $y-z$ slice at $x=50000$.

The resulting numerical grids were 17732 nodes (coarse) and 68442 nodes (fine). Most of the initial results were obtained using the coarse grid because of the much shorter computational time This allowed determination of significant modeling issues and resolution of problems. When satisfactory results were obtained, the fine grid was used to compute final results.

As noted previously, "standard DFM" estimates hydraulic head and $\ln (\mathrm{Kh})$ at each numerical node. In addition to the random component of $\ln (\mathrm{Kh})$, the trend was modeled as a constant bias for each hydrostratigraphic unit, which was estimated with an a priori uncertainty of 0.2 to 0.3 . Although the software is capable of estimating up to 4th-order spatial polynomials, there was no reason to expect significant trends in the conductivities so that only a bias was modeled. The log vertical anisotropy was treated as an unknown bias to be estimated (a priori value $=5.0$ with uncertainty $=0.5$ ). Again this could have been estimated as a higher order spatial polynomial but was not believed to be necessary. The horizontal anisotropy was fixed at 1.0.

The calculation of hydraulic conductivity from mud fraction data as described in section 4.4 is subject to both systematic and random errors. Calculation of vertical conductivity is believed to be particularly subject to errors. Biases on log horizontal and vertical conductivity were estimated in the DFM to allow for adjustments to average conductivity and recharge. The prior values were assumed to be zero with a 1 -sigma uncertainty of 0.3 . (Note that a bias in log is equivalent to a multiplicative error, i.e. scale factor error: $\mathrm{e}^{0.3}=1.35$ ).

Recharge is another modeling parameter potentially subject to errors. In the $\mathrm{A} / \mathrm{M}$ Area, recharge was modeled in four separate zones (Figure 6.1): the general area (16 inches/year), paved areas ( 3 inches/year), the A-14 outfall ( 30 inches/year) and the A-1 outfall ( 21 inches/year). While the two outfalls have high recharge, the area is small and there are few wells nearby, so that the model is not sensitive to these recharge values (i.e. the observability of the recharges is poor). Thus these two values were fixed in DFM while the general and paved area recharge values were estimated using a 1 -sigma uncertainty of $1 \mathrm{inch} /$ year.

Boundary conditions (BC) for the modeled area are poorly known because of a lack of wells outside the area. There are no monitoring wells north and east of the model boundaries and only a limited number of wells to the south and west (and these are not close). In this report, north, south, east and west refer to the SRS site coordinate. Following the methods used in previous studies, we initially assumed that the eastern boundary was no-flow for all three aquifers. Because some head data was available to the northwest of the site, we initially fit contours to all the data for each aquifer and used the interpolated values at the western boundaries as fixed head boundary conditions. For the north and south boundaries, we tried both no-accumulation (second derivative of head perpendicular to boundary is zero) and head-dependent flux (HDF) conditions where DFM estimated the source heads. Both methods produced satisfactory results. Since the no-accumulation $\mathrm{BC}$ is uncommon in groundwater modeling and use of the HDF gives DFM more 
WSRC-OS-97-00002

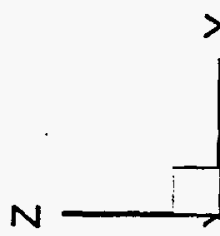

$-$
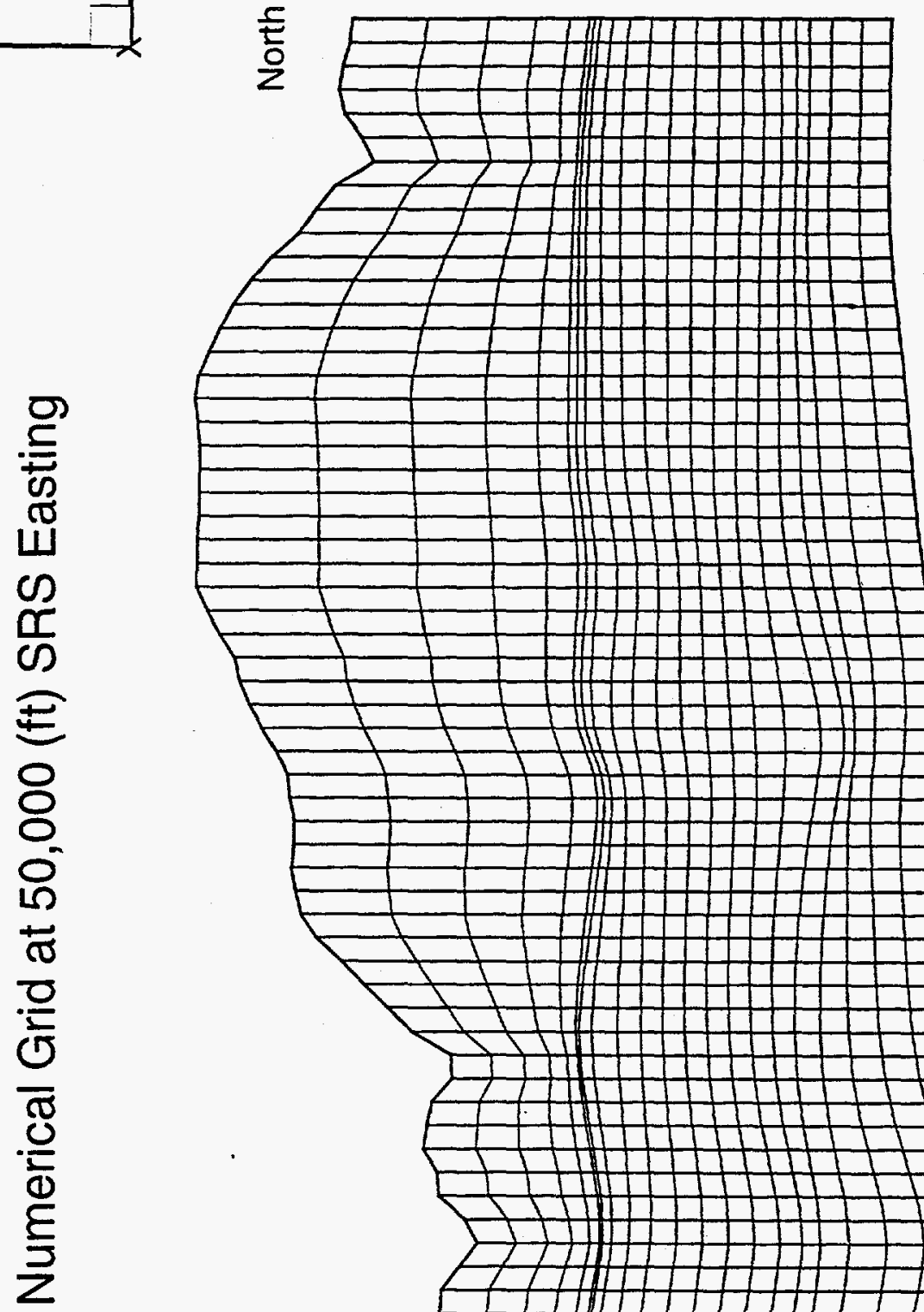

을
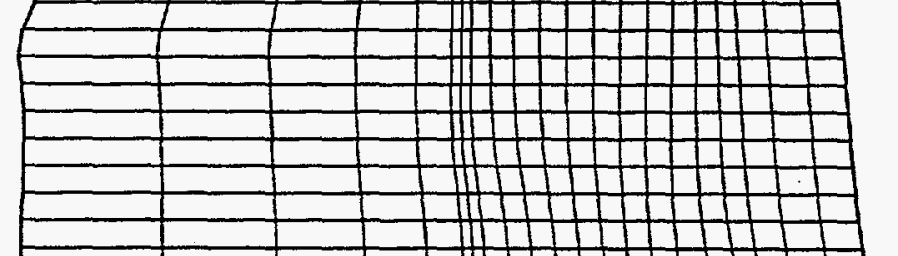

0

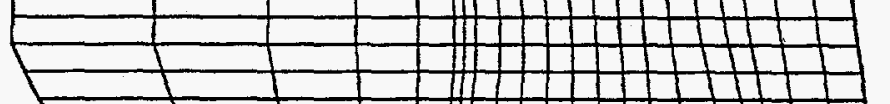

क

正
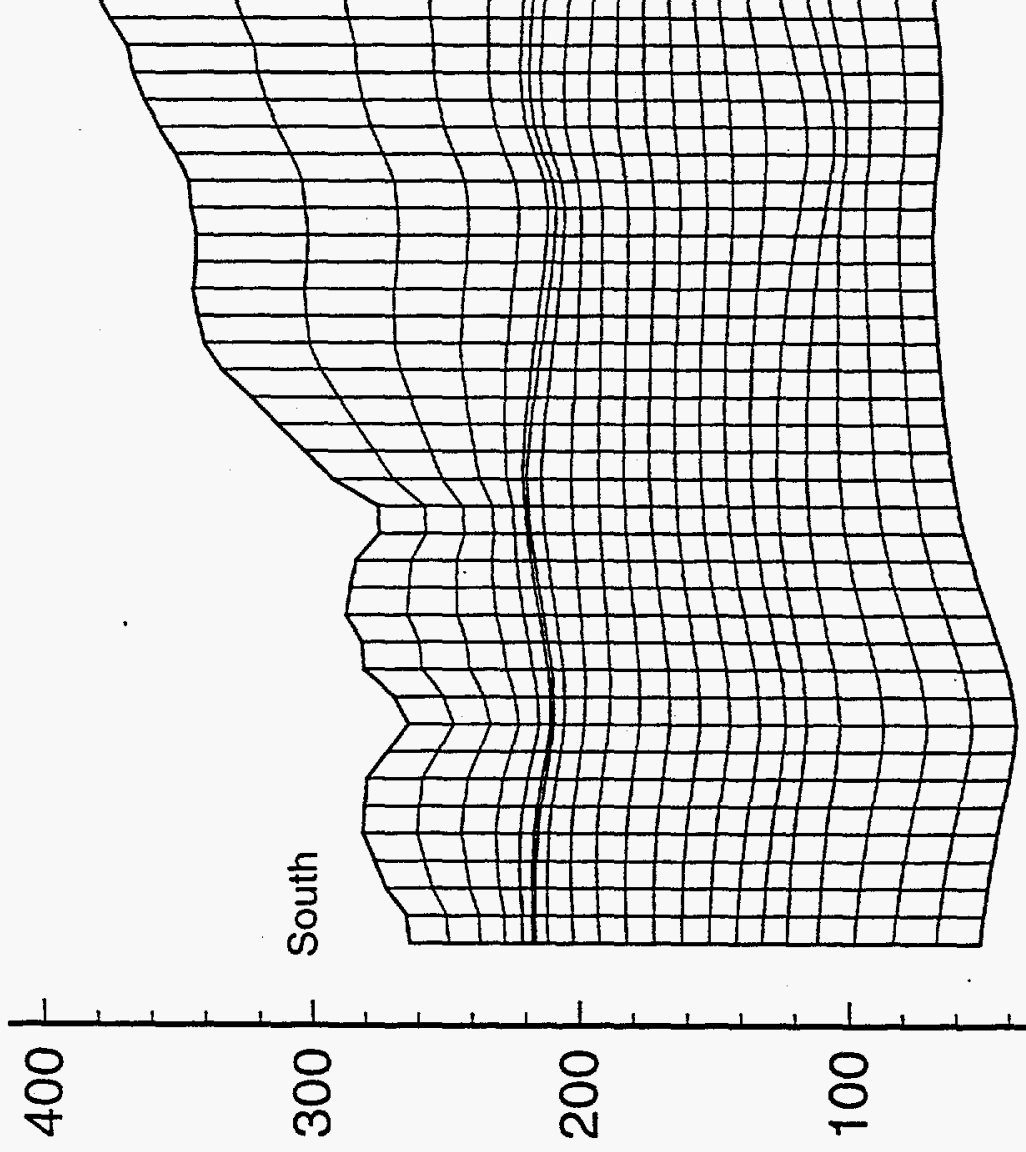

요

운

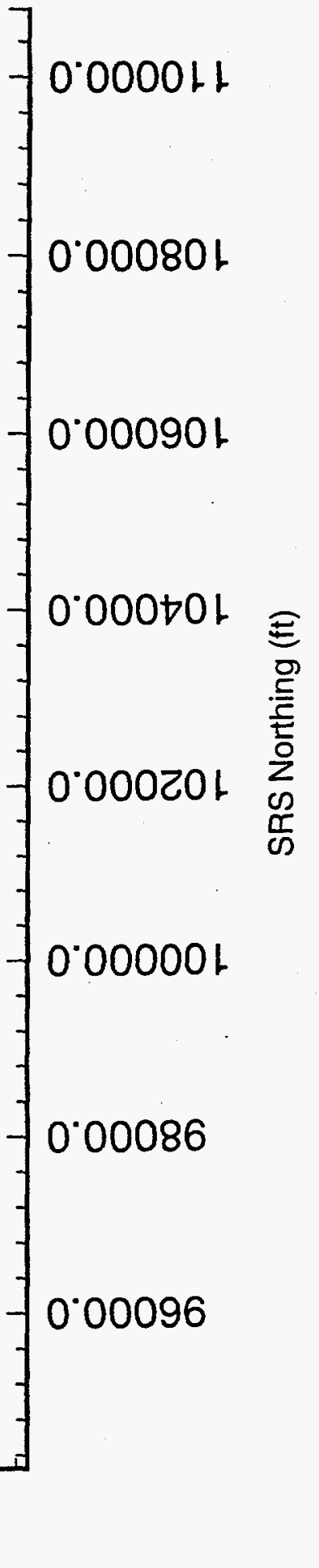

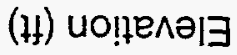

Figure 6.1 Fine grid at SRS Easting 50,000 (ft) 
control of the model, the HDF was selected as the preferred model. As will be explained later, the HDF was eventually used for all four model sides for all three aquifers.

The bottom layer of the model grid was defined as a fixed head BC using the interpolated heads (Figure 6.3) computed from fitting head data from the Crouch Branch Aquifer, which is listed in Table 6.2. Although the well information in this aquifer is sparse and thus the computed heads may not be accurate, the sensitivity of our model to those boundary conditions is small (because the bottom unit is mostly clay).

The HDF for each boundary and aquifer was defined in zones of about 2000 to 6000 feet horizontally where the extent of the zone was determined by the variation of head in the zone. All zones used the same leakance $\left(0.07\right.$ for an area of $\left.3800 \mathrm{ft}^{2}\right)$ but the source heads for each zone were different. Since these were not known accurately, they were estimated in DFM using an a prior value determined from fits to the data The assumed 1-sigma uncertainty was 2 feet.

To summarize, the prior information used for the 67 estimated common parameters is (units of $\mathrm{ft} / \mathrm{d}$ ) is listed in Tables 6.3 to 6.8 .

Table 6.2 Crouch Branch Aquifer Head Data

\begin{tabular}{|c|c|c|c|}
\hline Well ID & $\begin{array}{c}\text { SRS Easting } \\
\text { (ft) }\end{array}$ & $\begin{array}{c}\text { SRS Northing } \\
\text { (ft) }\end{array}$ & $\begin{array}{c}\text { Head } \\
\text { (ft) }\end{array}$ \\
\hline ASB 8TA & 53124.69 & 106375.79 & 217.0 \\
\hline MSB 12TA & 47127.30 & 102266.70 & 193.7 \\
\hline MSB 12TB & 47133.00 & 102260.10 & 193.6 \\
\hline MSB 21TA & 47218.19 & 103980.89 & 165.2 \\
\hline MSB 27TA & 49486.80 & 104951.39 & 202.3 \\
\hline MSB 29TA & 51245.69 & 107330.39 & 213.5 \\
\hline MSB 30A & 48004.10 & 105727.39 & 199.5 \\
\hline MSB 31A & 50100.19 & 101979.29 & 199.1 \\
\hline MSB 33TA & 51734.00 & 98018.20 & 195.6 \\
\hline MSB 34TA & 50536.60 & 104905.79 & 202.5 \\
\hline MSB 34TB & 50537.89 & 104891.60 & 203.8 \\
\hline MSB 35TA & 50919.60 & 102101.60 & 201.9 \\
\hline MSB 36TA & 49503.00 & 100507.70 & 195.5 \\
\hline MSB 37A & 51439.80 & 105295.00 & 208.5 \\
\hline MSB 37TA & 514499.80 & 105301.29 & 208.3 \\
\hline MSB 38TA & 49810.39 & 102434.89 & 199.2 \\
\hline MSB 39TA & 48357.69 & 100830.60 & 193.6 \\
\hline MSB 40TA & 48277.19 & 97660.39 & 190.2 \\
\hline MSB 41TA & 53429.69 & 102176.50 & 207.4 \\
\hline MSB 42TA & 54581.69 & 104545.60 & 206.9 \\
\hline MSB 43TA & 49281.80 & 107275.79 & 204.2 \\
\hline
\end{tabular}




\begin{tabular}{|l|l|l|l|}
\hline MSB 47TA & 52219.00 & 106987.70 & 217.9 \\
\hline MSB 54TA. & 52985.80 & 108446.29 & 220.8 \\
\hline MSB 55TA & 52014.69 & 108322.79 & 215.3 \\
\hline MSB 66TA & 51096.69 & 105842.60 & 207.6 \\
\hline MSB 69TA & 52418.39 & 107772.50 & 216.8 \\
\hline MSB 82TA & 51964.19 & 107525.70 & 215.7 \\
\hline MSB 83TA & 52410.89 & 108416.29 & 216.8 \\
\hline
\end{tabular}

Table 6.3 Prior Estimate and Uncertainty of Trend Log Conductivity States

\begin{tabular}{|c|c|c|c|}
\hline Parameter & Unit & Prior Value & Prior 1-sigma \\
\hline mean $\ln (\mathrm{Kh})$ & "M-Area" Aquifer & 2.0 & 1.0 \\
\hline mean $\ln (\mathrm{Kh})$ & $\begin{array}{c}\text { "Lost Lake"/"Green } \\
\text { Clay" }\end{array}$ & 2.0 & 1.0 \\
\hline mean $\ln (\mathrm{Kh})$ & CB Confining Unit & 2.0 & 1.0 \\
\hline $\begin{array}{c}\text { mean } \\
\ln (\mathrm{Kh} / \mathrm{Kv})\end{array}$ & "M-Area" Aquifer & 5.0 & 0.5 \\
\hline $\begin{array}{c}\text { mean } \\
\ln (\mathrm{Kh} / \mathrm{Kv})\end{array}$ & $\begin{array}{c}\text { "Lost Lake"/"Green } \\
\text { Clay" }\end{array}$ & 5.0 & 0.5 \\
\hline $\begin{array}{c}\mathrm{mean} \\
\ln (\mathrm{Kh} / \mathrm{Kv})\end{array}$ & CB Confining Unit & 5.0 & 0.5 \\
\hline
\end{tabular}

Table 6.4 Prior Estimate and Uncertainty of Measurement Bias and Recharge States

\begin{tabular}{|c|c|c|}
\hline Parameter & Prior Value & Prior 1-sigma \\
\hline meas. bias $-\ln (\mathrm{Kh})$ & 0.0 & 0.3 \\
\hline meas. bias $-\ln (\mathrm{Kv})$ & 0.0 & 0.3 \\
\hline recharge - general area & 0.00342 & 0.00023 \\
\hline recharge - paved areas & 0.00068 & 0.00023 \\
\hline
\end{tabular}


Table 6.5 Prior Estimate and Uncertainty of North Boundary Source Head States

\begin{tabular}{|c|c|c|c|}
\hline Aquifer & Grid Columns & Prior Value & Prior 1-sigma \\
\hline M-area & $2-10$ & 225. & 2.0 \\
\hline M-area & $11-40$ & 230. & 2.0 \\
\hline M-area & $41-50$ & 230. & 2.0 \\
\hline Lost Lake & $2-10$ & 220. & 2.0 \\
\hline Lost Lake & $11-20$ & 224. & 2.0 \\
\hline Lost Lake & $21-28$ & 230. & 2.0 \\
\hline Lost Lake & $29-36$ & 230. & 2.0 \\
\hline Lost Lake & $37-44$ & 225. & 2.0 \\
\hline Lost Lake & $45-50$ & 222. & 2.0 \\
\hline middle sand & $2-10$ & 220. & 2.0 \\
\hline middle sand & $11-40$ & 220. & 2.0 \\
\hline middle sand & $41-50$ & 220. & 2.0 \\
\hline
\end{tabular}

Table 6.6 Prior Estimate and Uncertainty of South Boundary Source Head States

\begin{tabular}{|c|c|c|c|}
\hline Aquifer & Grid Columns & Prior Value & Prior 1-sigma \\
\hline M-area & $2-10$ & 195. & 2.0 \\
\hline M-area & $11-40$ & 195. & 2.0 \\
\hline M-area & $41-50$ & 195. & 2.0 \\
\hline Lost Lake & $2-14$ & 195. & 2.0 \\
\hline Lost Lake & $15-30$ & 195. & 2.0 \\
\hline Lost Lake & $31-50$ & 195. & 2.0 \\
\hline middle sand & $2-10$ & 195. & 2.0 \\
\hline middle sand & $11-40$ & 195. & 2.0 \\
\hline middle sand & $41-50$ & 195. & 2.0 \\
\hline
\end{tabular}

Table 6.7 Prior Estimate and Uncertainty of East Boundary Source Head States

\begin{tabular}{|c|c|c|c|}
\hline Aquifer & Grid Rows & Prior Value & Prior 1-sigma \\
\hline M-area & $1-10$ & 215. & 2.0 \\
\hline M-area & $11-20$ & 227. & 2.0 \\
\hline M-area & $21-30$ & 235. & 2.0 \\
\hline M-area & $31-40$ & 235. & 2.0 \\
\hline M-area & $41-50$ & 235. & 2.0 \\
\hline M-area & $51-61$ & 235. & 2.0 \\
\hline Lost Lake & $1-10$ & 203. & 2.0 \\
\hline Lost Lake & $11-20$ & 215. & 2.0 \\
\hline Lost Lake & $21-30$ & 220. & 2.0 \\
\hline Lost Lake & $31-40$ & 220. & 2.0 \\
\hline Lost Lake & $41-50$ & 220. & 2.0 \\
\hline
\end{tabular}




\begin{tabular}{|c|c|c|c|}
\hline Lost Lake & $51-61$ & 220. & 2.0 \\
\hline middle sand & $1-10$ & 197.0 & 2.0 \\
\hline middle sand & $11-20$ & 210.0 & 2.0 \\
\hline middle sand & $21-30$ & 213.0 & 2.0 \\
\hline middle sand & $31-40$ & 213.0 & 2.0 \\
\hline middle sand & $41-50$ & 213.0 & 2.0 \\
\hline middle sand & $51-61$ & 212.0 & 2.0 \\
\hline
\end{tabular}

Table 6.8 Prior Estimate and Uncertainty of West Boundary Source Head States

\begin{tabular}{|c|c|c|c|}
\hline Aquifer & Grid Rows & Prior Value & Prior 1-sigma \\
\hline M-area & $1-10$ & 210. & 2.0 \\
\hline M-area & $11-20$ & 215. & 2.0 \\
\hline M-area & $21-30$ & 215. & 2.0 \\
\hline M-area & $31-40$ & 215. & 2.0 \\
\hline M-area & $41-50$ & 220. & 2.0 \\
\hline M-area & $51-61$ & 220. & 2.0 \\
\hline Lost Lake & $1-10$ & 197. & 2.0 \\
\hline Lost Lake & $11-20$ & 202. & 2.0 \\
\hline Lost Lake & $21-30$ & 210. & 2.0 \\
\hline Lost Lake & $31-40$ & 215. & 2.0 \\
\hline Lost Lake & $41-50$ & 215. & 2.0 \\
\hline Lost Lake & $51-61$ & 220. & 2.0 \\
\hline middle sand & $1-10$ & 199.0 & 2.0 \\
\hline middle sand & $11-20$ & 198.0 & 2.0 \\
\hline middle sand & $21-30$ & 198.0 & 2.0 \\
\hline middle sand & $31-40$ & 199.0 & 2.0 \\
\hline middle sand & $41-50$ & 199.0 & 2.0 \\
\hline middle sand & $51-61$ & 200.0 & 2.0 \\
\hline
\end{tabular}




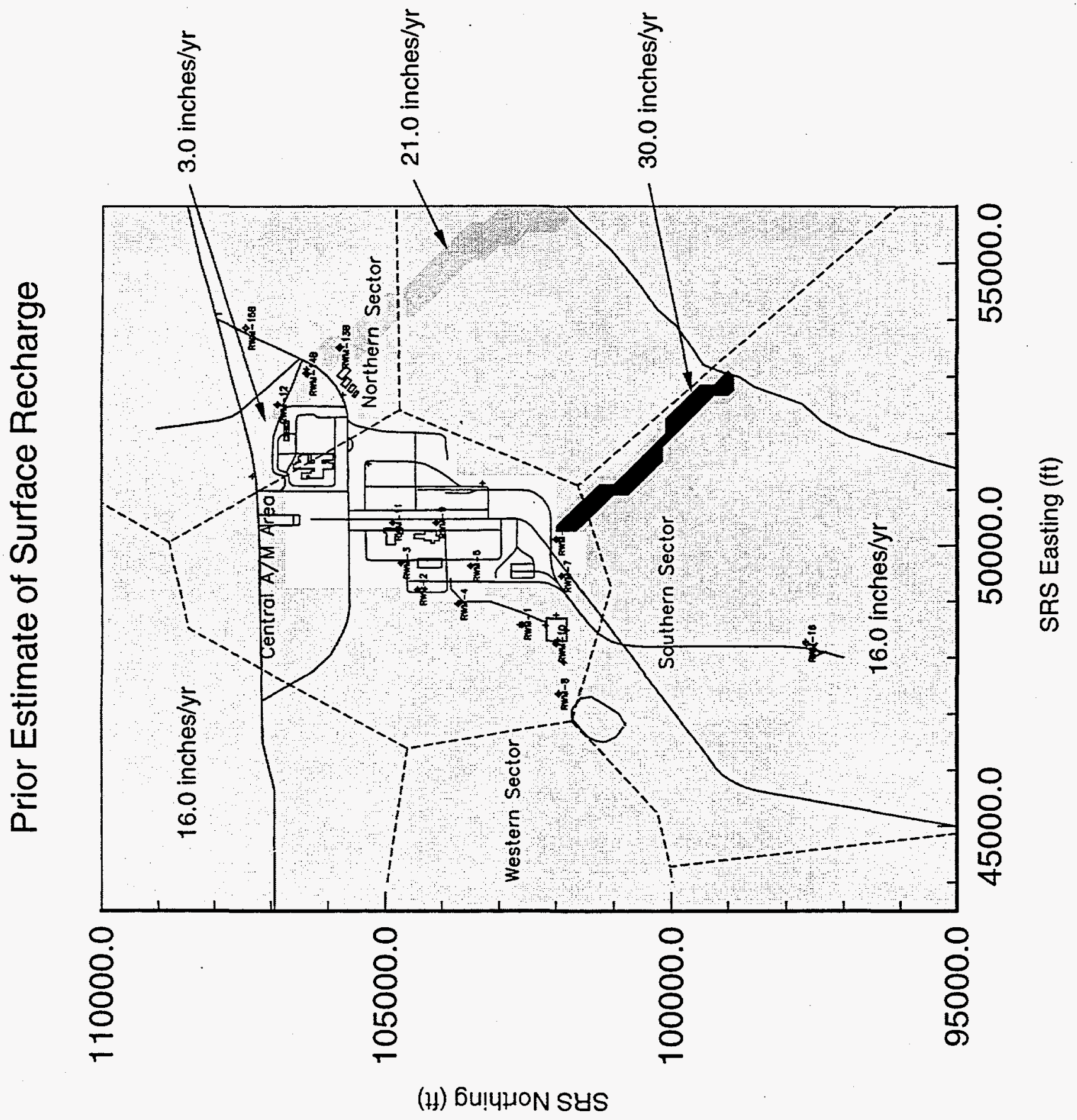

Figure 6.2 Prior estimate of surface recharge. 
Head Contours (ft) and Data for Crouch Branch Aquifer

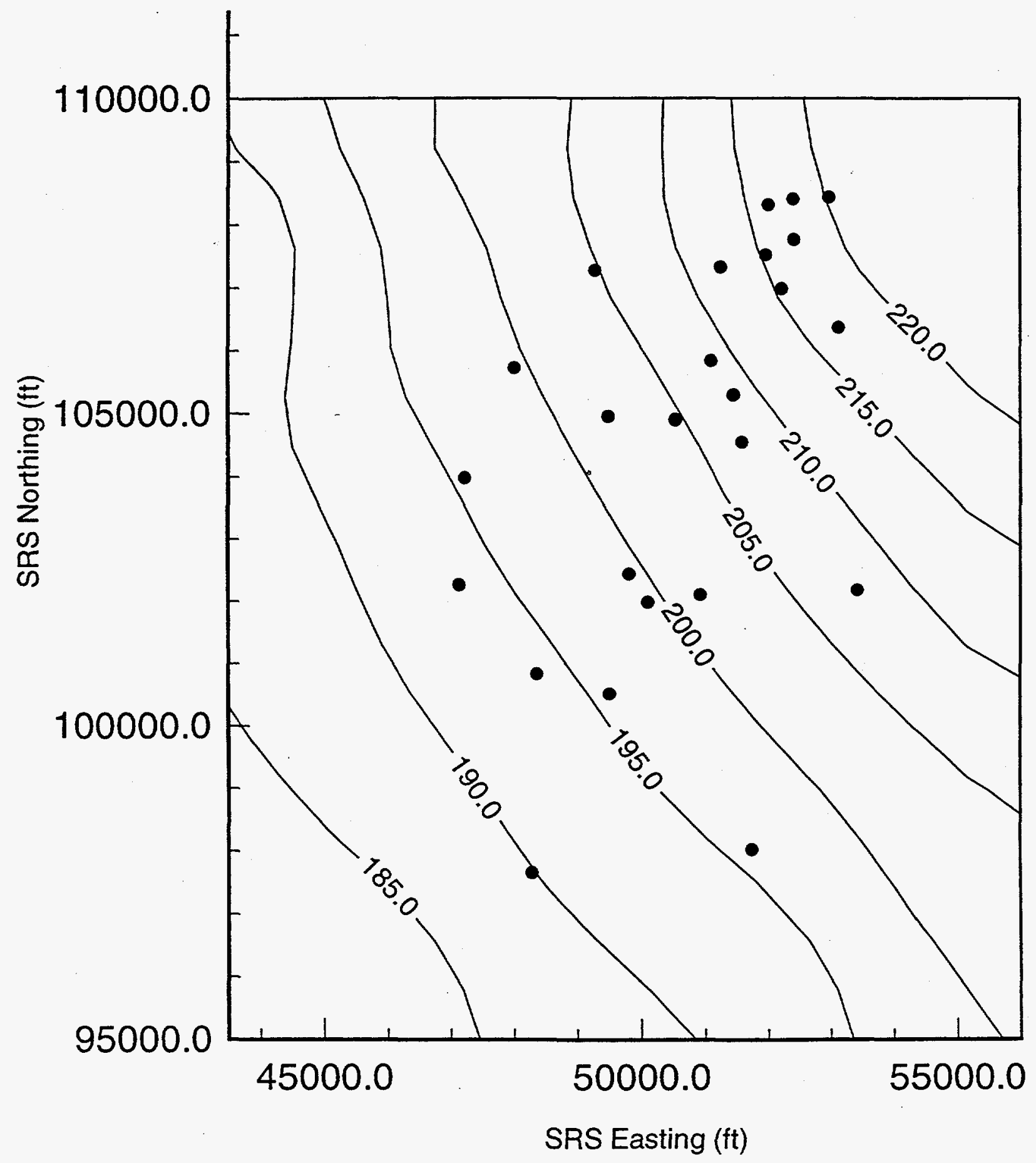

Figure 6.3 Head contours and data for Crouch Branch Aquifer. 
The 12 remediation pumping wells (RWM-1 to RWM-12) were modeled in DFM as fractured flow along a vertical column from the well screen top to bottom. This fractured flow is implemented in VAM3DF by multiplying the finite element geometric factor between the nodes by a "large number" such that the difference in head between the nodes is about 0.10 feet. The pumping was modeled in DFM as a flow from the grid node corresponding to the bottom-of-screen. Modeling the flow at the bottom-of-screen does create a minor problem in that the pathline will continue below the well bottom because of the downward head gradient in the aquifers. Thus it was necessary to stop the pathlines at the screen bottom. Table 6.9 lists the well node locations (fine grid) and average pumping rates (cu-ft/day) for 1994 (Note that the head data used in the analysis was for 1995 and it is assumed that the pumping rates for 1995 are not substantially different from 1994). The bottom of the "M-Area" Aquifer Zone is layer 7 in our model grid, so that 4 of the wells draw some water from the "M-Area" Aquifer Zone. However, most of drawdown is from the "Lost Lake" Aquifer Zone.

Table 6.9 Location and Pumping Rates (cu-ft/day) of Recovery Wells

\begin{tabular}{|c|c|c|c|c|c|}
\hline Well & grid columm & grid row & top layer & bottom layer & pumping rate \\
\hline RWM-1 & 21 & 31 & 5 & 11 & 3471 \\
\hline RWM-2 & 24 & 39 & 8 & 15 & 4277 \\
\hline RWM-3 & 26 & 40 & 8 & 15 & 11279 \\
\hline RWM-4 & 23 & 36 & 7 & 16 & 7624 \\
\hline RWM-5 & 26 & 35 & 8 & 16 & 9352 \\
\hline RWM-6 & 27 & 29 & 6 & 15 & 12517 \\
\hline RWM-7 & 25 & 29 & 7 & 14 & 7246 \\
\hline RWM-8 & 16 & 29 & 10 & 19 & 6588 \\
\hline RWM-9 & 29 & 37 & 8 & 17 & 5686 \\
\hline RWM-10 & 20 & 29 & 8 & 18 & 3347 \\
\hline RWM-11 & 29 & 41 & 10 & 16 & 17028 \\
\hline RWM-12 & 37 & 49 & 10 & 14 & 9193 \\
\hline
\end{tabular}

As explained in section 4.2 , the value of $\ln (\mathrm{Kh})$ at each node is assumed to consist of a trend (bias) component and a random field component which is different at each node. As shown above, the bias is defined separately for each hydrostratigraphic unit. Likewise, the random component is assumed to be spatially correlated within each hydrostratigraphic unit but uncorrelated between units. The random field model is defined by the process standard deviation and correlation distances (distance at which the correlation falls to 1/e) in each orthogonal direction. The statistics of the random field model used for the A/M Area is given in Table 6.10. Note that random variation in the "green clay/Lost Lake" unit was assumed to be slightly larger than in the other units, and the correlation distances were slightly shorter to allow for possible holes in the clay. 
Table 6.10 Statistics of Random Field Model for $\ln (\mathrm{Kh})$

\begin{tabular}{|c|c|c|c|c|}
\hline $\begin{array}{c}\text { Hydrostratigraphic } \\
\text { Unit }\end{array}$ & 1-sigma & $\begin{array}{c}\text { Correlation x } \\
\text { (ft) }\end{array}$ & $\begin{array}{c}\text { Correlation y } \\
\text { (ft) }\end{array}$ & $\begin{array}{c}\text { Correlation z } \\
\text { (ft) }\end{array}$ \\
\hline "M-Area" aquifer & 1.0 & 1000 & 1000 & 15 \\
\hline $\begin{array}{c}\text { "Green Clay"/Lost } \\
\text { Lake" }\end{array}$ & 1.2 & 800 & 800 & 10 \\
\hline CB Confining Unit & 1.0 & 1000 & 1000 & 15 \\
\hline
\end{tabular}

Preprocessing of the head and conductivity measurements was described in Sections 3.1 and 4.4. The error standard deviation assumed for the head measurements was 0.9 feet, which is believed to be a reasonable estimate of the errors in calibration plus the temporal variability in the measurement. The error standard deviations assigned for the log conductivity measurements is dependent upon the number of individual mud fraction measurements averaged to compute the value at the grid node. Since the $\ln (\mathrm{Kh})$ measurements are computed as the log of the arithmetic average of the $\mathrm{Kh}$ values near the node, the variance should decrease as the log of the inverse of the number of measurements averaged if all individual $\mathrm{Kh}$ values have random errors of equal variance. That assumption will not be strictly true, but it is sufficiently close that we computed the standard deviation of the log average as $\sigma=6.32 / \sqrt{n}$ where $n$ is the number of 1 -foot conductivity measurements in the average. This gives a 1-sigma error of 2 (in $\log \mathrm{ft} / \mathrm{day}$ ) for a typical 10 sample average. The log vertical conductivity measurements were computed as a harmonic average of the 1-foot conductivity measurements. We also used the same formula for computing the standard deviation even though it should be more heavily weighted by the low conductivity samples.

\subsection{DFM ESTIMATION}

All groundwater modeling is an iterative process in which the analyst makes assumptions about the model and adjusts parameters until the resulting model heads, conductivities and other flow parameters match the given measurement data. The analyst must also check that the behavior of the model is "reasonable". For example, it should show some flow path between known contaminant sources and the observed groundwater contamination. While the general process of groundwater modeling using DFM is conceptually similar to the conventional trial-and-error process, it differs significantly in the mechanism. Most importantly, it allows the analyst to think about modeling issues at a much higher level than has been previously possible. Using trial-and-error modeling, the analyst must adjust individual values of boundary conditions, recharge values, conductivities (or transmissivities) and leakances until the model output matches the measurements. It is virtually impossible for the analyst to keep track of the model sensitivity and interactions for hundreds of parameters. Thus the number of parameters varied in the analysis is usually very limited (e.g. conductivity is specified in a small number of zones) and the trial-and-error continues until the available time or funds are exhausted.

In contrast, DFM modeling automates the process of finding the set of parameters which best fit the available measurements. Thus the analyst can think about the model at the conceptual level and decide whether the results are reasonable. The burden of finding the best set of model parameters is replaced with the alternate task of determining the appropriate parameters to 
estimate and the appropriate error model for those parameters and the measurement data. That is, the cost function (Equation 6.1) to be minimized involves a weighted sum of squared residual errors involving measurements, prior information, spatial continuity, and steady-state flow errors. Thus the results are dependent upon choosing the correct weighting for these terms. Ideally the weights should be chosen so that they are equal to the standard deviation of the error for that model, i.e., the measurement weights should be equal to the standard deviation of the random error in the measurement, the prior weight should reflect the expected error in the prior estimate, the spatial continuity weight should be a function of the average randomness of the field, and the flow weighting should be the expected error in the steady-state flow numerics. If all terms are weighted appropriately, the computed least squares error covariance should accurately represent the error in the estimates.

The measurement error standard deviation can usually be determined by analyzing the method used to obtain the measurement and by computing the effect of individual error sources. In some cases, it can also be determined simply by examining the scatter in repeat measurements. The spatial continuity model weighting can be computed (Yancey (1994)) if the process standard deviation and correlation distances are known. These can usually be estimated by examining the variability in the geological and hydrogeological structures from well and geophysical data. However, these parameters are perhaps the least certain, and are prime candidates to be used as tuning parameters if the resulting DFM estimates exhibit more or less variability than thought likely. The uncertainty in prior estimates can also be guessed from knowing the source of the information (e.g. recharge estimates usually include a range). Finally, the error in the steady-state flow model must be defined. We have usually used weighting equal to about 3 to $10 \%$ of the recharge (since the DFM flow error is computed in units of head change per time). Ideally this flow error should be zero, and the final RMS residual error is always much smaller than the specified weighting. However, use of a weighting value much smaller than $3 \%$ of the recharge usually results in numerical instabilities in the nonlinear iterations.

The preceding discussion was given to help explain the sequence of DFM runs that were made in modeling the A/M Area. Initially we used the no-flow (east) and fixed-head (west) boundary conditions and 6 hydrostratigraphic unit model as described previously, with no allowance for measurement biases. The resulting DFM estimate of recharge was unreasonably high (e.g. 30 inches/year) and produced a relatively poor fit to the 250 unedited head measurements (RMS $=2.4$ feet). Convergence of the nonlinear iterations was poor, which is another indication of fundamental conceptual problems with the model. The high recharge estimate suggested that the measurements of conductivity may have been biased high. Thus biases on $\ln (\mathrm{Kh})$ and $\ln (\mathrm{Kv})$ were estimated in DFM: the resulting estimates were in the range 0.4 to 1.1 (scale factors of 1.5 to 3.0 ). This led us to re-examine the method used to compute conductivity from mud fraction. It was noted that the calibration curve was very sensitive to the values assigned for missing mud fraction data. The calibration curve was changed to piecewise linear, which was somewhat less sensitive to the assumption for missing data because conductivity was assumed to be constant for mud fractions below $8 \%$. We also computed the vertical averaging of the mud fraction samples at the elevations of our grid nodes, and used the variable weighting formula described above (constant weighting was previously used). Large head residuals from a few wells suggested anomalous data, which led to the discovery of errors which were corrected. 
These changes produced considerably improved DFM results with average recharge estimates of about 21 to 24 inches/year and estimated measurement biases of 0.2 to 0.6 . However, convergence of the iterations and the fit to the data were not as good as desired. It was noted that the estimated "M-Area" and "Lost Lake" Aquifer Zone source heads on the eastern part of the north boundary were lower than the head, so that there was considerable flow to the north-east corner. This suggested that there might be flow across the northern section of the east boundary, and thus three HDF zones were created in the northern section of the east boundary. At the same time, the recharge model was changed from uniform recharge across the entire model area to four zones (as described above). This produced faster convergence and a much better fit to the data with considerable flow through the eastern boundary in both the "M-Area" and "Lost Lake" Aquifer Zones. We then extended the HDF model to the rest of the eastern boundary and to the western boundary. Only the "middle sand" zone was retained as no-flow on the east, and fixed head on the west. The "middle sand" zone was not modeled as HDF because less head data was available in this unit than in the others. At this point, a total of 58 "common" flow parameters were being estimated, in addition to the head and $\ln (\mathrm{Kh})$ at each node. These changes produced more reasonable estimates of recharge (19 inches/year overall and 10 inches/year in the paved areas).

It was next noted that the estimates of source heads for the two "Lost Lake" zones in the middle of the northern boundary had increased more than 5 feet above the prior estimate of 225 feet. This provided a source of water flowing southward through the middle of the "Lost Lake" Aquifer Zone. To allow the model greater flexibility, the prior estimate of these source heads were increased to 230 feet, the prior 1-sigma uncertainty was increased from 1.0 to 2.0 feet, and the 1 -sigma on the random variation of $\ln (\mathrm{Kh})$ in the "green clay" unit was increased from 0.5 to 1.0 ( $\mathrm{ln} \mathrm{ft} / \mathrm{day}$ ). The standard deviation for the "Green Clay" Confining Zone was increased because it was thought that the high flow from the northern boundary might actually be the result of a "hole" in the "Green Clay" (There are few wells in that area that would allow the model to determine the better of the two possibilities). These changes resulted in an RMS fit to the head data of 1.7 feet with only three head measurements edited. A slightly lower head residual RMS (1.5 feet) was also obtained using somewhat different parameters, but the resulting conductivities were unrealistically high in the region north of the central pumping wells.

Finally we examined alternate model structures including three and single hydrostratigraphic unit models, and a no-flow boundary condition on the bottom. Surprisingly the bottom no-flow condition did not significantly change the flow in the "Lost lake" Aquifer Zone, and the fit to the data was only slightly worse. The convergence of the single unit model was a problem, and the three unit model produced the best fit to the measurements. We concluded that that the three unit model with a fixed-head bottom boundary condition was the best model structure. The head residual RMS for this model was 1.0 feet.

All the above results were obtained using the coarse grid (500 foot horizontal spacing) because of the much shorter computational times. When the results using this grid were thought to be satisfactory, the final DFM results were obtained using the fine grid (250 foot spacing). The results were similar to those of the coarse grid, but provided better detail.

In addition to the described results obtained using standard DFM, multi-grid DFM results were also obtained using both the quasi-Newton and direct methods. The conductivity grid was defined 
with 1000 foot horizontal spacing in the central region and 1500 foot spacing near the perimeter, giving 168 nodes per layer. Eleven conductivity layers were used as follows: "M-Area" Aquifer - 3, "Green Clay/Lost Lake" - 4, Crouch Branch Confining Unit - 4. This conductivity grid has a total of 1848 nodes. As noted, somewhat longer clock times were required for multi-grid DFM runs than standard DFM for the coarse grid flow models, but were about $50 \%$ shorter for the fine grid models. The resulting data fit and the estimated parameters were about the same as for standard DFM.

\subsection{DFM ESTIMATES}

The final estimates of the common parameters computed using standard DFM are listed in Tables 6.11 to 6.16 . As explained before, the a posterior standard deviations were obtained from the comparable multi-grid DFM. Figures 6.4 to 6.7 show the estimated heads and head measurement locations for several layers corresponding to the "M-Area", "Upper Lost Lake", "Lower Lost Lake", and "middle sand" aquifers and Tables 6.17 to 6.20 list the head measurements. Figure 6.8 shows the pore velocity vectors for the "Upper Lost Lake" aquifer and Figure 6.9 shows the pore velocity vectors for the "Lower Lost Lake" aquifer. Note that there is a region of high north-south flow within the upper layers of the "Upper Lost Lake" zone starting near the northern region of the "Green Clay" and running to six pumping wells (RWM-2,3,4,5,9,11) in the central M-Area. Surprisingly, there is very little horizontal flow within the "Lost Lake" Aquifer Zone at the east, west and south perimeters of the M-Area. Also note that there are significant differences in the flow between the upper and lower layers within the "Lost Lake". Figure 6.10 shows the estimated heads, head measurements and pore velocities at the $\mathrm{y}-\mathrm{z}$ slice through the middle of the grid at $\mathrm{x}=49,750$ (ft). Table 6.21 lists the head measurements. Figures 6.11 and 6.12 show the estimated $\mathrm{Kv}$ for the "Green Clay" Confining Zone and "upper clay" zone. Figures 6.13 to 6.15 show the estimated $\mathrm{Kh}$ for the "M-Area" Aquifer Zone, "Upper Lost Lake" aquifer, and "Lower Lost Lake" aquifer. Notice the large variation in conductivity within the "Lost Lake" Aquifer Zone: the region of low conductivity near the "Lost Lake" and the high conductivity region north of the pumping wells. Since there are no well cores in the north central region, DFM increased the conductivity in order to allow the large flow to the extraction wells. Also note that the vertical conductivity in the "Green Clay" Confining Zone (Figure 6.11) is somewhat higher in this same region, thus allowing more water to flow directly from the "M-Area" to "Lost Lake" Aquifer Zone. Again these high conductivity estimates were not caused by the presence of conductivity measurements, but rather by the need to fit the head data. Thus it cannot be determined by direct measurement if the regions actually have high conductivity, although the small drop in head across the "Green Clay" Confining Zone at certain locations certainly suggests higher conductivity. Also note the lower "Green Clay" vertical conductivity near the pumping wells RWM-2,3,4 and 5. There are no cores near these locations. Apparently a reduction in conductivity was required to match the measured head gradient across the unit, which was larger than initially modeled. However, the error may be partly due to the lack of detail in modeling the drawdown near the well. Figure 6.15 shows the estimated Kh and measurements for the $\mathrm{y}-\mathrm{z}$ slice at $\mathrm{x}=53,000(\mathrm{ft})$ and Table 6.22 list the measurements. 
Table 6.11 Posterior Estimate and Uncertainty of Mean Log Conductivity States

\begin{tabular}{|c|c|c|c|}
\hline Parameter & Unit & Estimate & 1-sigma \\
\hline mean $\ln (\mathrm{Kh})$ & M-area aquifer & 1.99 & 0.13 \\
\hline mean $\ln (\mathrm{Kh})$ & green clay/Lost Lake & 2.31 & 0.09 \\
\hline mean $\ln (\mathrm{Kh})$ & CB confining & 0.46 & 0.17 \\
\hline mean $\ln (\mathrm{Kh} / \mathrm{Kv})$ & M-area aquifer & 6.50 & 0.13 \\
\hline mean $\ln (\mathrm{Kh} / \mathrm{Kv})$ & green clay/Lost Lake & 5.92 & 0.10 \\
\hline mean $\ln (\mathrm{Kh} / \mathrm{Kv})$ & CB confining & 5.79 & 0.17 \\
\hline
\end{tabular}

Table 6.12 Posterior Estimate and Uncertainty of Measurement Bias and Recharge States

\begin{tabular}{|c|c|c|}
\hline Parameter & Estimate & 1-sigma \\
\hline meas. Bias $-\ln (\mathrm{Kh})$ & -0.28 & 0.10 \\
\hline meas. bias $-\ln (\mathrm{Kv})$ & 1.29 & 0.10 \\
\hline recharge - general area & 0.00413 & 0.00021 \\
\hline recharge - paved areas & 0.00120 & 0.00022 \\
\hline
\end{tabular}

Table 6.13 Posterior Estimate and Uncertainty of North Boundary Source Head States

\begin{tabular}{|c|c|c|c|}
\hline Aquifer & Grid Columns & Estimate & 1-sigma \\
\hline M-area & $2-10$ & 221.7 & 2.00 \\
\hline M-area & $11-40$ & 230.0 & 1.98 \\
\hline M-area & $41-50$ & 229.8 & 2.00 \\
\hline Lost lake & $2-10$ & 219.0 & 1.98 \\
\hline Lost Lake & $11-20$ & 224.0 & 1.99 \\
\hline Lost Lake & $21-28$ & 230.5 & 1.97 \\
\hline Lost Lake & $29-36$ & 228.5 & 1.83 \\
\hline Lost Lake & $37-44$ & 223.1 & 1.80 \\
\hline Lost Lake & $45-50$ & 221.4 & 1.96 \\
\hline middle sand & $2-10$ & 220.0 & 2.00 \\
\hline middle sand & $11-40$ & 219.7 & 2.00 \\
\hline middle sand & $41-50$ & 219.9 & 2.00 \\
\hline
\end{tabular}


Table 6.14 Posterior Estimate and Uncertainty of South Boundary Source Head States

\begin{tabular}{|c|c|c|c|}
\hline Aquifer & Grid Columns & Estimate & 1-sigma \\
\hline M-area & $2-10$ & 195.0 & 2.00 \\
\hline M-area & $11-40$ & 195.0 & 2.00 \\
\hline M-area & $41-50$ & 195.0 & 2.00 \\
\hline Lost Lake & $2-14$ & 191.2 & 1.84 \\
\hline Lost Lake & $15-30$ & 190.6 & 1.68 \\
\hline Lost Lake & $31-50$ & 192.8 & 1.81 \\
\hline middle sand & $2-10$ & 194.8 & 2.00 \\
\hline middle sand & $11-40$ & 194.6 & 2.00 \\
\hline middle sand & $41-50$ & 195.0 & 2.00 \\
\hline
\end{tabular}

Table 6.15 Posterior Estimate and Uncertainty of East Boundary Source Head States

\begin{tabular}{|c|c|c|c|}
\hline Aquifer & Grid Rows & Estimate & 1-sigma \\
\hline M-area & $1-10$ & 215.0 & 2.00 \\
\hline M-area & $11-20$ & 227.0 & 2.00 \\
\hline M-area & $21-30$ & 235.0 & 2.00 \\
\hline M-area & $31-40$ & 235.1 & 2.00 \\
\hline M-area & $41-50$ & 235.0 & 2.00 \\
\hline M-area & $51-61$ & 234.8 & 2.00 \\
\hline Lost Lake & $1-10$ & 202.8 & 1.99 \\
\hline Lost Lake & $11-20$ & 214.8 & 1.99 \\
\hline Lost Lake & $21-30$ & 219.9 & 1.98 \\
\hline Lost Lake & $31-40$ & 221.0 & 1.49 \\
\hline Lost Lake & $41-50$ & 221.8 & 1.28 \\
\hline Lost Lake & $51-61$ & 218.0 & 1.78 \\
\hline middle sand & $1-10$ & 197.0 & 2.00 \\
\hline middle sand & $11-20$ & 210.0 & 2.00 \\
\hline middle sand & $21-30$ & 213.0 & 2.00 \\
\hline middle sand & $31-40$ & 213.0 & 2.00 \\
\hline middle sand & $41-50$ & 213.0 & 2.00 \\
\hline middle sand & $51-61$ & 211.9 & 2.00 \\
\hline
\end{tabular}


Table 6.16 Posterior Estimate and Uncertainty of West Boundary Source Head States

\begin{tabular}{|c|c|c|c|}
\hline Aquifer & Grid Rows & Estimate & 1-sigma \\
\hline M-area & $1-10$ & 210.7 & 1.99 \\
\hline M-area & $11-20$ & 215.4 & 1.95 \\
\hline M-area & $21-30$ & 214.8 & 2.00 \\
\hline M-area & $31-40$ & 214.9 & 2.00 \\
\hline M-area & $41-50$ & 219.8 & 2.00 \\
\hline M-area & $51-61$ & 219.3 & 1.98 \\
\hline Lost Lake & $1-10$ & 195.4 & 1.93 \\
\hline Lost Lake & $11-20$ & 200.1 & 1.82 \\
\hline Lost Lake & $21-30$ & 209.1 & 1.85 \\
\hline Lost Lake & $31-40$ & 216.5 & 1.52 \\
\hline Lost Lake & $41-50$ & 214.2 & 1.97 \\
\hline Lost Lake & $51-61$ & 217.2 & 1.83 \\
\hline middle sand & $1-10$ & 198.8 & 2.00 \\
\hline middle sand & $11-20$ & 197.7 & 2.00 \\
\hline middle sand & $21-30$ & 198.0 & 2.00 \\
\hline middle sand & $31-40$ & 199.1 & 2.00 \\
\hline middle sand & $41-50$ & 199.0 & 2.00 \\
\hline middle sand & $50-61$ & 199.9 & 2.00 \\
\hline
\end{tabular}




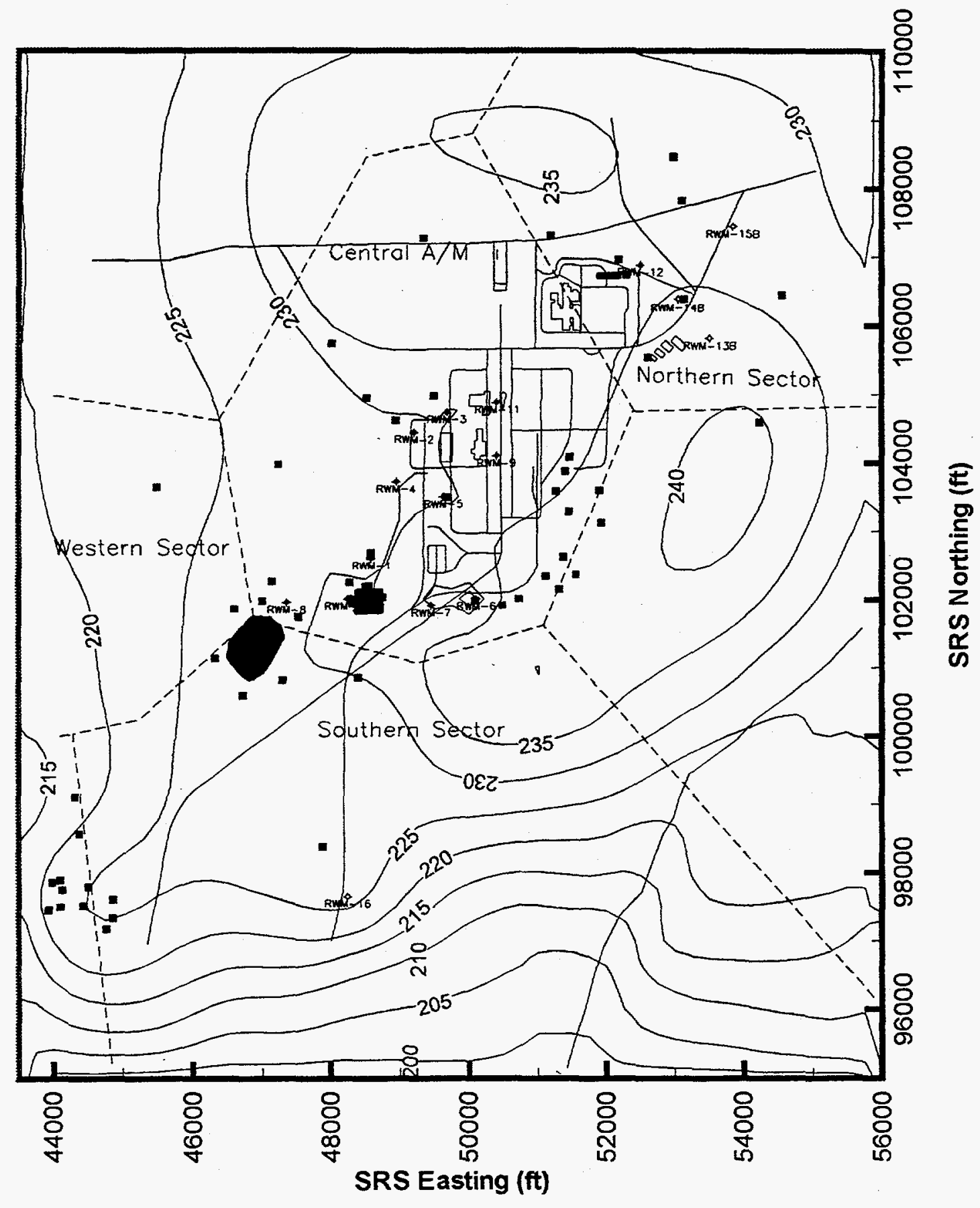

Figure 6.4 - Estimated Heads (ft) and Measurement Locations for "M-area" aquifer 


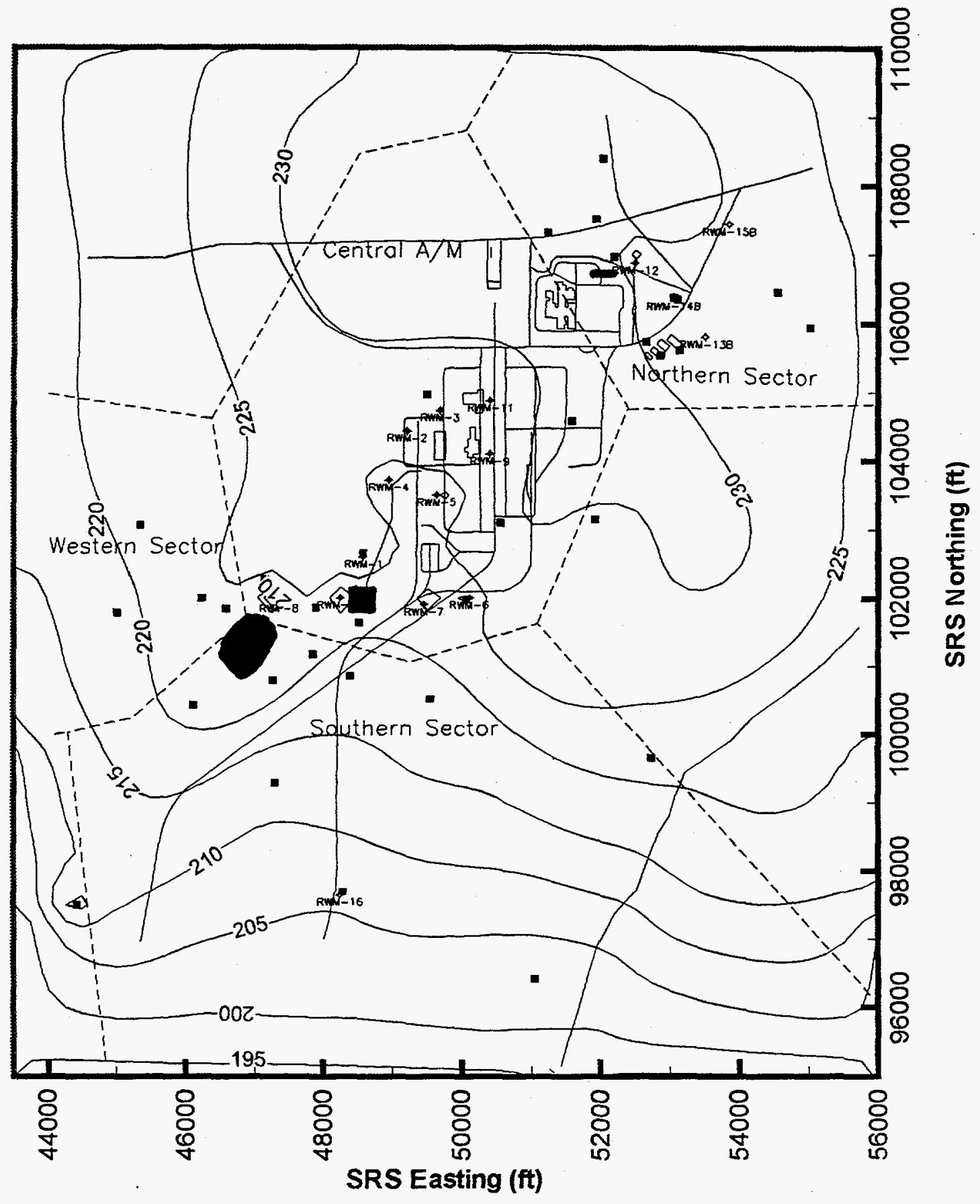

Figure 6.5 - Estimated Heads (ft) and Measurement Locations for "Upper Lost Lake" aquifer 


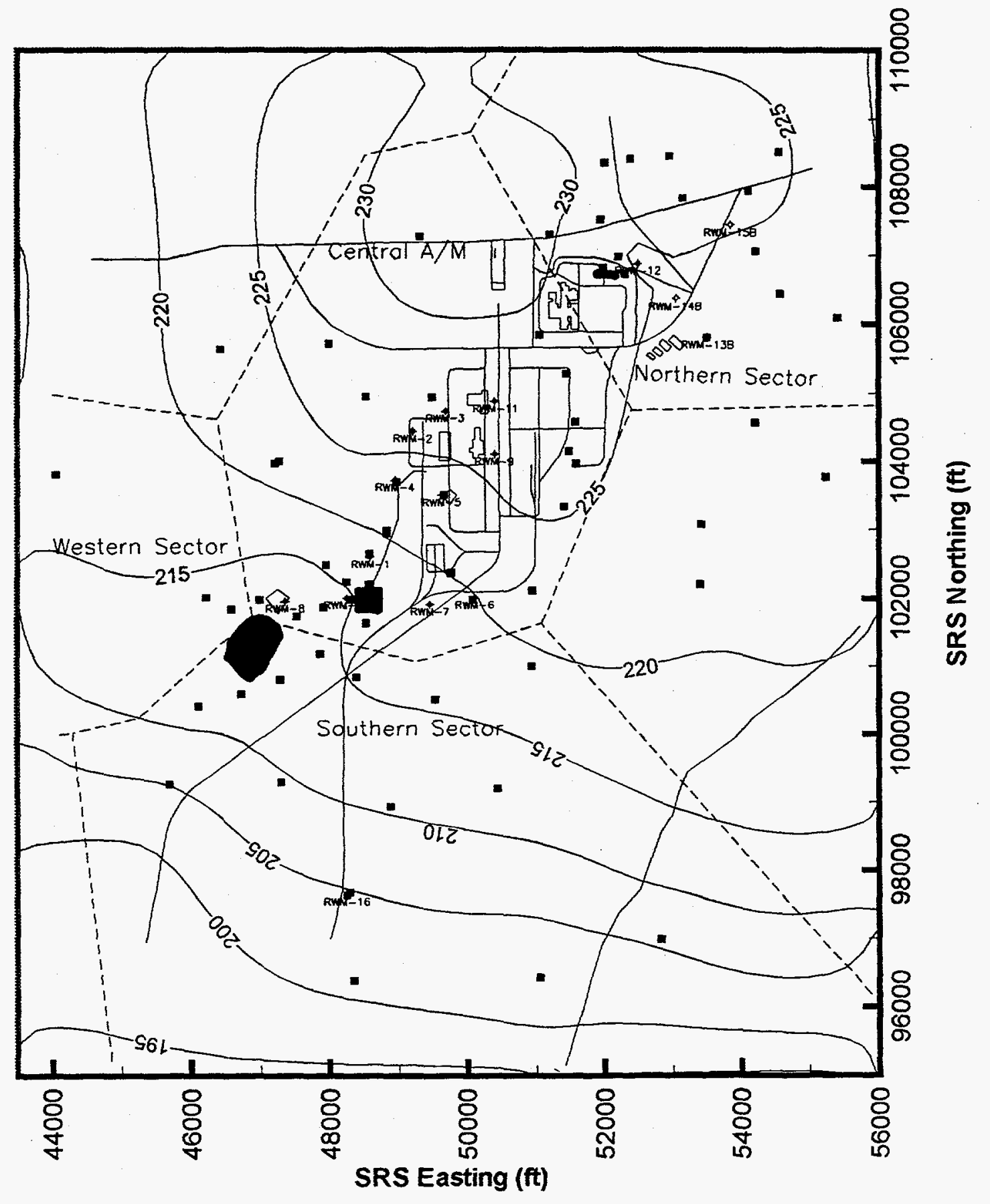

Figure 6.6 - Estimated Heads (ft) and Measurement Locations for "Lower Lost Lake" aquifer 


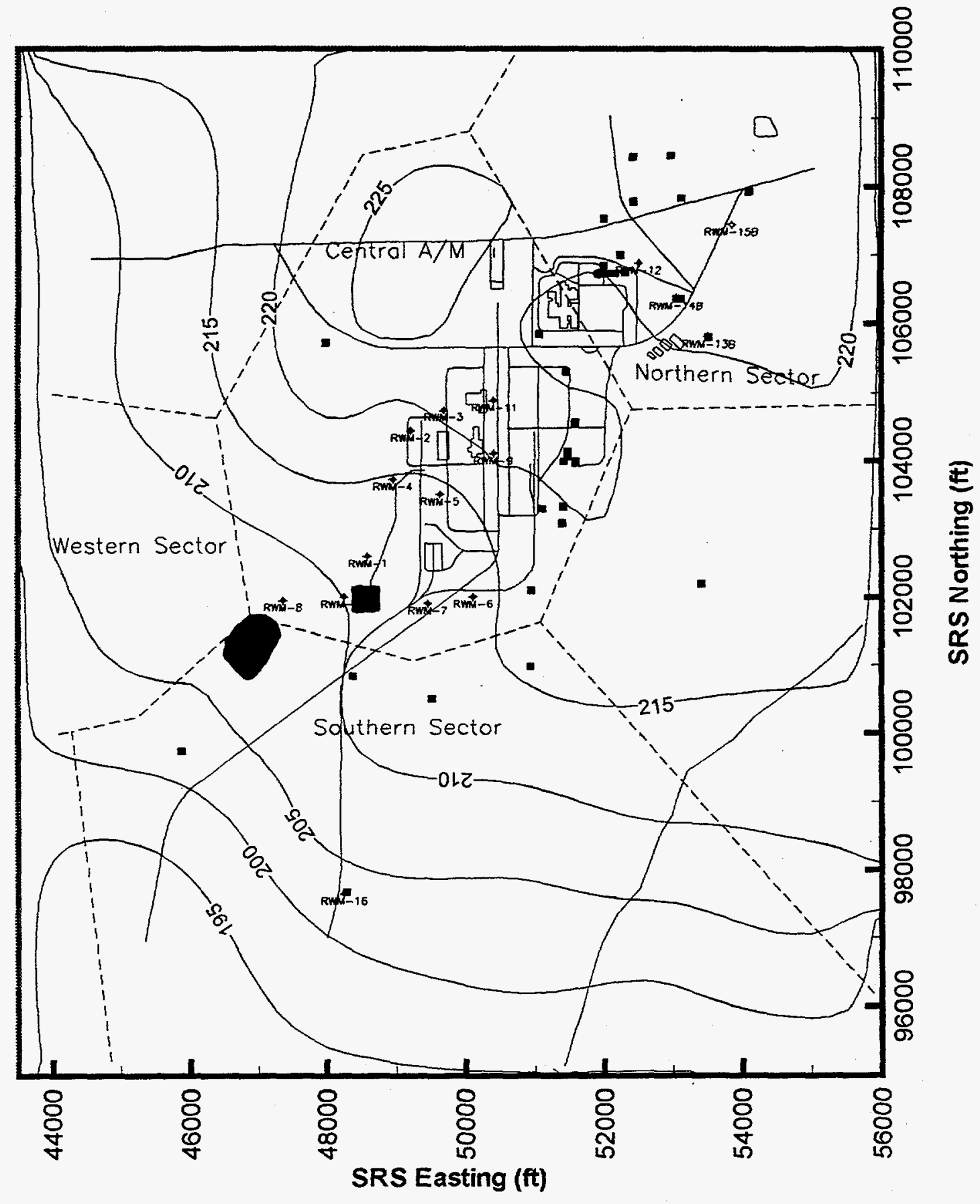

Figure 6.7 - Estimated Heads ( $\mathrm{ft}$ ) and Measurement Locations for "middle sand" aquifer 
Table 6.17 M-Area Aquifer Hydraulic Head Data

\begin{tabular}{|c|c|c|c|c|}
\hline Well ID & $\begin{array}{c}\text { SRS } \\
\text { Easting } \\
\quad(f t)\end{array}$ & $\begin{array}{l}\text { SRS } \\
\text { Northing } \\
\text { (ft) }\end{array}$ & Layer & $\begin{array}{c}\text { Head } \\
\text { Data } \\
\text { (ft) }\end{array}$ \\
\hline------- & -------- & -------- & ----- & ------ \\
\hline $\mathrm{ABP} \_7 \mathrm{D}$ & 43930.1 & 97449.7 & 5 & 224.41 \\
\hline$A B P \_8 D$ & 43984.1 & 97854.9 & 5 & 223.02 \\
\hline$A B P \_4 D D$ & 44101.3 & 97495.5 & 6 & 230.83 \\
\hline $\mathrm{ABP} \_6 \mathrm{D}$ & 44101.4 & 97889.7 & 6 & 223.32 \\
\hline$A B P_{\_} 2 D D$ & 44126.7 & 97753.7 & 6 & 223.34 \\
\hline$A R P \_1 A$ & 44317.4 & 99102.9 & 7 & 217.09 \\
\hline $\mathrm{ARP} \_4$ & 44374.8 & 98567.7 & 6 & 219.57 \\
\hline $\mathrm{ABP} \_1 \mathrm{DD}$ & 44433.6 & 97511.4 & 5 & 226.44 \\
\hline $\mathrm{ABP} \_3$ & 44509.3 & 97794.1 & 5 & 225.25 \\
\hline $\mathrm{MCB} \_8 \mathrm{D}$ & 44769.9 & 97180.6 & 5 & 226.42 \\
\hline$M C B \_9 D$ & 44858.8 & 97605.8 & 5 & 224.01 \\
\hline MCB_ 5 & 44863.9 & 97335.6 & 5 & 225.95 \\
\hline MSB_78D & 45482.2 & 103643.8 & 5 & 224.86 \\
\hline $\mathrm{MSB} \_6 \mathrm{~A}$ & 46319.9 & 101133.8 & 5 & 227.96 \\
\hline MSB_64D & 46598.5 & 101854.8 & 5 & 227.59 \\
\hline $\mathrm{MSB} \_7 \mathrm{~A}$ & 46726.1 & 100585.7 & 5 & 228.79 \\
\hline$M S B \_5 A$ & 46998.7 & 101971.5 & 5 & 228.60 \\
\hline MSB_12D & 47139.7 & 102262.2 & 5 & 234.08 \\
\hline MSB_21C & 47234.6 & 103973.0 & 5 & 229.77 \\
\hline$M S B \_8 A$ & 47293.2 & 100815.1 & 6 & 230.18 \\
\hline MSB_13C & 47521.9 & 101745.7 & 5 & 229.73 \\
\hline MSB_89C & 47881.6 & 98379.4 & 6 & 229.53 \\
\hline MSB_30C & 48013.7 & 105731.1 & 5 & 231.48 \\
\hline MSB__ $9 \mathrm{C}$ & 48273.0 & 102245.6 & 5 & 231.28 \\
\hline MSB__ $4 \mathrm{D}$ & 48311.7 & 102007.5 & 6 & 230.85 \\
\hline $\mathrm{MSB} \_4 \mathrm{~A}$ & 48313.0 & 101933.4 & 5 & 231.61 \\
\hline MSB_39D & 48396.0 & 100858.7 & 5 & 232.95 \\
\hline$M S B \_1 A$ & 48467.3 & 101833.7 & 5 & 232.35 \\
\hline MSB_22 & 48508.8 & 102186.5 & 5 & 231.74 \\
\hline MSB_28 & 48517.3 & 104941.8 & 6 & 231.27 \\
\hline$M S B \_3 D$ & 48524.6 & 102188.6 & 6 & 231.08 \\
\hline$M S B \_3 A$ & 48553.7 & 102189.9 & 5 & 232.71 \\
\hline MSB_IIF & 48577.0 & 102629.3 & 5 & 231.27 \\
\hline $\mathrm{MSB} \_2 \mathrm{~A}$ & 48746.4 & 102028.3 & 5 & 233.82 \\
\hline MSB_26 & 48941.7 & 104612.8 & 5 & 237.24 \\
\hline$M S B \_43 D D$ & 49341.2 & 107273.0 & 5 & 232.26 \\
\hline MSB__27 & 49487.7 & 104972.8 & 5 & 237.04 \\
\hline MSB_25 & 49668.9 & 103498.8 & 5 & 237.01 \\
\hline MSB_31C & 50089.9 & 101979.6 & 5 & 235.85 \\
\hline
\end{tabular}




$\begin{array}{lllll}\text { AOB_1 } & 50485.9 & 101910.7 & 5 & 237.73 \\ \text { AOB_2 } & 50724.7 & 102009.8 & 5 & 237.63 \\ \text { MSB_35D } & 50949.7 & 102122.4 & 4 & 243.48 \\ \text { ACB_4A } & 51116.2 & 102343.9 & 5 & 239.74 \\ \text { MSB_29DD } & 51191.3 & 107311.4 & 7 & 233.55 \\ \text { AMB_9D } & 51263.0 & 103585.2 & 6 & 235.40 \\ \text { ACB_3A } & 51313.3 & 102154.3 & 6 & 239.57 \\ \text { ACB_1A } & 51369.9 & 102622.9 & 5 & 238.68 \\ \text { AMB_8D } & 51400.5 & 103874.7 & 6 & 234.96 \\ \text { AMB_10D } & 51456.0 & 103293.4 & 5 & 236.64 \\ \text { AMB_5 } & 51467.2 & 104083.4 & 6 & 234.93 \\ \text { ACB_2A } & 51561.3 & 102367.4 & 5 & 239.78 \\ \text { AMB_12D } & 51901.6 & 103602.4 & 5 & 235.98 \\ \text { AMB_11D } & 51932.6 & 103132.3 & 5 & 237.31 \\ \text { MSB_47D } & 52184.0 & 106960.1 & 6 & 234.36 \\ M S B \_68 D & 52293.6 & 106741.4 & 6 & 234.34 \\ \text { ASB_1A } & 52614.0 & 105535.0 & 5 & 238.35 \\ \text { ASB_6A } & 52675.9 & 105716.0 & 5 & 237.87 \\ \text { ASB_2AR } & 52881.7 & 105550.5 & 5 & 239.65 \\ \text { MSB_54D } & 52984.5 & 108461.5 & 7 & 233.96 \\ \text { MSB_85D } & 53108.8 & 107822.8 & 7 & 233.68 \\ \text { ASB_3AR } & 53115.0 & 105605.1 & 5 & 240.25 \\ \text { ASB_8 } & 53136.6 & 106381.6 & 5 & 235.32 \\ \text { ASB_4 } & 53177.2 & 105935.7 & 4 & 239.47 \\ \text { ASB_9 } & 54226.2 & 104589.2 & 5 & 242.98 \\ \text { MSB_53D } & 54553.1 & 106448.2 & 5 & 234.46\end{array}$


Table 6.18 Upper Lost Lake Aquifer Hydraulic Head Data

\begin{tabular}{|c|c|c|c|c|}
\hline WeII ID & $\begin{array}{c}\text { SRS } \\
\text { Easting } \\
\text { (Et) }\end{array}$ & $\begin{array}{l}\text { SRS } \\
\text { Northing } \\
(\mathrm{ft})\end{array}$ & Layer & $\begin{array}{l}\text { Head } \\
\text { Data } \\
(f t)\end{array}$ \\
\hline$-\cdots-\cdots$ & -------- & -------- & ---- & ---- \\
\hline$A B P \_3 C$ & 44506.3 & 97778.2 & 12 & 198.23 \\
\hline MSB_70C & 45012.0 & 101785.2 & 11 & 218.54 \\
\hline $\mathrm{MCB} \_6 \mathrm{C}$ & 45207.7 & 97413.1 & 12 & 196.63 \\
\hline MSB_18B & 46115.7 & 100424.1 & 10 & 222.63 \\
\hline MSB_ $64 \mathrm{C}$ & 46589.2 & 101842.9 & 11 & 223.90 \\
\hline MSB_12C & 47138.4 & 102274.4 & 11 & 224.56 \\
\hline $\mathrm{MSB} \_8 \mathrm{C}$ & 47264.6 & 100793.2 & 10 & 221.51 \\
\hline MSB_79C & 47286.8 & 99290.2 & 10 & 211.01 \\
\hline MSB_63C & 47849.2 & 101174.6 & 10 & 222.35 \\
\hline MSB $62 \mathrm{C}$ & 47895.0 & 101857.2 & 10 & 224.84 \\
\hline $\mathrm{MSB} \_40 \mathrm{C}$ & 48283.5 & 97697.8 & 10 & 205.34 \\
\hline MSB_26A & 48440.7 & 104602.3 & 12 & 226.35 \\
\hline MSB_14B & 48519.1 & 101639.0 & 10 & 220.16 \\
\hline MSB_11C & 48579.4 & 102658.6 & 11 & 223.36 \\
\hline MSB_23B & 49286.4 & 104336.6 & 12 & 225.70 \\
\hline MSB_27A & 49487.8 & 104962.8 & 10 & 230.25 \\
\hline MSB_36C & 49537.2 & 100518.3 & 10 & 216.16 \\
\hline MSB_24A & 49845.3 & 104625.3 & 12 & 227.79 \\
\hline MSB_31CC & 50067.9 & 101983.1 & 11 & 220.65 \\
\hline$M S B \_34 B$ & 50534.9 & 104944.7 & 12 & 228.60 \\
\hline MSB_45B & 50555.3 & 103987.9 & 12 & 228.17 \\
\hline$M S B \_46 B$ & 50557.5 & 103102.4 & 11 & 227.09 \\
\hline MSB_44B & 51096.4 & 103296.2 & 12 & 226.48 \\
\hline MSB_42C & 51582.8 & 104581.9 & 11 & 231.90 \\
\hline MSB_33C & 51746.7 & 97984.8 & 12 & 211.15 \\
\hline$A M B \_11 B$ & 51919.5 & 103154.2 & 11 & 224.72 \\
\hline MSB_84C & 51973.7 & 108967.9 & 12 & 230.52 \\
\hline MSB_55C & 52029.7 & 108324.6 & 12 & 230.44 \\
\hline MSB__47C & 52195.5 & 106969.2 & 10 & 233.51 \\
\hline$A S B \_6 C$ & 52655.9 & 105736.8 & 11 & 225.53 \\
\hline$A S B \_5 C$ & 52837.8 & 105884.8 & 12 & 224.37 \\
\hline $\mathrm{ASB} \_2 \mathrm{CR}$ & 52862.7 & 105540.2 & 12 & 224.66 \\
\hline ASB_10CR & 52969.7 & 105655.4 & 11 & 224.63 \\
\hline RWM_14C & 53051.5 & 106380.8 & 11 & 225.52 \\
\hline$A S B \_8 C$ & 53101.0 & 106354.4 & 10 & 224.97 \\
\hline$A S B \_3 C R$ & 53130.4 & 105614.5 & 12 & 224.25 \\
\hline MSB_48C & 54077.0 & 107917.5 & 12 & 225.66 \\
\hline$A S B \_9 C$ & 54201.1 & 104568.1 & 12 & 222.13 \\
\hline MSB_77C & 54225.9 & 107078.3 & 12 & 225.26 \\
\hline
\end{tabular}


MSB_53C $54540.5 \quad 106456.2 .11 \quad 224.53$

ABW_1 . $55016.4 \quad 105939.9 \quad 11 \quad 226.92$ 
Table 6.19 Lower Lost Lake Aquifer Hydraulic Head Data

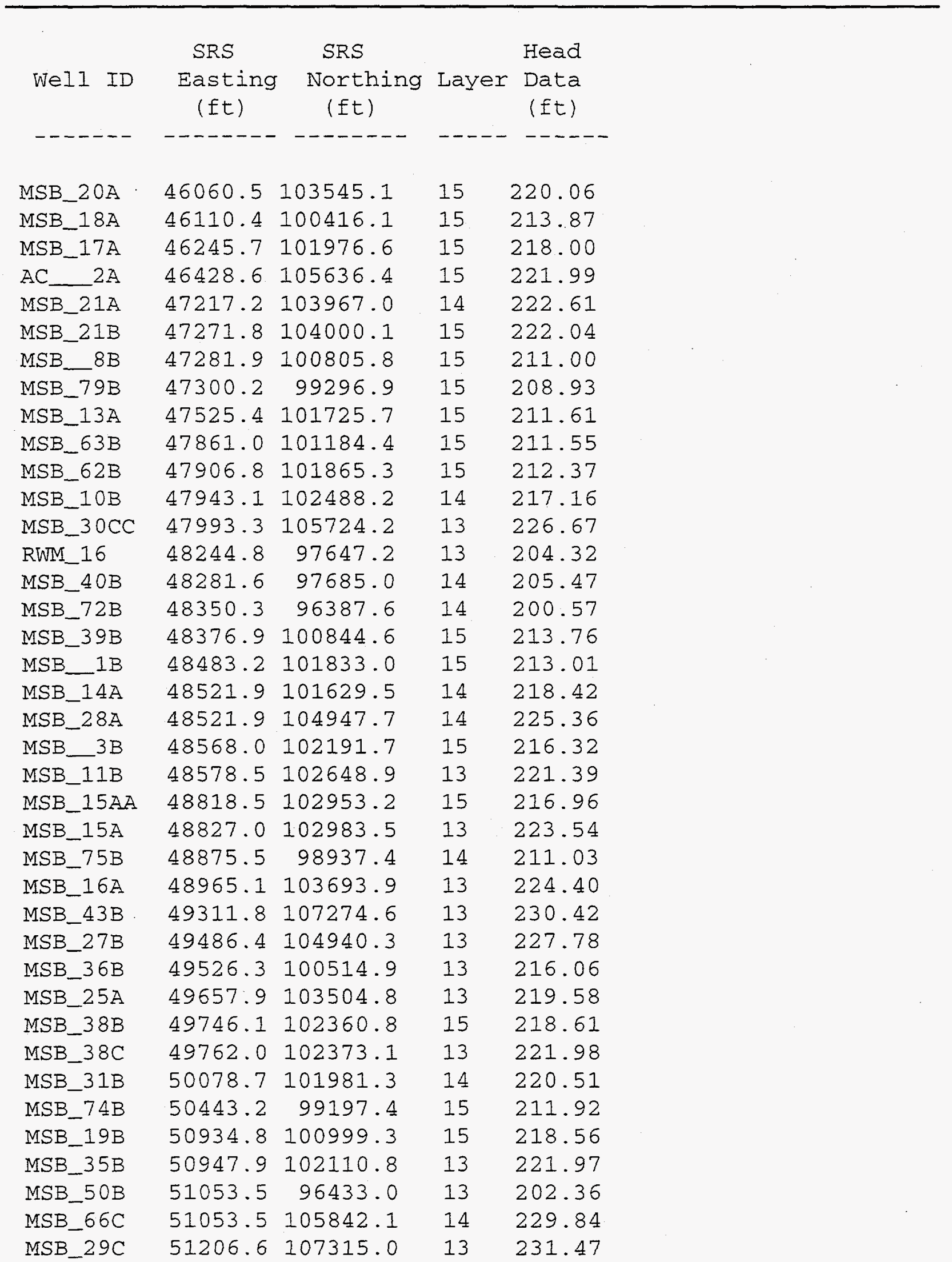




$\begin{array}{lrrrr}\text { AMB_10B } & 51418.3 & 103337.3 & 15 & 225.08 \\ \text { MSB_37C } & 51439.8 & 105283.2 & 13 & 229.64 \\ \text { AMB_4B } & 51482.7 & 104145.6 & 15 & 226.09 \\ \text { MSB_42B } & 51582.8 & 104569.8 & 14 & 227.54 \\ \text { AMB_7B } & 51590.3 & 103972.0 & 14 & 226.57 \\ \text { MSB_82C } & 51949.4 & 107521.9 & 14 & 229.61 \\ \text { MSB_67C } & 51988.6 & 106819.8 & 13 & 228.39 \\ \text { MSB_55B } & 52006.2 & 108342.4 & 15 & 223.19 \\ \text { MSB_47B } & 52207.2 & 106978.5 & 13 & 226.78 \\ \text { MSB_68C } & 52304.9 & 106730.5 & 13 & 226.60 \\ \text { MSB_83C } & 52384.7 & 108405.3 & 14 & 228.76 \\ \text { MSB_51B } & 52818.0 & 96992.7 & 13 & 205.19 \\ \text { MSB_54C } & 52955.7 & 108447.4 & 14 & 228.55 \\ \text { MSB_85C } & 53151.4 & 107835.2 & 14 & 225.99 \\ \text { MSB_41C } & 53410.6 & 102203.9 & 15 & 219.03 \\ \text { MSB_52B } & 53418.4 & 103077.7 & 14 & 220.09 \\ \text { RWM_13C } & 53502.2 & 105809.7 & 14 & 223.49 \\ \text { MSB_48B } & 54112.2 & 107945.0 & 14 & 225.18 \\ \text { ASB_9B } & 54215.3 & 104564.7 & 14 & 221.75 \\ \text { MSB_77B } & 54217.4 & 107065.8 & 14 & 223.23 \\ \text { MSB_86C } & 54560.5 & 108500.4 & 15 & 226.17 \\ \text { MSB_53B } & 54574.3 & 106443.6 & 15 & 223.64 \\ \text { MSB_81B } & 55230.4 & 103762.7 & 15 & 222.16 \\ \text { MSB_61C } & 55406.6 & 106091.1 & 13 & 224.97\end{array}$


Table 6.20 Middle Sand Aquifer Hydraulic Head Data

\begin{tabular}{|c|c|c|c|c|}
\hline & SRS & SRS & & Head \\
\hline WeII ID & $\begin{array}{l}\text { Easting } \\
(\mathrm{ft})\end{array}$ & $\begin{array}{l}\text { Northing } \\
(\mathrm{ft})\end{array}$ & Layer & $\begin{array}{c}\text { Data } \\
\text { (ft) }\end{array}$ \\
\hline------- & -------- & ------- & ---- & $-\ldots-$ \\
\hline $\mathrm{MSB} \_71 \mathrm{~B}$ & 44054.7 & 103801.6 & 16 & 218.05 \\
\hline MSB_73B & 45694.0 & 99270.3 & 17 & 202.75 \\
\hline $\mathrm{MSB} \quad 7 \mathrm{~B}$ & 46718.1 & 100597.6 & 16 & 208.90 \\
\hline $\mathrm{MSB} \_30 \mathrm{~B}$ & 47981.8 & 105719.9 & 17 & 226.59 \\
\hline MSB__ $9 A$ & 48242.5 & 102236.7 & 16 & 213.26 \\
\hline$M S B \_4 B$ & 48312.8 & 101978.3 & 17 & 213.53 \\
\hline MSB_11A & 48577.6 & 102638.9 & 16 & 215.70 \\
\hline MSB_26B & 48944.6 & 104646.7 & 16 & 221.25 \\
\hline$M S B \_43 A$ & 49293.7 & 107275.3 & 16 & 230.14 \\
\hline MSB_35A & 50945.2 & 102098.0 & 17 & 218.11 \\
\hline MSB_66B & 51064.6 & 105842.0 & 17 & 220.91 \\
\hline MSB_44A & 51106.9 & 103296.5 & 17 & 219.56 \\
\hline MSB_29B & 51217.5 & 107319.3 & 16 & 225.75 \\
\hline AMB_18A & 51418.8 & 103988.8 & 17 & 220.43 \\
\hline MSB_33B & 51741.9 & 97995.9 & 17 & 208.20 \\
\hline MSB_67B & 51989.6 & 106842.0 & 17 & 220.50 \\
\hline MSB_68B & 52308.5 & 106744.9 & 17 & 220.83 \\
\hline MSB_69B & 52432.9 & 107776.1 & 17 & 222.38 \\
\hline RWM_14B & 53044.7 & 106362.1 & 17 & 221.52 \\
\hline RWII_15B & 53849.0 & 107444.7 & 16 & 223.95 \\
\hline MSB_48A & 54099.8 & 107936.6 & 17 & 223.88 \\
\hline
\end{tabular}




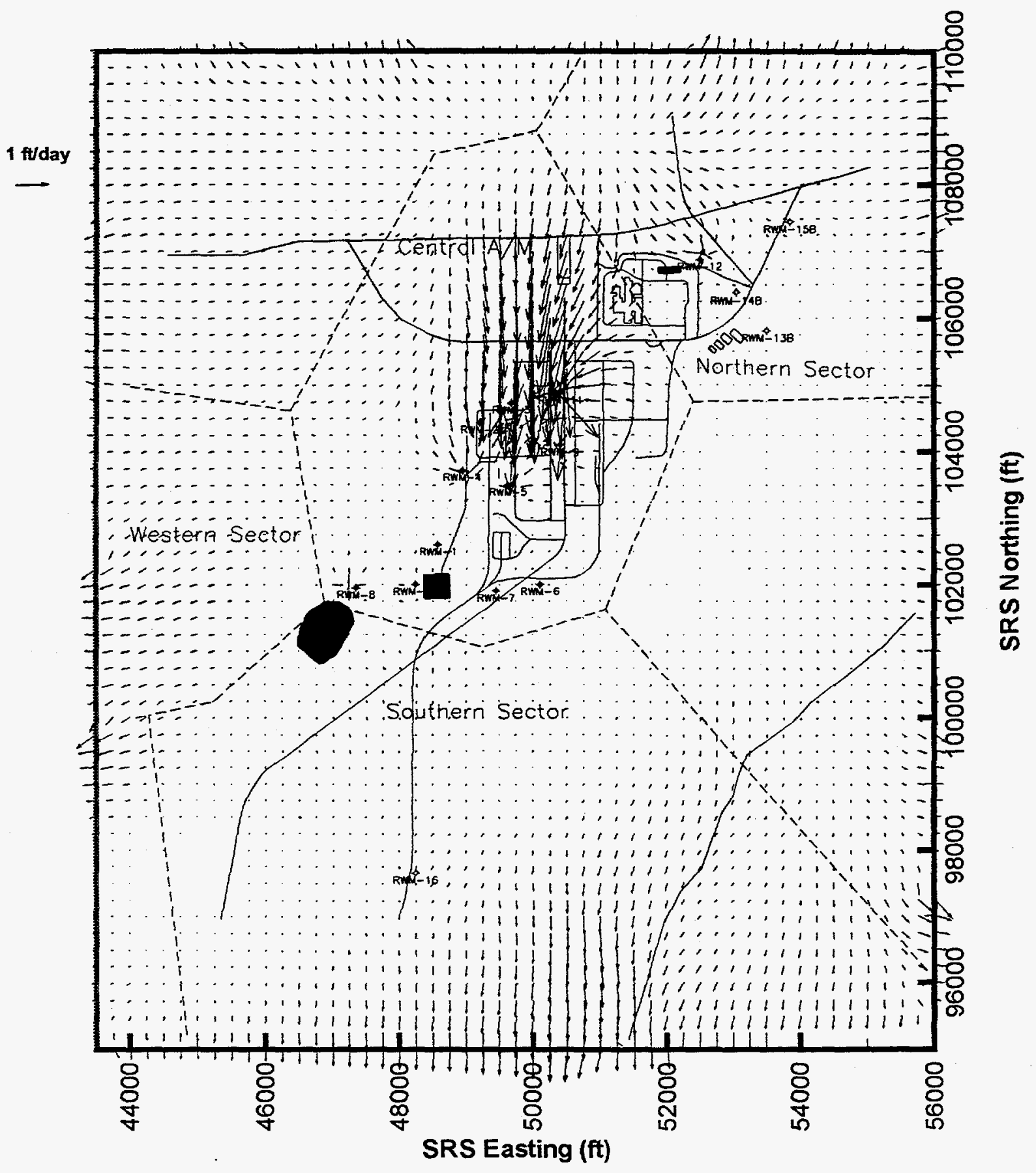

Figure 6.8 - Estimated Pore Velocity for "Upper Lost Lake" aquifer 


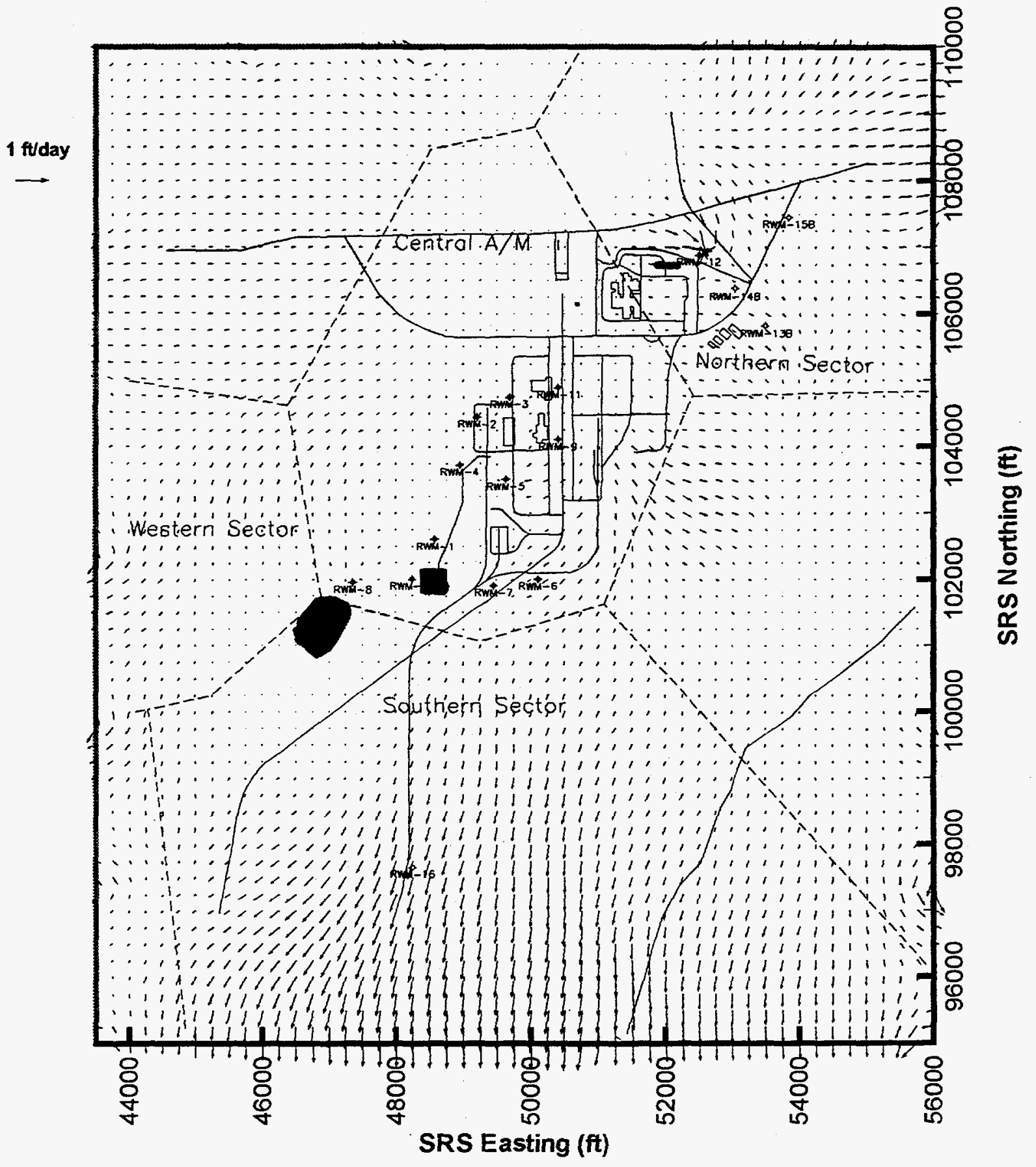

Figure 6.9 - Estimated Pore Velocity for "Lower Lost Lake" aquifer 


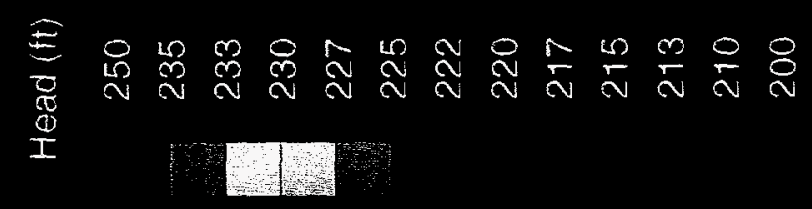

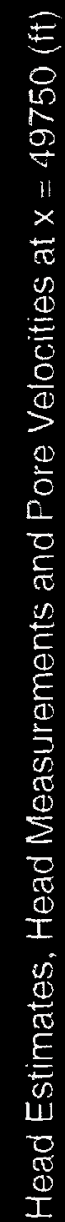

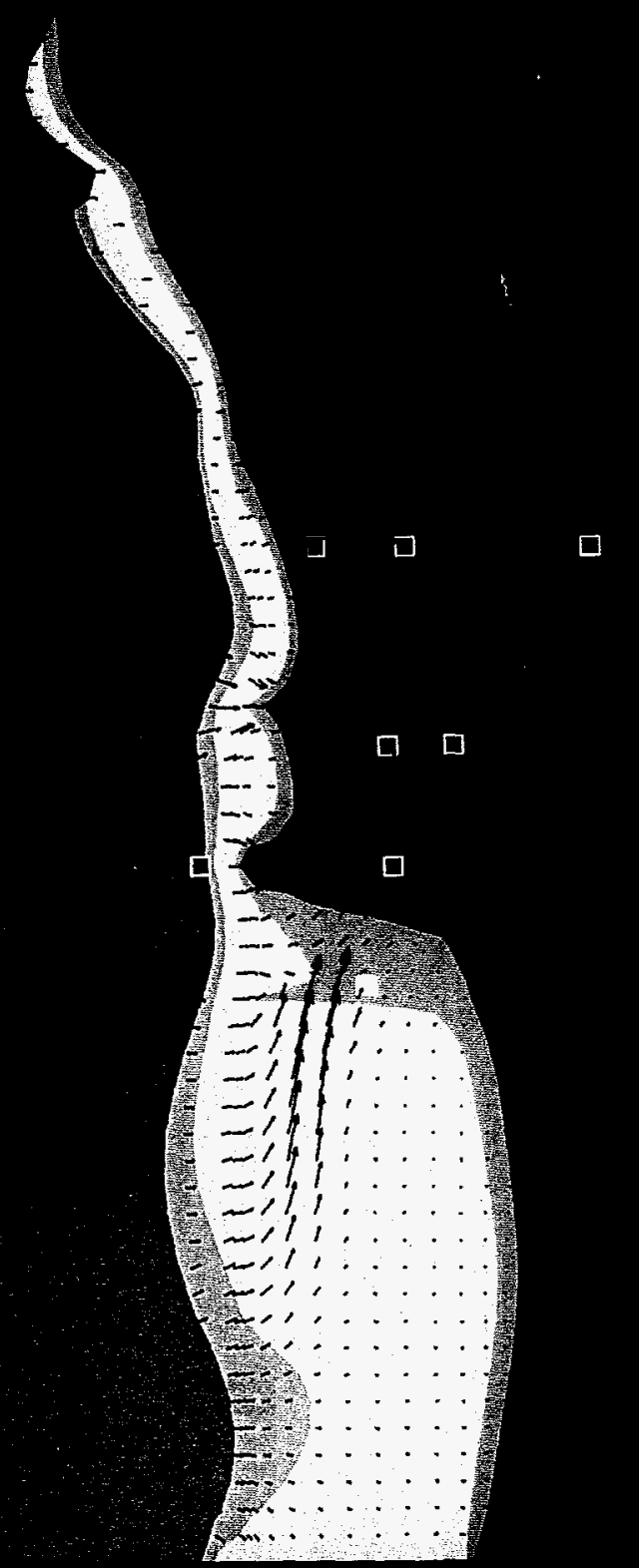

0.00096

0.00086

0.000001

$\stackrel{O}{\infty}$

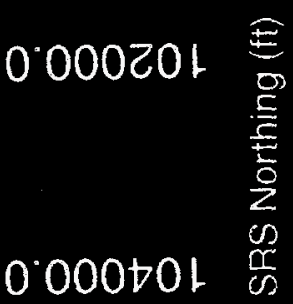

0.000901

0.000801

$0.00001 L$

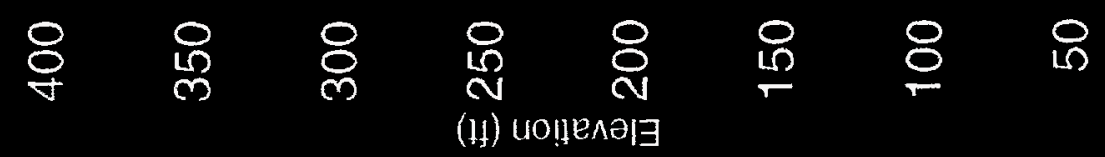

Figure 6.10 Head estimates, head measurements, and pore velocities at $\mathrm{x}=49,750(\mathrm{ft})$. 
Table 6.21 Head Data Near X $=49750(\mathrm{ft})$

\begin{tabular}{|c|c|c|c|c|}
\hline & SRS & SRS & & Head \\
\hline Well ID & $\begin{array}{c}\text { Easting } \\
\text { (ft) }\end{array}$ & $\begin{array}{l}\text { Northing } \\
\text { (ft) }\end{array}$ & $\begin{array}{c}\text { Elev. } \\
\text { (ft) }\end{array}$ & $\begin{array}{l}\text { Data } \\
\text { (ft) }\end{array}$ \\
\hline----- & ------- & $---\cdots---$ & ----- & ----- \\
\hline MSB_36A & 49514.9 & 100511.3 & 97.70 & 211.79 \\
\hline MSB_36B & 49526.3 & 100514.9 & 160.90 & 216.06 \\
\hline MSB_36C & 49537.2 & 100518.3 & 191.40 & 216.16 \\
\hline MSB_25A & 49657.9 & 103504.8 & 164.70 & 219.58 \\
\hline MSB_25 & 49668.9 & 103498.8 & 230.85 & 237.01 \\
\hline MSB_38B & 49746.1 & 102360.8 & 144.15 & 218.61 \\
\hline MSB_38C & 49762.0 & 102373.1 & 166.65 & 221.98 \\
\hline MSB_24A & 49845.3 & 104625.3 & 173.80 & 227.79 \\
\hline
\end{tabular}


(H) GUIYHON SUS
$\begin{array}{lll}\circ & 0 & 0 \\ 8 & 8 & 8 \\ 8 & 8 \\ 8 & 8 & 8 \\ 0 & 0 & 0\end{array}$
$\begin{array}{ll}0 & 0 \\ 8 & 8 \\ 8 & 8 \\ 8 & 0\end{array}$
$\begin{array}{ll}0 & 0 \\ 8 & 8 \\ 8 & 8 \\ ᄋ & 8 \\ 0 & \end{array}$
\begin{tabular}{lll}
$\circ$ & 0 & 0 \\
8 & 8 & 8 \\
8 & 8 & 8 \\
8 & 8 & 8 \\
\hline & 0 & 0
\end{tabular}

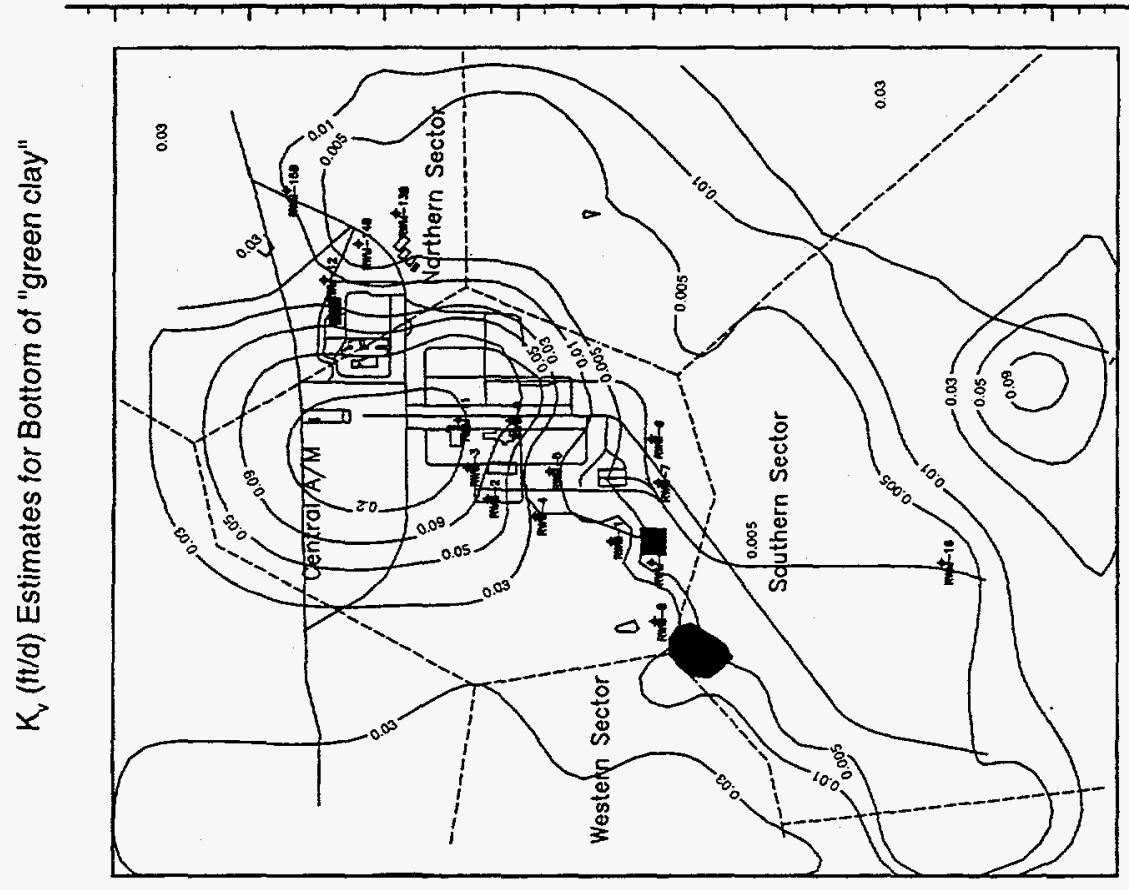

$-0.00095$

0.00079

10.00029

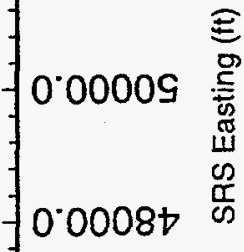

(H) БU!YHON SHS
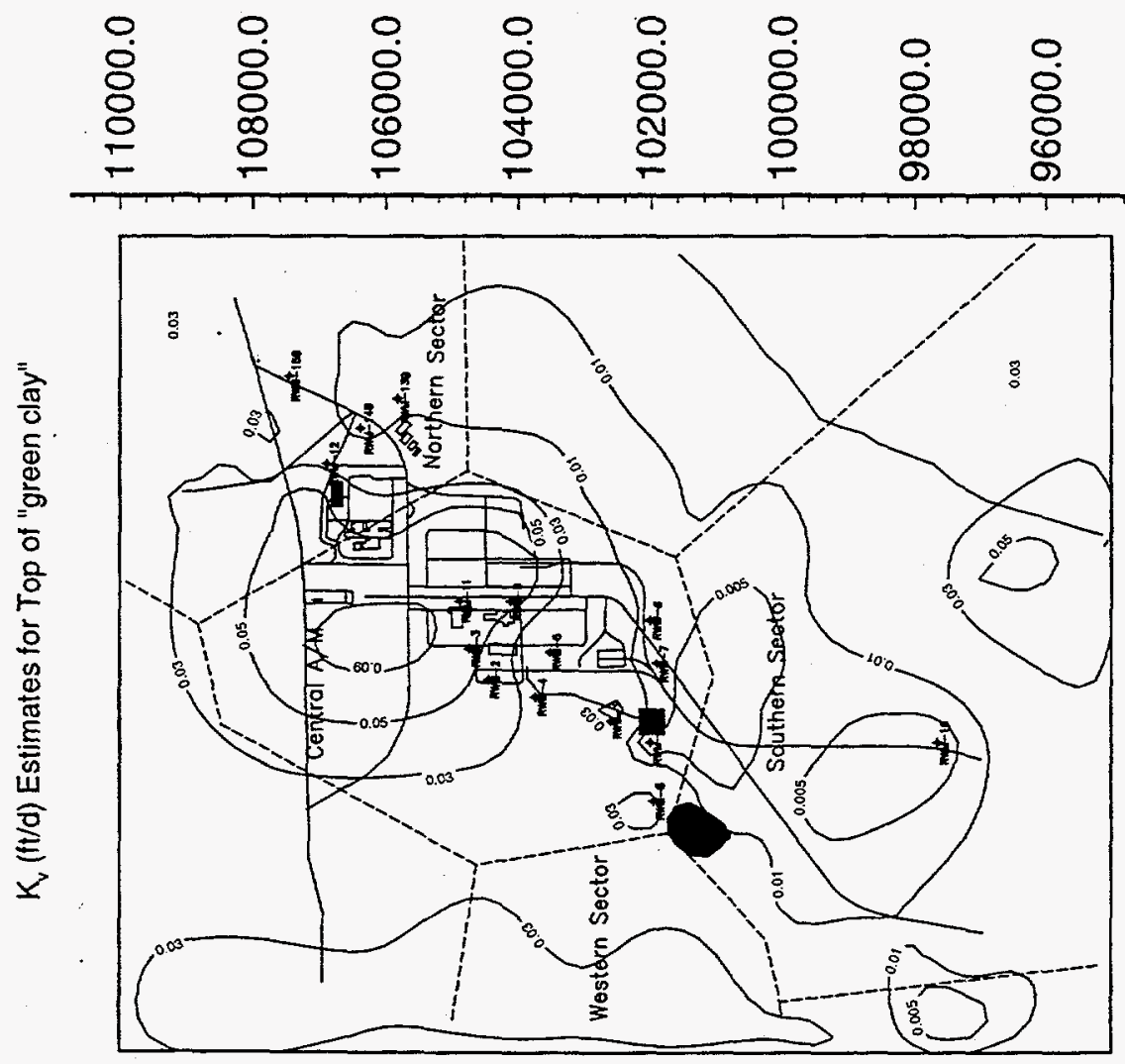

0.00099

$-0.000 t 9$

$-0.00029$

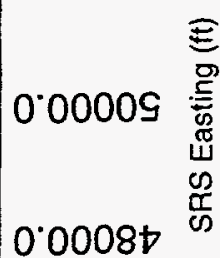

Figure $6.11 \mathrm{~K}_{\mathrm{v}}(\mathrm{ft} / \mathrm{d})$ estimates for "green clay." 
(1) Gu!4มоN S4S

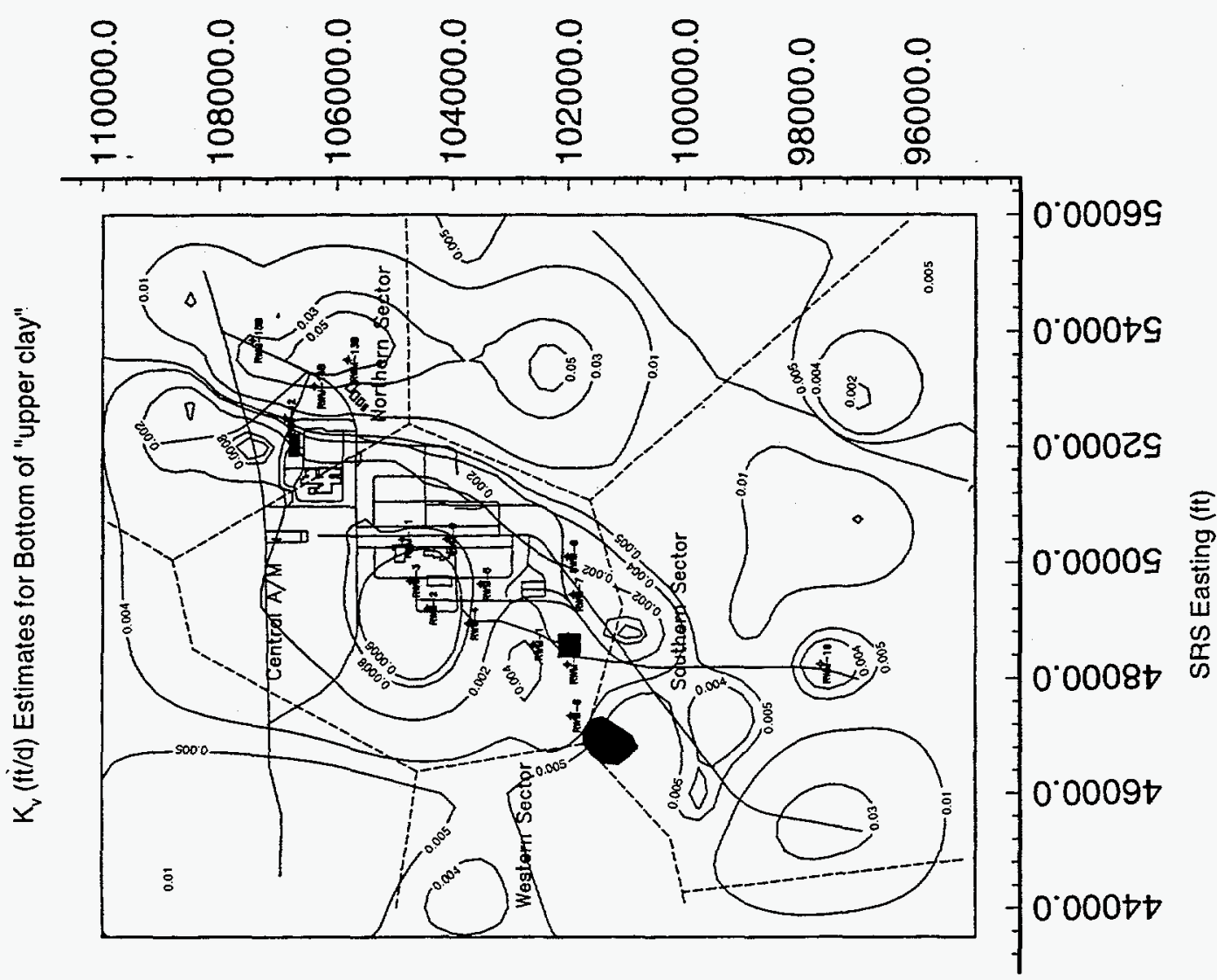

(4) Gu!4मON SצS

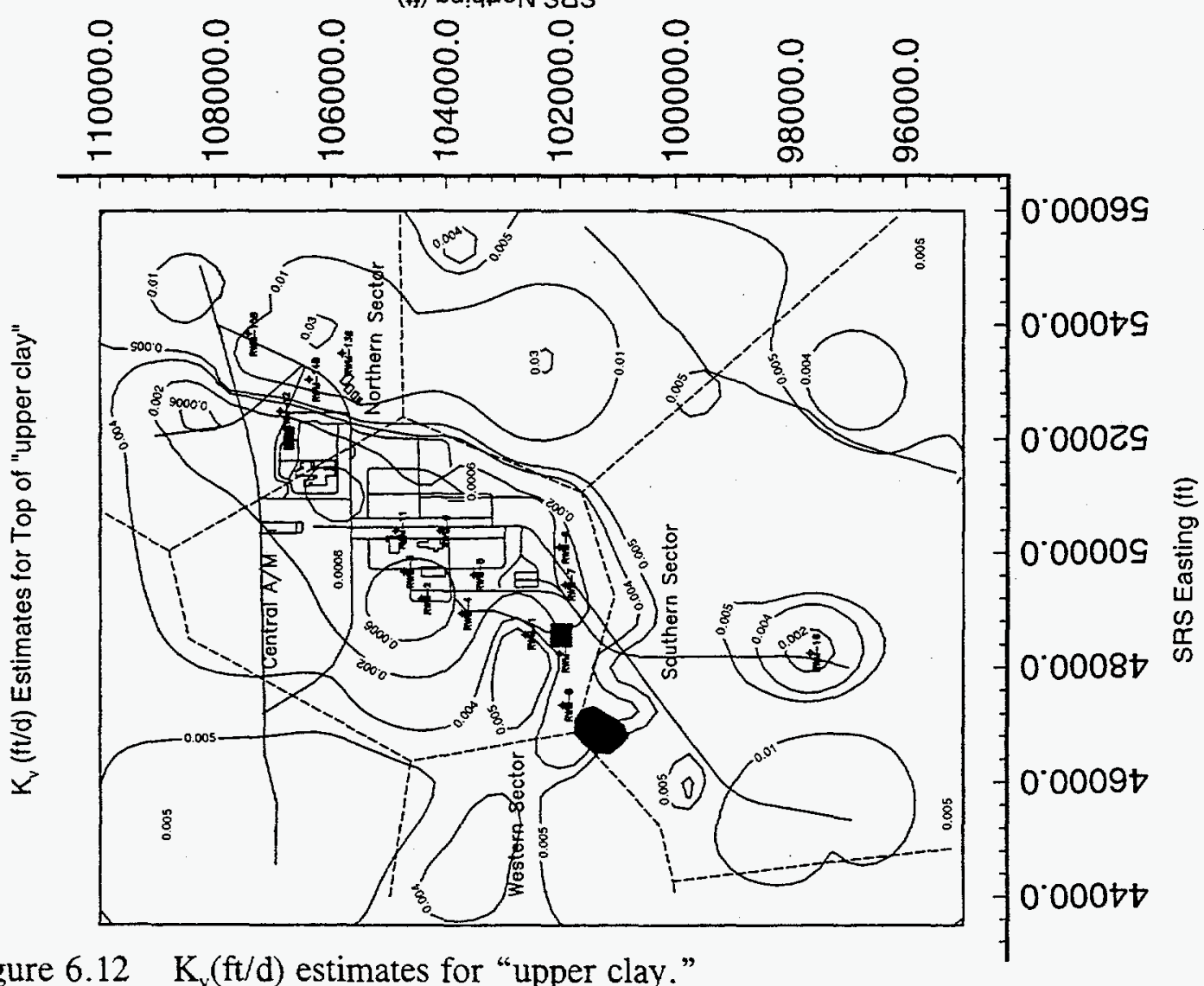

Figure 6.12 $\mathrm{K}_{\mathrm{v}}(\mathrm{ft} / \mathrm{d})$ estimates for "upper clay." 
$K_{h}(f t / d)$ Estimates for "M-Area" Aquifer

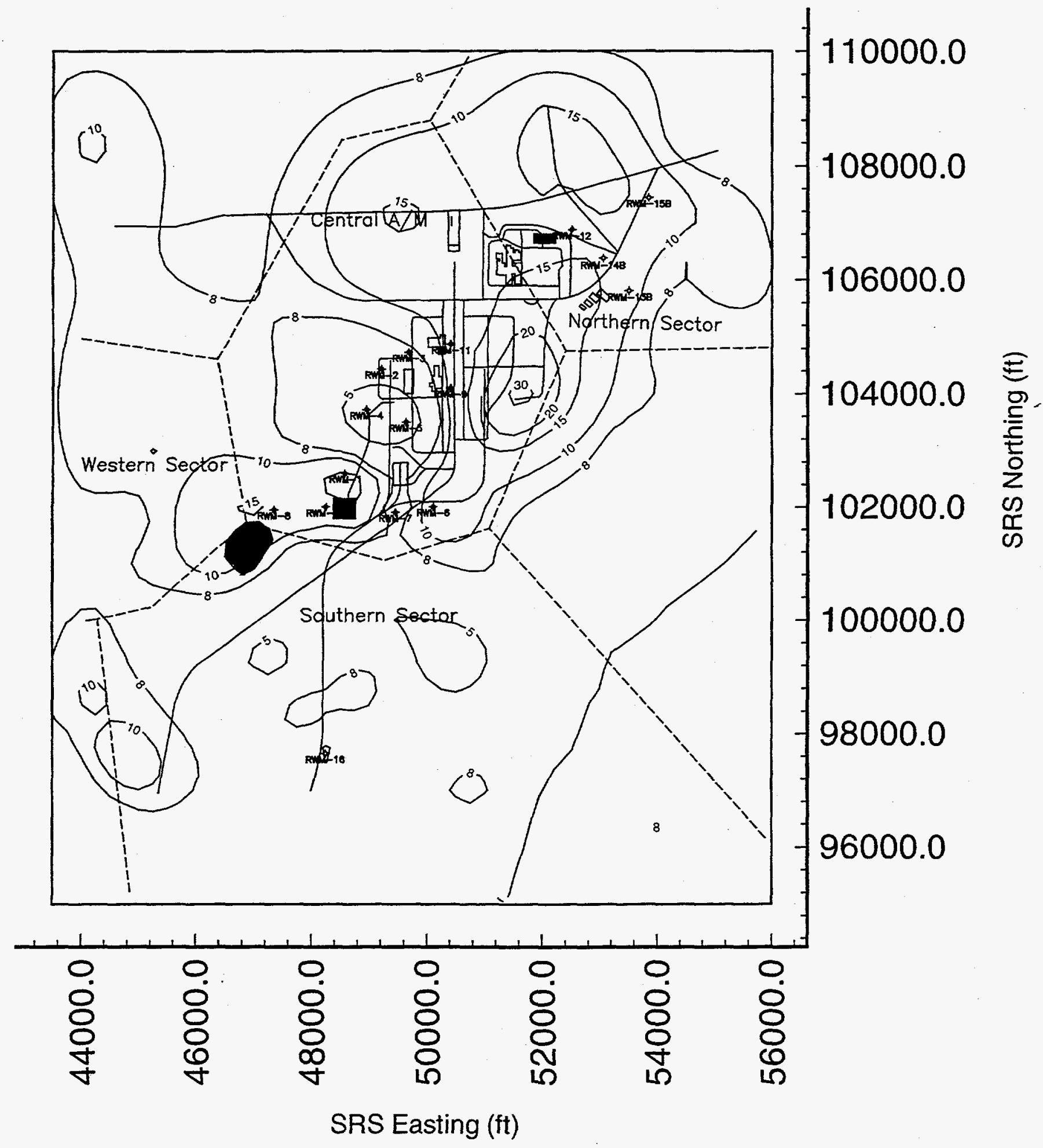

Figure 6.13 $\mathrm{K}_{\mathrm{h}}(\mathrm{ft} / \mathrm{d})$ estimates for "M-area" aquifer. 
$K_{h}(\mathrm{ft} / \mathrm{d})$ Estimates for "upper Lost Lake" Aquifer

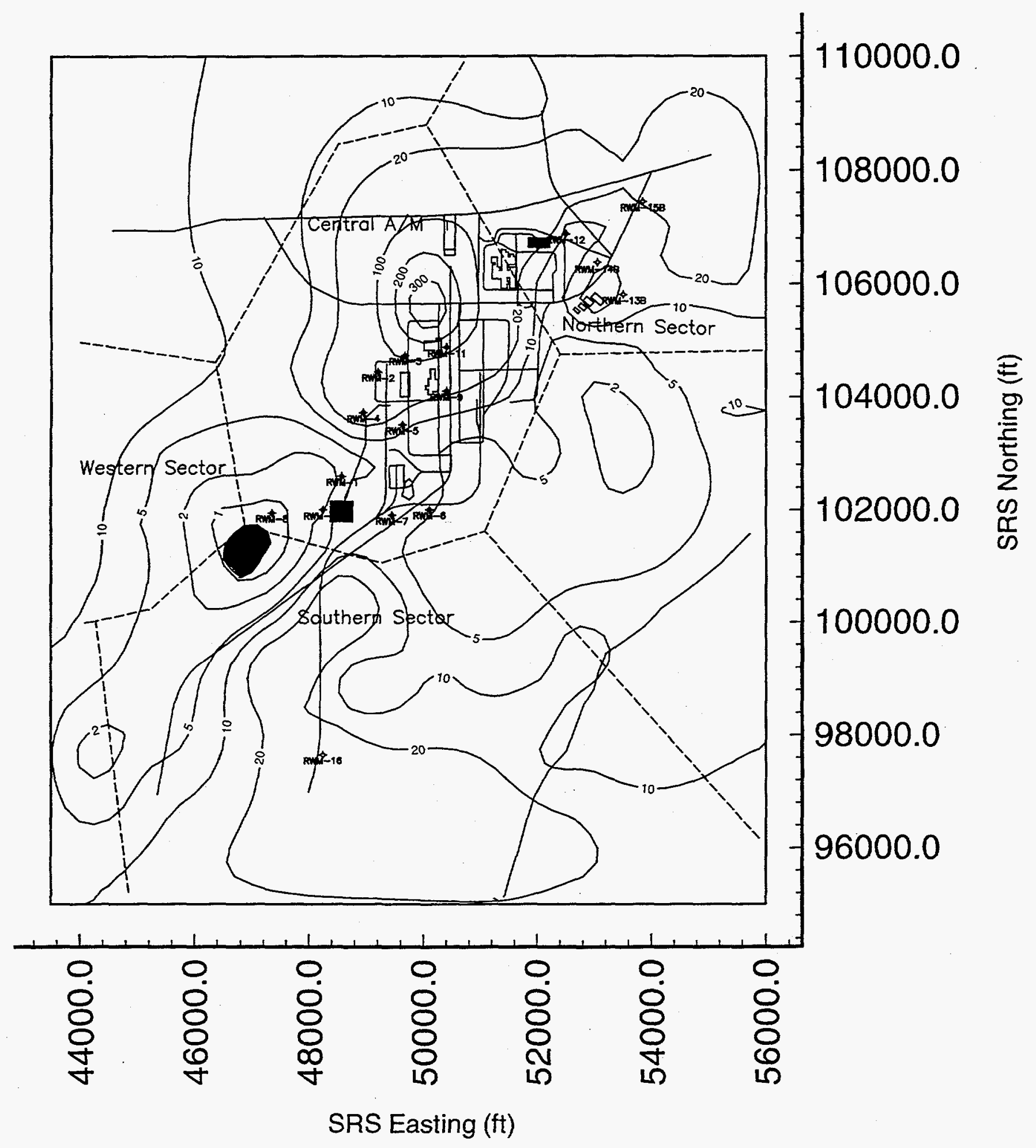

Figure 6.14 $\mathrm{K}_{\mathrm{h}}(\mathrm{ft} / \mathrm{d})$ estimates for "Upper Lost Lake" aquifer. 
$\mathrm{K}_{\mathrm{h}}(\mathrm{ft} / \mathrm{d})$ Estimates for "lower Lost Lake" Aquifer

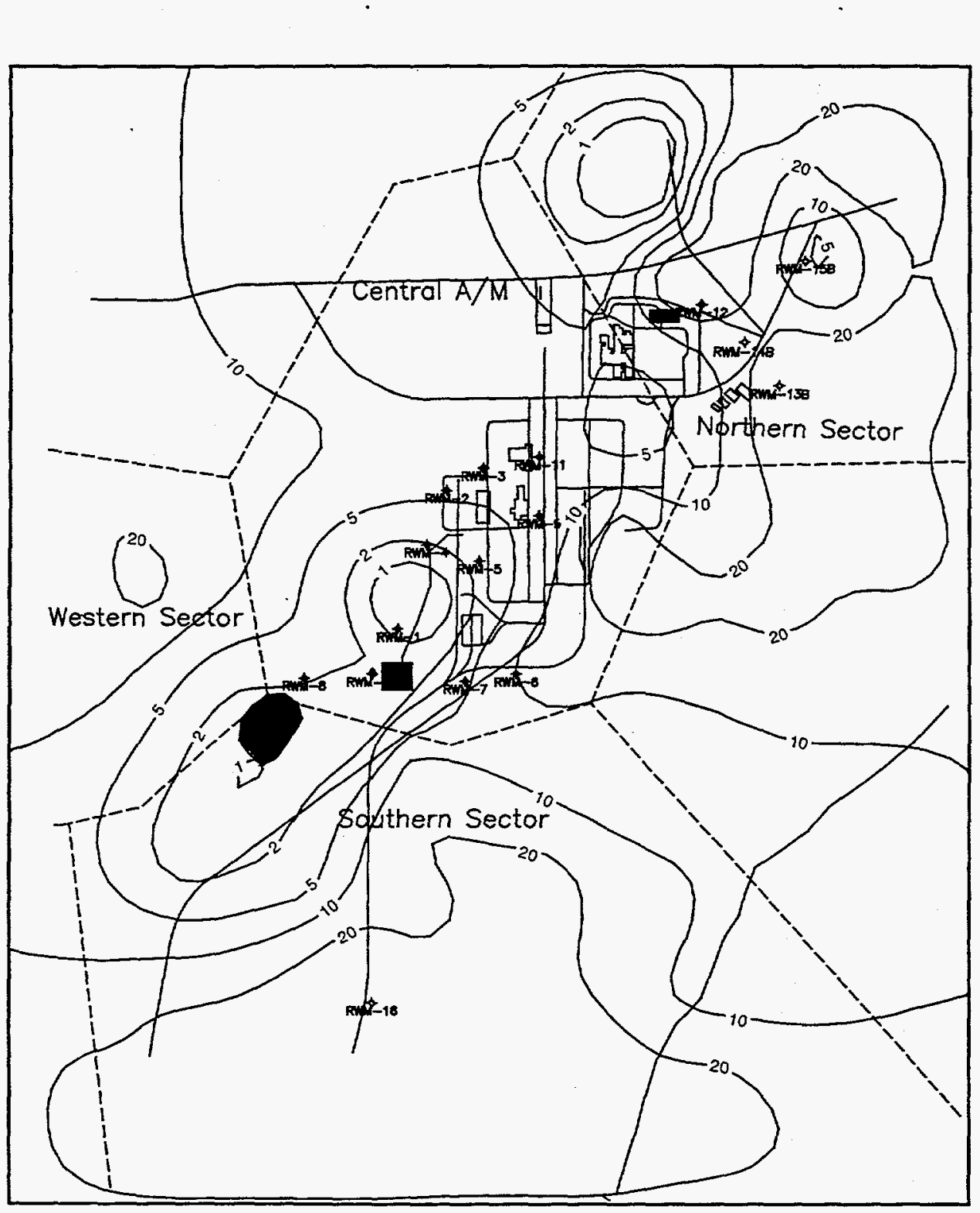

110000.0

108000.0

106000.0

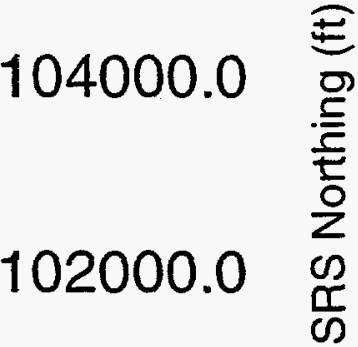

100000.0

98000.0

$-96000.0$

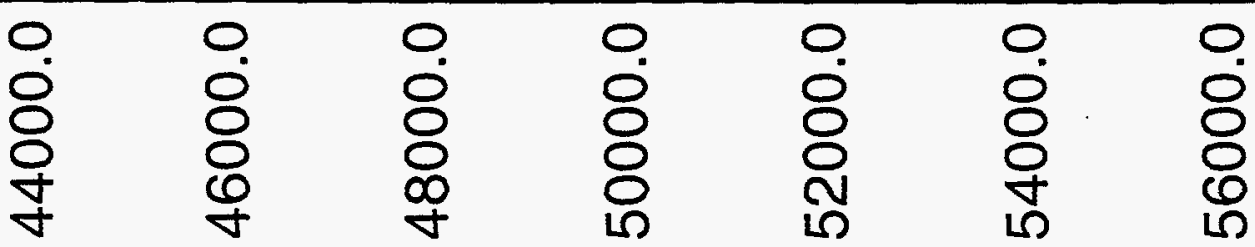

SRS Easting (ft)

Figure 6.15 $\mathrm{K}_{\mathrm{h}}(\mathrm{ft} / \mathrm{d})$ estimates for "Lower Lost Lake" aquifer. 


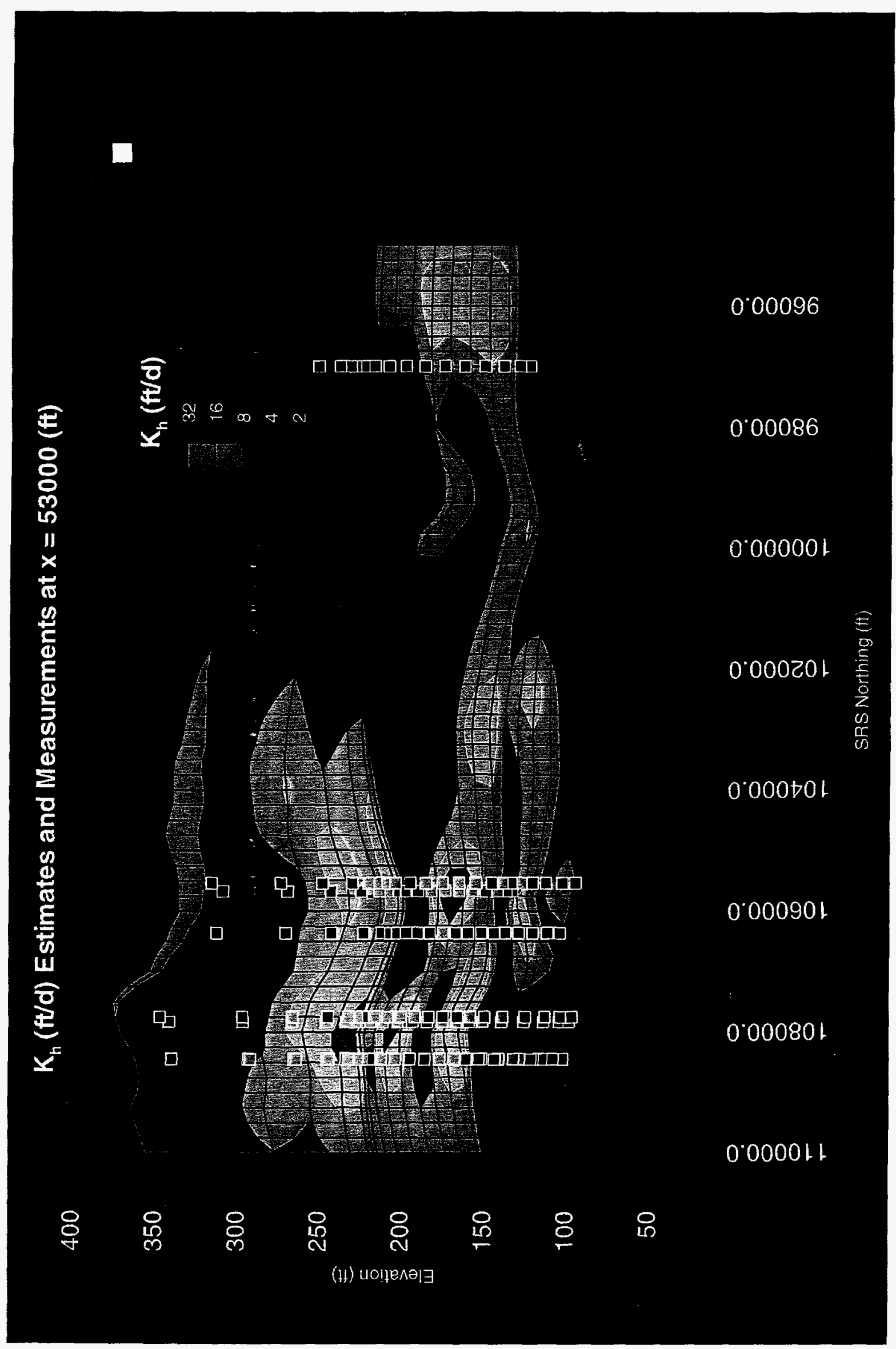

Figure 6.16 $\mathrm{K}_{\mathrm{h}}(\mathrm{ft} / \mathrm{d})$ estimates and measurement locations at $\mathrm{x}=53,000(\mathrm{ft})$. 
Table 6.22 Kh Data Near X $=53000(\mathrm{ft})$

\begin{tabular}{|c|c|c|c|c|}
\hline \multirow{2}{*}{$\begin{array}{l}\text { SRS } \\
\text { We11 ID }\end{array}$} & \multicolumn{2}{|c|}{ SRS } & \multicolumn{2}{|c|}{$\ln (K h)$} \\
\hline & $\begin{array}{c}\text { Easting } \\
\text { (ft) }\end{array}$ & $\begin{array}{l}\text { Northins } \\
\text { (ft) }\end{array}$ & $\begin{array}{c}\text { Elev. } \\
\text { (ft) }\end{array}$ & $\begin{array}{l}\text { Data } \\
\ln (\mathrm{ft} / \mathrm{d})\end{array}$ \\
\hline------ & -------- & ------- & ---- & ------ \\
\hline $\mathrm{MBC} \_1 \mathrm{SB}$ & 52780.0 & 108450.0 & 339.00 & 2.5118 \\
\hline $\mathrm{MBC} \_1 \mathrm{SB}$ & 52780.0 & 108450.0 & 291.50 & 8.6972 \\
\hline $\mathrm{MBC} \_1 \mathrm{SB}$ & 52780.0 & 108450.0 & 263.50 & 8.6538 \\
\hline $\mathrm{MBC} \_1 \mathrm{SB}$ & 52780.0 & 108450.0 & 243.50 & 9.6988 \\
\hline $\mathrm{MBC} \_1 \mathrm{SB}$ & 52780.0 & 108450.0 & 231.50 & 9.6988 \\
\hline $\mathrm{MBC} \_1 \mathrm{SB}$ & 52780.0 & 108450.0 & 226.00 & 9.6988 \\
\hline $\mathrm{MBC} \_1 \mathrm{SB}$ & 52780.0 & 108450.0 & 223.00 & 9.6988 \\
\hline $\mathrm{MBC} \_1 \mathrm{SB}$ & 52780.0 & 108450.0 & 218.50 & 1.5471 \\
\hline $\mathrm{MBC} \_1 \mathrm{SB}$ & 52780.0 & 108450.0 & 211.50 & 9.6988 \\
\hline $\mathrm{MBC} \ldots 1 \mathrm{SB}$ & 52780.0 & 108450.0 & 203.00 & 9.6988 \\
\hline $\mathrm{MBC} \_1 \mathrm{SB}$ & 52780.0 & 108450.0 & 194.00 & 3.2544 \\
\hline $\mathrm{MBC} \_1 \mathrm{SB}$ & 52780.0 & 108450.0 & 185.00 & 6.9588 \\
\hline $\mathrm{MBC} \_1 \mathrm{SB}$ & 52780.0 & 108450.0 & 176.00 & 9.6988 \\
\hline $\mathrm{MBC} \_1 \mathrm{SB}$ & 52780.0 & 108450.0 & 167.50 & 9.6988 \\
\hline $\mathrm{MBC} \_1 \mathrm{SB}$ & 52780.0 & 108450.0 & 160.00 & 4.1579 \\
\hline $\mathrm{MBC} \_1 \mathrm{SB}$ & 52780.0 & 108450.0 & 152.50 & .0006 \\
\hline $\mathrm{MBC} \_1 \mathrm{SB}$ & 52780.0 & 108450.0 & 143.00 & 7.0217 \\
\hline $\mathrm{MBC} \_1 \mathrm{SB}$ & 52780.0 & 108450.0 & 131.50 & 8.8906 \\
\hline $\mathrm{MBC} \_1 S B$ & 52780.0 & 108450.0 & 121.00 & .0164 \\
\hline $\mathrm{MBC} \_1 \mathrm{SB}$ & 52780.0 & 108450.0 & 113.00 & 1.3868 \\
\hline $\mathrm{MBC}_{2} \quad 1 \mathrm{SB}$ & 52780.0 & 108450.0 & 107.50 & .0464 \\
\hline MSB_51B & 52818.0 & 96992.7 & 251.10 & 2.6645 \\
\hline MSB_5IB & 52818.0 & 96992.7 & 238.10 & 3.2805 \\
\hline MSB_51B & 52818.0 & 96992.7 & 231.60 & 1.7437 \\
\hline MSB_51B & 52818.0 & 96992.7 & 227.10 & 6.1411 \\
\hline MSB_51B & 52818.0 & 96992.7 & 224.60 & 4.3579 \\
\hline MSB_51B & 52818.0 & 96992.7 & 222.10 & 1.4550 \\
\hline MSB_51B & 52818.0 & 96992.7 & 217.10 & .6427 \\
\hline MSB_51B & 52818.0 & 96992.7 & 208.60 & 1.1537 \\
\hline MSB_51B & 52818.0 & 96992.7 & 198.10 & .0711 \\
\hline MSB_51B & 52818.0 & 96992.7 & 186.60 & 4.4907 \\
\hline MSB_51B & 52818.0 & 96992.7 & 174.60 & 2.6039 \\
\hline MSB_51B & 52818.0 & 96992.7 & 162.60 & 5.2383 \\
\hline MSB_51B & 52818.0 & 96992.7 & 150.60 & 7.2500 \\
\hline MSB_51B & 52818.0 & 96992.7 & 139.10 & 3.8651 \\
\hline MSB_51B & 52818.0 & 96992.7 & 129.10 & .6332 \\
\hline$M S B \_51 B$ & 52818.0 & 96992.7 & 123.10 & .0004 \\
\hline $\mathrm{ASB} \_2 \mathrm{CR}$ & 52862.7 & 105540.2 & 315.10 & 3.8806 \\
\hline $\mathrm{ASB} \_2 \mathrm{CR}$ & 52862.7 & 105540.2 & 273.10 & 7.4038 \\
\hline
\end{tabular}




\begin{tabular}{|c|c|c|c|c|}
\hline $\mathrm{ASB} \_2 \mathrm{CR}$ & 862.7 & 105540.2 & 247.60 & \\
\hline $\mathrm{ASB} \_2 \mathrm{CR}$ & 2862.7 & 105540.2 & 229.60 & 379 \\
\hline $\mathrm{ASB} \_2 \mathrm{CR}$ & 62.7 & 10.2 & 219.60 & \\
\hline$S B \_2 C R$ & 862.7 & 0.2 & 214.60 & 88 \\
\hline$S B \_2 C R$ & 62.7 & 0.2 & 210.10 & 88 \\
\hline$S B \_2 C R$ & 2862.7 & 0.2 & 03.10 & 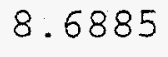 \\
\hline$A S B \_2 C R$ & 2862.7 & 0.2 & 94.10 & 3 \\
\hline $\mathrm{ASB} \_2 \mathrm{CR}$ & 862.7 & 40.2 & 184.60 & 8.7 \\
\hline $\mathrm{ASB} \_2 \mathrm{CR}$ & 52862.7 & 0.2 & 174.60 & 9.6 \\
\hline $\mathrm{ASB} \_2 \mathrm{CR}$ & 2.7 & & 164.60 & \\
\hline$A S B \_2 C R$ & 2.7 & 0.2 & 154.60 & 20 \\
\hline $\mathrm{ASB} \_2 \mathrm{CR}$ & .7 & & 145.10 & 18 \\
\hline $\mathrm{ASB} \_2 \mathrm{CR}$ & 2.7 & 0.2 & 37.60 & 9.6 \\
\hline $\mathrm{ASB} \_2 \mathrm{CR}$ & .7 & & 31.60 & \\
\hline $\mathrm{ASB} \_2 \mathrm{CR}$ & 2.7 & 0.2 & 126.10 & 8 \\
\hline $\mathrm{ASB} \_2 \mathrm{CR}$ & .7 & & .10 & 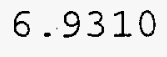 \\
\hline $\mathrm{ASB} \_2 \mathrm{CR}$ & 2.7 & 0.2 & 12.10 & 6.4 \\
\hline $\mathrm{ASB} \_2 \mathrm{CR}$ & 2.7 & & .10 & .5 \\
\hline $\mathrm{ASB} \_2 \mathrm{CR}$ & 2.7 & 0.2 & 4.10 & 7.5 \\
\hline$A S B \_10 C$ & .5 & & 8.30 & 2 \\
\hline$A S B \_10 C$ & .5 & 3.1 & 268.80 & 5.5 \\
\hline$A S B \_10 C$ & & & .30 & \\
\hline$A S B \_10 C$ & .5 & 3.1 & .80 & 4.7 \\
\hline ASB_10C & & & .80 & 3.9 \\
\hline $\mathrm{ASB} \_10 \mathrm{C}$ & .5 & 3.1 & .80 & 9.6 \\
\hline$A S B \_10 C$ & & & .30 & 7.1 \\
\hline$A S B \_10 C$ & .5 & 3.1 & .80 & 4.1 \\
\hline$A S B \_10 C$ & .5 & 3.1 & 189.30 & 5 \\
\hline$A S B \_10 C$ & .5 & 3.1 & 181.30 & 9.6 \\
\hline$A S B \_10 C$ & 5.5 & 3.1 & 172.80 & 9.6 \\
\hline ASB_10C & .5 & 3.1 & 164.30 & 9.6 \\
\hline ASB_10C & 5.5 & 83.1 & 156.30 & 8.4 \\
\hline$A S B \_10 C$ & 52 & 3.1 & 148.30 & 6.1 \\
\hline$A S B \_10 C$ & .5 & 3.1 & 141.30 & 8. \\
\hline ASB_10C & .5 & 3.1 & 135.80 & 5.8 \\
\hline$A S B \_10 C$ & 5.5 & 73.1 & 132.30 & 4.8 \\
\hline $\mathrm{MBC} \_2 \mathrm{SB}$ & 5.0 & 0.0 & 346.20 & 2.5 \\
\hline $\mathrm{MBC} \_2 \mathrm{SB}$ & 5.0 & 0.0 & 296.20 & 4.7922 \\
\hline $\mathrm{MBC} \_2 \mathrm{SB}$ & 5.0 & 107750.0 & 265.70 & 8.1743 \\
\hline $\mathrm{MBC} \_2 \mathrm{SB}$ & 529 & 107750.0 & 244.20 & 7.6523 \\
\hline $\mathrm{MBC} \_2 \mathrm{SB}$ & 5.0 & 107750.0 & 231.70 & 9.6988 \\
\hline $\mathrm{MBC} \_2 \mathrm{SB}$ & 52975.0 & 107750.0 & 226.20 & 9.6988 \\
\hline $\mathrm{MBC} \_2 \mathrm{SB}$ & 52975.0 & 107750.0 & 222.20 & 6.0557 \\
\hline $\mathrm{MBC} \_2 \mathrm{SB}$ & 52975.0 & 107750.0 & 215.70 & 9.0340 \\
\hline $\mathrm{MBC} \_2 \mathrm{SB}$ & 52975.0 & 107750.0 & 207.70 & 9.0340 \\
\hline $\mathrm{MBC} \_2 \mathrm{SB}$ & 52975.0 & 107750.0 & 199.70 & 8 \\
\hline $\mathrm{MBC} \_2 \mathrm{SB}$ & 52975.0 & 107750.0 & 191.20 & 8.5165 \\
\hline
\end{tabular}




\begin{tabular}{|c|c|c|c|c|}
\hline$B C \_\quad 2 S B$ & 52975.0 & 107750.0 & 182.70 & 10 \\
\hline $\mathrm{MBC} \_2 \mathrm{SB}$ & 52975.0 & 107750.0 & 174.20 & 7.5534 \\
\hline $\mathrm{MBC} \_2 \mathrm{SB}$ & 52975.0 & 50.0 & 165.70 & \\
\hline $\mathrm{MBC} \_2 \mathrm{SB}$ & 52975.0 & 107750.0 & 157.70 & 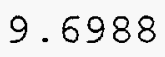 \\
\hline $\mathrm{MBC} \_2 \mathrm{SB}$ & 2975.0 & 107750.0 & 149.20 & .5 \\
\hline $\mathrm{MBC} \_2 \mathrm{SB}$ & 3975.0 & 07750.0 & 138.20 & 3.2 \\
\hline $\mathrm{MBC} \_2 \mathrm{SB}$ & 2975.0 & 07750.0 & 124.70 & 3.7 \\
\hline $\mathrm{MBC} \_2 \mathrm{SB}$ & 2975.0 & 07750.0 & 112.70 & 1.4 \\
\hline $\mathrm{MBC} \_2 \mathrm{SB}$ & 52975.0 & 07750.0 & 103.20 & 4.2 \\
\hline $\mathrm{MBC}$ & 2975.0 & 07750.0 & 96.20 & 6.4 \\
\hline MSB_54TA & 2985.8 & 08446.3 & 339.10 & \\
\hline$M S B \_54 T A$ & 2985.8 & 108446.3 & 292.60 & 2.4 \\
\hline $\mathrm{MSB} \_54 \mathrm{TA}$ & 2985.8 & 108446.3 & 264.60 & 3.3 \\
\hline$M S B \_54 T A$ & 2985.8 & 108446.3 & 244.60 & 3.9 \\
\hline MSB_54TA & 2985.8 & 108446.3 & 233.10 & 4.3 \\
\hline MSB_54TA & 52985.8 & 108446.3 & 228.10 & 3.2 \\
\hline MSB_54TA & 2985.8 & 108446.3 & 225.10 & \\
\hline MSB_54TA & 52985.8 & 108446.3 & 220.60 & 3.9 \\
\hline MSB_54TA & 52985.8 & 108446.3 & 213.60 & 9.6 \\
\hline$M S B \_54 T A$ & 52985.8 & 108446.3 & 204.60 & 6.3 \\
\hline MSB_54TA & 52985.8 & 108446.3 & 194.60 & 2.4 \\
\hline MSB_54TA & 52985.8 & 108446.3 & 185.10 & 4.3 \\
\hline MSB_54TA & 52985.8 & 108446.3 & 175.60 & 9.6 \\
\hline MSB_54TA & 52985.8 & 108446.3 & 166.10 & 7.6 \\
\hline $\mathrm{MSB} \_54 \mathrm{TA}$ & 52985.8 & 108446.3 & 158.60 & \\
\hline $\mathrm{MSB} \_54 \mathrm{TA}$ & 52985.8 & 108446.3 & 151.10 & 5.8 \\
\hline MSB_54TA & 52985.8 & 108446.3 & 140.60 & 8.5 \\
\hline$M S B \_54 T A$ & 52985.8 & 108446.3 & 127.60 & 8.2 \\
\hline $\mathrm{MSB} \_54 \mathrm{TA}$ & 5.8 & 5.3 & 116.10 & \\
\hline $\mathrm{MSB} \_54 \mathrm{TA}$ & 52985.8 & 108446.3 & 107.60 & 2.4 \\
\hline $\mathrm{MSB} \_54 \mathrm{TA}$ & 35.8 & 108446.3 & .60 & \\
\hline RWM_14B & 53044.7 & 106362.1 & 312.00 & 1.2 \\
\hline RWM_14B & 44.7 & 106362.1 & 00 & 5.1 \\
\hline RWM_14B & 53044.7 & 106362.1 & 242.00 & 3.4 \\
\hline RWM_14B & 44.7 & 106362.1 & .50 & 3.5 \\
\hline RWM_14B & 53044.7 & 106362.1 & 211.50 & 5.3 \\
\hline RWM_14B & 44.7 & 106362.1 & .00 & 3.8 \\
\hline RWM_14B & 53044.7 & 106362.1 & 202.00 & 2.4303 \\
\hline RWM_14B & 44.7 & 106362.1 & 196.50 & .6288 \\
\hline RWM_14B & 53044.7 & 106362.1 & 189.50 & 3.963 \\
\hline RWM_14B & 53044.7 & 106362.1 & 182.00 & 6.7666 \\
\hline RWM_14B & 53044.7 & 106362.1 & 174.00 & 9.698 \\
\hline RWM_14B & 53044.7 & 106362.1 & 166.50 & 2.5702 \\
\hline RWM_14B & 53044.7 & 106362.1 & 159.00 & 6.6792 \\
\hline RWM_14B & 53044.7 & 106362.1 & 151.00 & 5.8241 \\
\hline RWM_14B & 53044.7 & 106362.1 & 143.50 & 3.267 \\
\hline RWM_14B & 53044.7 & 106362.1 & 136.50 & 3.41 \\
\hline
\end{tabular}


RWM_14B $\quad 53044.7 \quad 106362.1 \quad 128.50 \quad 7.2066$

RWM_14B - $53044.7 \quad 106362.1 \quad 120.00 \quad 7.4187$

RWM_14B $53044.7 \quad 106362.1111 .00 \quad 4.7541$

RWM_I4B $53044.7 \quad 106362.1 \quad 103.50 \quad 4.8695$ 
Another prominent feature is the lower HDF source/drain heads on the western boundary and thus higher flow across the boundary, particularly in the northern region. As previously noted, the use of the HDF model on the east boundary of the "Lost Lake" Aquifer Zone results in significant flow across the northern portion of the boundary, which differs from previous models. As expected, the horizontal flow in the "M-Area" Aquifer Zone is relatively small, partly because of the lower conductivity. The higher head below the two outfall regions can be seen. The general flow is away from the A/M Areas.

Compared to the prior estimates, DFM has had decreased the average horizontal and vertical conductivity in the "M-Area" and "Lost Lake" Aquifer Zones slightly, increased the general area recharge slightly (from 15 to 18.2 inches/year), increased the "paved area" recharge significantly (from 3 to 5.3 inches/year), estimated respectively a large positive $(+264 \%)$ and small negative $(-24 \%)$ bias on the vertical and horizontal log conductivity measurements, and adjusted the HDF source/drain heads at a few locations. The estimated average horizontal conductivities in the "M-Area" and "Green Clay/Lost Lake" units were 7.3 and 10.0 feet/day respectively. The corresponding estimated average vertical conductivities were 0.011 and $0.027 \mathrm{feet} /$ day. These horizontal values are slightly lower than the pump test data, but not unreasonable. The generally lower conductivity in the upper aquifers appears to be necessary to handle the nominal recharge. Note, however, that there is significant spatial variation in the estimated conductivity, particularly in the "Lost Lake" Aquifer Zone.

Since the low a prior estimate for the paved area recharge was only an educated guess, the slightly higher DFM estimate is reasonable. The method used to compute conductivity from mud fraction using pump and laboratory test data is subject to considerable uncertainty-much larger estimated biases would not be surprising. The primary locations in which the "Lost Lake" Aquifer Zone HDF source heads were adjusted from the prior were the middle north (lower), south (lower), north east (lower), and south west (lower). Thus there is slightly more water flow out the "Lost Lake" Aquifer Zone boundaries than initially expected. However, the estimated heads at the boundaries are not significantly different than expected. None of the HDF source heads for the "M-Area" Aquifer Zone were significantly altered. Also note that the a posteriori 1-sigma for the source heads was reduced below the prior 1-sigma of 2.00 at only a few locations. These were the same locations at which the a posterior value deviated from the prior. The model is relatively insensitive to the source heads on much of the boundary, and thus the observability of the source heads at these locations is weak.

\subsection{DATA FIT RESIDUALS}

The least squares cost function for the final DFM estimate was 2576. The residual sum-of-squares contributions to the cost function are listed in Table 6.23. Note that the steady-state flow error contribution for standard DFM is slightly greater than zero. The total weighted residual RMS was 1.04 where the residual statistics for each measurement type and zone are listed in Table 6.24. The fit to head data is excellent, although 4 measurements were edited. The fit to the $\ln (\mathrm{Kh})$ measurements was very good, but the large variation in the $\ln (\mathrm{Kv})$ measurements could not be fit accurately with a model that attempts to fit both the horizontal and vertical conductivity measurements (with fixed anisotropy for each unit) and also performs spatial smoothing. Note that mean of the $\ln (\mathrm{Kv})$ residuals is positive in the upper two units but negative in the "Crouch Branch" Confining Unit. Since the residual is defined as the measured value minus 
the computed value (including the estimated bias), a positive residual implies that the measurement is larger than the computed conductivity. The average $\ln (\mathrm{Kv})$ measurement is much lower in the "Crouch Branch" Confining Unit than in the upper zones, which may partially explain the bias in the residuals. Since DFM estimated a mean bias of 1.29 on all the $\ln (\mathrm{Kv})$ measurements, the average difference between measured and model computed $\ln (\mathrm{Kv})$ in the "Crouch Branch" Confining Unit is found to be $-0.64+1.29=+0.65$. In other words, DFM has estimated $\ln (\mathrm{Kv})$ about 0.65 lower than the data.

Table 6.23 Residual Sum-of-Square Contributions

\begin{tabular}{|c|c|c|}
\hline $\begin{array}{c}\text { Residual } \\
\text { Component }\end{array}$ & Number of Terms & Sum-of-squares \\
\hline prior & 67 & 70 \\
\hline spatial continuity & 68442 & 913 \\
\hline $\begin{array}{c}\text { steady-state flow } \\
\text { error }\end{array}$ & 68442 & 887 \\
\hline Measurements & 3028 & 3282 \\
\hline Total & & 5152 \\
\hline
\end{tabular}

Table 6.24 Measurement Residual Statistics

\begin{tabular}{|l|l|l|l|l|l|}
\hline Type & zone & number & edited & mean & RMS \\
\hline head & "M-Area" & 66 & 1 & -0.08 & 0.96 \\
\hline & $\begin{array}{l}\text { "Green-Clay/ } \\
\text { Lost lake" }\end{array}$ & 134 & 1 & -0.05 & 0.99 \\
\hline & $\begin{array}{l}\text { CB Confining } \\
\text { Unit }\end{array}$ & 46 & 2 & 0.22 & 0.82 \\
\hline & total & 246 & 4 & -0.01 & 0.95 \\
\hline & "M-Area" & 489 & 0 & -0.45 & 1.38 \\
\hline $\ln (\mathrm{Kh})$ & $\begin{array}{l}\text { "Green-clay/ } \\
\text { Lost Lake" }\end{array}$ & 613 & 0 & -0.25 & 1.38 \\
\hline & $\begin{array}{l}\text { CB Confining } \\
\text { Unit }\end{array}$ & 289 & 0 & -0.07 & 2.43 \\
\hline & total & 1391 & 0 & -0.27 & 1.65 \\
\hline & & & 0 & & 2.45 \\
\hline $\ln (\mathrm{Kv})$ & "M-Area" & 489 & 0 & 0.58 & 2.84 \\
\hline & $\begin{array}{l}\text { "Green-Clay/ } \\
\text { Lost Lake" }\end{array}$ & 613 & 0 & 0.64 & 2.50 \\
\hline & $\begin{array}{l}\text { CB Confining } \\
\text { Unit }\end{array}$ & 289 & 0 & -0.64 & 2.64 \\
\hline & total & 1391 & 0 & 0.35 & \\
\hline & & & & & \\
\hline
\end{tabular}


Figures 6.17 to 6.19 show the head residuals for the three aquifer zones. Note that most of the head residuals in the "M-Area" Aquifer Zone are small, but there is one large positive residual in the south west (which was edited) and one large positive residual in the southern central region. The first did not match those of nearby wells, and the second was very close to recovery well RWM-1. A positive residual indicates higher water levels than modeled.

Figure 6.18 shows the head residuals in the "Lost Lake" Aquifer Zone. There is one large negative residual which was edited near recovery well RWM-8. There is also a moderately positive residual in the north east which does not match other close wells. Most head residuals for the "Crouch Branch" Confining Unit are relatively small, as shown in Figure 6.19. Two positive residuals near the bottom did not match the boundary heads and were edited. Figure 6.20 shows the head residuals in a $y-z$ slice, which better illustrates the vertical stratification of the errors.

Figures 6.21 and 6.22 show all the log conductivity residuals (In feet/day) in $y-z$ slices. (Residuals are not shown in plan view since they only display the top-most residual). From the $y-z$ slice, it is seen that residual patterns appear in layers, but the effect is not pronounced. The many large positive vertical conductivity residuals near the "Green Clay" Confining Zone may suggest holes. There does not appear to be strong evidence of a continuous clay layer within the "Lost Lake" Aquifer Zone.

\subsection{FLOW PATHLINES}

Figures 6.23 and 6.24 show 50 3D pathlines with starting locations in the "M-Area" Aquifer Zone. The selected starting locations were based on potential sources of contaminants, and on capture zones shown in Jackson and Aleman (1994). Note that 23 of the 50 pathlines terminate in pumping wells. Another three were probably captured by pumping wells, but the integration did not model the pumping with sufficient accuracy, as discussed in Section 6.2. The pathlines which exit the bottom of the grid are mostly in the western part of the M-Area, or north of the A-area. Figure 6.8 and 6.9 show that the "Lost Lake" Aquifer Zone pore velocities near the perimeter of the M-Area are very small and the velocity in the northeast corner is more toward the east than presented by Jackson and Aleman (1995). Likewise, the velocity in the west is more toward the boundary than previously shown.

Figure 6.25 shows the capture zones for recovery wells RWM-1 through RWM-12. Most of the Central Sector is captured. The capture zones are similar to the results of Jackson and Aleman (1995) except on the west side of the Central Sector. Due to the low horizontal conductivity in the "Lost Lake" Aquifer Zone north of the Lost Lake (Figures 6.14 and 6.15), RWM-8 and RWM-10 have the smallest capture zones.

Note that the vertical velocities in the central area is very small, and that those particles which avoid the pumping wells will take from 60 to more than 100 years before exiting the bottom of the "Crouch Branch" Confining Unit. The DFM estimate of $\ln (\mathrm{Kv})$ in this unit is about 0.5 smaller than the average $\ln (\mathrm{Kv})$ measurement, so that model $\mathrm{Kv}$ is smaller than the measurements by about $40 \%$. DFM is trying to reduce vertical conductivity, but the measurements are keeping this high. Thus the mud fraction to conductivity mapping must be modified if it is believed that the flow through the confining unit is too great. 


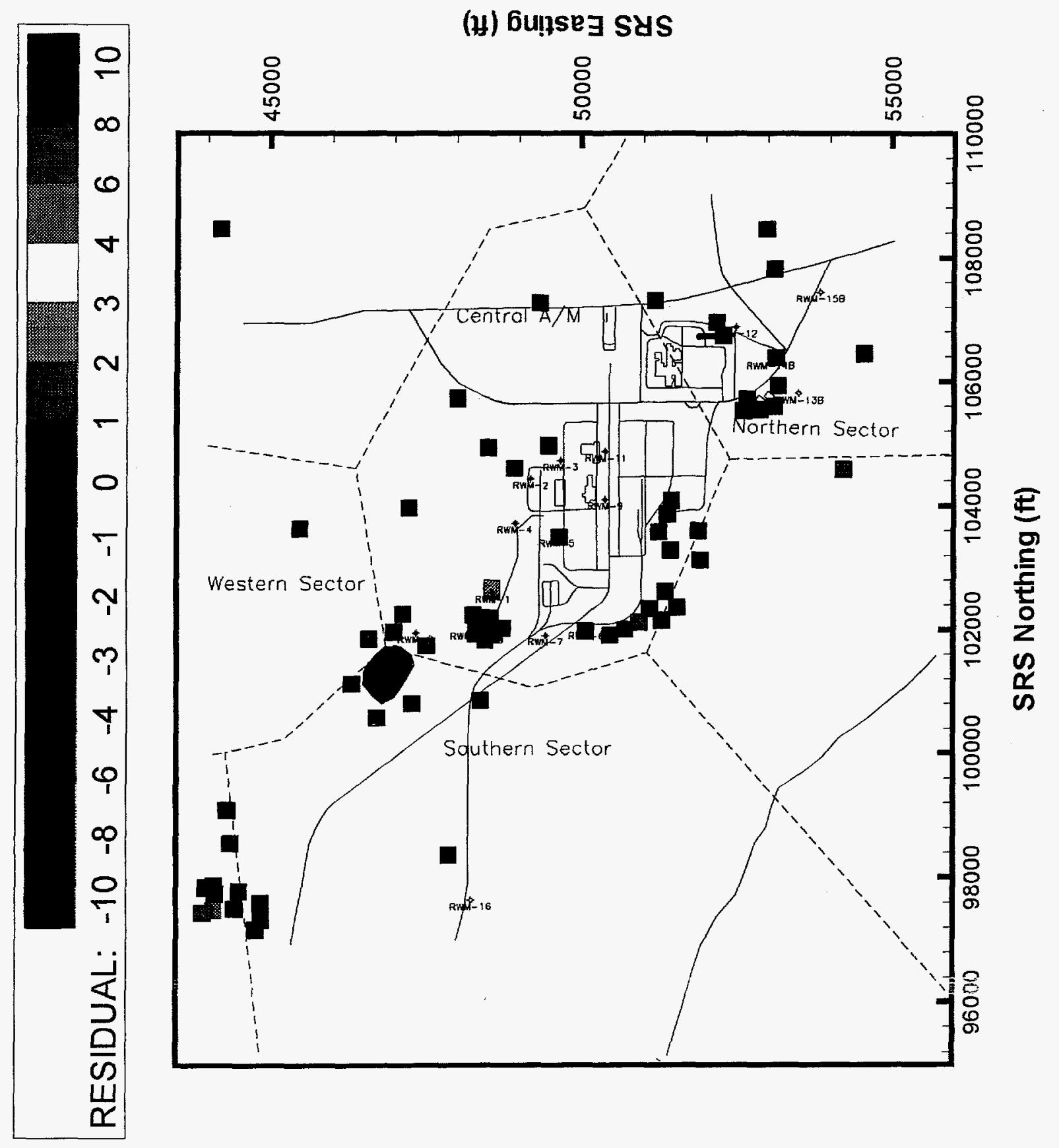

Figure 6.17 - "M-Area" Aquifer Head Residuais 


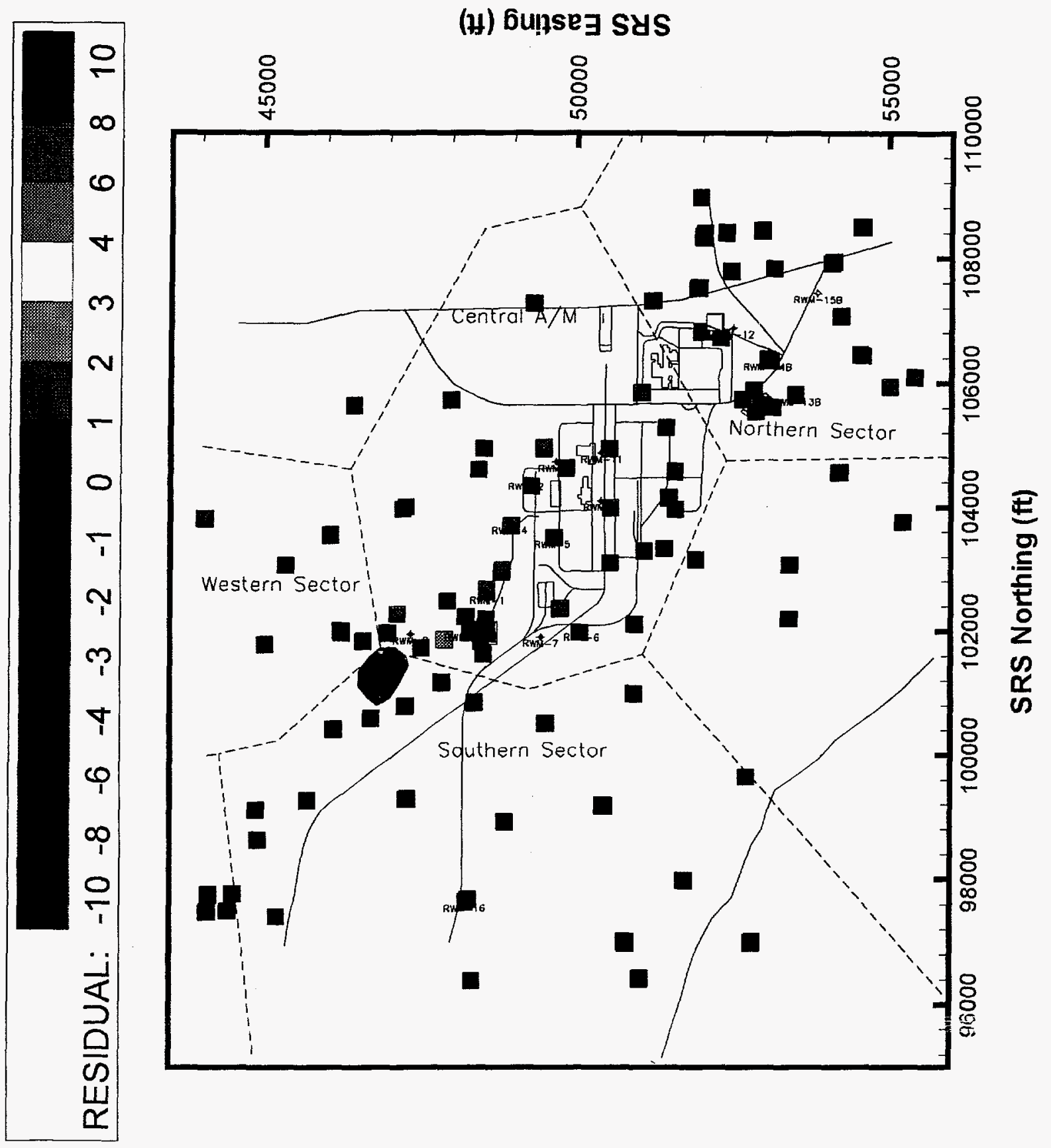

Figure 6.18 - "Lost Lake" Aquifer Head Residuais 


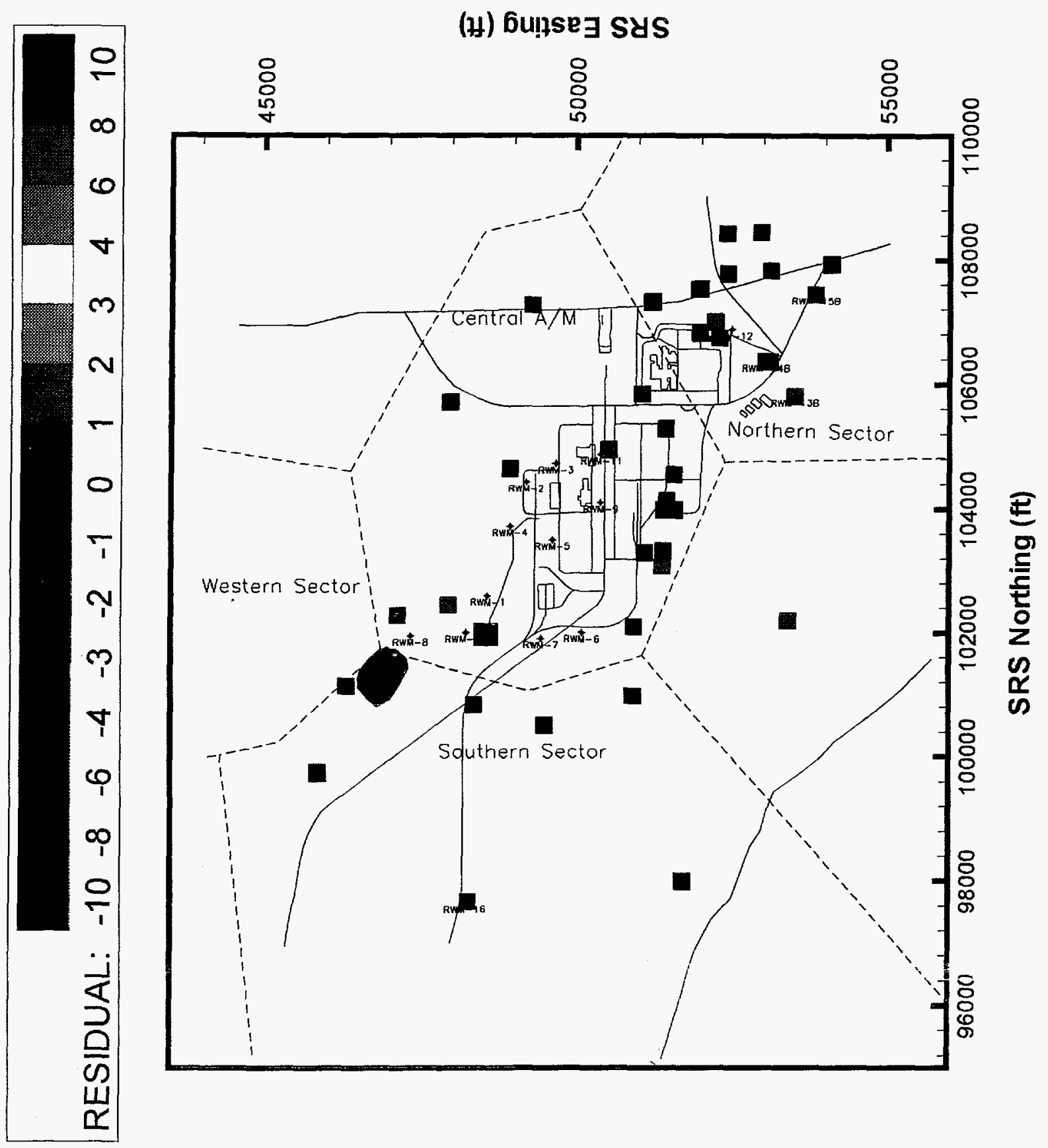

Figure 6.19 - "Crouch Branch Confining Unit" Head Residuals 


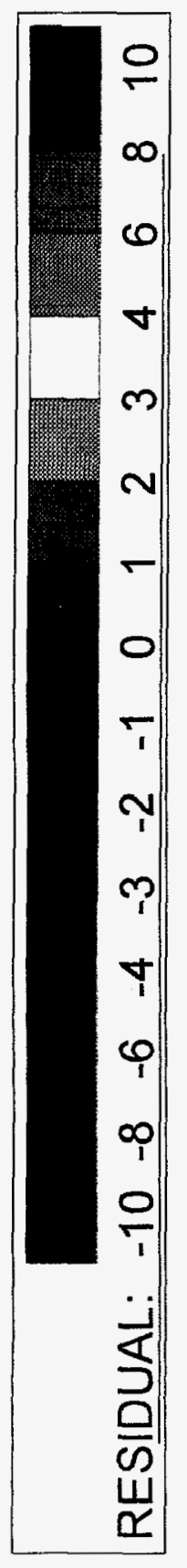

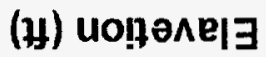

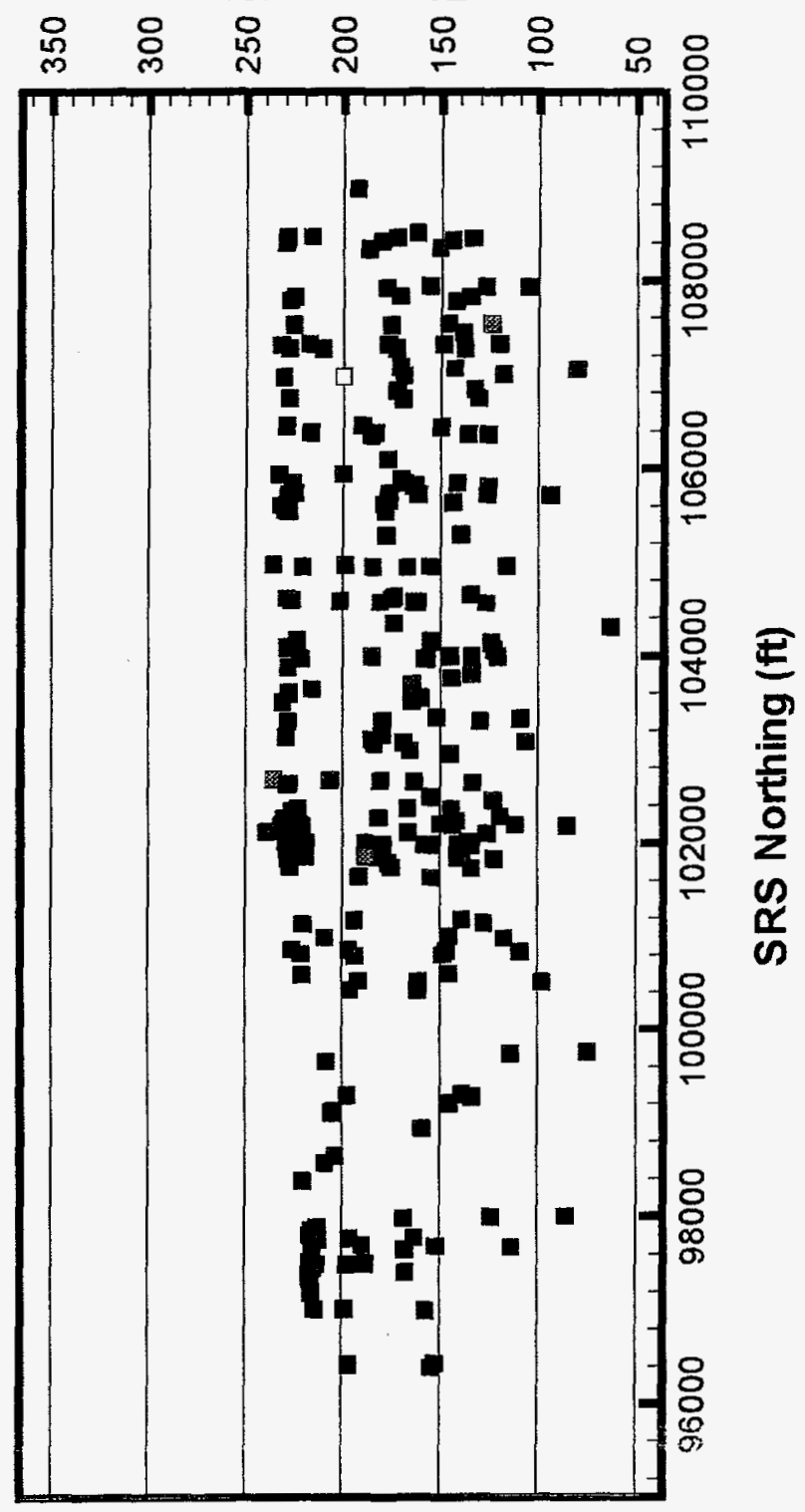

Figure 6.20 - Head Residuals - Y-Z View 

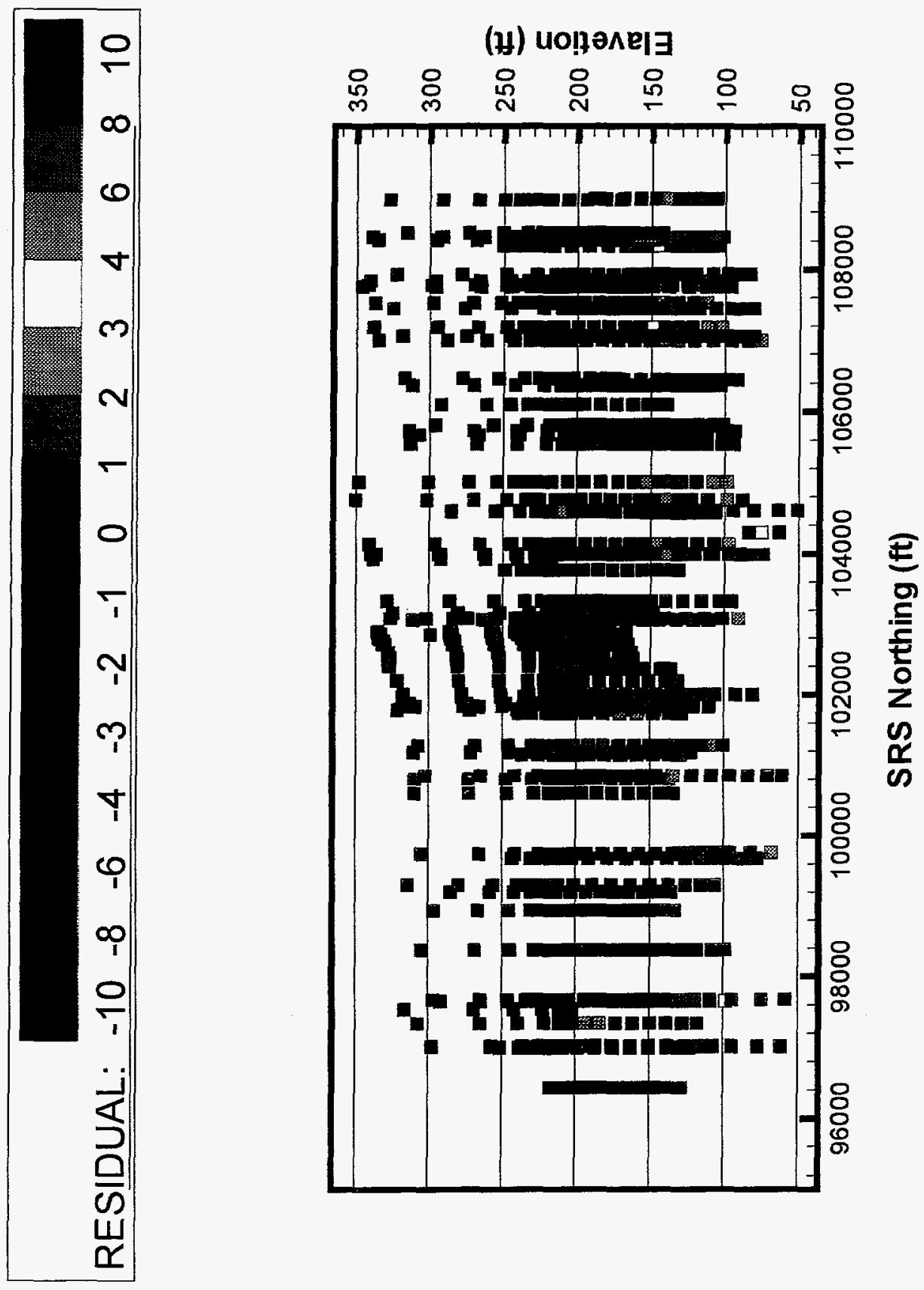

Figure 6.21 - $L n\left(K_{h}\right)$ Residuals - Y-Z View 


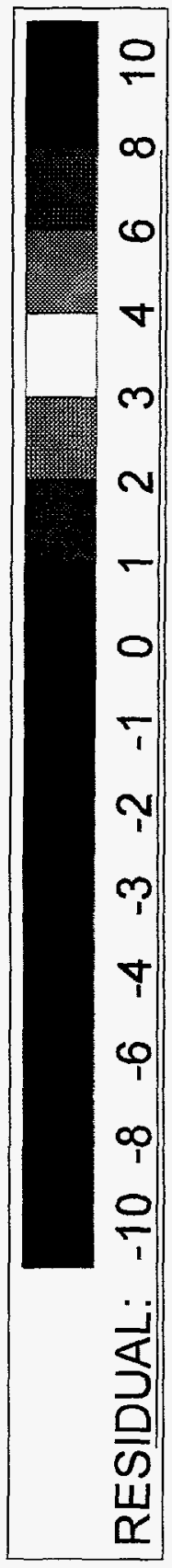

(4) บo!ฺә^еㅋ

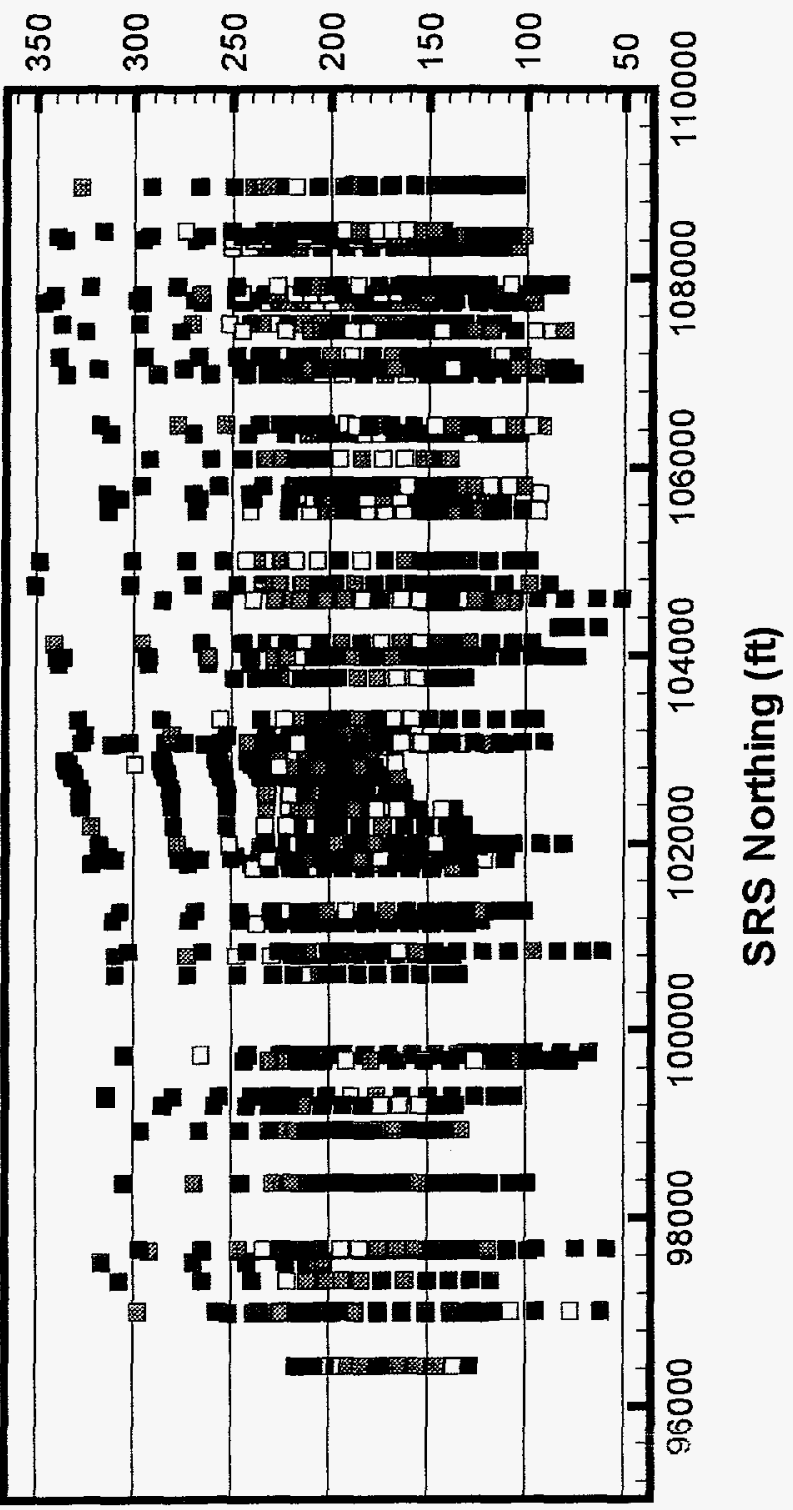

Figure 6.22 - $\operatorname{Ln}\left(\mathrm{K}_{\mathrm{v}}\right)$ Residuals - Y-Z View 
(y) 6unsez sys

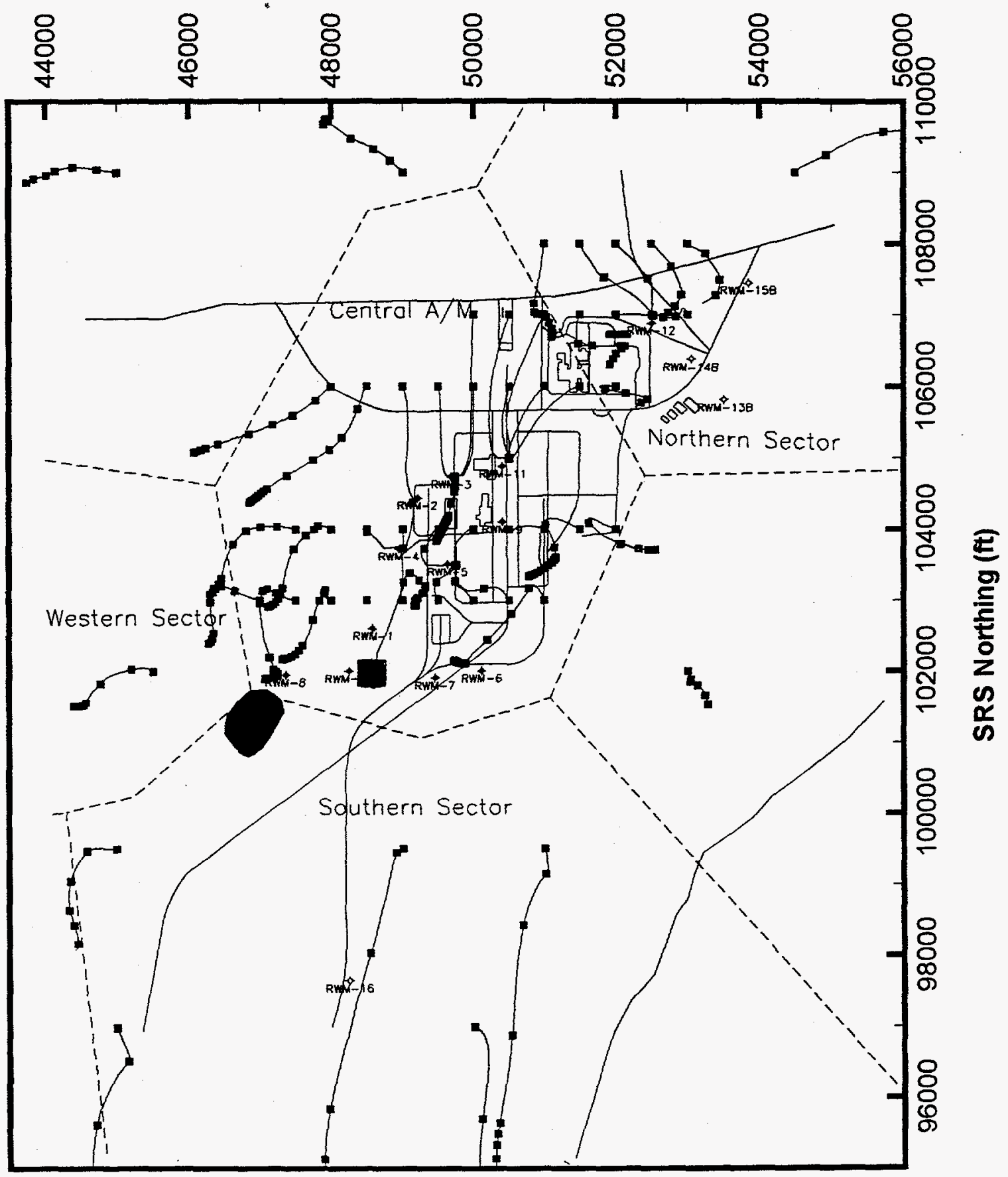

Figure 6.23 - 3D Pathlines for Standard DFM Plan View (markers at 10 year intervals) 


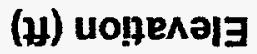

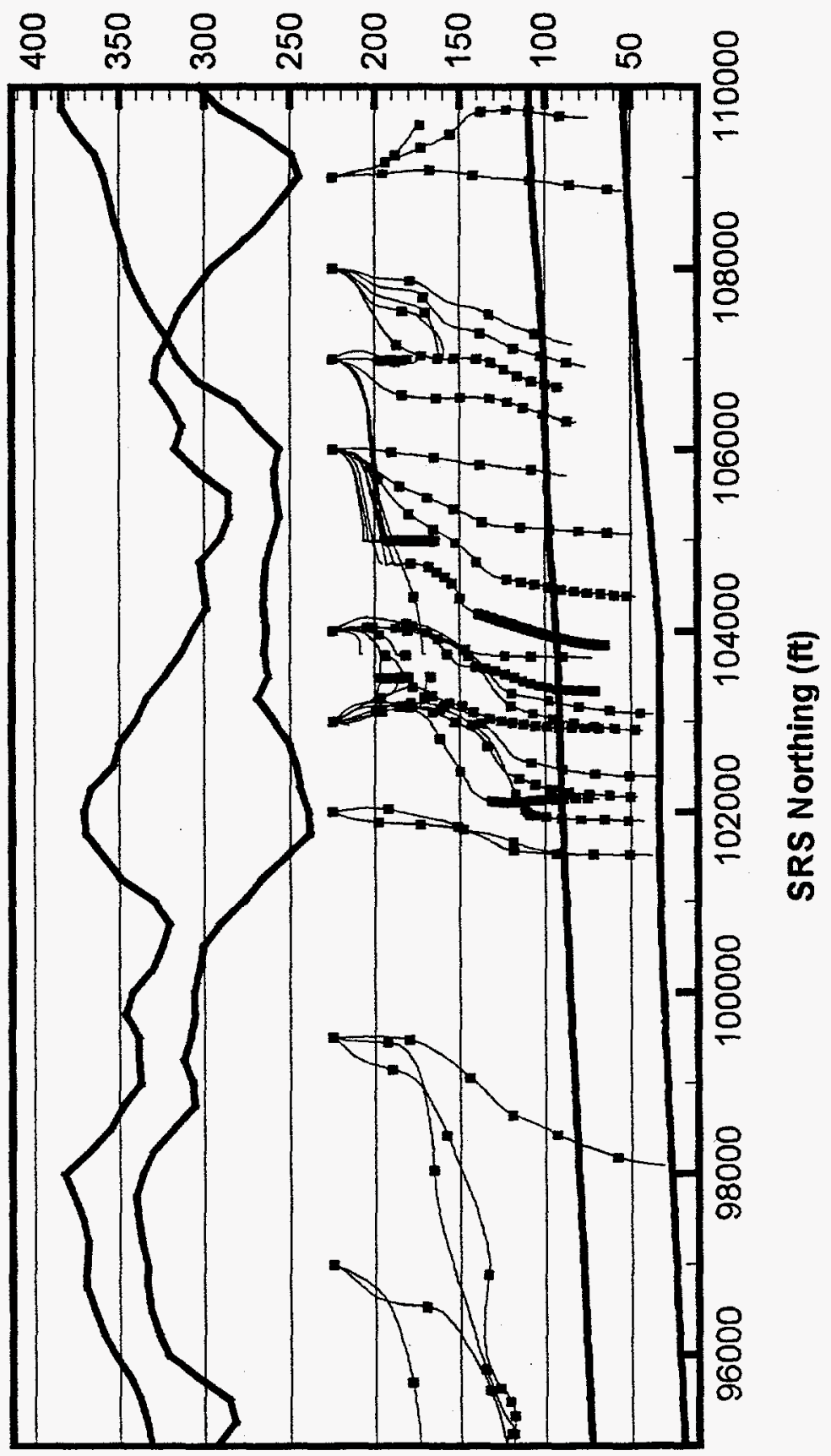

Figure 6.24 - 3D Pathlines for Standard DFM $Y-Z$ View (markers at 10 year intervals) 


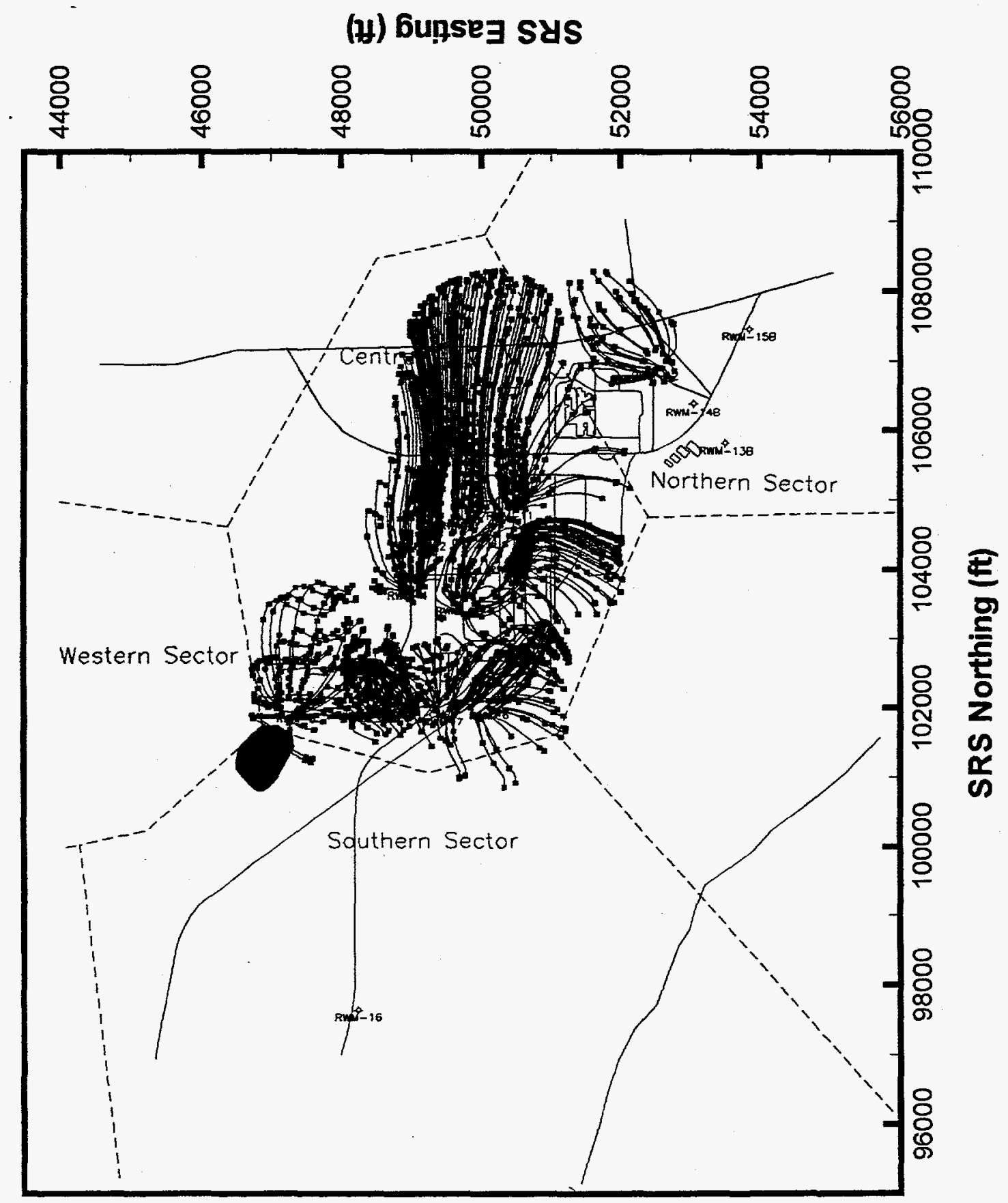

Figure 6.25

Capture Zones for Recovery Wells RWM-1 through RWM-12 


\subsection{SPATIAL VARIABILITY IN FLOW PATTERNS}

The flow pathlines shown in Section 6.0 were generated using the nominal flow model as computed by standard DFM. Since the parameters used for this flow model are estimates based on imperfect measurements, the parameters cannot be known with complete certainty. The covariance matrix computed in least squares estimation will provide an assessment of the parameter uncertainty if the model structure is correct. Unfortunately, it is not computationally practical to compute this covariance in standard DFM for models with more than about 20000 nodes. However, this can be computed in multi-grid DFM.

Figures 7.1 to 7.3 show the estimated head contours, velocity vectors and estimated log conductivity contours using multi-grid DFM. Figures 7.4 and 7.5 show the flow pathlines for the same starting locations used previously. Although there are some differences, these plot compare favorably with those in section 6 for standard DFM. Thus the variability in flow pathlines computed for multi-grid DFM should be representative of that for standard DFM.

The state error covariance matrix computed in multi-grid DFM can be used to compute the variation in pathlines which will result from errors in the measurements and prior information. The method used to compute the variation in the flow velocities from the state covariance matrix was documented in Gibbs and Jones (1995). Basically the square root of the information matrix is used with Gaussian random vectors to generate Monte Carlo samples of the states. Those samples will have a mean equal to the state estimate and covariance equal to the DFM computed covariance matrix. Those states (representing the flow model parameters) are used to compute steady-state flow solutions, from which the flow velocities are computed. Those velocities are then integrated to generate sample pathlines.

Figures 7.6 shows the plan view of 40 Monte Carlo pathline samples. Note that in the central region the distribution of pathlines is relatively tight, indicating that the flow model is known accurately. However, near the east and west boundaries and in the north east, the pathline distribution is much greater, indicating that flow model in subject to uncertainty. This is not surprising since the lack of data near the boundaries and unknown boundary condititons will result in uncertainty in the flow velocity.

A wide spread in pathlines near potential contaminant sources is an indication that more monitoring wells may be needed to properly determine flow directions. Design of successful remediation depends upon knowledge of the flow and the possible variation in that flow. Thus the flow pattern and variability may be used to analyze remediation alternatives. 


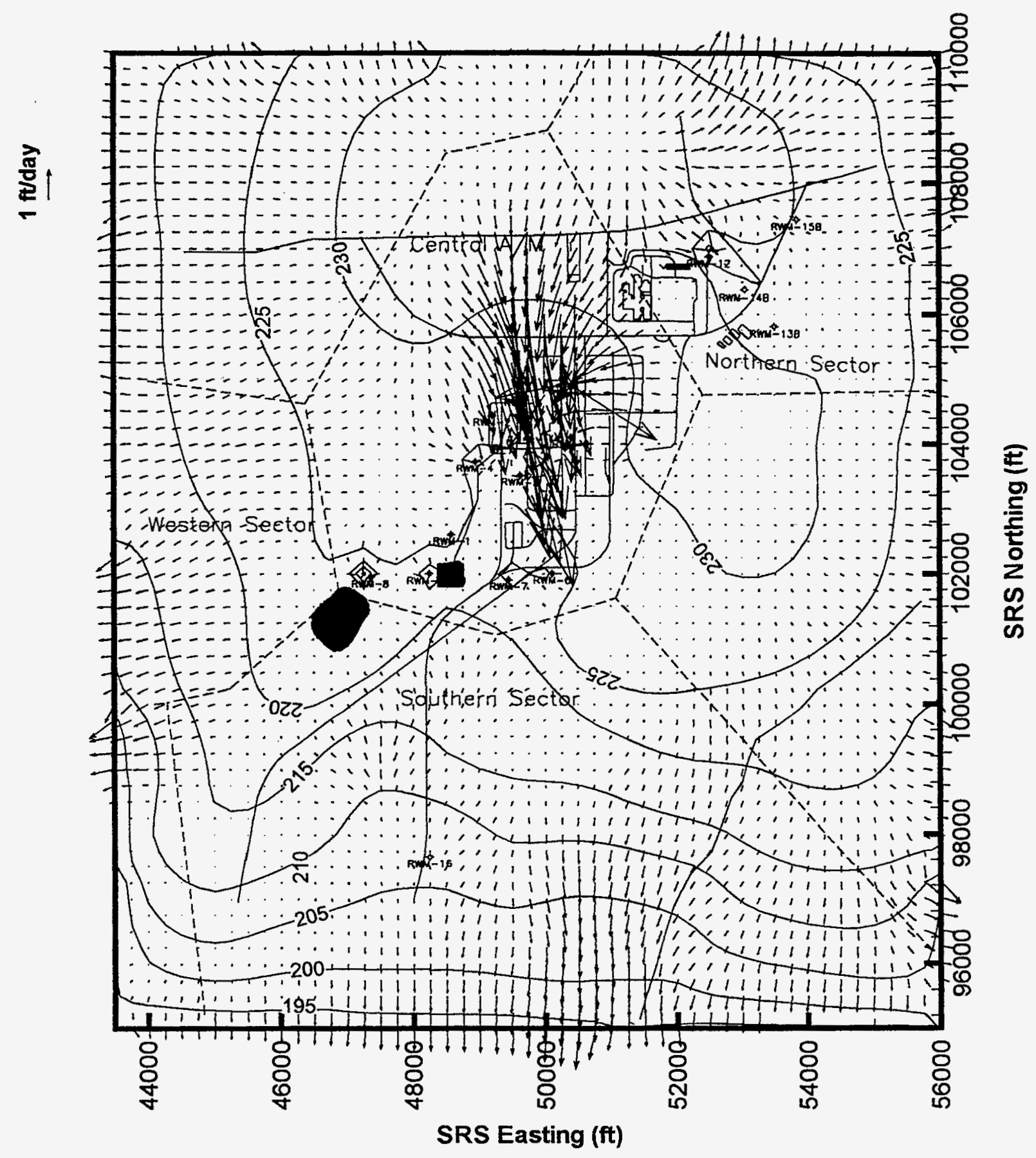

Figure 7.1 - Estimated Head and Pore Velocity for the "Upper Lost Lake" Aquifer (Multi-Grid DFM) 


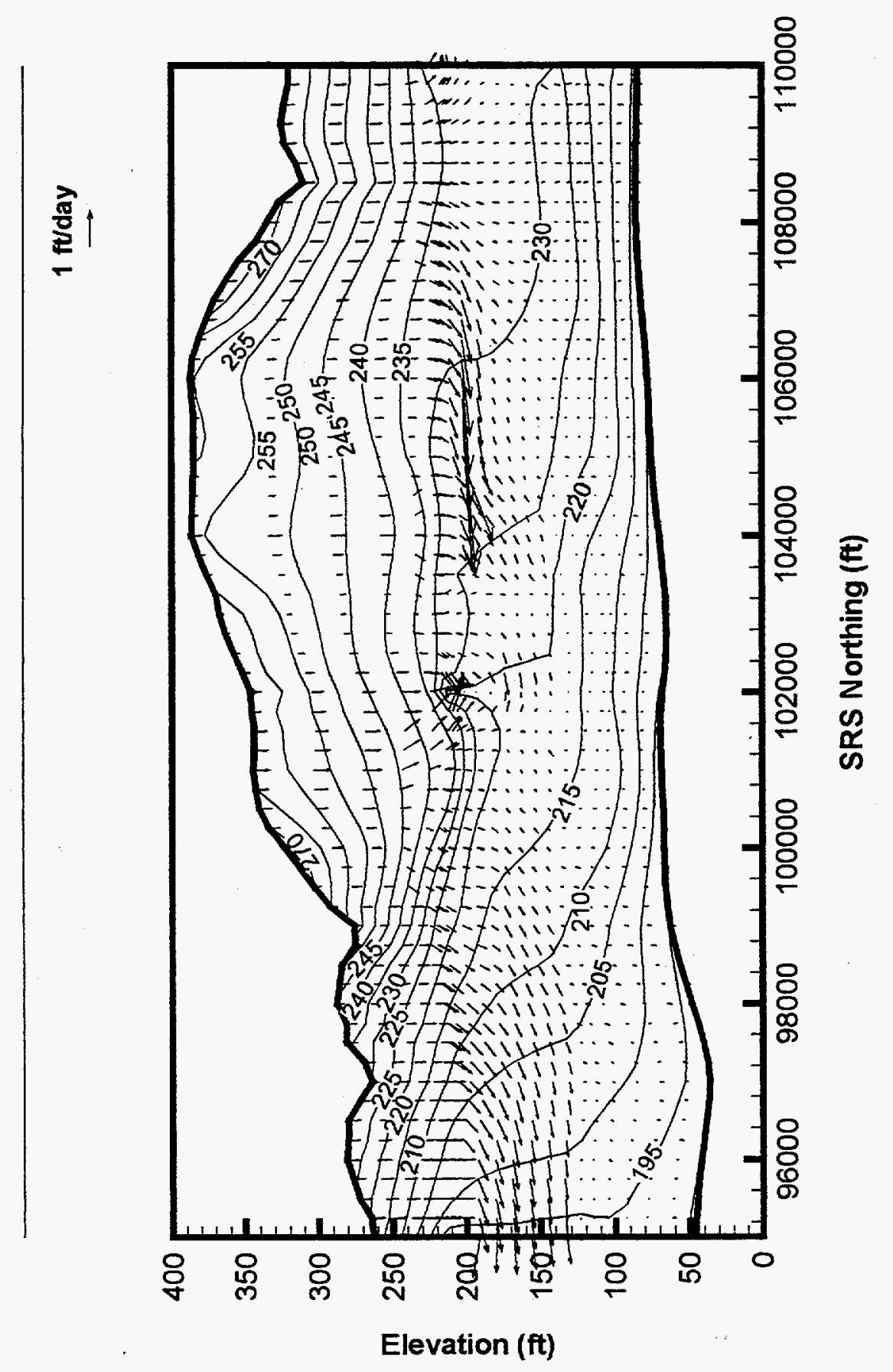

Figure 7.2 - Estimated Head and Pore Velocity at $x=50,000 \mathrm{ft}$ (Multi-Grid DFM) 


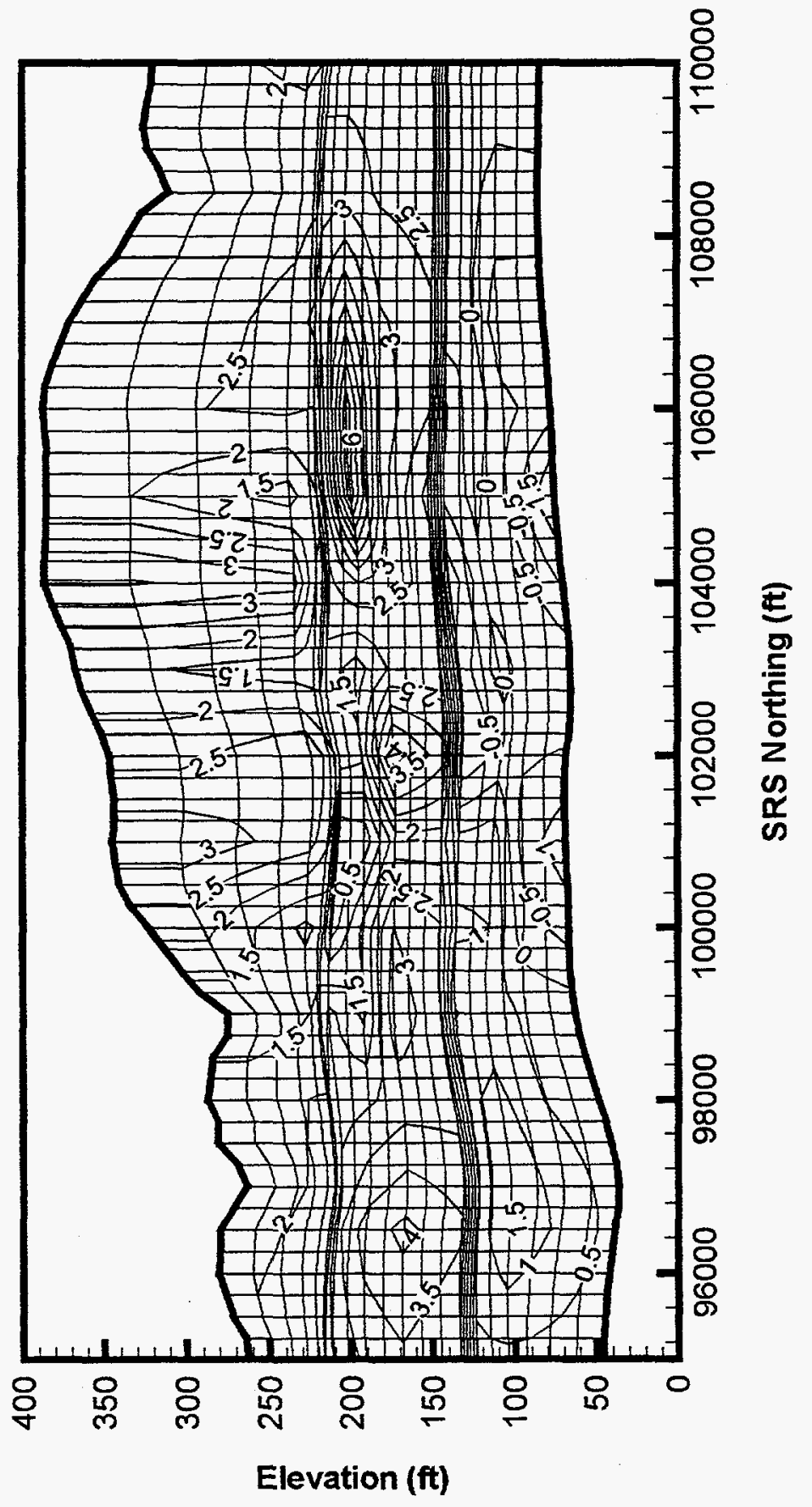

Figure 7.3 - Estimated $\operatorname{Ln}\left(K_{h}\right)$ at $x=50,000 \mathrm{ft}$ (Multi-Grid DFM) 
(y) 6unsez sys

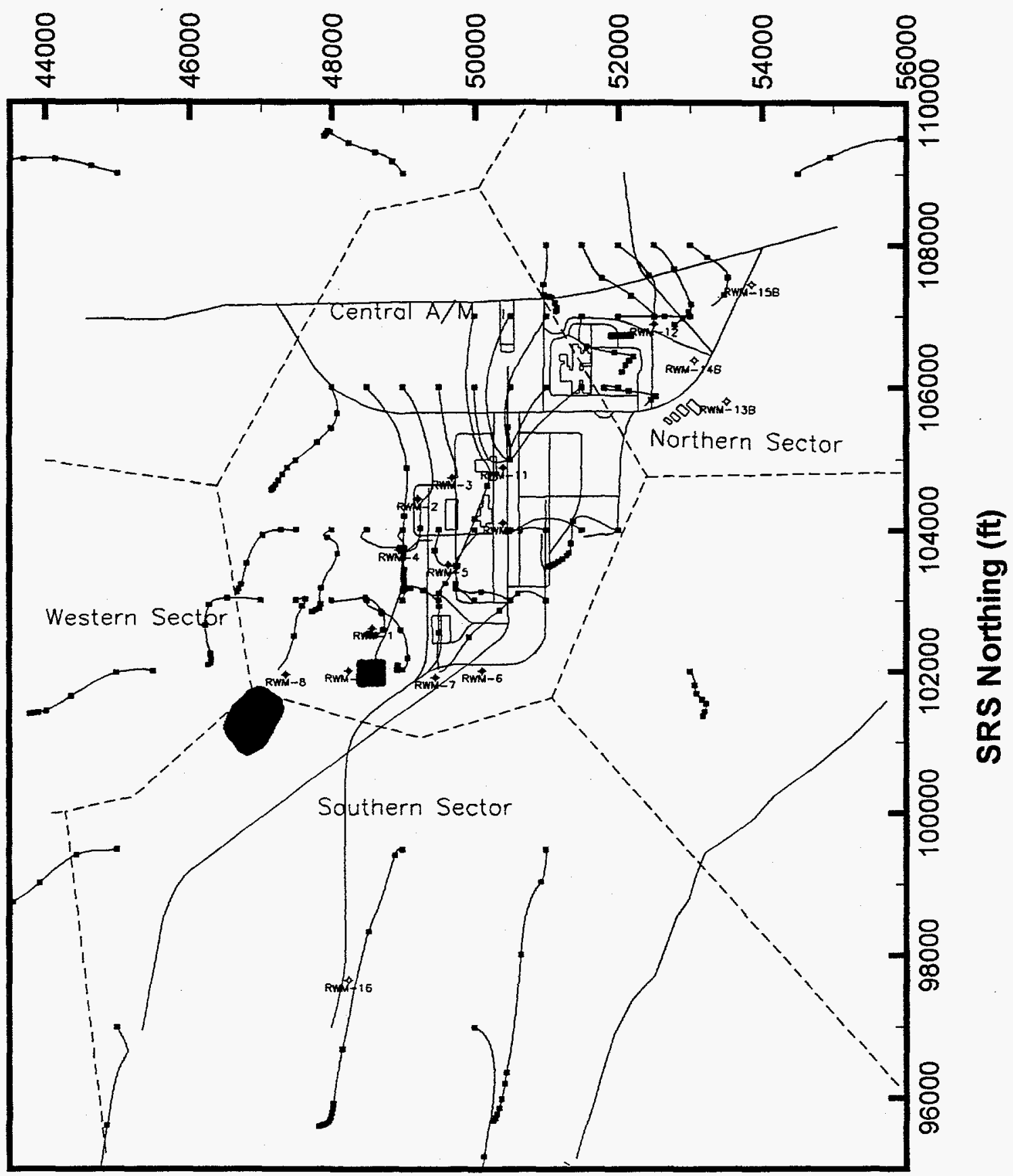

Figure 7.4 - 3D Pathlines for Multi-grid DFM Plan View, markers at 10 year intervals 


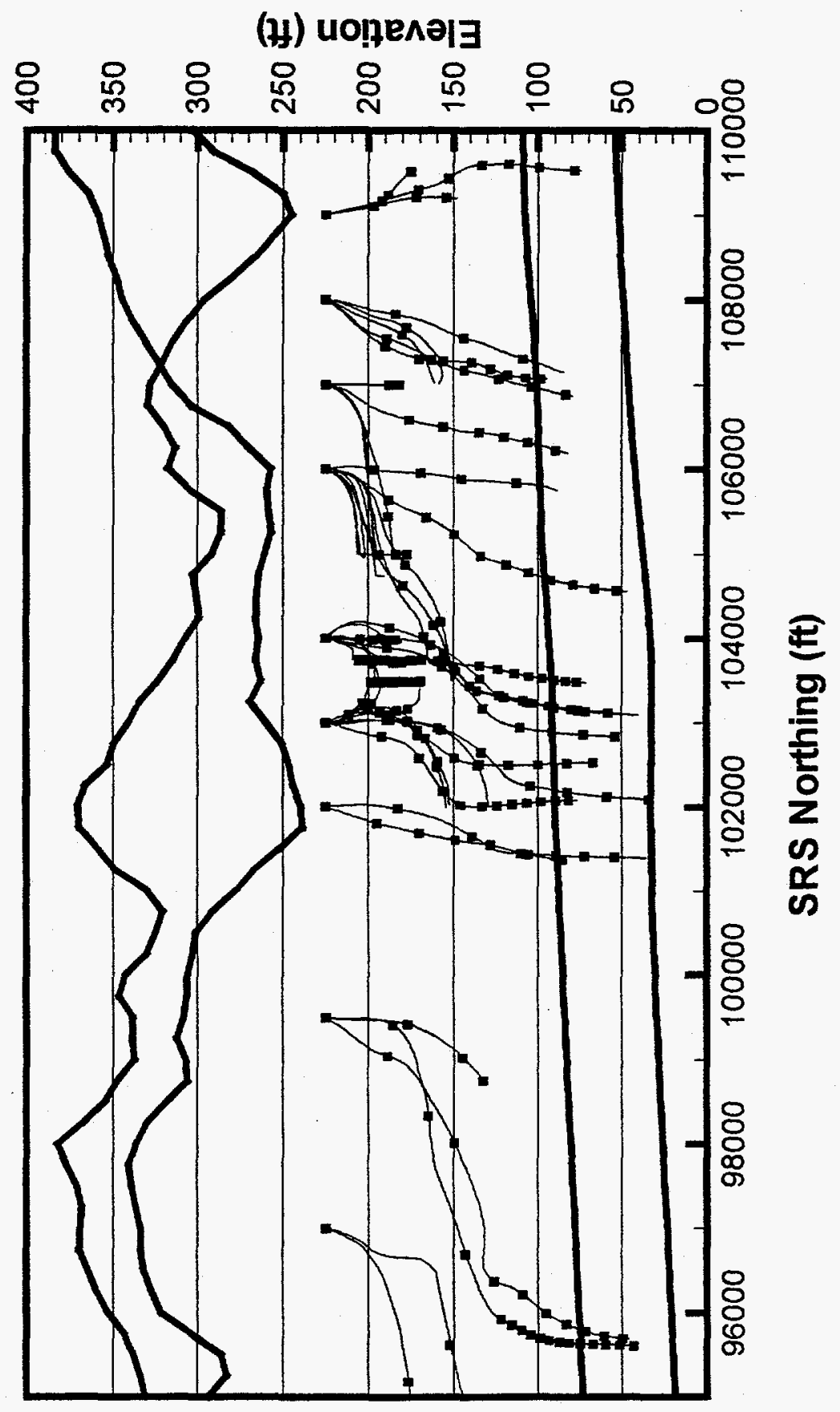

Figure 7.5 - 3D Pathlines for Multi-grid DFM Y-Z View, markers at 10 year intervals 


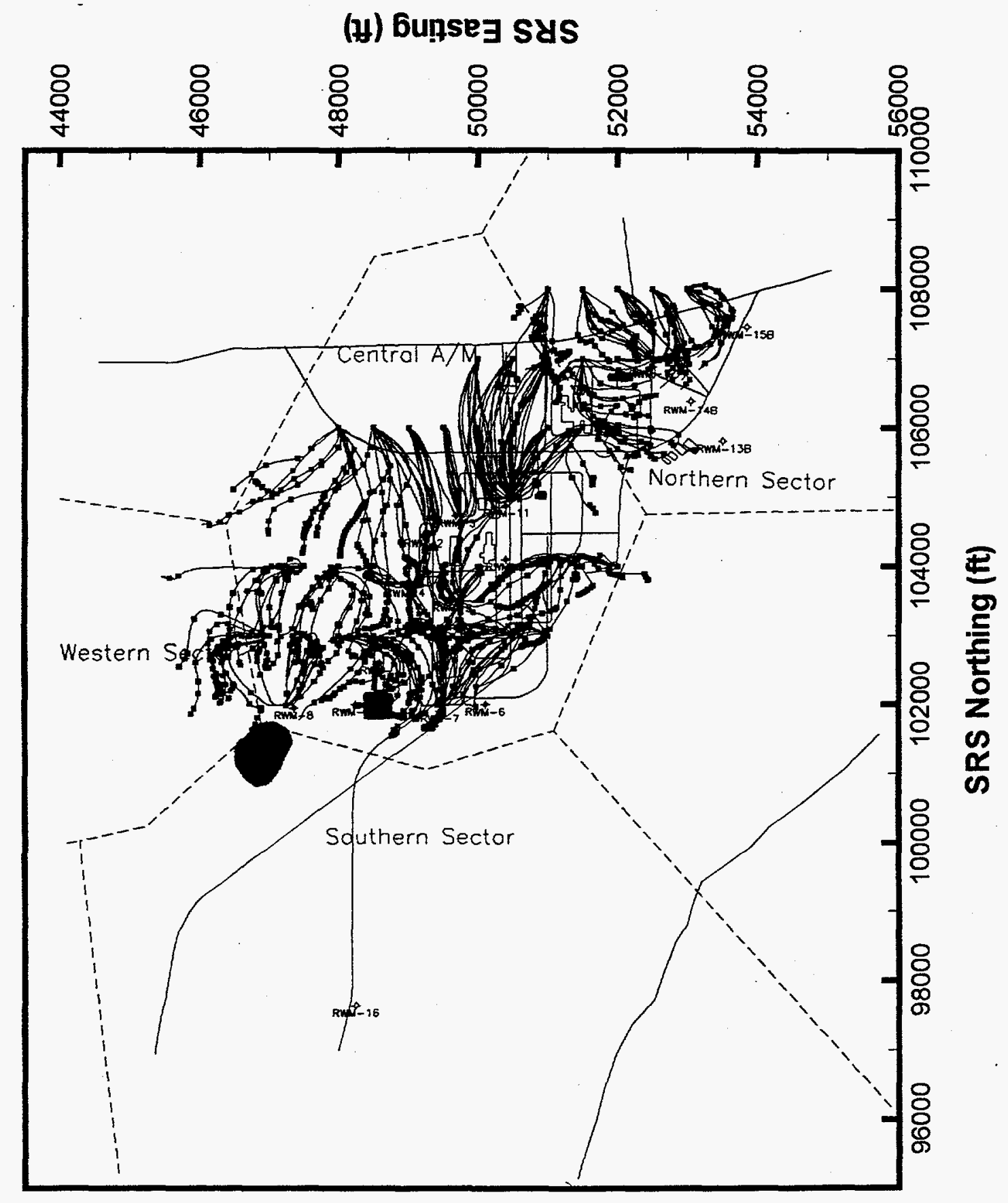

Figure 7.6 - Variation in 3D Pathlines 10 Monte Carlo Samples, Plan View 


\subsection{CONCLUSIONS}

In the $\mathrm{A} / \mathrm{M}^{-}$Area, well and cone penetrometer picks were used by DFM to delineate the hydrostratigraphy and define the hydrostratigraphic units in a grid for numerical modeling. Local hydraulic conductivity data were combined with hydraulic head data and prior information on recharge/boundary conditions by DFM. Model calibration provided estimates for hydraulic conductivity fields in each hydrostratigraphic layer, different recharge values in the general area and the paved areas, and source heads in the head-dependent flux boundary conditions. DFM also quantified parameter uncertainties and determined the impact of the uncertainties on the model prediction of flow patterns. The following are specific conclusions:

- The "Lost Lake" Aquifer Zone displays the greatest heterogeneity in hydraulic conductivity.

- Flow pattern estimates were driven by pumping in the Central A/M Area. The model can be used to determine flow patterns for future pumping alternatives.

- The model matches the available data very well. In particular, the RMS residual difference between head data and model predicted head was $1.0 \mathrm{ft}$.

- Recharge estimates were slightly higher than the prior estimates but not unreasonable.

- The model has significant flow through the "Green Clay" Confining Zone north of the MArea which continues south through the "Lost Lake" Aquifer Zone towards the recovery wells.

- The computed capture zones for the recovery wells are consistent with Jackson and Aleman (1995), except in the western portion of the central sector.

- Different combinations of parameters such as conductivity, recharge, and source heads sometimes produce nearly the same data fit, producing parameter uncertainties. Parameter uncertainties were computed, and the impact of parameter uncertainties on flow patterns was determined. The flow uncertainty is greatest in the eastern part of the northern sector and along the western boundary of the central sector.

- Acquisition of monitoring well and borehole data from the General Administration Area (703-A) will improve the A/M Area flow model.

Areas of potential future work suggested by the current effort are the following:

Customize DFM software to constrain the estimation of clay surfaces by the known distribution of DNAPL's to provide a tool to determine target zones for DNAPL remediation.

- Customize DFM software to incorporate contaminant data for flow and transport model calibration to support corrective action programs for the dissolved plume.

- Customize DFM software to calibrate multi-phase models for vadose zone remediation. 


\subsection{REFERENCES}

Aadland, R.K., and Bledsoe, H.W., 1990. Classification of Hydrostratigraphic Units at the Savannah River Site, South Carolina (U). Westinghouse Savannah River Company, Savannah River Site, Aiken, SC, WSRC-RP-90-987 (Distribution Category: UC).

Aadland, R.K., Gellici, J.A., and Thayer, P.A., 1995. Hydrogeologic framework of west-central South Carolina, South Carolina Department of Natural Resources, Water Resources Division Report 5, 200 p. +47 plates.

Beaudoin, C.M., Schreuder, P.J., and Haselow, J.S. (1991). “A Groundwater Flow Model for the A/M Area of the SRS (U)," WSRC-RP-91-0585, Westinghouse Savannah River Company, Aiken, South Carolina 29808.

Bierman, G.J., 1977. Factorization Methods for Discrete Sequential Estimation, Academic Press.

Chin, M.M., Goad, C.C., and Martin T.V., 1972. "Geodyn Systems Description - Volume 1", Wolf Research and Development Corporation, September.

Geraghty \& Miller, Inc., 1987. Evaluation of Recovery-well Efficiency, Specific Capacity and Transmissivity Estimation. Prepared for E.I. DuPont deMours \& Company, Savannah River Plant, 33 p.

Gibbs, B.P. and Hughes, L., 1995. Data Fusion Modeling: Theory and Practice, CMR/95-313, Coleman Research Corporation, Columbia, Maryland 21046.

Gibbs, B.P. and Jones, W.F., 1995. Flow and Transport Model of the SRS Old Burial Grounds Using Data Fusion Modeling, Coleman Research Corporation, CMB/95-328, November.

Gibbs, B.P., 1995. "Application of nonlinear Model-Based Predictive Control to Fossil Power Plants", Coleman Research Corporation, CMB/95-326, March.

Hamming, R.W. "Numerical Methods for Scientists and Engineers," Dover Publications, Inc., New York, 1973.

Hiergesell, R.A. (1993). "Hydrologic Analysis of Data from the Lower Lost Lake Aquifer Zone of the Steed Pond Aquifer at Recovery Well RWM-16(U)," WSRC-TR-92-529, Westinghouse Savannah River Company, Aiken, South Carolina 29808.

Hiergesell, R.A., (1994). "Hydrologic Analysis of Data from the Lower Lost Lake Aquifer at Recovery Well RWM-12(U)," WSRC-TR-93-666, Westinghouse Savannah River Company, Aiken, South Carolina 29808.

Hiergesell, R.A., Nichols, R.L., Ridgeway, R., May, C.P., Pemberton, B. (1994). "Report of Recovery Well Testing in the Northern A/M Area (U)," WSRC-94-0425, Westinghouse Savannah River Company, Aiken, South Carolina 29808.

Hiergesell, R.A., Pemberton, B.E. (1995). "Final Report: Aquifer Testing with 2-Inch Diameter Wells in the A/M Area Southern Sector: WSRC-TR-95-0365, Westinghouse Savannah River Company, Aiken, South Carolina 29808. 
Huyakorn, P.S., Thomas, S.D., and Thompson, B.M., 1984. "Techniques for Making Finiteelements Competitive in Modeling Flow in Variably Saturated Porous Media," Water Resources Research, 20(8):1099-1115.

Huyakorn, P.S., Springer, E.P., Guvanasen, V., and Wadsworth, T.D., 1986. "A ThreeDimensional Finite Element Model for Simulating Water Flow in Variably Saturated Porous Media,"Water Resources Research, 22(12):1790-1808.

Huyakorn, P.S. and Panday, S., 1992. VAM3DCG: Variably Saturated Analysis Model in Three-dimensions with Preconditioned Conjugate Gradient Matrix Solvers: Documentation and User's Guide, version 2.4, HydroGeoLogic, Inc., Virginia, 274 p.

Huyakorn, P.S. and Panday, S., 1995. VAM3DF: Variably Saturated Analysis Model in ThreeDimensions for the Data Fusion System: Documentation and User's Guide, version 1.0, HydroGeoLogic, Inc., Virginia, $278 \mathrm{p}$.

Intera, 1993. "A Transiently Calibrated Regional Groundwater Flow Model for the A/M Area" Report dated September 30, 1993 by Intera Inc., 6850 Austin Center Blvd., Suite 300, Austin, Texas 78759.

Jackson, D.G and Aleman, S.E., 1995. "Three Dimensional Zone of Capture Analysis for the A/M Area (U)." WSRC-RP-95-0843, Westinghouse Savannah River Company, Aiken, South Carolina 29808.

Jackson, D.G., to Ehrke, L., (October 7, 1994). "Three-Dimensional Groundwater Flow Model for A/M Area," Inter-Office-Memorandum SRTC-ESS-94-774.

Looney, B.B., M.W. Grant and C.M. King, 1987, Estimation of geochemical parameters for assessing subsurface transport at the Savannah River Plant, DPST-85-904.

McDonald, M.G., and Harbaugh, A.W., 1983. A Modular Three-Dimensional Finite-Difference Ground-Water Flow Model. U.S. Geological Survey Open-File Report 83-875.

Papadopulos \& Associates, S.S., 1987. "Evaluation of the Effectiveness of the M-Area Extraction System September 1985 to October 1986," "Prepared for E.I. DuPont deNemours \& Company by S.S. Papadopulos \& Associates, Rockville, Maryland 20852.

Press, W.H., Teukolsky, S.A., Vetterling, W.I., and Flannery, B.P., 1992. Numerical Recipes in FORTRAN, 2nd Edition, Cambride University Press, New York, NY.

Riha, B.D., 1993. Predicting saturated hydraulic conductivity for unconsolidated soils from commonly measured textural properties, M.S. Thesis, Clemson University, Clemson, SC, $87 \mathrm{p}$.

Shinn, J.D., Bratton, W.L. (1992). "Piezo-Resistivity Electric Cone Penetration Technology Investigation of the M-BASIN at the Savannah River Site, Aiken, South Carolina," Applied Research Associates, Inc., South Royalton, Vermont 05068.

Vandergraft, J., 1987. Efficient Optimization Methods for Maximum Likelihood Parameter Estimation, 24th IEEE Conference on Decision and Contol, Ft. Lauderdale, FL, December. 
WSRC, 1995a. "FACT: Subsurface Flow and Contaminant Transport Documentation and User's Guide (U)." WSRC-TR-95-0223, Westinghouse Savannah River Company, Aiken, South Carolina 29808.

WSRC, 1995b. "The Savannah River Site's Groundwater Monitoring Program, First Quarter 1995 (U)." ESH-EMS-950393, Westinghouse Savannah River Company, Aiken, South Carolina 29808.

WSRC, 1995c. "The Savannah River Site's Groundwater Monitoring Program, Second Quarter 1995 (U)." ESH-EMS-950394, Westinghouse Savannah River Company, Aiken, South Carolina 29808.

WSRC, 1995d. “The Savannah River Site's Groundwater Monitoring Program, Third Quarter 1995 (U)." ESH-EMS-950395, Westinghouse Savannah River Company, Aiken, South Carolina 29808.

WSRC, 1995e. "The Savannah River Site's Groundwater Monitoring Program, Fourth Quarter 1995 (U).” ESH-EMS-950396, Westinghouse Savannah River Company, Aiken, South Carolina 29808.

Yancy, W.E., 1994. A Random Field Model for Spatial Continuity: Development Notes, Coleman Research Corporation, Columbia, Maryland 21046. 
WeII ID

\begin{tabular}{|c|c|}
\hline$A B P$ & $1 \overline{\mathrm{A}}$ \\
\hline $\mathrm{ABP}$ & $1 \mathrm{DD}$ \\
\hline $\mathrm{ABP}$ & $2 \mathrm{~A}$ \\
\hline $\mathrm{ABP}$ & $2 D D$ \\
\hline $\mathrm{ABP}$ & 3 \\
\hline$A B P$ & $3 C$ \\
\hline$A B P$ & 4 \\
\hline$A B P$ & $4 D D$ \\
\hline$A B P$ & $6 D$ \\
\hline $\mathrm{ABP}$ & $7 D$ \\
\hline$A B P$ & $8 D$ \\
\hline ABW & 1 \\
\hline$A C$ & $1 \mathrm{~A}$ \\
\hline$A C$ & IB \\
\hline$A C$ & $2 \mathrm{~A}$ \\
\hline$A C$ & $3 A$ \\
\hline$A C$ & $3 B$ \\
\hline$A C B$ & IA \\
\hline $\mathrm{ACB}$ & $2 \mathrm{~A}$ \\
\hline $\mathrm{ACB}$ & $3 A$ \\
\hline $\mathrm{ACB}$ & $4 A$ \\
\hline AMB & $4 A$ \\
\hline $\mathrm{AMB}$ & $4 B$ \\
\hline AMB & $4 D$ \\
\hline AMB & 5 \\
\hline AMB & $7 \mathrm{~A}$ \\
\hline AMB & $7 B$ \\
\hline$A M B$ & $8 D$ \\
\hline AMB & $9 D$ \\
\hline$A M B$ & $10 \mathrm{~A}$ \\
\hline AMB & $10 \mathrm{~B}$ \\
\hline AMB & $10 D$ \\
\hline AMB & $11 B$ \\
\hline AMB & $11 D$ \\
\hline AMB & $12 \mathrm{D}$ \\
\hline AMB & $13 \AA R$ \\
\hline AMB & $17 \mathrm{~A}$ \\
\hline AMB & $18 \mathrm{~A}$ \\
\hline$A O B$ & 1 \\
\hline$A O B$ & 2 \\
\hline ARP & $1 \mathrm{~A}$ \\
\hline ARP & 2 \\
\hline ARP & 3 \\
\hline ARP & 4 \\
\hline ASB & $1 \mathrm{~A}$ \\
\hline ASB & $2 \mathrm{AR}$ \\
\hline ASB & $2 \mathrm{CR}$ \\
\hline ASB & $3 A R$ \\
\hline ASB & $3 \mathrm{CR}$ \\
\hline ASB & 4 \\
\hline ASB & $5 C$ \\
\hline ASB & $6 \mathrm{~A}$ \\
\hline ASB & 67 \\
\hline
\end{tabular}

SRS East (ft)

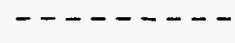

44425.60 44433.60 44118.80 44126.70 44509.30 44506.30 44096.00 44101.30 44101.40 43930.10 43984.10 55016.40 42238.80 42250.50 46428.60 42119.80 42113.60 51369.90 51561.30 51313.30 51116.20 51469.80 51482.70 51489.00 51467.20 51591.00 51590.30 51400.50 51263.00 51410.00 51418.30 51456.00 51919.50 51932.60 51901.60 51396.00 51465.40 51418.80 50485.90 50724.70 44317.40 44876.10 44903.70 44374.80 52614.00 52881.70 52862.70 53115.00 53130.40 53177.20 52837.80 52675.90 52643.90
SRS North (ft)

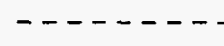

97501.60

97511.40

97764.30

97753.70

97794.10

97778.20

97489.70

97495.50

97889.70

97449.70

97854.90

105939.90

105865.00

105862.80

105636.40

100989.10

100996.50

102622.90

102367.40

102154.30

102343.90

104131.60

104145.60

104154.70

104083.40

103987.10

103972.00

103874.70

103585.20

103326.40

103337.30

103293.40

103154.20

103132.30

103602.40

103082.00

104056.70

103988.80

101910.70

102009.80

99102.90

99119.80

98638.20

98567.70

105535.00

105550.50

105540.20

105605.10

105614.50

105935.70

105884.80

105716.00

105727.00
Middle of Screen Head (ft) (ft)

187.90

225.07

226.44

226.44

196.10

223.74

212.25

223.34

225.25

162.80

198.23

197.50

223.55

213.20

230.83

223.32

224.41

223.02

226.92

214.20

214.27

221.99

211.04

212.95

238.68

239.78

239.57

239.74

220.56

226.09

234.52

234.93

220.55

226.57

234.96

235.40

219.66

225.08

236.64

224.72

237.31

235.98

220.56

220.53

220.43

237.73

237.63

217.09

219.77

222.42

219.57

238.35

239.65

224.66

240.25

224.25

239.47

224.37

237.87

219.84 


\begin{tabular}{|c|c|}
\hline ASB & $6 \dot{C}$ \\
\hline ASB & $6 \mathrm{TA}$ \\
\hline ASB & 8 \\
\hline $\mathrm{ASB}$ & $8 \mathrm{~A}$ \\
\hline$A S B$ & $8 B$ \\
\hline ASB & $8 \mathrm{C}$ \\
\hline$A S B$ & $8 \mathrm{TA}$ \\
\hline ASB & 9 \\
\hline $\mathrm{ASB}$ & $9 B$ \\
\hline$A S B$ & $9 \mathrm{C}$ \\
\hline ASB & $10 \mathrm{CR}$ \\
\hline IDP & $3 A$ \\
\hline IDP & $3 B$ \\
\hline LFW & 20 \\
\hline LFW & 26 \\
\hline LFW & 27 \\
\hline LFW & 29 \\
\hline LFW & 30 \\
\hline LEW & 31 \\
\hline LEW & 32 \\
\hline LFW & 33 \\
\hline LFW & 34 \\
\hline LFW & 35 \\
\hline LFW & $43 B$ \\
\hline LFW & $43 C$ \\
\hline LFW & $43 D$ \\
\hline LEW & $74 \mathrm{C}$ \\
\hline LFW & $74 D$ \\
\hline LFW & $75 \mathrm{C}$ \\
\hline LFW & $75 D$ \\
\hline $\mathrm{MCB}$ & 5 \\
\hline $\mathrm{MCB}$ & $6 \mathrm{C}$ \\
\hline $\mathrm{MCB}$ & $8 D$ \\
\hline $\mathrm{MCB}$ & $9 D$ \\
\hline MSB & $1 \mathrm{~A}$ \\
\hline MSB & IB \\
\hline MSB & $2 \mathrm{~A}$ \\
\hline MSB & $3 A$ \\
\hline MSB & $3 B$ \\
\hline MSB & $3 D$ \\
\hline MSB & $4 \mathrm{~A}$ \\
\hline MSB & $4 B$ \\
\hline MSB & $4 \mathrm{D}$ \\
\hline MSB & $5 A$ \\
\hline MSB & $5 B$ \\
\hline MSB & $6 \mathrm{~A}$ \\
\hline MSB & $6 \mathrm{~B}$ \\
\hline MSB & $7 \mathrm{~A}$ \\
\hline MSB & $7 B$ \\
\hline MSB & $8 A$ \\
\hline MSB & $8 B$ \\
\hline MSB & $8 C$ \\
\hline MSB & $9 A$ \\
\hline
\end{tabular}

SRS East (ft)

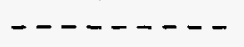

52655.90

52671.30

53136.60

53117.50

53109.60

53101.00

53124.70

54226.20

54215.30

54201.10

52969.70

37781.10

37785.30

45582.90

45633.80

45596.10

45503.30

45170.90

44869.00

44935.90

44973.00

45016.90

45378.80

45240.50

45234.90

45244.50

45097.80

45098.00

45357.00

45355.60

44863.90

45207.70

44769.90

44858.80

48467.30

48483.20

48746.40

48553.70

48568.00

48524.60

48313.00

48312.80

48311.70

46998.70

46983.60

46319.90

46321.60

46726.10

46718.10

47293.20

47281.90

47264.60

48242.50
SRS North (ft)

$-\cdot-\cdot--\cdot$

105736.80

105749.50

106381.60

106369.30

106362.30

106354.40

106375.80

104589.20

104564.70

104568.10

105655.40

85104.30

85119.50

85262.60

85654.60

85839.10

86372.70

86318.40

86262.20

85836.80

85633.80

85409.50

85237.40

86459.20

86480.60

86443.20

85813.80

85828.10

85856.80

85868.00

97335.60

97413.10

97180.60

97605.80

101833.70

101833.00

102028.30

102189.90

102191.70

102188.60

101933.40

101978.30

102007.50

101971.50

101971.10

101133.80

101148.50

100585.70

100597.60

100815.10

100805.80

100793.20

102236.70
Middle of Screen Head

(ft)

(ft) (ft)

176.15

225.53

37.25

215.54

216.60

80.70

235.32

220.59

221.89

224.97

217.07

242.98

221.75

222.13

224.63

167.43

158.65

159.67

162.31

162.99

165.92

165.81

167.95

163.29

162.14

160.87

159.88

165.91

166.20

166.53

163.29

163.46

163.19

163.72

225.95

196.63

226.42

224.01

232.35

213.01

233.82

232.71

216.32

231.08

231.61

213.53

230.85

228.60

211.29

227.96

208.50

228.79

208.90

230.18

211.00

221.51

213.26 


\begin{tabular}{|c|c|}
\hline MSB & $9 \bar{C}$ \\
\hline MSB & $10 \mathrm{~A}$ \\
\hline MSB & $10 \mathrm{~B}$ \\
\hline MSB & IIA \\
\hline MSB & $11 \mathrm{~B}$ \\
\hline MSB & $11 \mathrm{C}$ \\
\hline MSB & $I I D$ \\
\hline MSB & $11 \mathrm{E}$ \\
\hline MSB & $I I F$ \\
\hline MSB & $12 \mathrm{~A}$ \\
\hline MSB & $12 \mathrm{C}$ \\
\hline MSB & $12 \mathrm{D}$ \\
\hline MSB & $12 \mathrm{TA}$ \\
\hline MSB & $12 \mathrm{~TB}$ \\
\hline MSB & $13 \mathrm{~A}$ \\
\hline MSB & $13 \mathrm{~B}$ \\
\hline MSB & $13 \mathrm{C}$ \\
\hline MSB & $14 \mathrm{~A}$ \\
\hline MSB & $14 \mathrm{~B}$ \\
\hline MSB & $15 \mathrm{~A}$ \\
\hline MSB & $15 \mathrm{AA}$ \\
\hline MSB & $16 \mathrm{~A}$ \\
\hline MSB & $17 \mathrm{~A}$ \\
\hline MSB & $17 \mathrm{~B}$ \\
\hline MSB & $17 \mathrm{BB}$ \\
\hline MSB & $18 \mathrm{~A}$ \\
\hline MSB & $18 \mathrm{~B}$ \\
\hline MSB & $19 \mathrm{~A}$ \\
\hline MSB & $19 \mathrm{~B}$ \\
\hline MSB & $19 \mathrm{C}$ \\
\hline MSB & $20 \mathrm{~A}$ \\
\hline MSB & $21 A$ \\
\hline $\mathrm{MSB}$ & $21 B$ \\
\hline MSB & $21 \mathrm{C}$ \\
\hline MSB & $21 \mathrm{TA}$ \\
\hline MSB & 22 \\
\hline MSB & $23 B$ \\
\hline MSB & $23 \mathrm{TA}$ \\
\hline MSB & $24 \mathrm{~A}$ \\
\hline MSB & 25 \\
\hline MSB & $25 \mathrm{~A}$ \\
\hline MSB & 26 \\
\hline MSB & $26 \mathrm{~A}$ \\
\hline MSB & $26 B$ \\
\hline MSB & 27 \\
\hline MSB & $27 \mathrm{~A}$ \\
\hline MSB & $27 \mathrm{~B}$ \\
\hline MSB & $27 \mathrm{TA}$ \\
\hline MSB & 28 \\
\hline MSB & $28 \mathrm{~A}$ \\
\hline MSB & $29 A$ \\
\hline MSB & $29 B$ \\
\hline MSB & $29 \mathrm{C}$ \\
\hline
\end{tabular}

SRS East (ft)

- - - - - - - -

48273.00

47954.40

47943.10

48577.60

48578.50

48579.40

48579.70

48579.60

48577.00

47138.20

47138.40

47139.70

47127.30

47133.00

47525.40

47523.50

47521.90

48521.90

48519.10

48827.00

48818.50

48965.10

46245.70

46237.70

46220.80

46110.40

46115.70

50934.40

50934.80

50942.40

46060.50

47217.20

47271.80

47234.60

47218.20

48508.80

49286.40

49225.80

49845.30

49668.90

49657.90

48941.70

48440.70

48944.60

49487.70

49487.80

49486.40

49486.50

48517.30

48521.90

51236.40

51217.50

51206.60
SRS North (Et)

- - - - - - -

102245.60

102451.80

102488.20

102638.90

102648.90

102658.60

102669.50

102678.50

102629.30

102283.20

102274.40

102252.20

102266.70

102260.10

101725.70

101735.70

101745.70

101629.50

101639.00

102983.50

102953.20

103693.90

101976.60

101994.60

102009.50

100416.10

100424.10

100983.00

100999.30

100992.10

103545.10

103967.00

104000.10

103973.00

103980.90

102186.50

104336.60

104298.80

104625.30

103498.80

103504.80

104612.80

104602.30

104646.70

104972.80

104962.80

104940.30

104951.40

104941.80

104947.70

107326.80

107319.30

107315.00
Middle of Screen Head (ft)

(ft) (ft)

$\ldots \ldots$ (ft)

231.28

231.28

122.70

214.66

154.90

217.16

215.70

221.39

223.36

229.70

239.73

231.27

212.38

224.56

234.08

193.71

193.66

211.61

203.29

229.73

218.42

220.16

223.54

216.96

224.40

218.00

226.43

214.33

213.87

222.63

215.84

218.56

239.31

220.06

222.61

222.04

229.77

195.22

231.74

225.70

202.75

227.79

237.01

219.58

237.24

226.35

221.25

237.04

230.25

227.78

202.32

231.27

225.36

221.56

225.75

231.47 
WeII ID

\begin{tabular}{|c|c|}
\hline MSB & 290 \\
\hline MSB & 29DD \\
\hline MSB & $29 \mathrm{TA}$ \\
\hline MSB & $30 \mathrm{~A}$ \\
\hline MSB & $30 A A$ \\
\hline MSB & $30 B$ \\
\hline MSB & $30 \mathrm{C}$ \\
\hline MSB & $30 \mathrm{CC}$ \\
\hline MSB & $31 \mathrm{~A}$ \\
\hline MSB & $31 B$ \\
\hline MSB & $31 \mathrm{C}$ \\
\hline MSB & $31 \mathrm{CC}$ \\
\hline MSB & 32 \\
\hline MSB & $33 \mathrm{~A}$ \\
\hline MSB & $33 B$ \\
\hline MSB & $33 \mathrm{C}$ \\
\hline MSB & $33 \mathrm{TA}$ \\
\hline MSB & $34 \mathrm{~A}$ \\
\hline MSB & $34 \mathrm{~B}$ \\
\hline MSB & $34 \mathrm{TA}$ \\
\hline MSB & $34 \mathrm{~TB}$ \\
\hline MSB & $35 \mathrm{~A}$ \\
\hline MSB & $35 B$ \\
\hline MSB & $35 D$ \\
\hline MSB & $35 \mathrm{TA}$ \\
\hline MSB & $36 \mathrm{~A}$ \\
\hline MSB & $36 \mathrm{~B}$ \\
\hline MSB & $36 \mathrm{C}$ \\
\hline MSB & $36 \mathrm{TA}$ \\
\hline MSB & $37 \mathrm{~A}$ \\
\hline MSB & $37 \mathrm{~B}$ \\
\hline MSB & $37 \mathrm{C}$ \\
\hline MSB & $37 \mathrm{TA}$ \\
\hline MSB & $38 B$ \\
\hline MSB & $38 \mathrm{C}$ \\
\hline MSB & $38 \mathrm{TA}$ \\
\hline MSB & $39 A$ \\
\hline MSB & $39 B$ \\
\hline MSB & $39 C$ \\
\hline MSB & $39 D$ \\
\hline MSB & $39 \mathrm{TA}$ \\
\hline MSB & $40 \mathrm{~A}$ \\
\hline MSB & $40 B$ \\
\hline MSB & $40 \mathrm{C}$ \\
\hline MSB & $40 \mathrm{TA}$ \\
\hline MSB & $4 I A$ \\
\hline MSB & $4 I B$ \\
\hline MSB & $4 I C$ \\
\hline MSB & $41 \mathrm{TA}$ \\
\hline MSB & $42 \mathrm{~A}$ \\
\hline MSB & $42 B$ \\
\hline MSB & $42 \mathrm{C}$ \\
\hline MSB & $42 \mathrm{TA}$ \\
\hline
\end{tabular}

SRS East (ft)

51226.90

51191.30

51245.70

48004.10

47970.50

47981.80

48013.70

47993.30

50100.20

50078.70

50089.90

50067.90

52733.90

51738.00

51741.90

51746.70

51734.00

50534.90

50534.90

50536.60

50537.90

50945.20

50947.90

50949.70

50919.60

49514.90

49526.30

49537.20

49503.00

51439.80

51450.00

51439.80

51449.80

49746.10

49762.00

49810.40

48367.30

48376.90

48386.70

48396.00

48357.70

48279.40

48281.60

48283.50

48277.20

53424.10

53417.80

53410.60

53429.70

51582.30

51582.80

51582.80

51581.70
SRS North (ft)

$\ldots . . . .$.

107323.30

107311.40

107330.40

105727.40

105715.70

105719.90

105731.10

105724.20

101979.30

101981.30

101979.60

101983.10

99655.60

98006.70

97995.90

97984.80

98018.20

104954.90

104944.70

104905.80

104891.60

102098.00

102110.80

102122.40

102101.60

100511.30

100514.90

100518.30

100507.70

105295.00

105289.50

105283.20

105301.30

102360.80

102373.10

102434.90

100837.60

100844.60

100852.10

100858.70

100830.60

97672.80

97685.00

97697.80

97660.40

102184.40

102194.50

102203.90

102176.50

104557.90

104569.80

104581.90

104545.60
Middle of Screen Head

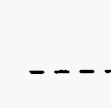

(ft)

(ft)

217.30

233.55

61.25

31.90

93.60

125.90

231.48

161.20

17.00

154.80

235.85

179.05

208.10

85.60

123.50

168.20

20.75

116.10

184.50

$-96.90$

70.80

126.00

166.50

243.48

35.55

97.70

160.90

191.40

50.90

70.90

139.50

178.00

32.45

144.15

166.65

29.35

108.90

146.80

196.80

232.95

47.05

113.40

151.90

189.60

26.35

85.10

111.40

149.70

24.05

126.60

163.50

201.50

43.15
233.26

233.55

213.52

199.56

224.98

226.59

231.48

226.67

199.13

220.51

235.85

220.65

224.47

205.34

208.20

211.15

195.69

220.51

228.60

202.57

203.88

218.11

221.97

243.48

201.94

211.79

216.06

216.16

195.53

208.58

220.91

229.64

208.38

218.61

221.98

199.23

211.05

213.76

217.15

232.95

193.63

203.65

205.47

205.34

190.29

218.32

218.57

219.03

207.45

220.89

227.54

231.90

206.93 


\begin{tabular}{|c|c|}
\hline MSB & $43 \bar{A}$ \\
\hline MSB & $43 B$ \\
\hline MSB & $43 D$ \\
\hline MSB & $43 D D$ \\
\hline MSB & $43 \mathrm{TA}$ \\
\hline MSB & $44 \mathrm{~A}$ \\
\hline MSB & $44 B$ \\
\hline MSB & $45 B$ \\
\hline MSB & $46 B$ \\
\hline MSB & $47 B$ \\
\hline MSB & $47 \mathrm{BB}$ \\
\hline MSB & $47 \mathrm{C}$ \\
\hline MSB & $47 D$ \\
\hline MSB & $47 \mathrm{TA}$ \\
\hline MSB & $48 \mathrm{~A}$ \\
\hline MSB & $48 B$ \\
\hline MSB & $48 \mathrm{C}$ \\
\hline MSB & $48 \mathrm{TA}$ \\
\hline MSB & $49 A$ \\
\hline MSB & $49 B$ \\
\hline MSB & $50 B$ \\
\hline MSB & $50 D$ \\
\hline MSB & $51 B$ \\
\hline MSB & $51 D D$ \\
\hline MSB & $52 B$ \\
\hline MSB & $53 B$ \\
\hline MSB & $53 \mathrm{C}$ \\
\hline MSB & $53 D$ \\
\hline MSB & $54 B$ \\
\hline MSB & $54 \mathrm{C}$ \\
\hline MSB & $54 D$ \\
\hline MSB & $54 \mathrm{TA}$ \\
\hline MSB & $55 B$ \\
\hline MSB & $55 \mathrm{C}$ \\
\hline MSB & $55 D$ \\
\hline MSB & $55 \mathrm{TA}$ \\
\hline MSB & $56 D$ \\
\hline MSB & $61 \mathrm{C}$ \\
\hline MSB & $62 B$ \\
\hline MSB & $62 \mathrm{C}$ \\
\hline MSB & $63 B$ \\
\hline MSB & $63 C$ \\
\hline MSB & $64 \mathrm{~B}$ \\
\hline MSB & $64 \mathrm{C}$ \\
\hline MSB & $64 \mathrm{D}$ \\
\hline MSB & $66 \mathrm{~B}$ \\
\hline MSB & $66 \mathrm{C}$ \\
\hline MSB & $66 D$ \\
\hline MSB & $66 \mathrm{TA}$ \\
\hline MSB & $67 \mathrm{~B}$ \\
\hline MSB & $67 \mathrm{C}$ \\
\hline MSB & $68 \mathrm{~B}$ \\
\hline MSB & $68 \mathrm{C}$ \\
\hline
\end{tabular}

SRS East (ft)

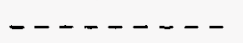

49293.70

49311.80

49322.00

49341.20

49281.80

51106.90

51096.40

50555.30

50557.50

52207.20

52234.40

52195.50

52184.00

52219.00

54099.80

54112.20

54077.00

54089.20

45864.60

45868.20

51053.50

51044.10

52818.00

52830.30

53418.40

54574.30

54540.50

54553.10

52970.50

52955.70

52984.50

52985.80

52006.20

52029.70

52032.50

52014.70

44207.90

55406.60

47906.80

47895.00

47861.00

47849.20

46579.70

46589.20

46598.50

51064.60

51053.50

51044.00

51096.70

51989.60

51988.60

52308.50

52304.90
SRS North (ft)

-.-.-.-.

107275.30

107274.60

107274.20

107273.00

107275.80

103296.50

103296.20

103987.90

103102.40

106978.50

106999.70

106969.20

106960.10

106987.70

107936.60

107945.00

107917.50

107925.80

99759.00

99737.80

96433.00

96416.70

96992.70

97006.20

103077.70

106443.60

106456.20

106448.20

108446.80

108447.40

108461.50

108446.30

108342.40

108324.60

108391.40

108322.80

108463.50

106091.10

101865.30

101857.20

101184.40

101174.60

101831.00

101842.90

101854.80

105842.00

105842.10

105841.80

105842.60

106842.00

106819.80

106744.90

106730.50
Middle of Screen Head

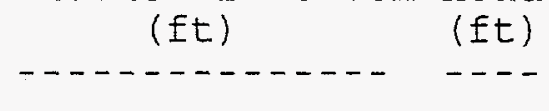

137.70

230.14

172.70

230.42

210.50

232.26

37.65

129.60

179.60

185.00

184.80

168.70

117.95

199.80

234.36

52.60

127.05

155.95

177.80

105.15

74.35

113.50

152.40

202.37

157.20

225.60

168.60

149.95

190.20

234.46

133.60

172.80

233.96

78.10

150.35

186.95

233.92

88.90

221.73

176.90

138.65

187.60

138.55

193.45

121.95

178.85

227.59

141.55

168.55

232.21

33.15

132.50

172.50

130.65

169.35
232.35

232.26

204.28

219.56

226.48

228.17

227.09

226.78

221.82

233.51

234.36

217.95

223.88

225.18

225.66

223.67

198.47

204.19

202.36

202.37

205.19

212.28

220.09

223.64

224.53

234.46

223.46

228.55

233.96

220.81

223.19

230.44

233.92

215.37

221.73

224.97

212.37

224.84

211.55

222.35

210.49

223.90

227.59

220.91

229.84

232.21

207.63

220.50

228.39

220.83

226.60 
WeIl ID

\begin{tabular}{|c|c|}
\hline MSB & $68 D$ \\
\hline MSB & $69 B$ \\
\hline MSB & 690 \\
\hline MSB & $69 \mathrm{TA}$ \\
\hline MSB & $70 \mathrm{C}$ \\
\hline MSB & $71 B$ \\
\hline MSB & $72 B$ \\
\hline MSB & $73 B$ \\
\hline MSB & $74 B$ \\
\hline MSB & $75 B$ \\
\hline $\mathrm{MSB}$ & $76 \mathrm{C}$ \\
\hline MSB & $77 \mathrm{~B}$ \\
\hline MSB & $77 \mathrm{C}$ \\
\hline MSB & $77 \mathrm{TA}$ \\
\hline MSB & $78 D$ \\
\hline MSB & $79 B$ \\
\hline MSB & $79 \mathrm{C}$ \\
\hline MSB & $81 B$ \\
\hline MSB & $82 \mathrm{~A}$ \\
\hline MSB & $82 \mathrm{~B}$ \\
\hline MSB & $82 \mathrm{C}$ \\
\hline MSB & $82 \mathrm{D}$ \\
\hline MSB & $82 \mathrm{TA}$ \\
\hline MSB & $83 B$ \\
\hline MSB & $83 \mathrm{C}$ \\
\hline MSB & $83 \mathrm{TA}$ \\
\hline MSB & $84 \mathrm{C}$ \\
\hline MSB & $85 B$ \\
\hline MSB & $85 \mathrm{C}$ \\
\hline MSB & $85 D$ \\
\hline MSB & $86 \mathrm{C}$ \\
\hline MSB & $88 D$ \\
\hline MSB & $89 \mathrm{C}$ \\
\hline RWM & 1 \\
\hline RWM & 2 \\
\hline RWM & 3 \\
\hline RWM & 4 \\
\hline RWM & 5 \\
\hline RWM & 6 \\
\hline RWM & 7 \\
\hline RWM & 8 \\
\hline RWM & 9 \\
\hline RWM & 10 \\
\hline RWM & 11 \\
\hline RWM & 12 \\
\hline RWM & $13 \mathrm{~B}$ \\
\hline RWM & $13 \mathrm{C}$ \\
\hline RWM & $14 B$ \\
\hline RWM & $14 \mathrm{C}$ \\
\hline RWM & $15 B$ \\
\hline RWM & 16 \\
\hline RW & 7 \\
\hline RW & \\
\hline
\end{tabular}

SRS East (ft)

52293.60

52432.90

52462.00

52418.40

45012.00

44054.70

48350.30

45694.00

50443.20

48875.50

45344.00

54217.40

54225.90

54208.90

45482.20

47300.20

47286.80

55230.40

51978.40

51993.30

51949.40

51934.60

51964.20

52421.40

52384.70

52410.90

51973.70

53122.70

53151.40

53108.80

54560.50

50793.50

47881.60

48575.10

49205.50

49680.00

48948.20

49628.00

50107.40

49449.50

47353.30

50400.00

48244.10

50400.20

52500.10

53516.30

53502.20

53044.70

53051.50

53849.00

48244.80

41407.00

41415.20
SRS North (ft)

-.......

106741.40

107776.10

107784.30

107772.50

101785.20

103801.60

96387.60

99270.30

99197.40

98937.40

103061.60

107065.80

107078.30

107053.80

103643.80

99296.90

99290.20

103762.70

107529.50

107533.40

107521.90

107518.10

107525.70

108426.70

108405.30

108416.30

108967.90

107827.00

107835.20

107822.80

108500.40

97012.30

98379.40

102599.10

104434.10

104730.20

103719.30

103502.20

102001.50

101904.60

101948.20

104099.80

102000.90

104875.00

106879.20

105803.30

105809.70

106362.10

106380.80

107444.70

97647.20

103776.70

103772.20
Middle of Screen Head (ft) (ft)

234.34

234.34

222.38

234.09

216.86

218.54

218.05

200.57

202.75

211.92

211.03

221.69

223.23

225.26

222.84

224.86

208.93

211.01

222.16

222.11

221.76

229.61

233.85

215.72

223.15

228.76

216.87

230.52

222.96

225.99

233.68

226.17

205.16

229.53

213.89

228.06

212.78

214.46

214.72

211.13

202.33

209.26

230.06

192.41

215.16

217.29

221.67

223.49

221.52

225.52

223.95

204.32

212.10

208.55 
WeII ID

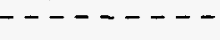

RW 2

RW 2A

RW 2B

RW $3 \mathrm{~A}$

RW $3 B B$

RW 4

RW $4 \mathrm{BB}$

RW 5

RW 6

RW 8

RW 9

RW $9 A$

RW 9B

RW $10 B B$

RW 11

RW IIBB

RW 12A

RW 12B

RW $12 \mathrm{C}$

RW 13A

RW 13B

RW 13C

RW $14 \mathrm{~A}$

RW 14B

RW 15A

RW 15B

RW 15C

RW 16B

RW 16C

RW $17 \mathrm{BB}$
SRS East

(ft)

-........

41627.20

41634.60

41631.70

41851.20

41845.60

41612.40

41609.20

41240.00

41243.90

40455.90

39688.40

39692.90

39697.60

40940.50

40874.20

40871.20

39013.30

39020.30

39023.10

40668.20

40675.80

40682.70

41538.60

41548.10

41234.70

41252.50

41245.10

42825.80

42841.80

42463.80
SRS North

(ft)

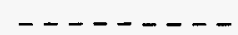

103721.80

103720.80

103729.70

103516.40

103526.60

103359.90

103347.90

103418.20

103602.70

103470.00

103259.80

103251.10

103241.60

103399.10

103693.20

103703.40

103710.30

103702.70

103712.50

103001.00

102993.60

102986.50

102831.30

102836.10

104778.00

104772.90

104774.90

103772.00

103772.40

103956.10
Middle of Screen Head

(ft)

(ft)

- - -

214.35

93.50

157.70

178.10

125.25

213.71

129.05

211.99

211.99

209.41

181.30

119.35

157.50

121.65

210.18

124.50

108.80

151.20

198.00

98.70

158.25

210.30

118.80

158.00

102.70

156.70

213.30

165.00

215.87

127.25
214.35

207.71

208.79

214.06

209.70

213.71

208.35

211.99

211.99

209.41

200.68

199.90

201.21

206.71

210.18

206.84

195.45

190.63

198.00

202.56

204.52

210.30

204.52

206.42

210.39

210.46

213.30

215.47

215.87

213.07 


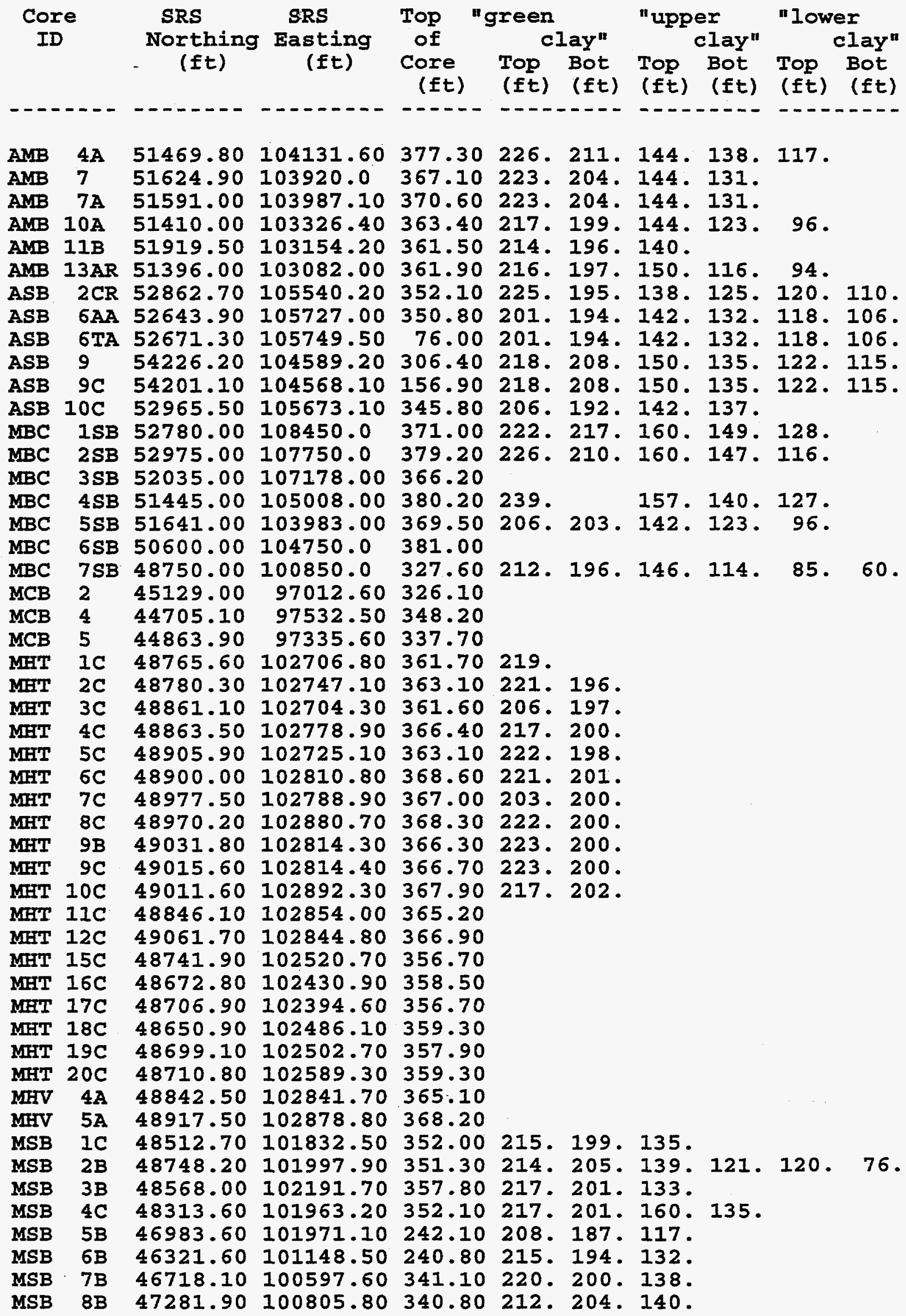




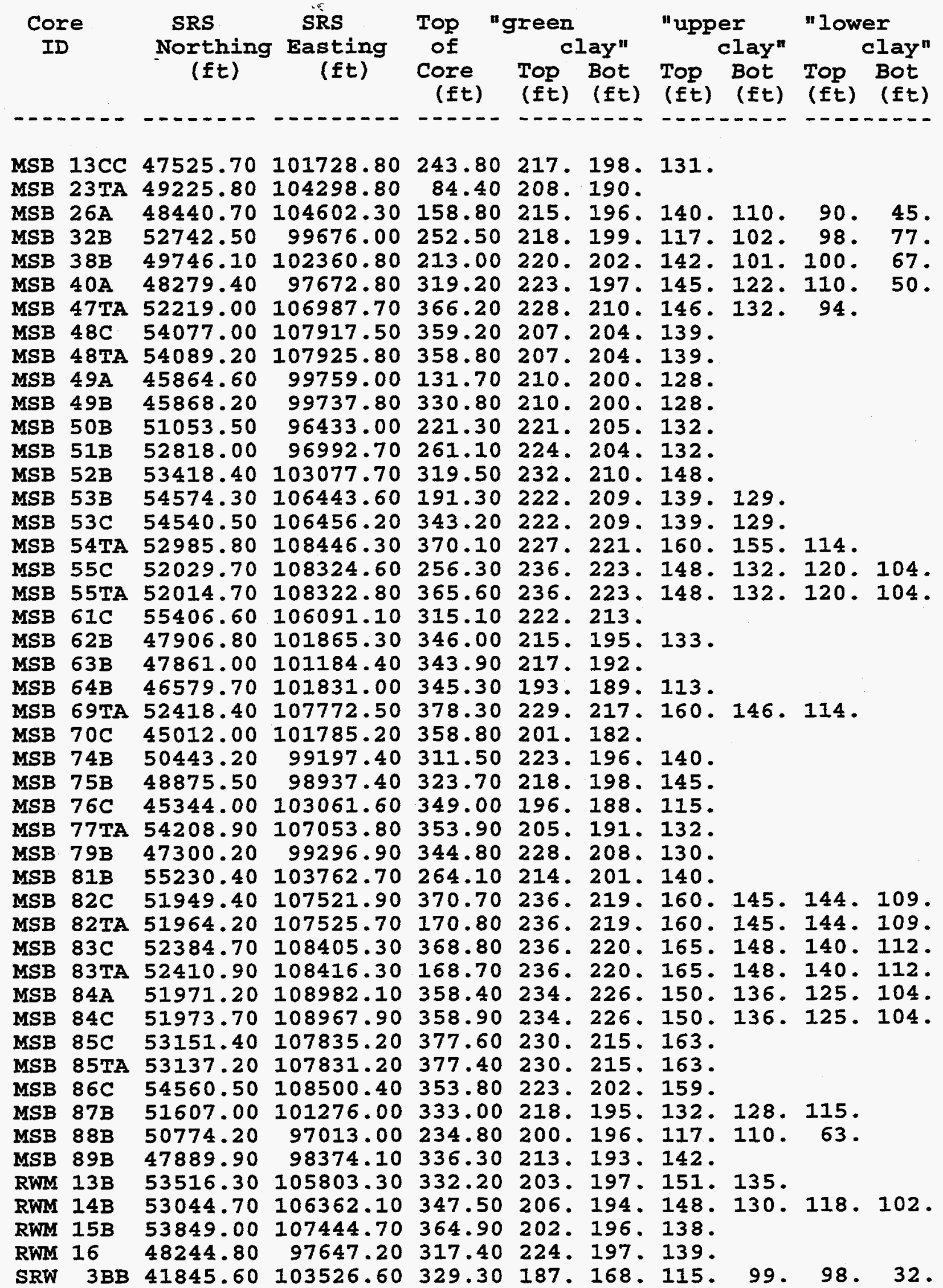




\begin{tabular}{|c|c|c|c|c|c|c|c|c|c|}
\hline \multirow[t]{2}{*}{$\begin{array}{l}\text { Core } \\
\text { ID }\end{array}$} & \multirow{2}{*}{$\begin{array}{l}\text { SRS } \\
\text { Northing } \\
\text { (ft) }\end{array}$} & \multirow{2}{*}{$\begin{array}{c}\text { SRS } \\
\text { Easting } \\
\quad(f t)\end{array}$} & \multirow{2}{*}{$\begin{array}{l}\text { Top } \\
\text { of } \\
\text { Core } \\
\text { (ft) }\end{array}$} & \multicolumn{2}{|c|}{$\begin{array}{l}\text { "green } \\
\text { clay" }\end{array}$} & \multicolumn{2}{|c|}{$\begin{array}{l}\text { "upper } \\
\text { clay" }\end{array}$} & \multicolumn{2}{|c|}{$\begin{array}{c}\text { "lower } \\
\text { clay" }\end{array}$} \\
\hline & & & & $\begin{array}{l}\text { Top } \\
\text { (ft) }\end{array}$ & $\begin{array}{l}\text { Bot } \\
\text { (ft) }\end{array}$ & $\begin{array}{l}\text { Top } \\
\text { (ft) }\end{array}$ & $\begin{array}{l}\text { Bot } \\
\text { (Et) }\end{array}$ & $\begin{array}{l}\text { Top } \\
\text { (ft) }\end{array}$ & $\begin{array}{l}\text { Bot } \\
\text { (Et) }\end{array}$ \\
\hline
\end{tabular}

SRW 4BB $41609.20 \quad 103347.90 \quad 317.60 \quad 186.172 .115 .99 .98 .37$.

SRW 8BB $40464.90 \quad 103468.00 \quad 286.30 \quad 197.177 .118 .99 .98 .20$.

SRW 10BB 40940.50 103399.10 299.70 183. 170. 111. 99. 98. 33.

SRW 17BB 2463.80 103956.10 330.30 171. 164. 114. 99. 98. 25. 


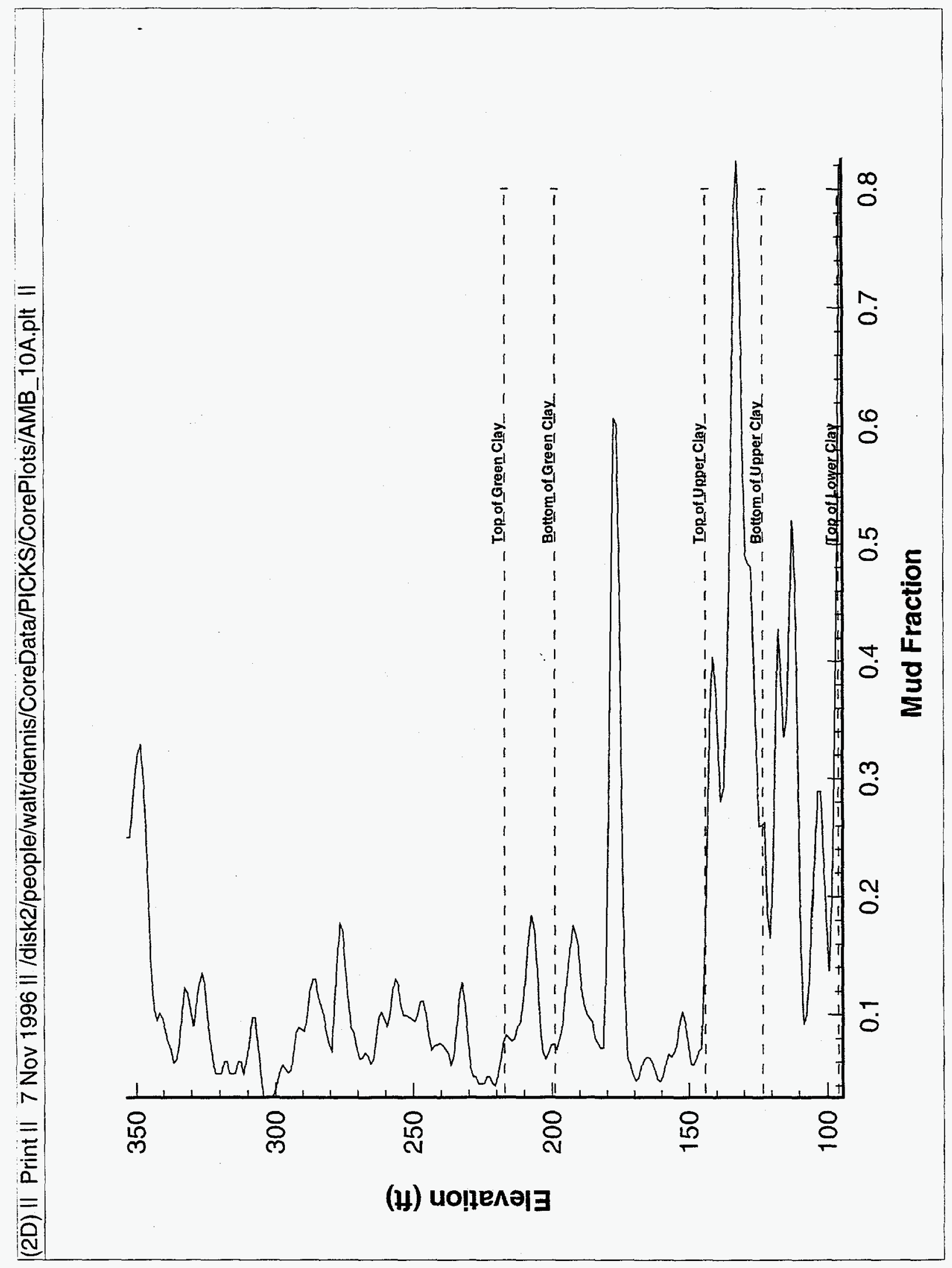




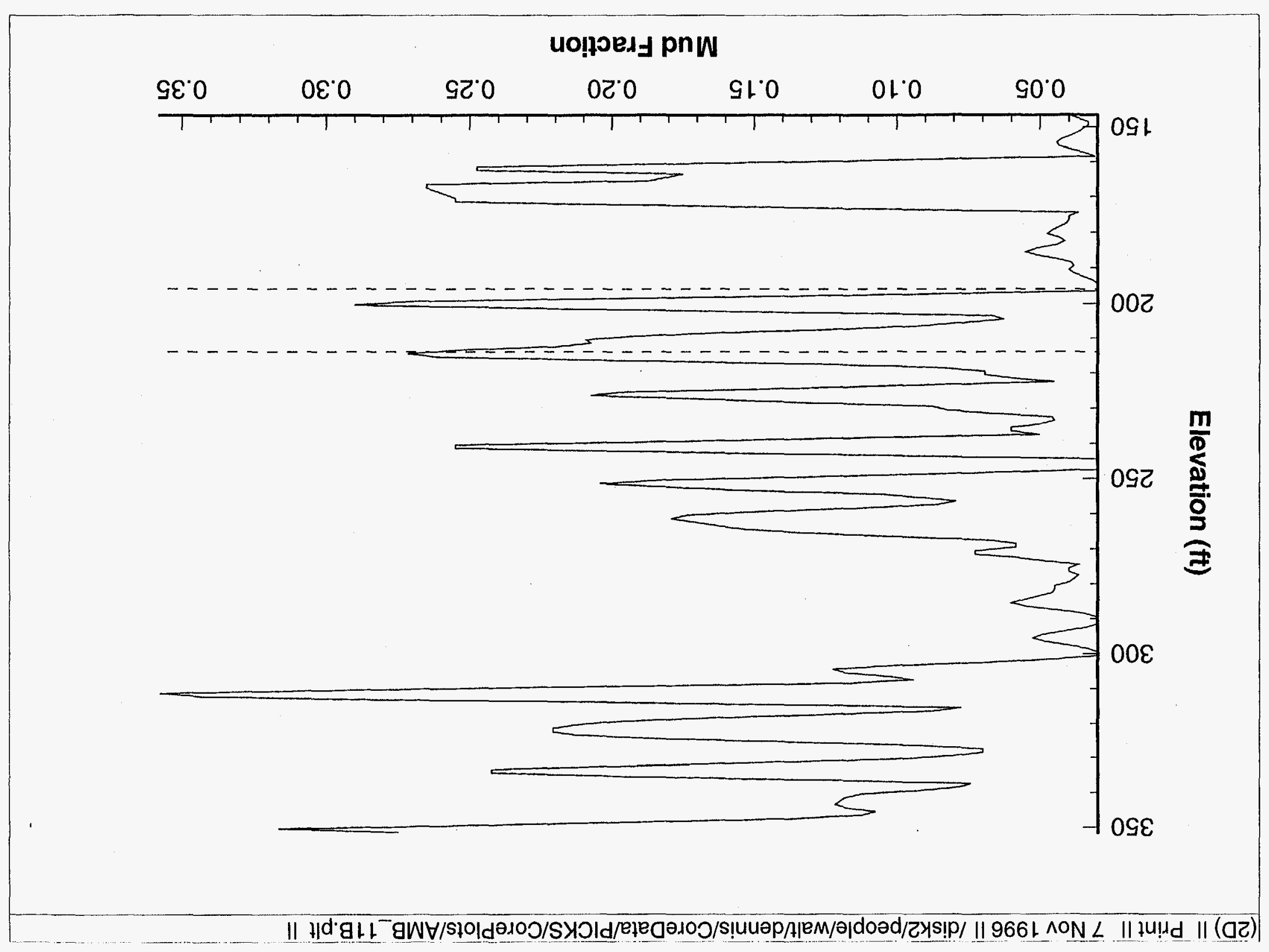




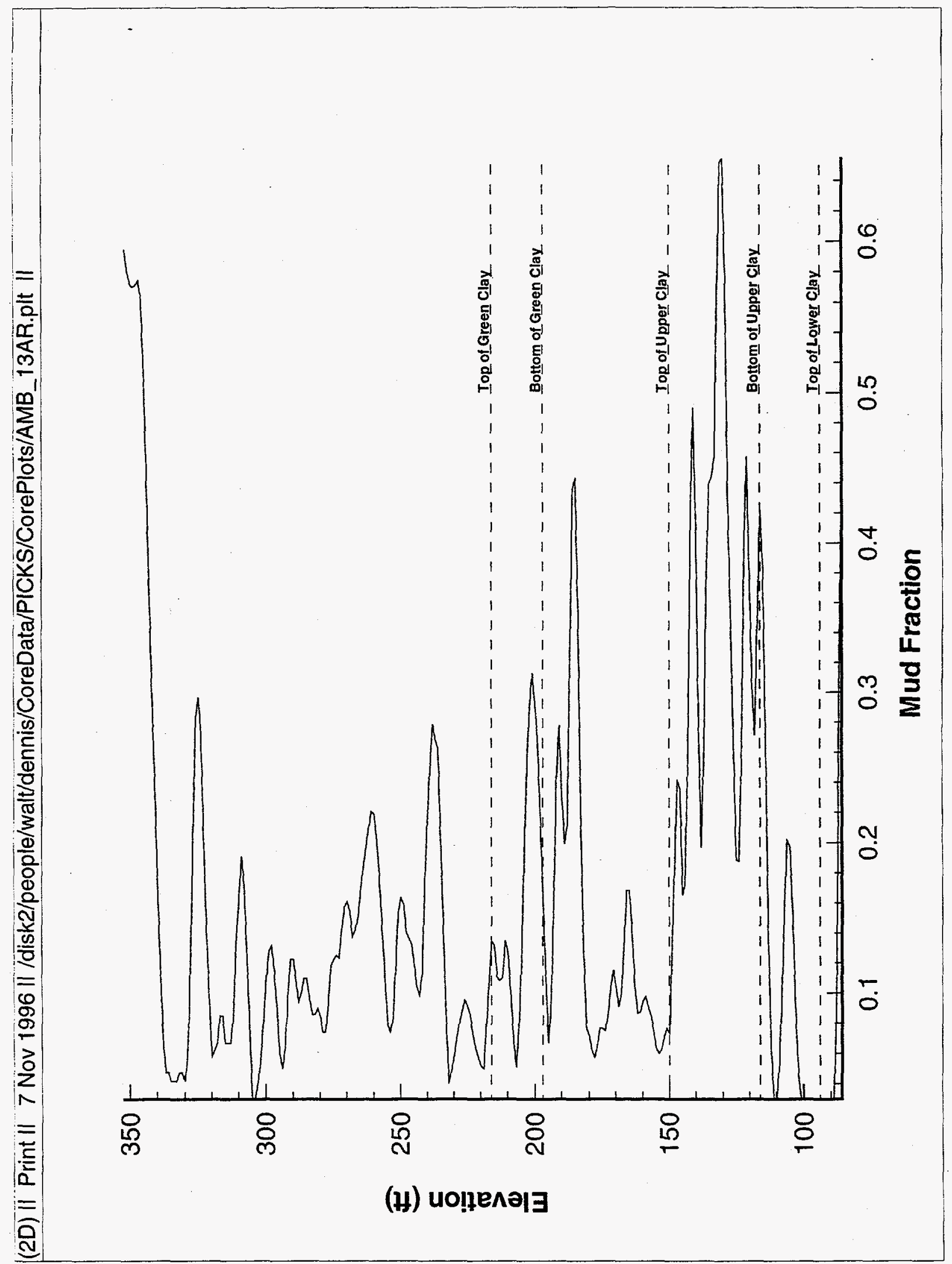




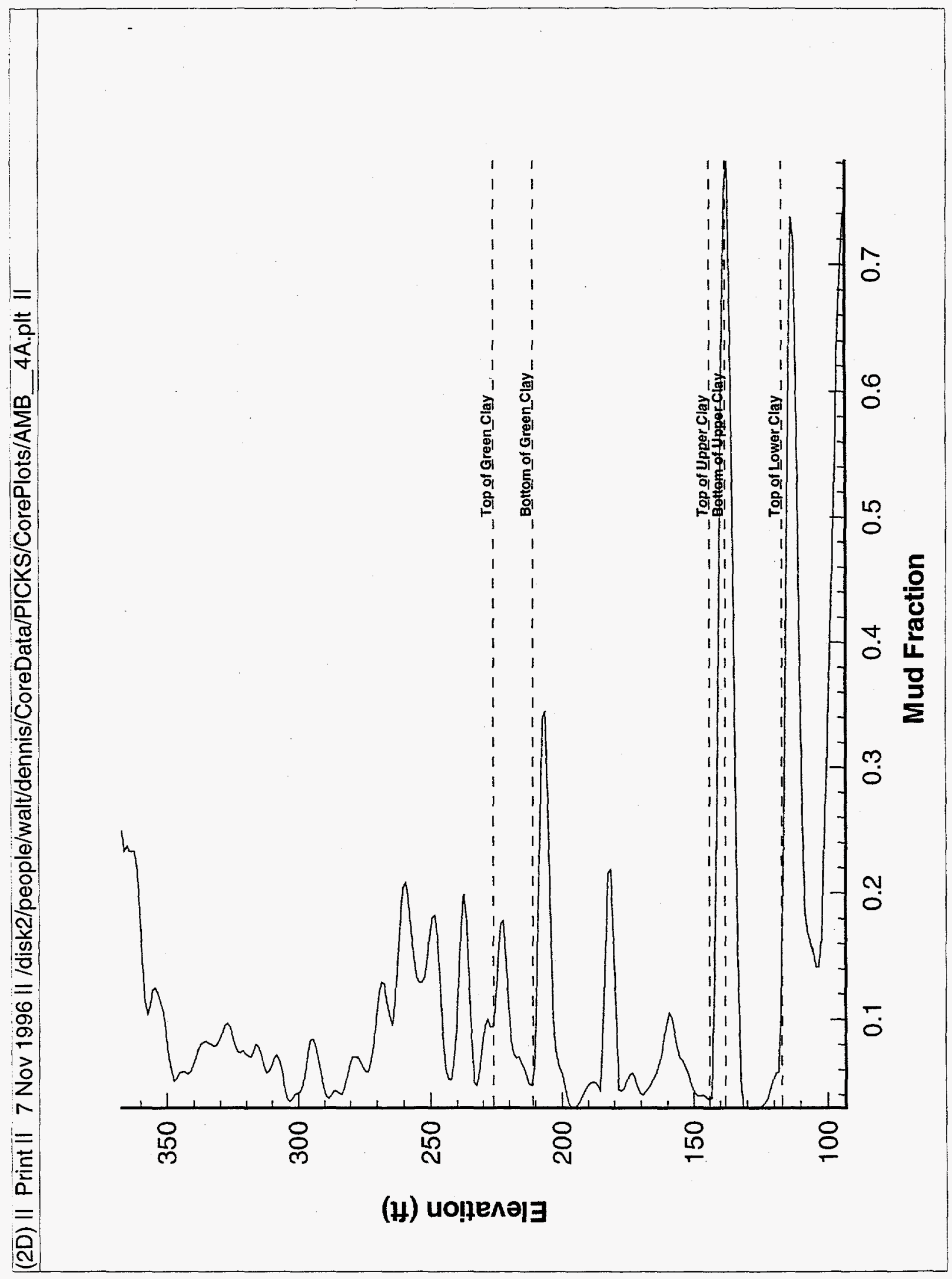




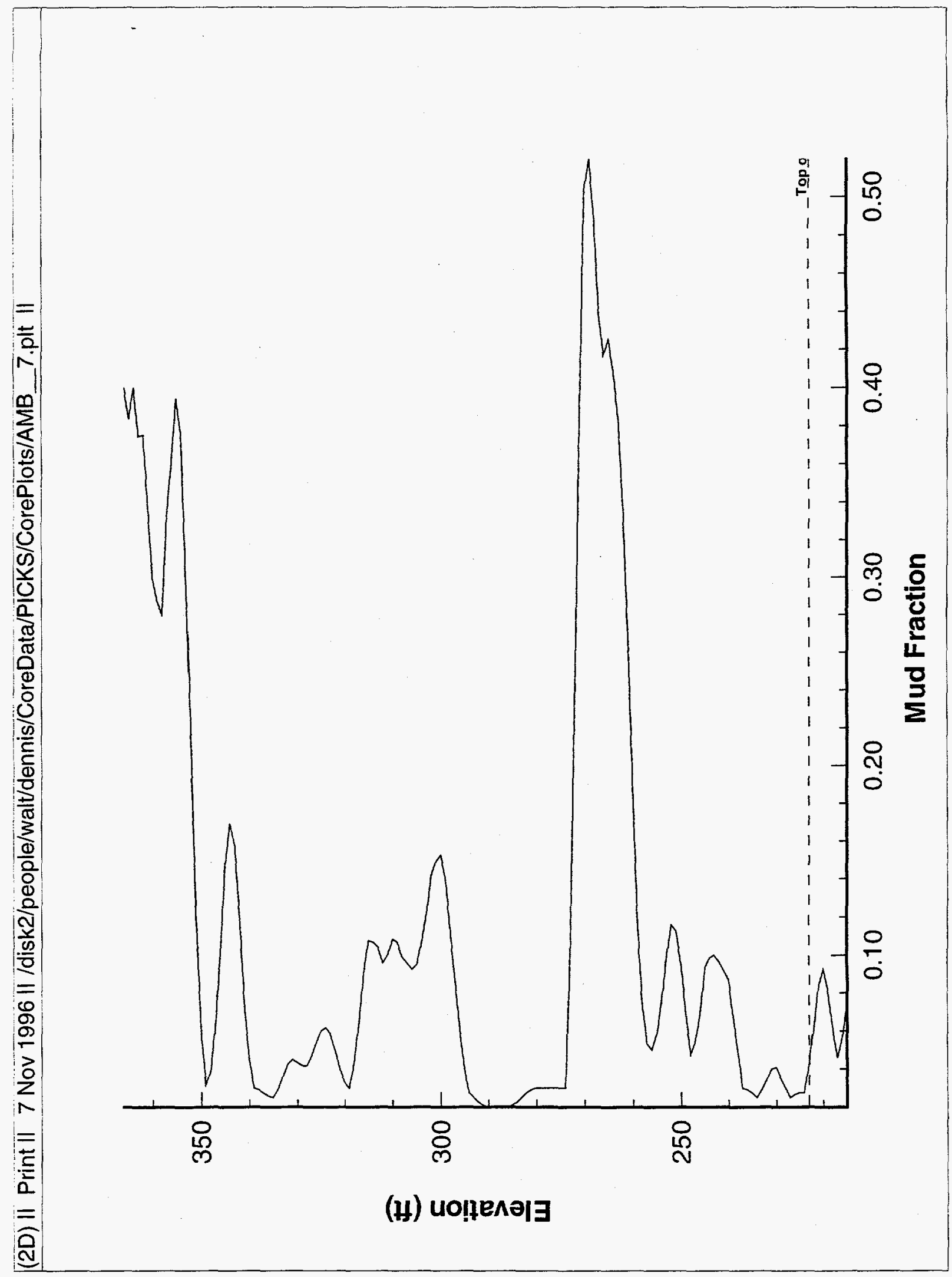




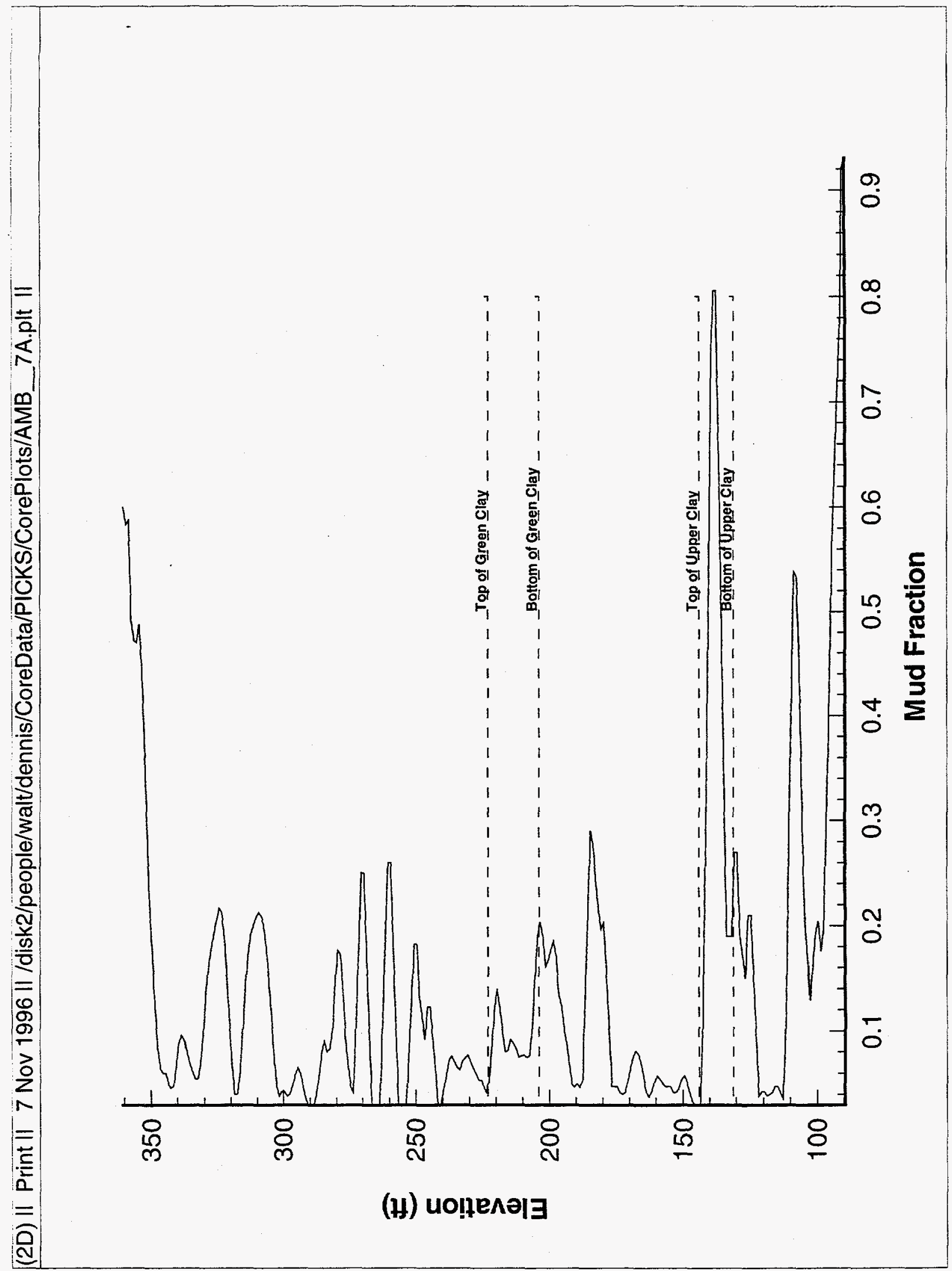




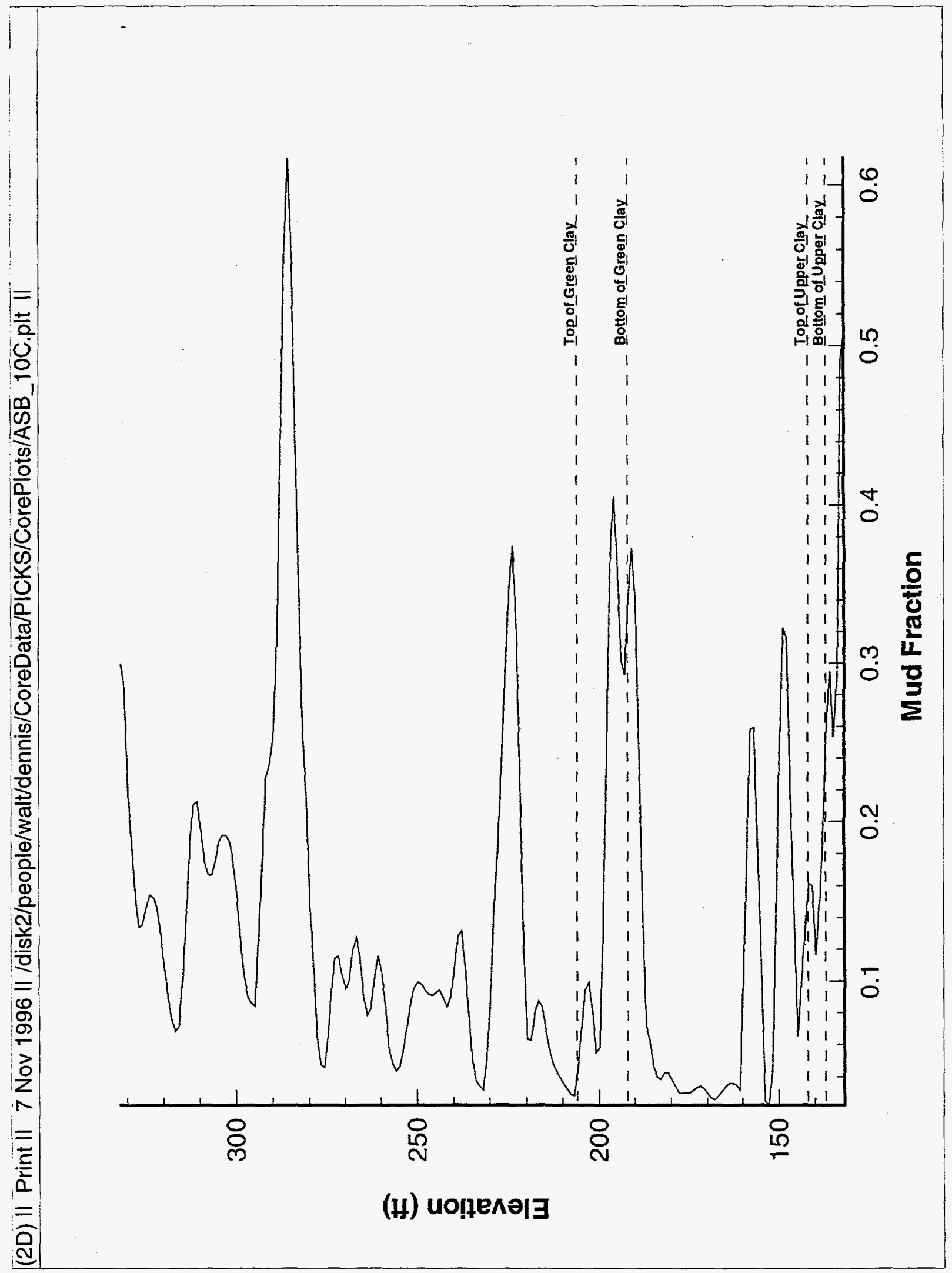




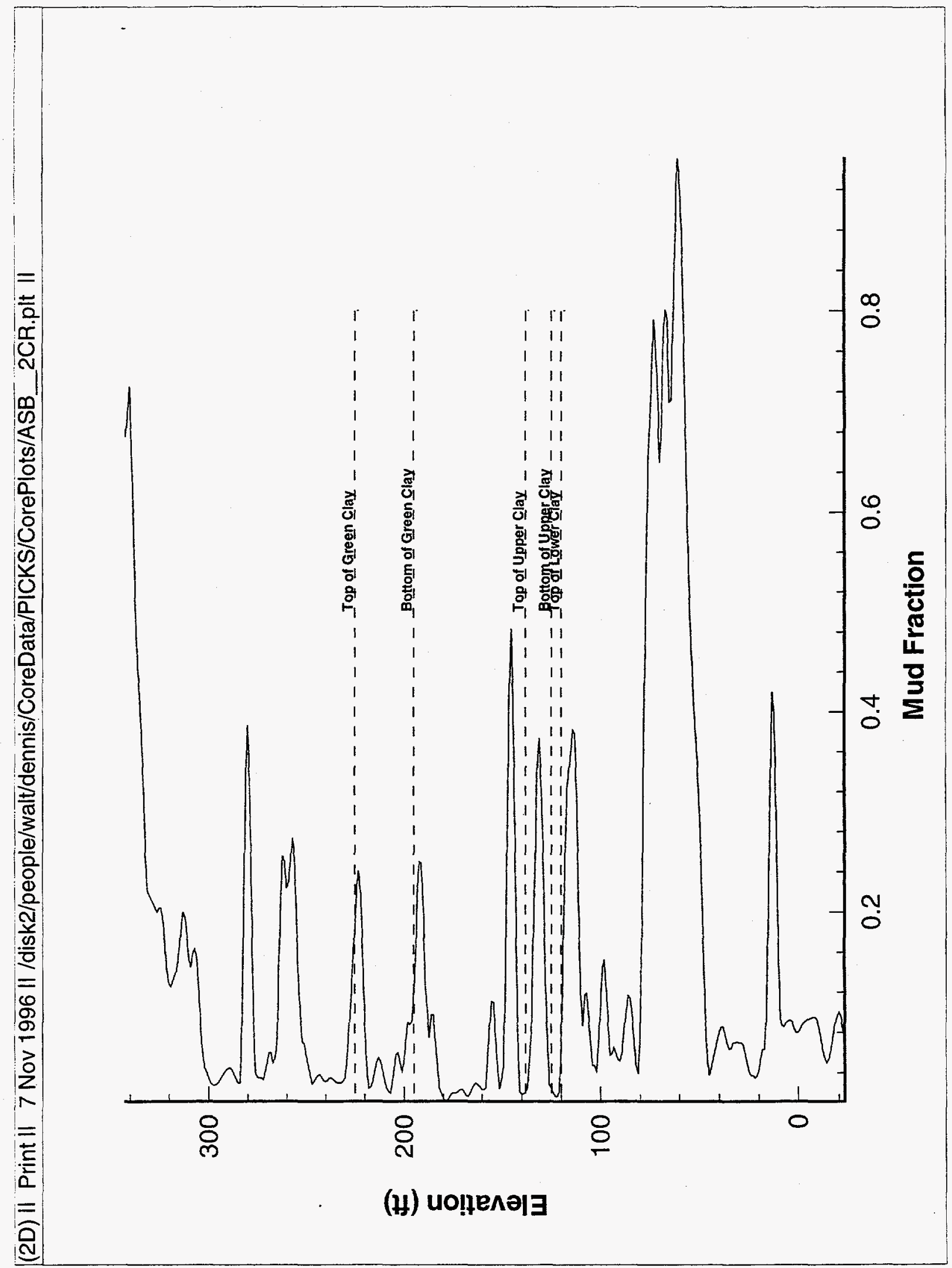




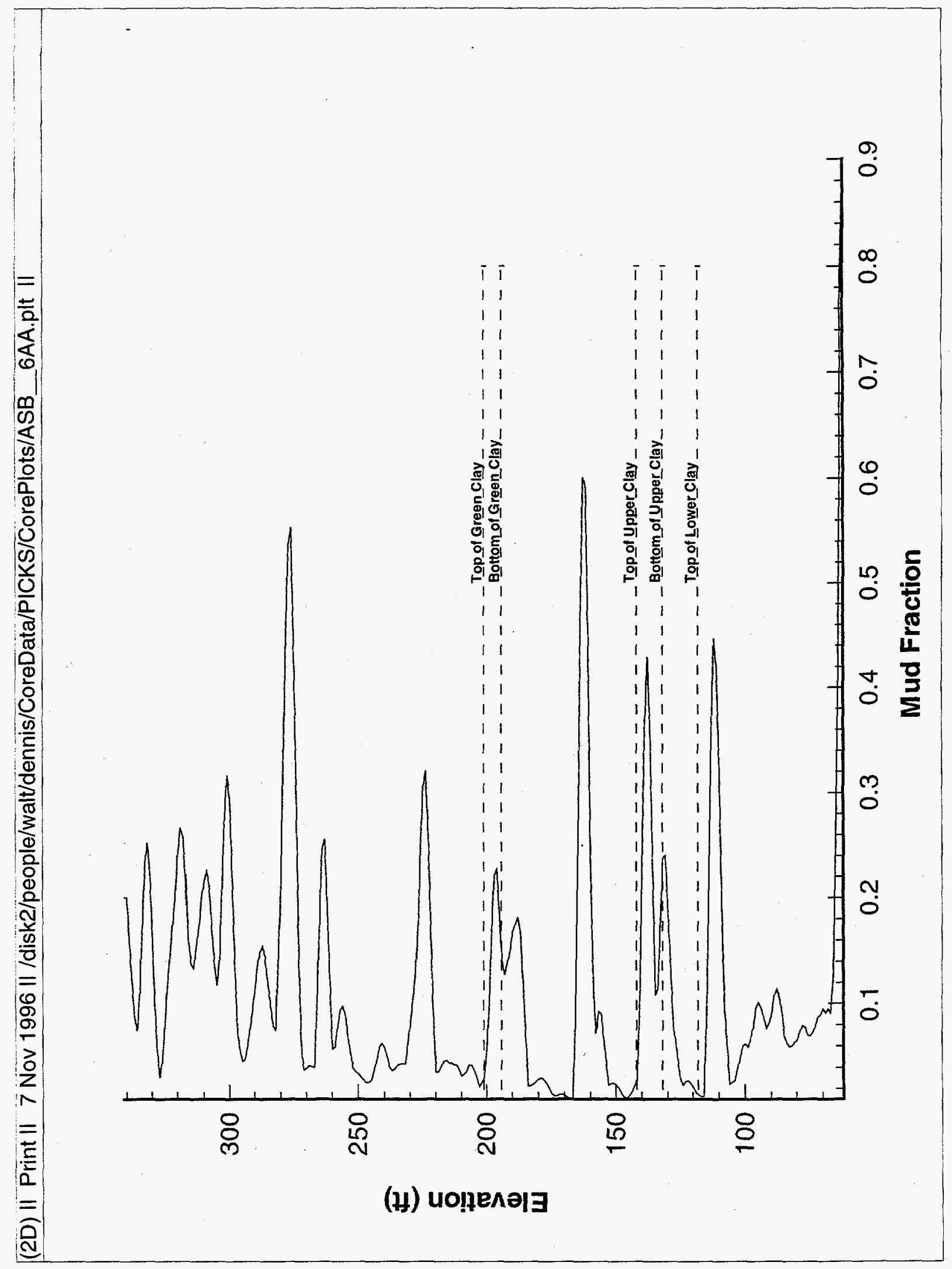




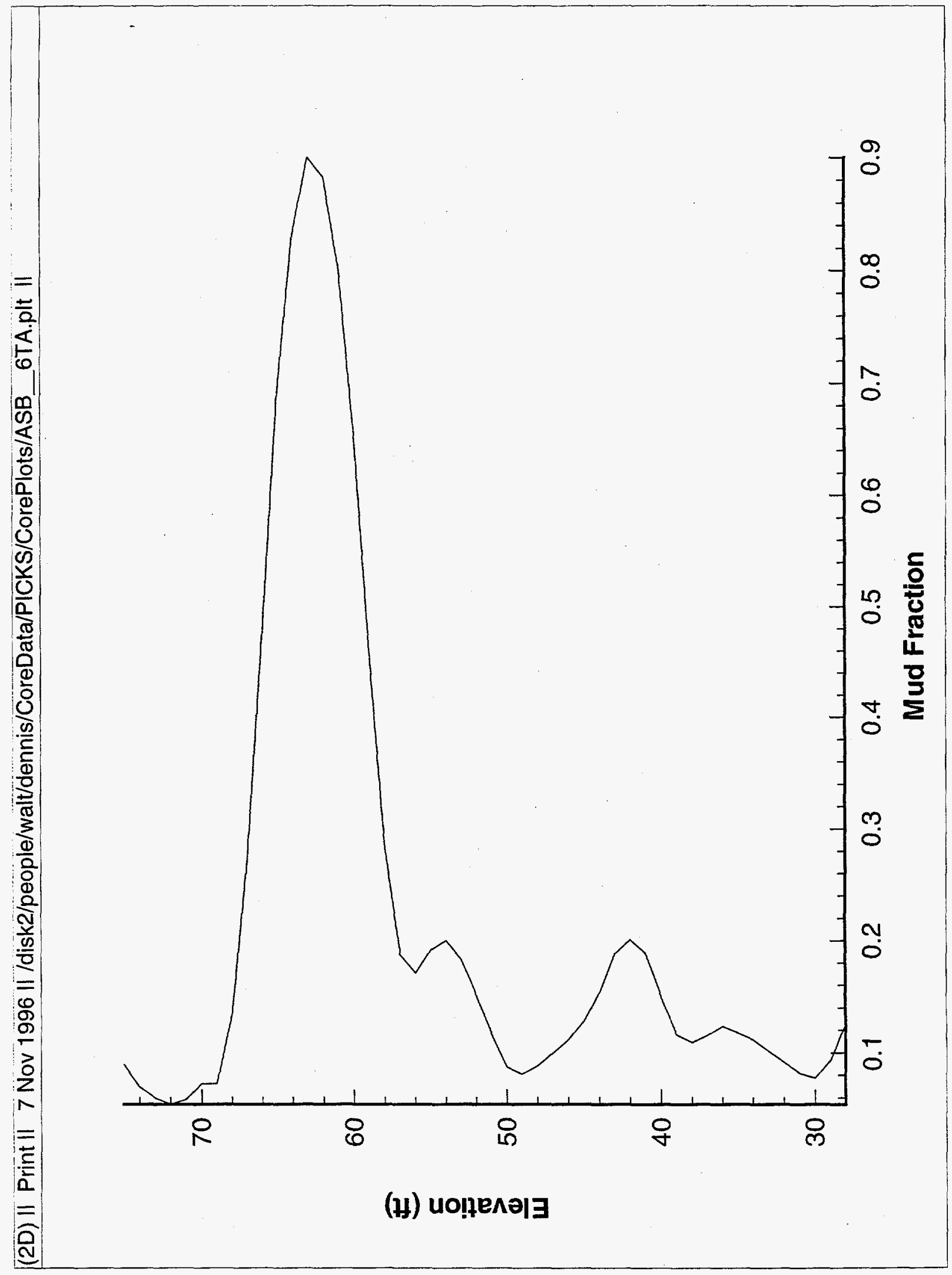




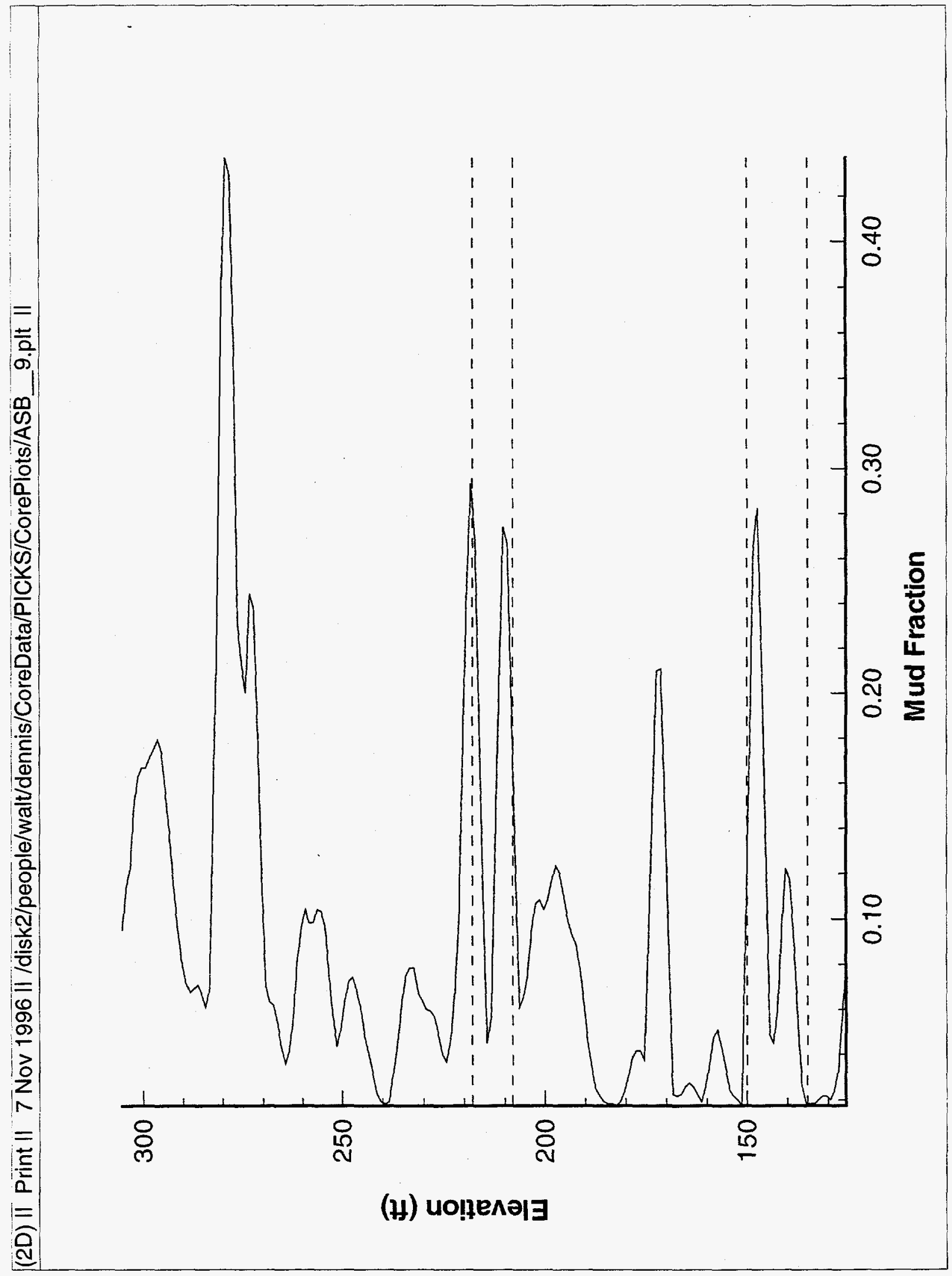




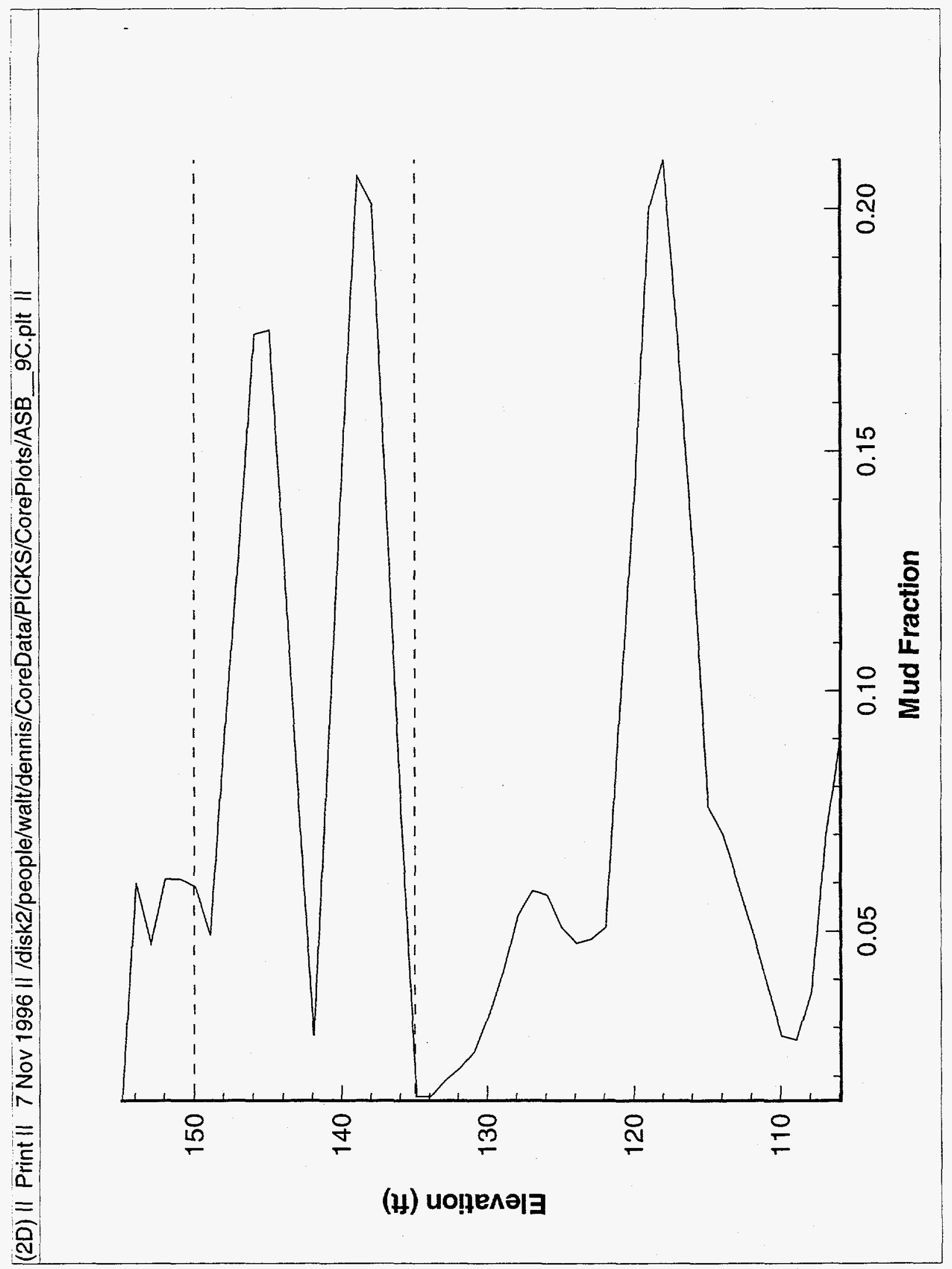




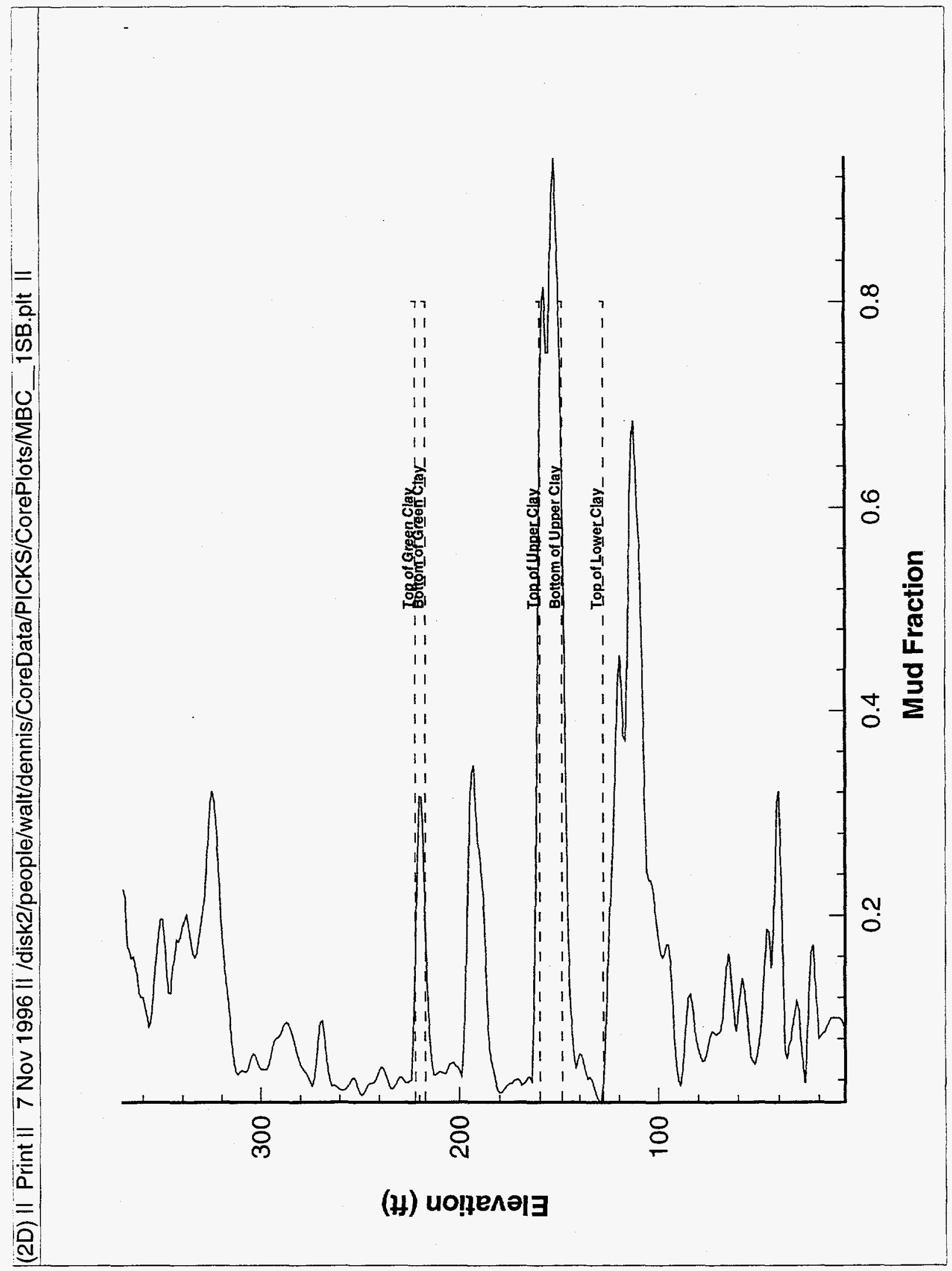




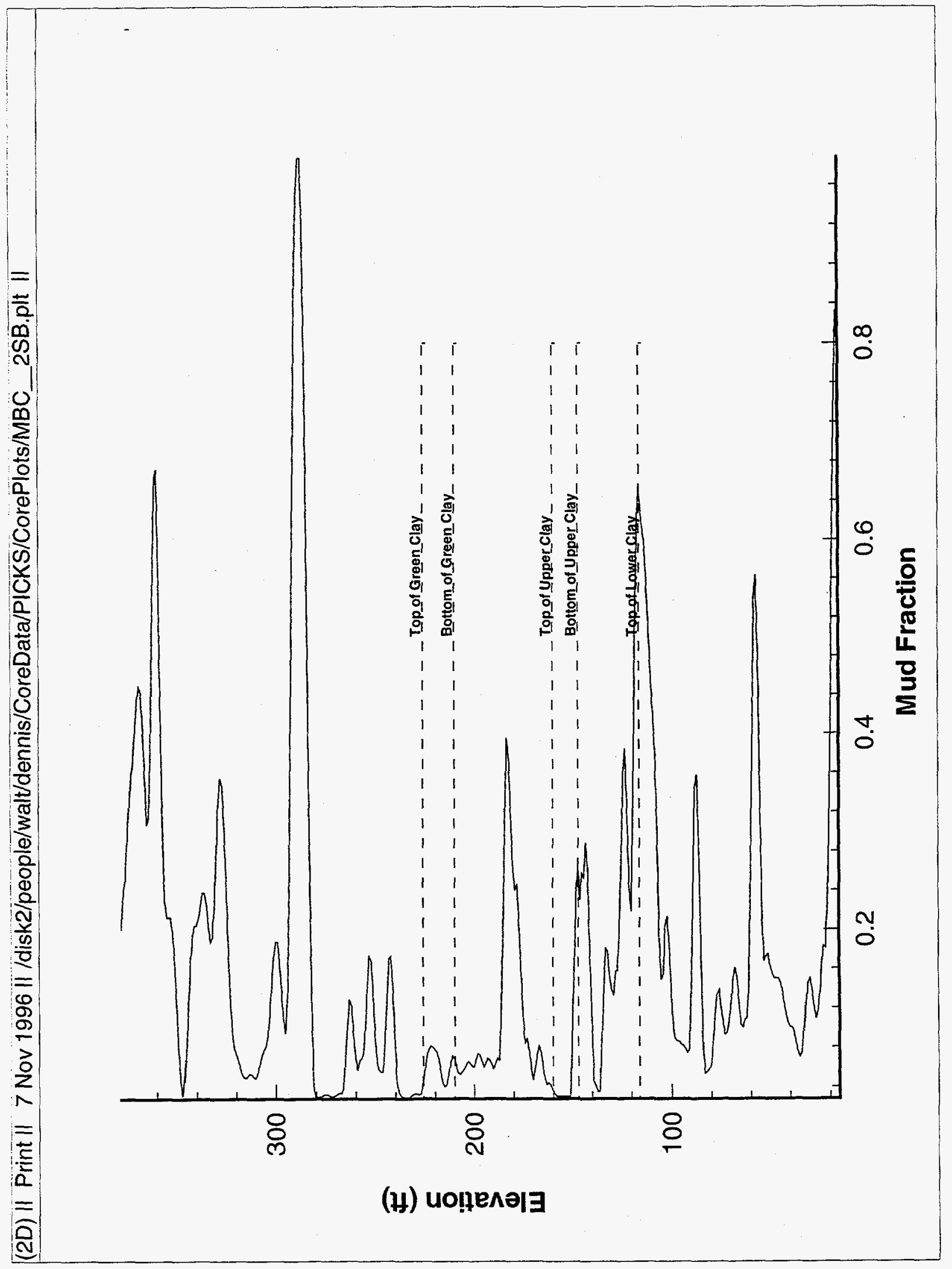




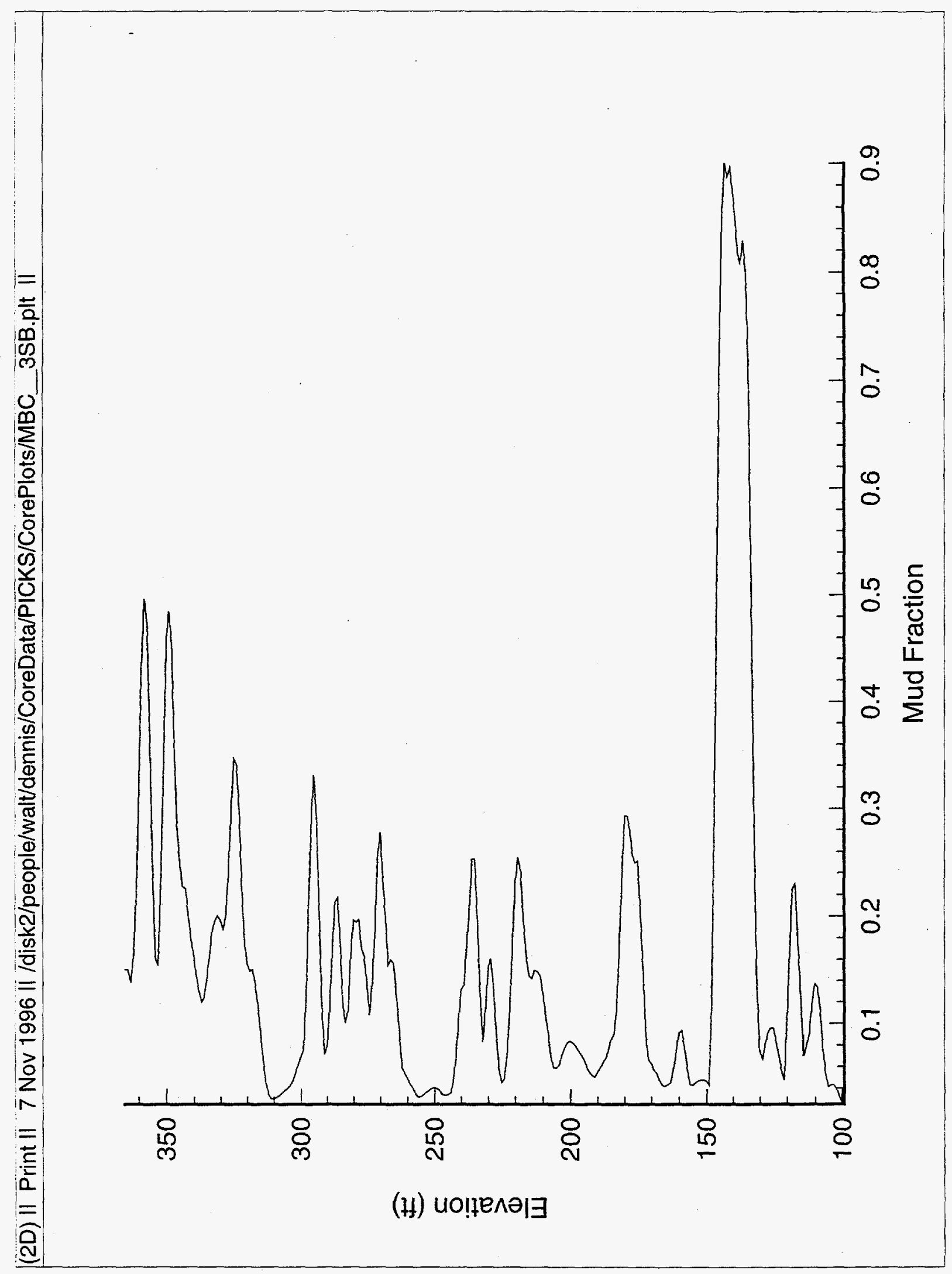




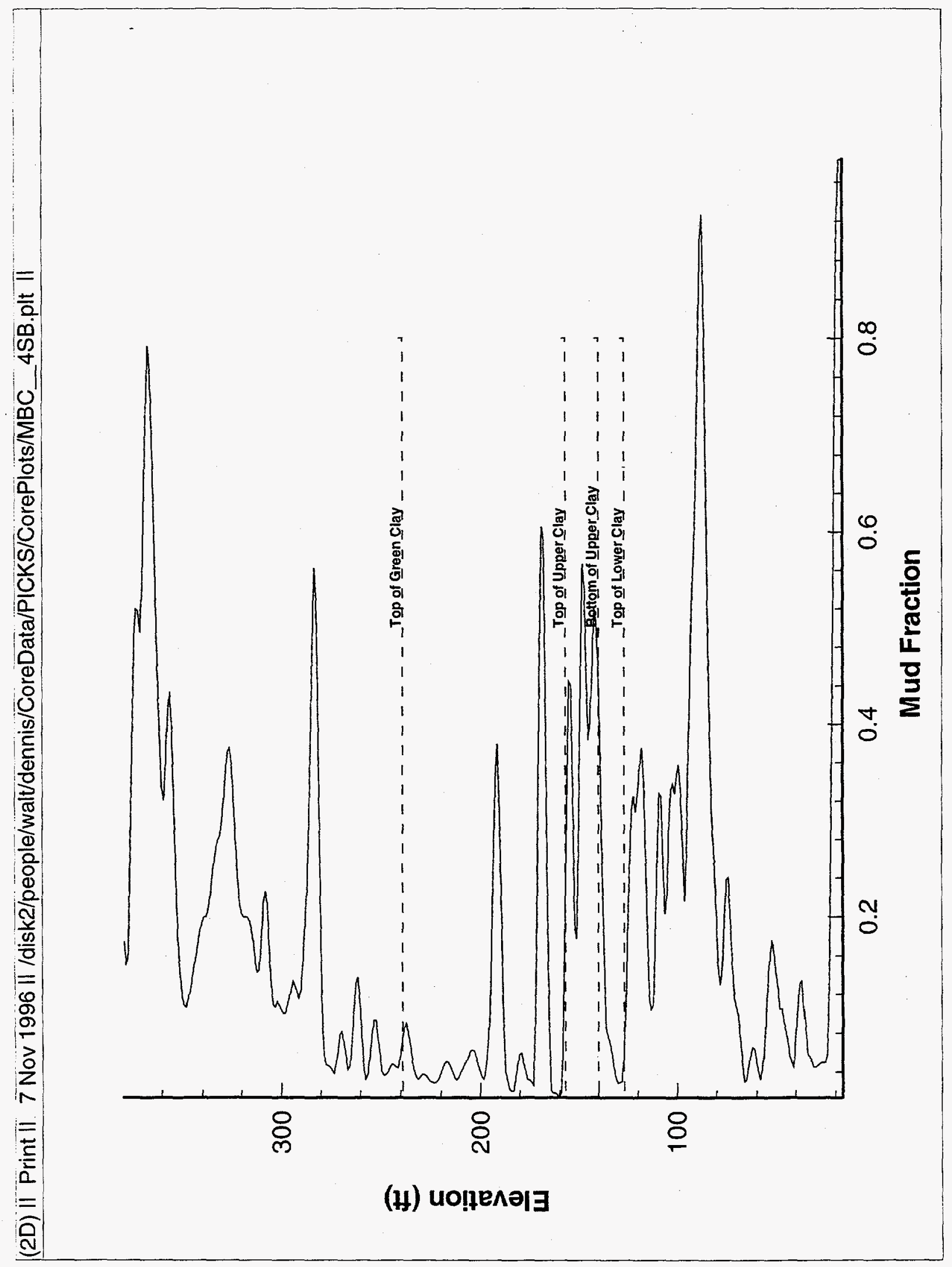


(2D) II Print II 7 Nov 1996 II /disk2/people/walt/dennis/CoreData/PICKS/CorePlots/MBC_5SB.plt II

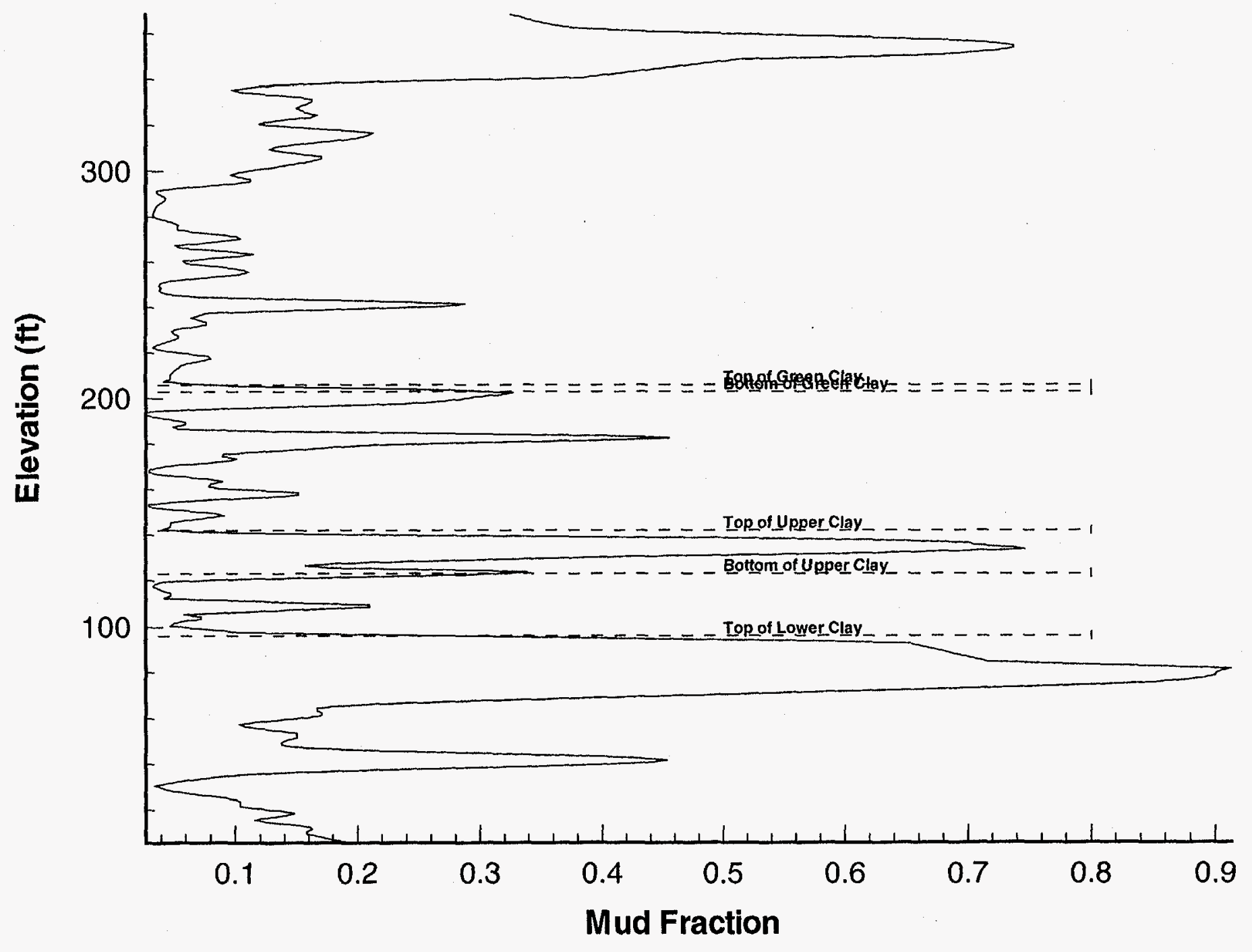


(2D) II Print I| 7 Nov 1996 || /disk2/people/walt/dennis/CoreData/PICKS/CorePlots/MBC_6SB.plt ii

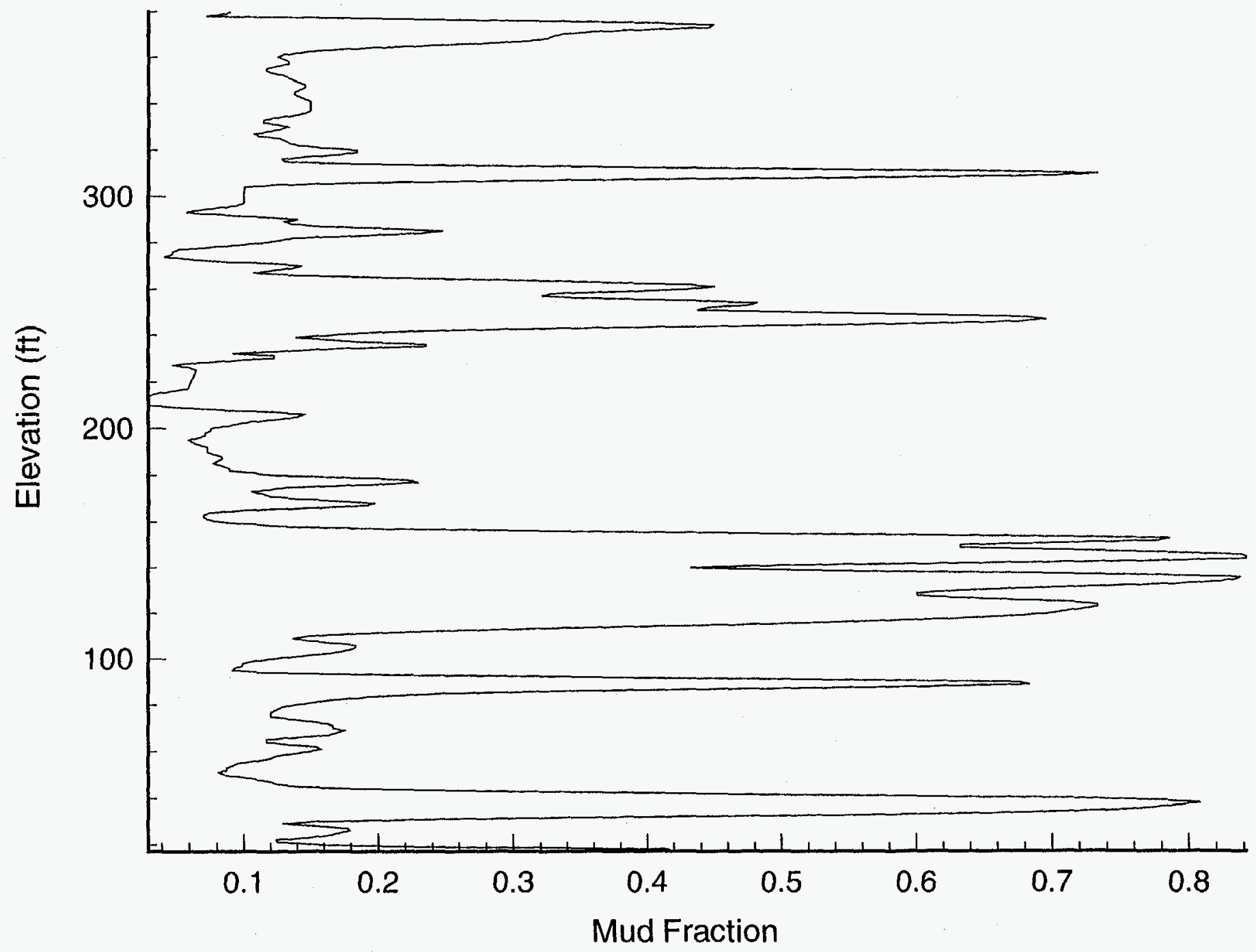




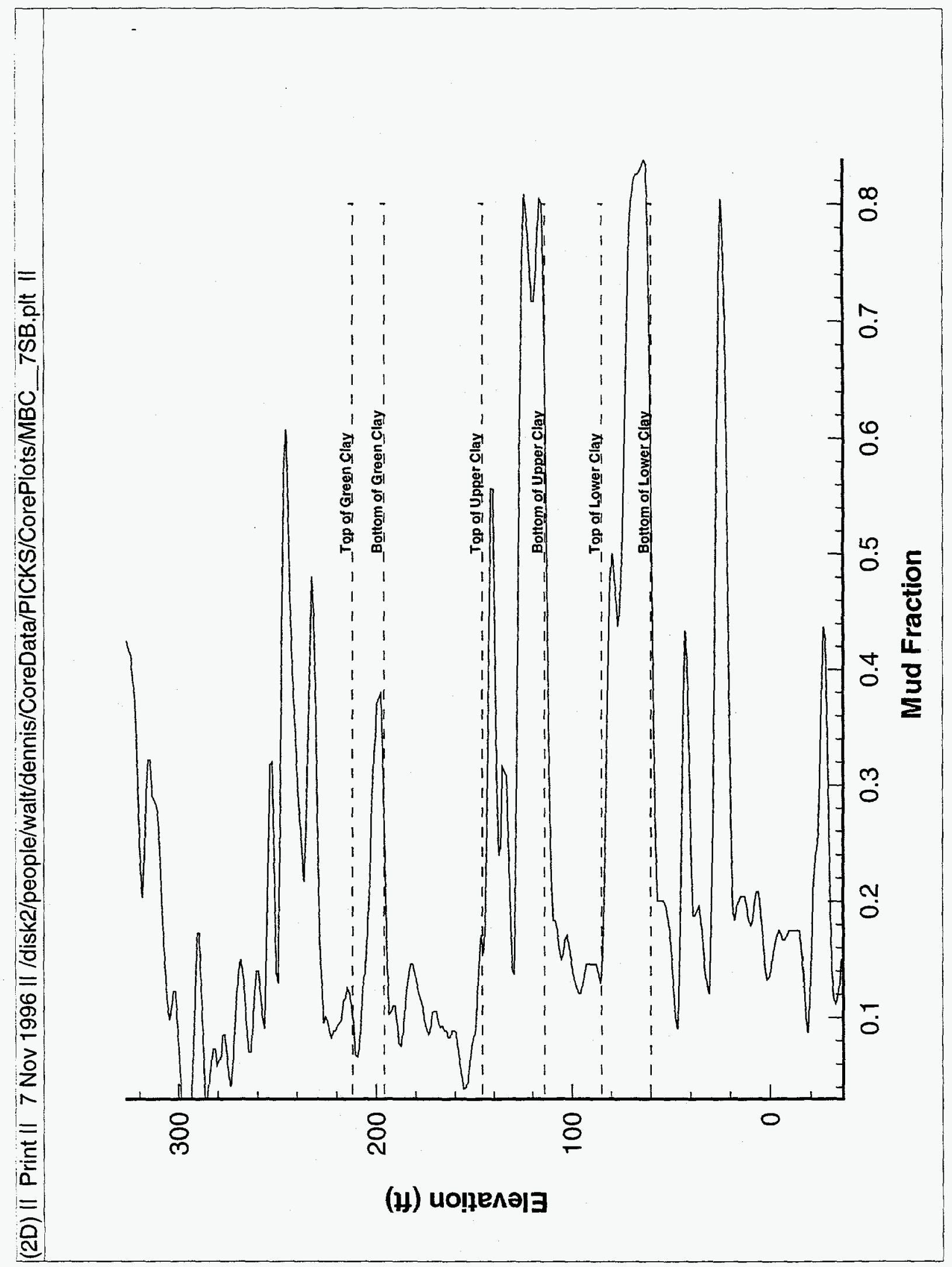




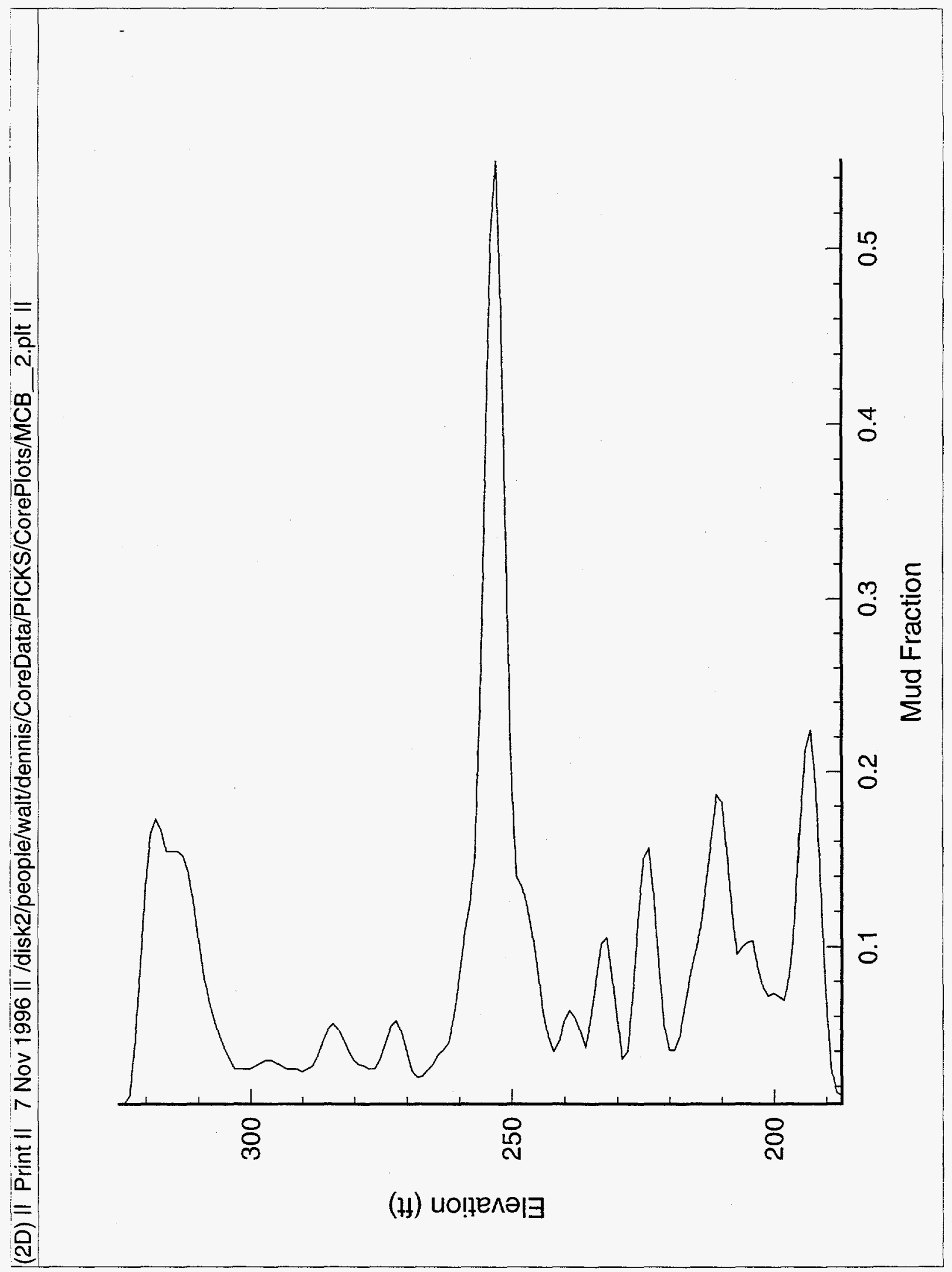




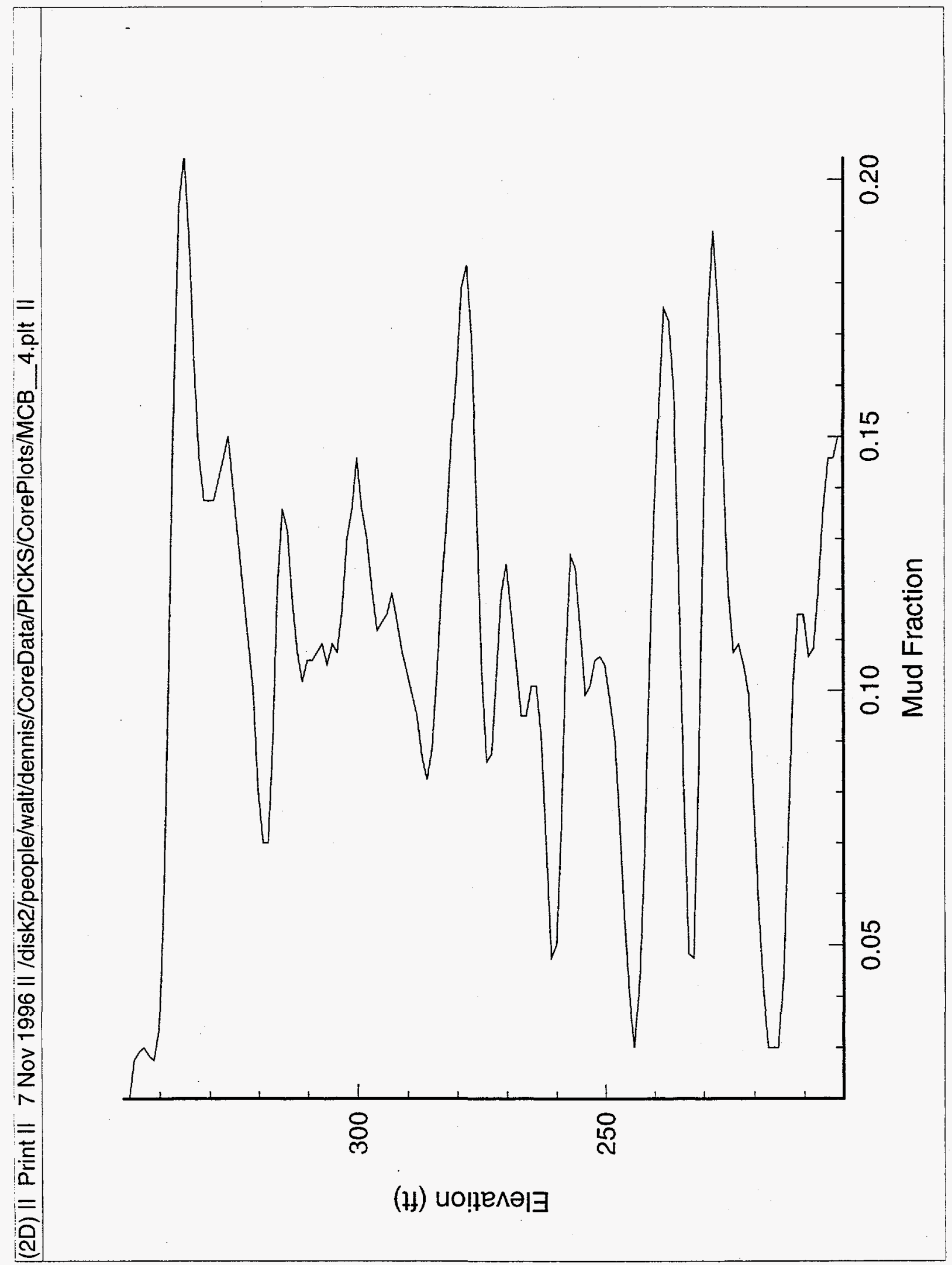




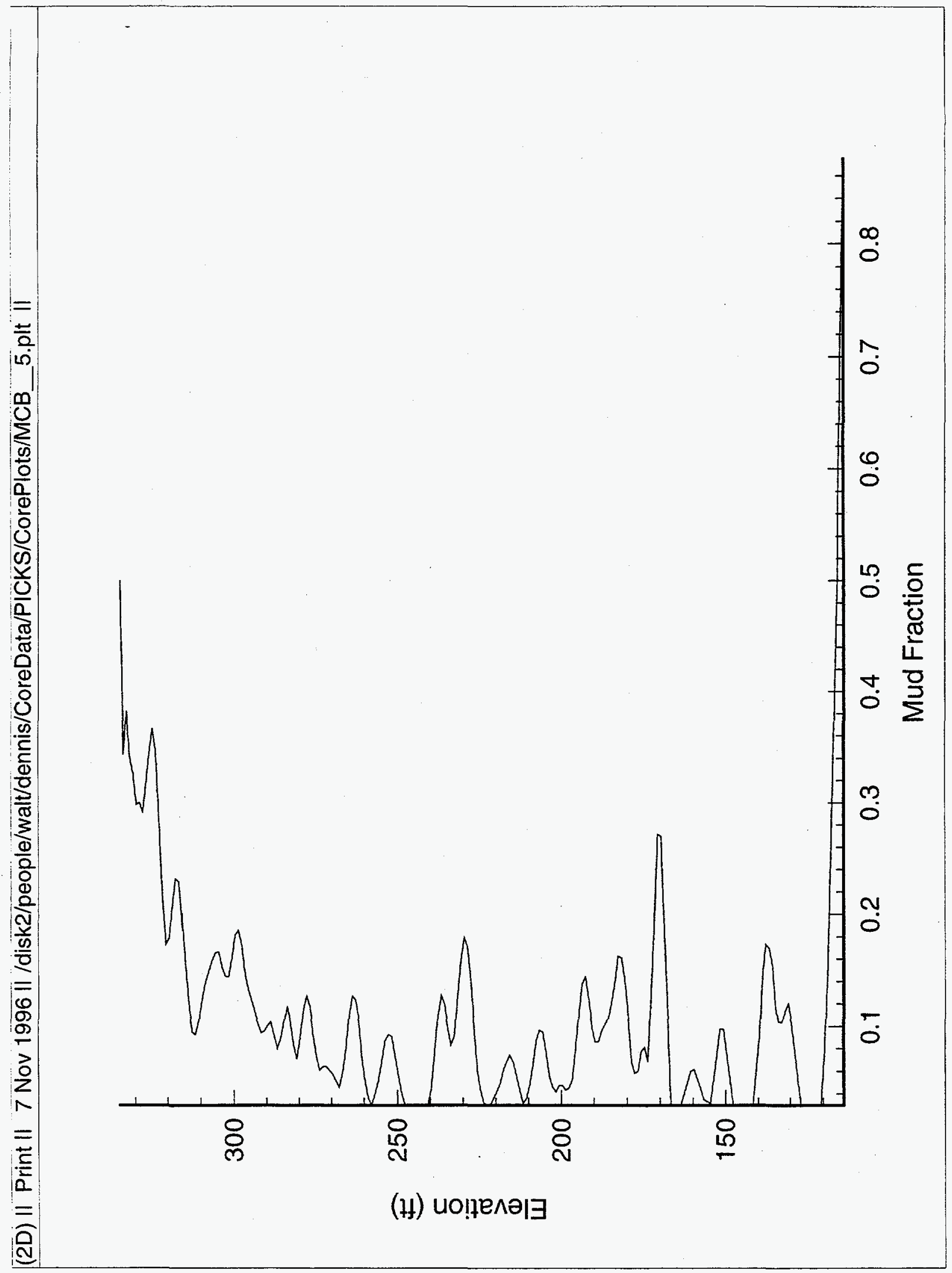




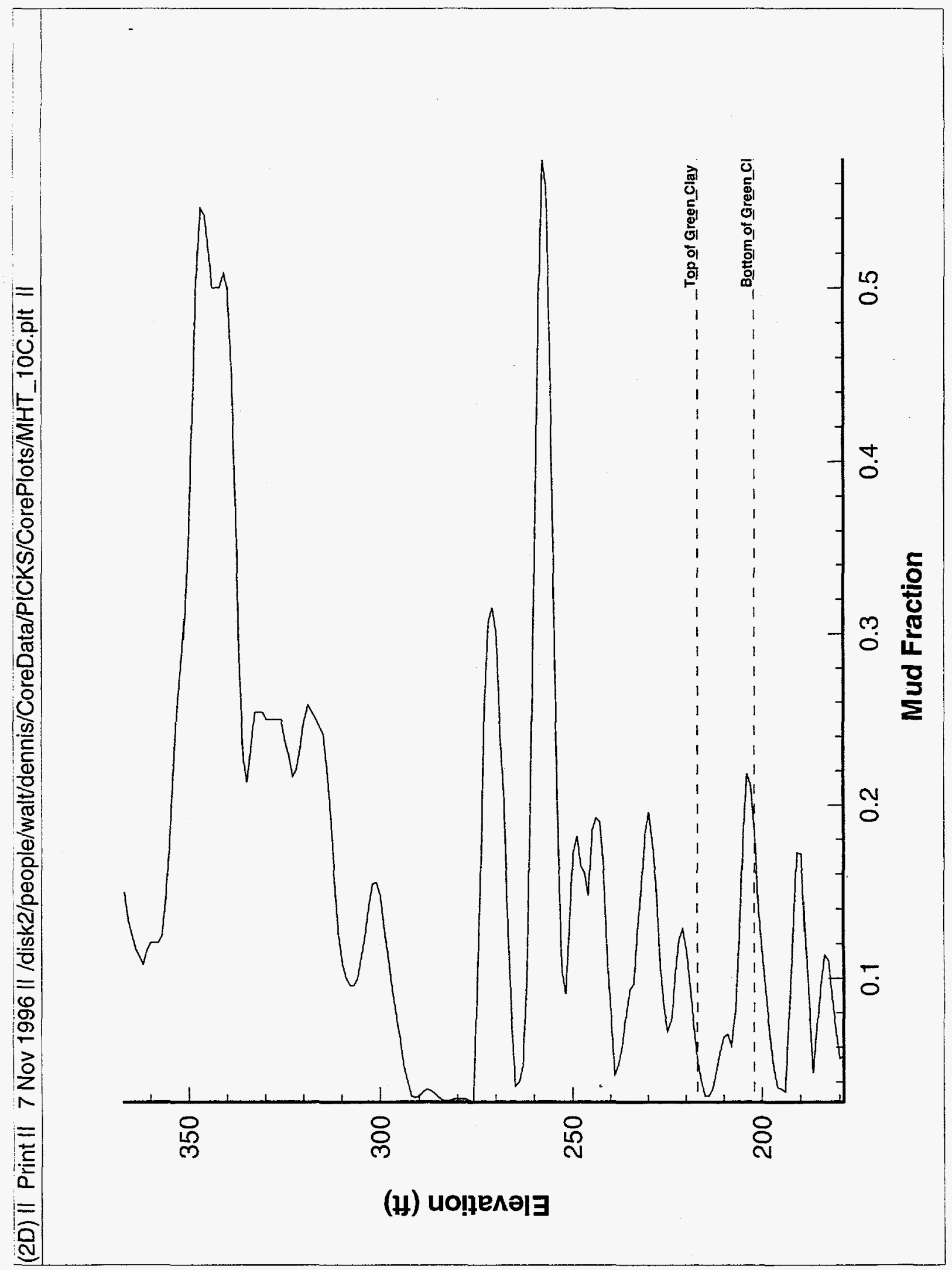




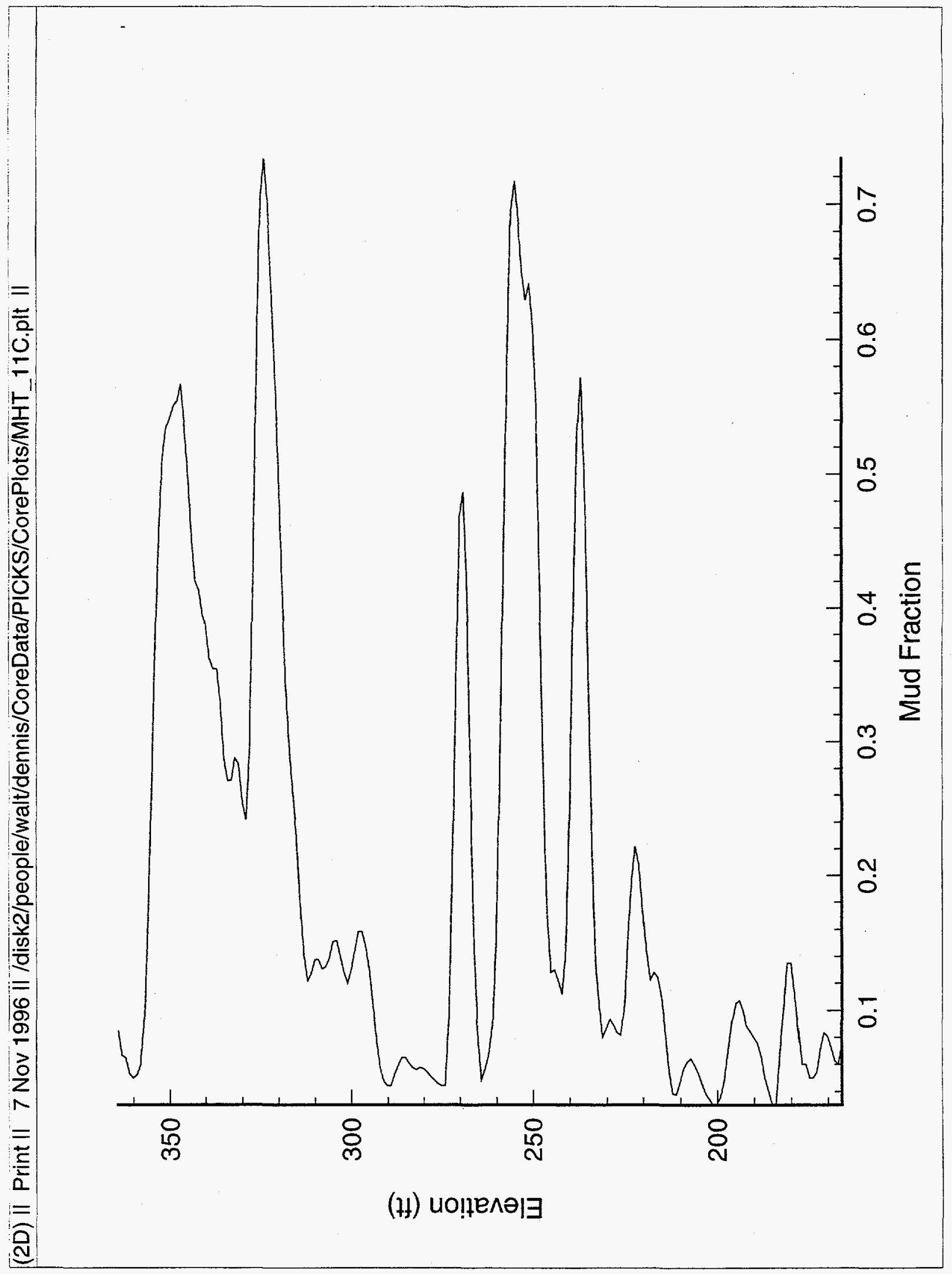




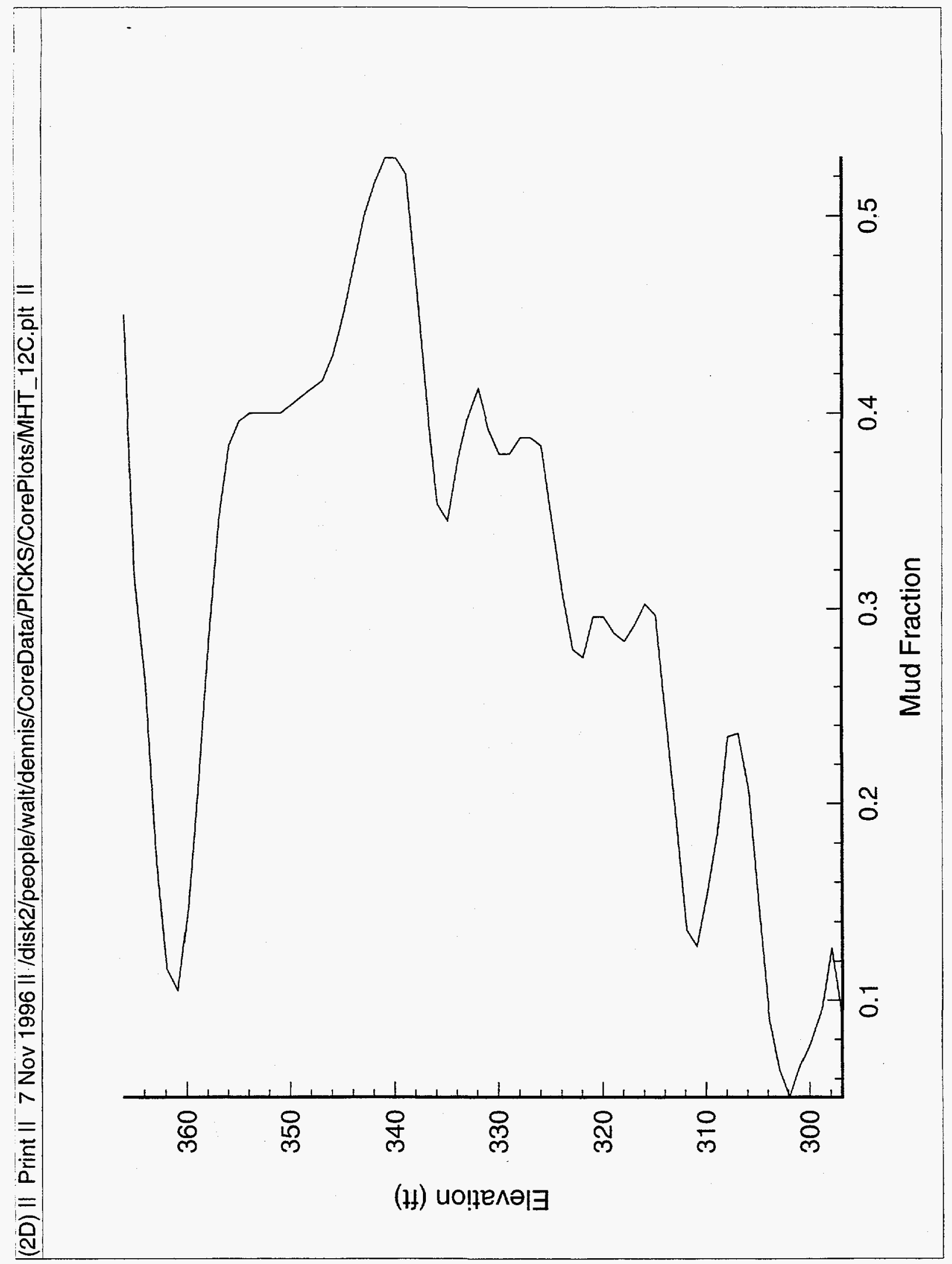




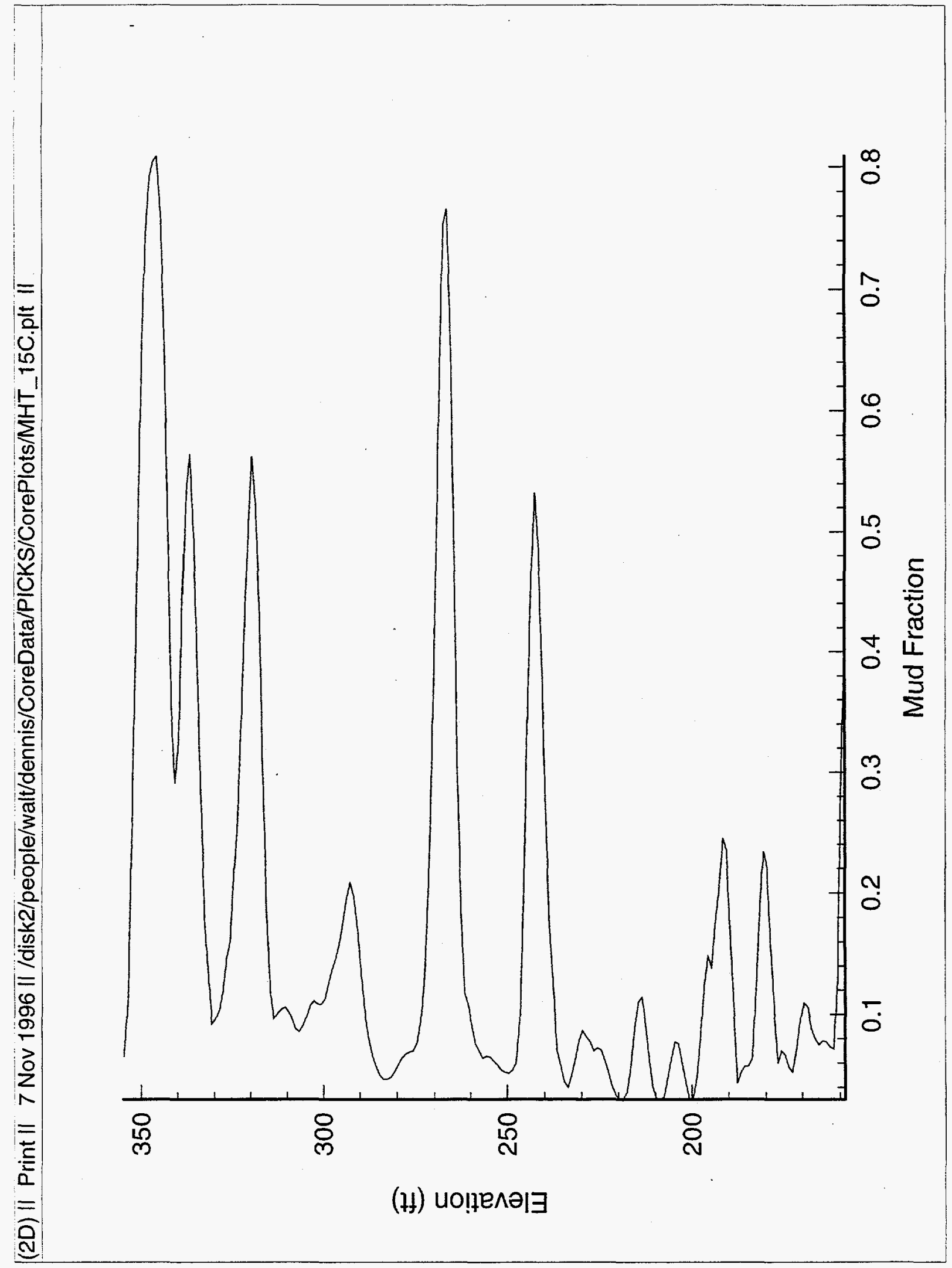




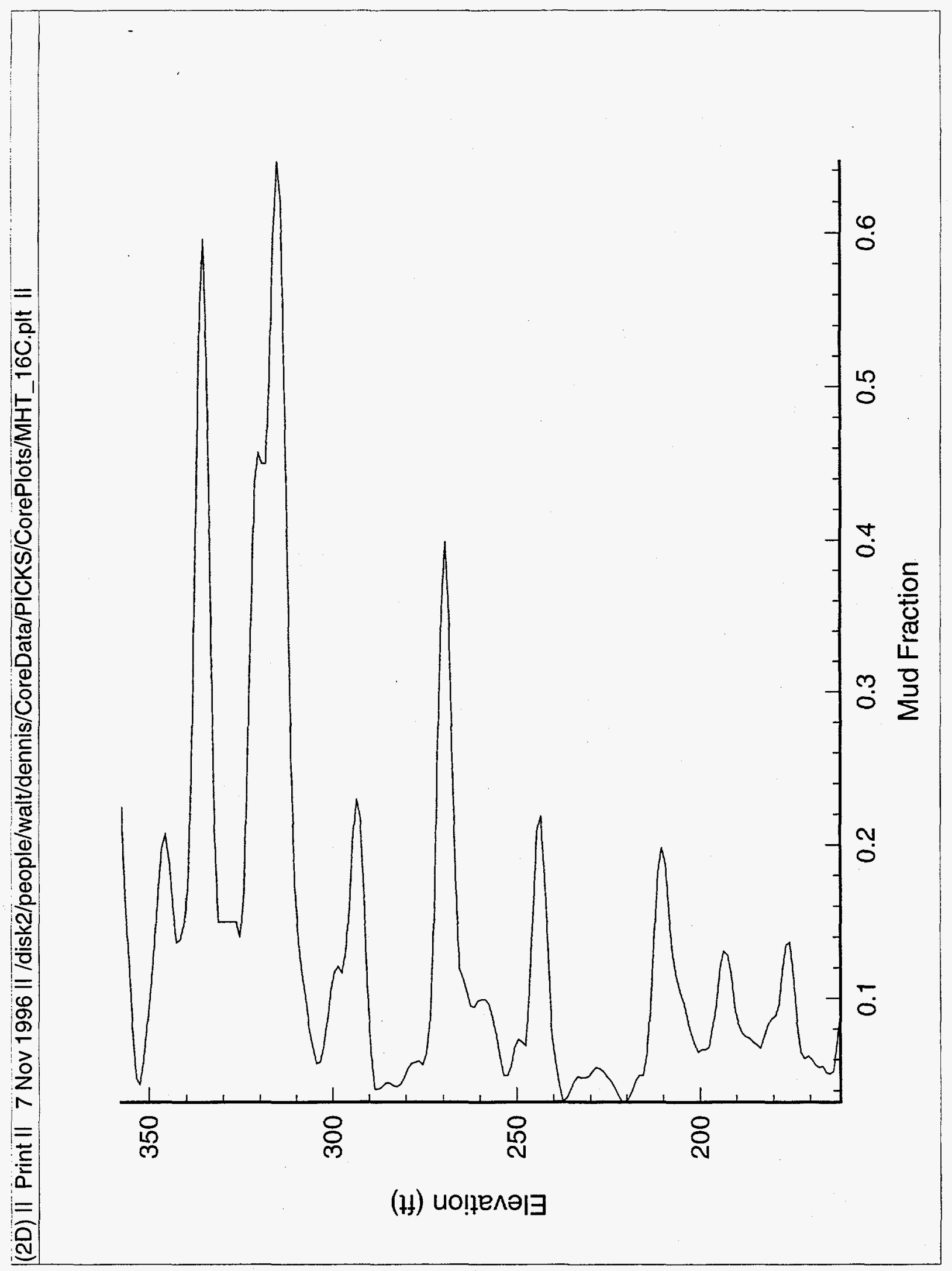




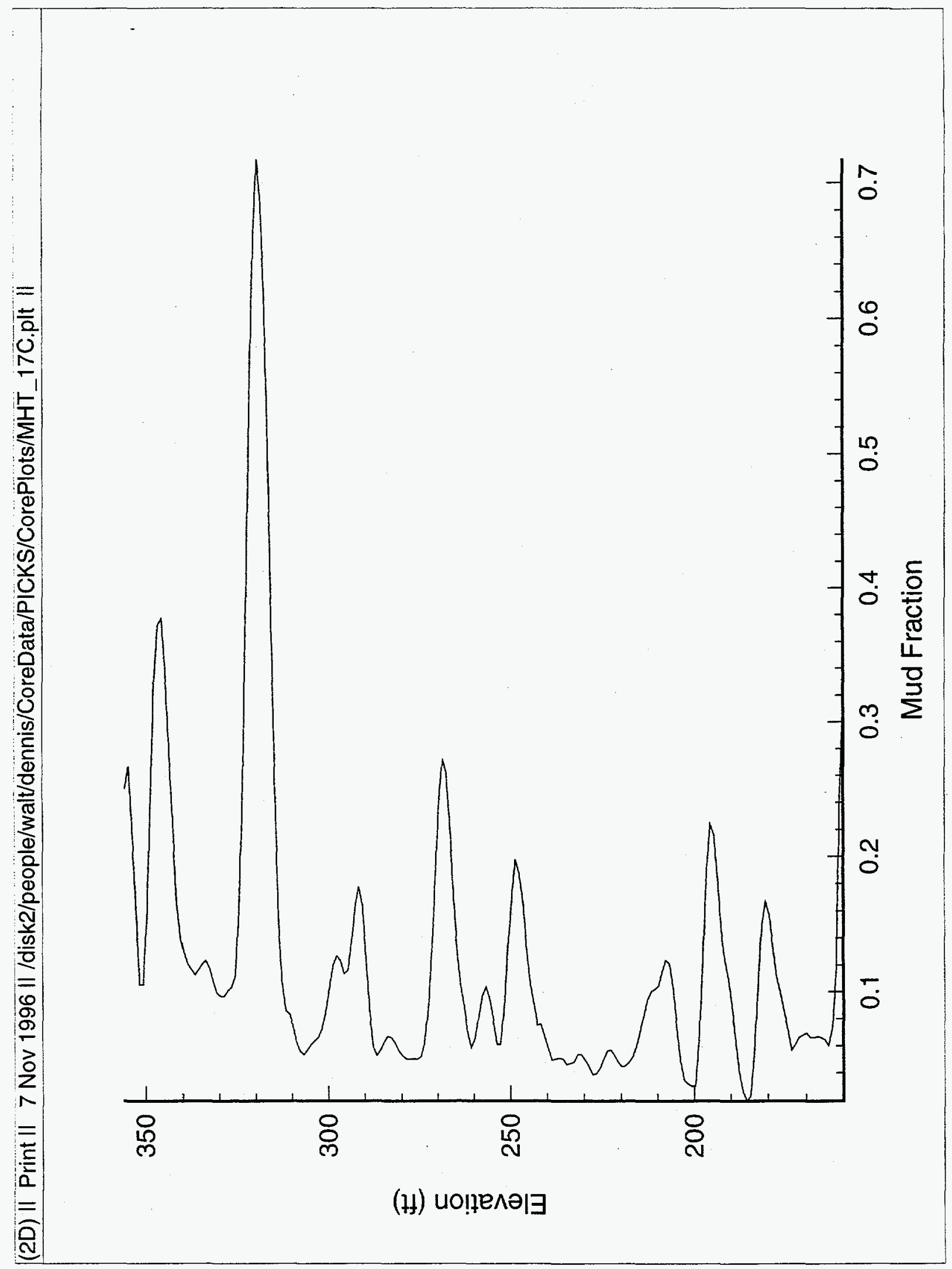




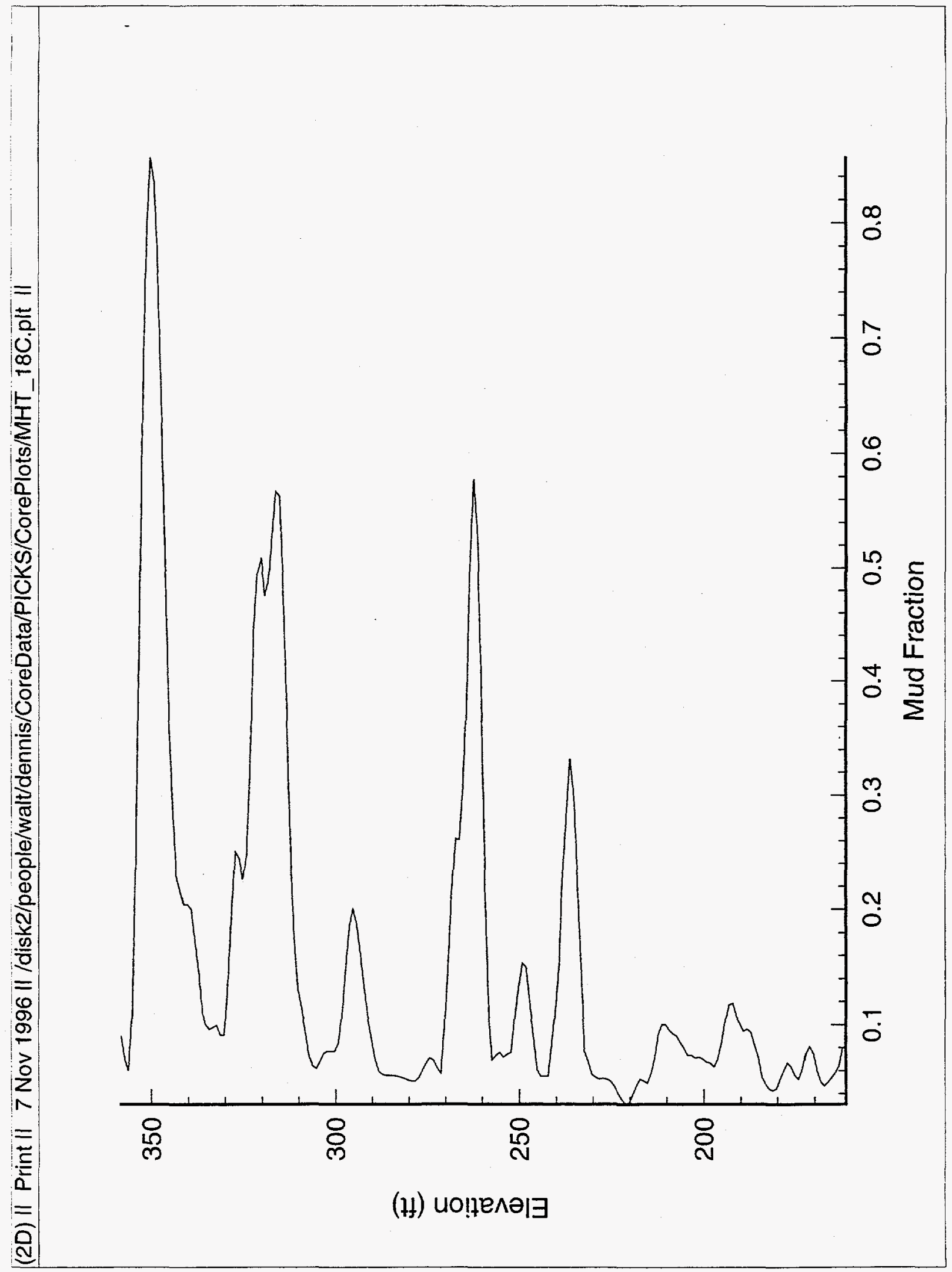




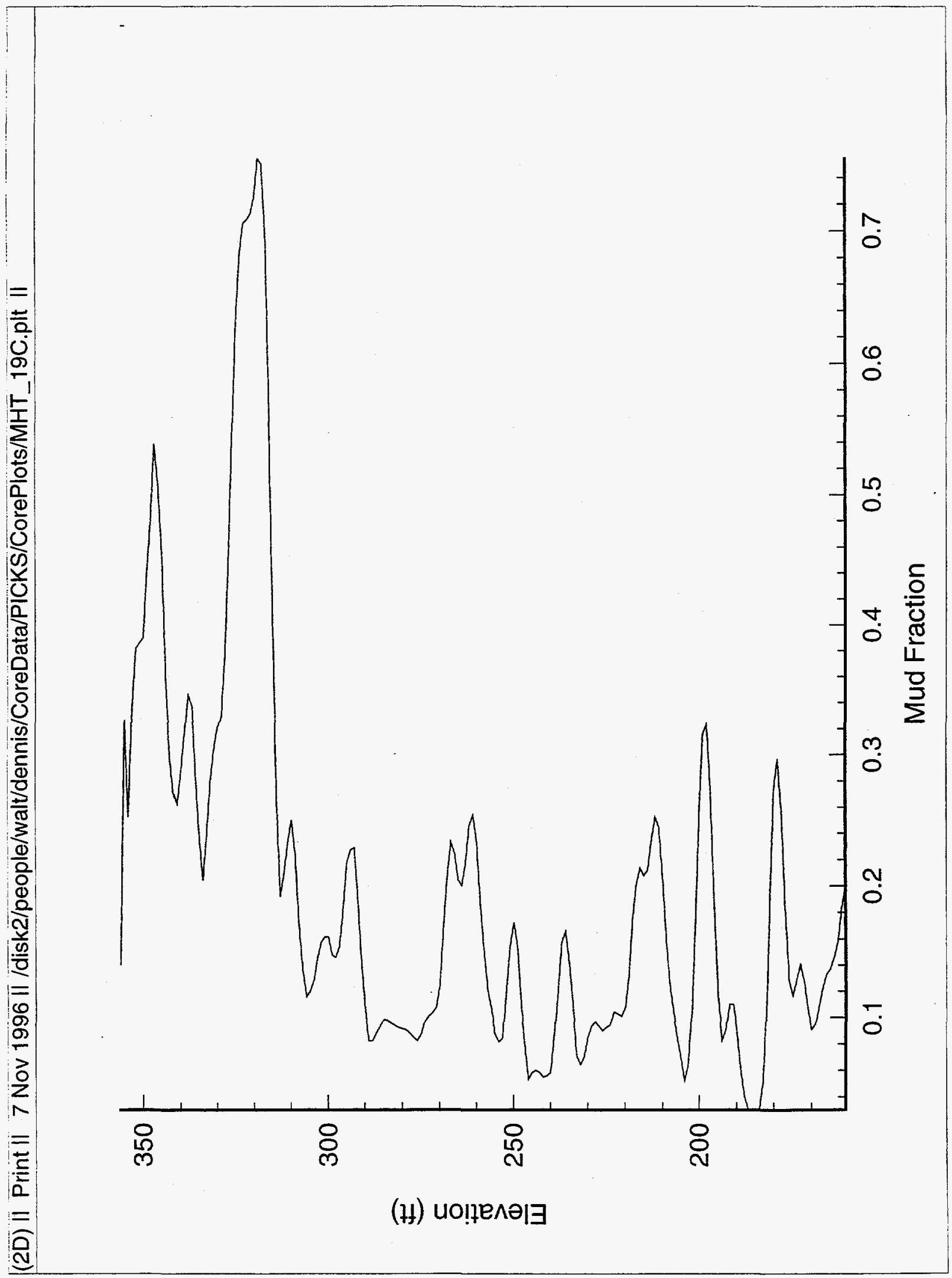




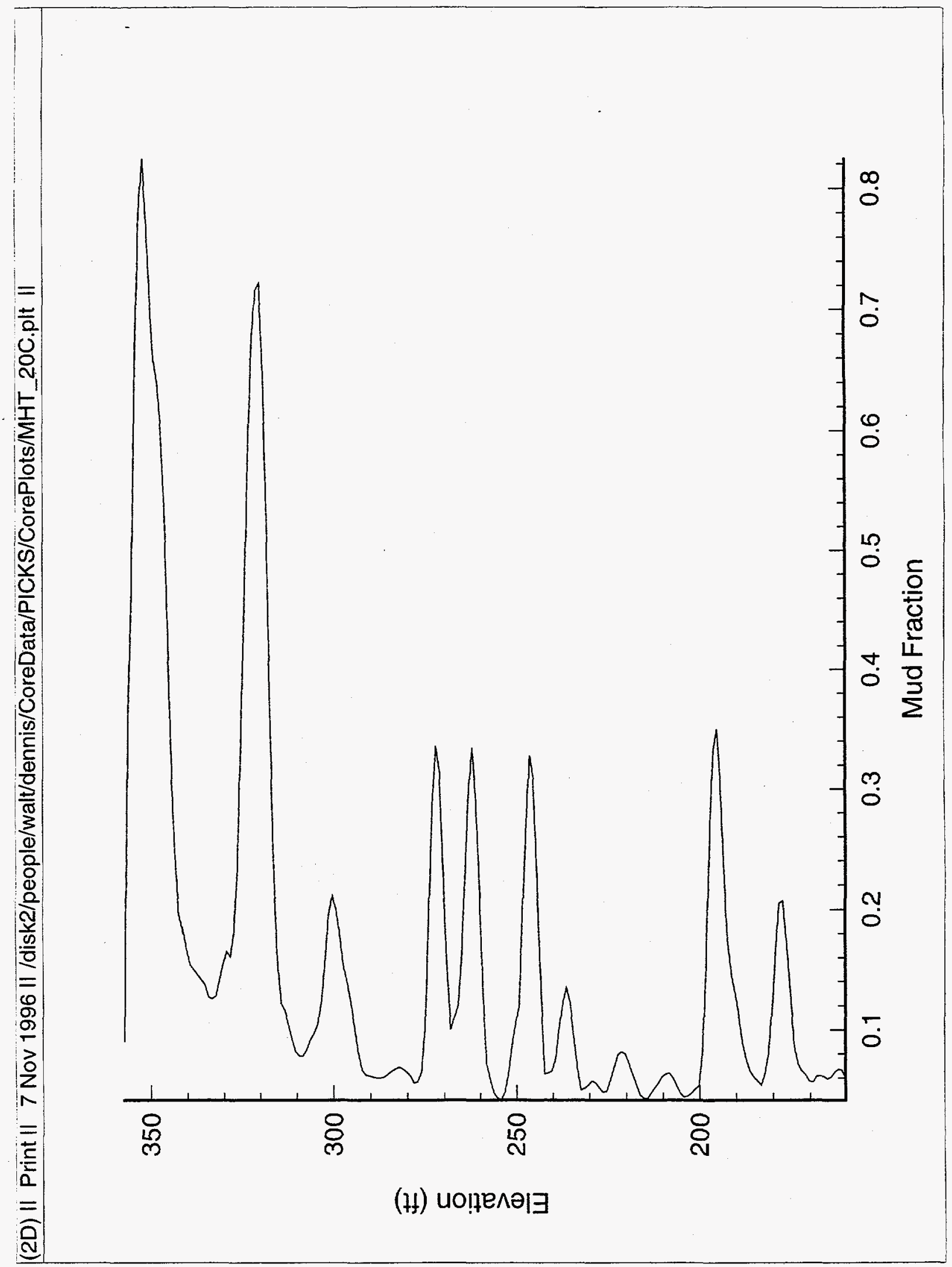


(2D) II Print II 7 Nov 1996 II/disk2/people/walt/dennis/CoreData/PICKS/CorePlots/MHT__C.plt II

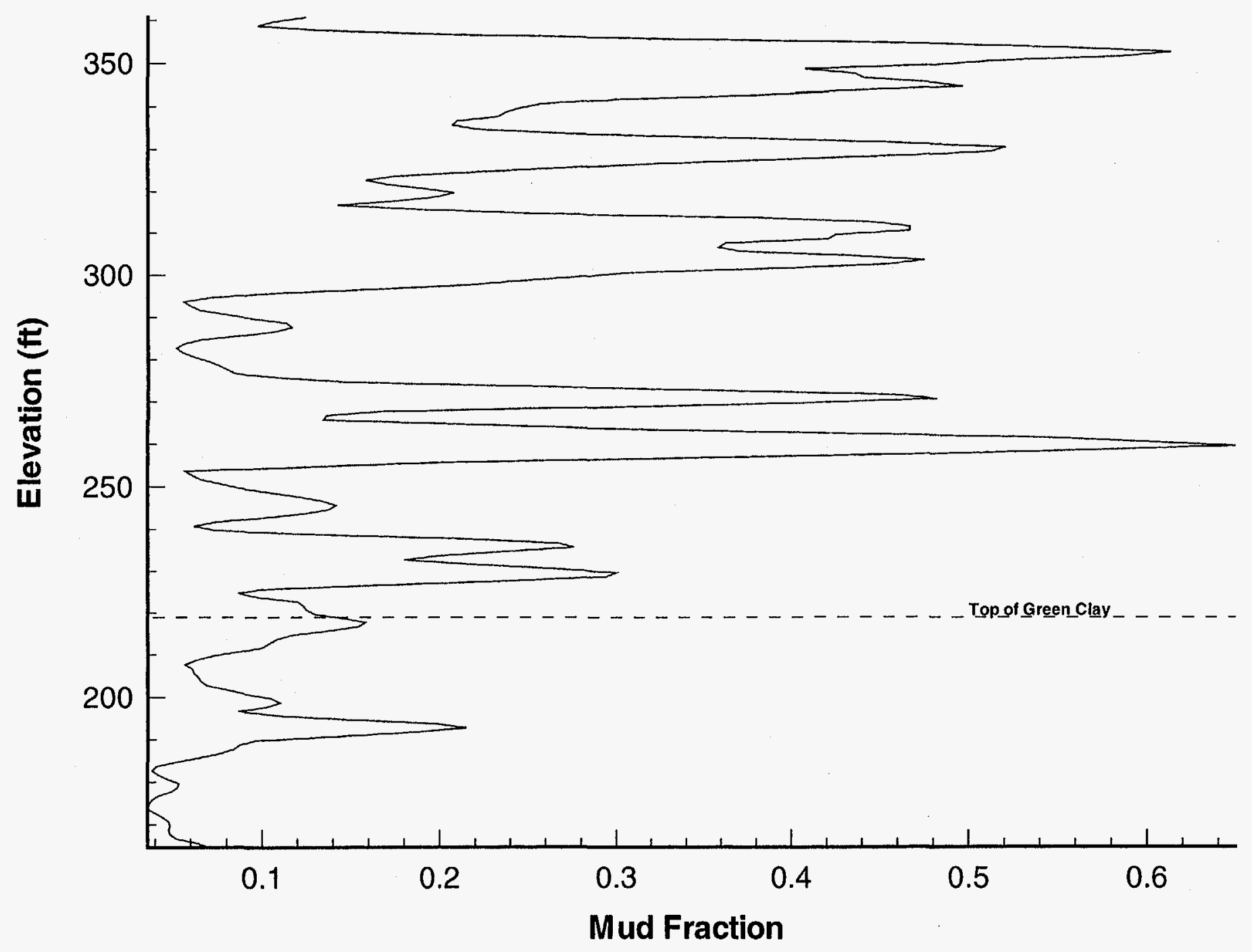




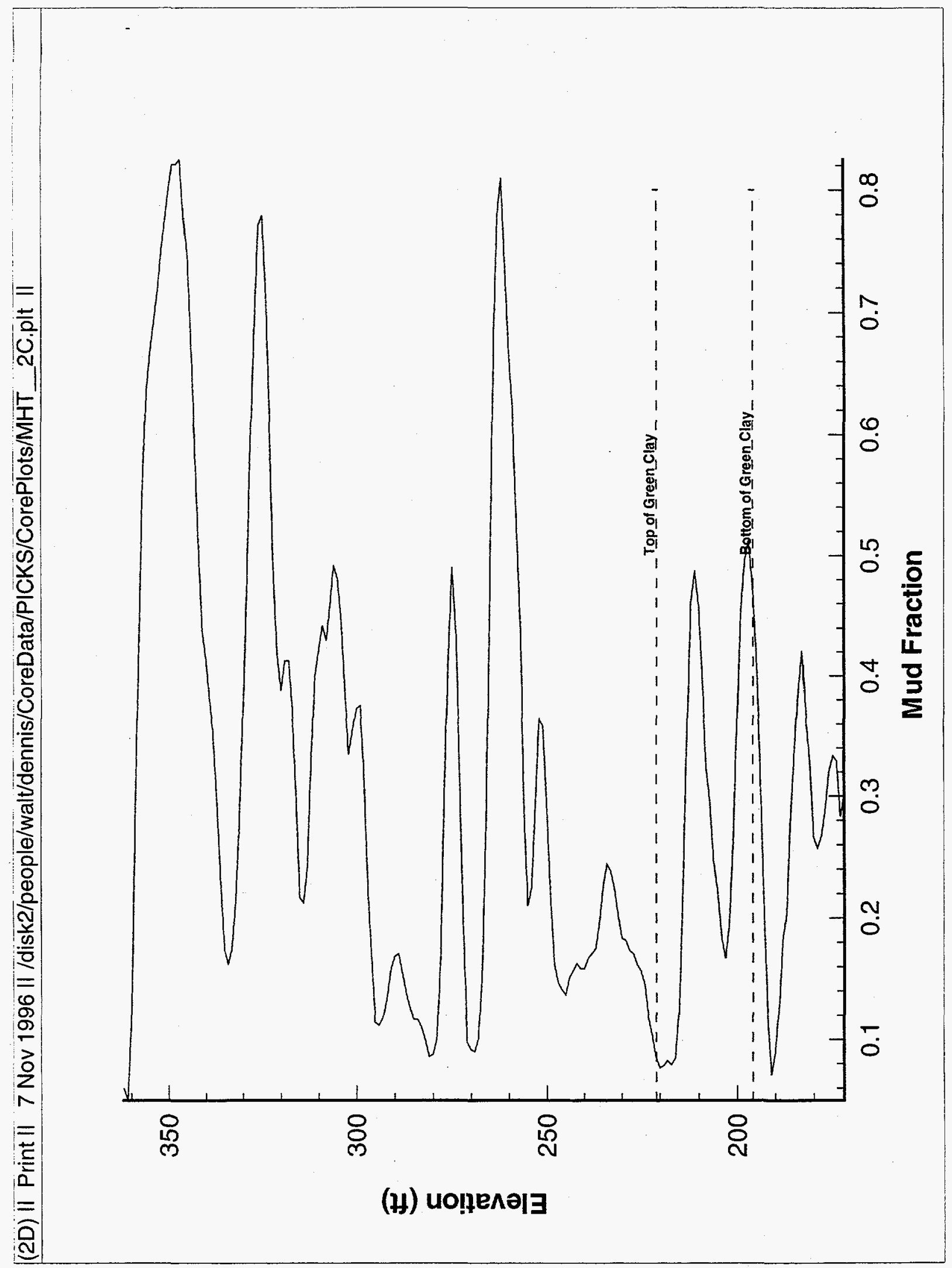




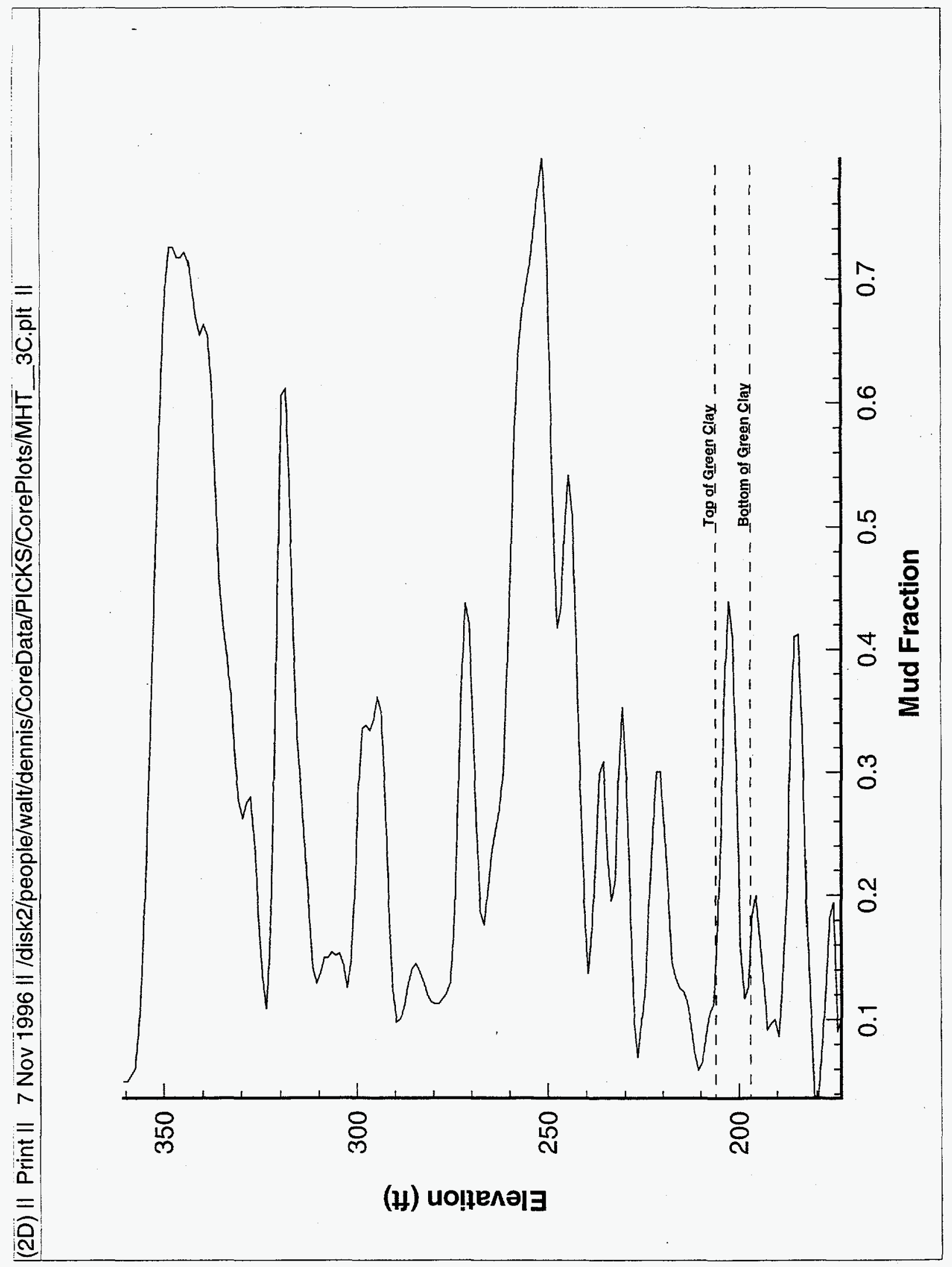




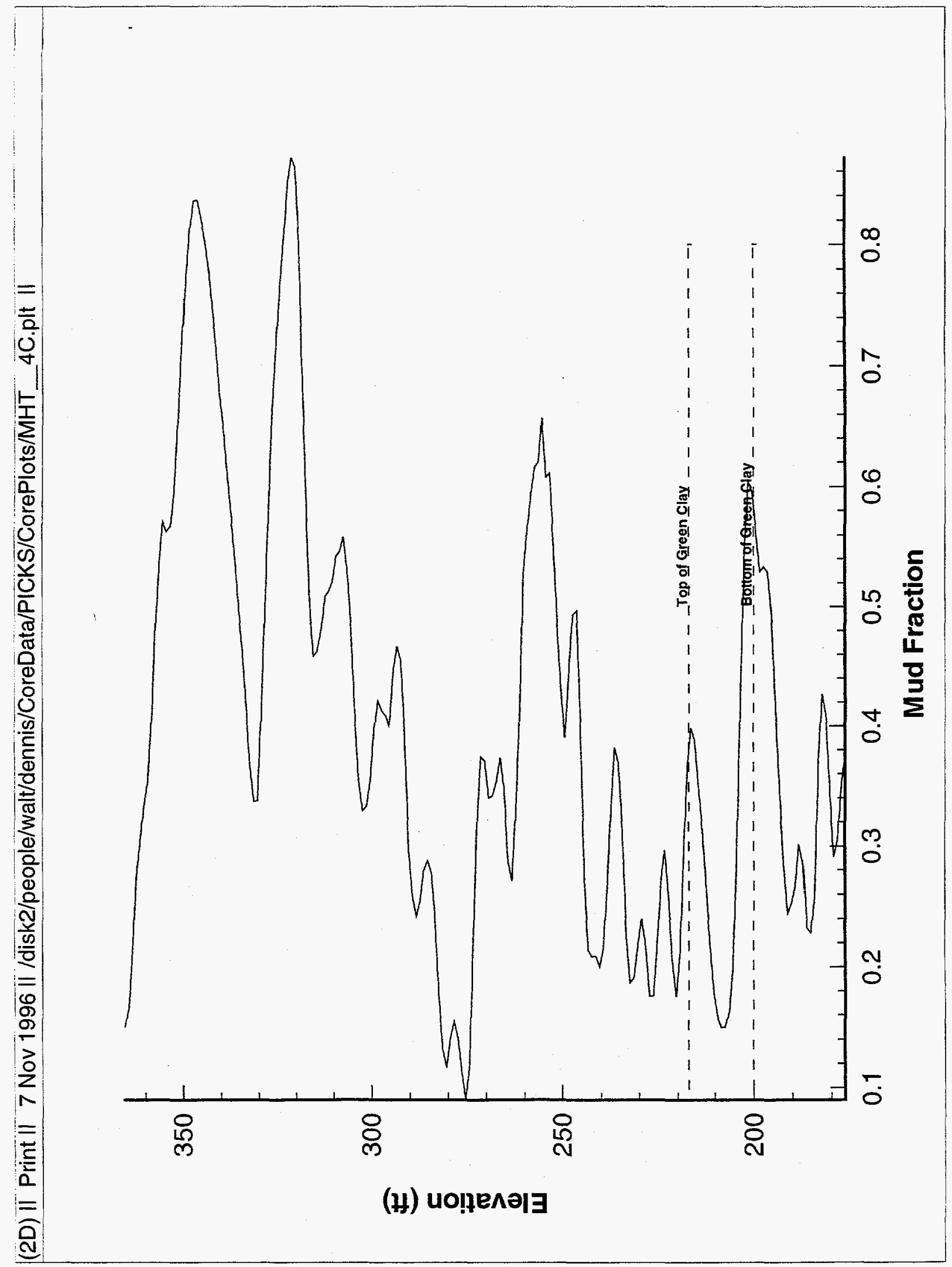


(2D) II Print II 7 Nov 1996 II /disk2/people/walt/dennis/CoreData/PICKS/CorePlots/MHT_5C.plt II

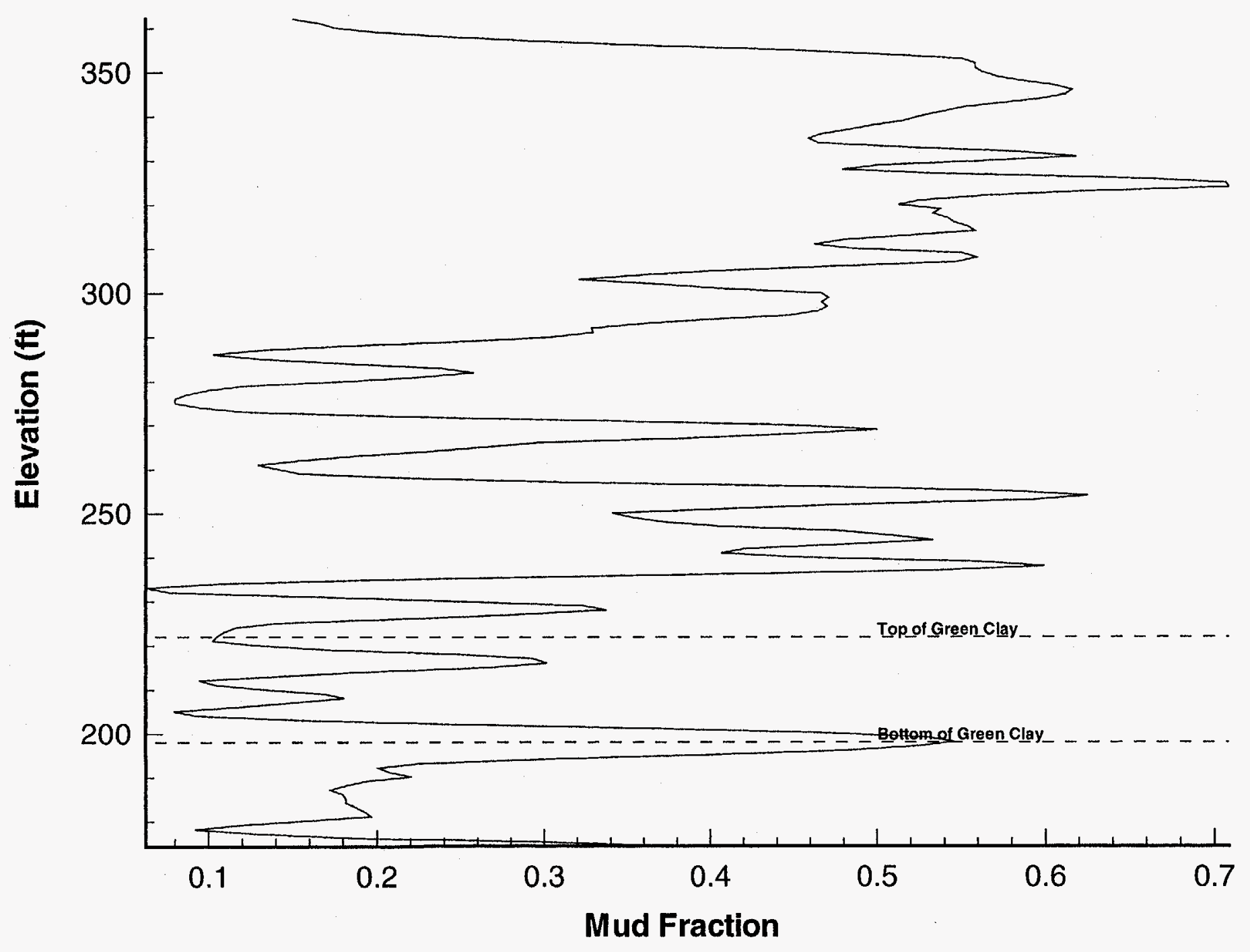




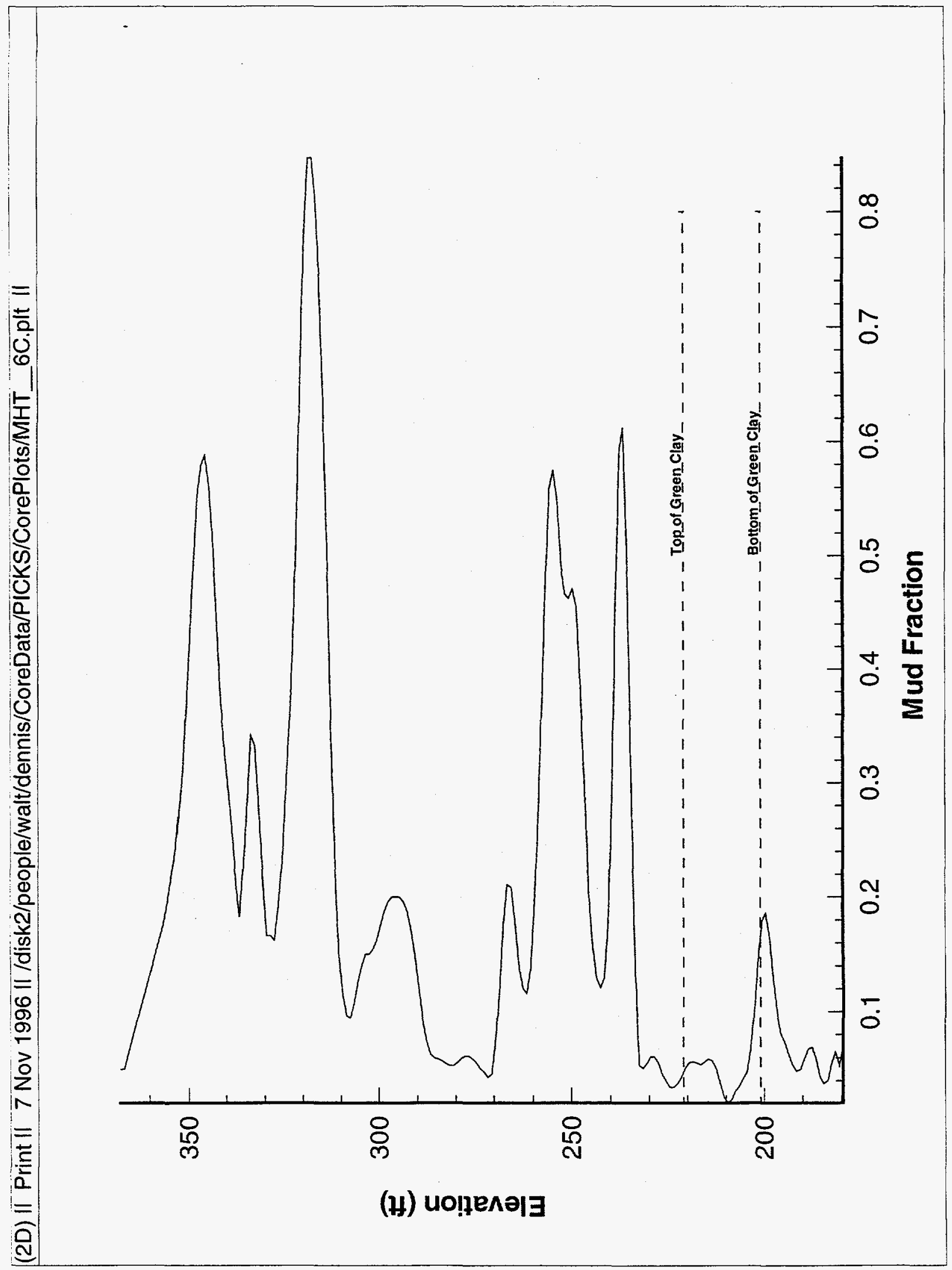




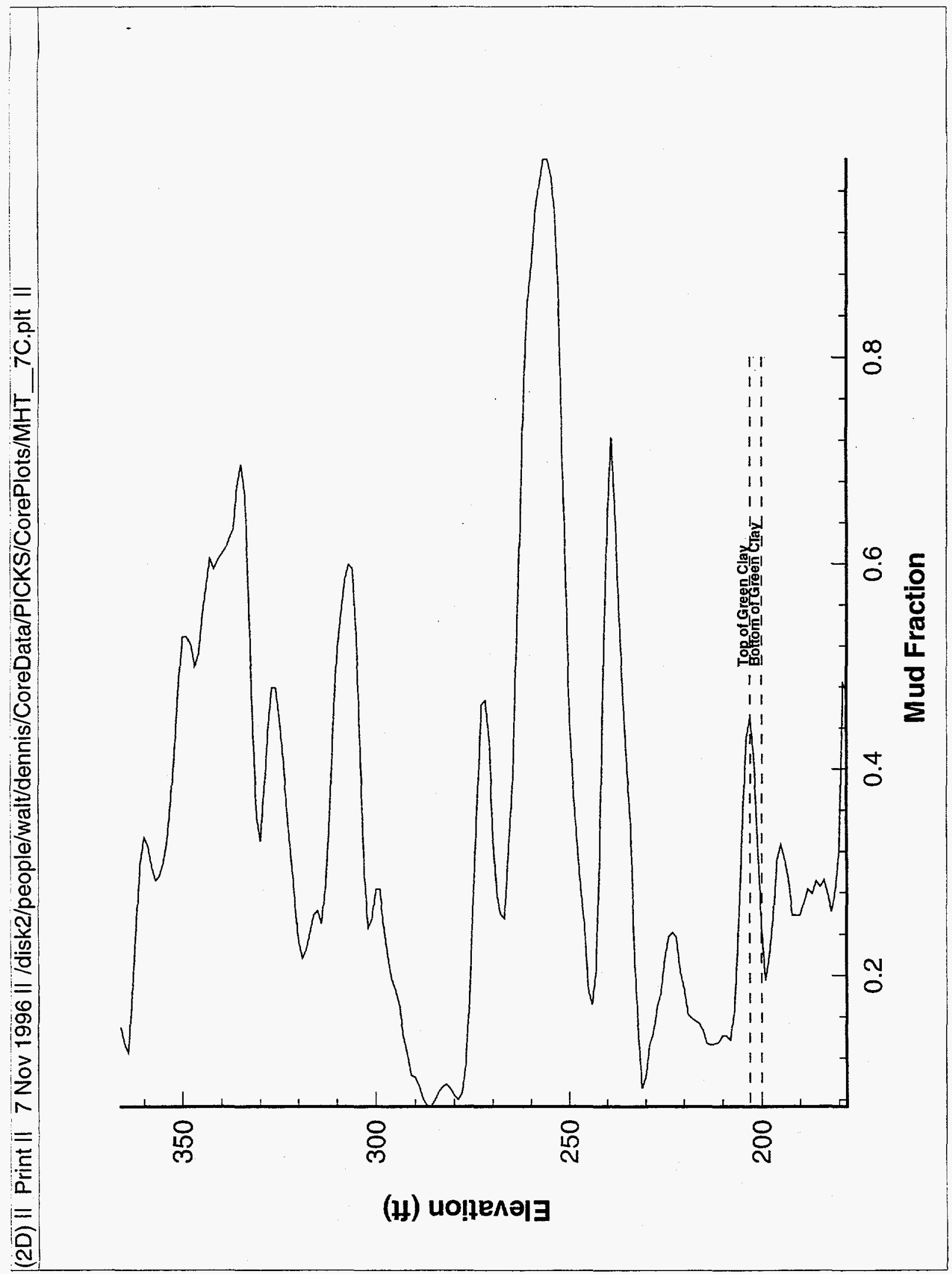




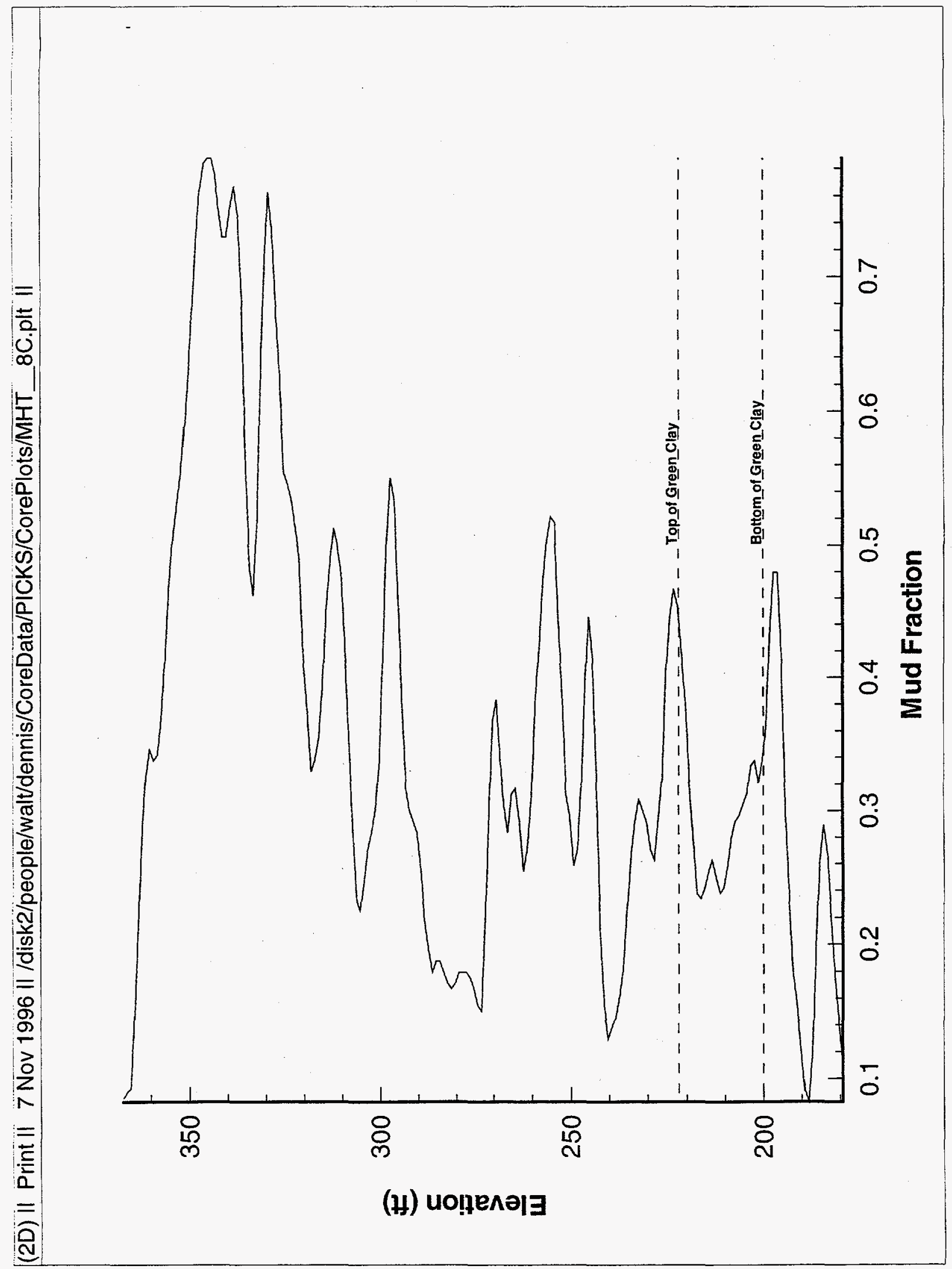




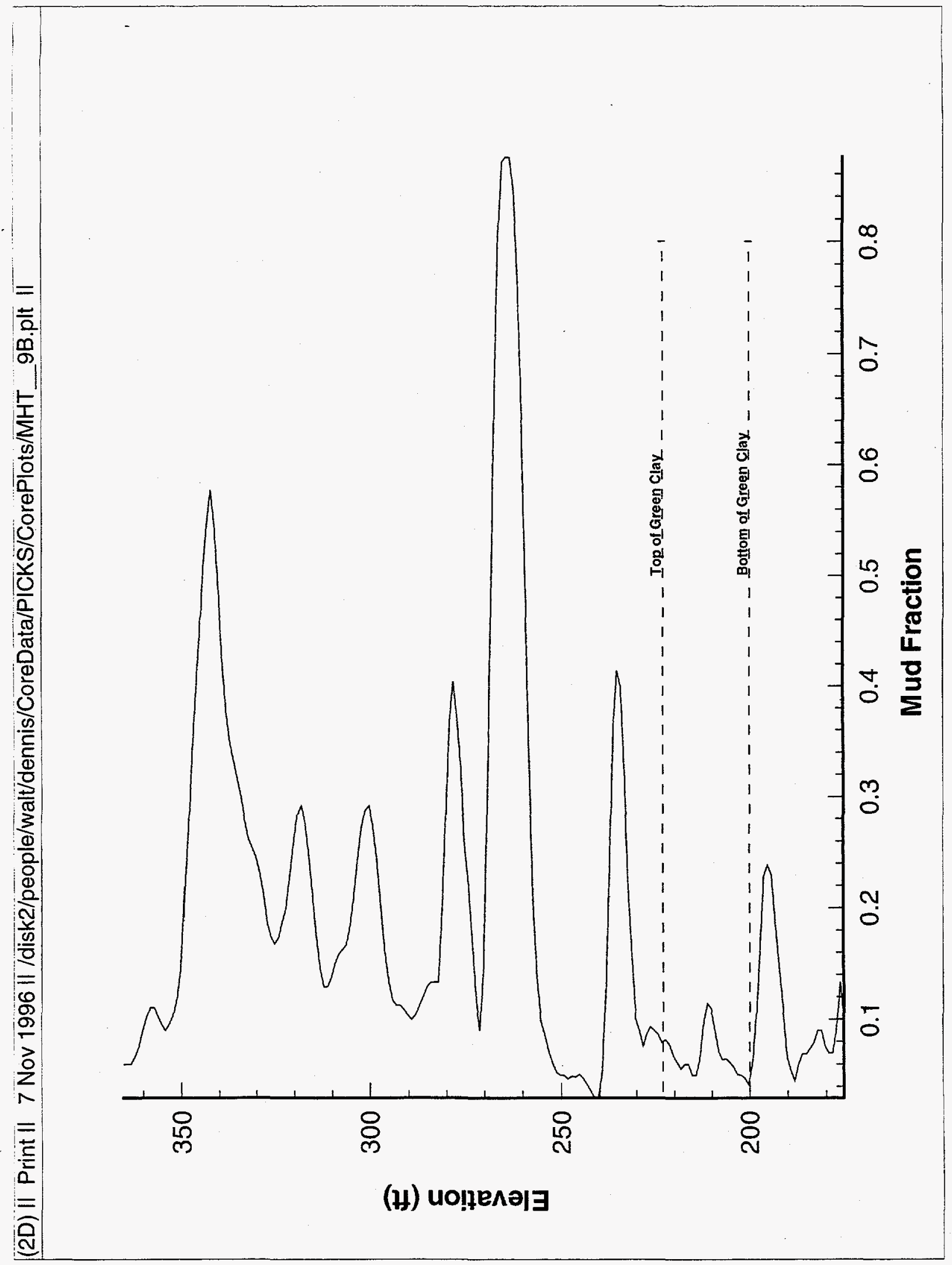




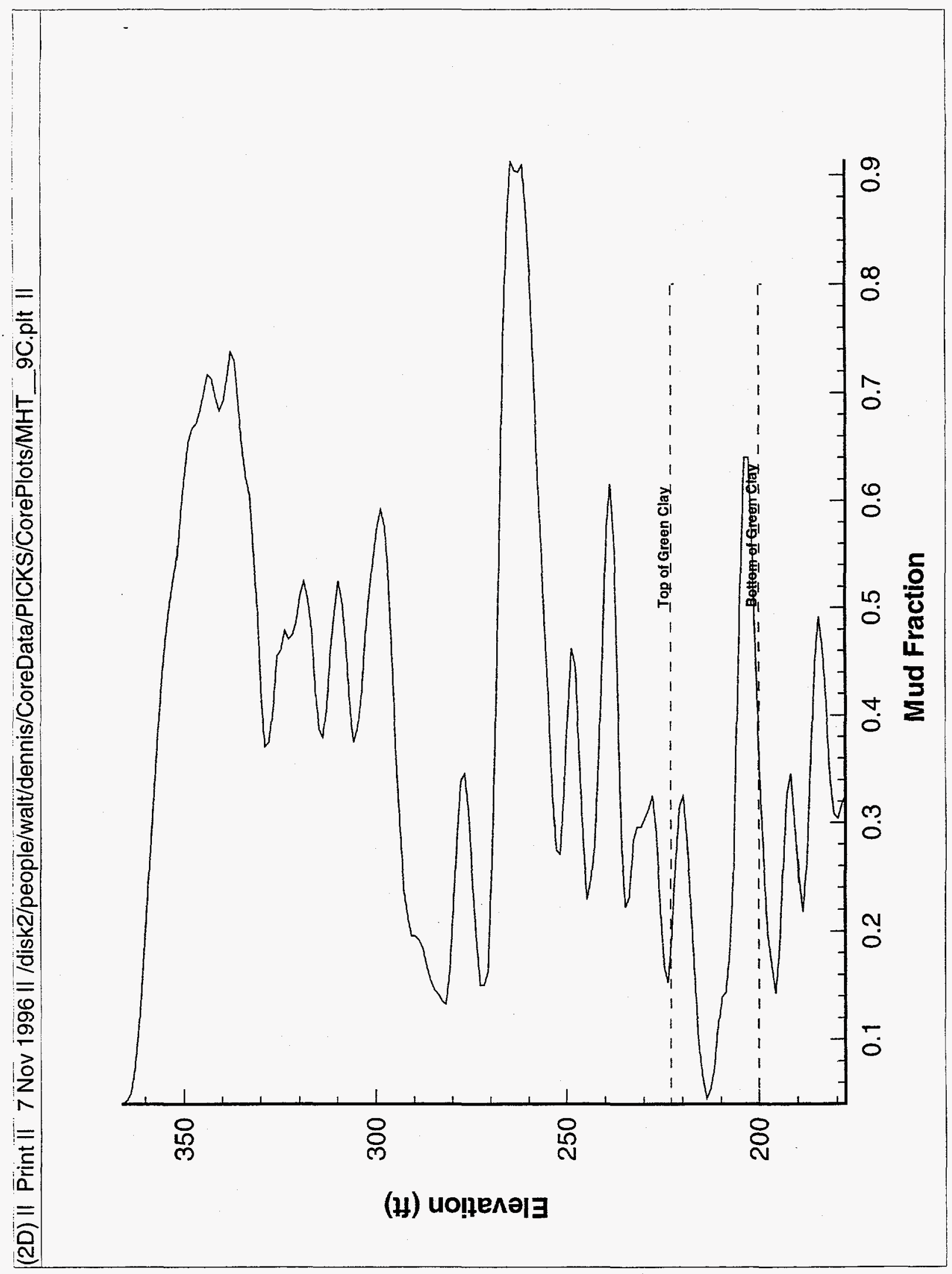


(2D) II Print II 7 Nov 1996 II /disk2/people/walt/dennis/CoreData/PICKS/CorePlots/MHV_4A.plt II

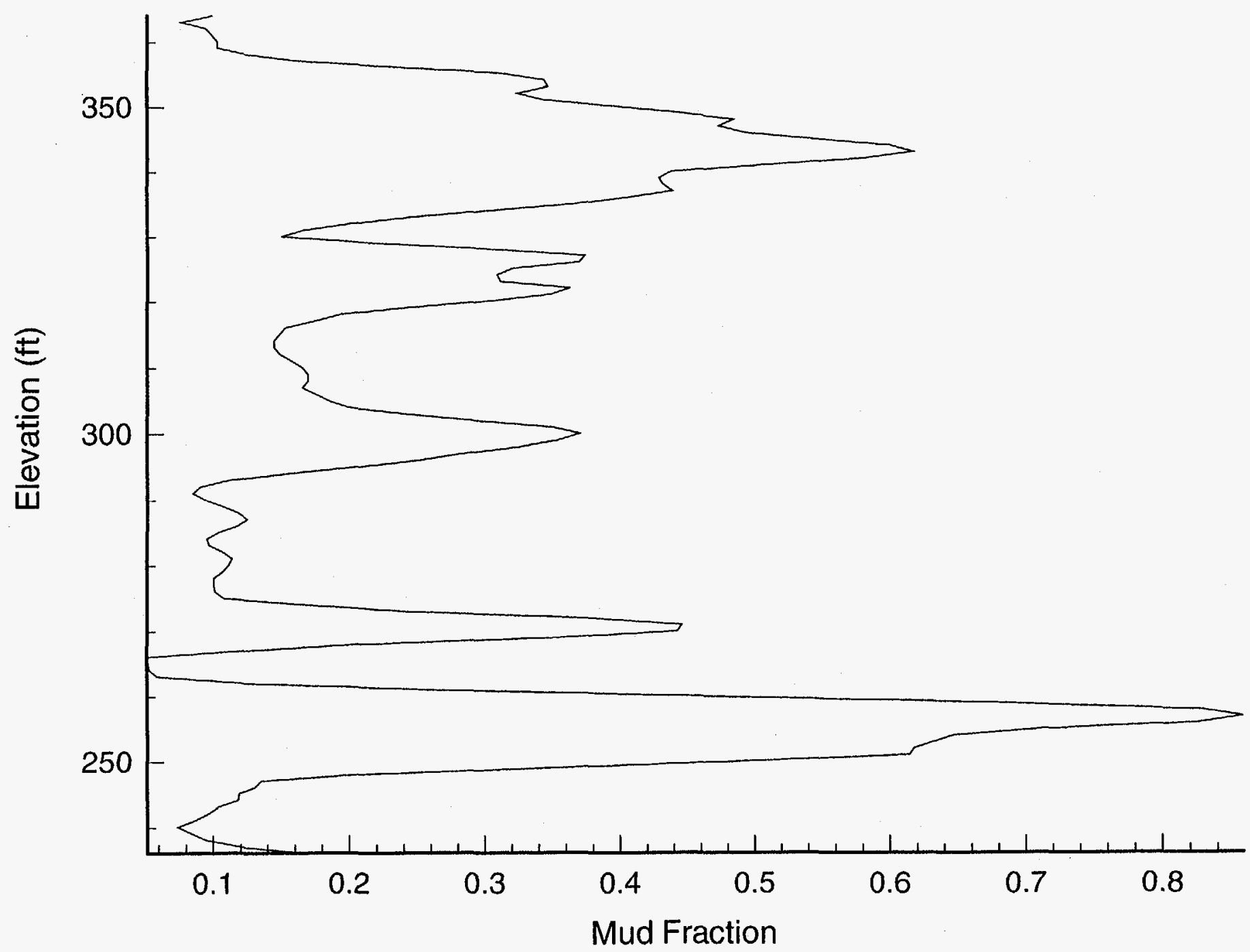




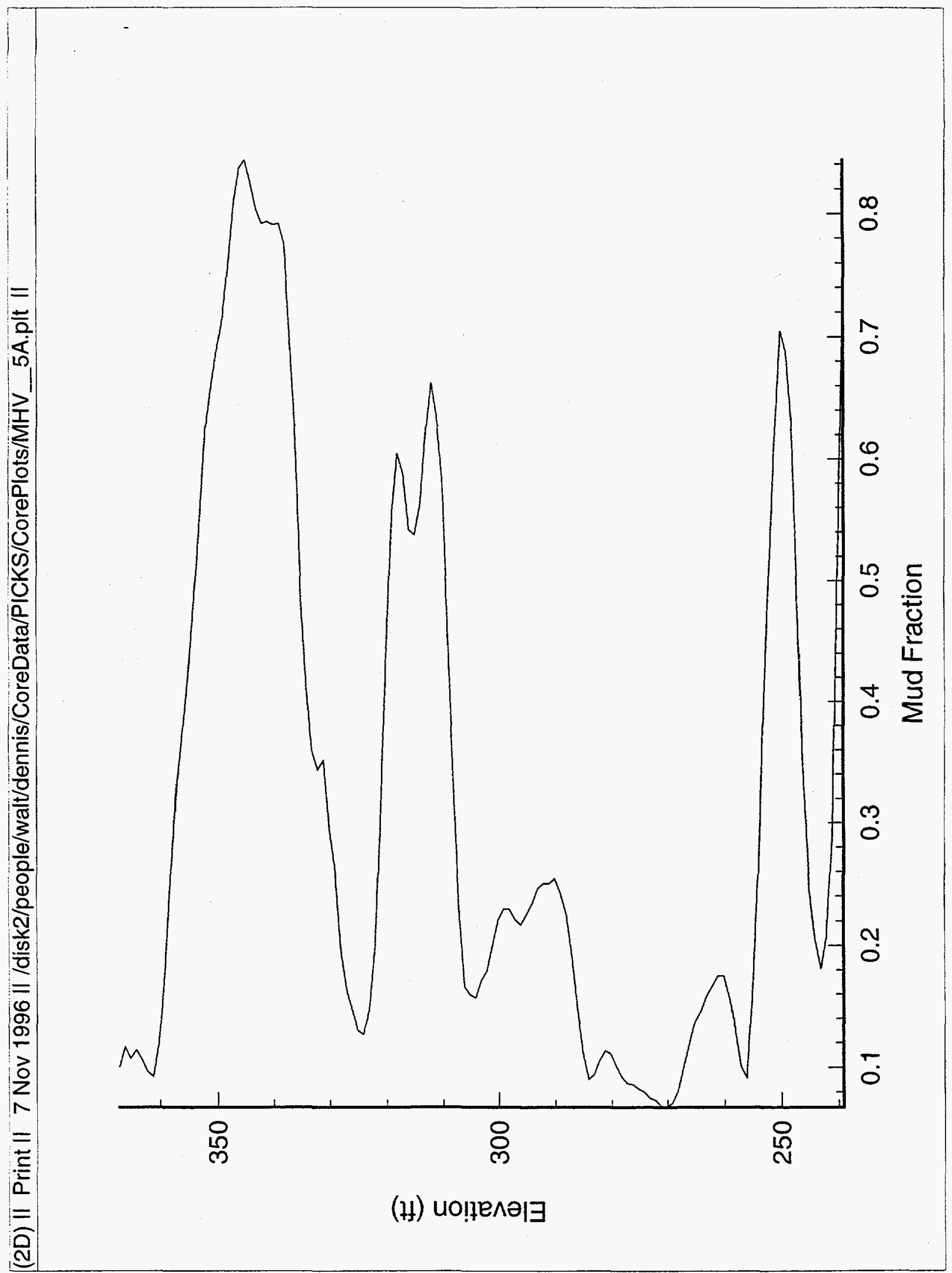




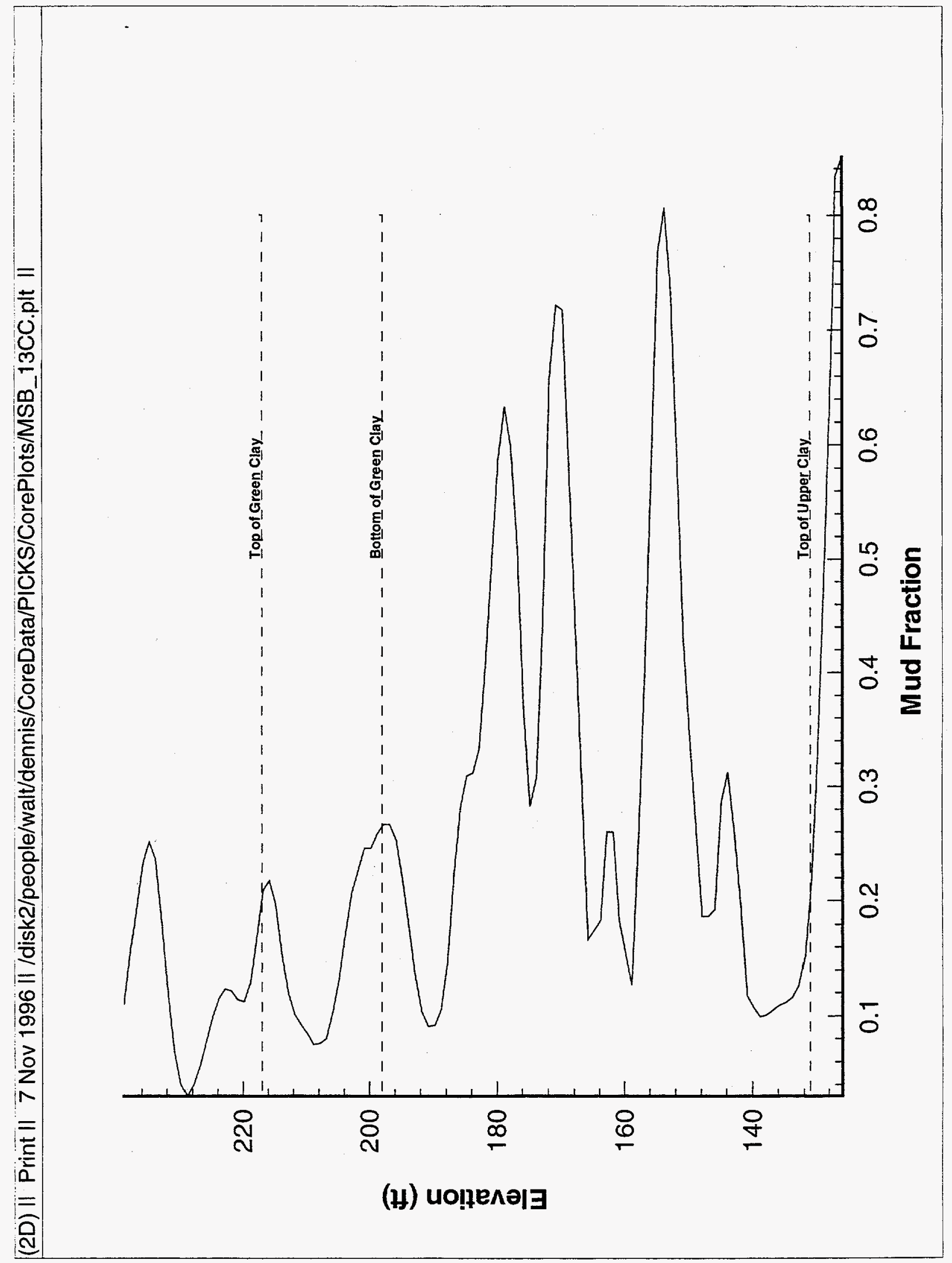




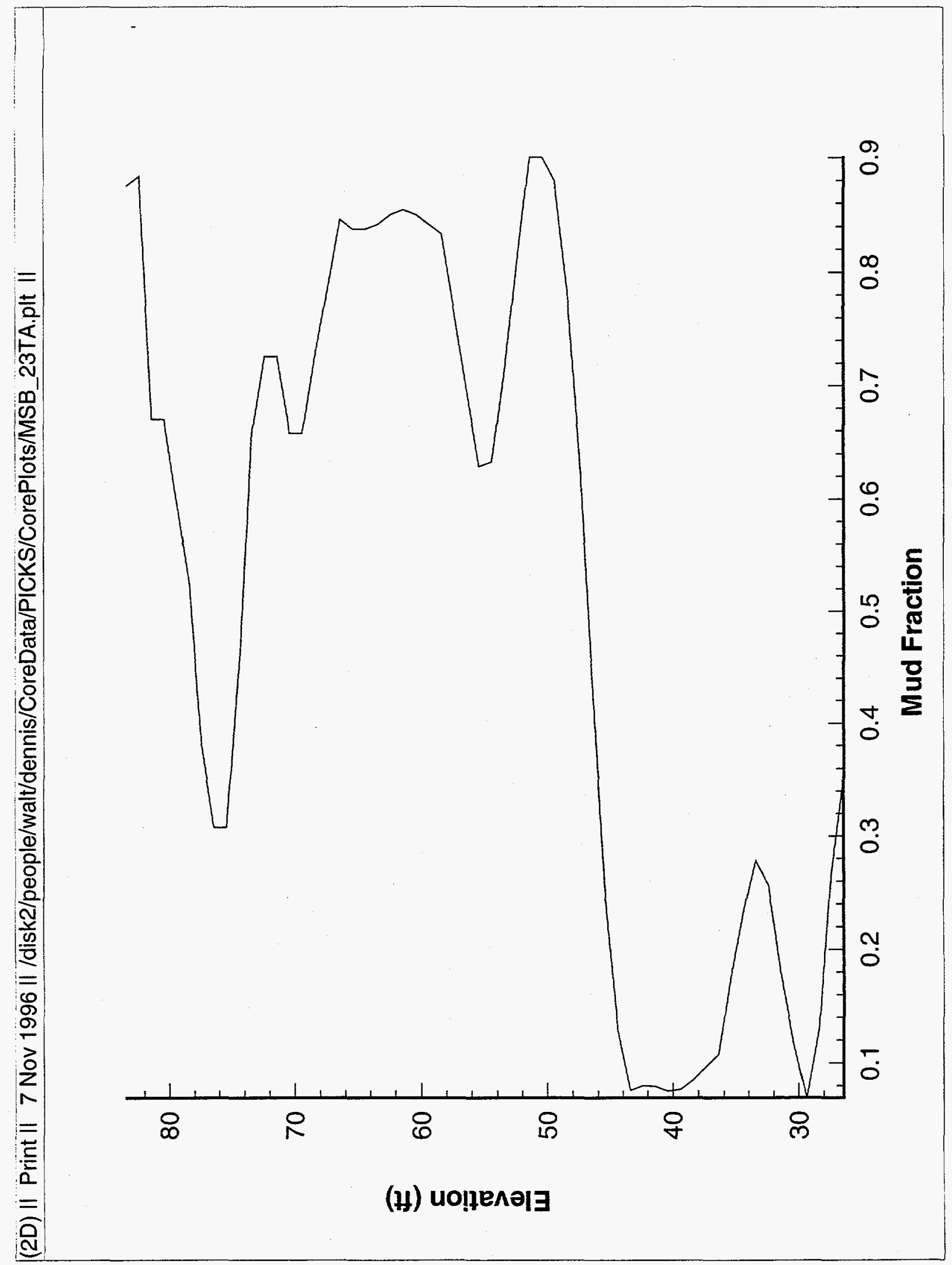




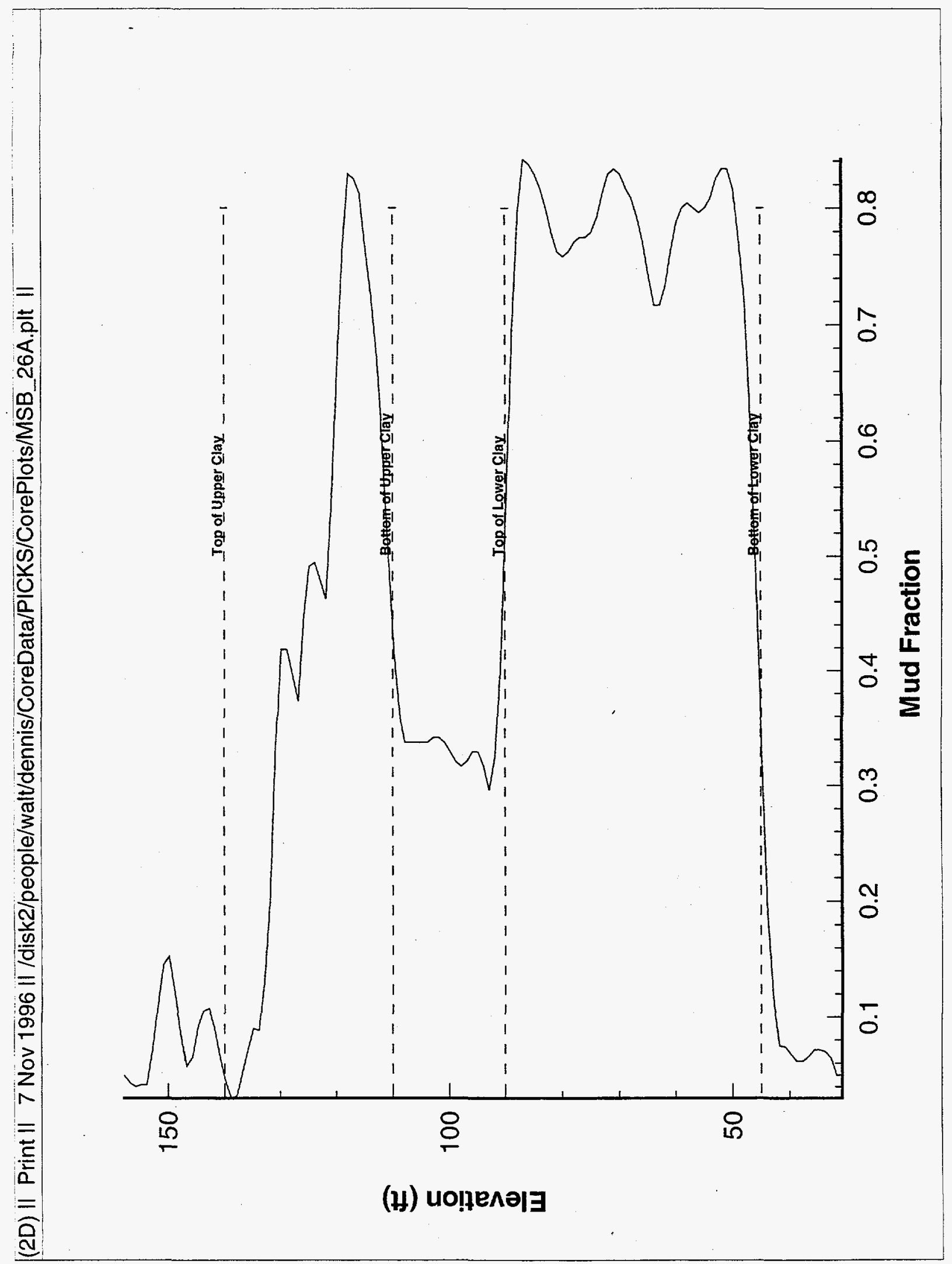




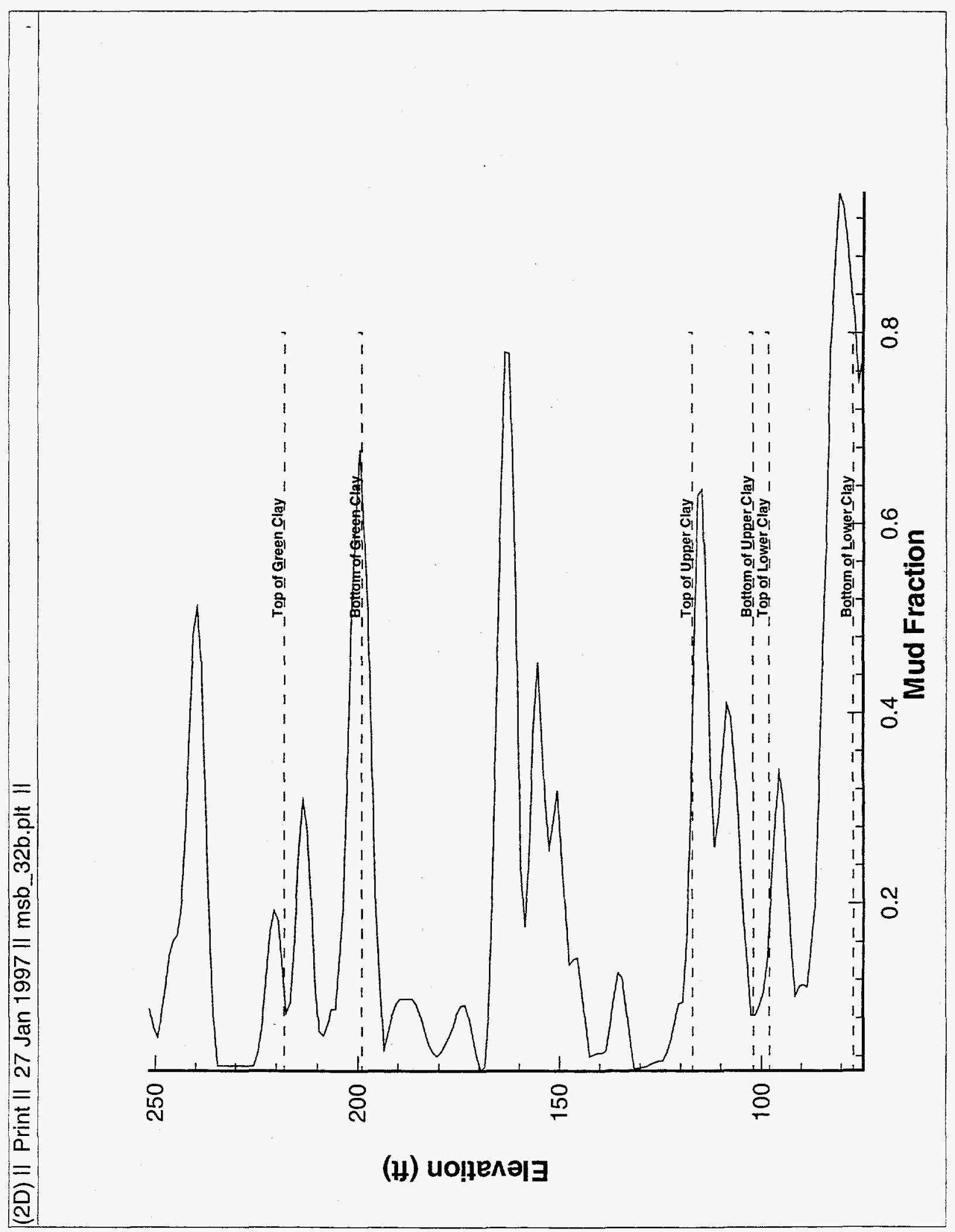




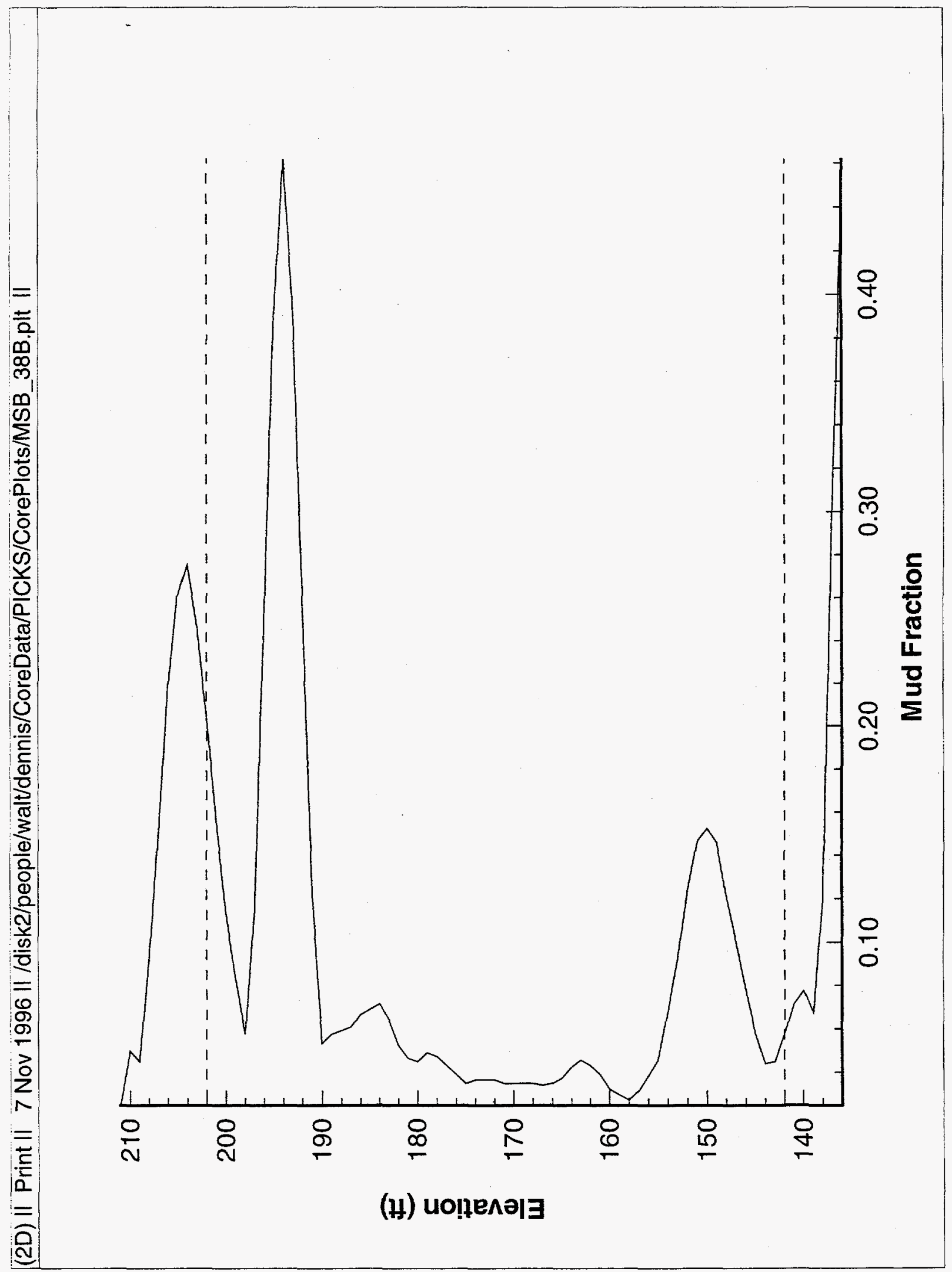




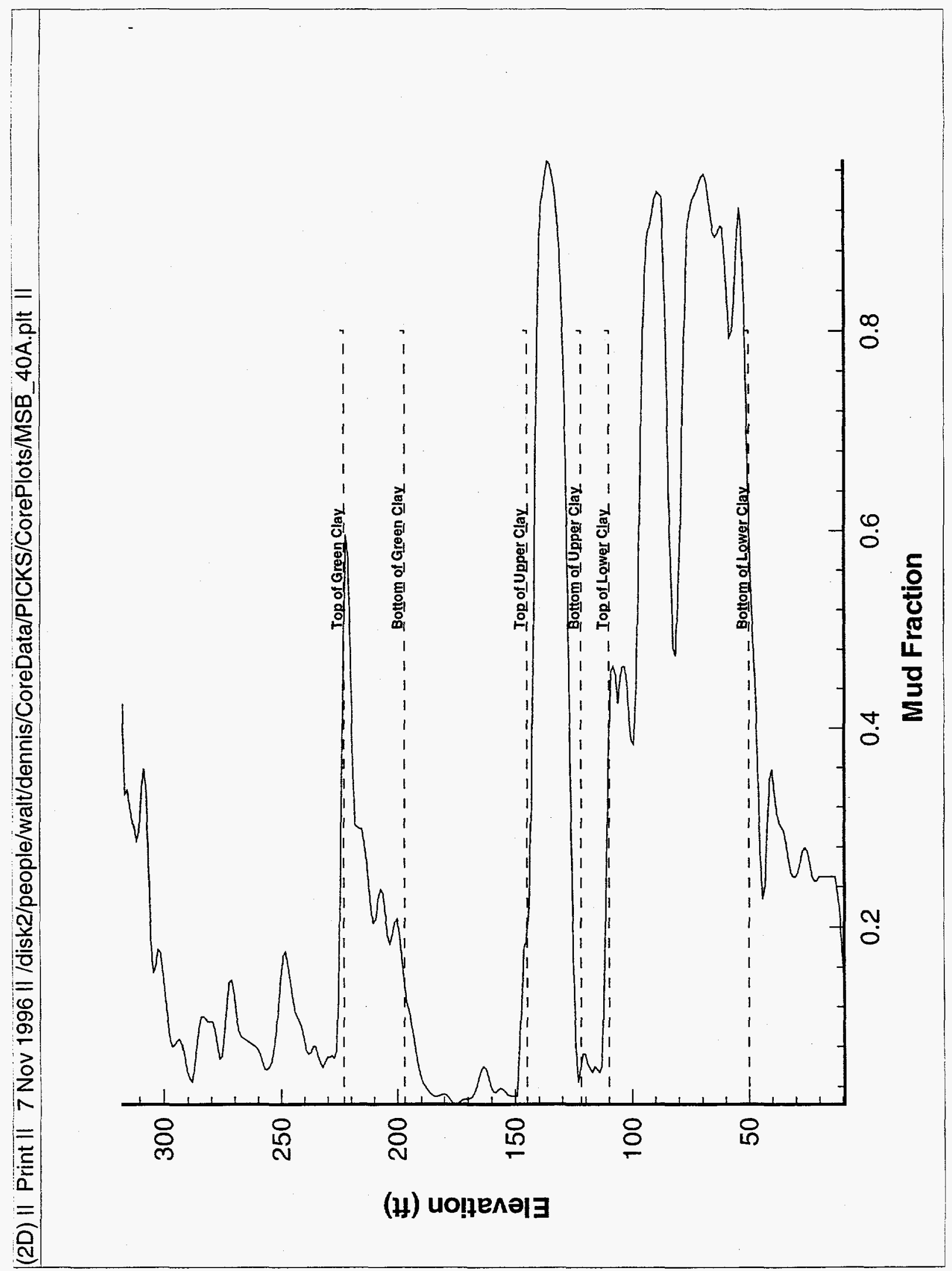




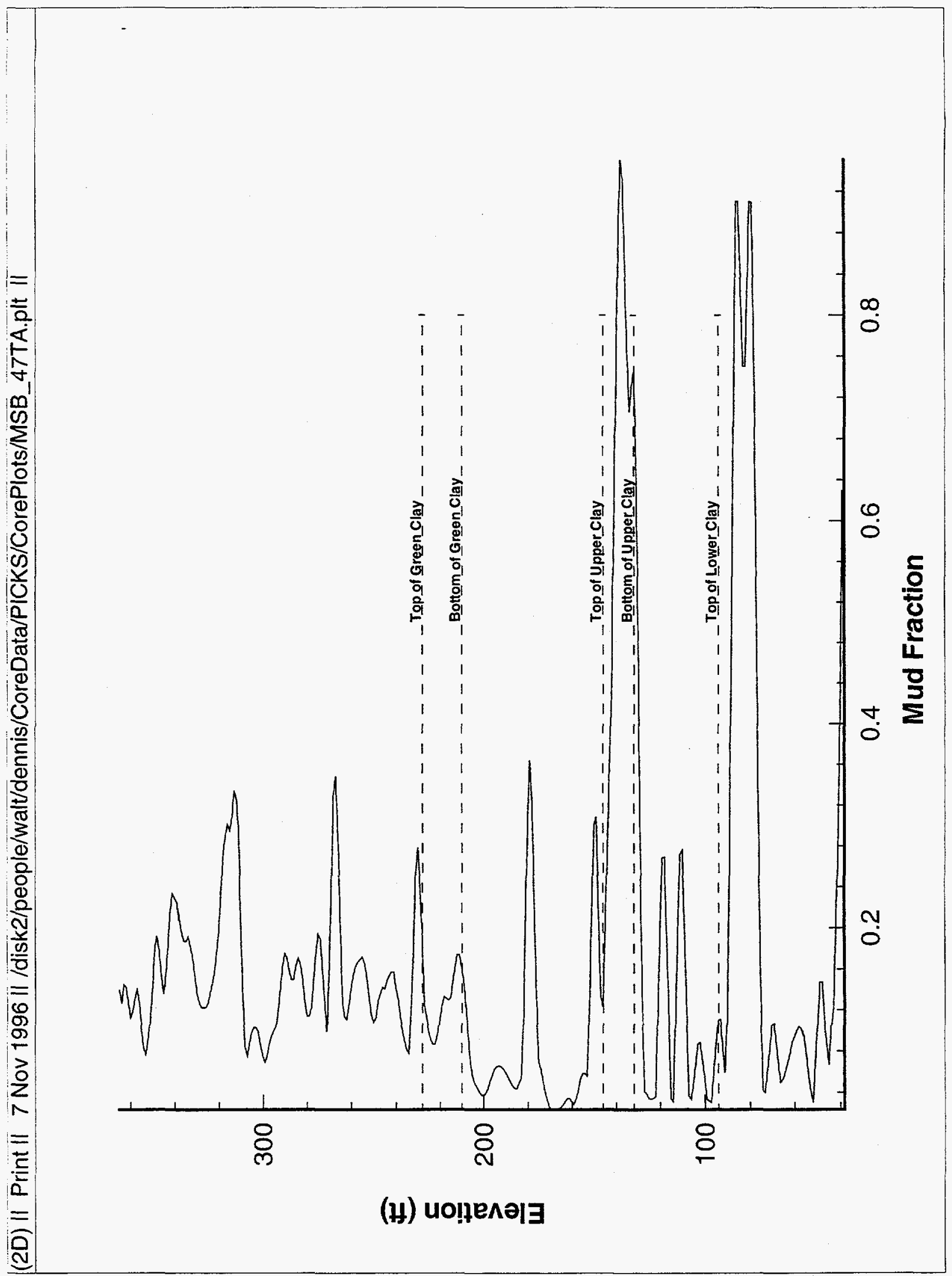




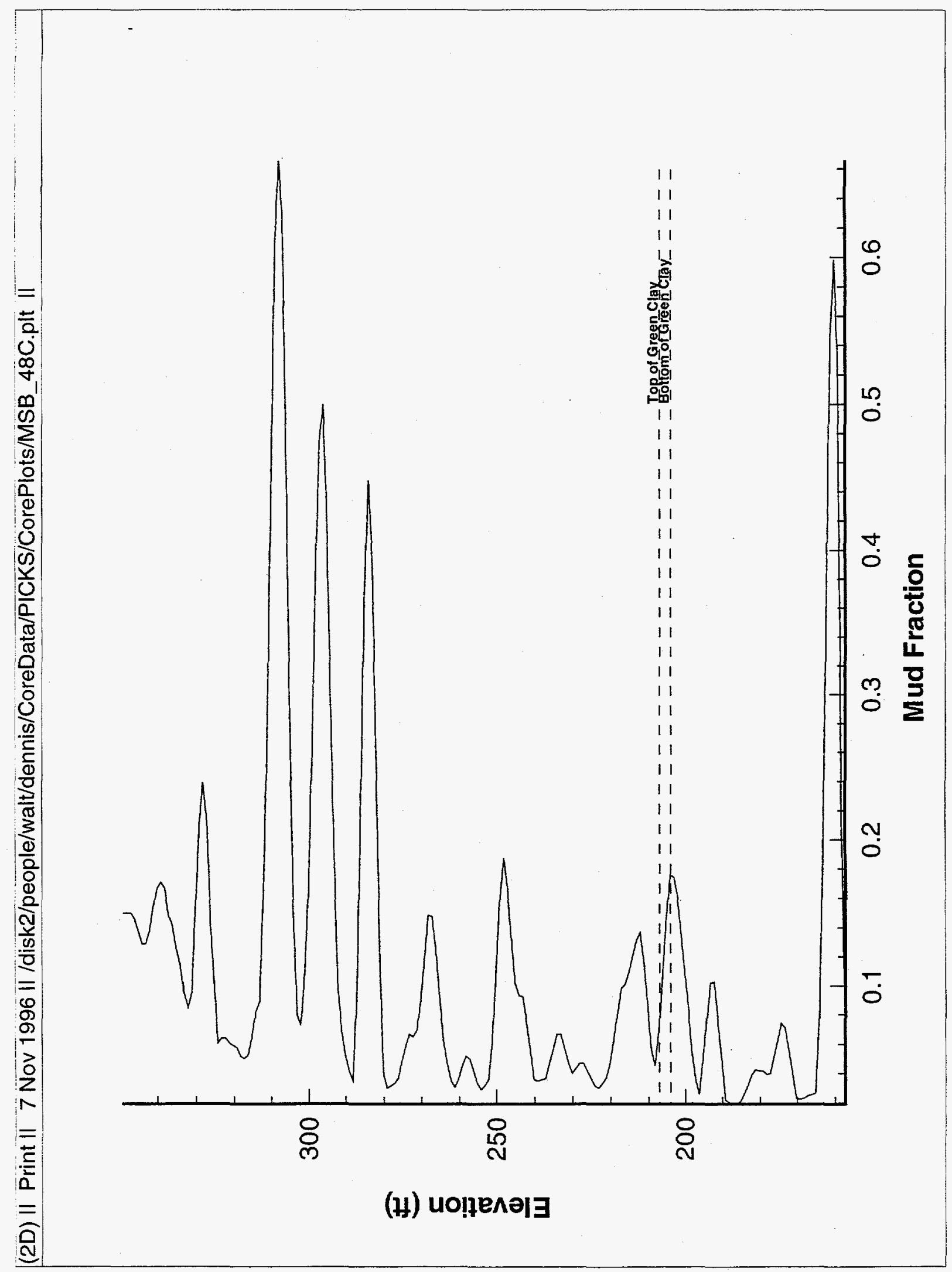




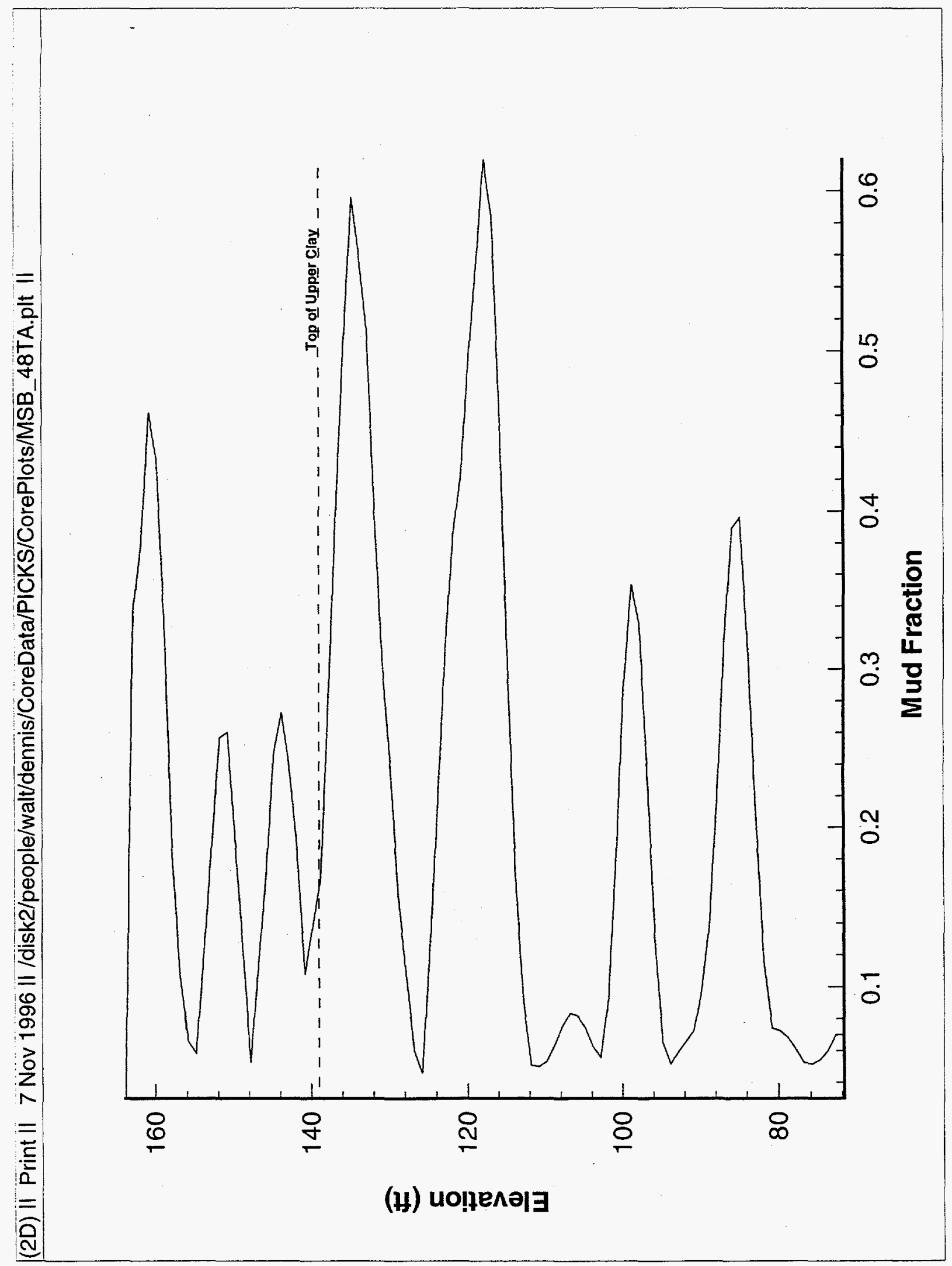




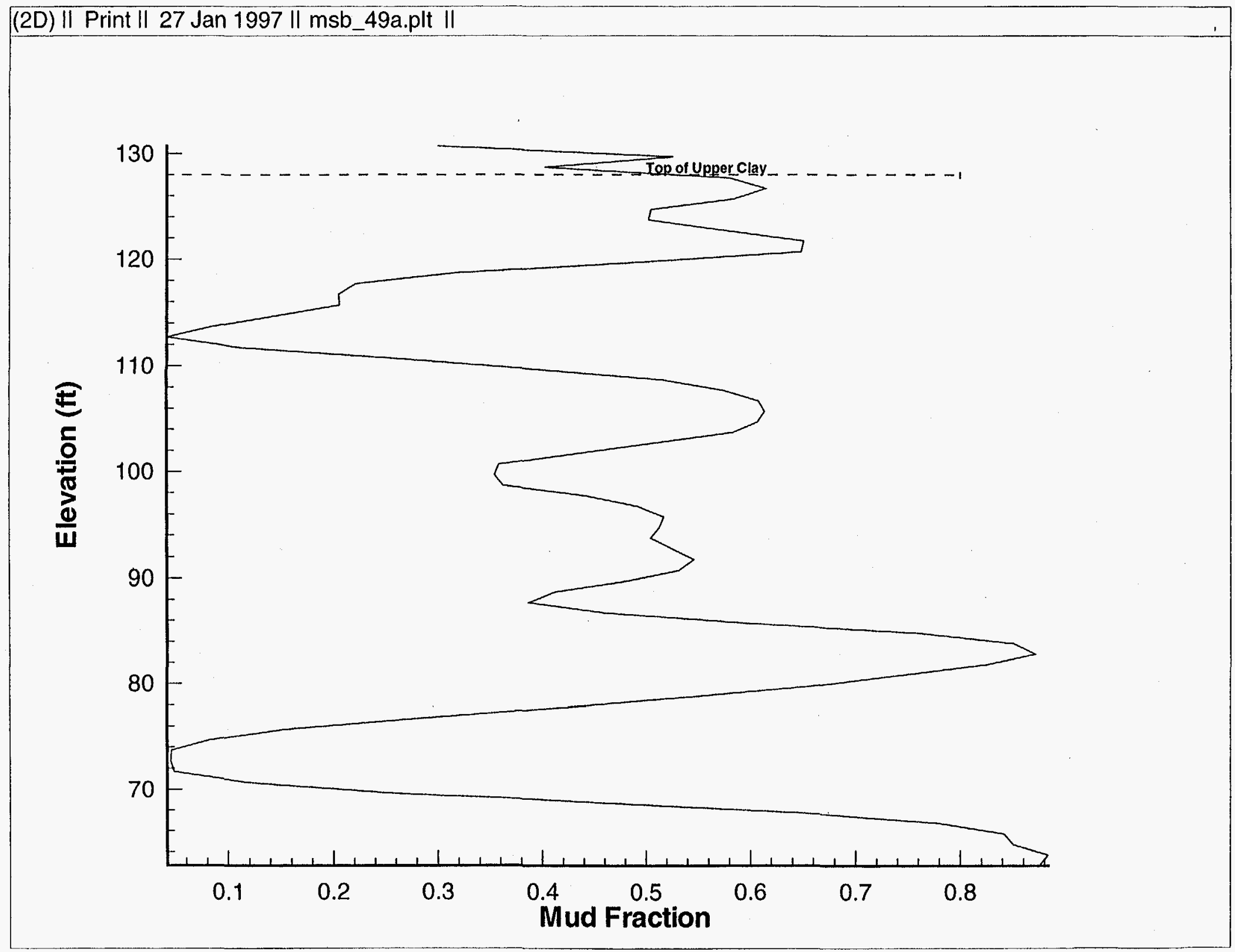




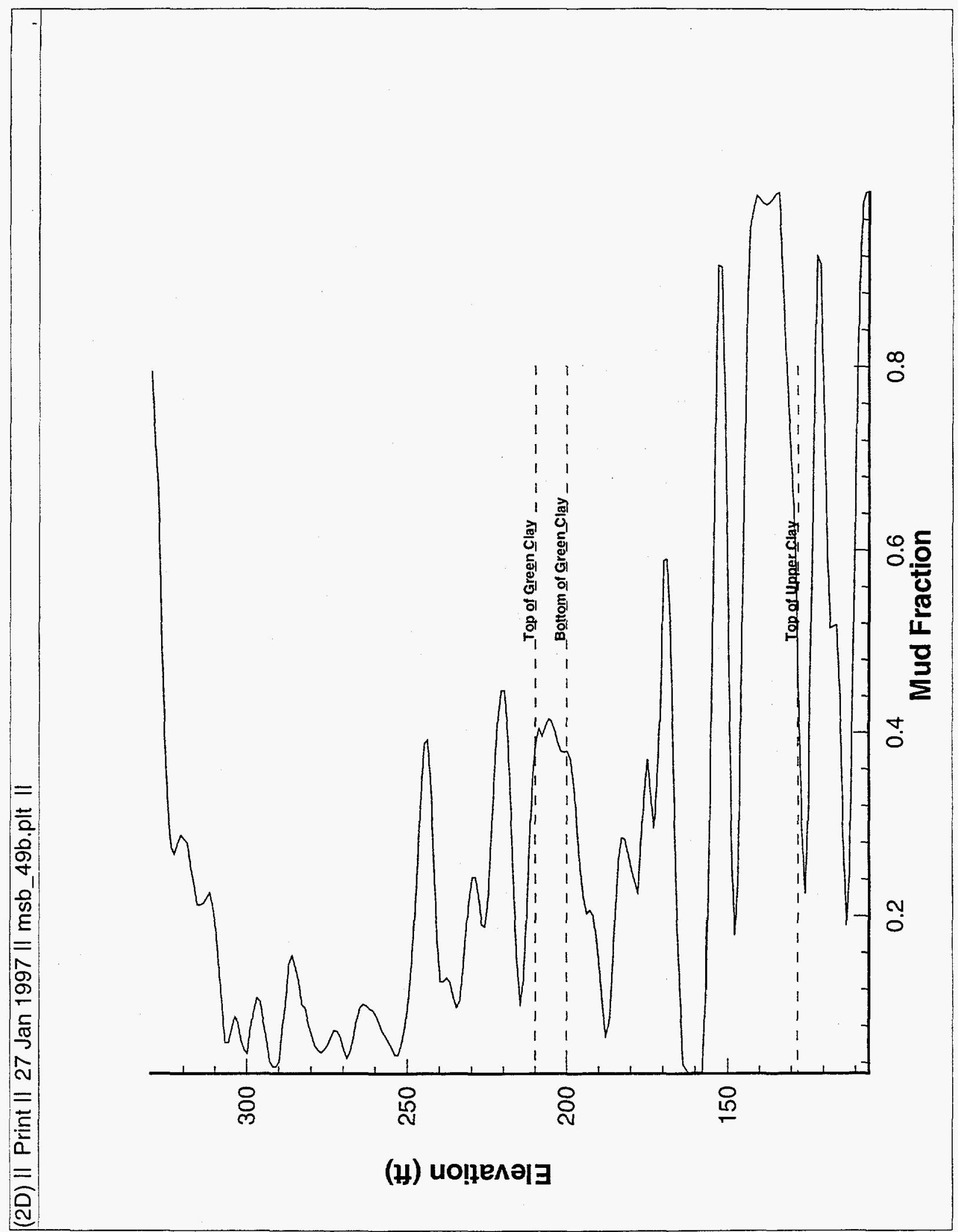




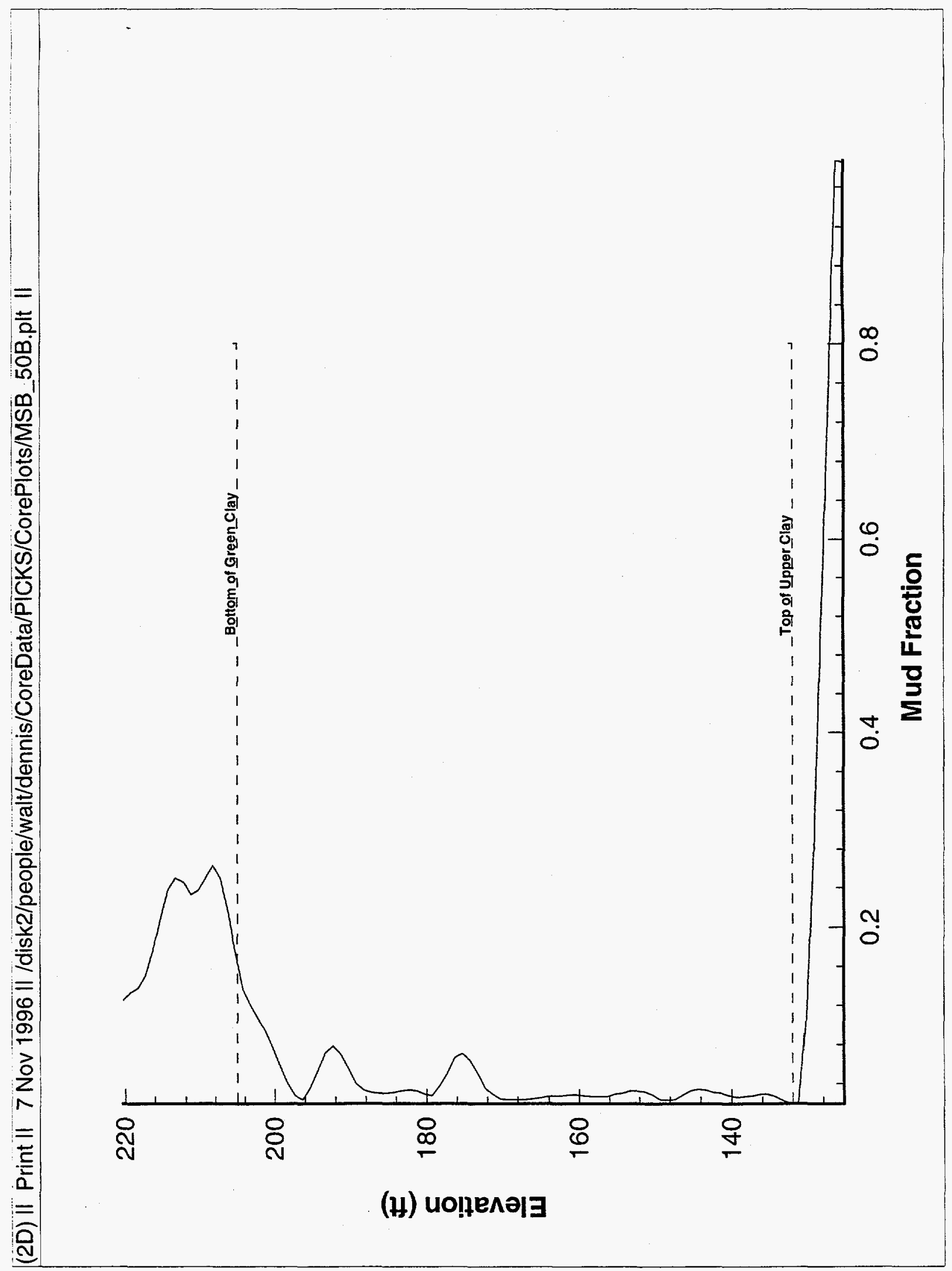




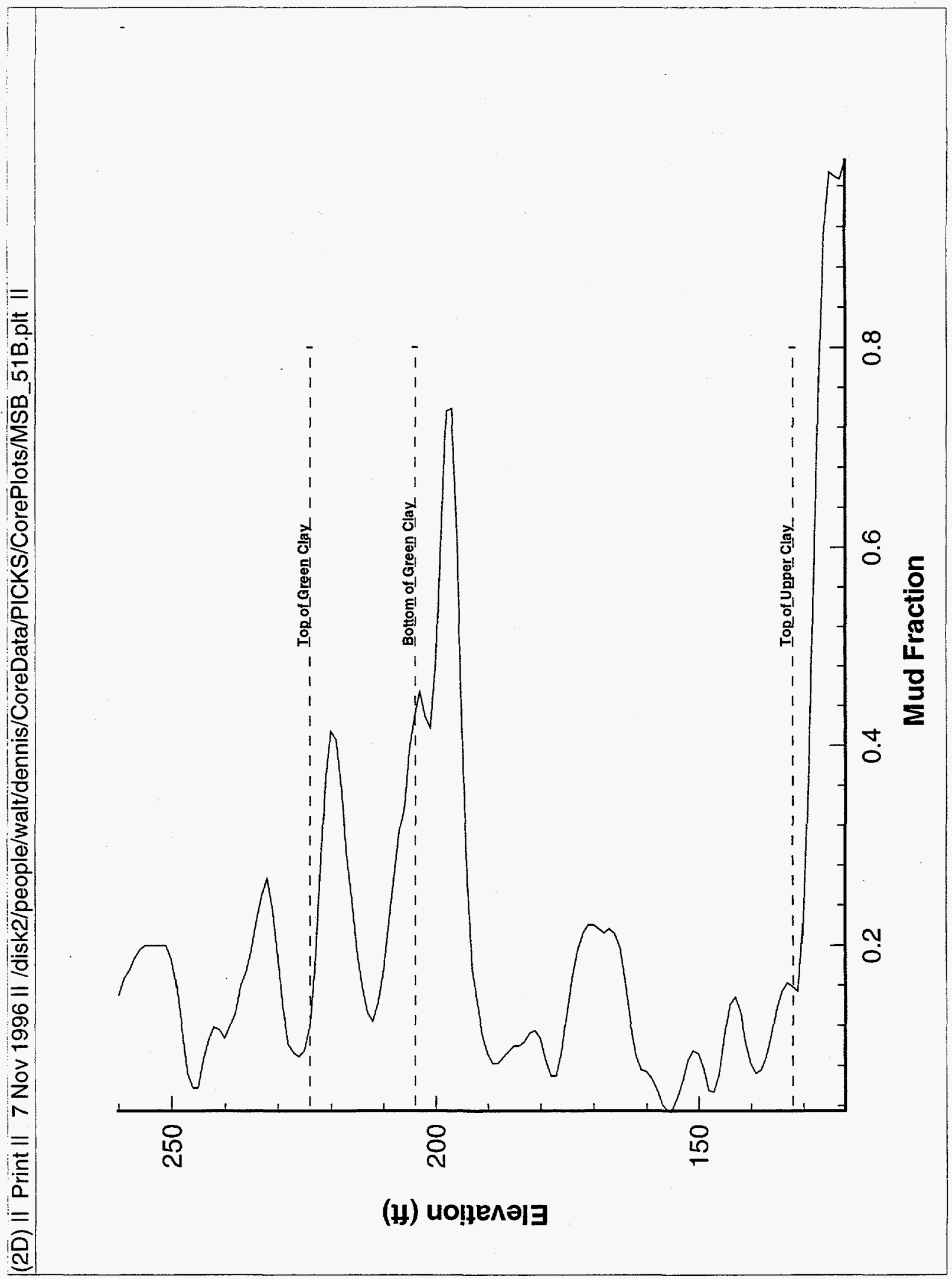




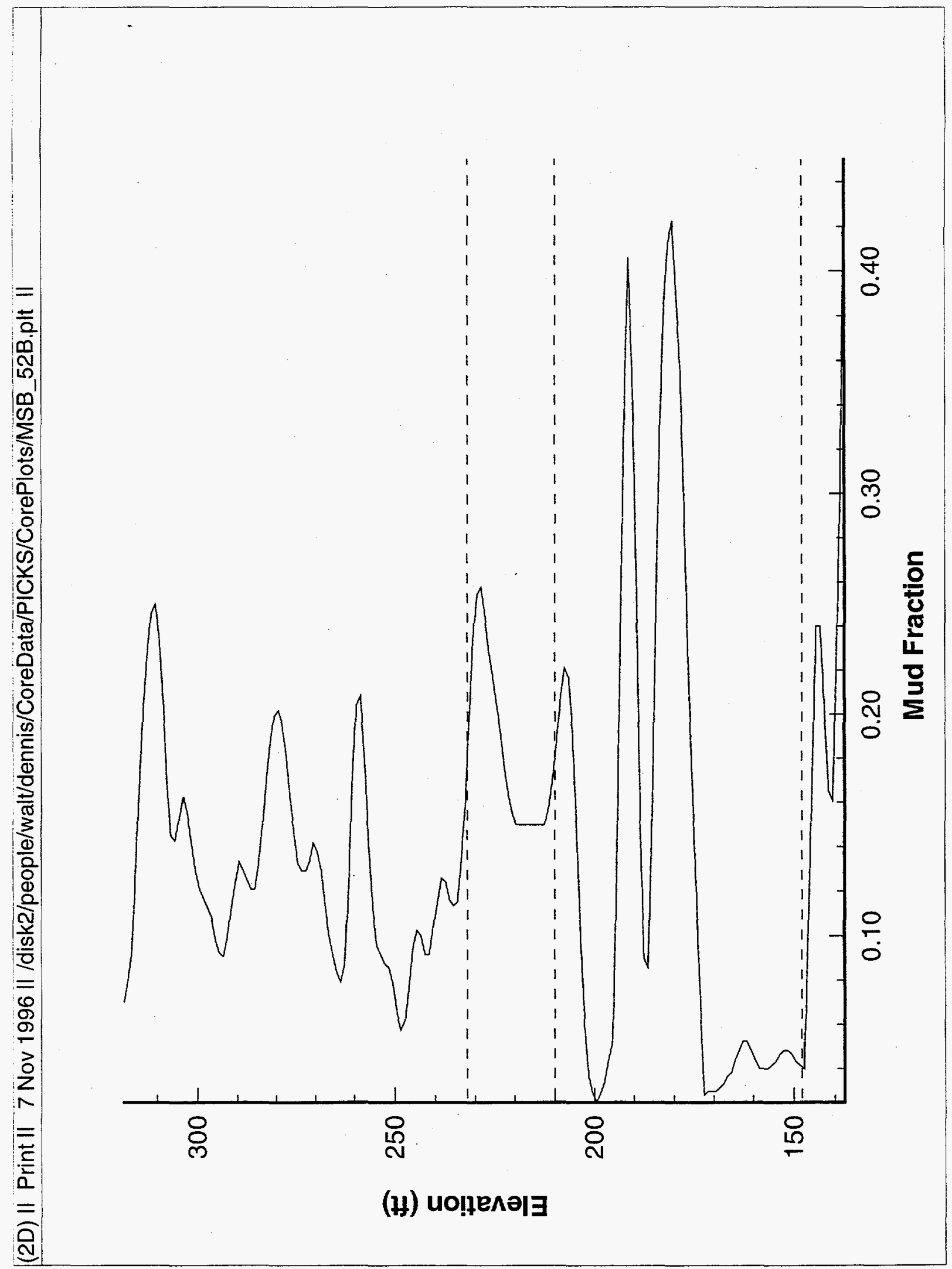




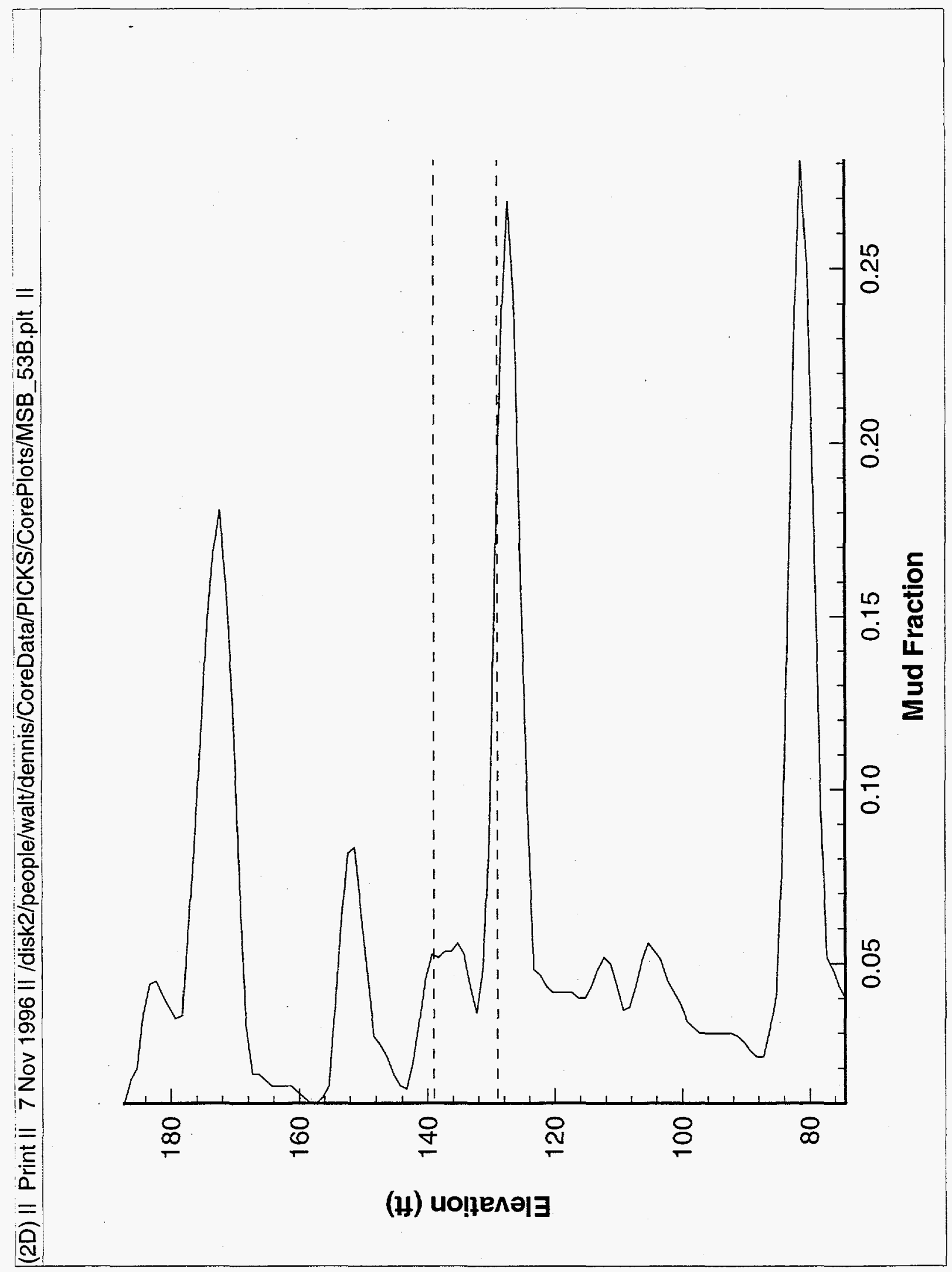




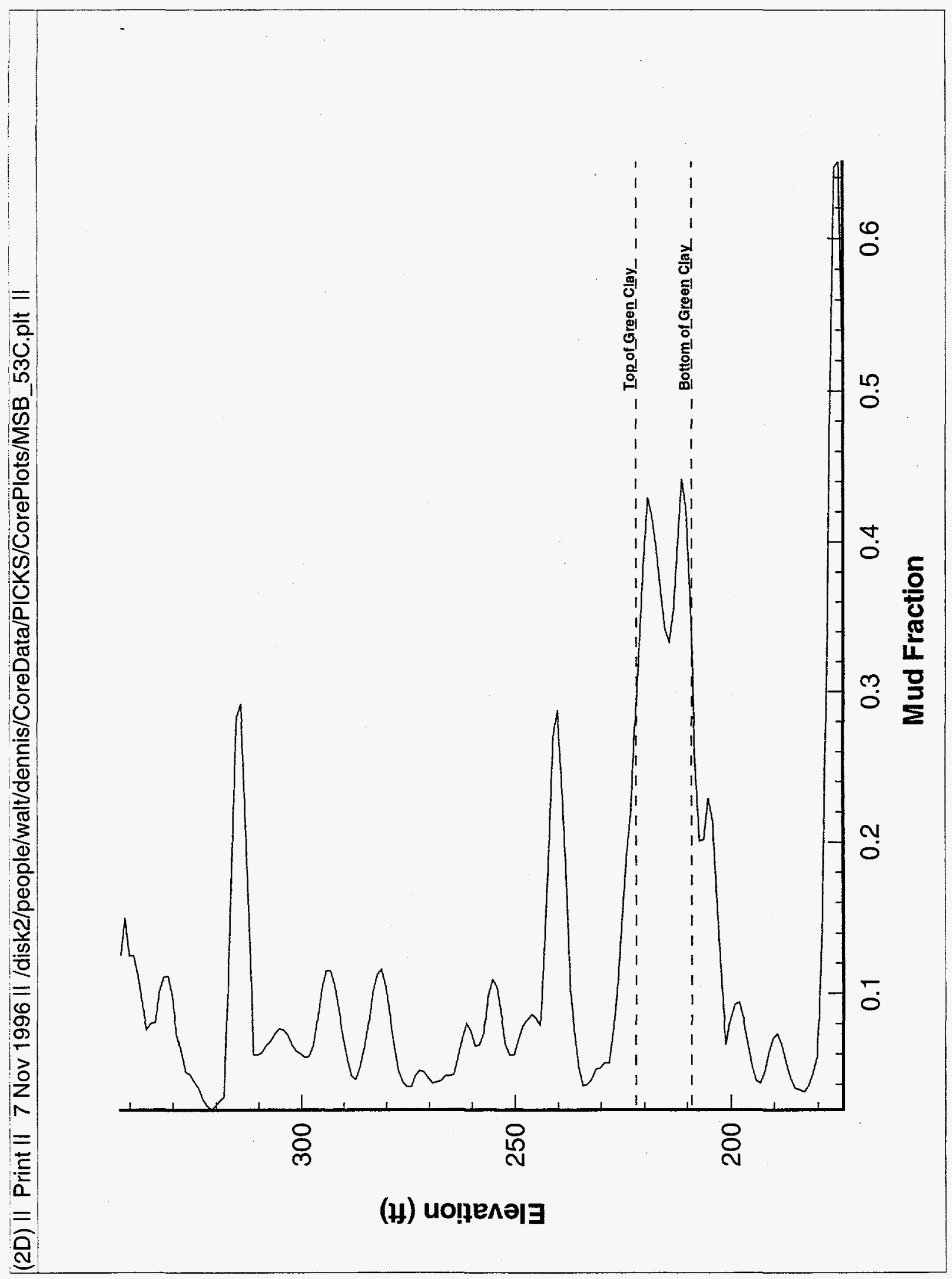




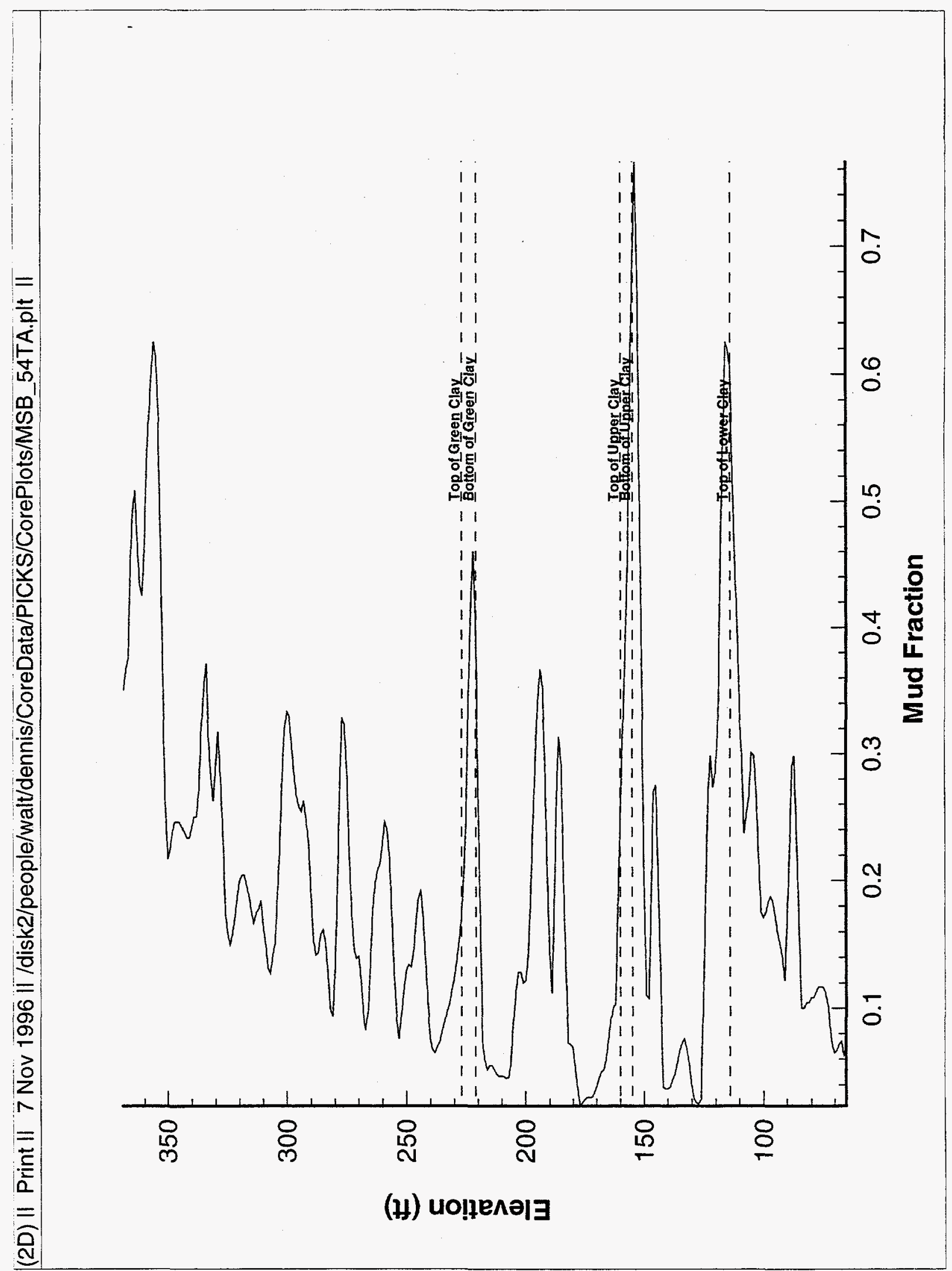




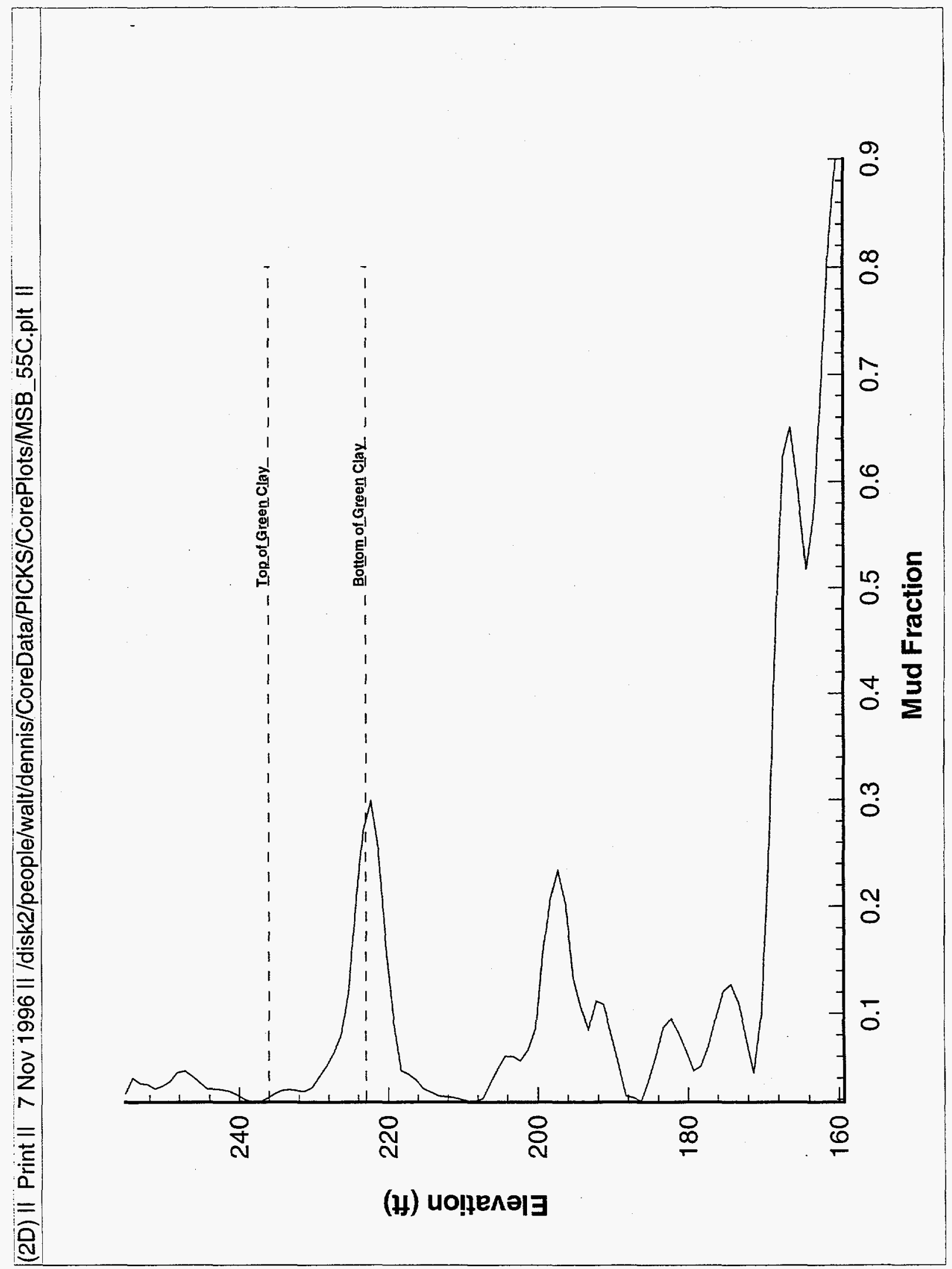




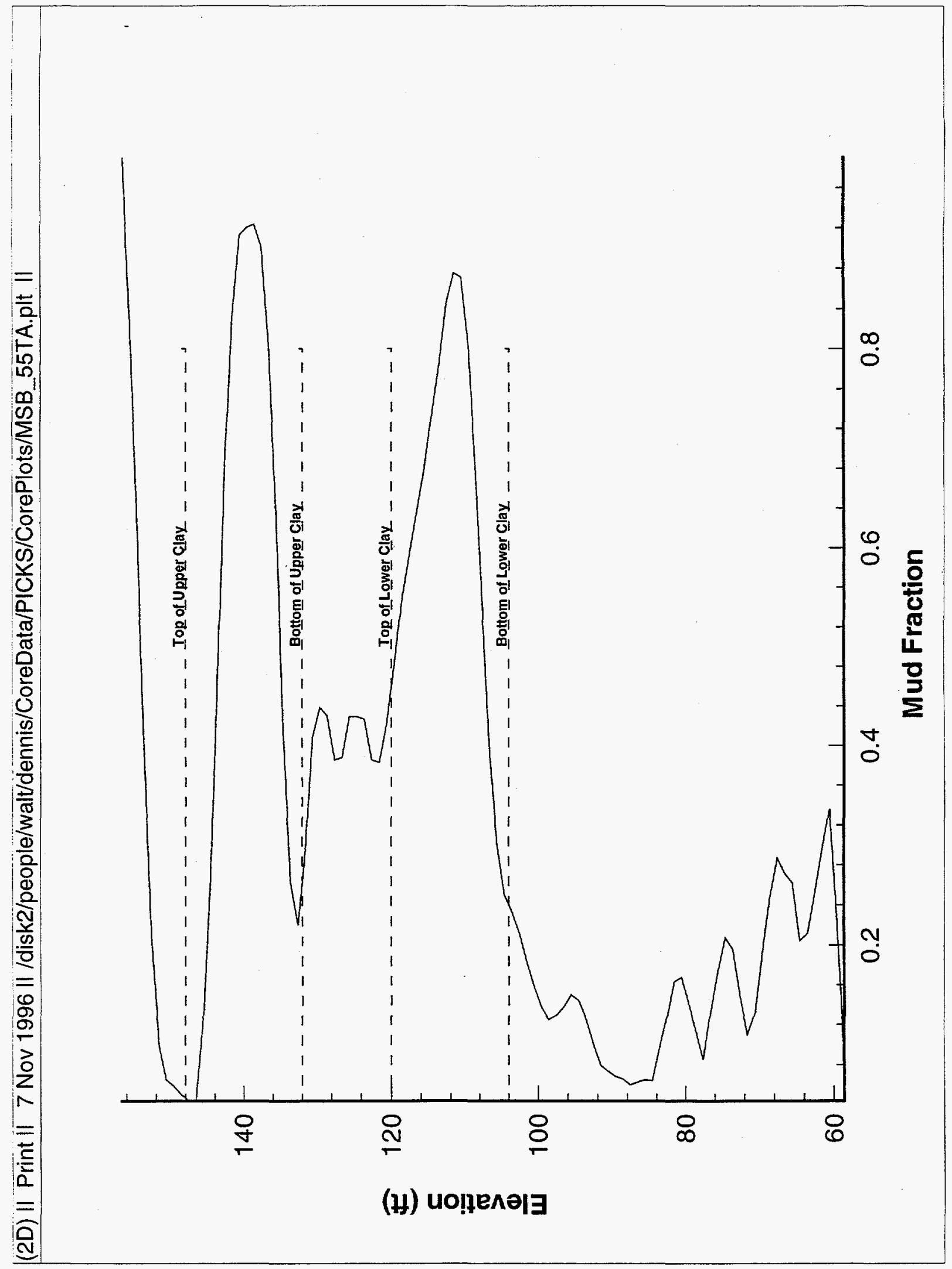




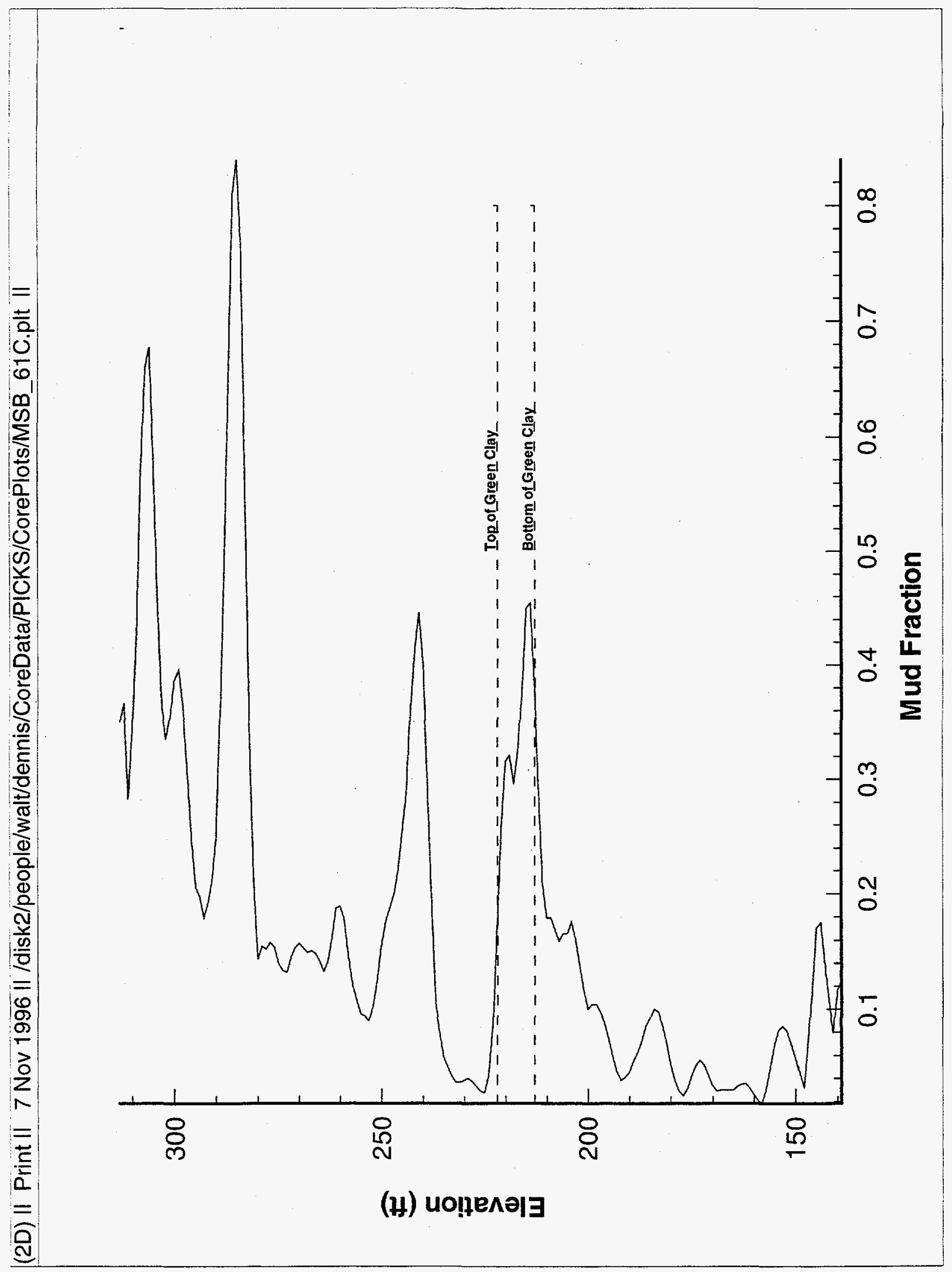




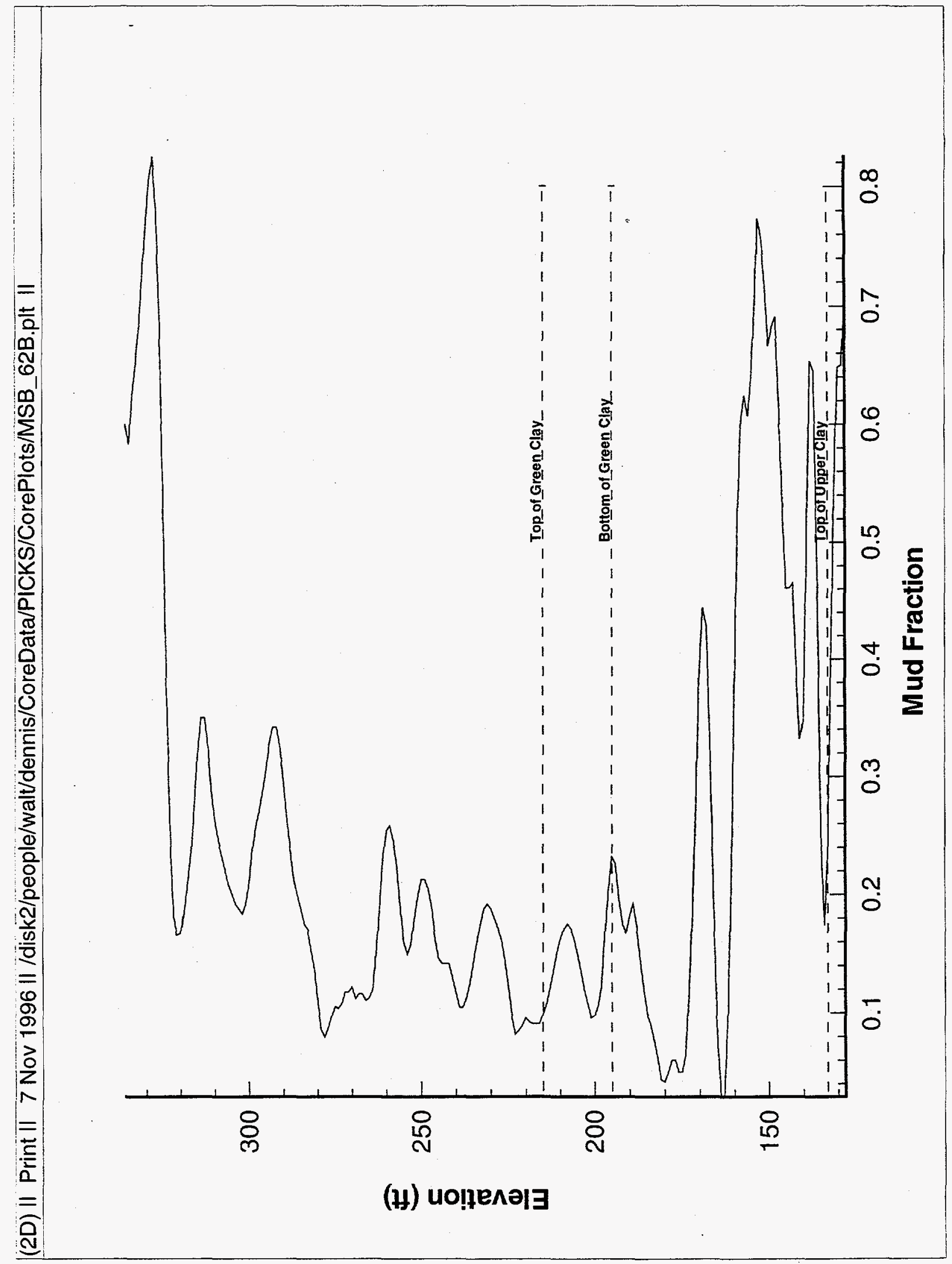


(2D) I| Print II 7 Nov 1996 || /disk2/people/walt/dennis/CoreData/PICKS/CorePlots/MSB_63B.plt II

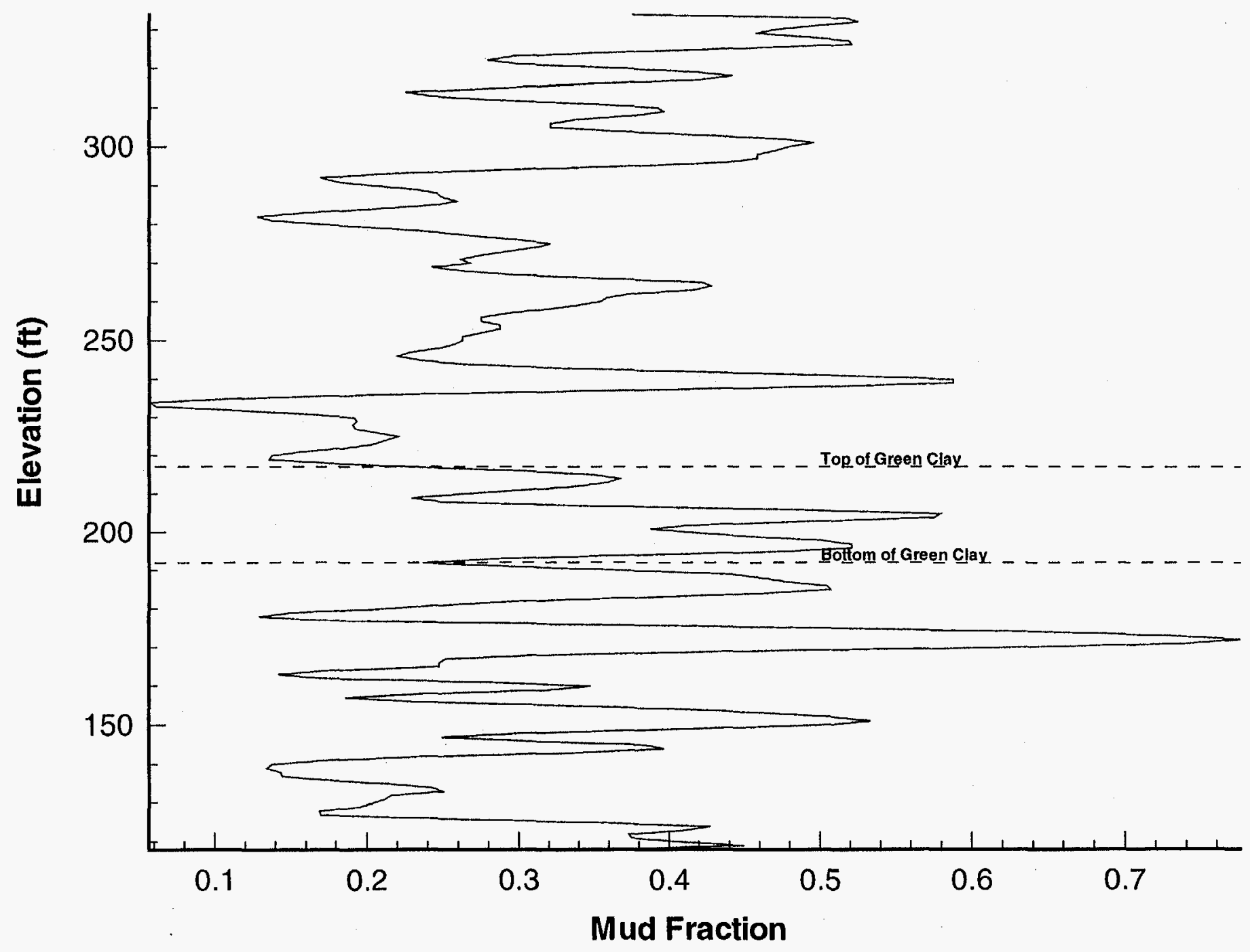




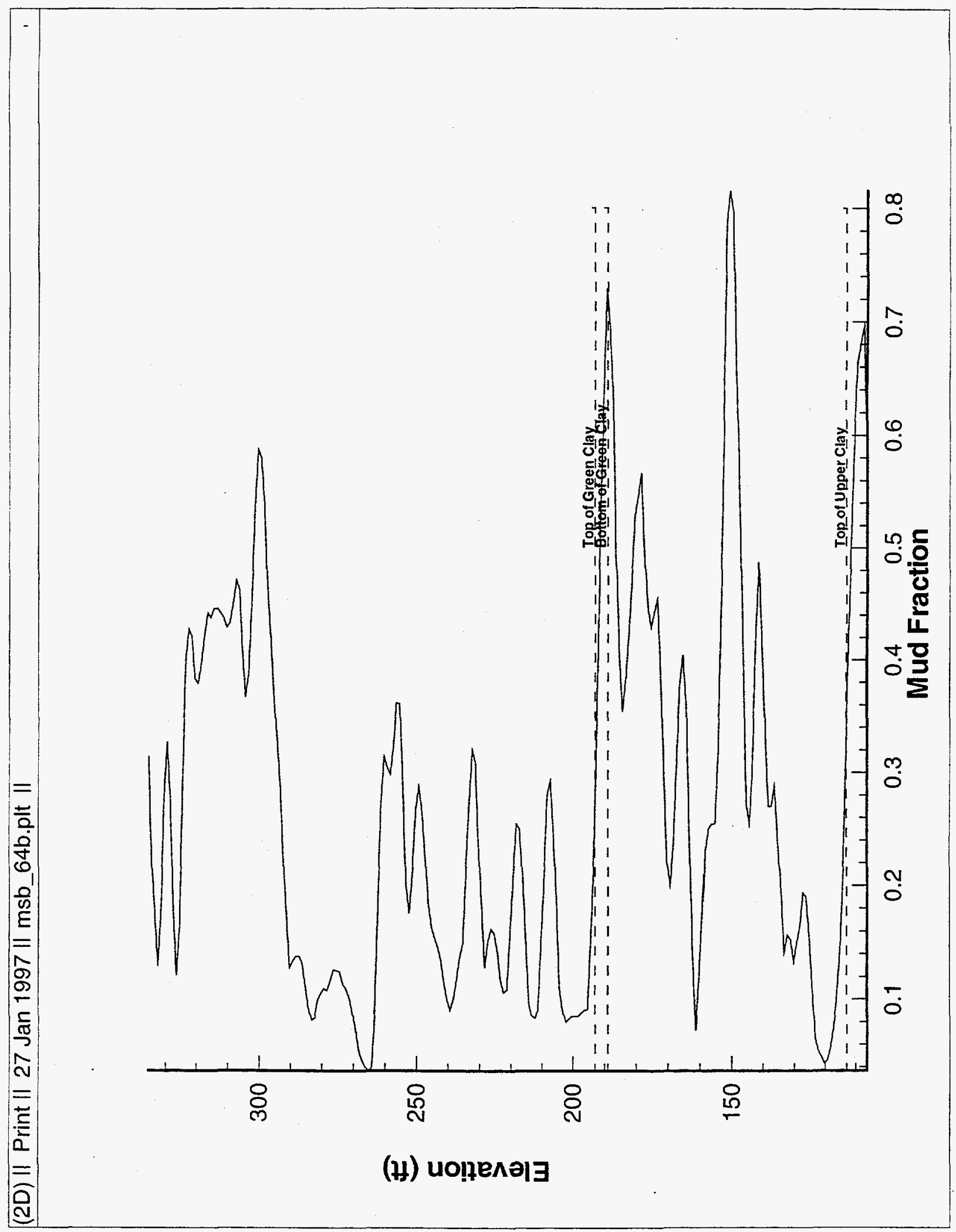




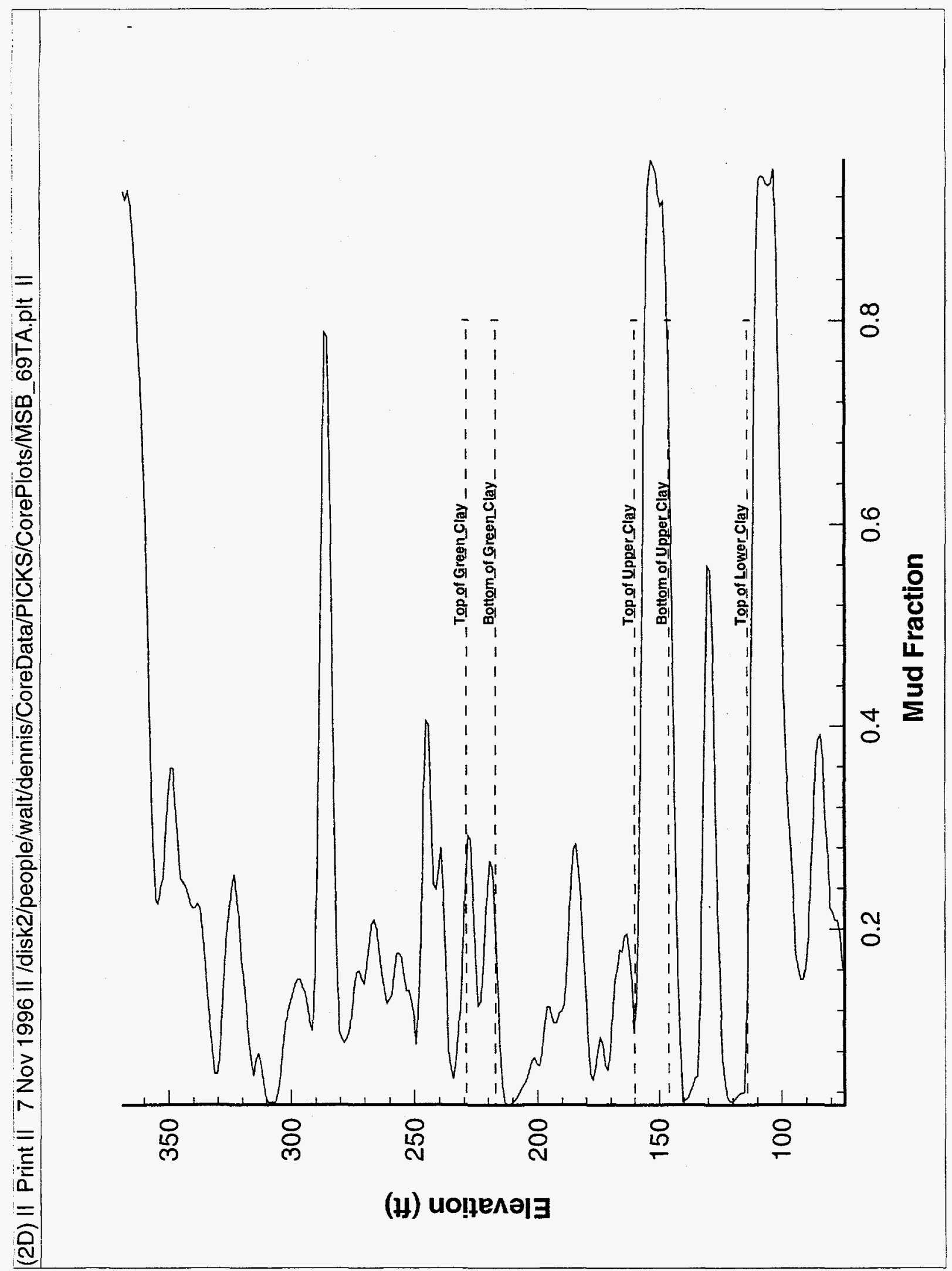


WSRC-OS-97-00002

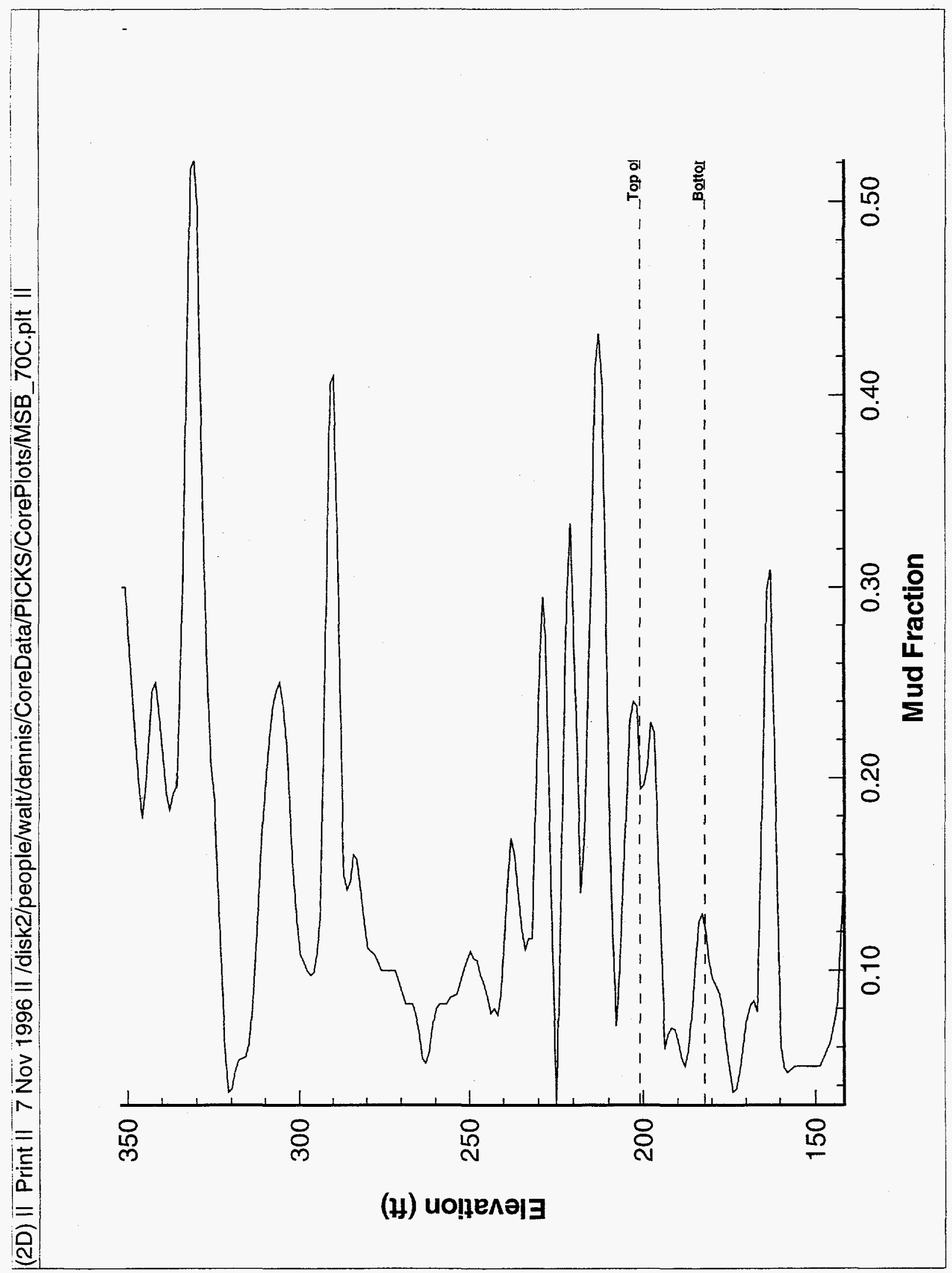




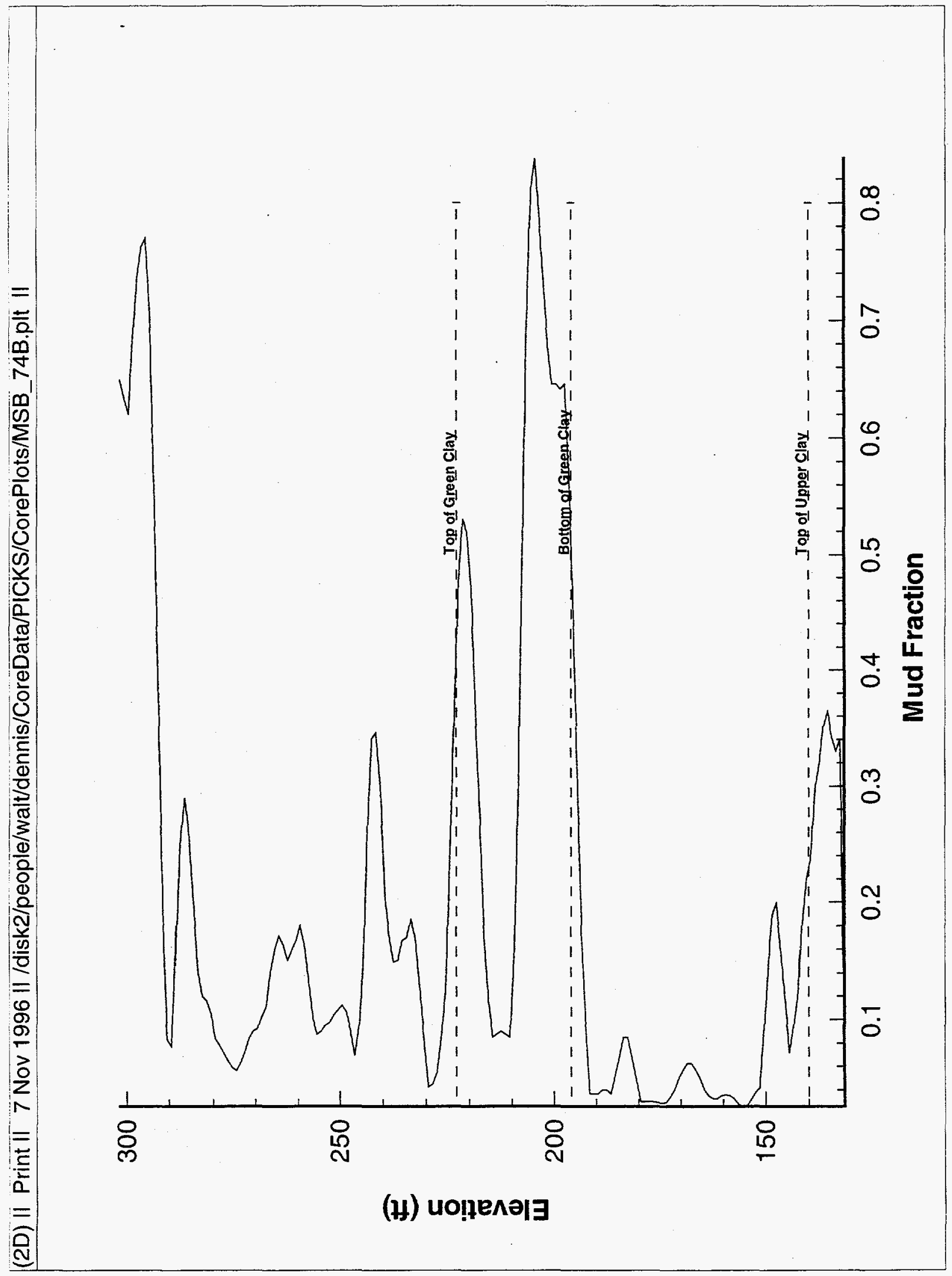


(2D) I| Print I| 7 Nov 1996 || /disk2/people/walt/dennis/CoreData/PICKS/CorePlots/MSB_75B.plt II

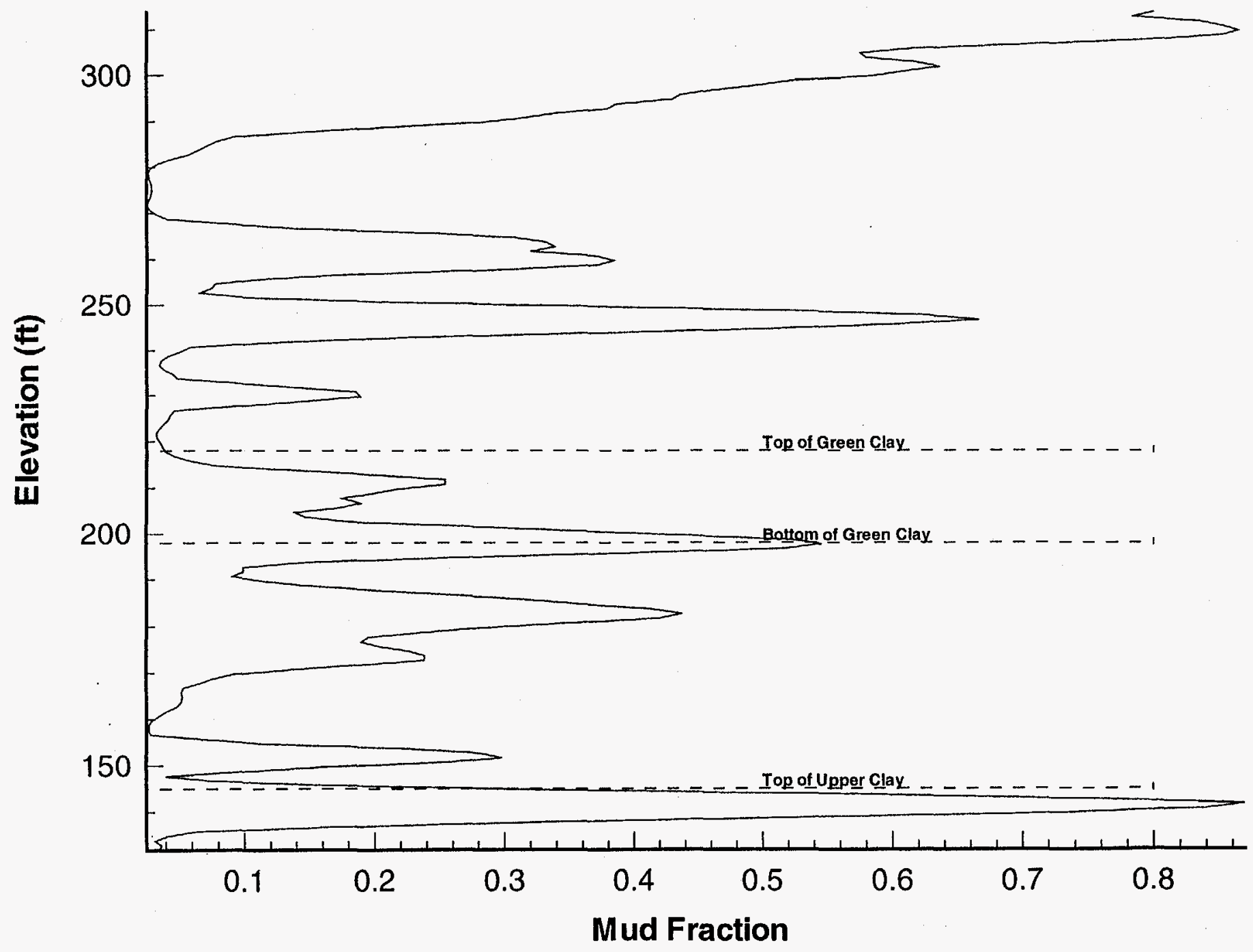




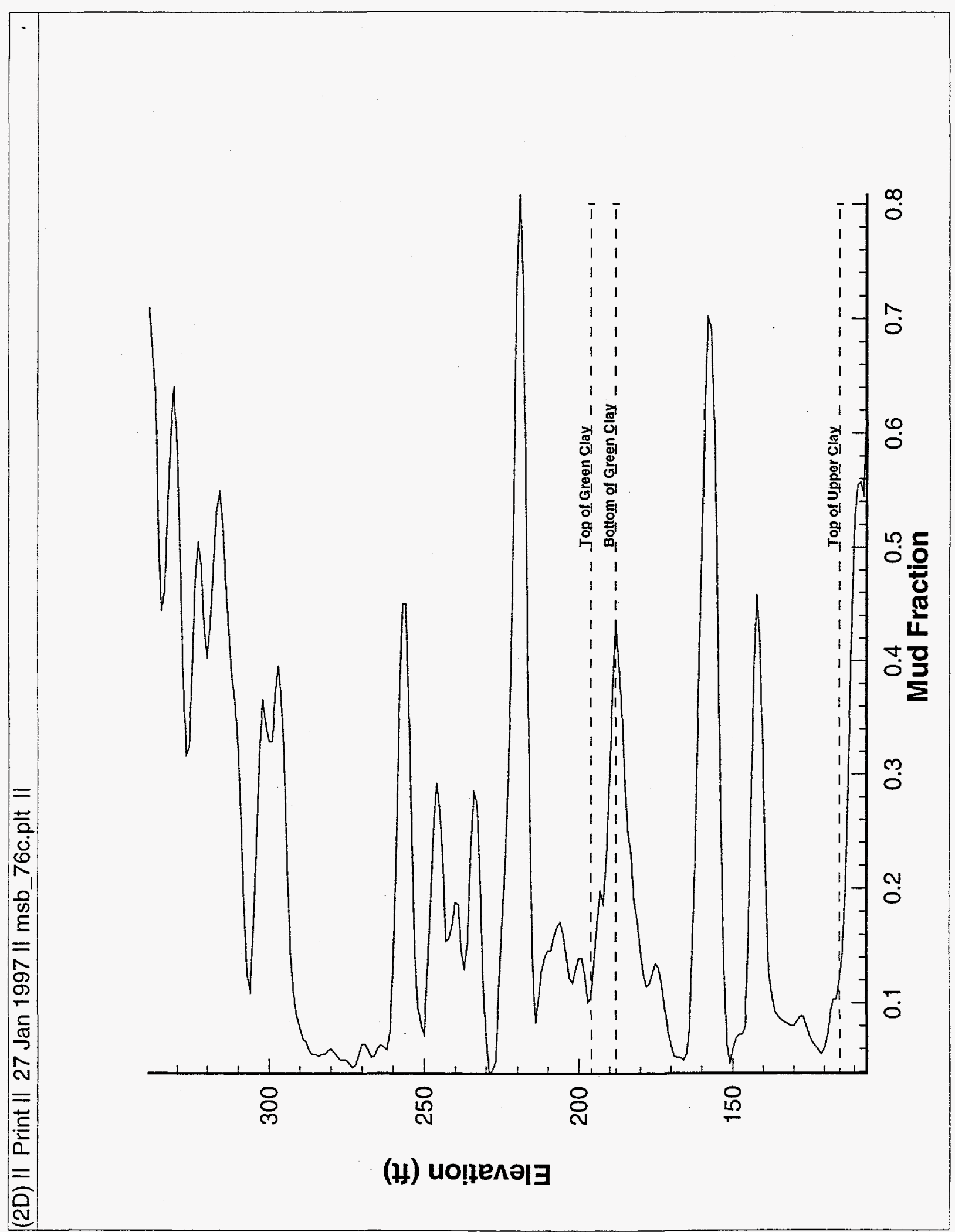




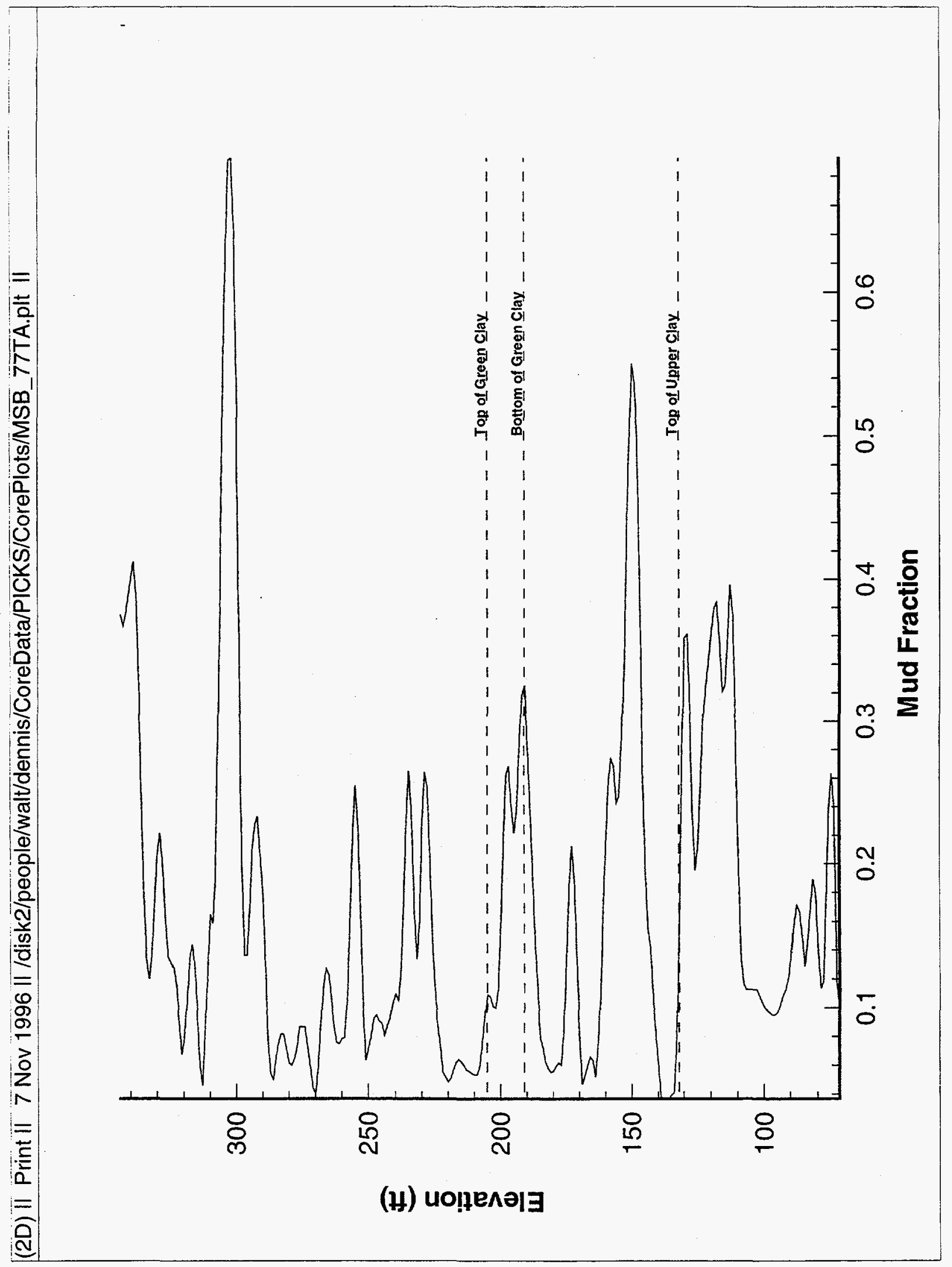




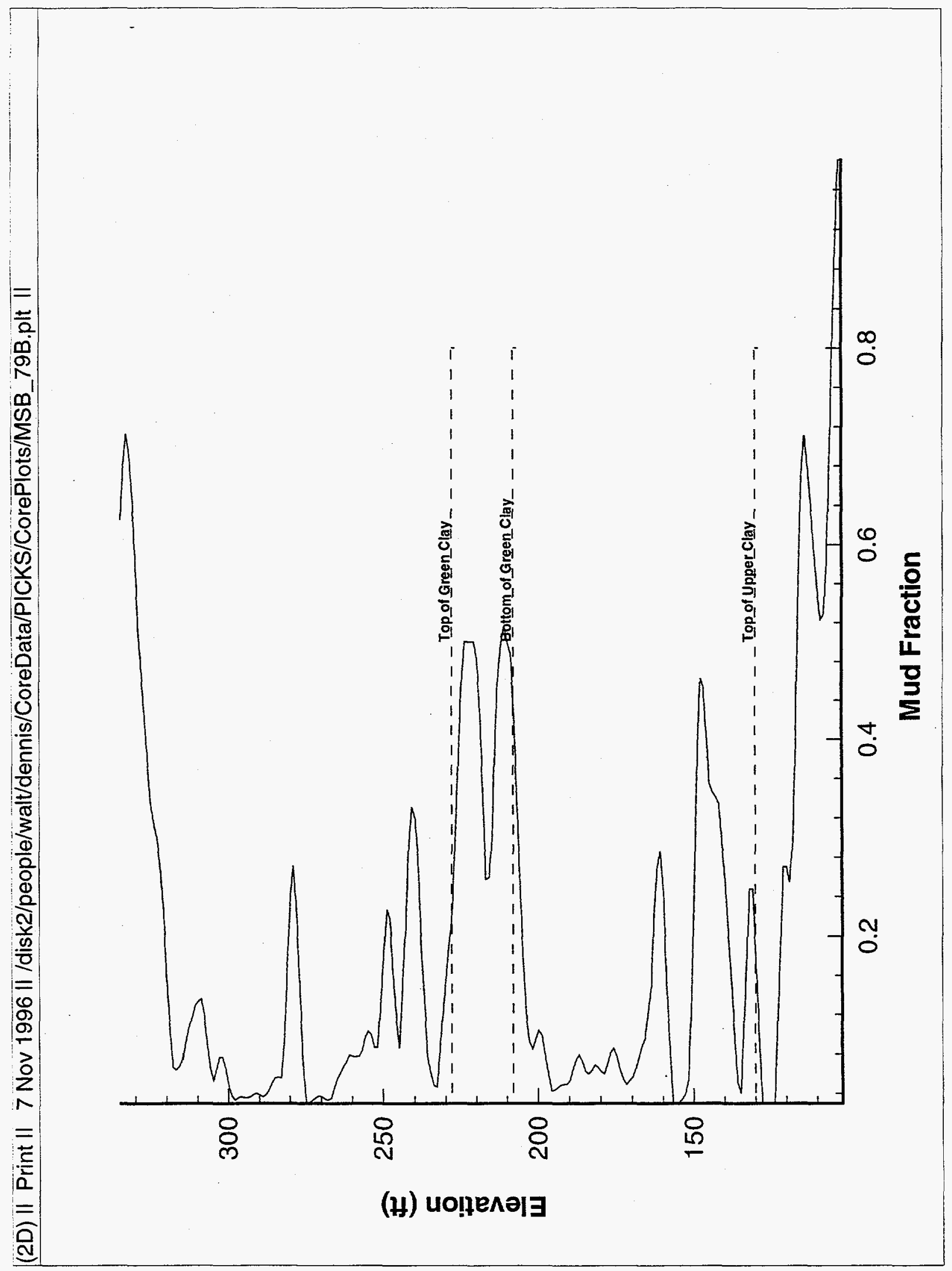




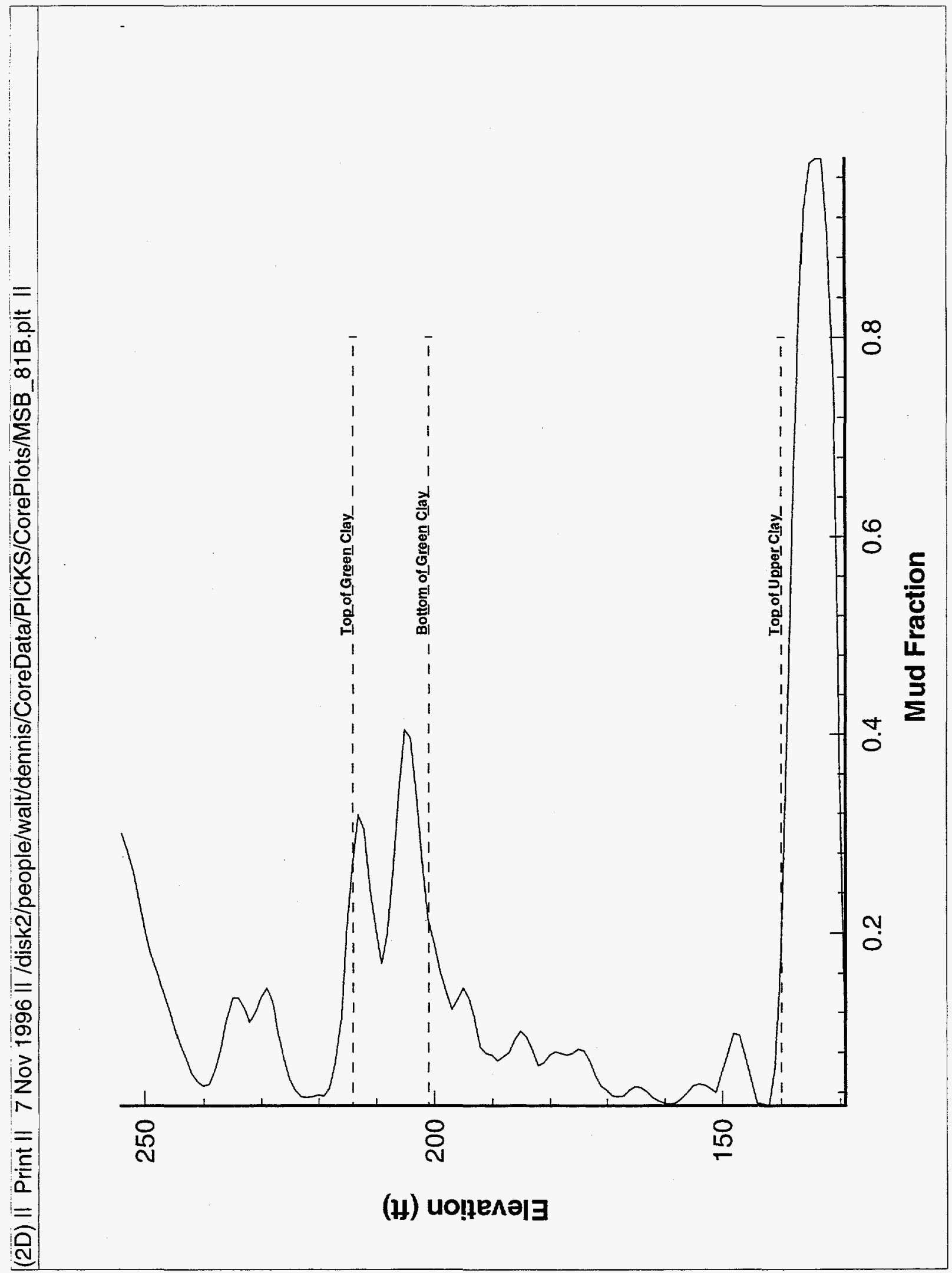




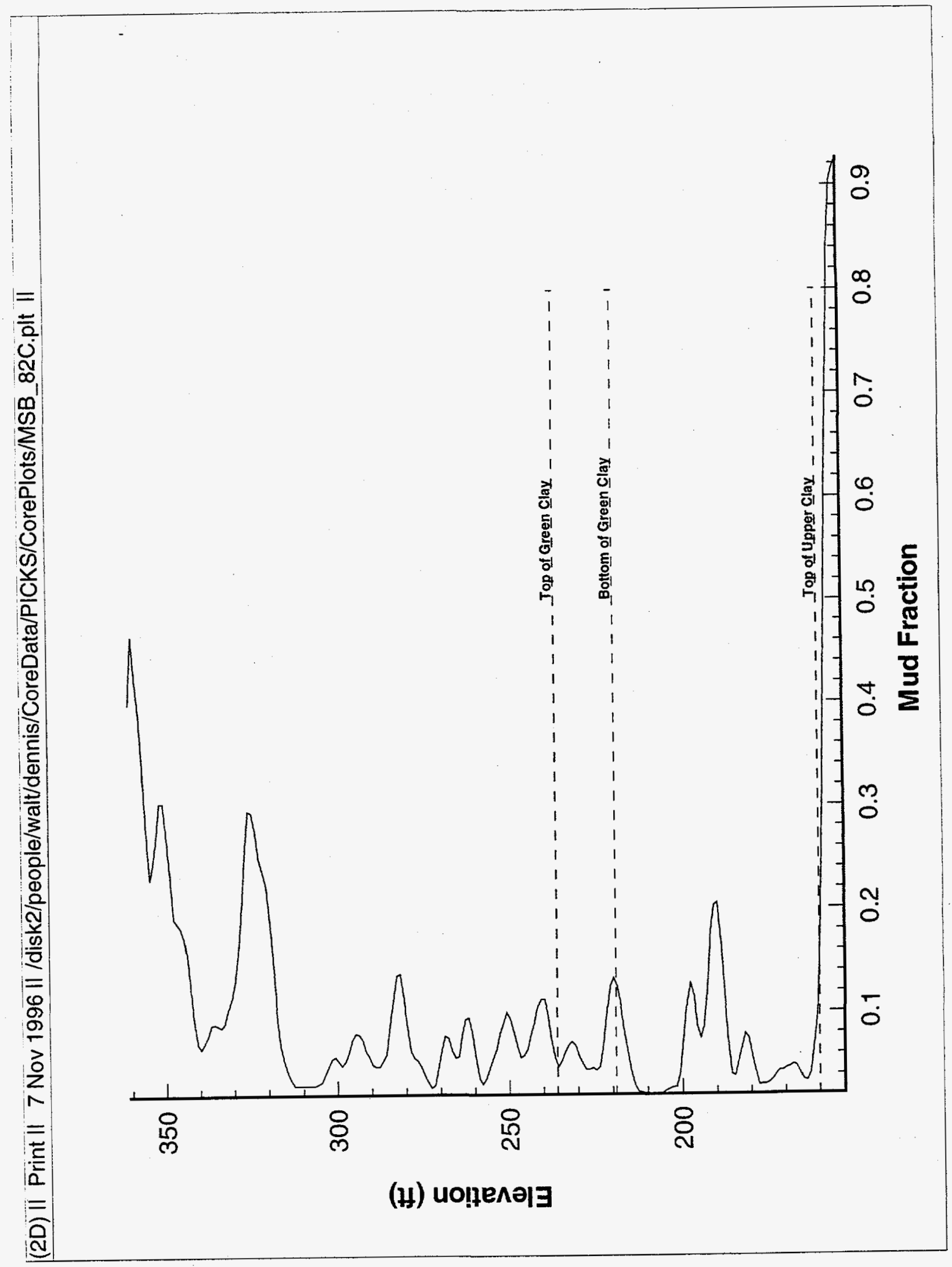




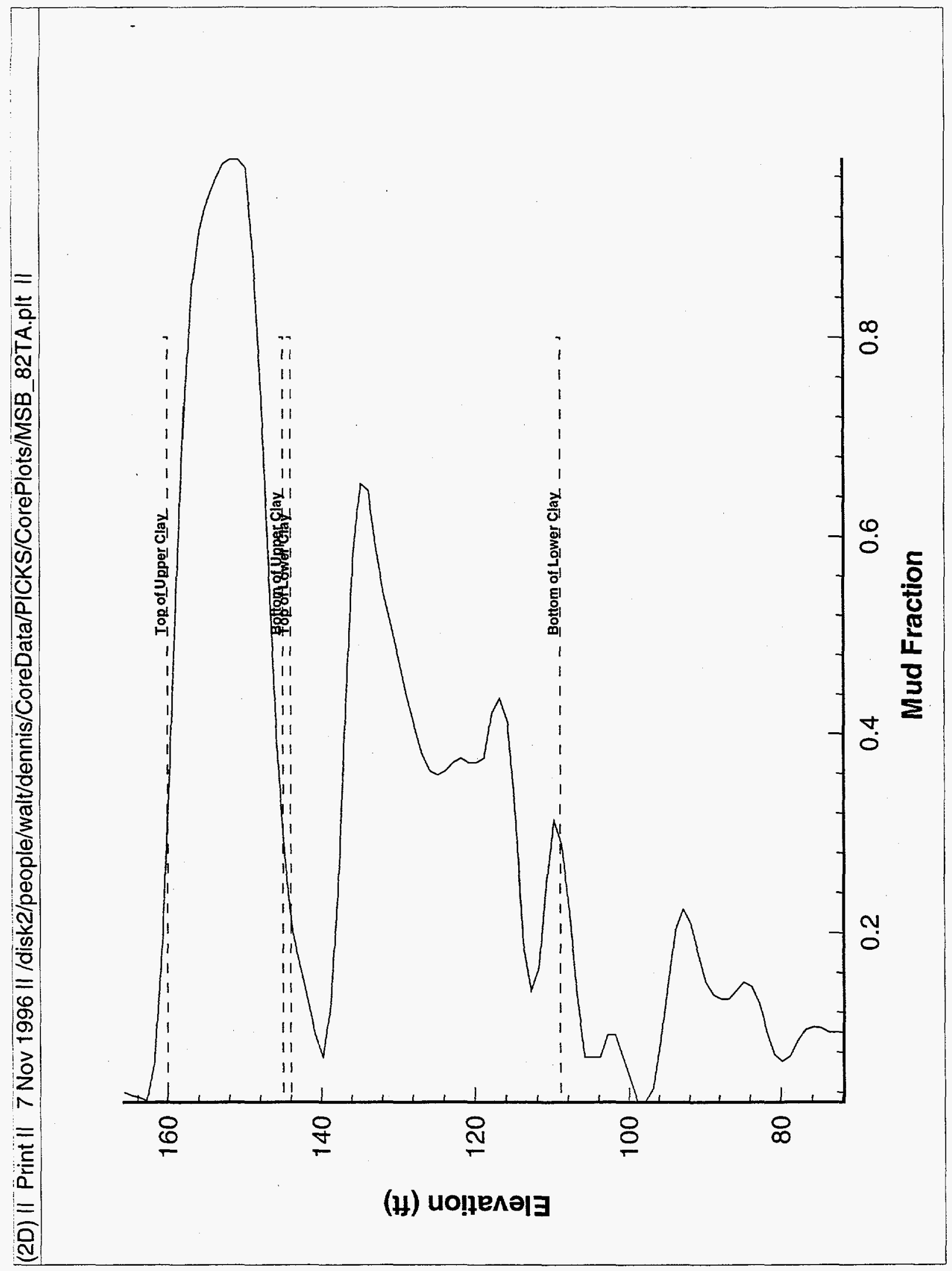


(2D) II Print II 7 Nov 1996 || /disk2/people/walt/dennis/CoreData/PICKS/CorePlots/MSB_83C.plt II

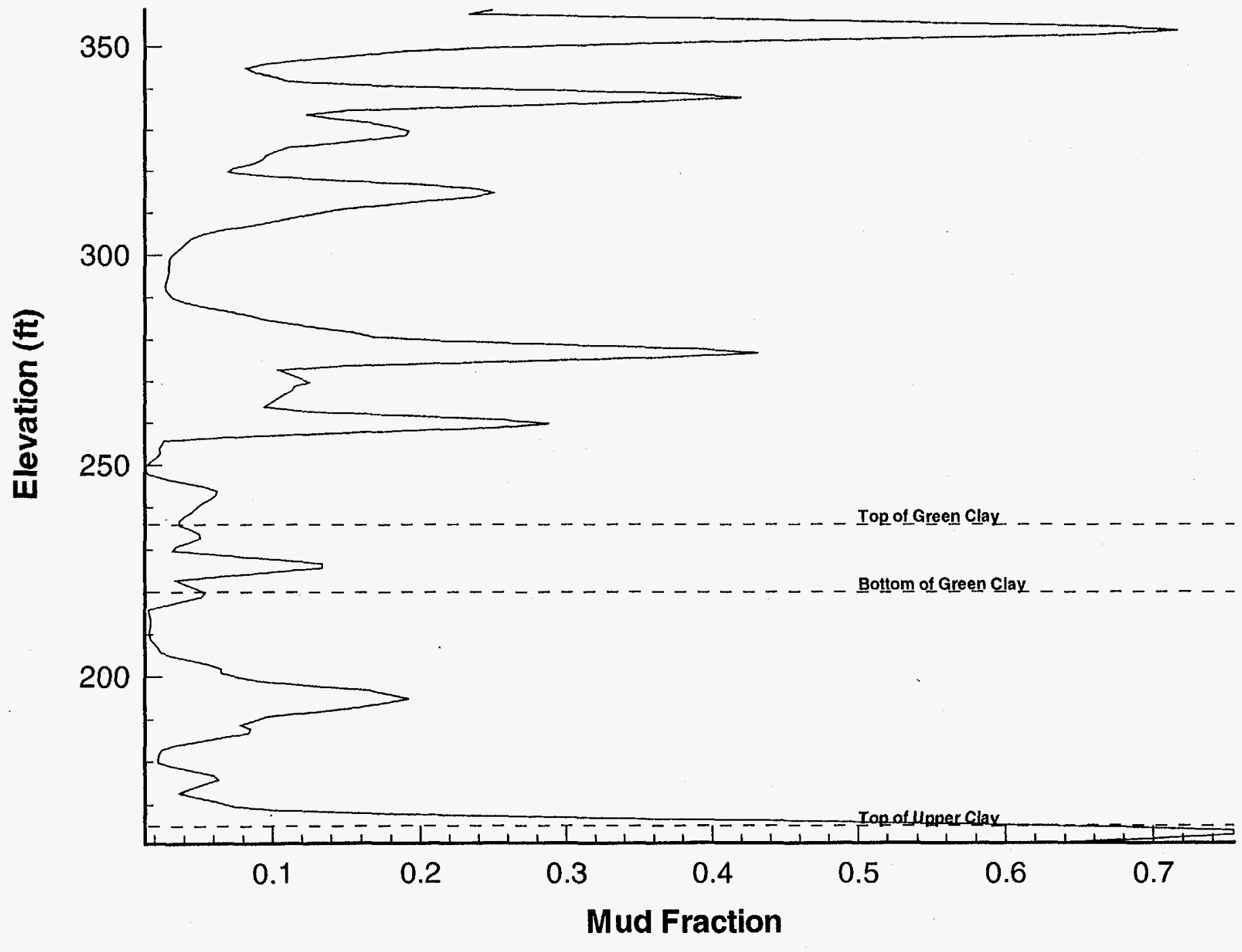




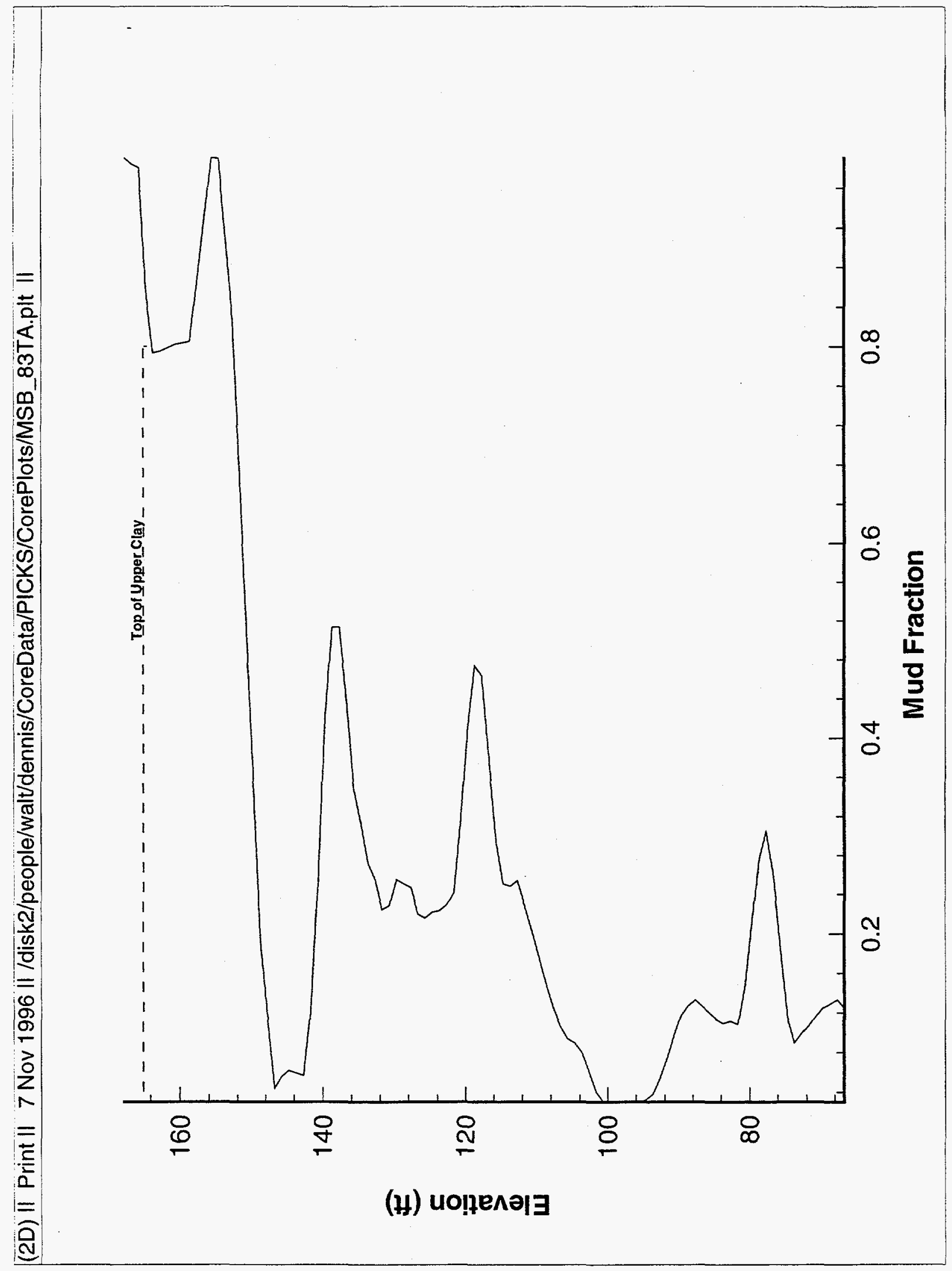




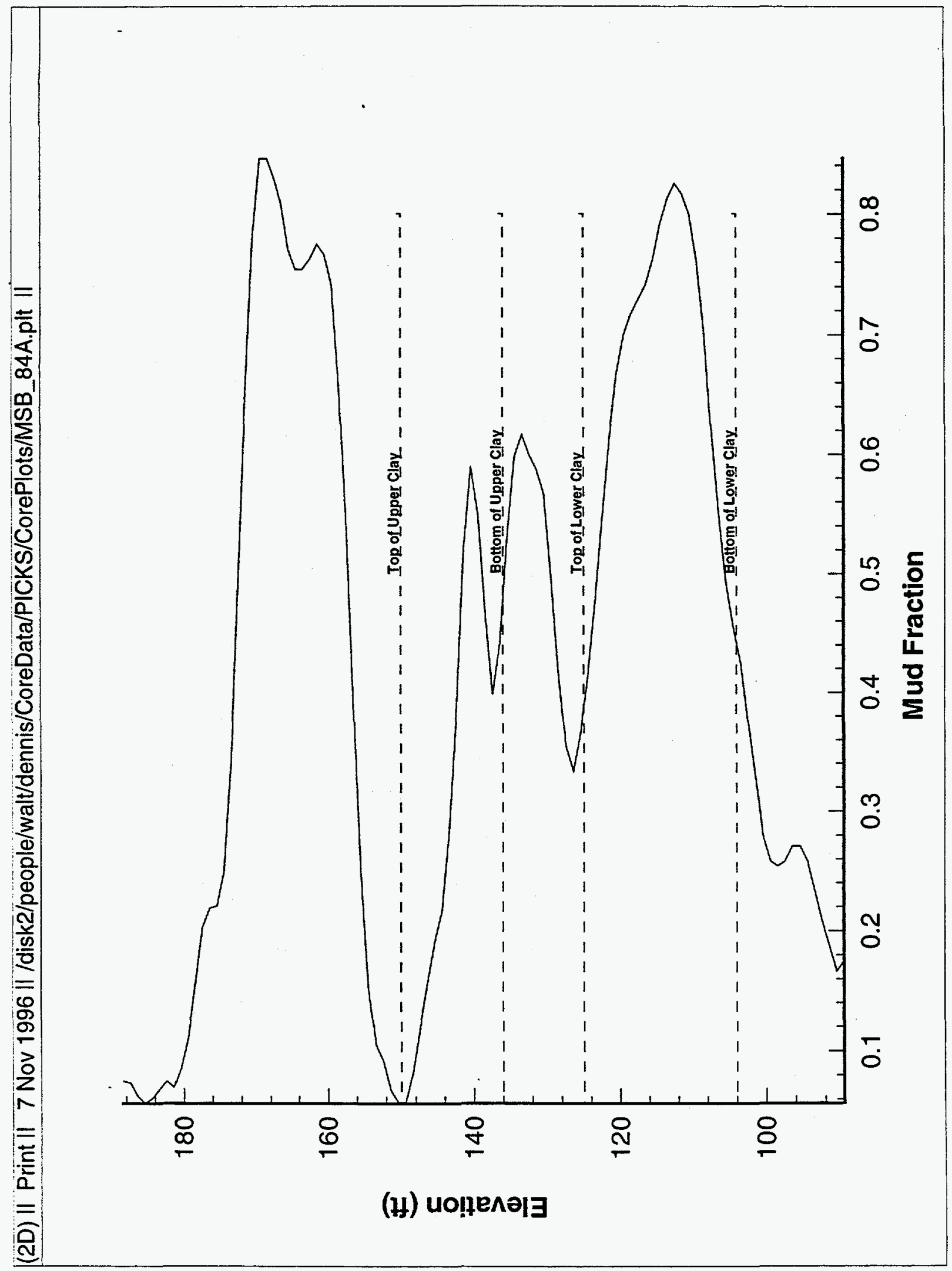




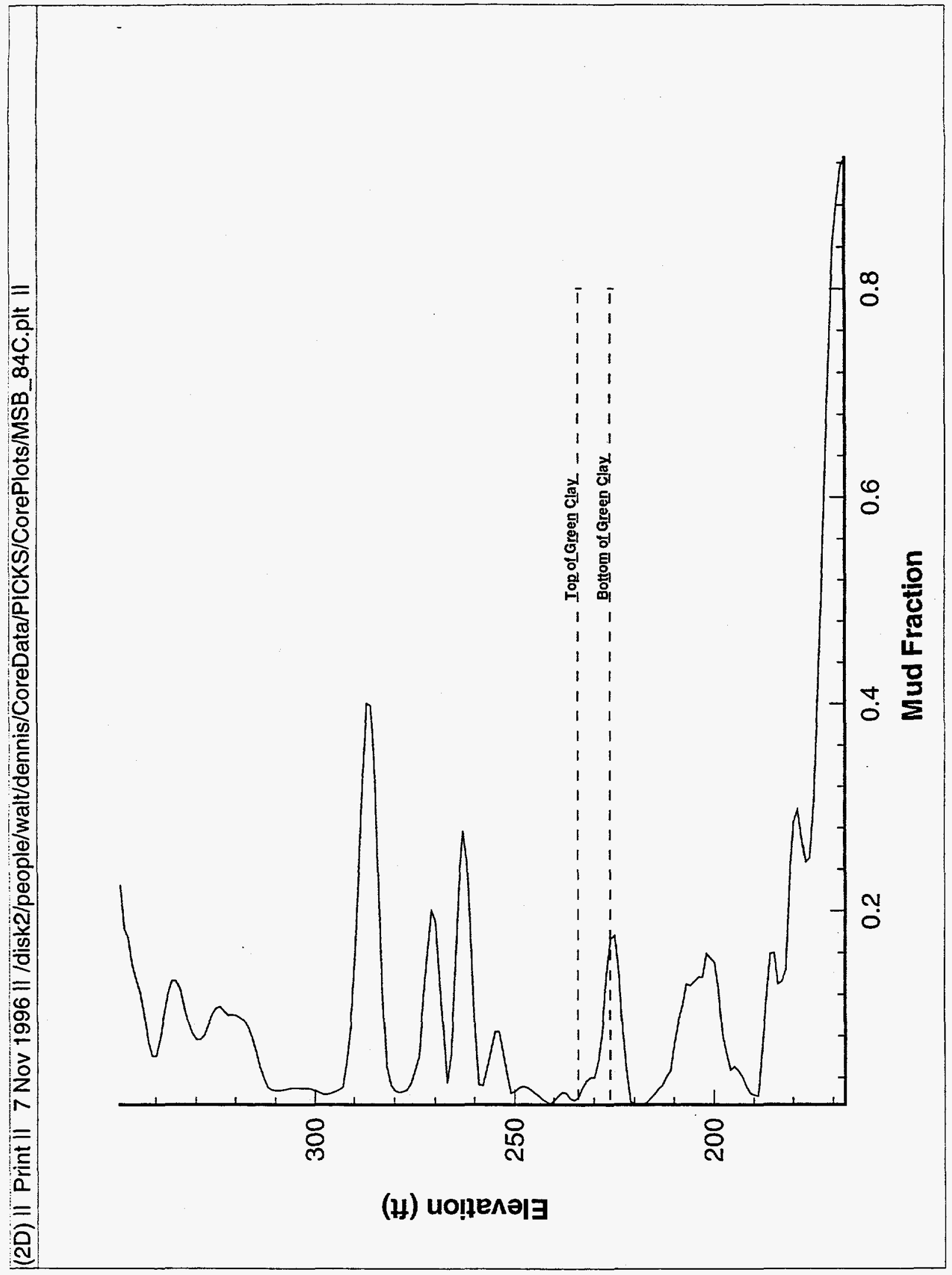




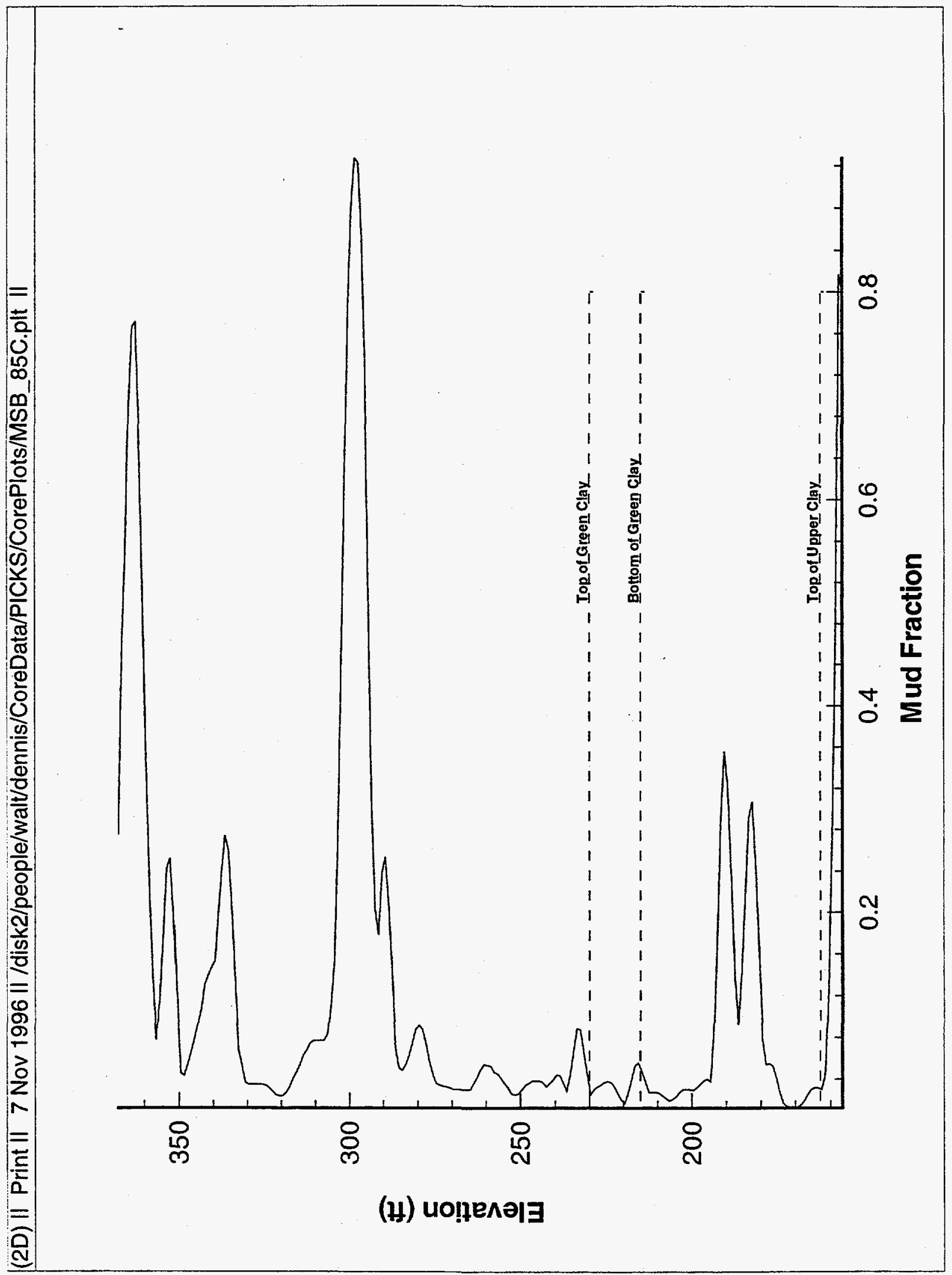




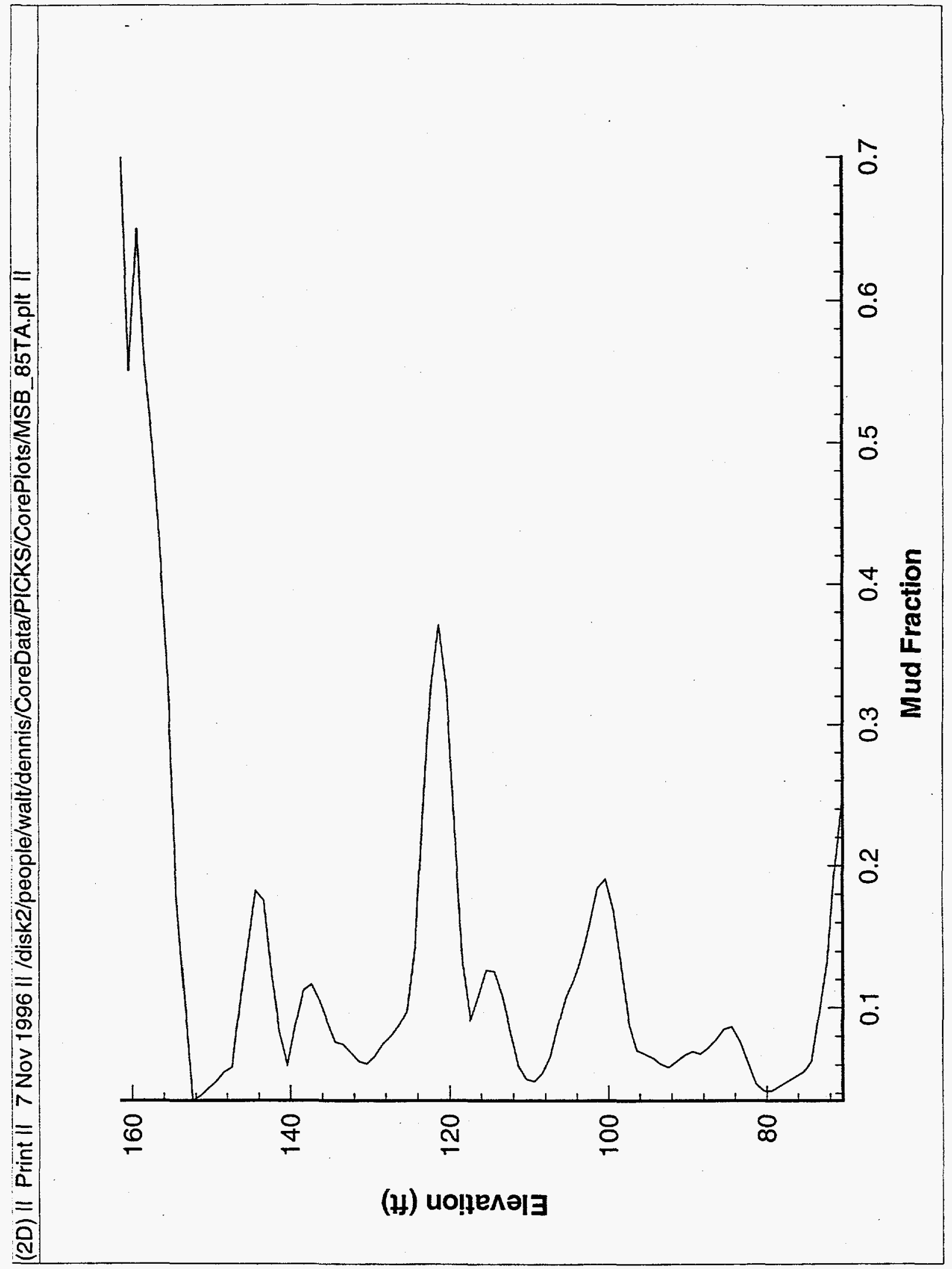




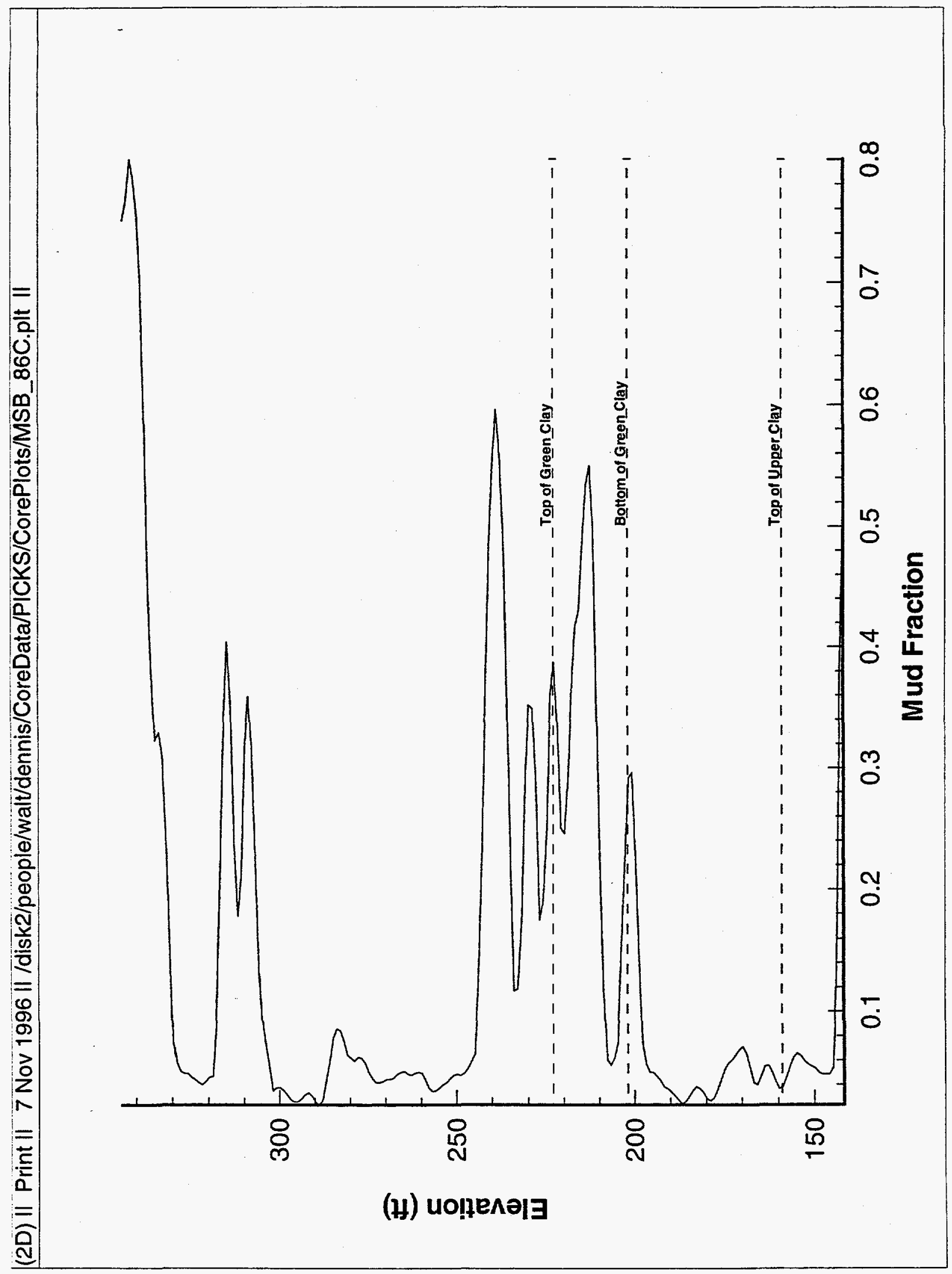




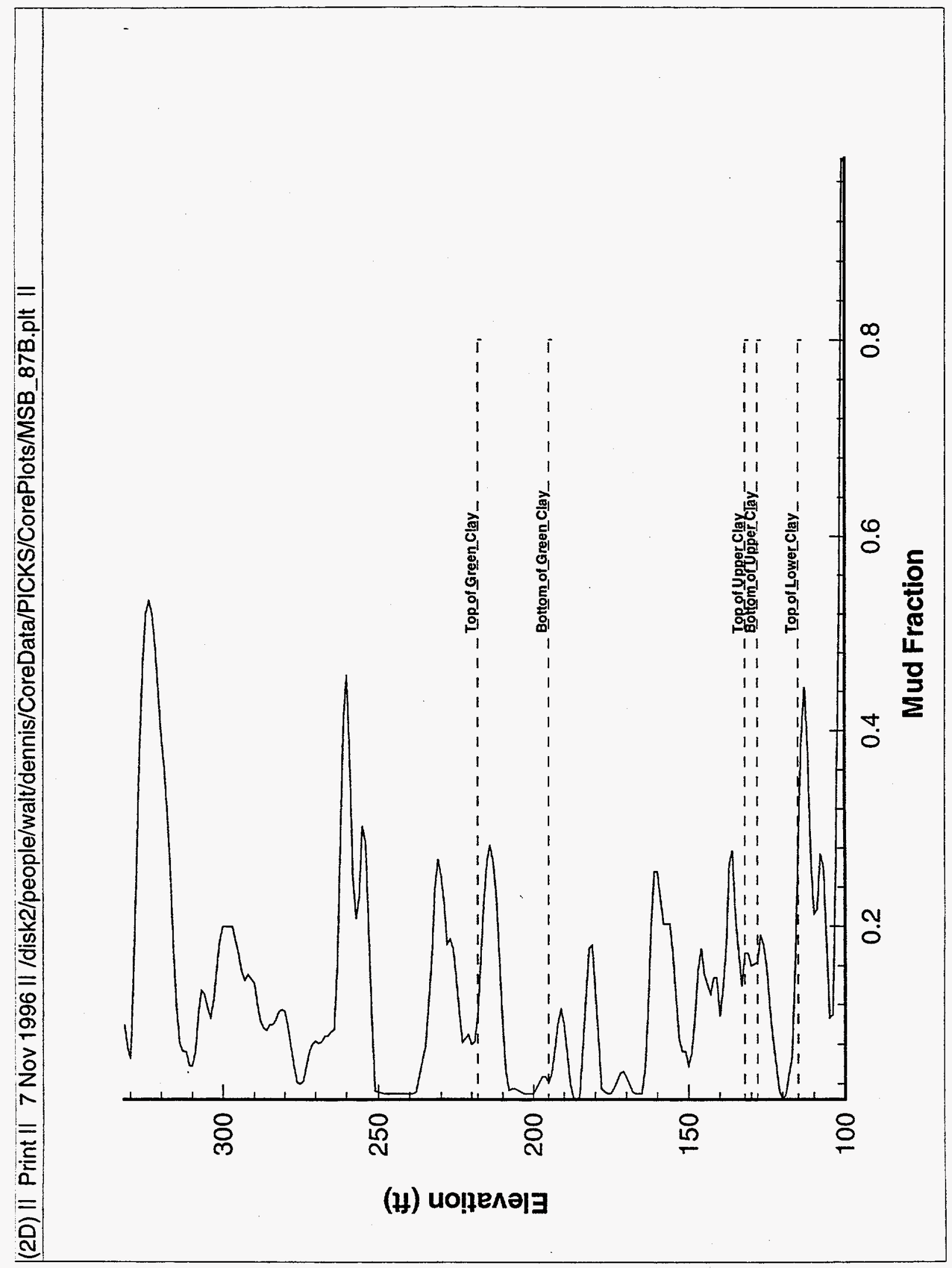




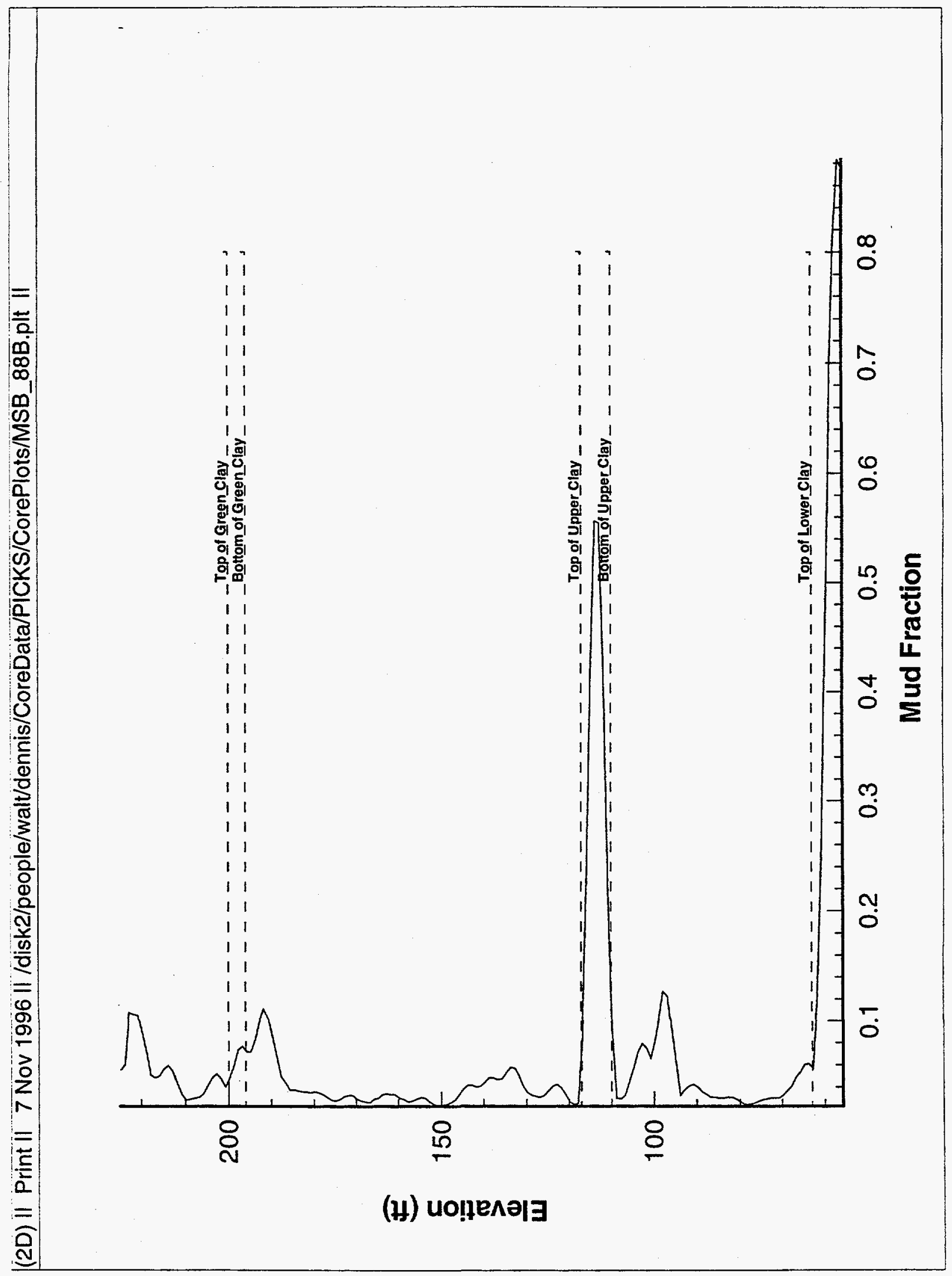




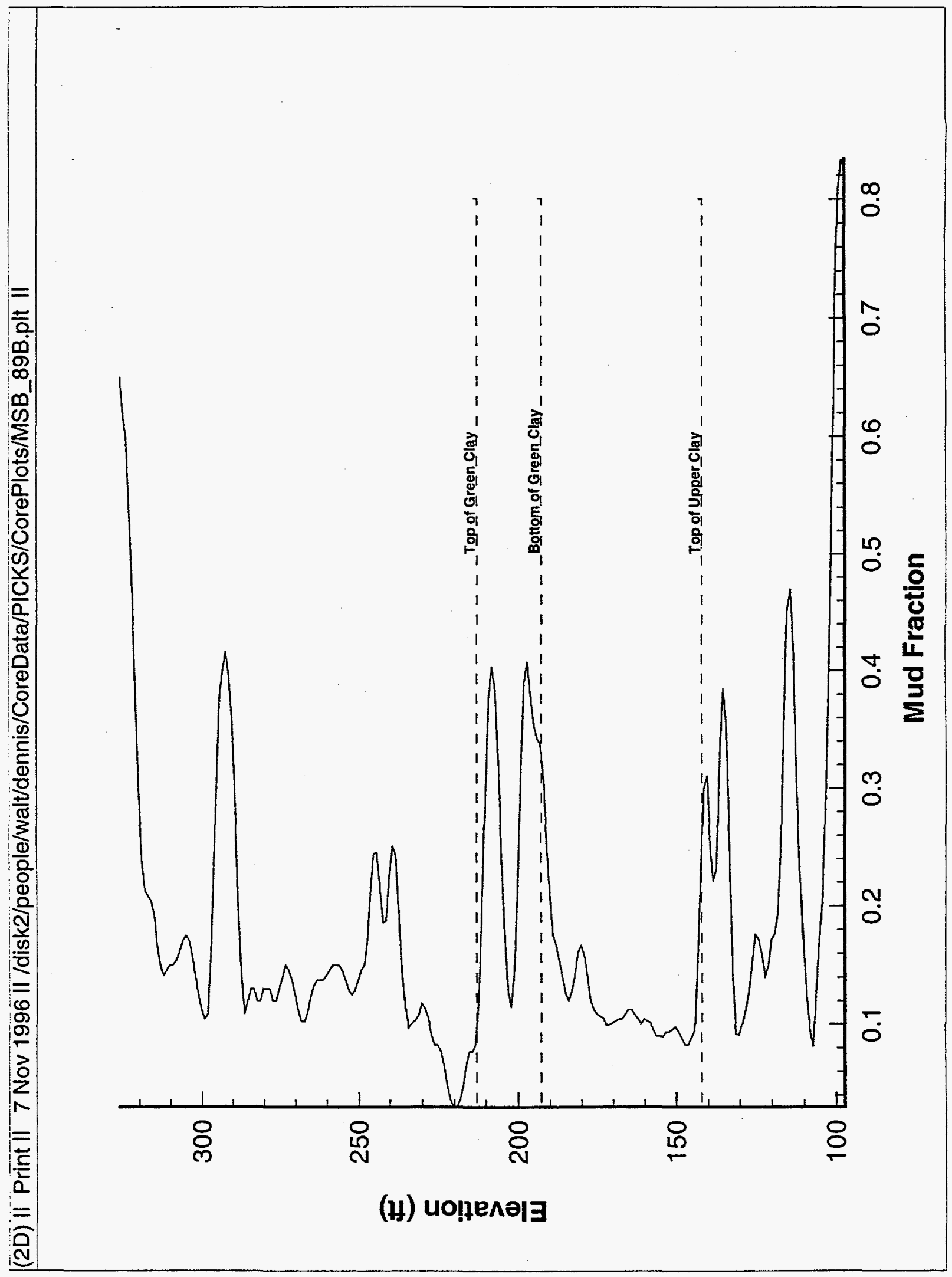




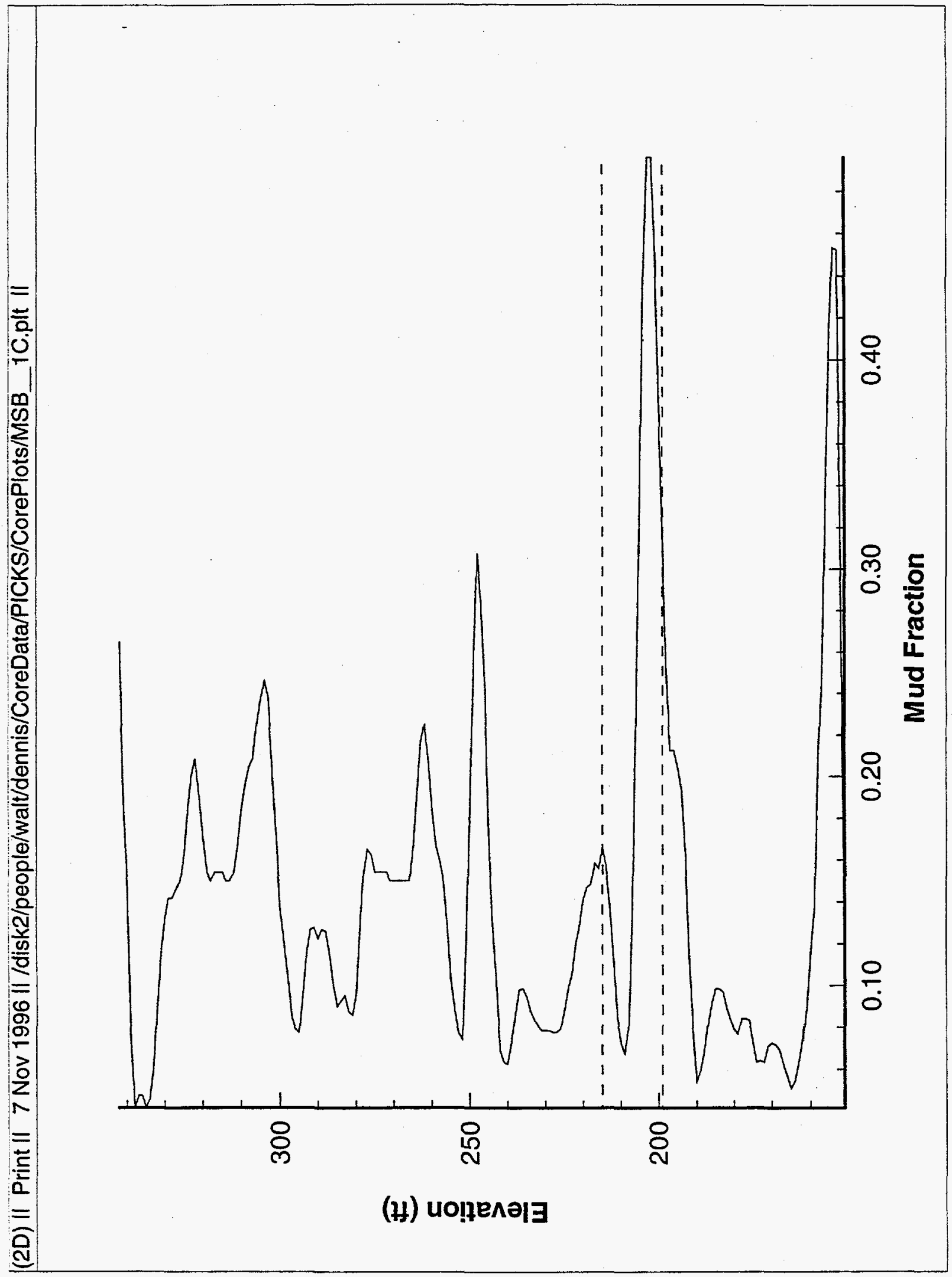




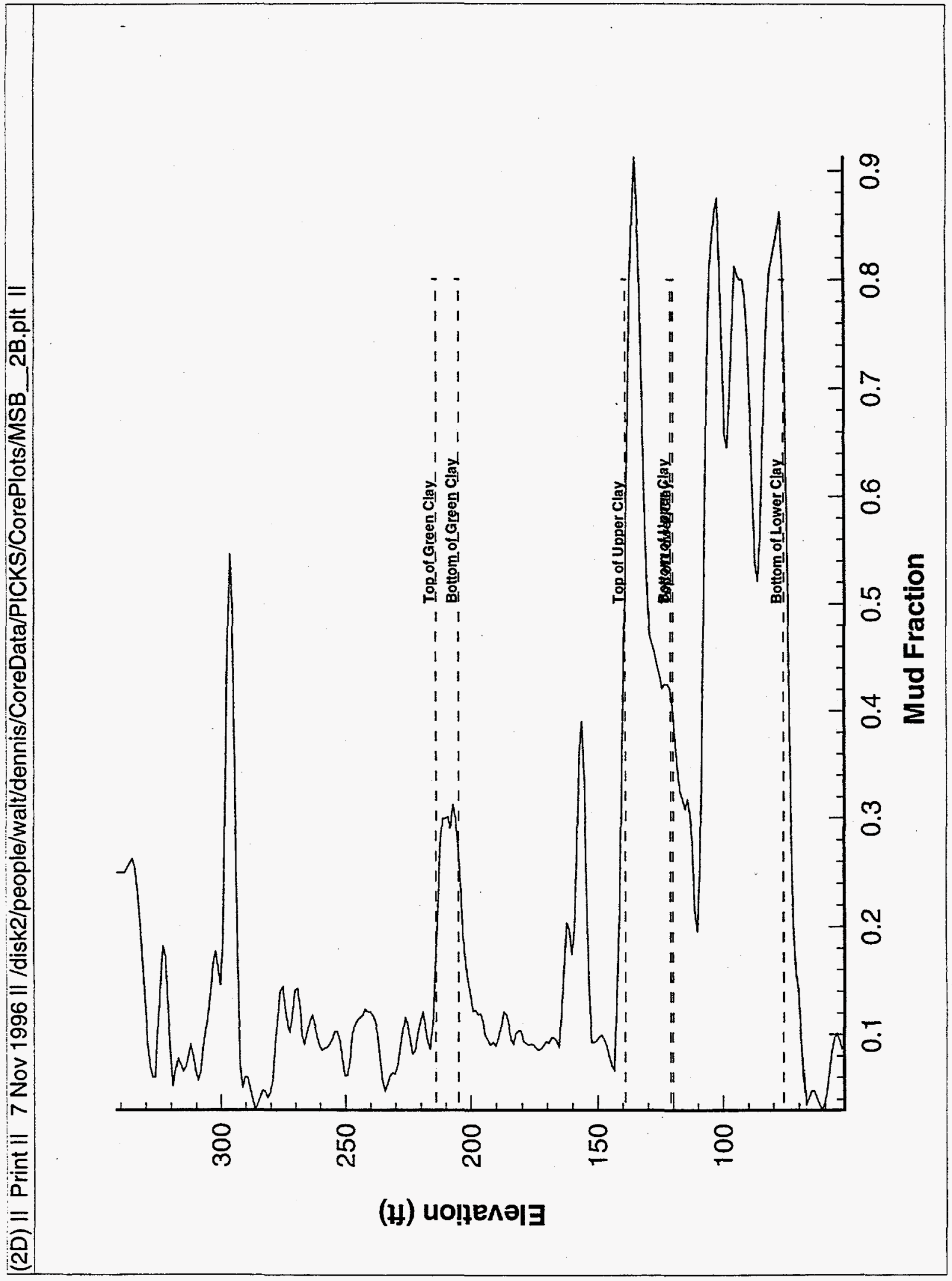




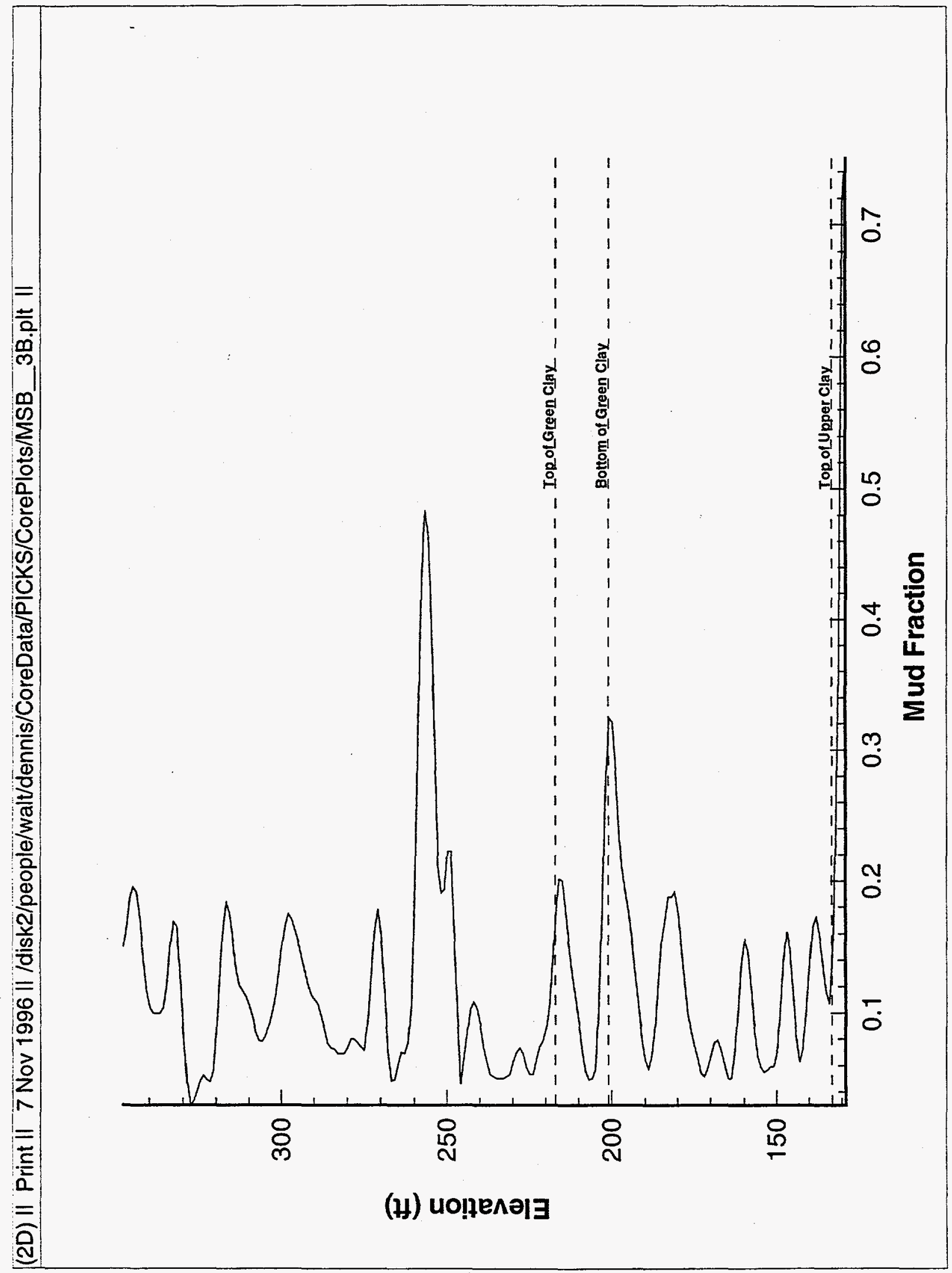




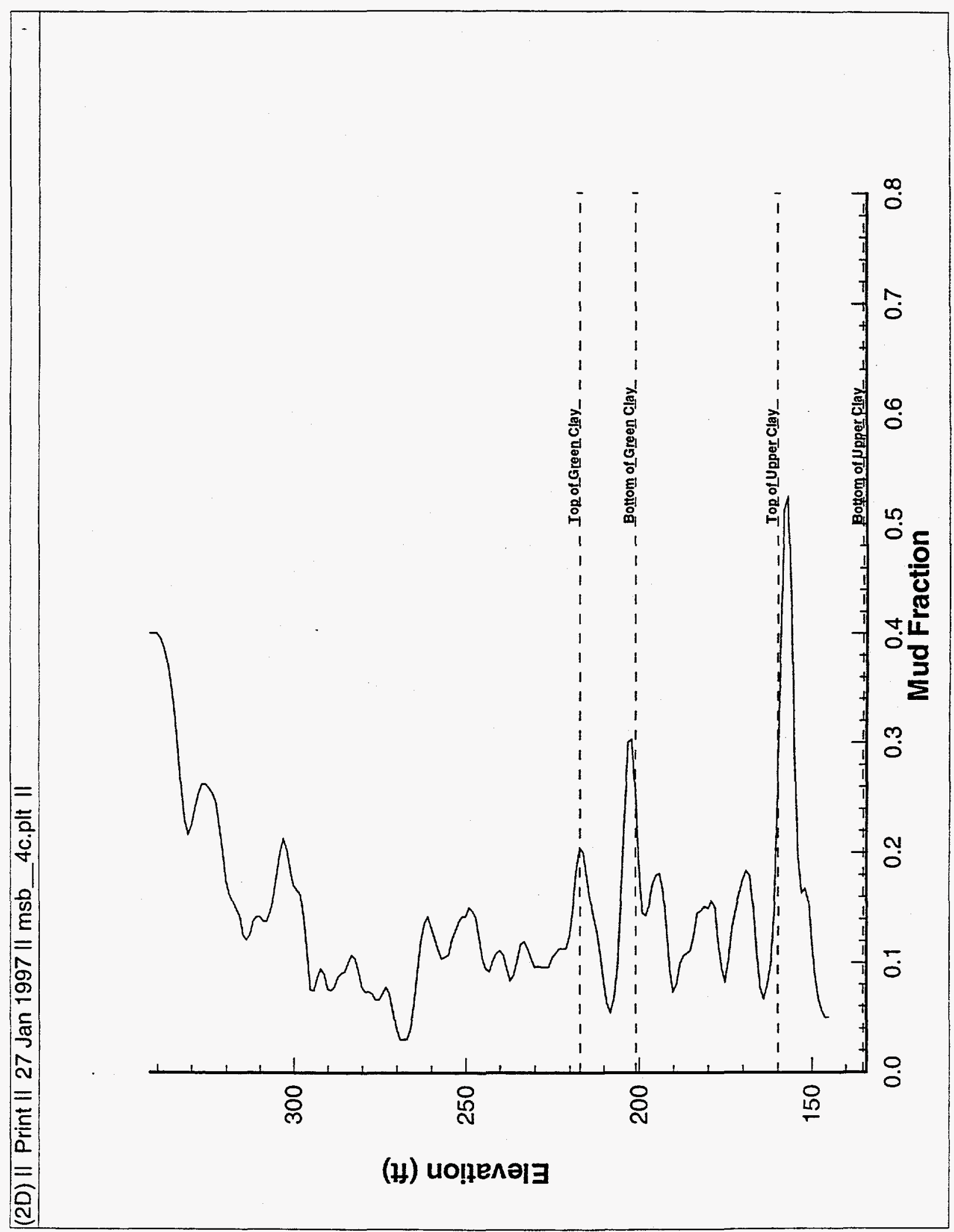




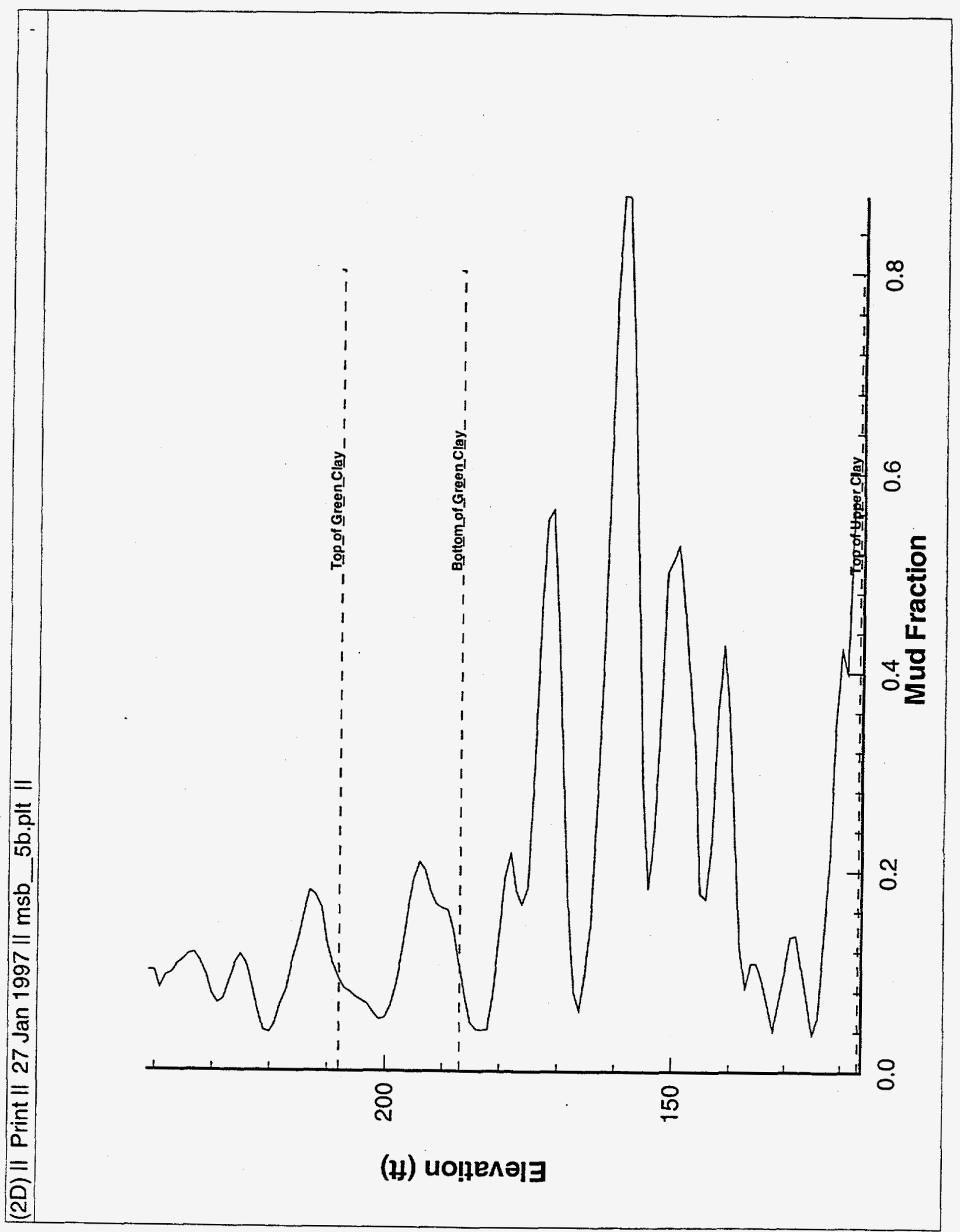




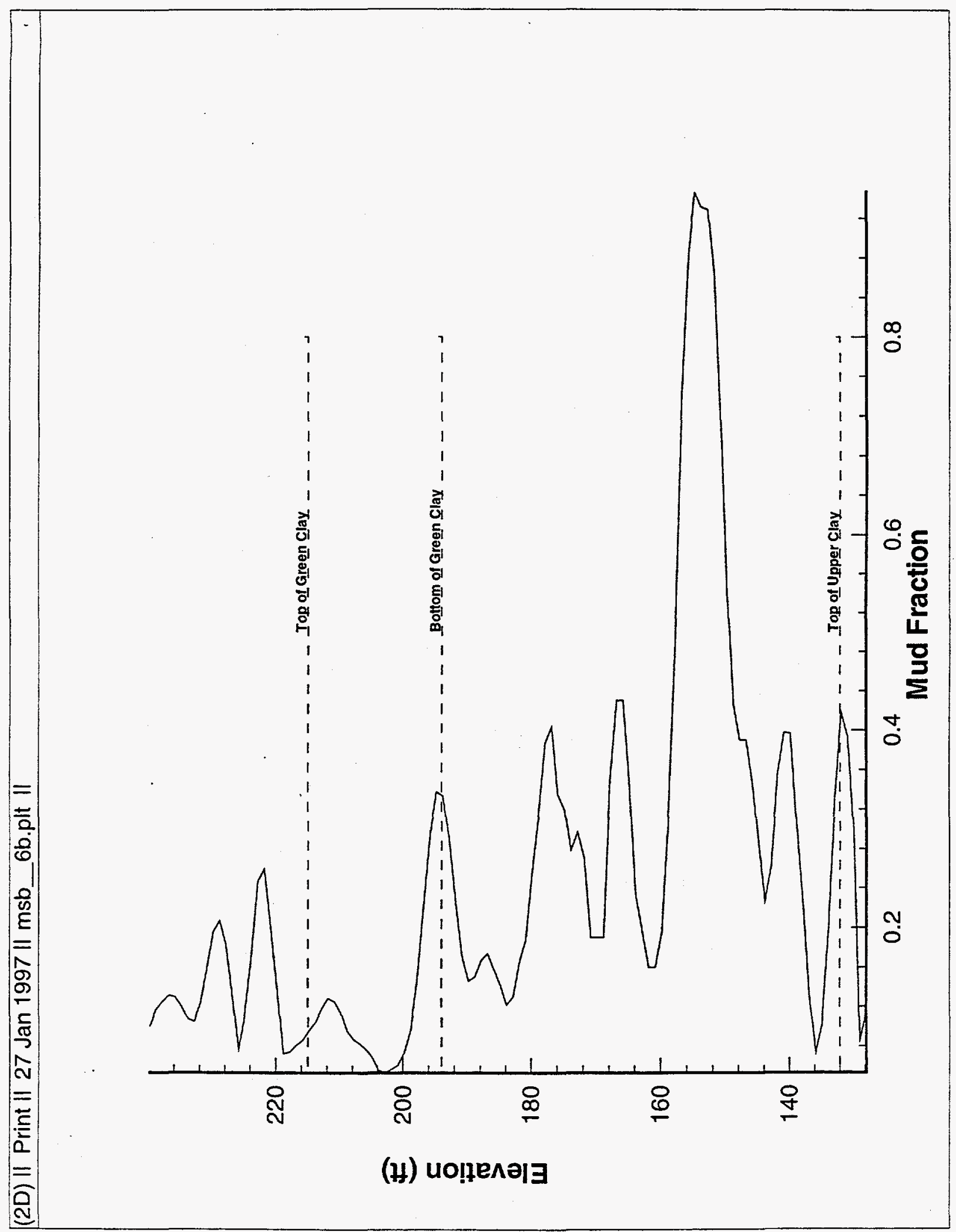




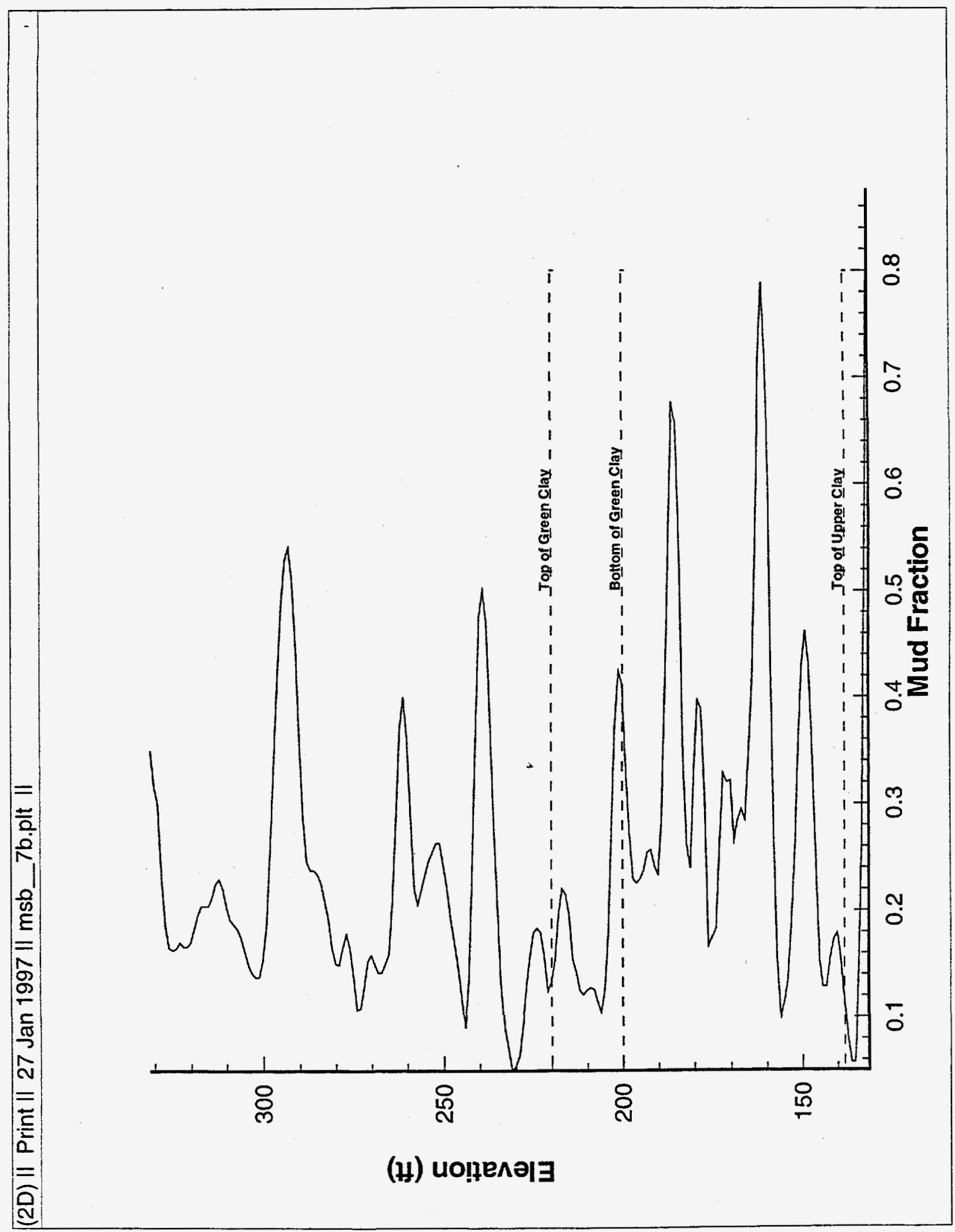




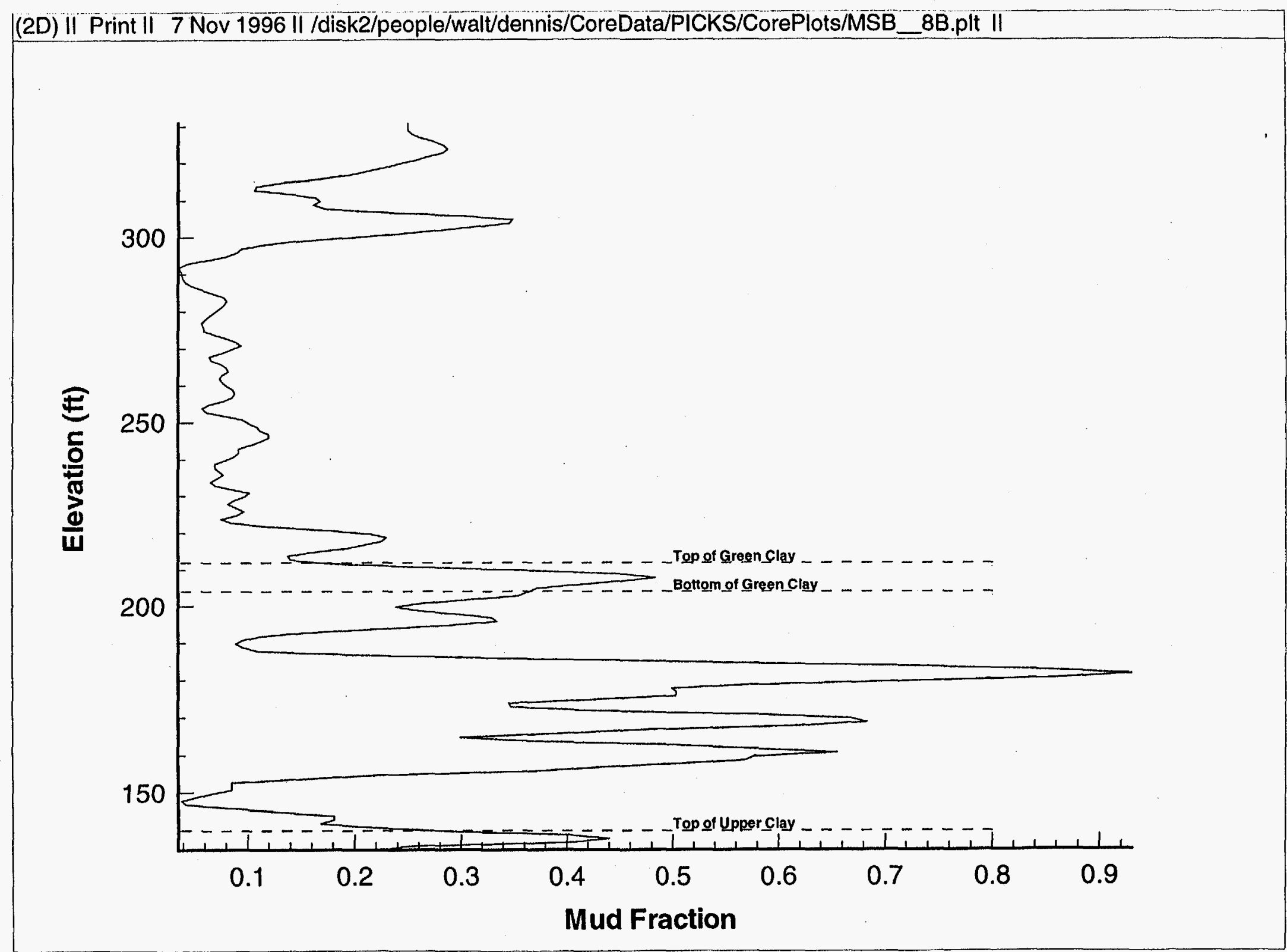




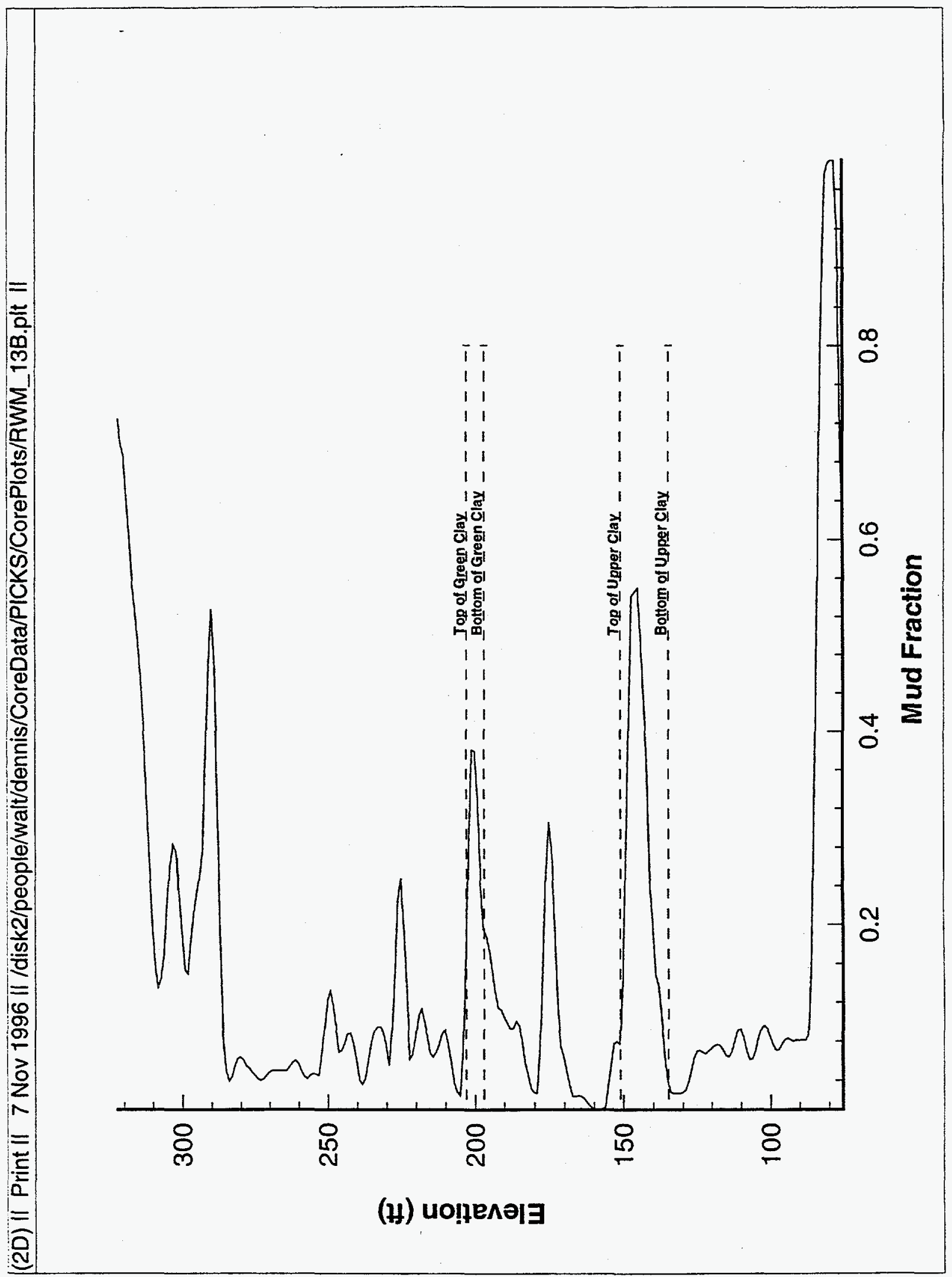




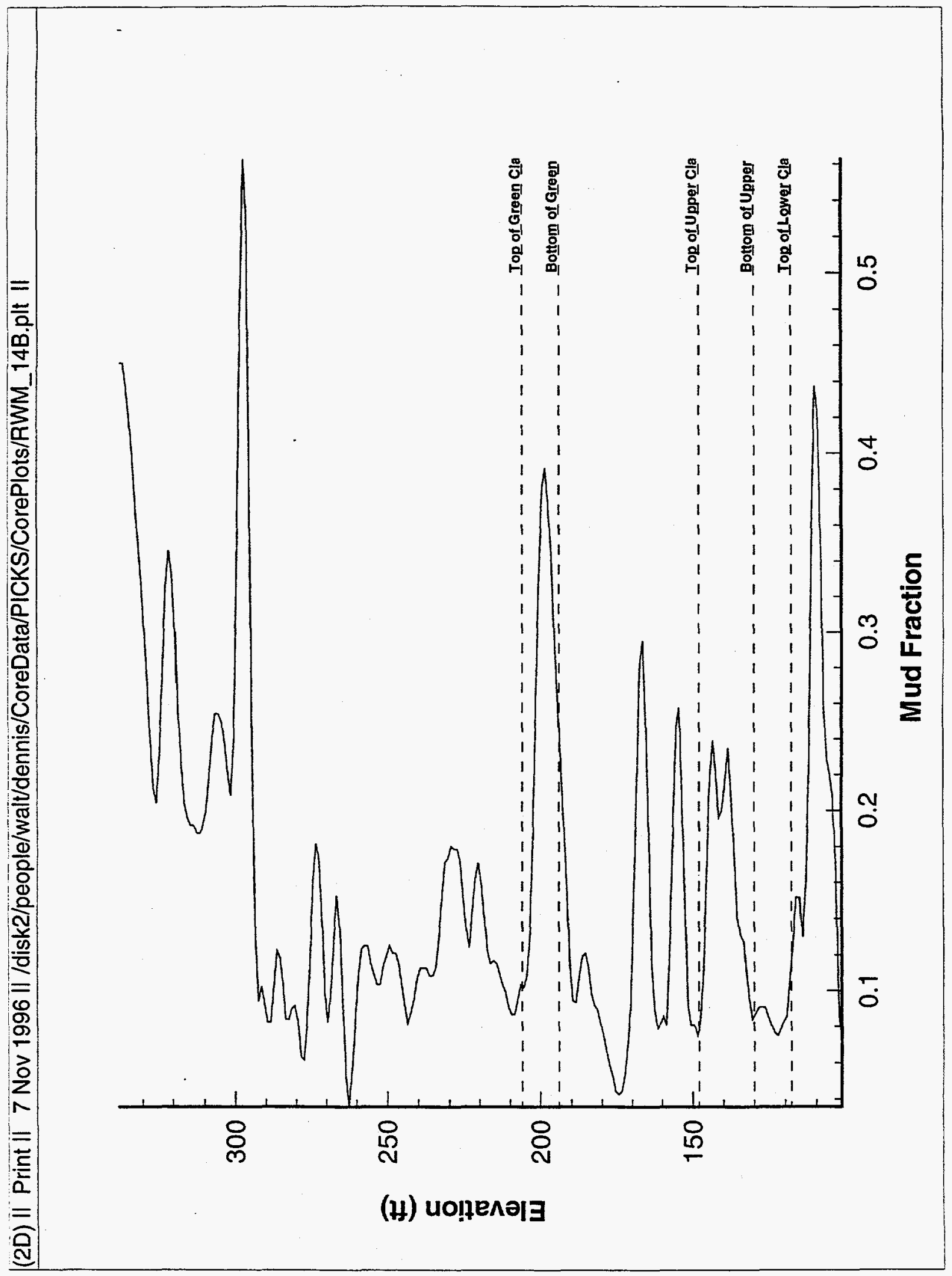




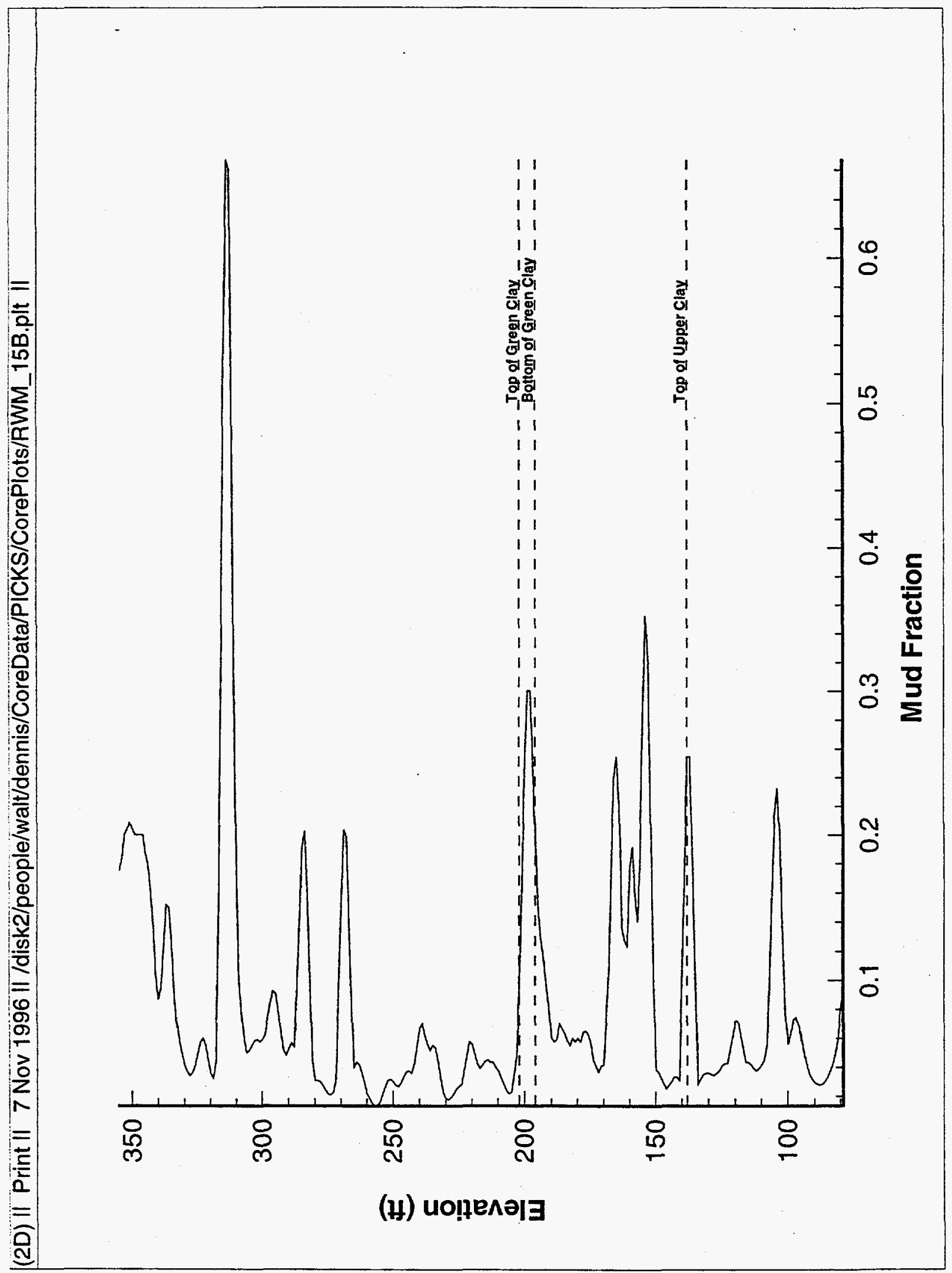




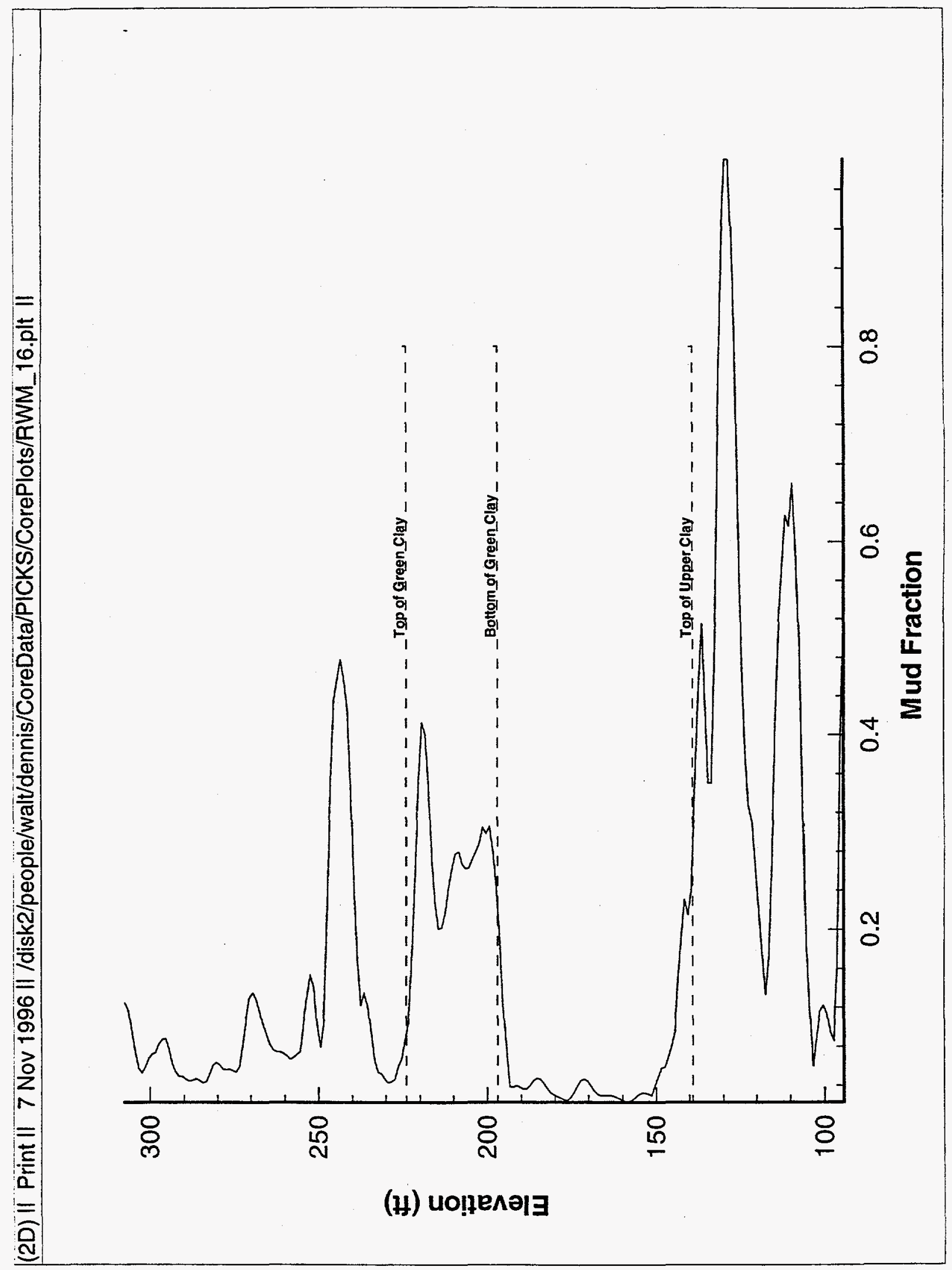




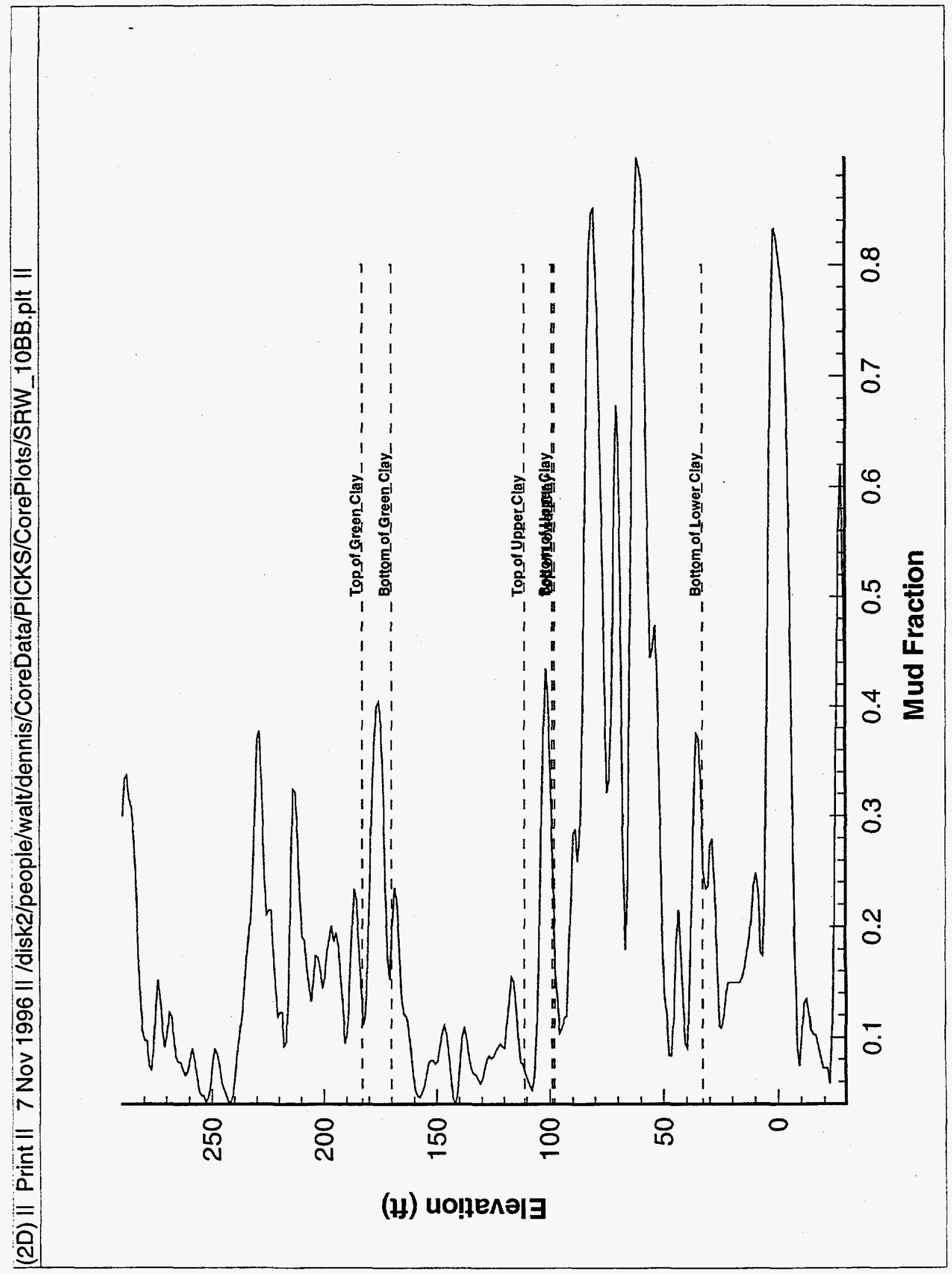




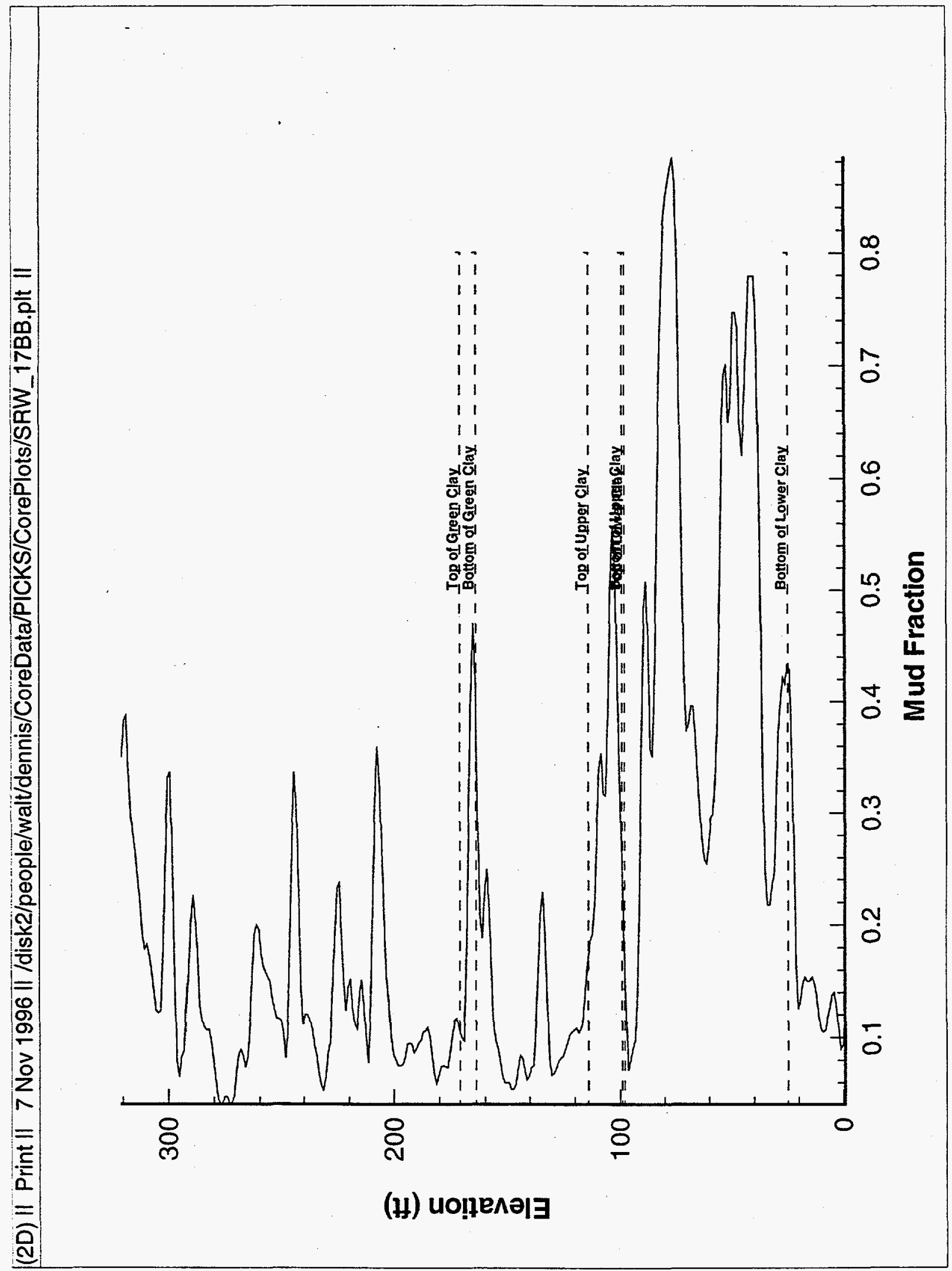




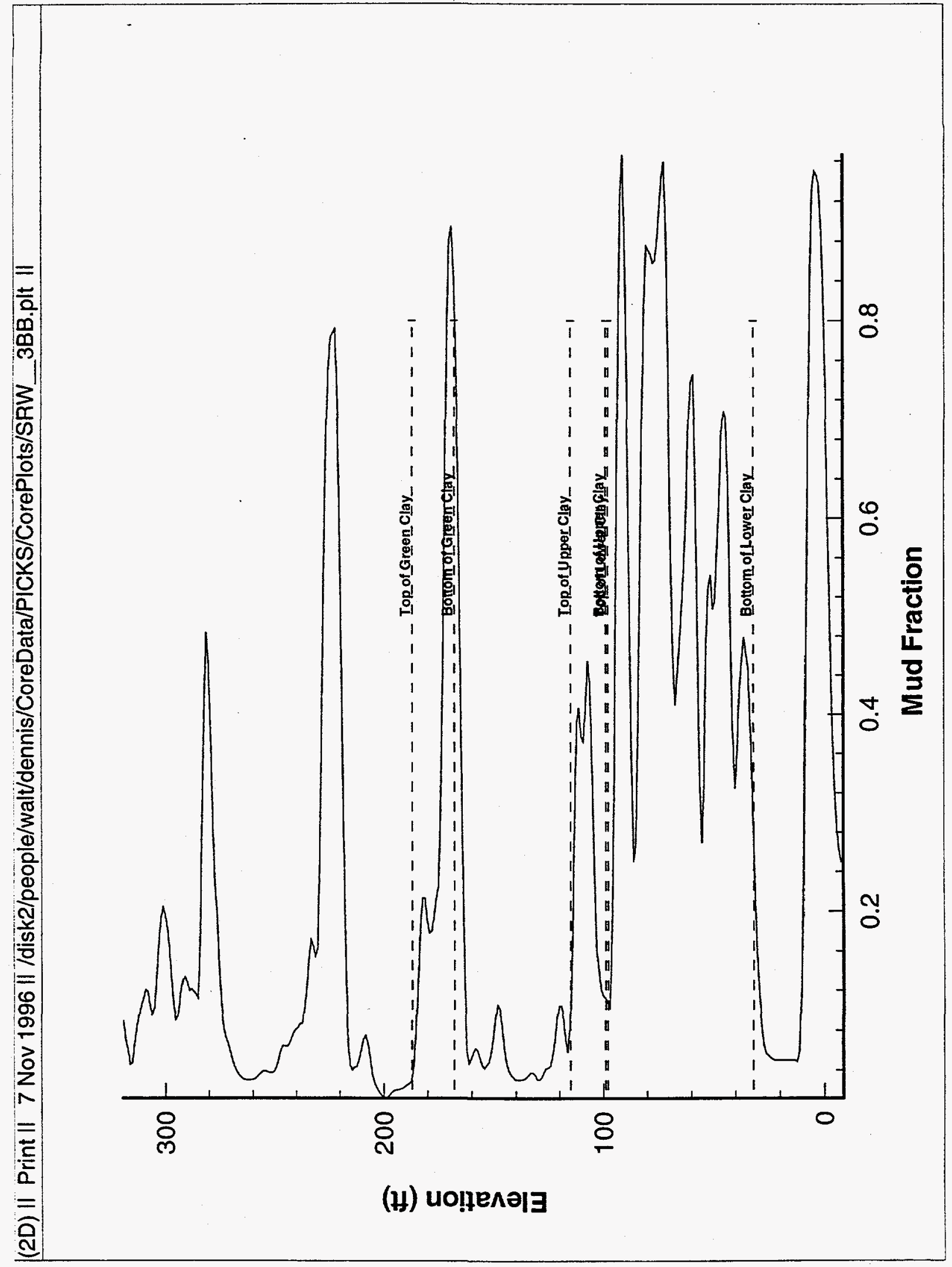




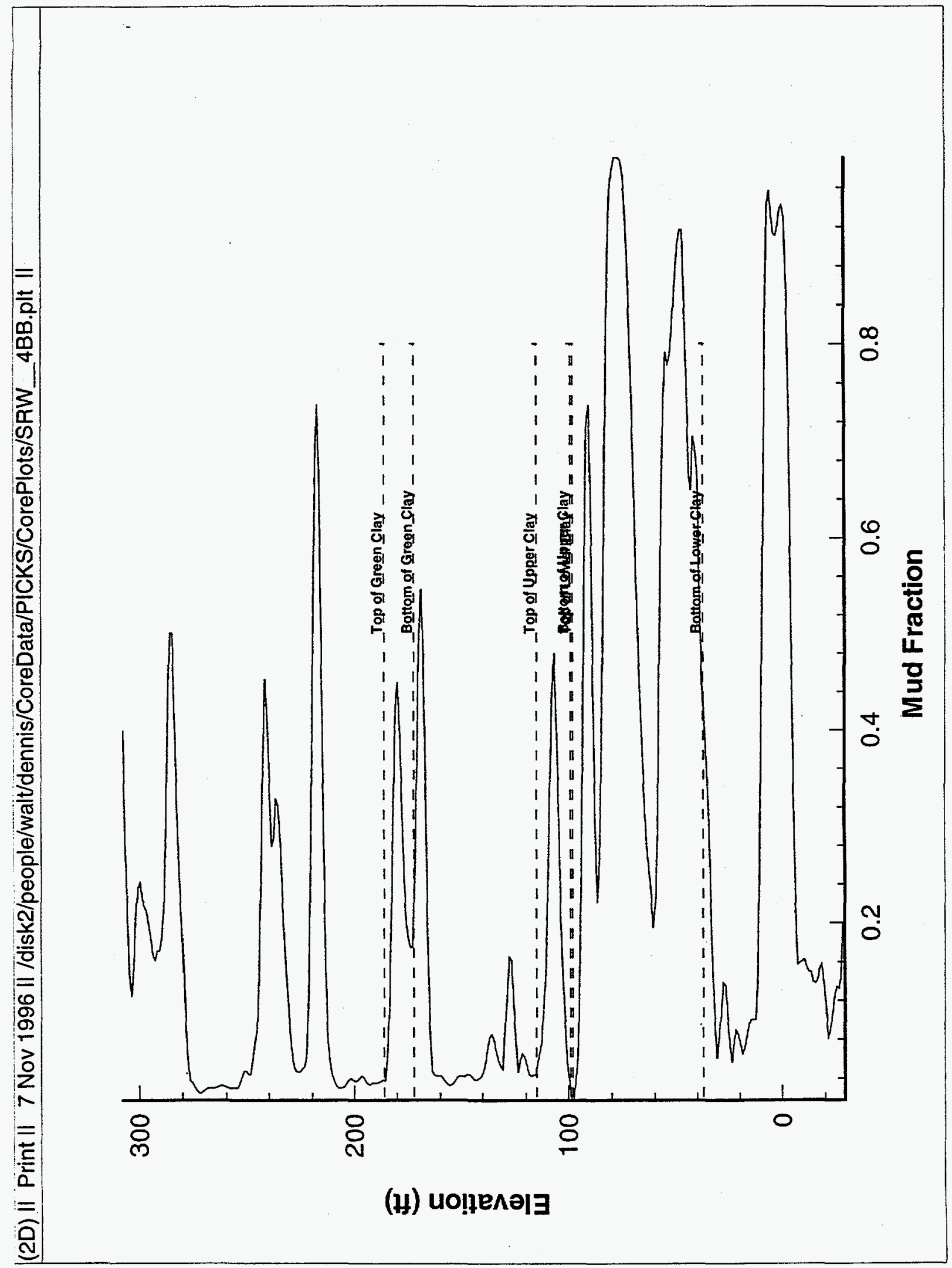




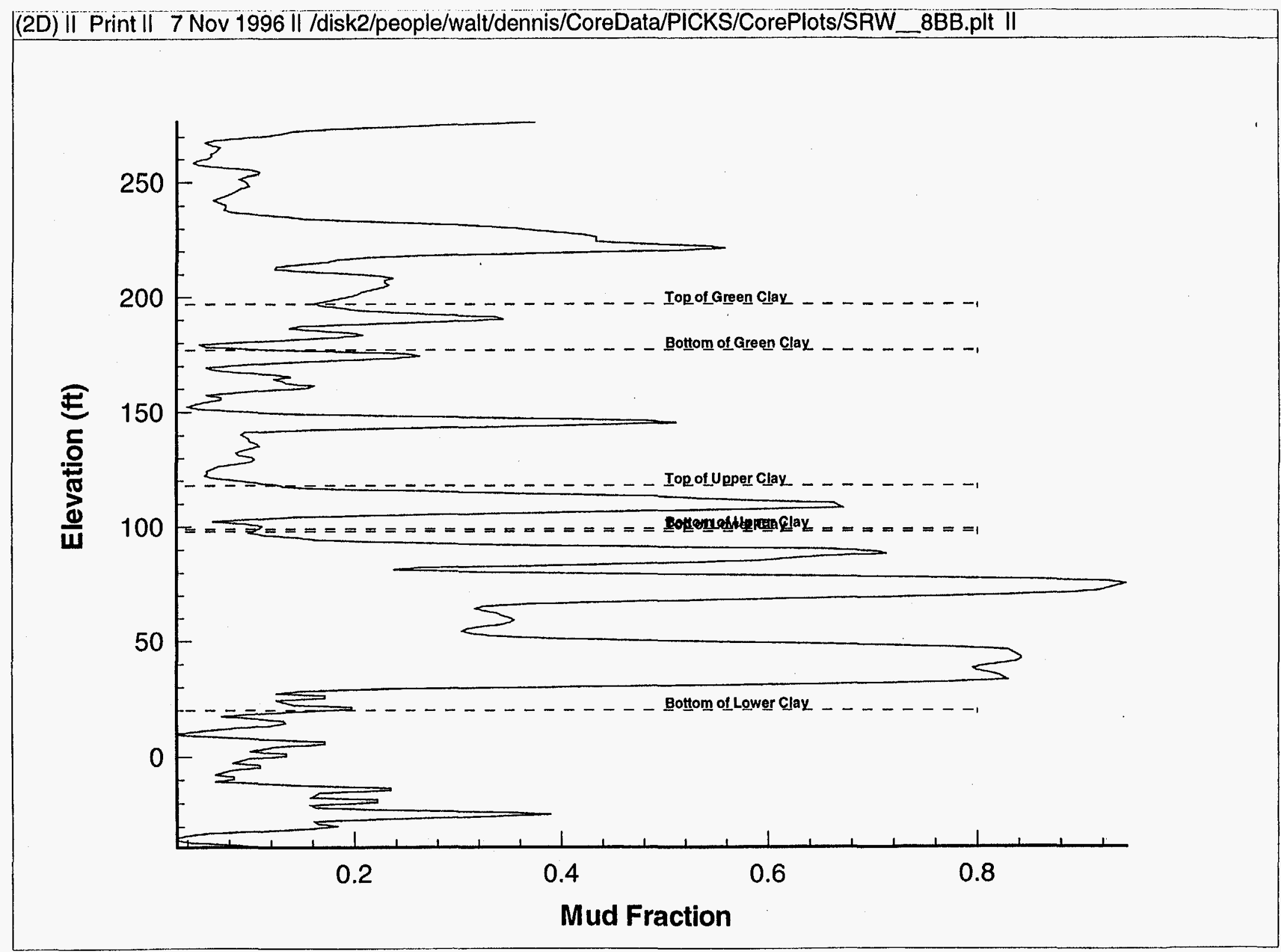


Geological Modeling Residuals

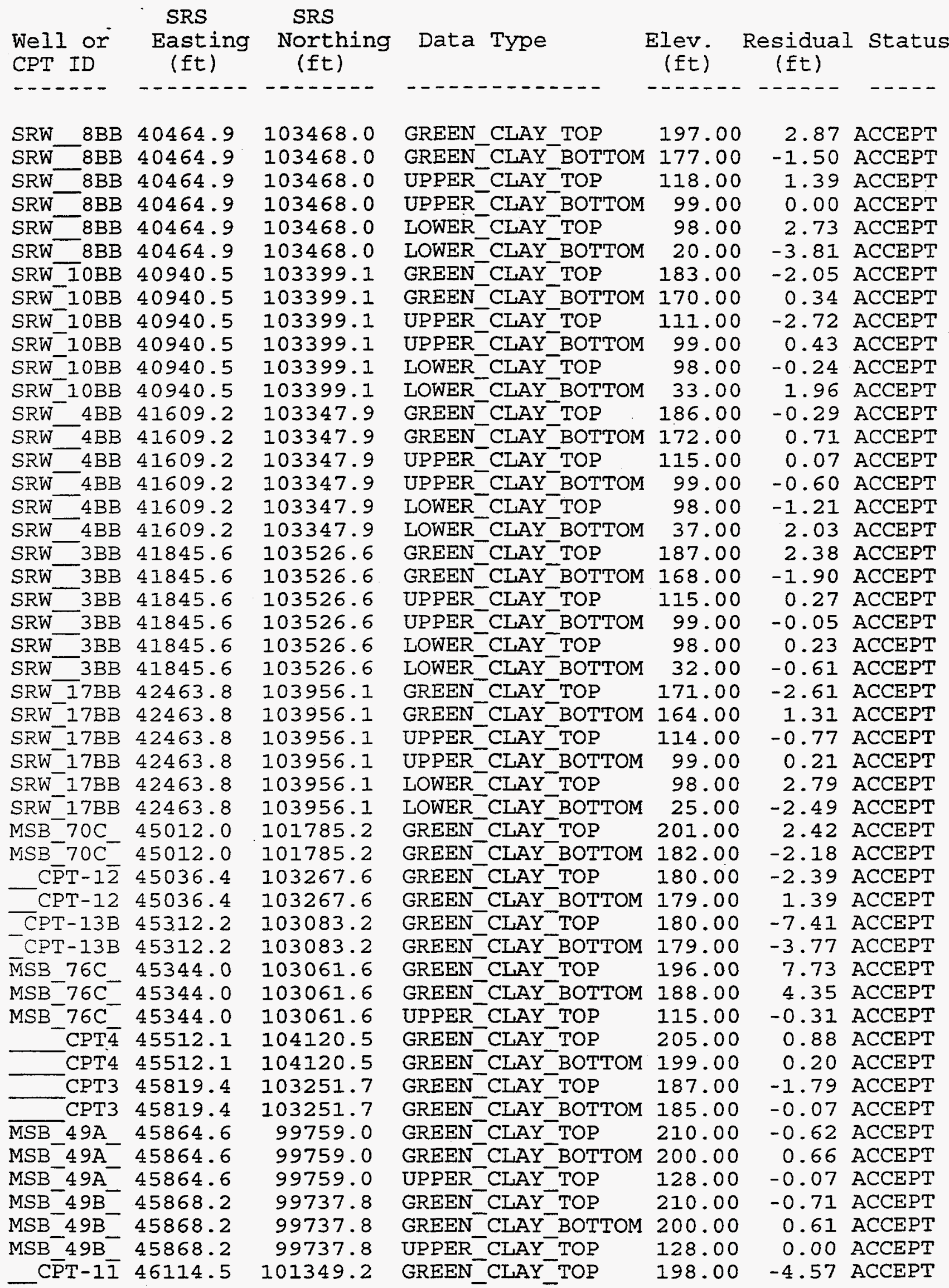


Geological Modeling Residuals

\begin{tabular}{|c|c|c|c|c|c|c|c|}
\hline $\begin{array}{l}\text { IE } 11 \text { or } \\
\text { ST ID }\end{array}$ & $\begin{array}{l}\text { SRS } \\
\text { Easting } \\
\text { (ft) }\end{array}$ & $\begin{array}{l}\text { SRS } \\
\text { Northing } \\
\text { (Et) }\end{array}$ & Data & Type & $\begin{array}{l}\text { Elev. } \\
\text { (ft) }\end{array}$ & $\begin{array}{l}\text { Residual } \\
\text { (ft) }\end{array}$ & \\
\hline & 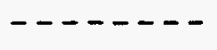 & & & & & 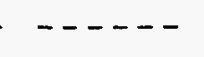 & \\
\hline$\perp \perp$ & .4 .5 & .2 & GRELIV & Y_BOTTOM & 115 & 3.23 & $A C C E P$ \\
\hline $6 B$ & 321.6 & 3.5 & GREEN & CLAY TOP & 00 & 5.37 & ACCEPT \\
\hline $6 \mathrm{~B}$ & 321.6 & 011 & GREEN & _CLAY_BOTTOM & 00 & 60 & ACCEPT \\
\hline $6 \mathrm{~B}$ & 5321.6 & 1011 & UPPER & CLAY_TOP & 132.00 & 0.89 & ACCEPT \\
\hline$\overline{C P T}-1 \overline{4}$ & 6433.0 & 1027 & GREEN & CLAY'TOP & 198.00 & -0.66 & ACCEPT \\
\hline CPT- 14 & 46433.0 & 102736.3 & GREEN & Y_BOTTOM & .00 & 1.21 & ACCEPT \\
\hline$\overline{S B}[64 B$ & 46579.7 & 101831.0 & GREEN & TOP & 00 & -4.02 & ACCEPT \\
\hline $\mathrm{SB}_{-}^{-} 64 \mathrm{~B}^{-}$ & 9.7 & 1.0 & GREEN & ГTOM & 1 & 2.33 & ACCEPT \\
\hline $\mathrm{MSB}^{-} 64 \mathrm{~B}$ & 6579.7 & 1.0 & UPPER & $C I$ & .00 & -1.44 & $\mathrm{AC}$ \\
\hline$C P \bar{T}-23 \bar{B}$ & 46596.2 & 2.5 & GREEN & CLAY & .00 & 0.23 & $\mathrm{PT}$ \\
\hline CPT-23B & 6596.2 & 2.5 & GREEN & TOM & .00 & 0.55 & $\mathrm{AC}$ \\
\hline$c$ & 46714.6 & 100 & GREEN & CI & .00 & -7.35 & PT \\
\hline C & 46714.6 & 5.8 & GREEN & $\mathrm{CI}$ & .00 & 2.07 & $\mathrm{PT}$ \\
\hline$S B$ & 46718.1 & .6 & GREEN & $\mathrm{CI}$ & .00 & 6.76 & $\mathrm{PT}$ \\
\hline $\mathrm{MSB}$ & 718.1 & 100 & GREEN & $C I$ & 2 & -1.63 & $\mathrm{AC}$ \\
\hline $7 B$ & 467 & 100 & UPPER & $C I$ & .00 & 0.27 & AC \\
\hline$[S B$ & 469 & 101 & GREEN & $C L$ & .00 & 3.93 & AC \\
\hline $5 \mathrm{~B}$ & 46 & 101 & GREEN & CI & .00 & -3.67 & AC \\
\hline ISB & 465 & .1 & UPPER & $\mathrm{C}$ & .00 & -0.31 & 1 \\
\hline $8 B$ & 472 & 100 & GREEN & $C L$ & .00 & -1.82 & PT \\
\hline$S B$ & 472 & 100 & GREEN & $C I$ & 00 & 1.94 & PT \\
\hline MSB & 472 & 1008 & UPPER & CI & .00 & 0.41 & AC \\
\hline$S B=79 B$ & 473 & 96.9 & GREEN & $C L$ & .00 & 2.05 & $\mathrm{AC}$ \\
\hline $9 \mathrm{~B}$ & 473 & 99296.9 & GREEN & CLAY BOTTOM & .00 & -0.79 & ACCEPT \\
\hline$S B \_79 B$ & 47300.2 & 99296.9 & UPPER & CLAY_TOP & .00 & -0.59 & ACCEPT \\
\hline $\mathrm{SB}-13 \mathrm{C} \overline{\mathrm{C}}$ & 47525.7 & 101728.8 & GREEN & CLAY_TO & 7.00 & 2.06 & ACCEPT \\
\hline $13 \mathrm{CC}$ & 47525.7 & 101728.8 & GREEN & _CLAY_BOTTOM & 198.00 & -0.69 & ACCEPT \\
\hline $13 \mathrm{CC}$ & 47525.7 & 101728.8 & UP. & CLAY_TOP & 131.00 & 0.34 & ACCEPT \\
\hline CPT7 & 47586.2 & 102444.4 & GR & Y_T & .00 & -3.91 & $\mathrm{ACC}$ \\
\hline CPT7 & 47586.2 & 102444.4 & GR & TOM & .00 & 44 & $A C$ \\
\hline PT -9 & 47696.7 & 100993.0 & GR & CLAY_T & .00 & -3.98 & $A C$ \\
\hline CPT - 9 & 47696.7 & 100993.0 & GR & _CLAY_BC & .00 & 2.98 & $A C$ \\
\hline $63 B$ & 47861.0 & 101184.4 & GR & CLAY_TC & .00 & 4.78 & $\mathrm{AC}$ \\
\hline $53 B$ & 47861.0 & .4 & GR & CLAY_BOT & 00 & -4.34 & AC \\
\hline ZPT5 & 47863.3 & .7 & G & 7 & 00 & -1.63 & $A C$ \\
\hline CPT5 & 47863.3 & .7 & GR & Y_B & 0 & 42 & $A C$ \\
\hline СPT2 & 7884.8 & .6 & N & $I_{-}$ & 0 & -2.78 & $\mathrm{AC}$ \\
\hline СРT2 & 884.8 & 1 & N. & Y_BOTTOM & $18+2>$ & 3 & RT \\
\hline $89 B$ & 889.9 & . 1 & GR & 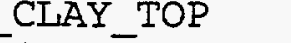 & 2 & 9 & $A C$ \\
\hline SB & 47889.9 & $=1$ & GR & _BOTTOM & 1. & 2 & $A C$ \\
\hline SB & 889.9 & 4.1 & UP & Y TOP & $14 \div 2$ & 9 & PT \\
\hline $52 B$ & 906.8 & 65.3 & GREEN & CLAY TOP & 27 & 8 & $\mathrm{PT}$ \\
\hline $62 B$ & 47906.8 & 65.3 & GR & CLAY_BOTTOM & 1 & -2.31 & $\mathrm{AC}$ \\
\hline$S B$ & 47906.8 & 101865.3 & UPPER & CL & 11 & 18 & ACCEPT \\
\hline WM & 48244.8 & 97647.2 & GREEN & CLAY_TOP & 22 & 10 & ACCEPT \\
\hline WM & 8244.8 & 97647.2 & GREEN & CLAY_BOTTOM & 1. & -1.04 & ACCEPT \\
\hline WM & 8244.8 & 97647 & UPPER & CLAY TOP & & -2.54 & ACCEPT \\
\hline$S E$ & 8279 & 97672.8 & GREEN & _CLAY_TOP & & & ACCEPT \\
\hline SB & 48279.4 & 97672.8 & GREEN & _CLAY_BOTTOM & & -0 & ACCEPT \\
\hline SE & 48279 & 97672.8 & UPPER & CT & & & ACCEPT \\
\hline $1 S B$ & 48279 & 97672 & UPPER & BOTr & 122.00 & -0.99 & ACCEPT \\
\hline
\end{tabular}


Geological Modeling Residuals

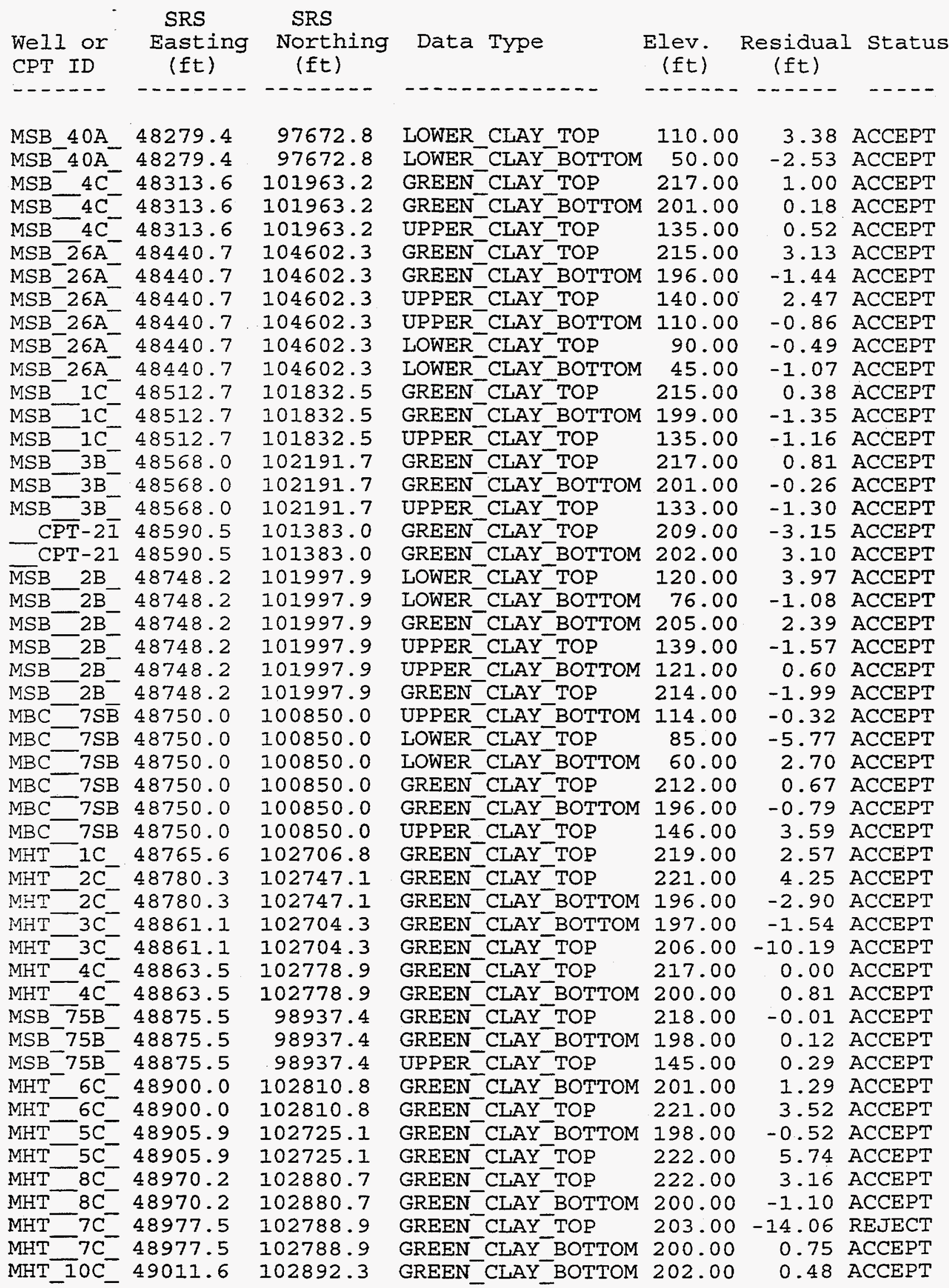


Geological Modeling Residuals

SRS SRS

\begin{tabular}{|c|c|c|c|c|c|c|c|}
\hline $\begin{array}{l}\text { WeII or } \\
\text { CPT ID }\end{array}$ & $\begin{array}{l}\text { Easting } \\
\text { (ft) }\end{array}$ & $\begin{array}{l}\text { Northing } \\
\text { (ft) }\end{array}$ & Data & Type & $\begin{array}{l}\text { Elev. } \\
\text { (Et) }\end{array}$ & $\begin{array}{c}\text { Residual } \\
\text { (ft) }\end{array}$ & $\operatorname{cac} a$ \\
\hline & & & & & & & \\
\hline IT & 011.6 & 2.3 & GREEN & CLAY & 217 & -2.28 & ACCEP \\
\hline $\mathrm{HT}$ & 49015.6 & 4.4 & GREEN & CLAY & 223.00 & 5.24 & ACCEP \\
\hline $\mathrm{HT}^{-}$ & 49015.6 & 102814.4 & GREEN & _CLAY_BOTTOM & 200.00 & 0.02 & CCEPI \\
\hline $\mathrm{HT}$ & 49031.8 & 4.3 & GREEN & CLAY'TOP & 223.00 & 5.04 & $C C$ \\
\hline $\mathrm{HT}_{-}^{-}$ & 49031.8 & 4.3 & GREEN & CLAY_BOTTOM & 200.00 & -0.20 & $C C E P$ \\
\hline$S B \overline{2} 3 T \bar{A}$ & 49225.8 & 8.8 & GREEN & CLAY TOP & 208.00 & -0.27 & $\mathrm{CC}$ \\
\hline $\mathrm{B}^{-} 23 \mathrm{TA}$ & 4922 & 8.8 & GREEN & CLAY_BOTTOM & 190.00 & -0.87 & $C c$ \\
\hline$B_{-3}^{-3} B$ & 497 & .8 & UPPER & _CLAY_BOTTOM & 101.00 & -5.77 & $-1+3+3$ \\
\hline $38 B$ & 497 & .8 & IOWER & CLAY'TOP & 100.00 & 0.32 & $\mathrm{Cc}$ \\
\hline $3 B-38 B$ & 497 & .8 & LOWER & CLAY_BOTTOM & 67.00 & 1.03 & $\mathrm{CO}$ \\
\hline $38 \mathrm{~F}$ & .1 & & GREEN & 3OTTOM & 202.00 & -1.17 & CC \\
\hline $5 B-38 \mathrm{E}$ & 497 & .8 & GREEN & CLAY & .00 & 1.98 & CC \\
\hline $\mathrm{SB}_{-}^{-} 38 \mathrm{E}$ & 497 & .8 & UPPER & _CLAY_TOP & .00 & 4.14 & $C \mathrm{C}$ \\
\hline$T-7$ & 501 & 1 & GREEN & CLAY & 00 & -3.68 & $\mathrm{CC}$ \\
\hline $\mathrm{CPT}-17$ & 50 & & GREEN & CLAY ВOTTOM & 00 & 57 & C \\
\hline $\bar{S} B \_74 B$ & .2 & & GREEN & CLAY'TOP & .00 & 2.28 & CC. \\
\hline$B-74 B$ & .2 & & GREEN & CLAY_BOTTOM & 00 & -1.92 & CCEP \\
\hline $74 \mathrm{~B}$ & .2 & & UPI & CLAY_TOP & Do & 34 & CCEPT \\
\hline $88 B$ & 2 & & GR & CLAY_TOP & 0 & -4.35 & CCEPJ \\
\hline $88 \mathrm{~B}$ & .2 & & $\mathrm{EN}$ & TOM & 0 & 4 & $\mathrm{CCl}$ \\
\hline 881 & 50 & 9 & UP & $P$ & 00 & -2.30 & CCEP \\
\hline 88 & 50 & 9 & $E R$ & RTOM & $1:$ & 3 & CEP \\
\hline $88 B$ & $50^{\circ}$ & & LOWER & $P$ & 0 & 0 & CEP \\
\hline 501 & 51 & & UPPER & CLA & 0 & 2 & CEPI \\
\hline $50 \mathrm{E}-\mathrm{r}-\mathrm{r}$ & 51053.5 & .0 & GREEN & CLAY & 0 & 49 & CCEPT \\
\hline $50 \mathrm{~B}$ & 51053.5 & .0 & GREEN & CIAYY & .00 & -0.39 & CCEPT \\
\hline $\mathrm{B}_{-1} 13 \mathrm{AR}$ & .0 & .0 & GREEN & CLAY & 0 & 52 & CCEPT \\
\hline$B-13 A R$ & 1396. & .0 & GREEN & CLAY_- & 00 & -0.56 & CCEPI \\
\hline B_I3AR & 139 & .0 & JPPER_ & CLAY_I & 00 & 28 & ACCEPT \\
\hline I3AR & 1396. & .0 & UPPER & CLAY_B & 00 & -3.60 & ACCEPI \\
\hline I3AR & 1396.0 & .0 & LOWER & CLAY_TOP & .00 & -0.99 & CCEPI \\
\hline $10 \mathrm{~A}$ & 1410.0 & .4 & GREEN_ & _CLAY_TOP & 00 & 25 & CCEPT \\
\hline $10 \mathrm{~A}$ & 141 & .4 & GREEN & CLAY_BOTTOM & 00 & 48 & CCEPT \\
\hline $10 \mathrm{~A}$ & 514 & 4 & ER & CIAYY & 00 & -1.08 & CCEPT \\
\hline $10 \mathrm{~A}$ & 51410.0 & & $E R$ & CLAY_BOTTOM & 00 & 01 & CCEPT \\
\hline 102 & 51410 & & SOWER & CLAY TOP & 0 & -0.36 & CCEPT \\
\hline $8 C_{-}$ & 4 & & $\mathrm{EN}$ & CLAYY & 0 & 34 & CCEPI \\
\hline$B C$ & 5144 & & LOWER & CLAY & 7 & 0.63 & CCEPT \\
\hline$A S B$ & 5144 & & UPPER & CLAY & 0 & 65 & ACCEPT \\
\hline $4 S B$ & 4 & 3.0 & UPPER & CLAYYBOTTOM & 10 & -1.48 & CCEPT \\
\hline $\mathrm{MB}$ & 1 & 6 & GREEN & CLAY_TOP & 22 & 13 & CCEPT \\
\hline$M B$ & 1 & 6 & GREEN & CLAY BOTTOM & & 0.50 & CCEPT \\
\hline $\mathrm{MB}$ & $8+2$ & & UPPER & CLAY & & -4.70 & CCEPI \\
\hline MB & 14698 & & UPPER & ЗОТTОМ & & & CCEPI \\
\hline$M R$ & 14698 & & LOWER & CLAY & & 90 & CCEPT \\
\hline$M P$ & 501 & & TIPPFR & CLAY_BOTTOM & & 2.29 & CCEPI \\
\hline$M R$ & 597 & & GREEN & CLAY TOP & & .00 & ACCEPI \\
\hline & & & GREEN & CLAY & & -0.32 & CCEPT \\
\hline & & & ו & D & & 36 & ACCEPT \\
\hline$S E$ & & 107 & GREEN & CLAY_TOP & 218.00 & 1.34 & $A C L E P$ \\
\hline
\end{tabular}


Geological Modeling Residuals

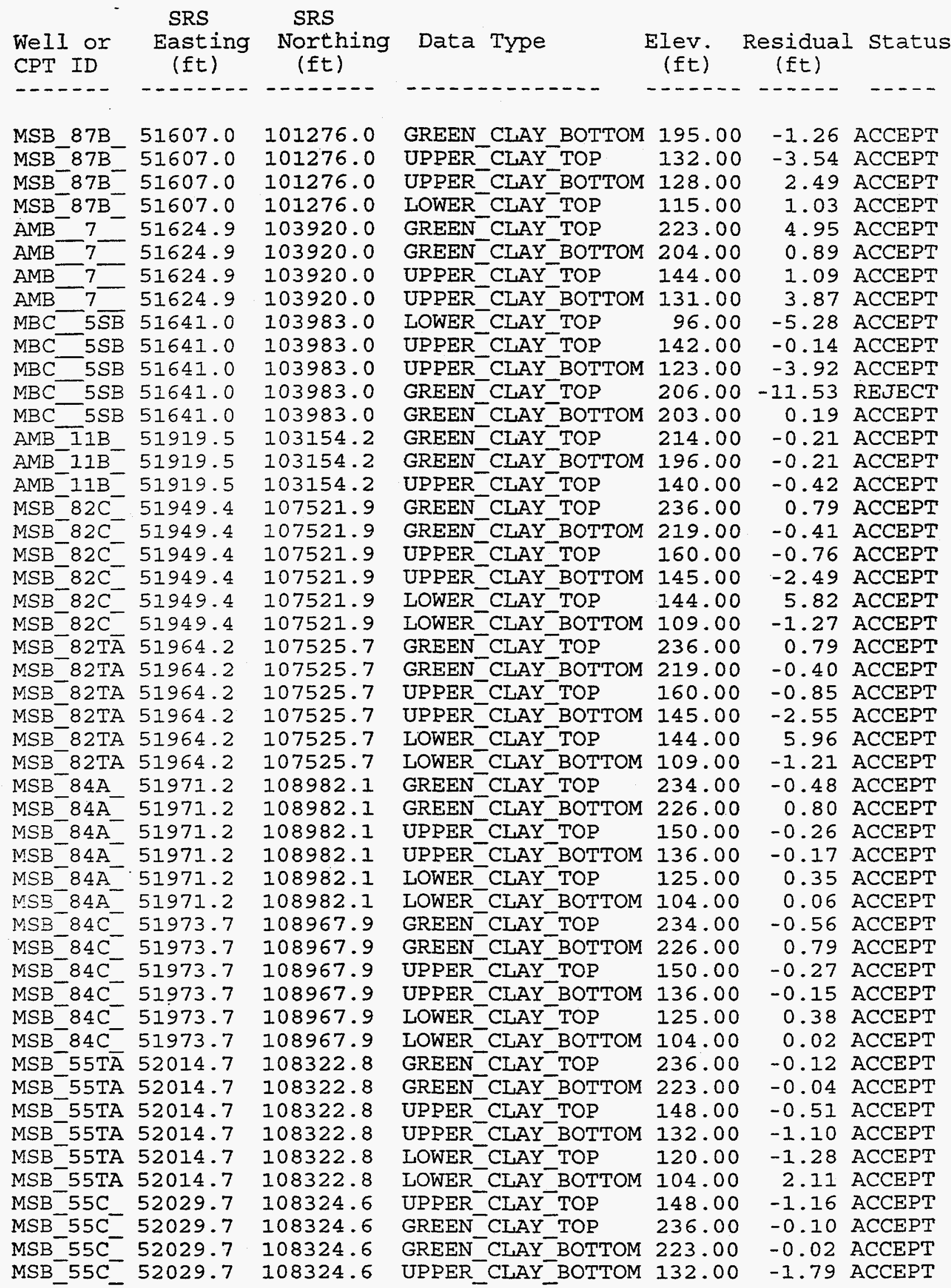


Geological Modeling Residuals

SRS SRS

We11 or CPT ID

$------$

MSB 55C MSB $55 \mathrm{C}^{-}$

52029.7

MSB 47TA 52219.0

MSB 47TA 52219.0

MSB 47TA 52219.0

MSB 47TA 52219.0

MSB $83 \mathrm{C}$

MSB $83 C^{-}$

MSB $83 C^{-}$

52384.7

52384.7

52384.7

MSB $83 T \overline{T A} 52410.9$

MSB 83TA 52410.9

MSB 83TA 52410.9

MSB 69TA 52418.4

MSB 69TA 52418.4

MSB 69TA 52418.4

MSB 69TA 52418.4

MSB 69TA 52418.4

$A S B-6 A A \quad 52643.9$

$A S B-6 A A \quad 52643.9$

$A S B-6 A A \quad 52643.9$

$A S B-6 A A \quad 52643.9$

ASB 6AA 52643.9

$A S B$ 6TA 52671.3

ASB - 6TA 52671.3

ASB 6TA 52671.3

$A S B$ 6TA 52671.3

$A S B-6 T A$ 52671.3

MSB 32B 52742.5

$M S B-32 B-52742.5$

MSB 32B 52742.5

$\mathrm{MSB}^{-} 32 \mathrm{~B}^{-} 52742.5$

$\mathrm{MSB}^{-} 32 \mathrm{~B}^{-} 52742.5$

$M S B-32 B^{-} 52742.5$

$\mathrm{MBC}^{-} 1 S \overline{\mathrm{B}} 52780.0$

$\mathrm{MBC}$ 1SB 52780.0

$\mathrm{MBC}$ ISB 52780.0

$\mathrm{MBC}$ - ISB 52780.0

$M B C \quad 1 S B \quad 52780.0$

MSB 51B 52818.0

MSB 51B 52818.0

MSB 51B 52818.0

$\mathrm{ASB}{ }^{-} \mathrm{CC} \overline{\mathrm{R}} 52862.7$

ASB - 2CR 52862.7

ASB 2CR 52862.7

$A S B-2 C R \quad 52862.7$

$\mathrm{ASB}$ 2CR 52862.7

$A S B$ IOC 52965.5

$\mathrm{ASB}^{-} 10 \mathrm{C}^{-} 52965.5$

ASB_10C_ 52965.5
SRS

Northing Data Type

(ft)

108324.6

108324.6

106987.7

106987.7

106987.7

106987.7

106987.7

108405.3

108405.3

108405.3

108416.3

108416.3

108416.3

107772.5

107772.5

107772.5

107772.5

107772.5

105727.0

105727.0

105727.0

105727.0

105727.0

105749.5

105749.5

105749.5

105749.5

105749.5

99676.0

99676.0

99676.0

99676.0

99676.0

99676.0

108450.0

108450.0

108450.0

108450.0

108450.0

96992.7

96992.7

96992.7

105540.2

105540.2

105540.2

105540.2

105540.2

105673.1

105673.1

105673.1
LOWER CLAY TOP 120.00

LOWER CLAY BOTTOM 104.00

GREEN CLAY BOTTOM 210.00

UTPPER CLAY TOP 146.00

LOWER CIAAY TOP

94.00

GREEN CLAY TOP

228.00

UPPER CLAY BOTTOM 132.00

GREEN_CLAY TOP

236.00

GREEN_CLAY_BOTTOM 220.00

UPPER CLAY TOP

165.00

UPPER CLAY TOP

165.00

GREEN_CLAY TOP

236.00

GREEN CLAY BOTTOM 220.00

GREEN CLAY BOTTOM 217.00

UPPER CLAY TOP

160.00

UPPER CLAY BOTTOM 146.00

LOWER CLAY TOP

114.00

GREEN CLAY TOP

229.00

GREEN CLAY TOP

201.00

GREEN CLAY BOTTOM 194.00

UPPER CLAY TOP

142.00

UPPER CLAY BOTTOM 132.00

LOWER CLAY TOP

118.00

UPPER CLAY TOP

142.00

GREEN CLAY TOP

201.00

GREEN CLAY BOTTOM 194.00

UPPER_CLAY_BOTTOM 132.00

LOWER_CLAY -TOP

118.00

LOWER CLAY BOTTOM 77.00

UPPER CLAY TOP

117.00

UPPER CLAY BOTTOM 102.00

LOWER CLAY TOP

98.00

GREEN CLAY BOTTOM 199.00

GREEN CLAY TOP

218.00

LOWER CIAYY TOP

128.00

UPPER CLAY BOTTOM 149.00

GREEN CLAY BOTTOM 217.00

UPPER CLAY TOP

GREEN CLAY TOP

160.00

UPPER CLAY TOP

222.00

GREEN CLAY TOP

132.00

GREEN CLAY BOTTOM

224.00

GREEN CLAY TOP

204.00

GREEN CLAYY BOTTOM

225.00

UPPER CLAY TOP

138.00

UPPER CLAY BOTTOM 125.00

LOWER CLAY TOP

UPPER CLAY TOP

120.00

GREEN CLAY TOP

142.00

206.00

GREEN_CIAY_BOTTOM 192.00
-1.66 ACCEPT

1.72 ACCEPT

-0.89 ACCEPT

1.12 ACCEPT

-6.93 ACCEPT

1.28 ACCEPT

4.14 ACCEPT

2.14 ACCEPT

-1.48 ACCEPT

2.55 ACCEPT

1.83 ACCEPT

2.53 ACCEPT

-1.22 ACCEPT

1.11 ACCEPT

1.34 ACCEPT

2.85 ACCEPT

-5.07 ACCEPT

-1.53 ACCEPT

-4.65 ACCEPT

1.59 ACCEPT

-0.06 ACCEPT

0.98 ACCEPT

-0.03 ACCEPT

-0.43 ACCEPT

-3.38 ACCEPT

2.75 ACCEPT

0.52 ACCEPT

-0.54 ACCEPT

1.91 ACCEPT

-0.96 ACCEPT

-2.33 ACCEPT

0.49 ACCEPT

-0.06 ACCEPT

-0.07 ACCEPT

3.33 ACCEPT

-1.64 ACCEPT

0.89 ACCEPT

-2.75 ACCEPT

-4.19 ACCEPT

0.00 ACCEPT

0.62 ACCEPT

-0.51 ACCEPT

10.48 REJECT

-4.57 ACCEPT

-1.65 ACCEPT

-3.91 ACCEPT

2.27 ACCEPT

-1.68 ACCEPT

-1.64 ACCEPT

-1.42 ACCEPT 
Geological Modeling Residuals

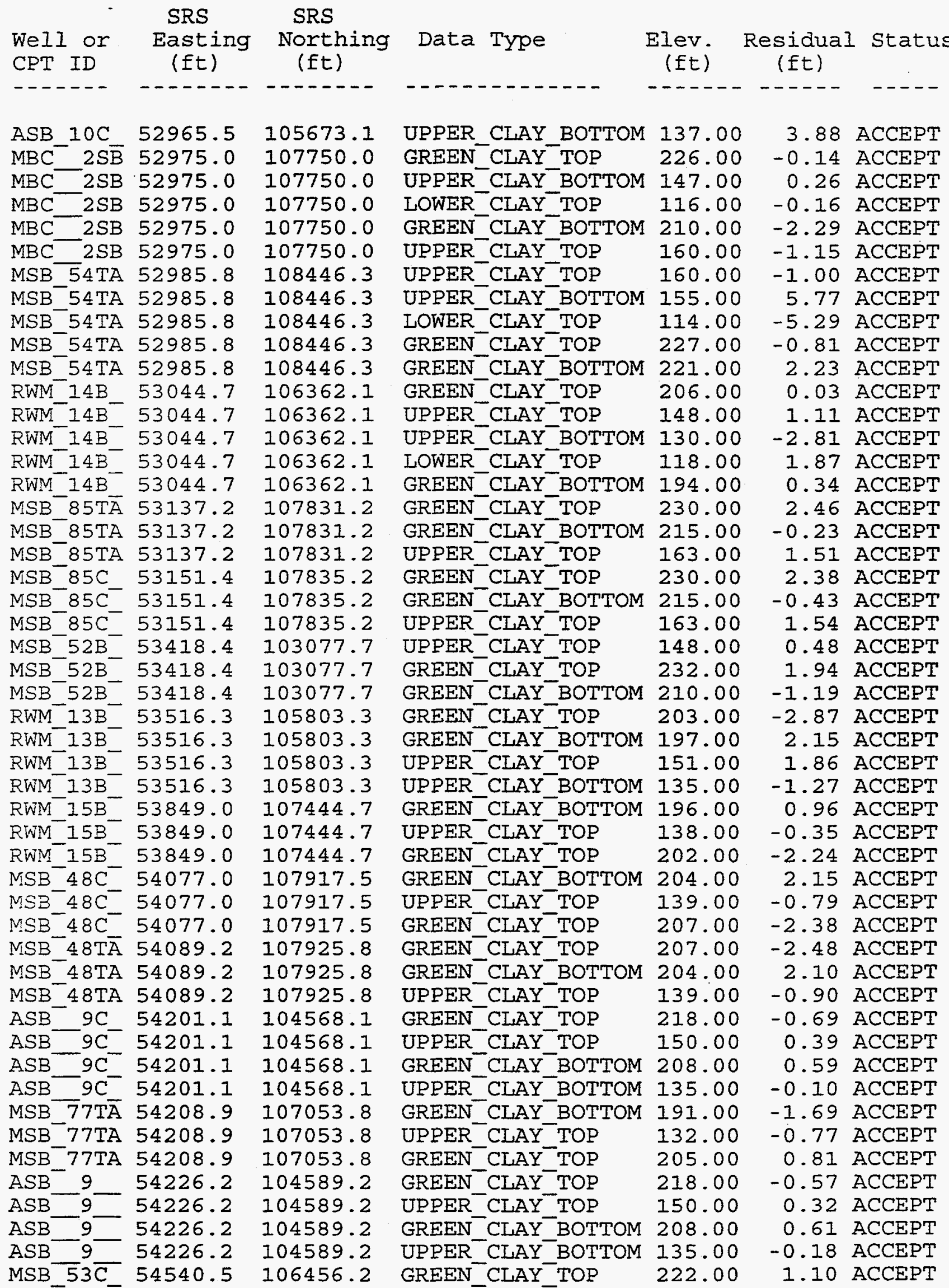


Hydraulic Head Residuals

\begin{tabular}{|c|c|c|c|c|c|c|}
\hline Wëll ID & $\begin{array}{l}\text { SRS } \\
\text { Easting } \\
\text { (ft) }\end{array}$ & $\begin{array}{l}\text { SRS } \\
\text { Northing } \\
\text { (ft) }\end{array}$ & Layer & $\begin{array}{l}\text { Head } \\
\text { Data } \\
\text { (ft) }\end{array}$ & $\begin{array}{l}\text { Residual } \\
\text { (ft) }\end{array}$ & I status \\
\hline & ------ & ------- & -- & $--\cdots$ & $-\cdots-\cdots$ & - \\
\hline $\mathrm{ABP}$ & 43930.1 & 97449.7 & 5 & 224.41 & 1.24 & ACCEPT \\
\hline $\mathrm{ABP}$ & 43984.1 & 97854.9 & 5 & 223.02 & -.29 & ACCEPT \\
\hline$M S B-\overline{7} 1 B$ & 44054.7 & 103801.6 & 16 & 218.05 & 1.14 & ACCEPT \\
\hline$A B P$ & 44096.0 & 97489.7 & 8 & 223.55 & .32 & ACCEPT \\
\hline $4 \mathrm{DD}$ & 44101.3 & 97495.5 & 6 & 230.83 & 6.85 & REJECT \\
\hline $\mathrm{ABP}$ & 44101.4 & 97889.7 & 6 & 223.32 & .03 & ACCEPT \\
\hline$A B P$ & 44118.8 & 97764.3 & 8 & 223.74 & .93 & ACCEPT \\
\hline $2 \mathrm{DD}$ & 44126.7 & 97753.7 & 6 & 223.34 & -.41 & ACCEPT \\
\hline MSB_56D & 44207.9 & 108463.5 & 4 & 221.73 & -1.56 & ACCEPT \\
\hline ARP & 44317.4 & 99102.9 & 7 & 217.09 & .20 & ACCEPT \\
\hline ARP & 44374.8 & 98567.7 & 6 & 219.57 & -1.20 & ACCEPT \\
\hline $\mathrm{ABP}$ & 44425.6 & 97501.6 & 10 & 225.07 & .37 & ACCEPT \\
\hline$A B P$ & 44433.6 & 97511.4 & 5 & 226.44 & .71 & ACCEPT \\
\hline $\mathrm{ABP}$ & 44506.3 & 97778.2 & 12 & 198.23 & -.50 & ACCEPT \\
\hline $\mathrm{ABP}$ & 44509.3 & 97794.1 & 5 & 225.25 & -.54 & ACCEPT \\
\hline $\mathrm{MCB}$ & 44769.9 & 97180.6 & 5 & 226.42 & .93 & ACCEPT \\
\hline $\mathrm{MCB}$ & 44858.8 & 97605.8 & 5 & 224.01 & -2.08 & ACCEPT \\
\hline $\mathrm{MCB}$ & 44863.9 & 97335.6 & 5 & 225.95 & .13 & ACCEPT \\
\hline$A R P$ & 44876.1 & 99119.8 & 8 & 219.77 & -.22 & ACCEPT \\
\hline ARP & 44903.7 & 98638.2 & 8 & 222.42 & .52 & ACCEPT \\
\hline $\mathrm{MSB}_{-70 \mathrm{C}}$ & 45012.0 & 101785.2 & 11 & 218.54 & .16 & ACCEPT \\
\hline $\mathrm{MCB}$ & 45207.7 & 97413.1 & 12 & 196.63 & -2.45 & ACCEPT \\
\hline MSB $76 \mathrm{C}$ & 45344.0 & 103061.6 & 9 & 221.69 & -.07 & ACCEPT \\
\hline$M S B \_78 D$ & 45482.2 & 103643.8 & 5 & 224.86 & -.75 & ACCEPT \\
\hline MSB_73B & 45694.0 & 99270.3 & 17 & 202.75 & -.75 & ACCEPT \\
\hline MSB_49A & 45864.6 & 99759.0 & 21 & 198.47 & .92 & ACCEPT \\
\hline MSB_s & 45868.2 & 99737.8 & 19 & 204.19 & .18 & ACCEPT \\
\hline MSB_2OA & 46060.5 & 103545.1 & 15 & 220.06 & -.28 & ACCEPT \\
\hline MSB_18A & 46110.4 & 100416.1 & 15 & 213.87 & .11 & ACCEPT \\
\hline MSB_18B & 46115.7 & 100424.1 & 10 & 222.63 & .11 & ACCEPT \\
\hline MSB_17BB & 46220.8 & 102009.5 & 18 & 214.33 & .14 & ACCEPT \\
\hline MSB_I7B & 46237.7 & 101994.6 & 9 & 226.43 & .94 & ACCEPT \\
\hline MSB_I IA & 46245.7 & 101976.6 & 15 & 218.00 & -.39 & ACCEPT \\
\hline MSB & 46319.9 & 101133.8 & 5 & 227.96 & .19 & ACCEPT \\
\hline $\mathrm{MSB}$ & 46321.6 & 101148.5 & 19 & 208.50 & .18 & ACCEPT \\
\hline $\mathrm{AC}$ & 46428.6 & 105636.4 & 15 & 221.99 & -.09 & ACCEPT \\
\hline MSB_6 $4 \mathrm{~B}$ & 46579.7 & 101831.0 & 19 & 210.49 & $-1 \cdot 10$ & ACCEPT \\
\hline MSB_64C & 46589.2 & 101842.9 & 11 & 223.90 & -.15 & ACCEPT \\
\hline MSB_64D & 46598.5 & 101854.8 & 5 & 227.59 & -1.67 & ACCEPT \\
\hline MSB & 46718.1 & 100597.6 & 16 & 208.90 & -.67 & ACCEPT \\
\hline MSB & 46726.1 & 100585.7 & 5 & 228.79 & -.30 & ACCEPT \\
\hline MSB & 46983.6 & 101971.1 & 18 & 211.29 & .24 & ACCEPT \\
\hline MSB & 46998.7 & 101971.5 & 5 & 228.60 & -1.32 & ACCEPT \\
\hline MSB_I $2 \mathrm{~A}$ & 47138.2 & 102283.2 & 19 & 212.38 & .90 & ACCEPT \\
\hline MSB_12C & 47138.4 & 102274.4 & 11 & 224.56 & 1.59 & ACCEPT \\
\hline MSB_12D & 47139.7 & 102262.2 & 5 & 234.08 & -.85 & ACCEPT \\
\hline MSB_21A & 47217.2 & 103967.0 & 14 & 222.61 & -.08 & ACCEPT \\
\hline MSB_2IC & 47234.6 & 103973.0 & 5 & 229.77 & -1.27 & ACCEPT \\
\hline MSB & 47264.6 & 100793.2 & 10 & 221.51 & .21 & ACCEPT \\
\hline MSB_ $21 B$ & 47271.8 & 104000.1 & 15 & 222.04 & .14 & ACCEPT \\
\hline
\end{tabular}


Hydraulic Head Residuals

\begin{tabular}{|c|c|c|c|c|c|c|}
\hline Wèll ID & $\begin{array}{l}\text { SRS } \\
\text { Easting } \\
\text { (ft) }\end{array}$ & $\begin{array}{l}\text { SRS } \\
\text { Northing } \\
\text { (ft) }\end{array}$ & Layer & $\begin{array}{l}\text { Head } \\
\text { Data } \\
\text { (ft) }\end{array}$ & $\begin{array}{l}\text { Residual } \\
\text { (ft) }\end{array}$ & I stat \\
\hline & & - & & -- & ------- & - \\
\hline MSB & 47281.9 & 100805.8 & 15 & 211.00 & -.17 & ACCEPT \\
\hline MSB $\overline{7} 9 \mathrm{C}$ & 47286.8 & 99290.2 & 10 & 211.01 & -.56 & ACCEPT \\
\hline $\mathrm{MSB}^{-} 8 \mathrm{~A}$ & 47293.2 & 100815.1 & 6 & 230.18 & -.02 & ACCEPT \\
\hline MSB_79B & 47300.2 & 99296.9 & 15 & 208.93 & .11 & ACCEPT \\
\hline MSB_13C & 47521.9 & 101745.7 & 5 & 229.73 & -.89 & ACCEPT \\
\hline MSB_13B & 47523.5 & 101735.7 & 12 & 203.29 & -5.42 & REJECT \\
\hline MSB_13A & 47525.4 & 101725.7 & 15 & 211.61 & .86 & ACCEPT \\
\hline MSB $63 \mathrm{C}$ & 47849.2 & 101174.6 & 10 & 222.35 & -.09 & ACCEPT \\
\hline MSB $63 B$ & 47861.0 & 101184.4 & 15 & 211.55 & -.63 & ACCEPT \\
\hline $\mathrm{MSB}^{-} 89 \mathrm{C}$ & 47881.6 & 98379.4 & 6 & 229.53 & .36 & ACCEPT \\
\hline MSB_62C & 47895.0 & 101857.2 & 10 & 224.84 & 2.13 & ACCEPT \\
\hline MSB_62B & 47906.8 & 101865.3 & 15 & 212.37 & -.43 & ACCEPT \\
\hline MSB $10 \mathrm{~B}$ & 47943.1 & 102488.2 & 14 & 217.16 & -.33 & ACCEPT \\
\hline $\mathrm{MSB} 10 \mathrm{~A}$ & 47954.4 & 102451.8 & 18 & 214.66 & 1.15 & ACCEPT \\
\hline $\mathrm{MSB}-30 \mathrm{AA}$ & 47970.5 & 105715.7 & 20 & 224.98 & 8.16 & REJECT \\
\hline $\mathrm{MSB}-30 \mathrm{~B}$ & 47981.8 & 105719.9 & 17 & 226.59 & .65 & ACCEPT \\
\hline $\mathrm{MSB} 30 \mathrm{CC}$ & 47993.3 & 105724.2 & 13 & 226.67 & -1.02 & ACCEPT \\
\hline $\mathrm{MSB} 30 \mathrm{C}$ & 48013.7 & 105731.1 & 5 & 231.48 & -.46 & ACCEPT \\
\hline$M S B \quad 9 A$ & 48242.5 & 102236.7 & 16 & 213.26 & -1.66 & ACCEPT \\
\hline RWM 16 & 48244.8 & 97647.2 & 13 & 204.32 & -.51 & ACCEPT \\
\hline MSB & 48273.0 & 102245.6 & 5 & 231.28 & -.35 & ACCEPT \\
\hline MSB $40 \mathrm{~A}$ & 48279.4 & 97672.8 & 19 & 203.65 & .59 & ACCEPT \\
\hline $\mathrm{MSB} 4 \mathrm{OB}$ & 48281.6 & 97685.0 & 14 & 205.47 & .59 & ACCEPT \\
\hline MSB_4OC & 48283.5 & 97697.8 & 10 & 205.34 & -.23 & ACCEPT \\
\hline MSB & 48311.7 & 102007.5 & 6 & 230.85 & .20 & ACCEPT \\
\hline MSB & 48312.8 & 101978.3 & 17 & 213.53 & -.81 & ACCEPT \\
\hline MSB & 48313.0 & 101933.4 & 5 & 231.61 & -.91 & ACCEPT \\
\hline $\mathrm{MSB}-72 \mathrm{~B}$ & 48350.3 & 96387.6 & 14 & 200.57 & -.45 & ACCEPT \\
\hline $\mathrm{MSB}$ & 48367.3 & 100837.6 & 18 & 211.05 & .81 & ACCEPT \\
\hline$M S B \_39 B$ & 48376.9 & 100844.6 & 15 & 213.76 & -.93 & ACCEPT \\
\hline MSB_39C & 48386.7 & 100852.1 & 9 & 217.15 & -2.43 & ACCEPT \\
\hline MSB_39D & 48396.0 & 100858.7 & 5 & 232.95 & -.63 & ACCEPT \\
\hline MSB_26A & 48440.7 & 104602.3 & 12 & 226.35 & -.59 & ACCEPT \\
\hline $\mathrm{MSB}$ & 48467.3 & 101833.7 & 5 & 232.35 & -.75 & ACCEPT \\
\hline $\mathrm{MSB}$ & 48483.2 & 101833.0 & 15 & 213.01 & -2.14 & ACCEPT \\
\hline MSB_22 & 48508.8 & 102186.5 & 5 & 231.74 & .00 & ACCEPT \\
\hline MSB_28 & 48517.3 & 104941.8 & 6 & 231.27 & .20 & ACCEPT \\
\hline MSB_14B & 48519.1 & 101639.0 & 10 & 220.16 & -.09 & ACCEPT \\
\hline MSB & 48521.9 & 101629.5 & 14 & 218.42 & 1.74 & ACCEPT \\
\hline MSB & 48521.9 & 104947.7 & 14 & 225.36 & -1.73 & ACCEPT \\
\hline MSB _ 3D & 48524.6 & 102188.6 & 6 & 231.08 & .19 & ACCEPT \\
\hline MSB_ $3 A$ & 48553.7 & 102189.9 & 5 & 232.71 & .24 & ACCEPT \\
\hline MSB_ $3 B$ & 48568.0 & 102191.7 & 15 & 216.32 & .03 & ACCEPT \\
\hline MSB_IIF & 48577.0 & 102629.3 & 5 & 231.27 & .79 & ACCEPT \\
\hline MSB_IIA & 48577.6 & 102638.9 & 16 & 215.70 & -.34 & ACCEPT \\
\hline MSB_11B & 48578.5 & 102648.9 & 13 & 221.39 & -1.29 & ACCEPT \\
\hline MSB_IIC & 48579.4 & 102658.6 & 11 & 223.36 & 4.24 & ACCEPT \\
\hline MSB_IIE & 48579.6 & 102678.5 & 5 & 239.73 & 1.59 & ACCEPT \\
\hline MSB_IID & 48579.7 & 102669.5 & 8 & 229.70 & .78 & ACCEPT \\
\hline MSB_ $2 \mathrm{~A}$ & 48746.4 & 102028.3 & 5 & 233.82 & -2.08 & ACCEPT \\
\hline MSB_15AA & 48818.5 & 102953.2 & 15 & 216.96 & 1.04 & ACCEPT \\
\hline
\end{tabular}


Hydraulic Head Residuals

\begin{tabular}{|c|c|c|c|c|c|c|}
\hline el1 ID & $\begin{array}{l}\text { SRS } \\
\text { Easting } \\
\text { (ft) }\end{array}$ & $\begin{array}{l}\text { SRS } \\
\text { Northing } \\
\text { (ft) }\end{array}$ & La] & $\begin{array}{l}\text { Head } \\
\text { Data } \\
(f t)\end{array}$ & $\begin{array}{l}\text { Residu } \\
\text { (ft) }\end{array}$ & \\
\hline & & & & - & & \\
\hline$S B \_15 A$ & 48827.0 & 102983.5 & 13 & 223.54 & 1.04 & ACCEPT \\
\hline $75 B$ & 48875.5 & 98937.4 & 14 & 211.03 & .45 & ACCEPT \\
\hline$S B-26$ & 48941.7 & 104612.8 & 5 & 237.24 & -.01 & ACCEPT \\
\hline $\mathrm{SB}^{-} 26 \mathrm{~B}$ & 48944.6 & 104646.7 & 16 & 221.25 & -.57 & ACCEPT \\
\hline $\mathrm{SB}^{-1} 16 \mathrm{~A}$ & 48965.1 & 103693.9 & 13 & 224.40 & 1.66 & ACCEPT \\
\hline $\mathrm{SB}-23 \mathrm{TA}$ & 49225.8 & 104298.8 & 22 & 202.75 & .23 & ACCEPT \\
\hline$S B-23 B$ & 49286.4 & 104336.6 & 12 & 25.70 & -.38 & ACCEPT \\
\hline$S B-43 A$ & 93.7 & 107275.3 & 16 & 30.14 & .42 & ACCEPT \\
\hline $5 B-43 B$ & 49311.8 & 107274.6 & 13 & 30.42 & -.97 & ACCEPT \\
\hline $\mathrm{SB}^{-} 43 \mathrm{D}$ & 49322.0 & 107274.2 & 8 & 32.35 & .21 & ACCEPT \\
\hline $\mathrm{SB}^{-} 43 \mathrm{DD}$ & 49341.2 & 107273.0 & 5 & 32.26 & -1.58 & ACCEPT \\
\hline $1 S B-27 B$ & 49486.4 & 104940.3 & 13 & 27.78 & -.16 & ACCEPT \\
\hline ISB 27 & 49487.7 & 104972.8 & 5 & 37.04 & -.12 & ACCEPT \\
\hline $\operatorname{MSB}-27 \mathrm{~A}$ & 49487.8 & 104962.8 & 10 & 230.25 & 1.79 & ACCEPT \\
\hline $1 S B-36 A$ & 49514.9 & 100511.3 & 19 & 211.79 & .40 & ACCEPT \\
\hline $1 \mathrm{SB}^{-} 36 \mathrm{~B}$ & 49526.3 & 100514.9 & 13 & 216.06 & .46 & $\dot{A C}$ \\
\hline $\mathrm{MSB}-36 \mathrm{C}$ & 49537.2 & 100518.3 & 10 & 16.16 & -.96 & ACCEPT \\
\hline $\mathrm{MSB}^{-2} 2 \mathrm{~A}$ & 49657.9 & 103504.8 & 13 & 19.58 & -.37 & ACCEPT \\
\hline $\mathrm{MSB}^{-} 25$ & 49668.9 & 103498.8 & 5 & 37.01 & .74 & ACCEPT \\
\hline $1 \mathrm{SB}^{-} 38 \mathrm{~B}$ & 49746.1 & 102360.8 & 15 & 18.61 & $-1: 14$ & ACCEPT \\
\hline $1 \mathrm{SB}^{-} 38 \mathrm{C}$ & 49762.0 & 102373.1 & 13 & 21.98 & 1.39 & ACCEPT \\
\hline $1 S B-24 A$ & 49845.3 & 104625.3 & 12 & 27.79 & .61 & $A C$ \\
\hline $\mathrm{MSB} 31 \mathrm{CC}$ & 67.9 & 33.1 & 1. & 220.65 & .65 & $A C$ \\
\hline$M S B-31 B$ & 8.7 & 31.3 & 14 & 20.51 & .84 & $A C$ \\
\hline $\mathrm{MSB} 31 \mathrm{C}$ & 9.9 & 79.6 & 5 & 5.85 & .71 & $\mathrm{AC}$ \\
\hline $1 S B-74 B$ & 3.2 & 7.4 & 15 & 1.92 & -.26 & $\mathrm{AC}$ \\
\hline $\mathrm{AOB}^{-1} \quad 1$ & 85.9 & 1019 & 5 & 7.73 & -.96 & PPT \\
\hline $1 \mathrm{SB} 34 \mathrm{~A}$ & 50534.9 & 1049 & 20 & 20.51 & .92 & $\mathrm{AC}$ \\
\hline MSB_34B & 4.9 & 1049 & 12 & 228.60 & .90 & ACCEPT \\
\hline $\mathrm{MSB} 445 \mathrm{~B}$ & 5.3 & 103 & 12 & 228.17 & 1.24 & ACCEPT \\
\hline MSB_46B & 7.5 & 103 & 11 & 27.09 & 77 & ACCEPT \\
\hline $\mathrm{AOB}$ & 4.7 & 102 & 5 & 7.63 & -1.64 & ACCEPT \\
\hline MSB_88D & 93.5 & 2.3 & 8 & 05.16 & -.29 & ACCEPT \\
\hline MSB_19A & 4.4 & 3.0 & 18 & 5.84 & .17 & ACCEPT \\
\hline MSB_19B & 4.8 & 9.3 & 15 & 218.56 & -.11 & ACCEPT \\
\hline MSB_19C & 2.4 & 2.1 & 8 & 9.31 & .92 & ACCEPT \\
\hline MSB_35A & 5.2 & 38.0 & 17 & 8.11 & -.51 & EPT \\
\hline MSB_35B & 47.9 & 10.8 & 13 & 1.97 & -.64 & ACCEPT \\
\hline MSB_35D & 9.7 & 102122.4 & 4 & 243.48 & 1.20 & ACCEPT \\
\hline MSB_66D & 51044.0 & 105841.8 & 8 & 232.21 & 1.08 & ACCEPT \\
\hline $\mathrm{MSB}^{-} 50 \mathrm{D}$ & 4.1 & 16.7 & 9 & 202.37 & -.56 & ACCEPT \\
\hline $\mathrm{MSB}-50 \mathrm{~B}$ & 51053.5 & 96433.0 & 13 & 202.36 & -.07 & ACCEPT \\
\hline MSB_66C & 51053.5 & 105842.1 & 14 & 229.84 & .29 & ACCEPT \\
\hline MSB_66B & 51064.6 & 105842.0 & 17 & 220.91 & -1.02 & ACCEPT \\
\hline MSB_44B & 51096.4 & 103296.2 & 12 & 226.48 & -.39 & ACCEPT \\
\hline $\mathrm{MSB}^{-} 44 \mathrm{~A}$ & 51106.9 & 103296.5 & 17 & 219.56 & -1.10 & ACCEPT \\
\hline$A C B=4 A$ & 51116.2 & 102343.9 & 5 & 239.74 & .79 & ACCEPT \\
\hline$M S B \_29 D$ & 51191.3 & 107311.4 & 7 & 233.55 & -.21 & ACCEPT \\
\hline MSB_29C & 51206.6 & 107315.0 & 13 & 231.47 & .44 & ACCEPT \\
\hline MSE & 51217.5 & 107319.3 & 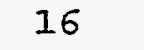 & 225.75 & 1.08 & ACCEPT \\
\hline
\end{tabular}


Hydraulic Head Residuals

\begin{tabular}{|c|c|c|c|c|c|c|}
\hline Well ID & $\begin{array}{l}\text { SRS } \\
\text { Easting } \\
\text { (ft) }\end{array}$ & $\begin{array}{l}\text { SRS } \\
\text { Northing } \\
\text { (ft) }\end{array}$ & Layer & $\begin{array}{l}\text { Head } \\
\text { Data } \\
\text { (ft) }\end{array}$ & $\begin{array}{l}\text { Residual } \\
\text { (ft) }\end{array}$ & \\
\hline & & & & & & \\
\hline$S B \_29 D$ & 51226.9 & 107323.3 & 9 & 233.26 & .73 & ACCEPT \\
\hline $\mathrm{SB} 29 \mathrm{~A}$ & 51236.4 & 107326.8 & 20 & 221.56 & .78 & ACCEPT \\
\hline $\mathrm{MB}^{-} 9 \mathrm{D}$ & 51263.0 & 103585.2 & 6 & 235.40 & -.01 & ACCEPT \\
\hline $\mathrm{CB}-3 \mathrm{~A}$ & 51313.3 & 102154.3 & 6 & 239.57 & .43 & ACCEPT \\
\hline $1 \mathrm{~A}$ & 51369.9 & 102622.9 & 5 & 238.68 & -.51 & ACCEPT \\
\hline $\mathrm{MB} 13 \mathrm{AR}$ & 51396.0 & 103082.0 & 19 & 220.56 & 1.57 & ACCEPT \\
\hline $\mathrm{MB} \quad 8 \mathrm{D}$ & 51400.5 & 103874.7 & 6 & 234.96 & .20 & ACCEPT \\
\hline $\mathrm{MB} 10 \mathrm{~A}$ & 51410.0 & 103326.4 & 18 & 219.66 & .24 & ACCEPT \\
\hline $\mathrm{MB}-10 \mathrm{~B}$ & 51418.3 & 103337.3 & 15 & 225.08 & -.08 & ACCEPT \\
\hline $\mathrm{MB} 18 \mathrm{~A}$ & 51418.8 & 103988.8 & 17 & 220.43 & -1.48 & ACCEPT \\
\hline $\mathrm{SB}^{-3} 3 \mathrm{C}$ & 51439.8 & 105283.2 & 13 & 229.64 & .88 & ACCEPT \\
\hline$S B-37 B$ & 51450.0 & 105289.5 & 18 & 220.91 & .15 & ACCEPT \\
\hline$M B-10 D$ & 51456.0 & 103293.4 & 5 & 236.64 & -.35 & ACCEPT \\
\hline $\mathrm{MB}^{-17 \mathrm{~A}}$ & 51465.4 & 104056.7 & 18 & 220.53 & -.05 & ACCEPT \\
\hline $\mathrm{MB}^{-} 5$ & 51467.2 & 104083.4 & 6 & 234.93 & .57 & ACCEPT \\
\hline$A M B-4 A$ & 51469.8 & 104131.6 & 19 & 220.56 & -.04 & ACCEPT \\
\hline $\mathrm{AMB}-4 \mathrm{~B}$ & 51482.7 & 104145.6 & 15 & 226.09 & -.08 & ACCEPT \\
\hline$A M B-4 D$ & 51489.0 & 104154.7 & 8 & 234.52 & .96 & ACCEPT \\
\hline $\mathrm{ACB}$ & 51561.3 & 102367.4 & 5 & 239.78 & .45 & ACCEPT \\
\hline$M S B \overline{4} 2 A$ & 51582.3 & 104557.9 & 19 & 220.89 & .61 & ACCEPT \\
\hline $1 S B-42 B$ & 51582.8 & 104569.8 & 14 & 227.54 & .83 & ACCEPT \\
\hline $1 \mathrm{SB}^{-} 42 \mathrm{C}$ & 51582.8 & 104581.9 & 11 & 231.90 & 1.31 & ACCEPT \\
\hline $\mathrm{AMB}^{-} \quad 7 \mathrm{~B}$ & 0.3 & 103972.0 & 14 & 226.57 & .55 & ACCEPT \\
\hline $\mathrm{MB}-7 \mathrm{~A}$ & 51591.0 & 103987.1 & 18 & 220.55 & -.18 & ACCEPT \\
\hline $1 S B \overline{3} 3 A$ & 51738.0 & 6.7 & 20 & 205.34 & .50 & ACCEPT \\
\hline $\mathrm{SB}-33 \mathrm{~B}$ & 51741.9 & 5.9 & 17 & 208.20 & .09 & ACCEPT \\
\hline $\mathrm{SB}_{-} 33 \mathrm{C}$ & 16.7 & 4.8 & 12 & 211.15 & .88 & ACCEPT \\
\hline $\mathrm{AMB}^{-} 12 \mathrm{D}$ & 1.6 & 1036 & 5 & 235.98 & -.94 & ACCEPT \\
\hline $\mathrm{MB}_{-} 11 \mathrm{~B}$ & 19.5 & 103154.2 & 11 & 224.72 & -1.82 & ACCEPT \\
\hline$A M B \_11 D$ & 32.6 & 103132.3 & 5 & 237.31 & -1.33 & ACCEPT \\
\hline $\mathrm{SB}^{-} 82 \mathrm{D}$ & 34.6 & 107518.1 & 9 & 233.85 & .96 & ACCEPT \\
\hline $\mathrm{SB}-82 \mathrm{C}$ & 19.4 & 107521.9 & 14 & 229.61 & 1.24 & ACCEPT \\
\hline$S B-84 C$ & 73.7 & 108967.9 & 12 & 230.52 & -.84 & ACCEPT \\
\hline $\mathrm{ISB}_{-} 82 \mathrm{~A}$ & 78.4 & 107529.5 & 21 & 222.11 & 2.06 & ACCEPT \\
\hline ISB $67 \mathrm{C}$ & 51988.6 & 106819.8 & 13 & 228.39 & .13 & ACCEPT \\
\hline $\mathrm{SB}^{-} 67 \mathrm{~B}$ & 51989.6 & 106842.0 & 17 & 220.50 & -.91 & ACCEPT \\
\hline $\mathrm{SB}^{-} 82 \mathrm{~B}$ & 51993.3 & 107533.4 & 18 & 221.76 & -.51 & ACCEPT \\
\hline $\mathrm{SB}^{-} 5 \mathrm{~B}$ & 52006.2 & 108342.4 & 15 & 223.19 & -3.77 & ACCEPT \\
\hline $1 \mathrm{SB} 55 \mathrm{C}$ & 52029.7 & 108324.6 & 12 & 230.44 & -.91 & ACCEPT \\
\hline MSB $55 \mathrm{D}$ & 52032.5 & 108391.4 & 9 & 233.92 & -.06 & ACCEPT \\
\hline $\mathrm{MSB}^{-47 D}$ & 52184.0 & 106960.1 & 6 & 234.36 & .37 & ACCEPT \\
\hline $\mathrm{MSB}^{-} 4 \mathrm{CC}$ & 52195.5 & 106969.2 & 10 & 233.51 & 3.29 & ACCEPT \\
\hline $\mathrm{MSB}^{-} 4 \mathrm{7B}$ & 52207.2 & 106978.5 & 13 & 226.78 & .20 & ACCEPT \\
\hline $\mathrm{MSB}^{-} 47 \mathrm{BB}$ & 52234.4 & 106999.7 & 18 & 221.82 & 1.16 & ACCEPT \\
\hline $\mathrm{SB}^{-}-68 \mathrm{D}$ & 52293.6 & 106741.4 & 6 & 234.34 & -.08 & ACCEPT \\
\hline$M S B-68 C$ & 52304.9 & 106730.5 & 13 & 226.60 & .08 & ACCEPT \\
\hline$M S B-68 B$ & 52308.5 & 106744.9 & 17 & 220.83 & -.71 & ACCEPT \\
\hline MSB_83C & 52384.7 & 108405.3 & 14 & 228.76 & -.35 & ACCEPT \\
\hline MSB $83 B$ & 52421.4 & 108426.7 & 18 & 223.15 & -.20 & ACCEPT \\
\hline $1 \mathrm{SB}^{-} 69 \mathrm{~B}$ & 52432.9 & 107776.1 & 17 & 222.38 & -.90 & ACCEPT \\
\hline
\end{tabular}


Hydraulic Head Residuals

\begin{tabular}{|c|c|c|c|c|c|c|}
\hline WeII ID & $\begin{array}{l}\text { SRS } \\
\text { Easting } \\
\text { (ft) }\end{array}$ & $\begin{array}{l}\text { SRS } \\
\text { Northing } \\
\text { (ft) }\end{array}$ & Layer & $\begin{array}{l}\text { Head } \\
\text { Data } \\
\text { (ft) }\end{array}$ & $\begin{array}{l}\text { Residual } \\
\text { (ft) }\end{array}$ & Status \\
\hline & & & $\ldots$ & $\cdots-\cdots$ & & $-\cdots-$ \\
\hline MSB_69D & 52462.0 & 107784.3 & 8 & 234.09 & .53 & ACCEPT \\
\hline $\mathrm{ASB}^{-} \quad \mathrm{AA}$ & 52614.0 & 105535.0 & 5 & 238.35 & .39 & ACCEPT \\
\hline $\mathrm{ASB}$ & 52655.9 & 105736.8 & 11 & 225.53 & -.56 & ACCEPT \\
\hline $\mathrm{ASB}$ & 52675.9 & 105716.0 & 5 & 237.87 & -.39 & ACCEPT \\
\hline $\operatorname{MSB} \overline{32}$ & 52733.9 & 99655.6 & 9 & 224.47 & -.12 & ACCEPT \\
\hline$M S B-51 B$ & 52818.0 & 96992.7 & 13 & 205.19 & -.67 & ACCEPT \\
\hline$M S B-51 D D$ & 52830.3 & 97006.2 & 8 & 212.28 & -.37 & ACCEPT \\
\hline $\mathrm{ASB}^{-} \quad 5 \mathrm{C}$ & 52837.8 & 105884.8 & 12 & 224.37 & -.82 & ACCEPT \\
\hline $\mathrm{ASB}$ & 52862.7 & 105540.2 & 12 & 224.66 & -.64 & ACCEPT \\
\hline $\mathrm{ASB}$ & 52881.7 & 105550.5 & 5 & 239.65 & .50 & ACCEPT \\
\hline $\operatorname{MSB} \overline{5} 4 \mathrm{C}$ & 52955.7 & 108447.4 & 14 & 228.55 & .93 & ACCEPT \\
\hline $\mathrm{ASB} 10 \mathrm{CR}$ & 52969.7 & 105655.4 & 11 & 224.63 & -.33 & ACCEPT \\
\hline $\mathrm{MSB}^{-} 54 \mathrm{~B}$ & 52970.5 & 108446.8 & 19 & 223.46 & -.04 & ACCEPT \\
\hline $\mathrm{MSB} 54 \mathrm{D}$ & 52984.5 & 108461.5 & 7 & 233.96 & .15 & ACCEPT \\
\hline RWM $14 \mathrm{~B}$ & 53044.7 & 106362.1 & 17 & 221.52 & -.03 & ACCEPT \\
\hline RWM $14 \mathrm{C}$ & 53051.5 & 106380.8 & 11 & 225.52 & -.55 & ACCEPT \\
\hline $\mathrm{ASB}^{-} 8 \mathrm{C}$ & 53101.0 & 106354.4 & 10 & 224.97 & -1.42 & ACCEPT \\
\hline$M S B-85 D$ & 53108.8 & 107822.8 & 7 & 233.68 & .58 & ACCEPT \\
\hline $\mathrm{ASB}^{-} \quad 8 \mathrm{~B}$ & 53109.6 & 106362.3 & 19 & 221.89 & .99 & ACCEPT \\
\hline $\mathrm{ASB}$ & 53115.0 & 105605.1 & 5 & 240.25 & .23 & ACCEPT \\
\hline MSB $\overline{8} 5 \mathrm{~B}$ & 53122.7 & 107827.0 & 18 & 222.96 & -.14 & ACCEPT \\
\hline $\mathrm{ASB} \quad 3 \mathrm{CR}$ & 53130.4 & 105614.5 & 12 & 224.25 & -.42 & ACCEPT \\
\hline $\mathrm{ASB} 8$ & 53136.6 & 106381.6 & 5 & 235.32 & -.76 & ACCEPT \\
\hline MSB $\overline{8} 5 \mathrm{C}$ & 53151.4 & 107835.2 & 14 & 225.99 & -.64 & ACCEPT \\
\hline $\mathrm{ASB}^{-} 4$ & 53177.2 & 105935.7 & 4 & 239.47 & -.62 & ACCEPT \\
\hline MSB_ $41 \mathrm{C}$ & 53410.6 & 102203.9 & 15 & 219.03 & -.99 & ACCEPT \\
\hline $\mathrm{MSB}_{-}^{-} 4 \mathrm{~B}$ & 53417.8 & 102194.5 & 19 & 218.57 & 1.10 & ACCEPT \\
\hline $\mathrm{MSB}_{-52 \mathrm{~B}}$ & 53418.4 & 103077.7 & 14 & 220.09 & -1.23 & ACCEPT \\
\hline $\mathrm{MSB}_{-} 41 \mathrm{~A}$ & 53424.1 & 102184.4 & 22 & 218.32 & 9.93 & REJECT \\
\hline RWM $13 \mathrm{C}$ & 53502.2 & 105809.7 & 14 & 223.49 & .07 & ACCEPT \\
\hline RWM I IB & 53516.3 & 105803.3 & 19 & 221.67 & 1.73 & ACCEPT \\
\hline RWM 15B & 53849.0 & 107444.7 & 16 & 223.95 & -.37 & ACCEPT \\
\hline $\mathrm{MSB}_{-} 48 \mathrm{C}$ & 54077.0 & 107917.5 & 12 & 225.66 & -1.60 & ACCEPT \\
\hline $\mathrm{MSB} 48 \mathrm{TA}$ & 54089.2 & 107925.8 & 19 & 223.67 & .36 & ACCEPT \\
\hline $\mathrm{MSB}_{-}^{-} 48 \mathrm{~A}$ & 54099.8 & 107936.6 & 17 & 223.88 & -.30 & ACCEPT \\
\hline $\mathrm{MSB}_{-} 48 \mathrm{~B}$ & 54112.2 & 107945.0 & 14 & 225.18 & -.65 & ACCEPT \\
\hline $\mathrm{ASB} \quad 9 \mathrm{C}$ & 54201.1 & 104568.1 & 12 & 222.13 & -.71 & ACCEPT \\
\hline $\mathrm{MSB}-77 \mathrm{TA}$ & 54208.9 & 107053.8 & 22 & 222.84 & 1.38 & ACCEPT \\
\hline $\mathrm{ASB}^{-} \quad 9 \mathrm{~B}$ & 54215.3 & 104564.7 & 14 & 221.75 & -.22 & ACCEPT \\
\hline MSB_77B & 54217.4 & 107065.8 & 14 & 223.23 & -.95 & ACCEPT \\
\hline MSB_77C & 54225.9 & 107078.3 & 12 & 225.26 & -.91 & ACCEPT \\
\hline $\mathrm{ASB}^{-} \quad 9$ & 54226.2 & 104589.2 & 5 & 242.98 & 1.64 & ACCEPT \\
\hline$M S B=53 C$ & 54540.5 & 106456.2 & 11 & 224.53 & -.67 & ACCEPT \\
\hline MSB_53D & 54553.1 & 106448.2 & 5 & 234.46 & -.87 & ACCEPT \\
\hline MSB_86C & 54560.5 & 108500.4 & 15 & 226.17 & .96 & ACCEPT \\
\hline MSB_53B & 54574.3 & 106443.6 & 15 & 223.64 & .31 & ACCEPT \\
\hline $\mathrm{ABW}^{-} 1$ & 55016.4 & 105939.9 & 11 & 226.92 & 1.17 & ACCEPT \\
\hline$M S B \_81 B$ & 55230.4 & 103762.7 & 15 & 222.16 & .64 & ACCEPT \\
\hline$M S B=61 C$ & 55406.6 & 106091.1 & 13 & 224.97 & 1.50 & ACCEPT \\
\hline
\end{tabular}


APPENDIX A

HYDRAULIC HEAD MEASUREMENTS 


\section{APPENDIX B}

CORES AND HYDROSTRATIGRAPHIC DATA 


\section{APPENDIX C}

CORE MUD FRACTION PLOTS 
APPENDIX D

GEOLOGICAL RESIDUALS 


\section{APPENDIX E}

HYDRUALIC HEAD RESIDUALS 